svusvid

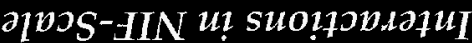
vusvid-lasv 7

иочาทวนdıวน рих

'8uñopoW'squautuadx马 :huquumhs vooN

pautт u 8

suozsolduI

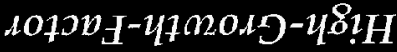

suolsolduI yooN

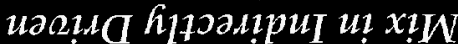
jon $n_{-1}$-laysnd fo stsousp?a

$\operatorname{vaoN}_{\text {uo squamuadx }}$

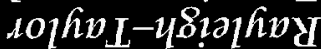

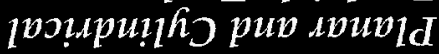

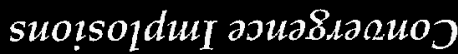

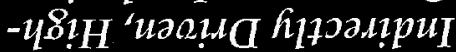
IIN 247 of
$728 . v_{L} u 0 ! 1 ? u \& I$

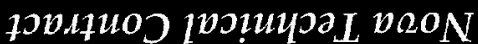
ayt of ио!тапроди

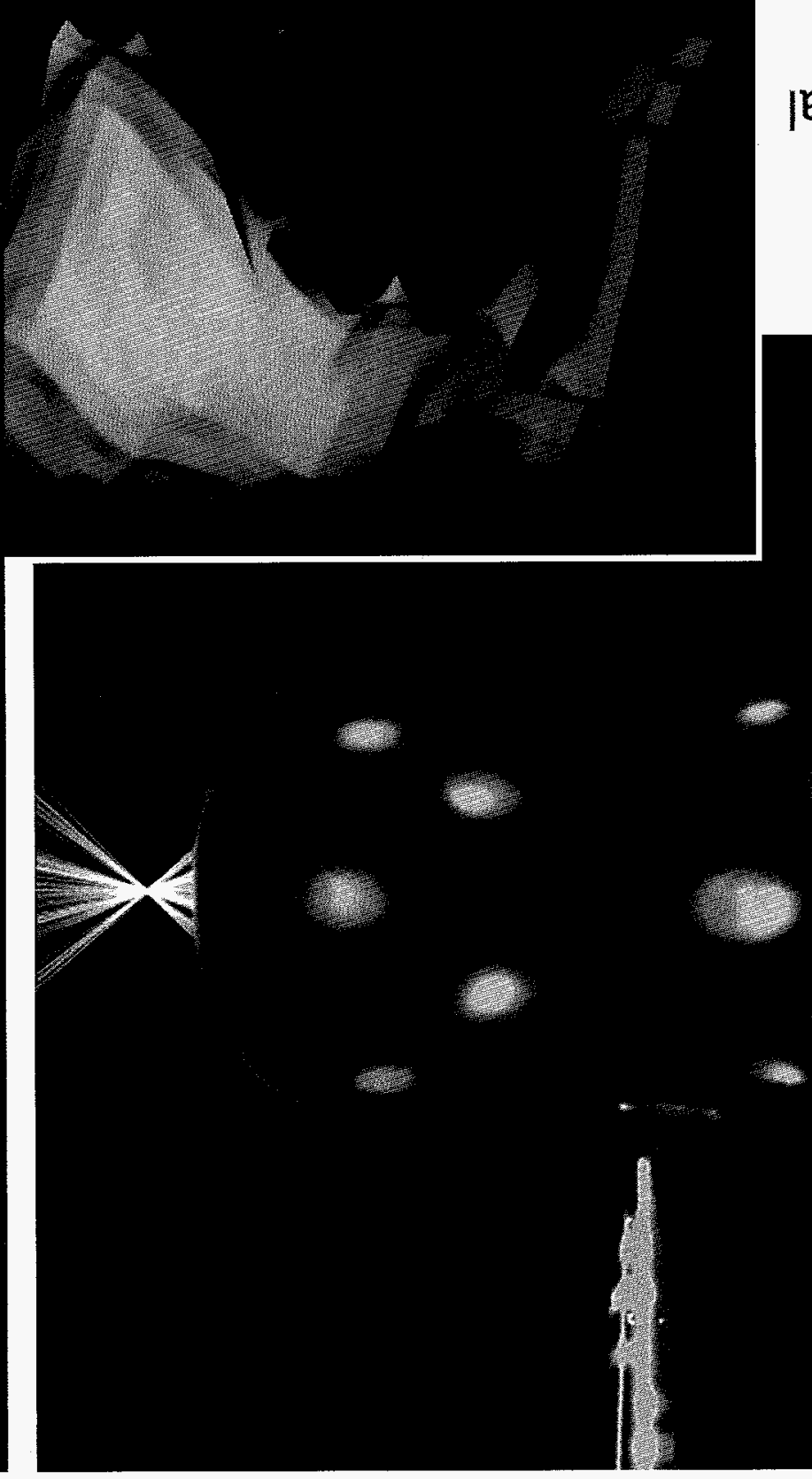

joentuOO

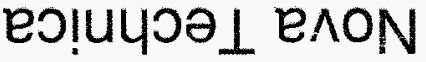
:ənss| ןeloəds

:ənsSl jeiogds 
The ICF Quarterly Report is published four times each fiscal year by the Inertial Confinement Fusion Program at the Lawrence Livermore National Laboratory. The journal reports selected current research within the ICF Program. Major areas of investigation presented here include fusion target theory and design, target fabrication, target experiments, and laser and optical science and technology. Questions and comments relating to the technical content of the journal should be addressed to the ICF Program Office, Lawrence Livermore National Laboratory, P.O. Box 5508, Livermore, CA 94551.

The Cover: The larger image (top) shows a 2.75-mm-long hohlraum as used in experiments on inertial confinement fusion at Lawrence Livermore National Laboratory. To create this image, researchers built the hohlraum wall extra-thin to allow viewing of the radiation (shown here by the spots) and to verify its predicted distribution. In the experiments, laser beams enter through holes on either end of the hohlraum and strike the inside, which produces $x$ rays that cause implosion of the fuel pellet in the hohlraum's center. For details, see "Nova Symmetry: Experiments, Modeling, and Interpretation" on p. 293 of this Quarterly.

The smaller image (bottom) shows an individual bubble from a two-dimensional planar array of singlemode Rayleigh-Taylor (RT) data taken on Nova. Individual bubbles on the array are $70 \mu \mathrm{m}$ apart. The interface described by the data is between low-density, hot ablated plasma and dense, cooler foil material. The work performed to measure the RT instability at the ablation front for $\mathrm{x}$-ray-driven targets is described in "Planar and Cylindrical Rayleigh-Taylor Experiments on Nova" on p. 232 of this Quarterly.

UCRL-LR-105821-95-4 Distribution Category UC-712 July-September 1995

Printed in the United States of America Available from

National Technical Information Service U.S. Department of Commerce 5285 Port Royal Road Springfield, Virginia 22161 Price codes: printed copy A03, microfiche A01.

This document was prepared as an account of work sponsored by an agency of the United States Government. Neither the United States Government nor the University of California nor any of their employees makes any warranty, express or implied, or assumes any legal liability or responsibility for the accuracy, completeness, or usefulness of any information, apparatus, product, or process disclosed, or represents that its use would not infringe privately owned rights.

Reference herein to any specific commercial products, process, or service by trade name, trademark, manufacturer, or otherwise, does not necessarily constitute or imply its endorsement, recommendation, or favoring by the United States Government or the University of

California. The views and opinions of authors expressed herein do not necessarily state or reflect those of the United States Government or the University of California and shall not be used for advertising or product endorsement purposes.

Work performed under the auspices of the U.S. Department of Energy by Lawrence Livermore National Laboratory under Contract W-7405-Eng-48. 


\section{DISCLAIMER}

This report was prepared as an account of work sponsored by an agency of the United States Government. Neither the United States Government nor any agency thereof, nor any of their employees, make any warranty, express or implied, or assumes any legal liability or responsibility for the accuracy, completeness, or usefulness of any information, apparatus, product, or process disclosed, or represents that its use would not infringe privately owned rights. Reference herein to any specific commercial product, process, or service by trade name, trademark, manufacturer, or otherwise does not necescarily constitute or imply its endorsement, recommendation, or favoring by the United States Government or any agency thereof. The views and opinions of authors expressed herein do not necessarily state or reflect those of the United States Government or any agency thereof. 


\section{DISCLAMIER}

Portions of this document may be illegible in electronic image products. Images are produced from the best available original document. 


\section{INERTIAL CONFINEMENT 隹USION Quarterly Report \\ July-September 1995 Volume 5, Number 4}

\section{In this issue:}

\section{Foreword}

Introduction to the Nova Technical Contract

We summarize the accomplishments of the Nova Technical Contract to complete a series of target physics objectives and to concentrate on advanced target design for ignition achieved in collaboration with Los Alamos National Laboratory.

\section{Ignition Target Design for the National Ignition Facility}

We designed a number of targets that we predict to produce ignition and burn for the National Ignition Facility. Each of the targets gives a different trade-off of the physics issues. A point-design target has been modeled in detail and is acceptably robust.

\section{Indirectly Driven, High-Convergence Implosions (HEP1)}

We describe a series of high convergence implosion experiments on Nova that were well characterized by a variety of diagnostics. Fuel-areal-density measurements, performed using a technique based on secondary neutron spectroscopy, demonstrate capsule convergence up to $24: 1$ and imploded fuel density of $19 \mathrm{~g} \mathrm{~cm}^{-3}$.

\section{Planar and Cylindrical Rayleigh-Taylor Experiments on Nova (HEP2)} The Rayleigh-Taylor instability is investigated in planar and cylindrical experiments in indirect drive on Nova. Perturbation growth is measured in ablatively accelerated planar foils with surface perturbations of various wavelengths, amplitudes, and shapes. Cylindrical experiments have been conducted to measure the integral effect of perturbation growth at the ablation front, feedthrough, growth at the inner surface during deceleration, and the initial effects of convergence.

\section{Diagnosis of Pusher-Fuel Mix in Indirectly Driven Nova Implosions (HEP3)}

We report measurements and analysis of controlled implosion performance degradation arising from the Rayleigh-Taylor instability in inertial confinement fusion capsules driven indirectly by the Nova laser. Variations in both capsule $x$ ray and neutron emission are observed, which are consistent with a model that describes mixing pusher material into fuel material.

Scientific Editor Iohin D lind

Editorial Staff Iason Carpenter Al Miguel Peter Murohy Dabbie P. Schieich

Design Staff Daniels. Moore

Art staff Linda I. Wiseman

\section{High-Growth-Factor Implosions (HEP4)}

High-growth-factor, low-entropy implosions approaching the hydrodynamic instability conditions expected of ignition-scale capsules have been designed and fielded at Nova. The level of instability growth, controlled by using pre-roughened capsules, is inferred from neutron yields which are compared with predictions from one-, two-, and three-dimensional simulations.

\section{Energy Coupling in Lined Hohlraums (HLP1, HLP2, and HLP7)}

We summarize the results and analysis of experiments, studying the energetics of laser-heated, lined hohlraums. Using the Nova laser, hohlraums lined with either $\mathrm{CH}$ or $\mathrm{Ni}$ were heated using 1-ns square, 3:1 contrast shaped pulses. X-ray drive, scattered light, and fast-electron production were studied as a function of laser irradiation conditions. We heated the hohlraums to the temperature goals defined in the Nova Technical Contract, but that temperature is lower than obtained on pure Au hohlraums for similar laser-drive pulses. Experiments indicate that the lower drive may be due to increased losses due to scattering light, because of higher levels of laser-plasma instabilities. 
Nova Symmetry: Experiments, Modeling, and Interpretation (HLP3 and HLP4)

Our two-dimensional LASNEX simulations of Nova's nine symmetry scaling data bases reproduce the fundamental features seen in experiments. In particular, we predict how we must change Nova's beam pointing to achieve best symmetry with various pulse shapes. This is the result of bulk-plasma-induced migration of the $x$-ray production region. We have observed and modeled this migration.

Laser-Plasma Interactions in NIF-Scale Plasmas (HLP5 and HLP6)

We have developed plasmas and laser beam smoothing that mimic many of the characteristics of those expected on the National Ignition Facility. Our experiments in such plasmas compare different beam smoothing options and show that scattering of light by laser-plasma instabilities can be reduced to tolerable levels.

\section{Appendix: Nova Technical Contract as presented to the $\mathbf{1 9 9 0}$ NAS Review of ICF \\ The Appendix provides more details of the objectives of the Nova Technical Contract as presented at the 1990 National Academy of Sciences review.}

\section{Facility Report}

Publications 


\section{FOREWORD}

This issue of the ICF Quarterly is dedicated to the design of inertial confinement fusion (ICF) ignition targets for the National Ignition Facility (NIF) and to accomplishments on the issues and goals established by the 1990 National Academy of Science review of ICF for accurately specifying the laser requirements for ignition. This set of issues and goals, called the Nova Technical Contract (NTC), has served as a valuable guide over the past five years for the Nova Target Physics Program. The significant progress on the NIF target designs and the NTC has been enhanced by the close cooperation between researchers at Los Alamos National Laboratory (LANL) and Lawrence Livermore National Laboratory (LLNL).

Overall, the original NTC objectives have been met, and the results are consistent with the requirements of ignition targets. In the Introduction, we summarize those objectives and their motivation in the context of the requirements for ignition. The article "Ignition Target Design for the National Ignition Facility" describes the NIF ignition target designs. Detailed LASNEX design calculations performed at both LLNL and LANL predict that a wide variety of target designs will achieve ignition on the NIF.

Seven articles summarize the principal accomplishments in the various elements of the NTC. The NTC consists of Hydrodynamically Equivalent Physics (HEP) goals and Hohlraum Laser-Plasma Physics (HLP) goals.

The HEP program addresses capsule physics issues associated with ignition. These include hydrodynamic instability in planar and spherical geometries, the effects of drive nonuniformities on capsule performance, and the physics associated with ignition in the absence of alpha-particle deposition. The article "Indirectly Driven, High-Convergence Implosions" describes the results of implosion experiments that used the Large Neutron Scattering Array (LaNSA) detector to measure imploded fuel conditions. These experiments provided an integrated test of symmetry and stability, which approach the requirements of NIF capsules. The article "Planar and Cylindrical Rayleigh-Taylor Experiments on Nova" describes the extensive work performed to look at the growth of hydrodynamic instability on an ablation front driven by x-ray ablation. The article "Diagnosis of Pusher-Fuel Mix in Indirectly Driven Nova Implosions" presents the results of initial experiments designed to demonstrate that $x$-ray spectroscopy could be used to examine the effects of hydrodynamic instability on the imploded fuel conditions in targets with relatively little hydrodynamic instability growth. The final article on the HEP goals, "High-Growth-Factor Implosions," describes the results of hydrodynamic instability on implosion experiments that used $x$-ray preheat shielding to control instability growth, as would be done on NIF targets. These targets have instability growth approaching that of NIF targets. The surface finish in these experiments was varied using laser ablation to provide a specified amplitude and spectral distribution of perturbations on the capsule. The HEP experimental results are consistent with calculations of the control of hydrodynamic instability required for ignition targets.

The HLP program addresses laser-plasma coupling, $x$-ray generation and transport, and the development of energy-efficient hohlraums that provide the appropriate spectral, temporal, and spatial x-ray drive. The article "Energy Coupling in Lined Hohlraums" summarizes the results of x-ray drive measurements and modeling for Nova hohlraums. These results are consistent with the drive calculated for NIF targets. The article "Nova Symmetry: Experiments, Modeling, and Interpretation" shows the results of experiments designed to address the key issue of hohlraum symmetry. Over a wide range of hohlraum types and pulse shapes, the experiments and modeling are consistent with the requirements of ignition targets. The article "Laser-Plasma Interactions in NIF-Scale Plasmas" addresses the issue of laser-plasma coupling in plasmas designed to match the characteristics of NIF hohlraum plasmas. These experiments and theoretical modeling show that with appropriate laser-beam smoothing, stimulated scattering levels can be kept to the few-percent level on NIF targets.

John D. Lindl Scientific Editor 


\title{
Introduction to THE Nova Technical ConTRACT
}

\author{
J. D. Lindl \\ J. D. Kilkenny
}

The 1990 National Academy of Sciences (NAS) final report ${ }^{1}$ recommended proceeding with the construction of a 1- to 2-MJ Nd-doped glass laser designed to achieve ignition in the laboratory (a laser originally called the Nova Upgrade, but now called the National Ignition Facility, or NIF, and envisioned as a national user facility). As a prerequisite, the report recommended completion of a series of target physics objectives on the Nova laser in use at the Lawrence Livermore National Laboratory (LLNL). Meeting these objectives, which were called the Nova Technical Contract (NTC), would demonstrate (the Academy committee believed) that the physics of ignition targets was understood well enough that the laser requirements could be accurately specified. Completion of the NTC objectives was given the highest priority (it was Recommendation 1.1) in the NAS report. The NAS committee also recommended a concentrated effort on advanced target design for ignition. As recommended in the report, completion of these objectives has been the joint responsibility of LLNL and the Los Alamos National Laboratory. Most of the articles in this issue of the ICF Quarterly were written jointly by scientists from both institutions.

Several of the NTC objectives required the completion of improvements to Nova's power balance and pointing accuracy and of new diagnostics and new target fabrication capabilities. These improvements were called "Precision Nova" and are documented in Ref. 2.

The original NTC objectives have been largely met. This Introduction summarizes those objectives and their motivation in the context of the requirements for ignition. The articles that follow describe the NIF ignition target designs and summarize the principal accomplishments in the various elements of the NTC. Reference 3 gives a much more extensive discussion of ignition requirements.

\section{Ignition Requirements}

The strong connection between the compression achievable in a spherical implosion and the ignition threshold was pointed out by Nuckolls et al. ${ }^{4}$ in 1972. Because the compression that can be achieved in an implosion is related to the implosion velocity $v_{\text {imp }}$, the ignition threshold depends strongly on $v_{\text {imp }}$. If a laser pulse shape can be achieved that maintains compressibility independent of $v_{\text {imp }}$, the ignition threshold varies $^{3,5}$ as $v_{\text {imp }}{ }^{-n}$, where $n \approx 5$ for the target type shown in Fig. 1.

The implosion of a shell such as that shown in Fig. 1 is driven by the ablation of material from the surface of the shell and can be described by a spherical rocket equation. The work $W$ done on the imploding shell is given by $W=\int P d V$, where $P$ is the pressure generated by ablation and $V$ is the volume enclosed by the shell. For a given shell mass, the implosion velocity is maximized by generating the highest possible ablation pressure on a shell that encloses the greatest possible volume.

The ablation pressure is related to the energy flux incident on the surface of the shell. In laser-driven inertial confinement fusion (ICF), laser-plasma interaction effects limit the incident flux to $\sim 10^{15} \mathrm{~W} / \mathrm{cm}^{2}$. In ion beam-driven ICF, the pressure is limited by the focused intensity achievable. In general, ablation pressures are limited to about 100 Mbar.

As the volume enclosed by a shell with fixed mass and density (and thus with a fixed volume of shell material) is increased, the shell must become thinner. Hydrodynamic instabilities during the acceleration and deceleration phases of the implosion limit the so-called in-flight aspect ratio to $R / \Delta R<25-35$, where $R=$ shell radius and $\Delta R=$ shell thickness as it implodes. For thin shells, the shell aspect ratio increases linearly with the 
volume enclosed. This limitation on shell aspect ratio, and the pressure limitation described above, together limit implosion velocities. If driver technology can be developed so that other details of an implosion, such as pulse shaping and pulse symmetry, can be controlled, these two limitations ultimately set the ignition threshold for laboratory fusion to a driver of about 1 to $2 \mathrm{MJ}$ for capsules with implosion velocities of 3 to $4 \times 10^{7} \mathrm{~cm} / \mathrm{s}$.

As shown in Fig. 2, two principal approaches are used with lasers to generate the energy flux required to drive the implosion. In the direct-drive approach, the laser beams are aimed directly at the target. The beam energy is absorbed by electrons in the target's outer, low-density corona, and they transport that energy to the denser shell material to drive the ablation and the resulting implosion. In the indirect-drive approach, the laser energy is absorbed and converted to $x$ rays by high- $Z$ material inside the hohlraum that surrounds the target. The NTC has concentrated on indirect drive.

Because of the $x$-ray conversion and transport step, indirect drive is less efficient than direct drive. However, ablation driven by electron conduction is in general more hydrodynamically unstable than ablation driven by $x$ rays. $^{3}$ (Indirect drive is less sensitive to hydrodynamic instability because $x$ rays generate a higher ablation rate than electrons.) Measures taken to mitigate hydrodynamic instability in direct-drive targets ${ }^{3,6}$ largely offset the efficiency advantage. Also, direct-drive targets are very sensitive to intensity variations within individual beams. These variations imprint perturbations on the target that are then amplified by hydrodynamic instability. If adequate beam uniformity can be achieved, calculations for current target designs ${ }^{6}$ indicate that direct-drive targets have about the same ignition threshold as indirect-drive targets, but that they can have about a factor of 2 higher gain. The NIF will be configured with a beam geometry capable of being used for either direct or indirect drive. ${ }^{3}$ Beam smoothing and hydrodynamic instability requirements for direct drive will be determined in an implosion physics program on the Omega Upgrade laser at the University of Rochester and in planar experiments on Nova and on the Nike laser at the Naval Research Laboratory.

Although indirect drive is less sensitive to individual beam nonuniformities than direct drive, beam placement inside the hohlraum must be accurately controlled to achieve adequate symmetry. As indicated in Fig. 1, typical capsule convergence ratios are $C_{\mathrm{r}}=R_{\mathrm{A}} / r_{\mathrm{hs}} \approx 25-35$, where $R_{\mathrm{A}}$ is the initial outer capsule radius and $r_{\mathrm{hs}}$ is the final compressed hot fuel radius (the "hot spot" radius). Achieving a convergence ratio this high requires $x$-ray fluxes uniform to 1 to $2 \%$. Use of a relatively large hohlraum (with a ratio of hohlraum radius to capsule radius of 3-4) greatly reduces imbalances in irradiation between points close together on the capsule surface; $;, 7$ imbalances between points farther apart can be controlled by hohlraum geometry and laser beam placement. In the NIF laser, two rings of beams, each with an independent pulse shape, will enter each end of the hohlraum. (In Nova, a single ring

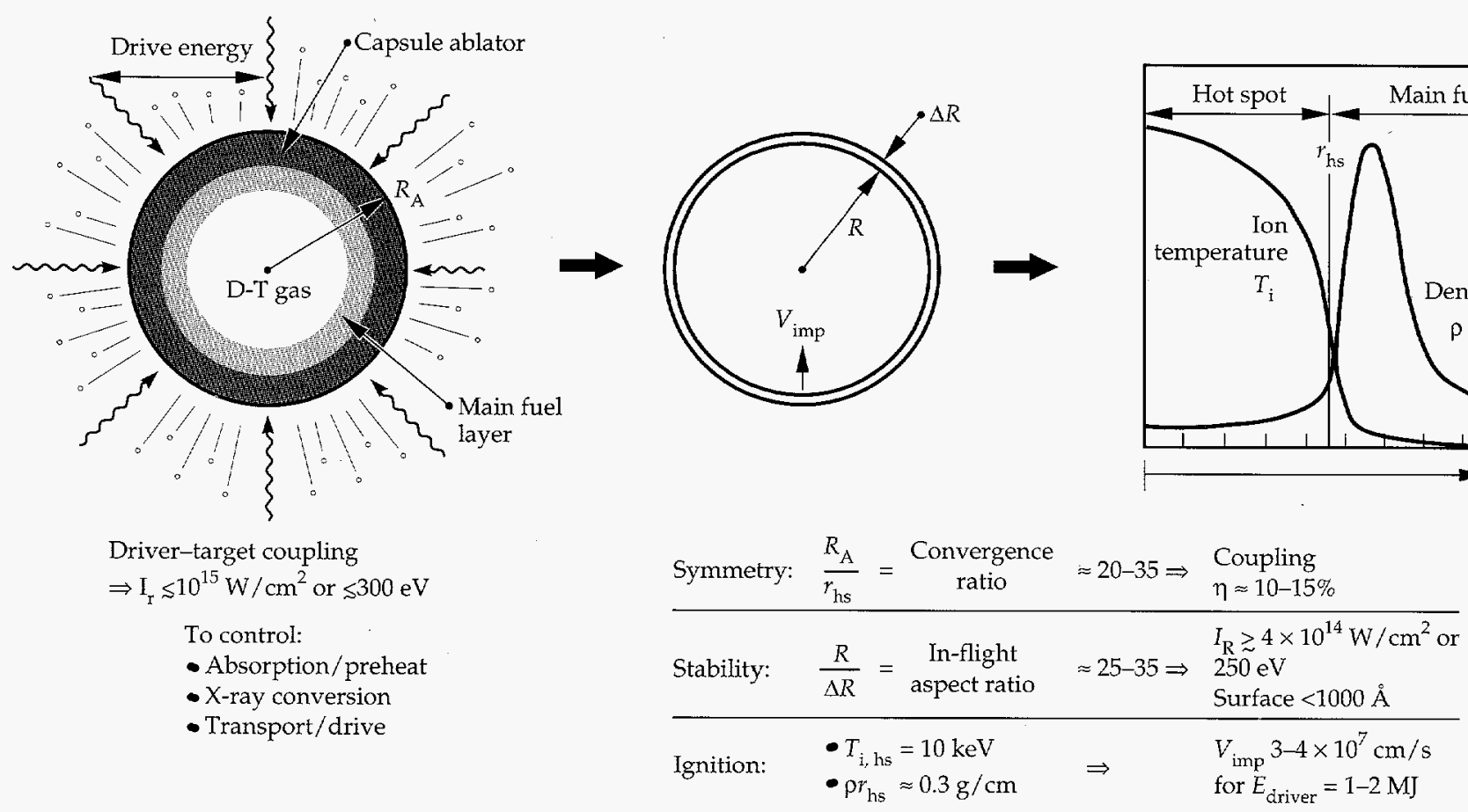

FIGURE 1. Physics specifications on current ICF ignition targets include constraints on drive intensity, symmetry, stability, and ignition. (02-08-0692-1865Cpb02) 
of five beams enters each end.) The two rings will allow "beam phasing," in which the power in the individual rings is varied independently to control time-dependent asymmetry. For a short-wavelength laser such as Nova (or NIF) (laser wavelength $\lambda=0.35 \mu \mathrm{m}$ in most experiments), about $70 \%$ of the incident laser energy is converted to $x$ rays by the high $Z$ hohlraum material, but symmetry requirements limit overall coupling efficiency to 10 to $15 \%$ of the laser energy into the capsule for typical ignition designs.

Since the hohlraum wall physics and the capsule physics are essentially the same for any $x$-ray source, much of what is learned on Nova is also applicable to ion-beam drivers. Because of this, Sandia National Laboratories has been able to carry out experiments on Nova to test pulse-shaping schemes important in lightion-driven targets. Indirect-drive laser experiments also provide-much of the target physics basis for the Heavy Ion Driver Program, supported by the DOE Office of Fusion Energy.

The laser requirements for ignition by indirect drive can be shown in a plot of laser power $P_{\mathrm{L}}$ vs laser energy (Fig. 3). As laser power increases for a given laser energy, the achievable hohlraum temperature $T_{R}$ increases. The ablation pressure increases approximately ${ }^{3}$ as $T_{R}^{3.5}$, so $v_{i m p}$ is a strong function of $T_{R}$. Generation of plasma in the hohlraum increases as $T_{R}$ increases; this results in laser-plasma collective effects that limit $T_{R}$ and the usable power that can be put into the hohlraum. This power depends on laser wavelength, laser beam spatial and temporal uniformity, pulse duration, hohlraum size, and other variables. At $T_{\mathrm{R}}=400 \mathrm{eV}$, for the long pulses required for ignition capsules, the hohlraum plasma density $n$ will approach $n / n_{c} \approx 1 / 4$ (the critical density $n_{\mathrm{c}}=10^{21} \lambda^{-2} \mathrm{~cm}^{-3}$, where $\lambda$ is the

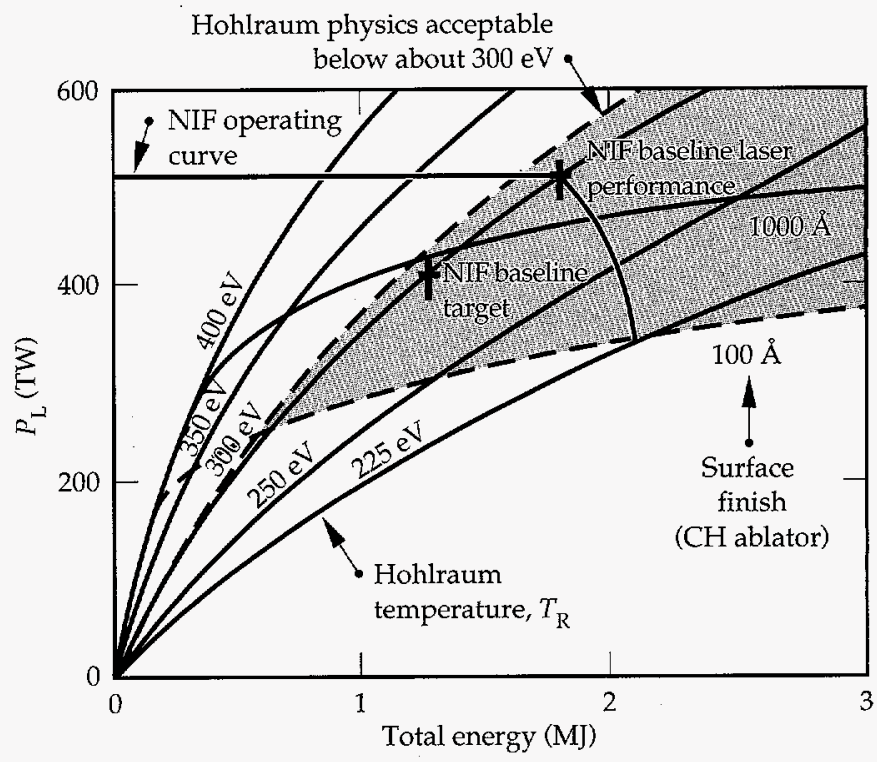

FIGURE 3. For laser-driven indirect-drive ignition targets, plasma physics issues constrain the achievable hohlraum temperature, and hydrodynamic instabilities (represented here by surface finish) establish the minimum required temperature at a given driver energy. The shaded region constitutes the accessible region in power-energy space where ignition with indirect-drive capsules is predicted. The NIF power-energy operating curve shown here has ample margin. (08-00-0693-2196Dpb01) (a) Direct (electron) drive

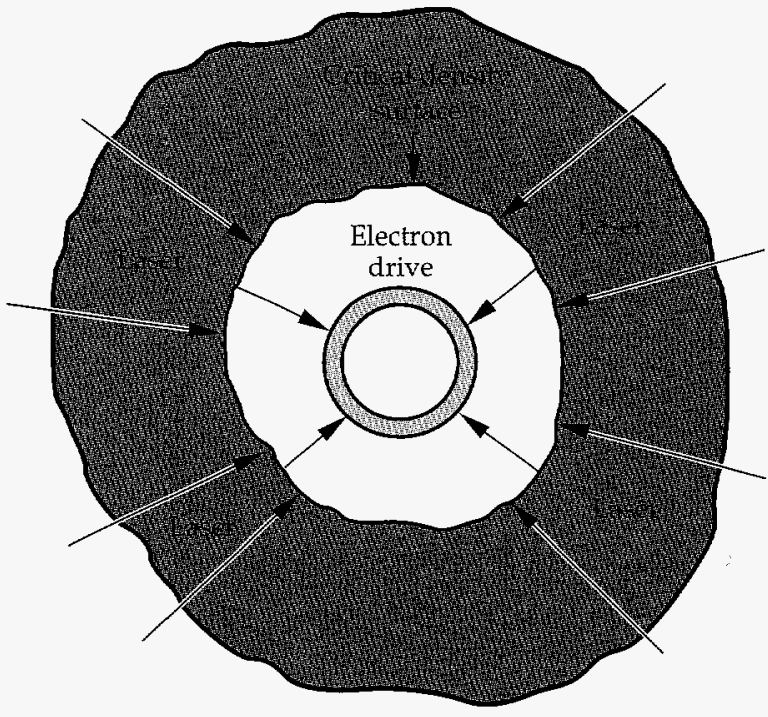

Advantage:

- Higher coupling efficiency (b) Indirect (x-ray) drive

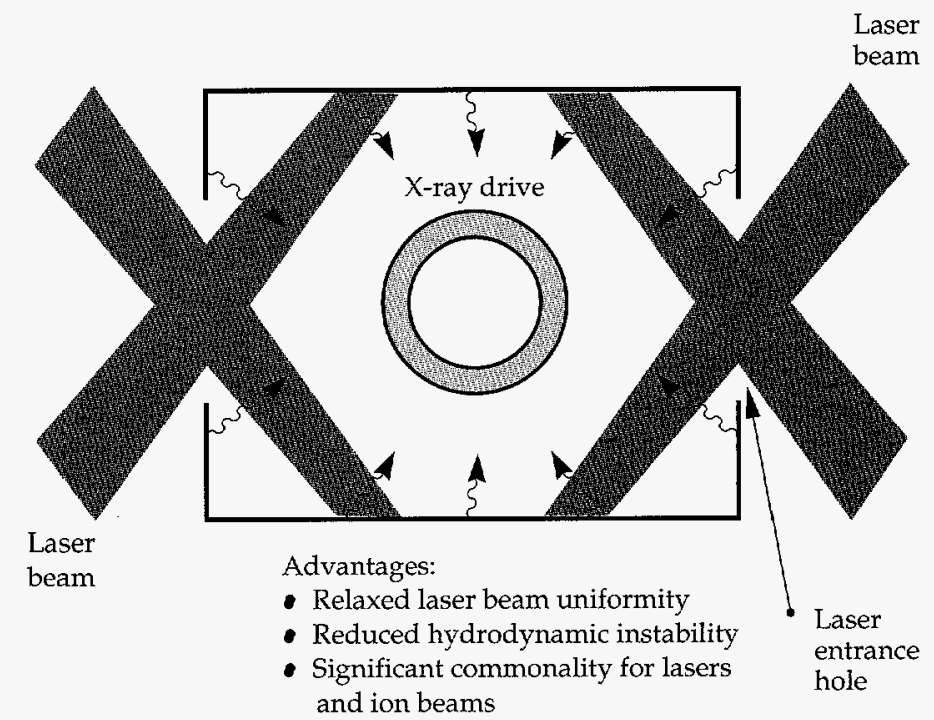

Laser beam

FIGURE 2. ICF uses either (a) electron conduction (direct drive) or (b) $x$ rays (indirect drive) to produce a high shell ablation pressure to drive an implosion. (08-00-0894-3312Apb01) 
laser wavelength in micrometers). Experiments ${ }^{3}$ at $\lambda=1 \mu \mathrm{m}$ suggest that this is an upper-limit temperature for ignition hohlraums. (It should be possible to achieve higher temperatures for short pulses with reduced plasma filling.) We limit peak hohlraum temperatures in current ignition target designs to $T_{R} \approx 300$ $\mathrm{eV}$, which limits plasma densities to $n / n_{c} \approx 0.1$. Above this temperature, it is likely that a significant fraction of laser light would be scattered out of the hohlraum or be absorbed in a way that results in the production of high-energy electrons that heat the fusion fuel and thereby reduce the achievable compression.

At a given driver energy, hydrodynamic instabilities place a lower limit on the temperature required to drive a capsule to ignition conditions. A larger capsule requires a lower implosion velocity, which can be achieved with a lower radiation temperature consistent with the shell aspect ratio constraints. The value of the required minimum temperature at a given energy will depend on the allowed shell aspect ratio, which depends on the smoothness of the capsule surface, currently limited to 200 to $300 \AA \mathrm{rms}$ for Nova capsules. Below a certain size, the required implosion velocity will exceed the velocity achievable within the temperature and capsule uniformity constraints, and ignition is not possible. Above this threshold energy, there is a region in power-energy space where ignition is feasible. This is the shaded area in Fig. 3, which encloses the region limited by $300-\mathrm{eV}$ hohlraum temperatures and $100-\AA$ capsule surface finishes.

Hohlraum Laser-Plasma Physics (HLP):

- Absorption

- Laser-plasma instabilities

- X-ray conversion

- Radiation transport/wall loss

- Radiation uniformity control

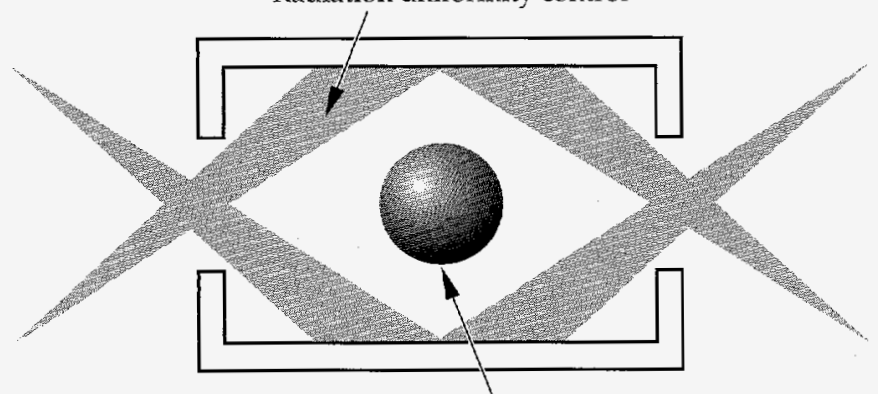

Hydrodynamically Equivalent Physics (HEP):

- Hydrodynamic stability

- Effects of drive asymmetry

- Ignition physics

FIGURE 4. The Nova program comprises two elements that address the hohlraum and capsule physics of ignition and high gain: hydrodynamically equivalent physics (HEP) and hohlraum laser-plasma physics (HLP). (05-00-0995-2136pb01)
The NIF laser is being designed to operate at an energy of $1.8 \mathrm{MJ}$ and a power of $500 \mathrm{TW}$; both values are about a factor of 2 above the threshold ignition values shown in Fig. 3, allowing for remaining uncertainty in the implosion process. Within the ignition region shown in Fig. 3, a wide variety of targets have been evaluated, as discussed in the article "Ignition Target Design for the National Ignition Facility," p. 215. The predicted yields of these targets range from about 1 to greater than $25 \mathrm{MJ}$.

\section{Elements of the Nova Technical Contract}

The objectives of the NTC have been to experimentally demonstrate and predictively model laser-plasma interaction, hohlraum characteristics, and capsule performance in targets that have been scaled in key physics variables from NIF targets. Since the Nova geometry consists of a single ring of five beams in each end of the hohlraum, Nova experiments have been limited to controlling only the time-averaged symmetry. To address the hohlraum and hydrodynamic constraints on indirect-drive ignition, the NTC comprises so-called Hydrodynamically Equivalent Physics (HEP) goals and Hohlraum Laser-Plasma Physics (HLP) goals, as shown in Fig. 4.

The HEP program addresses capsule physics issues associated with ignition. These include hydrodynamic instability in planar and spherical geometries, the effects of drive nonuniformity on capsule performance, and the physics associated with ignition (energy gain and energy loss to the fuel) in the absence of $\alpha$-particle deposition. The HEP program was subdivided into five subgoals:

- HEP1: Demonstrate fuel densities of 20 to $40 \mathrm{~g} / \mathrm{cm}^{3}$ using high-contrast pulse shaping with noncryogenic targets. The fuel density will be inferred from measurements of fuel areal density $\rho R$ using advanced neutron-based diagnostics.

- HEP2: Measure the reduced linear growth and early nonlinear behavior of the Rayleigh-Taylor (RT) instability at the ablation surface for $x$-ray-driven targets. Using planar targets, observe single-mode growth at the ablation surface by factors of $>30$, from which reductions by factors of 2 to 3 from the classical RT growth rate are inferred. Targets of various compositions will be used to confirm the modeling of plasma opacity as it affects $x$-raydriven hydrodynamics.

- HEP3: Using x-ray spectroscopy, demonstrate pusher/fuel mixing that is dependent on initial target surface quality. The targets will be low-growth (perturbations grow by a factor of $\sim 10$ ), low-convergence $\left(C_{r}<10\right)$ plastic capsules with a multimode spectrum of initial surface perturbations. 
- HEP4: Demonstrate quantitative understanding of implosion experiments to convergence ratios $C_{r} \approx 10$ with overall hydrodynamic instability growth factors of 100 to 500 for an $l$-mode spectrum similar to those characteristic of ignition target designs (for which maximum growth occurs for mode numbers $l \approx 30$ ).

- HEP5: Extend HEP4 experiments to convergence ratios $C_{r}=20-40$ with capsule performance consistent with Nova's symmetry limitations.

The HLP program addresses laser-plasma coupling, $x$-ray generation and transport, and the development of energy-efficient hohlraums that provide the appropriate spectral, temporal, and spatial $x$-ray drive. The HLP program was divided into seven subgoals:

- HLP1: Demonstrate acceptable coupling of laser light to $x$ rays in low-Z-lined hohlraums using shaped laser pulses and peak radiation temperatures up to $T_{\mathrm{R}} \approx 210 \mathrm{eV}$.

- HLP2: Demonstrate acceptable coupling of laser light to $x$ rays in lined hohlraums with peak radiation temperature $T_{R} \geq 270 \mathrm{eV}$ with 1-ns square pulses. Acceptable coupling for HLP1 and 2 was defined as follows:

- Absorption fraction $f_{\text {abs }}>90 \%$.

- Stimulated Brillouin scattering fraction $\mathrm{f}_{\mathrm{SBS}}<5-10 \%$.

- Suprathermal-electron fraction $f_{\text {hot }}<5 \%$ at $T_{\text {hot }} \geq 50 \mathrm{keV}$.

- Stimulated Raman scattering fraction $f_{\text {SRS }}<5 \%$.

- HLP3: Demonstrate an ability to measure and calculate energy balance in a hohlraum with emphasis on wall loss and albedo and an ability to diagnose and predict the (time-dependent) position of the laserproduced x-ray source within the hohlraum. Demonstrate an ability to characterize and model plasma evolution in a hohlraum.

- HLP4: Demonstrate symmetry control with low- and intermediate-Z-lined hohlraums. Achieve low-order $l$-mode $\left(P_{2}, P_{4}\right)$ time-integrated symmetry $\leq 2-4 \%$.

- HLP5: Demonstrate acceptable levels of scattering in large-scale plasmas that match the plasma conditions, beam geometry, and beam smoothing of ignition hohlraums as closely as possible. The plasmas should have density and velocity scalelengths $\sim 2 \mathrm{~mm}$, electron temperature $>1.5 \mathrm{keV}$, and $n / n_{\mathrm{c}}<0.15$.

Acceptable levels of scattering were defined as follows:

- Stimulated Brillouin scattering fraction $f_{\text {SBS }}$ (back, side) $<5-10 \%$.

- Stimulated Raman scattering fractions $f_{\mathrm{SRS}}$ (back, side) $<5-10 \%$ and $f_{\text {SRS }}$ (forward) $<5 \%$.

- HLP6: Evaluate the impact of laser beam filamentation on SBS and SRS and develop control techniques to the extent necessary to ensure acceptable levels of scattering.
- HLP7: Develop an improved understanding of x-ray conversion efficiency in hohlraums under conditions appropriate for NIF ignition targets.

Table 1 shows where each NTC element is discussed in detail in this Quarterly. The degree to which convergence ratio can be increased on Nova for HEP5 is yet to be determined. The appendix gives a more detailed statement of the objectives of the NTC as presented at the 1990 NAS Review. Because of the declassification of many aspects of ICF in 1993, the appendix includes many details, such as specific hohlraum temperature goals, left out of the unclassified summary contained in the NAS report. These goals have served as a very valuable guide to the Nova Target Physics Program for the past five years, and have largely been carried out as specified.

\section{Changes to the NTC Objectives and Plans for Further Work on Nova}

One notable change to the Nova targets has been made as we have learned from the results of more detailed NIF target designs. Ignition-scale hohlraums require some sort of a low- $Z$ fill to control the position of laser beam absorption and $x$-ray emission. At the time of the NAS report, ignition targets used low-Z liners on the inside of the hohlraum wall to create this plasma. These "lined hohlraum" targets, spelled out in the NTC, worked well in the Nova experiments

TABLE 1. List of NTC elements in this Quarterly.

\begin{tabular}{|c|c|c|}
\hline $\begin{array}{l}\text { Subgoal } \\
\text { discussion }\end{array}$ & $\begin{array}{l}\text { Article } \\
\text { title }\end{array}$ & $\begin{array}{l}\text { Page } \\
\text { no. }\end{array}$ \\
\hline \multicolumn{3}{|l|}{ HEP: } \\
\hline - HEP1 & $\begin{array}{l}\text { Indirectly Driven, High-Convergence } \\
\text { Implosions }\end{array}$ & 226 \\
\hline - HEP2 & $\begin{array}{l}\text { Planar and Cylindrical Rayleigh- } \\
\text { Taylor.Experiments on Nova }\end{array}$ & 232 \\
\hline - HEP3 & $\begin{array}{l}\text { Diagnosis of Pusher-Fuel Mix in } \\
\text { Indirectly Driven Nova Implosions }\end{array}$ & 265 \\
\hline - HEP4 & High-Growth-Factor Implosions & 271 \\
\hline \multicolumn{3}{|l|}{ HLP: } \\
\hline $\begin{array}{l}\text { - HLP1, HLP2, } \\
\text { and HLP7 }\end{array}$ & Energy Coupling in Lined Hohlraums & 281 \\
\hline - HLP3 and HLP4 & $\begin{array}{l}\text { Nova Symmetry: Experiments, } \\
\text { Modeling, and Interpretation }\end{array}$ & 293 \\
\hline - HLP5 and HLP6 & $\begin{array}{l}\text { Laser-Plasma Interactions } \\
\text { in NIF-Scale Plasmas }\end{array}$ & 305 \\
\hline Appendix & $\begin{array}{l}\text { Nova Technical Contract as presented } \\
\text { to the } 1990 \text { NAS Review of ICF }\end{array}$ & $A-1$ \\
\hline
\end{tabular}


described below. But detailed NIF target calculations predicted a significant asymmetric pressure pulse on the capsule when the liner plasma collapsed onto the hohlraum axis. Although this pulse may have been an artifact of calculations that are currently constrained to be axisymmetric, the baseline NIF target design has been switched from a liner to a low- $Z$ gas fill. Symmetry control with gas-filled targets has been demonstrated on Nova, but the experiments are still in progress and will not be described here.

Continuing work aimed at gaining further understanding of ignition targets is covered under a set of goals called the Target Physics Contract, which are extensions of goals in the NTC. Most of these goals should be reached within about a year.

HEP2 has been extended to include direct measurement of perturbation growth in spherical and/or cylindrical geometry and demonstration of a quantitative understanding of the three-dimensional (3-D) evolution of the RT instability. This objective has included the development of 3-D radiation hydrodynamics codes, which are now used in the planning and evaluation of experiments.

HEP4 and HEP5 are continuing, with emphasis on 3-D calculations of the effects of capsule surface and hohlraum flux perturbations on capsule performance. Target designs that minimize the flux asymmetry are being evaluated to determine the limits to the achievable convergence ratio on Nova.

The goals of the HLP program, including energetics, $x$-ray emission pattern and hohlraum-wall blowoff, and symmetry have been extended to include gas-filled hohlraums. HLP5 and HLP6 will continue to explore the limits to hohlraums imposed by laser-plasma interaction with various forms of laser-beam coherence control.

\section{Notes and References}

1. National Academy of Sciences Review of the Department of Energy's Inertial Confinement Fusion Program, Final Report (National Academy Press, Washington, DC, 1990)

2. H. T. Powell and D. L. Correll, ICF Quarterly Report 4(1), Lawrence Livermore National Laboratory, Livermore, CA, UCRL-LR-105821-94-1 (1994).

3. J. D. Lindl, Phys. Plasmas 2 (11), 3933-4024 (1995).

4. J. H. Nuckolls, L. Wood, A. Thiessen, and G. B. Zimmerman, Nature 239, 139 (1972).

5. W. K. Levedahl and J. D. Lindl, Energy Scaling of ICF Targets for Ignition and High Gain, Lawrence Livermore National Laboratory, Livermore, CA, X-Division Memorandum CLY-95-004 (1990).

6. C. Verdon, Bull. Am. Phys. Soc. II 38 (10), 2010 (1993).

7. S. W. Haan, Radiation Transport Between Concentric Spheres, Lawrence Livermore National Laboratory, Livermore, CA, X-Division Memorandum COPD 83-64 (1983). 


\section{IgNition TARget Design for the NATIONAL IGNITION FACILITY}

\begin{tabular}{|c|c|c|c|}
\hline S. W. Haan & P.A. Amendt & G. L. Strobel & D. B. Harris ${ }^{*}$ \\
\hline S. M. Pollaine & J.A. Futterman & M. Tabak & N. M. Hoffman \\
\hline J. D. Lindl & W. K. Levedahl & S. V. Weber & B. H. Wilde* \\
\hline L.J. Suter & M.D. Rosen & G. B. Zimmerman & W. S. Varnum ${ }^{*}$ \\
\hline R. L. Berger & D. P. Rowley & W. J. Krauser* & F.J. Swenson ${ }^{*}$ \\
\hline L. V. Powers & R. A. Sacks & D. C. Wilson* & P. A. Bradley* \\
\hline W. E. Alley & A. I. Shestakov & S. Coggeshall* & \\
\hline
\end{tabular}

\section{Introduction}

The goal ${ }^{1,2}$ of inertial confinement fusion (ICF) is to produce significant thermonuclear burn from a target driven with a laser or ion beam. To achieve that goal, the national ICF Program has proposed a laser capable of producing ignition and intermediate gain. ${ }^{3}$ The facility is called the National Ignition Facility (NIF). This article describes ignition targets designed for the $\mathrm{NIF}^{4}$ and their modeling. Although the baseline NIF target design, described herein, is indirect drive, the facility will also be capable of doing direct-drive ignition targets - currently being developed at the University of Rochester. ${ }^{5}$

Figure 1 illustrates the baseline target, which is typical of all our ignition targets. A spherical cryogenic capsule, composed of deuterium-tritium (DT) gas, DT solid fuel, and an ablator, is encased in a cylindrical Au hohlraum with two laser entrance holes (LEHs) at opposite ends. The hohlraum peak radiation temperature $\left(T_{R}\right)$ is $250-300 \mathrm{eV}$, with a shaped prepulse for a low-entropy implosion. The ablation pressure allows the fuel shell to reach a velocity of $3-4 \times 10^{7} \mathrm{~cm} / \mathrm{s}$. The central part of the DT is then compressed and heated, forming a hot spot that reaches ignition conditions of density times radius $\rho r \sim 0.3 \mathrm{~g} / \mathrm{cm}^{2}$ and ion temperature $\sim 10 \mathrm{keV}$. Then, $\alpha$ deposition "bootstraps" the central temperature to $>30 \mathrm{keV}$. The hot-spot density at ignition is typically $75-100 \mathrm{~g} / \mathrm{cm}^{3}$. The hot spot is tamped by a colder main fuel layer, with $\rho \Delta r \approx 1 \mathrm{~g} / \mathrm{cm}^{2}$ and density $\approx 1000 \mathrm{~g} / \mathrm{cm}^{3}$. The burn propagates into the main fuel layer, and $10-15 \%$ of the total DT mass is

*Los Alamos National Laboratory, Los Alamos, New Mexico

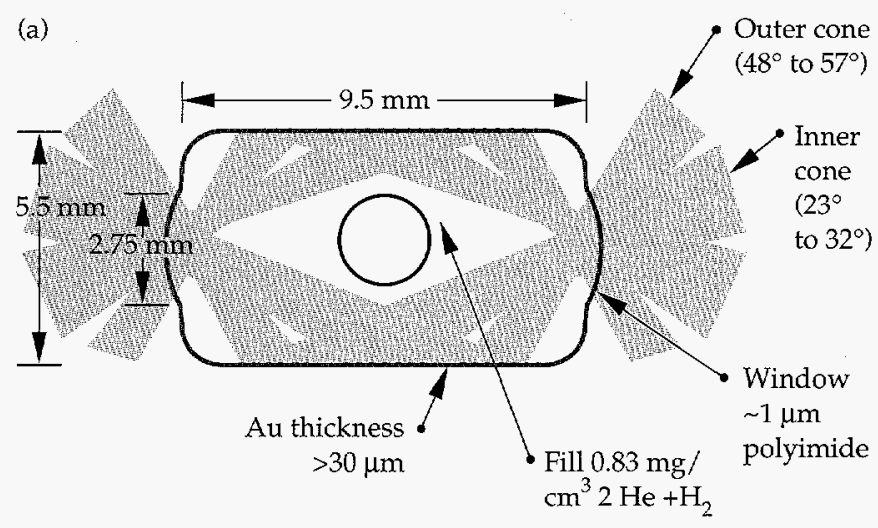

(b)

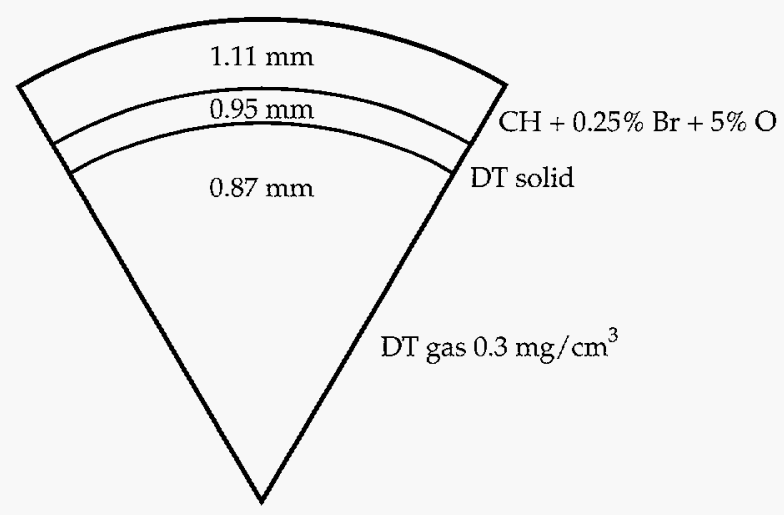

FIGURE 1. This ignition target (referred to as the point-design target or PT) uses $1.35 \mathrm{MJ}$ of laser energy. (a) Shows the entire target, and (b) shows details of the central spherical capsule. The DT fuel is in a cryogenic layer, surrounded by a CH ablator doped with $0.25 \% \mathrm{Br}$. The capsule is in the center of a Au hohIraum, which the incoming laser beams heat to $300 \mathrm{eV}$. The beams are arranged in two cones entering from opposite sides. (50-05-0494-1802 pb01) 
burned. The target shown in Fig. 1 produces $10-15 \mathrm{MJ}$ of yield in simulations, depending on the modeling used.

Assuming the laser meets other specifications, the two most important laser parameters determining the margin for ignition are the total energy and the peak power. ${ }^{6}$ Ignition requires both energy and power, as indicated in Fig. 2. The ignition region is bounded on one side by hydrodynamic instabilities. Ultimately, this boundary of the ignition region is determined by the capsule surface smoothness; Fig. 2 assumes the surface finish currently achieved on Nova capsules in modeling described in this article. On the other side, the ignition region is bounded by laser-plasma instabilities. Laser intensity and other parameters determining the instabilities (especially the electron density $n_{\mathrm{e}}$ ) depend primarily on the desired peak hohlraum $T_{R}$. Estimates of the laser-plasma instabilities, described here, indicate that laser-plasma instabilities will be acceptable in targets driven to at least $320 \mathrm{eV}$ (shown as the upper boundary of the ignition region in Fig. 2).

The smallest possible ignition target with this assumed surface finish, at $0.8 \mathrm{MJ}$ and $300 \mathrm{TW}$, would have no remaining margin for uncertainties or errors in the target modeling. We have specified the NIF at $1.8 \mathrm{MJ}$ and 500 TW to provide margin for such uncertainties. This margin is adequate to cover our estimates of energetically significant uncertainties, as described in this article.

\section{Baseline Ignition Targets}

Figure 1 shows the baseline design, referred to as the PT: "point-design target." Cryogenic hardware, not shown, is external to the hohlraum. The spherical capsule is a doped $\mathrm{CH}$ ablator around a shell of solid cryogenic DT. The solid DT layer is self-smoothing, because of the $\beta$-smoothing effect. ${ }^{7}$ The cryogenic temperature controls the density of the central DT gas. The hohlraum is filled with a 50-50 (atomic) mixture of $\mathrm{He}$ and $\mathrm{H}$. This gas conducts away the $\beta$ decay energy before the target is shot and maintains the open hohlraum cavity during the implosion. The mixture of gases minimizes stimulated Brillouin scattering (SBS).

The PT uses 1.35 MJ of $3 \omega$ light, which is intermediate between the full 1.8 MJ and the "ignition cliff" at $800 \mathrm{~kJ}$. Most of our modeling concentrates on this intermediate-scale target. It is sufficiently robust that we can make a good case for its ignition, while it leaves margin for uncertainty with a 1.8-MJ facility. Also, by using a relatively small target to set specifications for power balance, pointing, target fabrication quality, and so forth, we can be sure that the specifications are adequate for a range of likely targets.

Figure 3 shows an optimal $T_{\mathrm{R}}$ profile for the capsule, used as input to our capsule modeling, and an input laser profile. The target can tolerate moderate deviations from the nominal profile. For example, Fig. 4 shows the yield from integrated calculations (described later in more detail) as the duration of the peak power portion of the pulse is varied. Our robustness study of the PT is described in more detail below.

The light entering each LEH is in two cones, as shown in Fig. 1, and we can minimize time-dependent asymmetry in the $x$ radiation incident on the capsule by dynamically varying the relative power of the cones. About one third of the energy must go into the waist

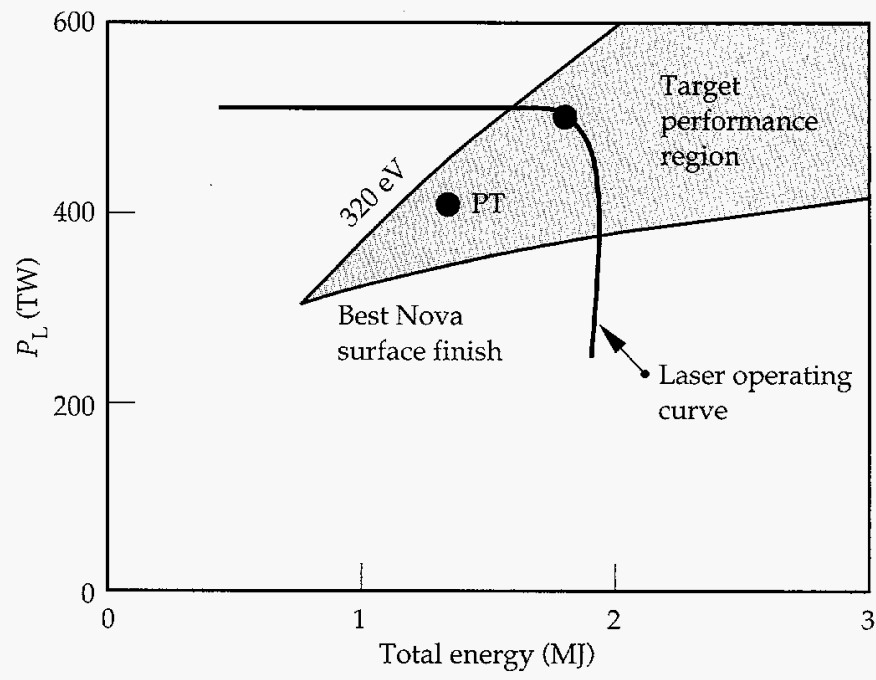

FIGURE 2. Total laser energy and peak power determine the margin for ignition. Powers and energies along the indicated curve will be accessible to the NIF, as currently planned. The upper dot illustrates the laser's nominal operating point (1.8 MJ, 500 TW); the lower dot illustrates the energy and power needed to drive the PT (1.35 MJ, 410 TW). (50-05-0494-1805pb01)

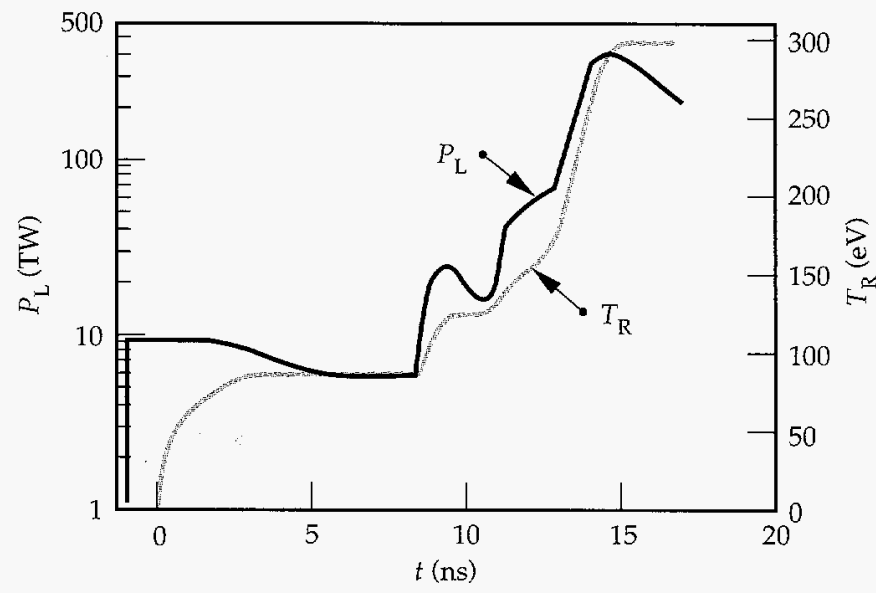

FIGURE 3. Temperature vs time optimal for the PT capsule, and laser power vs time to drive the target. The shaped pulse prior to peak drive is needed to compress the target, increasing the pressure in a controlled way before applying peak power. The gray curve is the radiation temperature vs time that drives capsule simulations, and the black curve is the laser power vs time used as input to hohlraum simulations. (50-05-0295-0395pb01) 
cones. The 192 beams are clustered in groups of four, so that there are effectively 48 spots. These are divided as 8 spots in each of the inner cones and 16 in the outer cones. We may use slightly separate wavelengths in

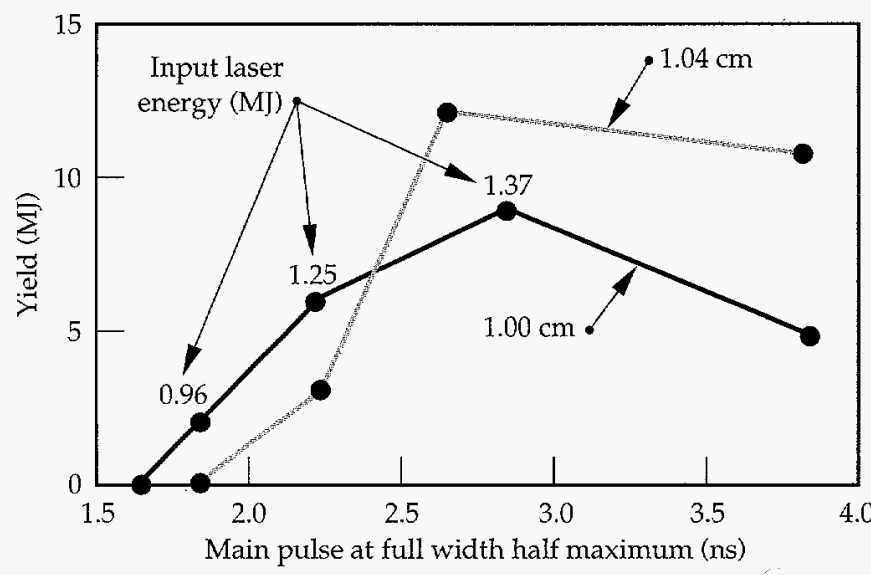

FIGURE 4. Yield vs duration of the peak power pulse, from integrated 2-D simulations. The pulse width changes by varying the cut-off time. The energy in a sample of the pulses is shown. The failure at short pulse appears to be due to asymmetry, not energetic failure to ignite. Two different hohlraum lengths, providing different symmetry "tunes," are shown. (50-05-0295-0388pb01)

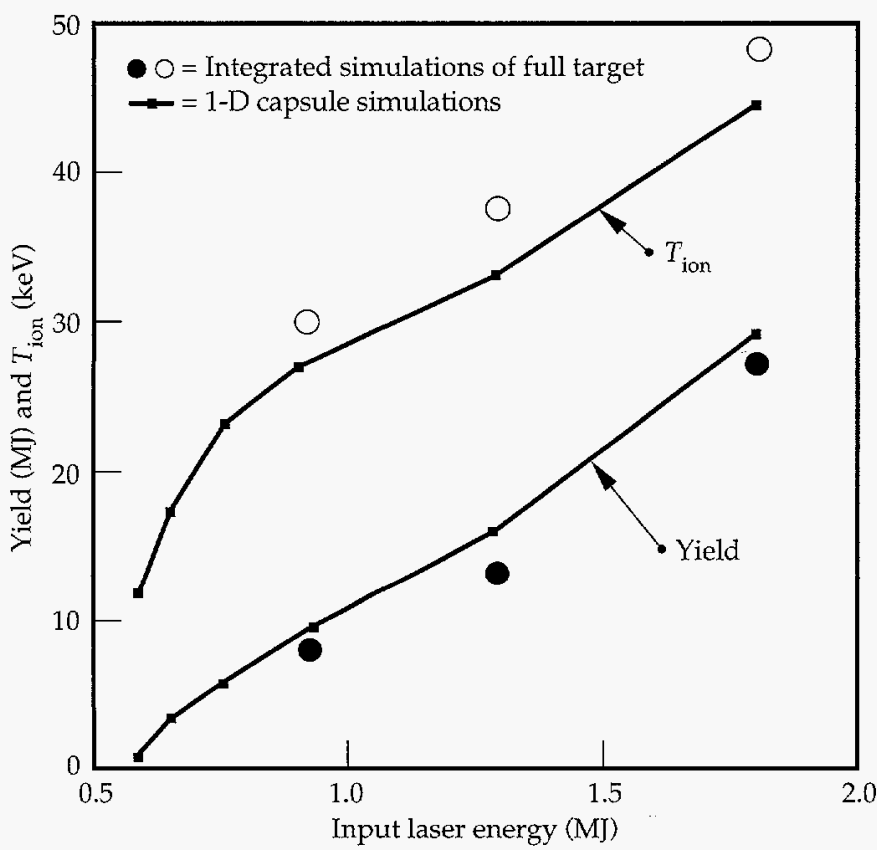

FIGURE 5. Yield and burn-weighted ion temperature for geometric scales of the PT. The lines show 1-D calculations of the capsule alone, in which linear dimensions and times are scaled together (the horizontal scale is effective energy, that is, $1.35 \mathrm{MJ}$ times the scale factor cubed; the dots are integrated calculations, plotted against the laser energy). For these, linear dimensions and times were scaled, and laser powers were scaled as the square of the scale factor. Hohlraum length and cone-to-cone power ratios were adjusted to recover symmetry. (50-05-0295-0389pb01) the four beams in each spot to limit laser-plasma instabilities. The four beams combine at an angle corresponding to an effective $f / 8$ optic.

Each beam is focused to an elliptical spot, with the minor axis in the plane of the laser ray and the hohlraum axis. This maximizes the LEH clearance while minimizing the laser intensity. The spot has a shape approximating a flat top (probably a sixth-order super-Gaussian), again to minimize the peak intensity while maximizing $\mathrm{LEH}$ clearance. The nominal spot is $500 \mu \mathrm{m} \times 1000 \mu \mathrm{m}$ at best focus. Such a spot can be made with recently developed kinoform phase plate techniques. ${ }^{8}$

The pulse shape shown in Fig. 3 creates four shocks, with the final shock bringing the ablator up to peak pressure with sufficiently low DT entropy. The entropy requirement implies a corresponding requirement on the precision of the pulse shaping. For optimal performance, the shocks must be timed within about 200 ps. Adequate shock timing may not be predictable a priori given uncertainties in opacity and equation-of-state (EOS), but is achievable with an experimental program using techniques currently in use on Nova. ${ }^{9}$

The $\mathrm{CH}$ ablator contains $0.25 \% \mathrm{Br}$ dopant. (Some Nova targets currently use $\mathrm{Br}$-doped $\mathrm{CH} .{ }^{10}$ ) The dopant is used to control the stability of the ablator/DT interface. It reduces the preheat in the $\mathrm{CH}$ and eliminates an unstable density step at the $\mathrm{CH} / \mathrm{DT}$ interface. The $\mathrm{CH}$ is assumed to contain $5 \% \mathrm{O}$ as an incidental fabrication by-product.

We conclude this section with a brief description of our baseline one-dimensional (1-D) capsule simulations. These simulations are performed with the LASNEX code $_{r}^{11}$ using Legendre decomposed radiation transport, 12 EOSs calculated in line with a "Quotidian EOS" package, ${ }^{13}$ and average-atom opacities calculated with the XSN code. ${ }^{14}$ We have also simulated the capsule implosion with other radiation transport schemes, but find no difference in the calculations. Implosion calculations use as a source nonPlanckian frequencydependent radiation determined from hohlraum simulations. The spectrum affects the short-wavelength hydrodynamic instability growth; other than this, the spectrum has little effect on target characteristics. We normally calculate the deposition of $\alpha$ particles produced by the burn with the multigroup diffusion package in LASNEX. ${ }^{15}$ Hatchett ${ }^{16}$ performed a baseline 1-D simulation of the PT using a Monte Carlo charged-particle transport code $\mathrm{e}^{17}$ and found that the ignition and burn are essentially the same as with multigroup chargedparticle diffusion.

\section{Other Possible Ignition Targets}

Modeling of a wide variety of other targets has been performed at various levels of detail. Several important aspects of the target can be varied, providing 
different trade-offs of the remaining uncertainties in our understanding.

We can vary the size of the target, and the energy it uses. Direct geometric scales of the PT produce good burn at any laser energy above about $700 \mathrm{~kJ}$, as shown in Fig. 5. This energy margin allows for loss of energy to stimulated scattering processes and laser coupling. Also, it allows us, if necessary, to change the relative size of the hohlraum and capsule. This allows a tradeoff of the capsule ignition physics, and hydrodynamic instability, with symmetry and hohlraum filling.

The knee at 600-800 kJ in Fig. 5 and the two thresholds at 1.6 and $1.8 \mathrm{~ns}$ in Fig. 4 are typical signatures of ignition. Without $\alpha$ deposition and thermonuclear bootstrapping, NIF targets would be expected to produce no more than $10-100 \mathrm{~kJ}$ of yield at the likely ion temperatures of less than about $10 \mathrm{keV}$. Experimental demonstration of ignition will be clear when we do a series of targets that scan through the threshold between such a non-bootstrapping mode and burn producing about $1 \mathrm{MJ}$ or more. This could be done by varying the target size, as shown in Fig. 5, or more likely by varying the implosion velocity (implicit in Fig. 4). Varying the $\mathrm{D}$ to $\mathrm{T}$ ratio will provide another easy-to-interpret lever on the burn rate. Curves of yield vs implosion velocity, for various $\mathrm{D}$ to $\mathrm{T}$ ratios, should show an inflection and a large increase in yield when the burn threshold is crossed for ratios near 50-50.

Various other target designs, described next, are at various scales in the ignition range.

Uncertainty in ablation characteristics can be addressed by using other ablator materials. In particular, Be generally performs better than $\mathrm{CH}$ as an ablator. (The PT uses a CH ablator because of fabrication experience with Nova. ${ }^{18}$ ) Be must be doped more heavily than $\mathrm{CH}$, and radially varying the doping allows for complete optimization. Designs exist in which the Be is doped with $\mathrm{Cu}$, which appears attractive from a fabrication point of view, and others with a mixture of $\mathrm{Na}$ and $\mathrm{Br}$. In the most highly optimized targets, the additional performance margin obtained by using Be instead of $\mathrm{CH}$ is equivalent to about $25 \mathrm{eV}$ in peak hohlraum $T_{R}$. The advantage is better at $250 \mathrm{eV}$ than at $300 \mathrm{eV}$.

Another important advantage of $\mathrm{Be}$ is more hydrodynamic stability. Using the modeling described below in the section on hydrodynamic instabilities, we have found a clear advantage to Be targets. Because it ablates faster, Be is more stable - the initial mass of the Be ablator is nearly twice that of the $\mathrm{CH}$. The outer surface roughness specification is somewhat looser for Be than for $\mathrm{CH}$, but the most important difference is in the required surface quality for the inner surface of the DT ice. Perturbations initially on the ice grow by coupling to the outer surface, the unstable ablation front, during acceleration; this coupling is much less effective through the more massive Be shell. As a result, a Be target (driven at $300 \mathrm{eV}$ ) can tolerate about four times larger perturbations initially on the inner surface of the ice than a $\mathrm{CH}$ target.

We can modify the convergence ratio (defined as the initial outer radius of the ablator divided by the ignition-time hot-spot radius) by varying the initial central DT gas density. Since reducing the convergence ratio reduces the final $\rho r$, it also reduces the yield. If the initial gas fill is increased the ignition is marginal, i.e., the yield is reduced from $15 \mathrm{MJ}$ nominal to about $1 \mathrm{MJ}$. These low-convergence targets require gas densities that would initially be in vapor equilibrium with liquid DT (as opposed to solid for the PT), and fielding them will require some modifications in the fabrication and fielding technology. The triple-point gas density, ${ }^{19}$ $0.68 \mathrm{mg} / \mathrm{cm}^{3}$, corresponds to a PT yield of $10 \mathrm{MJ}$.

Various peak drive temperatures are possible-high temperatures stress laser-plasma instabilities while minimizing hydrodynamic instabilities, and low temperatures provide the opposite trade-off. The baseline is $300 \mathrm{eV}$, a compromise between the two constraints. We have designed capsules driven at temperatures as high as $400 \mathrm{eV}$ that appear to be very resistant to hydrodynamic instabilities. Using doped Be as an ablator, we have designed targets driven at $250-\mathrm{eV}$ for which ignition is nearly as robust as with $\mathrm{CH}$ at $300 \mathrm{eV}$. Laser-plasma instabilities are estimated to be very benign in the $250-\mathrm{eV}$ hohlraum.

There is also a wide variety of possible pulse shapes. The pulse shown in Fig. 3 has four pulses or steps, each at a time and power to launch a shock, as needed, for the low-entropy implosion. Many other pulses can result in the same shocks in the fuel. We can use shorter pulses at higher powers (sometimes called "picket fence pulses"); we have used steps with the power held constant for a few nanoseconds in each step; at the other extreme, we have used pulses in which the power increases smoothly from an initial $10 \mathrm{TW}$ up to peak power. Each of these shapes represents a different trade-off of laser, hohlraum, and capsule physics.

We have also designed targets in which the solid DT fuel is supported in a foam layer. ${ }^{20}$ This may be an important option if $\beta$ layering is inadequate. DT-wettable foams of density $0.05 \mathrm{~g} / \mathrm{cm}^{3}$ with micrometer-scale cell structure have been fabricated, and our designs assume this density. The foam targets work nearly as well as the solid DT targets. If $\beta$ layering, or some other technique, can be used to maintain a pure DT layer about $10 \mu \mathrm{m}$ thick on the inside of a foam-supported main fuel layer, ignition occurs in clean DT and target performance is barely degraded by the presence of the foam. If all of the solid DT must be supported by foam, it is somewhat more difficult to ignite, although targets at the PT scale still ignite with some remaining margin.

There are numerous possible ignition designs that have attractive features, and may actually perform better 
than the point design, but that are not as closely connected to the existing experimental data. Direct-drive targets are an important option, pending experimental results from the Omega Upgrade at the University of Rochester. Other hohlraum designs are being investigated on Nova-for example, Au shields placed between the capsule and the LEHs can reduce the time-dependent asymmetry. Recent results on Nova for such a hohlraum are in excellent agreement with expectations based on simulations. ${ }^{21}$ As the NIF is being planned and built, experiments on Nova will continue to refine our understanding of the target physics and will allow us to optimize the NIF design further.

So far we have described the various target options that work according to current modeling; we have also found two target concepts where our modeling predicts difficulties:

- We considered lining the hohlraum with $\mathrm{CH}$, instead of the $\mathrm{He} / \mathrm{H}$ gas fill described earlier. However, we find that the lining stagnates on axis, creating a pressure spike that perturbs the capsule implosion unacceptably. Unfilled hohlraums have too much Au absorption of the light. We intend to consider alternate liners and unfilled hohlraums with short, low-temperature pulses.

- In principle, it seems possible to achieve ignition with noncryogenic gaseous DT. However, we have found that the implosion velocities required for ignition at the NIF scale result in high core temperatures before ignition $\rho r$ is reached, and then conduction from the hot spot is very high. In a cryogenic target, energy conducted from the hot spot into the DT pusher heats more DT-this increases the mass of the hot spot, and the energy is not lost. If the hot spot is surrounded by inert material, the energy is not only lost from the fuel, but it serves to degrade the compressibility of the pusher. Rayleigh-Taylor (RT) instabilities would also be much more problematic if the igniting fuel were surrounded with inert material. Therefore, we have been unable to calculate ignition successfully in noncryogenic capsules at the NIF scale.

\section{Hohlraum Design and Modeling}

The size of the hohlraum relative to the capsule is determined by a variety of trade-offs. The required profile of $T_{\mathrm{R}}$ vs time is determined by the capsule, and any hohlraum larger than some minimum size could provide the needed $T_{R}$ vs time profile. A larger hohlraum takes more laser energy and power, and the optimal size is a trade-off of the energy and power requirements and the need for symmetry and acceptable plasma filling. Our modeling indicates that the symmetry and laser-plasma instabilities are acceptable in the baseline hohlraum. Assuming a 1.8-MJ, 500-TW
NIF, there will be margin to increase the hohlraum size with the PT capsule, increasing the margin for laserplasma instabilities or asymmetry. If necessary, we can further increase this margin by using a smaller capsule, at the cost of either increasing hydrodynamic instabilities or developing Be-ablator fabrication technology.

Even with perfect laser pointing and beam-to-beam power balance, there is some asymmetry that we call the intrinsic asymmetry. This asymmetry arises because of the LEH and the bright laser-irradiated spots (the LEH alone causes a 15\% peak-to-valley asymmetry). As described in Ref. 6, the laser spots are placed to cancel the LEH asymmetry. The symmetry can be adjusted by changing the hohlraum length and the pointing of the beams. The quantities determining the intrinsic symmetry change in time: the LEH shrinks, the laser spots move due to plasma evolution, and the spots become less bright relative to the overall hohlraum brightness. With a single cone of beams, we found that the time-dependent asymmetry was too large. Two cones of beams can be arranged to provide adequate symmetry. Also, with two cones the time-dependent asymmetry can be corrected dynamically by varying the relative power in the cones as a function of time. This detailed symmetry tuning will have to be done with a time-dependent symmetry campaign similar to those being done on the Nova laser. ${ }^{22}$

To model the intrinsic asymmetry, we use a detailed two-dimensional (2-D) simulation with a radiationhydrodynamics code such as LASNEX. ${ }^{13}$ We use the best available radiation transport model for the hohlraum/capsule coupling, and simulations are continued all the way through burn. The simulations track the laser beams, calculating inverse Bremsstrahlung energy deposition and any refraction that occurs. We typically use XSN nonLTE multigroup opacities, ${ }^{16}$ although we have also performed simulations with an opacity table derived from the super transition array opacity model. ${ }^{23}$ Any coupling to the capsule via hydrodynamic pressure or electron conduction is included.

We have achieved adequate symmetry and good burn in such integrated simulations of a variety of designs: the PT at several scales as shown in Fig. 5, two Be designs driven at $250 \mathrm{eV}$ and at $300 \mathrm{eV}$, and a smooth-pulse 250-eV Be design. All except the PT scales shown in Fig. 5 use 1.1-1.5 MJ, at powers ranging from 365-500 TW. They give yields in the integrated simulations that are between $50-90 \%$ of clean 1-D yields and show unambiguous ignition.

Modeling asymmetry from imperfect power balance and pointing of the laser beams requires fully threedimensional (3-D) asymmetry. This asymmetry has been estimated analytically using laser-spot brightness and positions determined from the 2-D LASNEX simulations. Also, the asymmetry has been calculated in 3-D with a view-factor code. ${ }^{24}$ We have used fully integrated 
calculations (described earlier) to confirm the modeling and for some sensitivity studies. The actual asymmetry on the capsule is 3-D, and its effect on the implosion must be estimated with 2-D simulations of the implosion driven with an asymmetric radiation source.

We have imposed a wide variety of asymmetries on 2-D capsule implosions to ensure that the specified asymmetry levels are acceptable. Asymmetry can affect ignition in a variety of ways: the obvious kinematic effects of differing velocities; initiation of RT instability growth, especially evident during deceleration; mass flow toward less driven regions, seeding RT instability; irregular hot-spot compression, sometimes forming jets that protrude from the core and disrupt the imploded configuration; and delayed ignition, resulting in more RT growth. The maximum tolerable asymmetry depends on its temporal and spatial specifics. In summary, the capsule can tolerate less than about $1 \%$ time-averaged asymmetry, 5-10\% timedependent swings in asymmetry that last for $\sim 2 \mathrm{ns,}$ and larger swings if they last much less than $2 \mathrm{~ns}$.

We do not find much variation in sensitivity to asymmetry among the various targets we have designed. Smaller capsules are slightly more sensitive to asymmetries that couple to deceleration RT growth. The difference is not large, and symmetry sensitivity is not an issue that is important in deciding the overall trade-offs of laser size and power. Varying the hohlraum size, with a given capsule, is the symmetry issue likely to be more important in the trade-offs.

The 3-D view factor calculations indicate that with nominal pointing errors-each beam is to point within $50 \mu \mathrm{m}$ of its nominal position, rms deviation - the resulting additional asymmetry on the capsule will be significantly $<1 \%$. This pointing specification also ensures more than adequate clearance of the LEH. This requirement is similar to that met by the Nova laser (30 $\mu \mathrm{m} \mathrm{rms,}{ }^{25}$ which is $10 \mu \mathrm{rad}$, while $50 \mu \mathrm{m}$ rms on NIF is $7 \mu \mathrm{rad}$ because of the longer focal length).

The 3-D view factor calculations also indicate that $10 \% \mathrm{rms}$ power imbalance results in $<1 \%$ asymmetry on the capsule, provided the deviations are uncorrelated among the 192 beams. The tolerable power imbalance can be much larger than this, depending on its temporal dependence. If there are correlations between the beams' powers, a much tighter power balance requirement is necessary. Groups of eight beams, with each group entering the same area of the hohlraum, must be balanced within about $3 \%$. Generally the requirements are consistent with purely independent statistical deviations of the 192 beams; any correlations significantly beyond this may increase the asymmetry unacceptably.

These requirements on the laser are well within current Nova performance parameters of $3 \%$ rms energy imbalance, and 5-10\% power imbalance over time scales that are generally less than half the pulse length. ${ }^{26}$ This does not mean that symmetry in Nova hohlraums is as good as in NIF hohlraums; the looser requirements for NIF are a result of the larger number of beams.

Asymmetries might also arise from laser-plasma interaction processes or other phenomena, such as RT instability at the Au/He interface, which are currently predicted not to be significant but for which uncertainty remains. Light can be scattered or it can be absorbed more or less efficiently at different positions in the hohlraum. The effect in all cases is equivalent to a power balance change, a movement of the $x$-ray emission spots, or perhaps a spreading of the laser deposition spots (for small-angle side-scattering). Difficulties could arise only if these effects are so large that the irreproducible part of them is larger than the limits described here. If any of these processes occurs but is reproducible and not too large, the effect can be compensated for by changing the hohlraum design parameters. Estimates based on Nova experiments and appropriate theory and modeling indicate that these processes can be kept within acceptable limits. If not, our ultimate recourse will be to increase the hohlraum size, reduce the laser intensity, and correspondingly reduce the hohlraum drive temperature.

\section{Laser-Plasma Instabilities}

The most important laser-plasma scattering processes are SBS, stimulated Raman scattering (SRS), and filamentation. In SBS and SRS, the incident laser beam scatters from electron waves and ion waves, respectively, in the forward, side, or backscatter direction. Backscatter is calculated and observed to be the most unstable process, although sidescatter must be examined for its possible effect on capsule symmetry. SRS forward scatter is a very weak process; forward SBS is being evaluated for possible symmetry effects because of the exchange of energy between overlapping beams. ${ }^{27}$ Filamentation or whole-beam self focusing results from the refraction of the laser light into low-density regions, which are themselves produced by the pressure gradients from nonuniform laser heating or by ponderomotive forces. All of these processes are sensitive to the $n_{\mathrm{e}}$ and temperature, and laser intensity and wavelength. ${ }^{28}$ In addition, SBS is sensitive to the electron-ion temperature ratio, velocity gradient, and the fraction of light and heavy ions in multiple species plasma. ${ }^{29}$ Also, we have shown with 3-D filamentation simulations ${ }^{30}$ that filamentation is sensitive to the speckle length (the axial length of a diffraction-limited hot spot near the focal plane of the laser beam). The speckle length increases with the square of the $f$-number of the focusing system.

The laser must propagate through 3-5 $\mathrm{mm}$ of hot $\left(T_{\mathrm{e}} \sim 3-5 \mathrm{keV}\right.$ at peak power), low-density $\left(n_{\mathrm{e}} \leq 1 \times 10^{21} \mathrm{~cm}^{-3}\right.$ ), low-Z (mixture of $\mathrm{He}$ and $\mathrm{H}$ ) plasma. The density is about 0.05 critical over most of the beam path. For the inner ring of beams, the density reaches as high as $15 \%$ of critical for the last millimeter 
of pathlength. However, this far into the hohlraum the individual laser beam intensity has decreased substantially from its peak of $2 \times 10^{15} \mathrm{~W} / \mathrm{cm}^{2}$.

These scattering processes affect the target performance in several ways. Of course, energy scattered back out of the hohlraum is unavailable for $x$-ray conversion. The total energy lost comes out of the $\sim 50 \%$ energy margin shown in Fig. 2. The irreproducible part of this becomes a pulse-shape uncertainty, and any resultant geometrical nonuniformity can affect the symmetry of the irradiation on the capsule. These effects will be tolerable if the scattering is less than about $10 \%$. We do not expect hot electrons produced by SRS to have any effect on target performance.

We estimate that the scattering processes will be acceptable under these predicted plasma conditions. Experimentally verifying these estimates is an action part of the Nova program, described in "Laser-Plasma Interactions in NIF-Scale Plasmas (HLP5 and HLP6)" on pg. 305 of this Quarterly.

\section{Modeling of Hydrodynamic Instabilities}

The shell is subject to RT instability on the outside during its acceleration and on the inside during deceleration. There is also Richtmyer-Meshkov (RM) instability at all interfaces. (These instabilities are reviewed in Ref. 31.) Short-wavelength RT growth in these capsules is stabilized by ablation of material through the unstable interface and by the finite scale length on the ablation front. Experimental ${ }^{32}$ and calculational ${ }^{33}$ aspects of this stabilization are well documented. During deceleration, the growth of short wavelengths is also reduced from classical RT since the unstable interface is between two DT regions, the hot spot and the main fuel, and again there is ablation (driven by electron conduction in this case) and a finite gradient scale length. Also, perturbations that grow on the outside must couple through the shell to affect the ignition, and short wavelength modes couple less effectively. These effects all reduce the impact of the short wavelengths, so the system is only weakly nonlinear. The targets have been designed so that this is the case.

We have based our modeling on linear analysis that is as accurate as possible, with an extension into the weakly nonlinear regime, as necessary. The linear analysis is based on a decomposition of the surface perturbations into spherical harmonics, which are eigenmodes of the linear evolution. We determine the single-mode growth spectrum by running multiple 2-D simulations, each of one single mode in the linear regime throughout the simulation. This provides the most accurate calculation of all known effects, including stabilization, RM growth, and convergence effects. This set of calculations provides a spectrum of growth factors, which we combine with an assumed initial sur- face spectrum to determine the ignition-time perturbation. Using a nonlinear saturation model from Ref. 34, we determine whether the perturbations are nonlinear and estimate the nonlinear saturation. This results in a curve of ignition-time perturbation amplitude as a function of initial perturbation amplitude.

To test the weakly nonlinear analysis, we also run full simulations of multimode perturbations with realistic initial amplitudes. Currently simulations must be 2-D, and the number of modes that can be included is limited. We have run a variety of multimode simulations on several capsules, at solid angles ranging from relatively small conic sections to half-spheres. Results are consistent with the modeling described earlier, although further substantiation is an area of current work. Recent development of 3-D codes will allow testing of possible differences between 2- and 3-D evolution. 35

We must also estimate how the perturbations around the hot spot at ignition time will affect the ignition. The unstable interface is between relatively cold, dense DT and the hot, lower-density DT. Material mixing of different elements is not occurring, and there is only thermal mixing. The actual perturbations are 3-D, and multimode, and the weakly nonlinear perturbation growth analysis indicates that the spectrum is strongly dominated by mode numbers around $l=10-15^{\circ}$. The 3 -D character cannot be fully represented in any existing code; available 3-D codes do not include all of the relevant physical processes. There is experimental ${ }^{32}$ and calculational ${ }^{35}$ evidence that the multimode $3-D$ perturbation is probably an array of spikes penetrating in toward the hot-spot center, surrounding approximately hexagonal bubbles. In 2-D, we modeled this array of spikes and bubbles five ways: (1) We simulated a single bubble of appropriate solid angle surrounded by a curtain of material falling along a reflecting boundary condition. The circular cone represents approximately a multifaceted 3-D cone of similar size and gross shape. (2) We ran perturbations with the opposite sign: a spike on axis surrounded by a circular bubble. (3) We simulated perturbations on the waist that represent long circular ridges and curtains. (4) We continued through burn time the multimode 2-D simulations mentioned earlier. (5) We did 1-D modeling in which the thermal mixing caused by the perturbation growth is represented as an enhanced thermal conductivity in the perturbed region.

All of these approaches give similar results, regarding how large a spike can be tolerated before ignition is quenched. Combined with the modeling described earlier, this corresponds to a maximum tolerable initial ablator surface roughness of about $50 \mathrm{~nm}$ rms. This compares with $30 \mathrm{~nm}$ rms on current Nova capsules.

We have also considered the bubble penetration from the outside of the shell at peak velocity. We find that the surface finish requirements for shell integrity during acceleration and for ignition are similar. This 
equivalence depends weakly on the shape assumed for the spectrum of initial perturbations.

Because we have modeled the perturbation growth and its effects with a variety of different approaches, and get generally consistent results, we are fairly confident that our modeling is accurate. The modeling relies on 2-D code simulations of linear-regime perturbation growth, so it is very important that these be tested thoroughly. The dominant uncertainties are the dependence on the spectrum of the drive $x$ rays, and on zoning, resulting in a net uncertainty in the outer surface finish specification that we believe to be about a factor of two. Finally, of course, it is very important to test the modeling experimentally. A major fraction of the Nova program is oriented toward verifying this modeling, with a variety of experiments measuring perturbation growth and its effects in planar ${ }^{32}$ and spherical ${ }^{5,10,36}$ geometry. Results from these experiments have been consistent with the modeling.

The modeling described so far pertains to surface perturbations that are initially on the outside of the ablator. Of course, there will be perturbations on the other interfaces, as well as material inhomogeneity and other fabrication defects. Any of these can be modeled in a conceptually identical way, using LASNEX simulations that assume the existence of the perturbation of interest. We have determined that the capsule tolerates perturbations initially on the other interfaces, which are much larger than tolerable perturbations initially on the outside. Perturbations on the DT/CH interface are unlikely to be large enough to matter. Ignition does not occur if perturbations on the DT gas/solid interface are greater than about $3.0 \mu \mathrm{m}$ for the PT, and more than about $8 \mu \mathrm{m}$ for Be capsules, which are the same size as the PT and are driven at $300 \mathrm{eV}$. Current estimates $^{37}$ of the smoothness of $\beta$ layer surfaces are $\sim 1 \mu \mathrm{m}$. Solid DT in a low-density foam is somewhat smoother.

In summary, the PT has a factor of about two margin in surface finish beyond surface finishes on the best current Nova capsules. The requirement on the DT gas/solid interface also gives about a factor of 2 margin compared with roughness measured on recent DT ice surfaces.

\section{Robustness Studies}

We performed extensive studies of the robustness of the PT target following the initial design work, which we categorize into two studies discussed below.

\section{Robustness of Yield in Integrated Simulations}

Choosing a particular configuration as nominal, we varied the laser powers and pointing in detailed integrated calculations (described earlier). Figure 4 is an example of such a variation (although the calculations shown there used a laser pulse slightly different from what we chose as nominal for the full set of variations). Table 1 shows the sensitivities we found, and compares them with estimates of the reliability with which we can determine and maintain these parameters. This study produced three valuable results. (1) We verified that the target can tolerate plausible variations in the input parameters (shown in Table 1). This allays concern that the performance of the target is a finely tuned optimum that would be impossible to achieve experimentally. (2) We identified ways in which the configuration we chose as nominal was not in fact optimal (most importantly, we found significant room for improvement in the pointing we were using for the inner cone). We are currently doing a second iteration, with improved pointing and better optimization of the other parameters, which should show a margin of performance even larger than that shown in Table 1. Some results of the study with the new optimization are shown in Table 1. (3) This study provides a context for designing the experimental campaign to achieve ignition. Table 1 (and subsequent revisions) shows which parameters must be measured and maintained and to what accuracy.

We are also doing integrated simulations varying several parameters at once. We have varied both the inner and outer cone laser powers separately (in addition to the variations shown in Table 1), and see sensitivity similar

TABLE 1. Sensitivity analysis of the PT using integrated calculations of the entire hohlraum/capsule target.

\begin{tabular}{|c|c|c|}
\hline Laser parameter* & $\begin{array}{l}\text { Determined } \\
\text { sensitivities }\end{array}$ & $\begin{array}{c}\text { Preliminary } \\
\text { estimate }\end{array}$ \\
\hline Power during foot & $30 \%$ & $<5 \%$ \\
\hline Peak power & $35 \%$ & $<5 \%$ \\
\hline Second-rise timing & $500 \mathrm{ps}$ & $<100$ ps \\
\hline Third-rise timing & 500 ps & $<100$ ps \\
\hline Duration of peak power & 800 ps & $<100$ ps \\
\hline $\begin{array}{l}\text { Inner beam power during foot } \\
\text { (total power fixed) }\end{array}$ & $25 \%$ & $<5 \%$ \\
\hline $\begin{array}{l}\text { Inner beam power during peak } \\
\text { (total power fixed) }\end{array}$ & $35 \%$ & $<5 \%$ \\
\hline $\begin{array}{l}\text { Inner beam power during peak } \\
\text { (outer cone power fixed) }\end{array}$ & $25 \%$ & $<5 \%$ \\
\hline Pointing of inner beams & $85 \mu \mathrm{m}(200 \mu \mathrm{m})^{\dagger}$ & $<20 \mu \mathrm{m}$ \\
\hline Pointing of outer beams & $100 \mu \mathrm{m}(350 \mu \mathrm{m})^{\dagger}$ & $<20 \mu \mathrm{m}$ \\
\hline
\end{tabular}

${ }^{*}$ For each indicated parameter describing the laser input power, we tabulated the full-width at half maximum of the yield as that parameter was varied. We also tabluated estimates of the precision with which the parameters can be determined and maintained in an experimental campaign working toward ignition. In all cases, the indicated precision is dominated by estimates of experimental precision, and the corresponding specification on the laser itself is significantly smaller.

${ }^{+}$These values are for a new optimization with the $P_{6}$ asymmetry reduced. 
to that shown in Table 1. Ultimately we will explore the entire parameter space of possible variations, although we will use the new nominal design mentioned above instead of continuing to center the variations on our first preliminary optimization.

\section{Sensitivity to Combinations of Asymmetry, Pulse-Shaping Errors, and Hydrodynamic Instabilities}

To create a complete model for what could affect the implosion adversely, we utilized a series of capsuleonly simulations. We started by simulating the capsule with the asymmetry determined from the nominal integrated calculation, then added perturbed surfaces, and finally added asymmetric drive sources. The asymmetry can be made to match that in the integrated calculations by extracting from the integrated simulation the asymmetry in ablation pressure in the imploding capsule. (The asymmetry can be characterized with Legendre polynomial moments, with $P_{2}$ through $P_{6}$ contributing.) Then a matching radiation drive asymmetry, in a simulation of the capsule alone, produces an identically out-of-round implosion. This technique matches the asymmetry both for a nominal design and for the off-nominal integrated calculations represented in Table 1 . (We chose as nominal the same integrated calculation used as the central point for the variations presented in Table 1). We performed three variations given the "baseline" nominal asymmetric implosion.

1. Asymmetry alone. We found that the "nominal" asymmetry has about $50 \%$ margin in the $P_{6}$ moment and $>100 \%$ margin in the $P_{2}$ and $P_{4}$ moments. This suggests that the overall robustness can be improved by reoptimizing to minimize $P_{6}$. This is part of the reoptimization mentioned above, increasing further the margin of the PT.

2. Nominal asymmetry plus short-wavelength surface roughness. We performed implosions with the nominal asymmetry and perturbations on both the inner and outer capsule surfaces. The spectral features of the perturbations were based on characterization of $\beta$ layered DT for the ice roughness and of Nova $\mathrm{CH}$ capsule surface roughness for the outside. The capsule ignites and burns well with nominal asymmetry and nominal surface roughnesses of $30 \mathrm{~nm}$ on the $\mathrm{CH}$ and $1.0 \mu \mathrm{m}$ on the DT.

3. Off-nominal asymmetry and drive, plus shortwavelength surface roughness. In addition to the nominal asymmetry, we included further random asymmetry-up to $4.5 \% \mathrm{rms} P_{2}$ and $1.5 \% \mathrm{rms} P_{4}$. The additional asymmetry was a random function of time, with zero mean and about $2 \mathrm{~ns}$ typical period of variation. We also included variations in the drive profile (the net flux onto the capsule, $P_{0}$ ) of $\pm 5 \%$. The combination of asymmetry, drive profile errors, and surface roughness represents the most complete possible model of the implosion. We found that the additional asymmetry and profile variations had little effect on the yield; for example, with $30 \mathrm{~nm}$ outer roughness and $1.0 \mu \mathrm{m}$ inner roughness, we obtain $11.5 \mathrm{MJ}$ of yield with the nominal asymmetry plus the variations of $4.5 \% \mathrm{rms} P_{2}, 1.5 \% \mathrm{rms} P_{4}$, and $\pm 5 \% P_{0}$.

These robustness results are extremely encouraging assurance of the nominal design's performance.

\section{Summary}

Given the experimental substantiation from the Nova program, we have good reason to expect ignition with a 1.8-MJ, 500-TW laser. Such a laser will provide an adequate safety margin, above the ignition threshold indicated by modeling supported by Nova experiments. This margin is sufficient to cover estimated uncertainties.

We can compensate for the remaining uncertainties by adjusting the target design if necessary after additional Nova experiments, or after the NIF experiments begin. Some possible changes in the target design or performance will be energetically significant. These include:

- A factor of two in hydrodynamic instability growth (equivalent to a factor of two in surface finish, or a factor of two in the acceptable size of the bang-time perturbations) shifts the ignition cliff from $0.8 \mathrm{MJ}$ to about 1.0 MJ. Improvements in surface finish could probably recover the original margin.

- The combined uncertainties in $x$-ray conversion and hohlraum wall loss are less than about $20 \%$ in energy.

- SBS and SRS should be less than about $10 \%$, based on the experiments described in "Laser-Plasma Interactions in NIF-Scale Plasmas (HLP5 and HLP6)" on pg. 305 of this Quarterly.

- Achieving the correct power balance between the inner and outer cones of beams may require reducing the power in one or the other, so that it cannot run at its full power. This may result in a net energy loss of $10-15 \%$.

- An error in hohlraum optimization that requires increasing the $\mathrm{LEH}$ radius $50 \%$ would require an increase in laser energy of $15 \%$ to regain the same hohlraum temperature.

- Similarly, increasing the hohlraum area by $35 \%$ increases the required laser energy by $15 \%$.

Several other uncertainties are energetically insignificant. For example, the EOS and opacity of the $\mathrm{CH}$ ablator are sufficiently uncertain that we expect to adjust the details of the pulse shape phenomenologically, but this will not significantly affect the performance 
requirements from the laser or the target performance. These errors, in combined effect, are consistent with the factor of two margin provided by a 1.8-MJ, 500-TW laser.

There are some issues that we are addressing to substantiate this conclusion further and to progress with plans for the facility. We need to make a final decision regarding the optimal cone-to-cone energy ratio, and beam angles, which will be built into the target chamber and will be difficult to change once detailed facility design is in progress. To maximize our understanding of the options available to us, we are continuing to pursue other designs-e.g., hohlraums with shields between the capsule and the LEH. Finally, we also continue to pursue more detailed modeling of the PT. These results, along with the ongoing experimental program on Nova, will either lead to increasing confidence in the performance of the PT or will indicate what changes need to be made in the design.

\section{Notes and References}

1. J. H. Nuckolls, L. Wood, A. Thiessen, and G. B. Zimmerman, Nature 239, 129 (1972).

2. J. D. Lindl, R. L. McCrory, E. M. Campbell, Physics Today (Sept. 1992).

3. J. T. Hunt, K. R. Manes, J. R. Murray, P. A. Renard, et al., "Laser Design Basis for the National Ignition Facility," Lawrence Livermore National Laboratory, Livermore, CA, UCRL-JC117399 (1994).

4. S. W. Haan et al., Phys. Plasmas 2(6) (1995).

5. C. Verdon, Laboratory for Laser Energetics, Rochester, NY, private communication (1994)

6. J. D. Lindl, "Development of the Indirect-Drive Approach to Inertial Confinement Fusion and the Target Physics Basis for Ignition and Gain," UCRL-JC-119015218 (1995); submitted to Phys. Plasmas (1995).

7. A. J. Martin, R. J. Simms, and R. B. Jacobs, J. Vac. Sci. Technol. A 6(3), 1885 (1988).

8. S. N. Dixit, J. K. Lawson, K. R. Manes, H. T. Powell, and K. A. Nugent, Opt. Lett. 9, 417-419 (1994).

9. R. L. Kauffman, L. J. Suter, C. B. Darrow, J. D. Kilkenny, et al., Phys. Rev. Lett. 73, 2320-2323 (1994).

10. C. J. Keane, R. C. Cook, T. R. Dittrich, B. A. Hammel, et al., "Diagnosis of Pusher-Fuel Mix in Spherical Implosions Using $X$-Ray Spectroscopy," Lawrence Livermore National Laboratory, Livermore, CA, UCRL-JC-116787 (1994); submitted to Rev. Sci. Inst. (1995).

11. G. B. Zimmerman and W. L. Kruer, Comm. Plasmas Phys. Cont. Thermonuclear Fusion 2, 51 (1975).

12. D. S. Kershaw, "Flux Limiting in Nature's Own Way," Lawrence Livermore National Laboratory, Livermore, CA, UCRL-78378 (1976).
13. R. M. More, K. H. Warren, D. A. Young, and G. B. Zimmerman, Phys. Fluids 31(10), 3059 (1988).

14. W. A. Lokke and W. H. Grasberger, "XSNQ-U: A NonLTE Emission and Absorption Coefficient Subroutine," Lawrence Livermore National Laboratory, Livermore, CA, UCRL-52276 (1977).

15. E. G. Corman, W. B. Loewe, G. E. Cooper, and A. M. Winslow, Nucl. Fusion 15, 377 (1975).

16. S. P. Hatchett, Lawrence Livermore National Laboratory, Livermore, CA, private communication (1993).

17. G. B. Zimmerman, "Recent Developments in Monte-Carlo Techniques," Lawrence Livermore National Laboratory, Livermore, CA, UCRL-105616 (1990).

18. A. K. Burnham, J. Z. Grens, E. M. Lilley, J. Vac. Sci. Technol. A 5(6), 3417 (1987).

19. P. C. Souers, Hydrogen Properties for Fusion Energy (University of California Press, Berkeley, CA, 1986).

20. R. A. Sacks and D. H. Darling, Nuc. Fus. 27, 447 (1987).

21. P. A. Amendt, Lawrence Livermore National Laboratory, Livermore, $\mathrm{CA}$, private communication regarding symmetry tuning and increased $x$-ray drive in modified Nova hohlraums, to be published in Phys. Rev. Lett. (1995).

22. A. Hauer, et al., Phys. Plasmas 2(6) (1995).

23. A. Bar Shalom, J. Oreg, W. H. Goldstein, D. Shvarts, and A. Zigler, Phys. Rev. A 40, 3183 (1989).

24. R. C. Kirkpatrick and C. A. Wingate, Los Alamos National Laboratory, Los Alamos, NM, private communication (1980); R. C. Kirkpatrick, J. E. Tabor, E. L. Lindman, A. J. Cooper, "Indirect Solar Loading of Waste Heat Radiators," Proc. Space 88, S. W. Johnson and J. P. Wetzel, Eds. (the American Society of Civil Engineers, 1988) p. 964; D. S. Bailey (1981), D. H. Munro and G. B. Zimmerman (1993), Lawrence Livermore NationaI Laboratory, Livermore, CA, private communication.

25. J. E. Murray, M. C. Rushford, C. S. Vann, R. L. Saunders, and J. A. Caird, ICF Quarterly Report 4(1), 18, Lawrence Livermore National Laboratory, Livermore, CA, UCRL-LR-105821-94-1 (1993).

26. J. A. Caird, R. B. Ehrlich, O. L. Landen, C. W. Laumann, et al., ICF Quarterly Report 4(1), 10, Lawrence Livermore National Laboratory, Livermore, CA, UCRL-LR-105821-94-1 (1993).

27. K. Marsh, C. Joshi, and C. J. McKinstrie, BAPS 39, 1585, Minneapolis, MN, November 7-11, 1994.

28. W. L. Kruer, The Physics of Laser Plasma Interactions (Addison-Wesley, NY, 1998).

29. E. A. Williams, R. L. Berger, R. P. Drake, A. M. Rubenchik, et al., Physics of Plasmas 2, 129 (1995); S. C. Wilks, W. L. Kruer, J. Denavit, K. Estabrook, et al., "Nonlinear Theory and Simulations of Stimulated Brillouin Backscatter in Two and Three Species Plasmas," Lawrence Livermore National Laboratory, Livermore, CA, UCRL-JC-117313 (1995); accepted for publication in Phys. Rev. Lett.

30. R. L. Berger et al., Phys Fluids B 5, 2243 (1993).

31. S. W. Haan, "Hydrodynamic Instabilities on ICF Capsules," Lawrence Livermore National Laboratory, Livermore, CA, UCRL-JC-107592 (1991); to be published in Lecture Series on Inertial Fusion, Dept. of Astrophysical Sciences, Princeton University. 
32. B. A. Remington, S. V. Weber, S. W. Haan, J. D. Kilkenny, et al., Phys. Fluids B 5, 2589 (1993).

33. S. V. Weber, B. A. Remington, S. W. Haan, B. G. Wilson, and J. K. Nash, Phys. Plasmas 1, 3652 (1994); H. Takabe, K. Mima,

L. Montierth, and R. L. Morse, Phys. Fluids 28, 3676 (1985);

M. Tabak, D. H. Munro, and J. D. Lindl, Phys. Fluids B 2, 5

(1990); J. H. Gardner, S. E. Bodner, and J. P. Dahlburg, Phys. Fluids B 3, 1070 (1991).

34. S. Haan, Phys. Rev. A 39, 5812 (1989).

35. J. P. Dahlburg et al., Phys. Plasmas 2(6), (1995); M. Marinak, Lawrence Livermore National Laboratory, Livermore, CA, private communication (1994).

36. T. R. Dittrich, B. A. Hammel, C. J. Keane, R. McEachern, et al,, Phys. Rer. Lett. 73, 2324-2327 (1994).

37. G. W. Collins, E. R. Mapoles, J. Hoffer, J. Simpson, et al., ICF Quarterly Report 3(2), 81, Lawrence Livermore National Laboratory, Livermore, CA, UCRL-LR-105821-93-2 (1993). 


\title{
InDiRectly Driven, High-ConVERgence IMPLOSIONS (HEP1)
}

\author{
S. P. Hatchett \\ S. M. Lane \\ M. B. Nelson \\ M. D. Cable \\ C. Laumann \\ D. W. Phillion \\ J. A. Caird \\ R. A. Lerche \\ H. Powell \\ J. D. Kilkenny \\ T. J. Murphy \\ D. B. Ress \\ H. N. Kornblum \\ J. Murray
}

\section{Introduction}

High-gain inertial confinement fusion will most readily be achieved with hot-spot ignition, ${ }^{1,2}$ in which a relatively small mass of gaseous fuel at the center of the target is heated to $5-10 \mathrm{keV}$, igniting a larger surrounding mass of approximately isobaric fuel at higher density but lower temperature. Existing lasers are too low in energy to achieve thermonuclear gain, but hydrodynamically equivalent implosions using these lasers can demonstrate that the important, scalable parameters of ignition capsules are scientifically and technologically achievable. The experiments described in this article used gas-filled glass shells driven by $\mathrm{x}$ rays produced in a surrounding cavity, or hohlraum. These implosions achieved convergence ratios (initial capsule radius / final fuel radius) high enough to fall in the range required for ignition-scale capsules, and they produced an imploded configuration (high-density glass with hot gas fill) that is equivalent to the hot-spot configuration of an ignition-scale capsule. Other recent laser-driven implosions ${ }^{3,4}$ have achieved high shell density but at lower convergences and without a well defined hot spot. Still other experiments ${ }^{5,6}$ have used very-lowdensity gas fill to reach high convergence with unshaped drive (see below), but that approach results in a relatively low shell density. Moreover, even at the highest convergence ratios the implosions described here had neutron yields averaging $8 \%$ of that calculated for an idealized, clean, spherically symmetric implosion-much higher than previous high-convergence experiments.

As we discuss below, the implosions described here were characterized by a number of diagnostics. In particular, convergence ratios were directly determined by measurements of the areal density of the imploded fuel using a technique based on secondary-neutron spectroscopy. The implosions were modeled, with the inclusion of non-ideal effects, with detailed computer codes such as LASNEX, ${ }^{7}$ a coupled radiation transport, hydrodynamics, and burn-particle transport code. All observable quantities were in close agreement with these simulations, demonstrating good understanding of the implosions.

\section{Experimental Design}

The capsules, shown in Fig. 1, were indirectly driven gas-filled microballoons. We chose a relatively small capsule (capsule diameter $16 \%$ of hohlraum diameter) to limit the areal density of the imploded fuel, allowing the use of secondary neutrons for the determination of this quantity (see below). We used glass capsules filled with deuterium (D) or equimolar deuterium/tritium (DT). Capsule fill pressures varied from 25 to $200 \mathrm{~atm}$, which changed the capsule convergence for constant drive (see "Measuring Convergence"). Ten Nova beams $(2.1 \mathrm{~kJ}$ each at $0.35 \mu \mathrm{m})$ were incident on the interior of a uranium (U) hohlraum at $2 \times 10^{15} \mathrm{~W} / \mathrm{cm}^{2}$ and produced an $x$-ray drive flux on the surface of the capsule; the hohlraum geometry is shown in Fig. 1(b). Figure 2 shows the measured laser power $P_{L^{\prime}}$ corresponding brightness temperature of the $\mathrm{x}$-ray drive $T_{\text {rad, }}$ and the neutron production rate $R_{\mathrm{n}}$.

We measured $\mathrm{x}$-ray drive using a multichannel, $\mathrm{K}$ and L-edge-filtered $x$-ray spectrometer looking into the hohlraum at both directly illuminated laser spots and the indirectly illuminated wall, ${ }^{8}$ observed spectra were nearly Planckian. Subject to hydrodynamic instability limitations, ${ }^{9}$ we chose the $x$-ray drive vs time dependence, or pulse shape, to optimize the pressure-density trajectory of the capsule compression. Use of a glass shell and a $U$ hohlraum minimizes the $x$-ray preheating of the capsule. The capsule implosion is driven by pressure generated by ablation of the outer surface material. Under the conditions of these experiments- $x$-ray brightness temperature low enough that the ablation front is subsonic, time-scale short enough that the ablated material is optically thin to the driving $x$ rays-it can be shown ${ }^{10}$ that the ablation pressure is 
approximately given by $P_{\mathrm{abl}}=0.5 \sigma \mathrm{T}_{\mathrm{rad}}^{4} /\left(R \mathrm{~T}_{\mathrm{rad}} / \mu\right)^{1 / 2}$ where $\sigma$ is the Stefan-Boltzmann constant, $R$ is the gas constant and $\mu$ is the molecular weight. This gives ablation pressures of $8 \mathrm{Mbar}$ at the foot of the pulse $(t=0.2 \mathrm{~ns})$ and $110 \mathrm{Mbar}$ at the peak of the pulse $(t=1.4 \mathrm{~ns})$.

\section{Achieving High Convergence}

In implosions such as these, several factors limit the convergence ratio achievable. The most important are pusher entropy, drive asymmetry, and Rayleigh-Taylor (RT) instability. These factors must be minimized in the experiments and properly accounted for in models.

The first factor is pusher entropy. The less dense the pusher is near stagnation, the less efficiently it can couple its kinetic energy into compressing the fuel. X-ray preheat of the pusher raises its specific entropy; ablation pressure cannot then hold it at as high a density during inward acceleration, and at stagnation more of its kinetic energy will be spent compressing itself rather than compressing the fuel. $\mathrm{X}$-ray preheat is minimized by the use of a $U$ hohlraum, with the resulting nearPlanckian $\mathrm{x}$-ray spectrum, and a glass pusher, which

(a) 20-nm rms surface finish

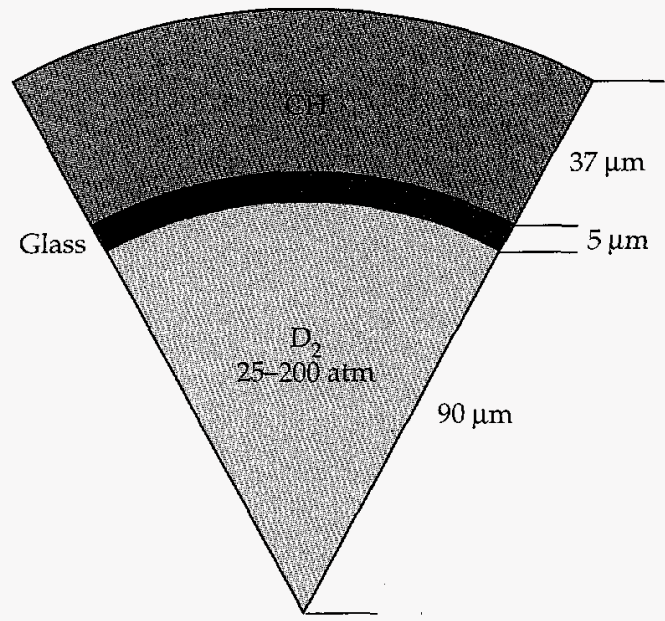

(b)

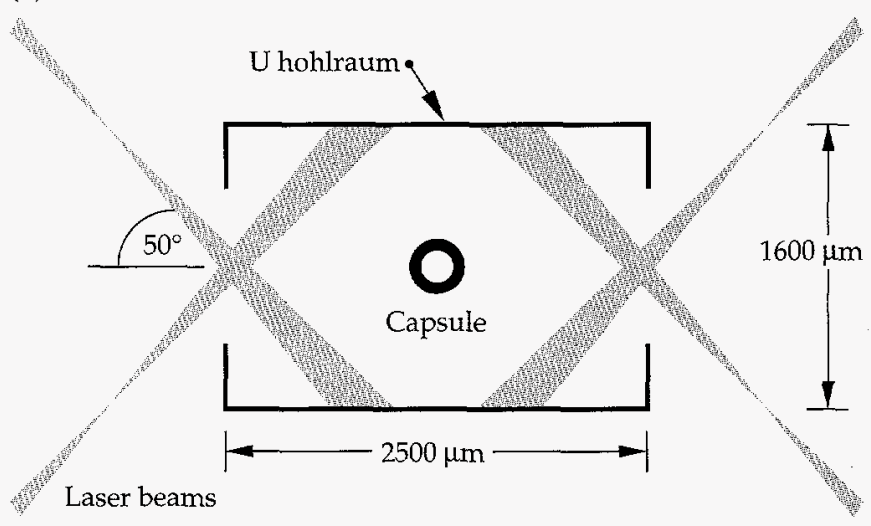

FIGURE 1. Capsule and indirect-drive hohlraum geometry. (50-05-0695-1562pb01) self shields from hard $x$ rays. Similarly, pulse-shaping is required to limit pusher entropy generation by shocks and to keep the pusher dense during inward acceleration. In the ideal, lowest entropy state, the pusher pressure results entirely from the Fermi degeneracy of the free electrons. In Figure 3, we plot the ratio of the pressure to the electron degeneracy pressure as a function

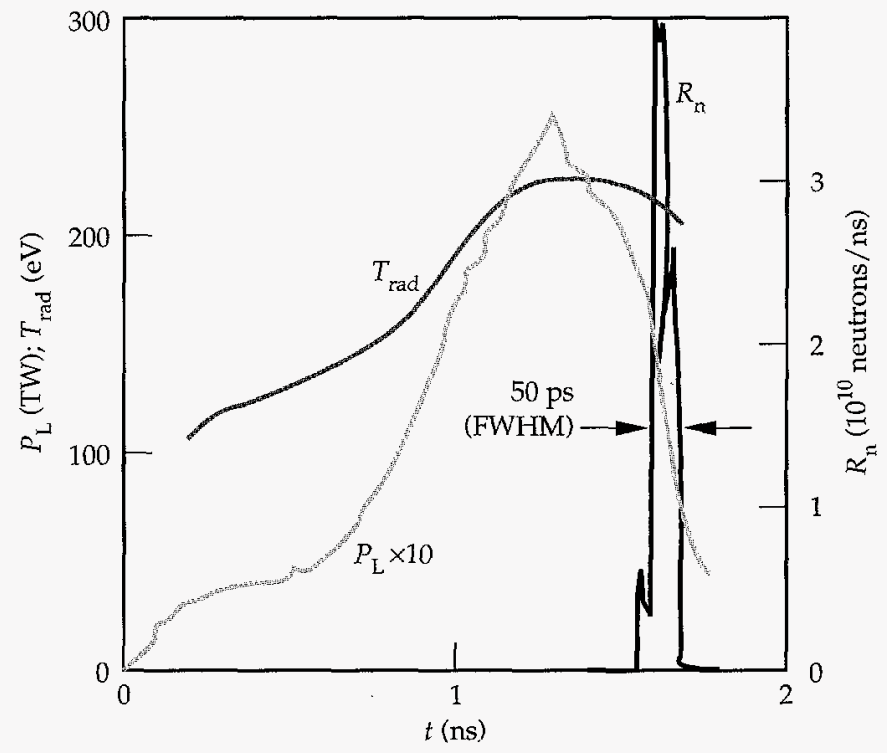

FIGURE 2. Observed laser power $P_{\mathrm{L}^{\prime}}$, hohlraum temperature $T_{\text {rad }}$ and neutron production rate $R_{\mathrm{n}}$ for 100 -atm DT-filled capsules. (50-05-0695-1563pb01)

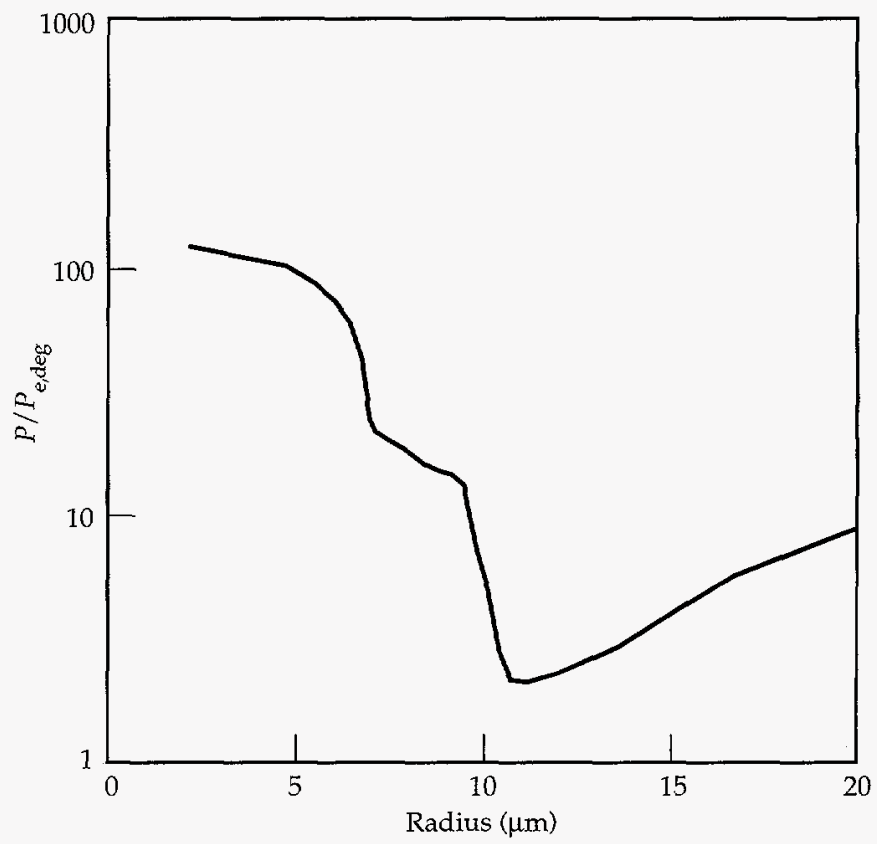

FIGURE 3. Ratio of pressure to electron degeneracy pressure in fuel and pusher of 100-atm fill capsules at bang time as calculated with the Haan mix model. The dense glass pusher, from 10 to $15 \mu \mathrm{m}$, is in a good, low-entropy state. $\quad(50-05-0695-1564 \mathrm{pb} 01)$ 
of radius through the fuel and pusher at the time of peak neutron production (bang time) in a model calculation of the implosion of a 100-atm DT-filled capsule. Figure 3 shows three more or less distinct regions: clean fuel $(0-7 \mu \mathrm{m})$, mixed fuel and glass $(7-10 \mu \mathrm{m})$, and dense glass pusher $(10-15 \mu \mathrm{m})$. The pusher has been compressed along a good low-entropy $P(\rho)$ trajectory, or adiabat, so that its pressure is only a factor of about two above the Fermi degeneracy pressure. This capsule has the sort of compressed configuration-isobaric with a hot spot surrounded by colder and denser matter-described in the introduction for a high-gain capsule implosion.

$X$-ray drive asymmetry is the second factor limiting convergence. The hohlraum does not produce a perfectly uniform drive distribution, but any position on the capsule "sees" radiation from all the various elements of the hohlraum (directly illuminated wall regions, indirectly illuminated regions, and entrance holes), so higher moments of the drive distribution are strongly smoothed. (Typically the drive symmetry is analyzed in terms of spherical harmonics, which reduce to Legendre polynomials $P_{l}$ with cylindrical symmetry.) The remaining systematic asymmetry is controlled by choosing the relative values of capsule radius, hohlraum dimensions, and the first bounce position of the laser beams along the hohlraum wall to minimize the $P_{2}$ and $P_{4}$ effects. $P_{1}$ and $P_{3}$ effects are eliminated by the left-right symmetry of the hohlraum. The remaining lower-moment asymmetry is time-dependent: both the albedo of the wall and the effective positions of the laser spots change as hot wall material moves into the hohlraum. By design with simulations, the configuration used in this work produces a $P_{2}$ asymmetry that changes sign, is at most $8 \%$, and averages to a very low value. Details of hohlraum design are being reported in "Nova Symmetry: Experiments, Modeling, and Interpretation (HLP3 and HLP4)" on p. 293 of this Quarterly.

A second source of time-dependent drive asymmetry is random variations due to imprecise laser beam-tobeam power balance and pointing. This is minimized by precise control of the laser. ${ }^{11,12}$ We maintain tolerances of $8 \%$ rms beam-to-beam power balance during the foot of the laser pulse and $4 \%$ power balance during the peak. Pointing tolerance is $\pm 30 \mu \mathrm{m}$ rms. This control gives a power balance on the capsule that from simulations is uniform to within $2 \% \mathrm{rms}$ at peak power and $4 \%$ in the foot. Early experiments demonstrated that this level of power balance is necessary for high convergence. A version of Figure 6 with the early data showed convergence declining as fill was reduced.

Finally, since the fuel is compressed at stagnation by a denser pusher, convergence may be limited by RT instability at the fuel-pusher interface, which leads to what is commonly called mix. Calculations show that perturbations on the interface are primarily seeded by the feed-through of growing perturbations at the ablation front, which in turn are seeded by initial ablator surface finish perturbations. We have applied Haan's multimode, moderately nonlinear mix model ${ }^{9}$ to calculations of the implosions of the 25- and 100-atm fill capsules. In this model, growth factors (final amplitude at fuel-pusher interface/initial amplitude at ablator surface) are calculated at a number of $l$-modes, initial amplitude $\propto P_{l}(\cos \theta)$, for perturbations small enough that the growth factors remain linear in the initial amplitudes. We then obtain an estimate of the rms depth of mix penetration by multiplying the initial surface-finish mode spectrum by the growth factors, applying a saturation model in which nearby modes contribute to the saturation of a particular mode, and then adding the saturated mode amplitudes in quadrature. There is an empirical parameter in the saturation model, which Haan calls $v$, that must be estimated from mix experiments. (In the presence of a dense spectrum of modes, mode $l$ has a saturation amplitude at radius $R_{0}$ of $v R_{0} / l^{2}$.) Haan's current best estimate of this parameter is $v=2$, which is the value we have used in our analysis.

Figure 4 shows the linear growth factors we calculated. At low $l$ values (long wavelengths), the growth factors are comparable for the 25- and 100-atm capsules, whereas at higher $l$ values the 100 -atm capsules have much larger growth factors. This is a reflection of much more feed-through in the 100 -atm case. When the first shock breaks out of the back of the glass pusher into the fuel, there is less decompression in the 100-atm case, leading to a thinner, denser pusher during inward acceleration. Were our pulse shape more highly

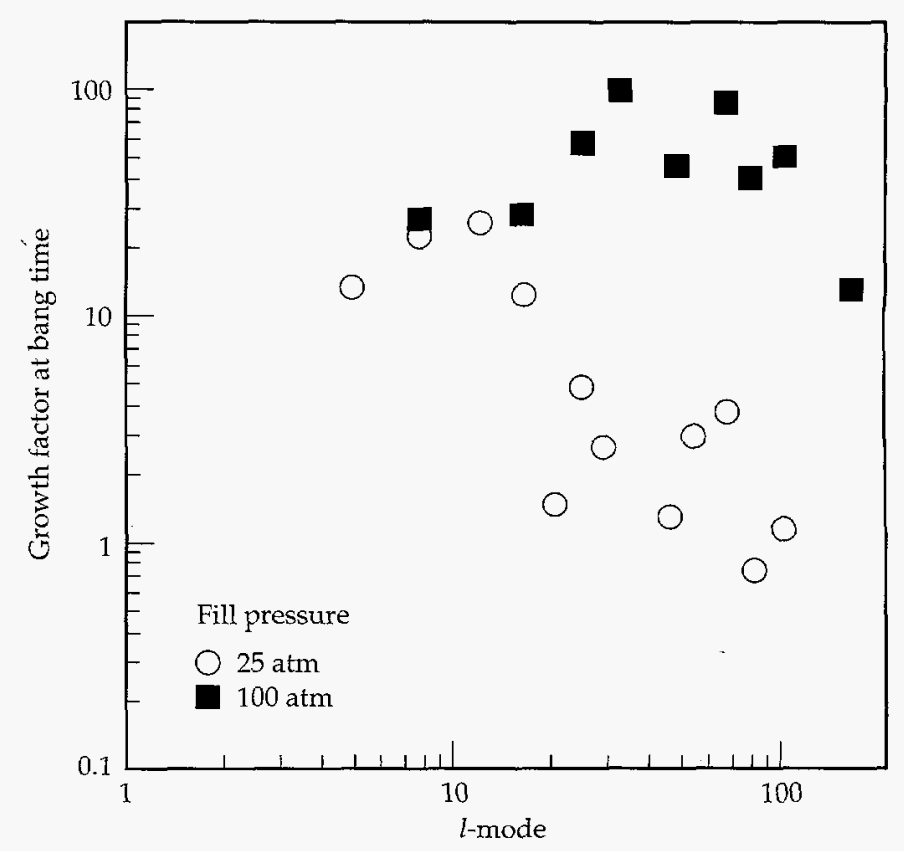

FIGURE 4. Calculated growth factors for surface perturbations on 25- and 100-atm fill capsules. (50-05-0695-1565pb01) 
tuned, the 25-atm shells would have been thinner, but then mix and shell breakup might have destroyed their convergence altogether. As it is, with saturation applied, most of the quadrature sum of amplitudes at bang time comes from $l \leq 20$ for either fill pressure.

One way of expressing the depth of mix penetration is in terms of the distance from the fuel-pusher interface to the fall line, which is the trajectory the interface would have if it never decelerated at stagnation. For our capsules, the Haan model gives a bang-time mix depth-defined as the height of bubble tops above the interface- of about $35 \%$ of the distance to the fall line. Mix depth is not a constant with time, but rather it peaks at about that fraction near bang-time. Were it a constant, we would have another, simpler mix model, known as a fracmix model. In either model, the spike penetration - the distance from interface to spike tips-is estimated at $(1+\alpha)$ times the bubble height, where $\alpha$ is the interface Atwood number.

\section{Measuring Convergence}

We determined burn-averaged fuel density and capsule convergence by measuring burn-averaged fuel areal density $\rho R \equiv \int_{\text {fuel }} \rho(r) d r$. If $\rho$ is uniform, as we assume, then $\rho=\rho_{0}\left(\rho R / \rho_{0} R_{0}\right)^{3 / 2}$, so a determination of $\rho R$ gives both $\rho$ and $R$. When we make comparisons later with calculations of $\rho$ and $R$, we shall calculate those quantities in exactly the same way, with $\rho R$ determined from the calculated secondary neutron

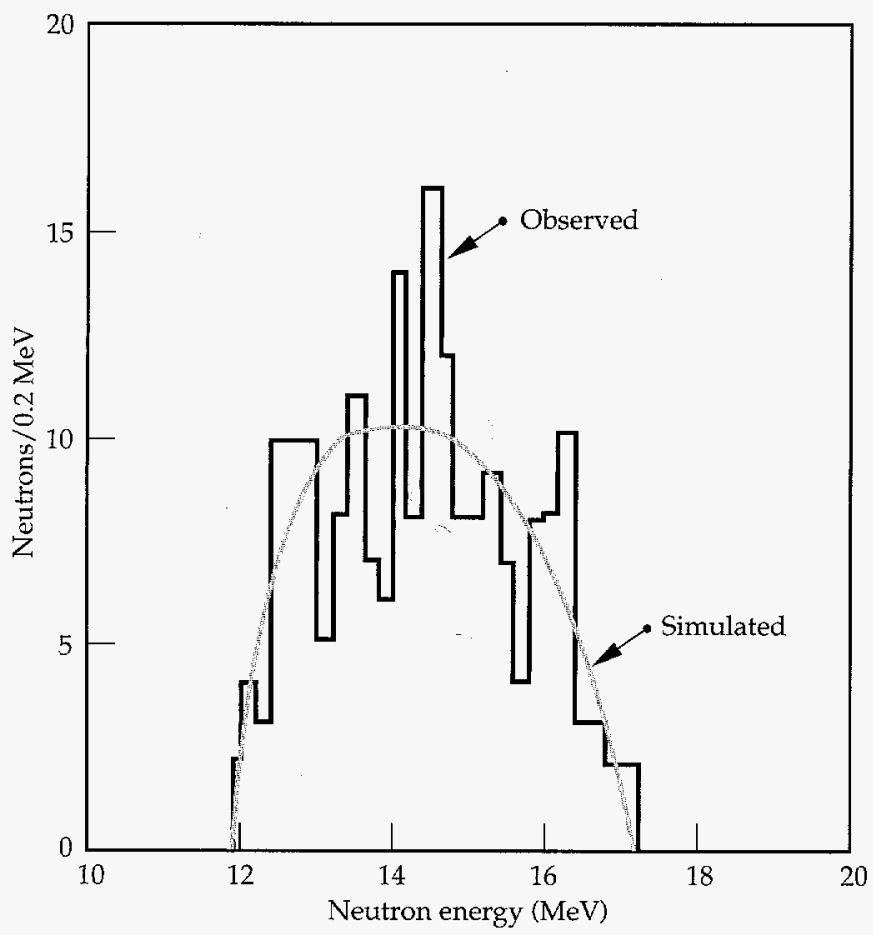

FIGURE 5. Secondary-neutron energy spectrum measured with array of neutron time-of-flight detectors (LaNSA). Observed is sum of spectra from all 25-atm capsules. (50-05-0695-1566 pb01) spectrum as described below for the experimental analysis. In fact, the uniform- $\rho$ assumption gives a slight underestimate of the actual convergence and density because the actual density must increase with radius as the temperature decreases. From simulations, this is about a $15 \%$ effect in the density for the 25-atm capsules and about a $25 \%$ effect in the 100-atm capsules.

We measured fuel $\rho R$ by the secondary-neutron technique. ${ }^{13-17}$ This technique relies on the observation of $12-17-\mathrm{MeV}$ secondary neutrons produced via the $D(T, n)^{4}$ He reaction in an initially pure $D$ fuel. The $1.01-\mathrm{MeV}$ tritium nuclei, or tritons, are produced in the primary fusion reaction $D(D, p) T$. If the tritons do not slow significantly as they traverse the fuel, then the fraction of tritons producing secondary neutrons is proportional to fuel $\rho R$. For the fuel conditions in this work, (low temperature with mixed pusher material), $\rho R$ values above a few $\mathrm{mg} / \mathrm{cm}^{2}$ cause significant triton slowing, and corrections must be made for the energy dependence of the $\mathrm{D}(\mathrm{T}, \mathrm{n})^{4} \mathrm{He}$ cross section. Cable and Hatchett ${ }^{13}$ have outlined how this can be done based on a measurement of the secondary-neutron energy spectrum. Since the cross section rises with decreasing triton energy, this correction typically results in a $\rho R$ value lower than that calculated for the case of little slowing. We measured the secondary-neutron energy spectrum with an array of neutron time-of-flight detectors (LaNSA). ${ }^{18}$ Figure 5 shows a spectrum obtained by summing all the 25-atm capsule data; the figure also shows the spectrum obtained from calculations of these implosions with the Haan mix model, as discussed further below.

Observed fuel areal densities, which ranged up to $16 \mathrm{mg} / \mathrm{cm}^{2}$, allowed us to determine the densities and convergences plotted in Fig. 6. For this figure, observed values were averaged over several implosions (two at $200 \mathrm{~atm}$, six at $100 \mathrm{~atm}$, and ten at $25 \mathrm{~atm}$ ), and the errors were dominated by statistics related to the number of the observed secondary neutrons. Figure 6 shows that the observed values are consistent with or better than those expected from simulations if the effects of fuel-pusher mixing are included at the level that current models ${ }^{9}$ predict given the capsules' surface finish. (The calculations labeled "clean 1-D" include no mix effects and assume perfect spherical symmetry; this is physically unrealistic but is commonly quoted as an "ideal" limit). Fuel-pusher mixing introduces two important effects: mixing of high-Z matter into the fuel enhances the triton slowing, and mixing of fuel outward into the pusher decreases the fuel convergence. Secondary-neutron spectroscopy allows us to quantify these effects since the secondary-neutron energy spectrum is dependent on the rate of the triton slowing. Figure 5 shows that the calculated and observed secondary spectra are in good agreement, which further supports the validity of the mix modeling. 


\section{Other Diagnostics and Discussion}

We determined primary neutron yield ${ }^{19}$ and pusher areal density ${ }^{20}$ by activation techniques. We determined burn duration and burn time relative to the start of the laser pulse with a scintillator/streak camera arrangement ${ }^{21}$ capable of measuring neutron production as a function of time with 20-ps resolution. Figure 2
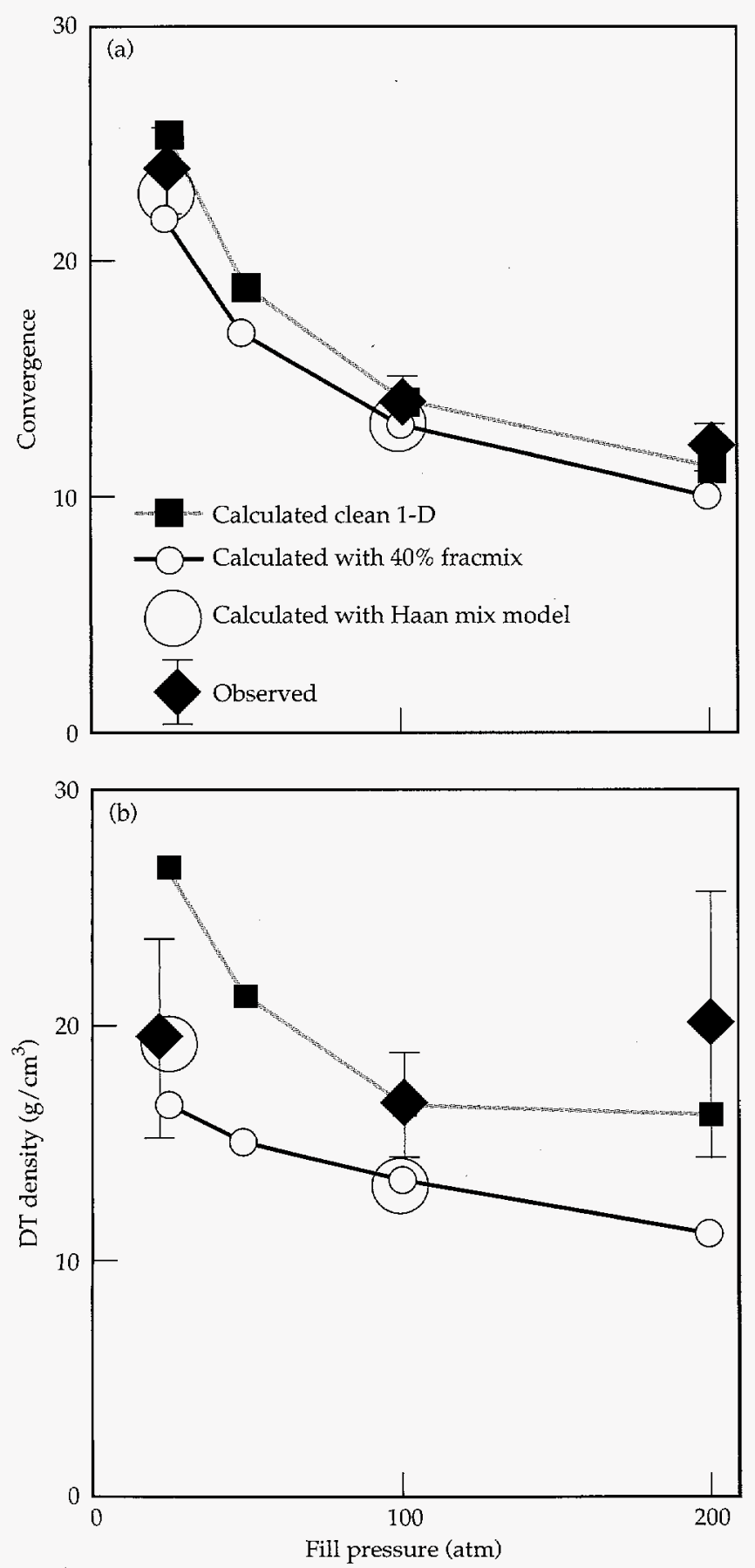

FIGURE 6. Observed and calculated (a) convergence and (b) density vs capsule fill pressure. Density is expressed as equivalent DT fill even when capsule is D-filled. (50-05-0695-1567pb01) shows an example of the measured reaction rate. We measured fuel ion temperature by observing the temperature-dependent Doppler broadening of the primaryneutron energy spectrum with a neutron time-of-flight system ${ }^{22}$ that was simpler and separate from that used for secondary-neutron spectroscopy.

Primary-neutron yields for these implosions were $2 \times 10^{7}$ to $1.4 \times 10^{8}$ with pure D fill (2.45-MeV neutrons) and $3 \times 10^{8}$ to $3 \times 10^{9}$ with equimolar DT fill (14-MeV neutrons); the lower yields were observed for the higher-convergence, lower-fill capsules. Figure 7 shows the individual capsule yields and $\rho R$ values, with the yield expressed as a fraction of that calculated for a clean, 1-D implosion. Also shown for comparison are the values calculated for these implosions with the Haan mix model.

The scatter in $\rho R$ is consistent with the measurement error, but scatter in the yield is larger since yield is very sensitive to the fuel ion temperature (roughly

(a) 100-atm capsules

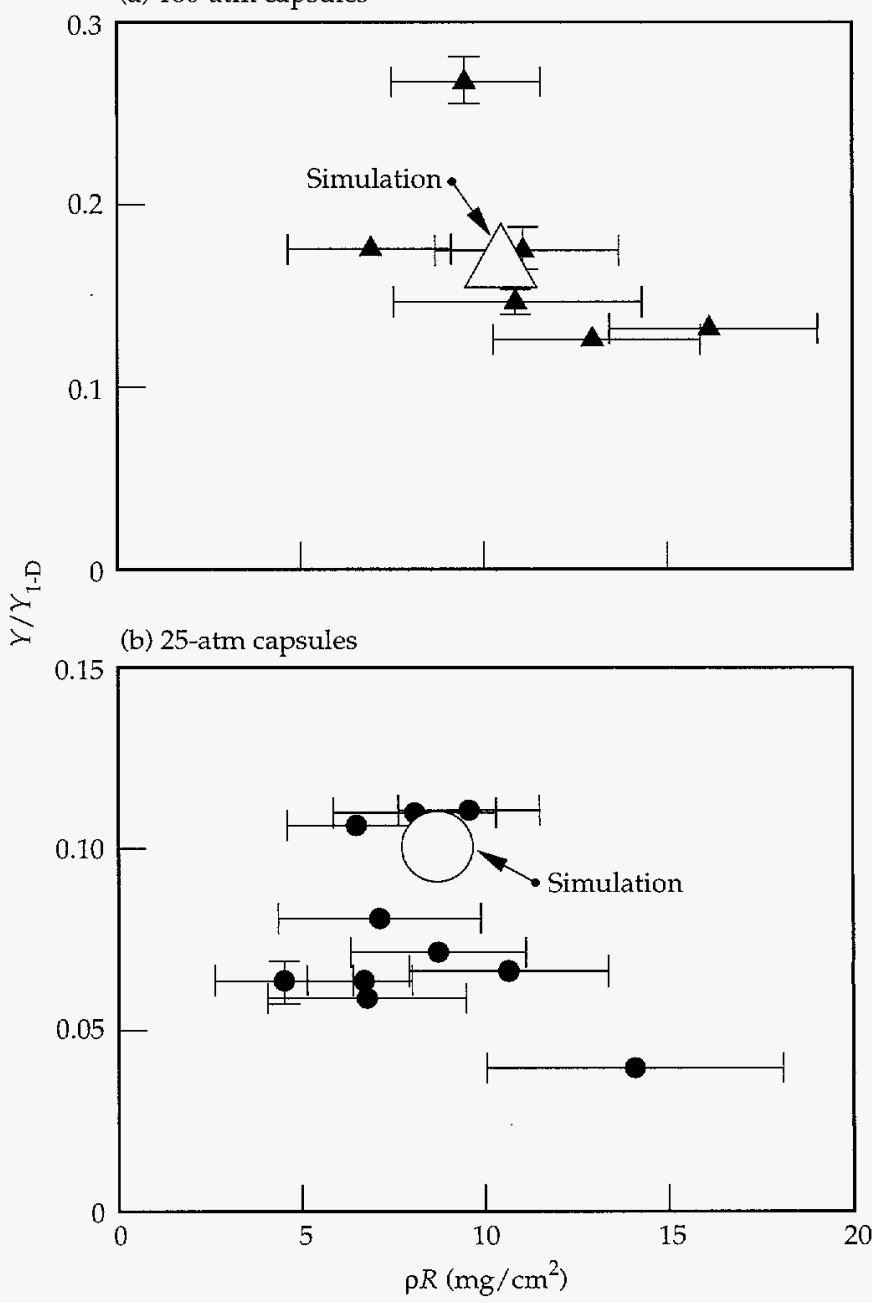

FIGURE 7. Observed neutron yields and $\rho R$ values for (a) 100 -atm and (b) 25-atm capsules. Yields are plotted as a fraction of simulated clean 1-D yield. $\quad(50-05-0695-1568 \mathrm{pb01})$ 
proportional to $T^{5}$ for this temperature range) and because the fuel temperature is affected by small variations in capsule surface finish, capsule dimensions and laser energy. (The yield would be much less sensitive to mix if the glass pusher were replaced by a layer of cryogenic liquid DT, as it is in most current ignitionscale capsule designs.)

Fuel ion temperatures were $0.9 \pm 0.4 \mathrm{keV}$ for all cases; at this temperature, the observed fuel density corresponds to a final fuel pressure of 16 Gbar.

The glass shell $\rho R$ was $73 \pm 16 \mathrm{mg} / \mathrm{cm}^{2}$ (100 atm) and $60 \pm 19 \mathrm{mg} / \mathrm{cm}^{2}(25 \mathrm{~atm})$ in a pair of shots at each fill. These values are about $1 \sigma$ from the simulated values of 54 and $81 \mathrm{mg} / \mathrm{cm}$, respectively. In the 25 -atm simulations, the peak burn-time glass density is $160 \mathrm{~g} / \mathrm{cm}^{3}$.

Burn duration for the 100-atm capsules was $50 \pm 15$ ps; burn occurred at $1600 \pm 100$ ps after the start of the laser pulse (see Fig. 2); simulations gave 33-ps burn duration occurring at $1603 \mathrm{ps}$. We see in both simulations and measurements from a separate, brief experimental series that shock breakout, which corresponds to initial fuel movement, does not occur until 1 ns after the start of the laser pulse. This gives an average implosion velocity of $1.4 \times 10^{7} \mathrm{~cm} / \mathrm{s}$; simulations show that peak velocity is $1.8 \times 10^{7} \mathrm{~cm} / \mathrm{s}$. Using the observed fuel density and burn duration, we obtain a confinement parameter of $n \tau=1.9 \pm 0.6 \times 10^{14} \mathrm{~s} / \mathrm{cm}^{3}$.

\section{Summary and Conclusion}

We have done a series of indirectly driven highconvergence implosions with the Nova laser fusion facility. These implosions were well characterized by a variety of measurements, and computer models are in good agreement with the measurements. We measured the imploded fuel areal density using a technique based on secondary-neutron spectroscopy. At capsule convergence ratios of 24 , comparable to what is required for the hot spot of ignition-scale capsules, these capsules achieved fuel densities of $19 \mathrm{~g} / \mathrm{cm}^{3}$. Independent measurements of density, burn duration, and ion temperature gave $n \tau \theta=1.7 \pm 0.9 \times 10^{14} \mathrm{keV}-\mathrm{s} / \mathrm{cm}^{3}$.

These experiments, which used better diagnostic techniques than previous work, have allowed detailed comparisons with simulations and have permitted a deeper understanding of the sensitivity of the implosion process to factors such as laser power balance. These implosions have provided an integrated test of our ability to control and model the implosion dynamics enough to achieve convergence ratios comparable to those required for the hot spot of an ignition-scale capsule.

\section{Notes and References}

1. J. Nuckolls, et al., Nature 239, 139 (1972).

2. J. D. Lindl, R. L. McCrory, and E. M. Campbell, Physics Today 45, 32 (1992).

3. F. J. Marshall, et al, Phys. Rev. A 40, 2547 (1989).

4. H. Azechi, et al., Laser Part. Beams 9, 193 (1991).

5. J. D. Kilkenny, et al., Plasma Physics and Controlled Nuclear Fusion Research (International Atomic Energy Agency, Vienna, 1989), vol. 3, p. 29.

6. H. Nishimura, et al., Plasma Physics and Controlled Nuclear Fusion Research 1992 (IAEA, Vienna, 1993), vol. 3, p. 97.

7. G. B. Zimmerman and W. L. Kruer, Comments Plasma Phys. Controlled Fusion 11, 51 (1975).

8. H. N. Kornblum, R. L. Kauffman, and J. A. Smith, Rev. Sci. Instrum. 57, 2179 (1986).

9. S. Haan, Phys. Rev. A 39, 5812 (1989).

10. S. P. Hatchett, Ablation Gas Dynamics of Low-Z Materials Illuminated by Soft X Rays, Lawrence Livermore National Laboratory, Livermore, CA, UCRL-JC-108348 (1991).

11. J.A. Caird, R.B. Ehrlich, O.L. Landen, et al., ICF Quarterly Report 4(1), 10-17, Lawrence Livermore National Laboratory, Livermore, CA, UCRL-LR-105821-94-1 (1994).

12. J.E. Murray, M.C. Rushford, C.S. Vann, et al., ICF Quarterly Report 4(1), 18-24, Lawrence Livermore National Laboratory, Livermore, CA, UCRL-LR-105821-94-1 (1994).

13. M. D. Cable and S. P. Hatchett, J. Appl. Phys. 62, 2223 (1987).

14. E. G. Gamalii, et al., JETP Lett. 21, 70 (1975).

15. T. E. Blue and D. B. Harris, Nucl. Sci. Eng. 77, 463 (1981).

16. H. Azechi, et al., Appl .Phys. Lett. 49, 555 (1986).

17. M. D. Cable, et al., Bull. Am. Phys. Soc. 31, 1461 (1986)

18. M. B. Nelson and M. D. Cable, Rev. Sci. Instrum. 63, 4874 (1992).

19. S. M. Lane, et al., Lawrence Livermore National Laboratory, Livermore, CA, UCRL 50021-86 (1986).

20. S. M. Lane and M. B. Nelson, Rev. Sci. Instrum. 61, 3298 (1990).

21. R. A. Lerche, D. W. Phillion, and G. L. Tietbohl, in Proceedings of SPIE 2002, P. W. Roehrenbech, Ed. (San Diego, 1993), p. 153.

22. T. J. Murphy and R. A. Lerche, Rev. Sci. Instrum. 63, 4883 (1992). 


\title{
Planar and Cylindrical Rayleigh-Taylor EXPERIMENTS ON NOVA (HEP2)
}

\author{
B. A. Remington \\ S. V. Weber \\ M. M. Marinak \\ W. W. Hsing \\ N. M. Hoffman
}

\section{Introduction}

A high-density fluid on top of a low-density fluid is Rayleigh-Taylor ${ }^{1}$ (RT) unstable. Driven by gravity, random perturbations at the interface between the two fluids will grow: fingers ("spikes") of the heavier fluid will poke through the lighter fluid, and bubbles of the lighter fluid will rise into the heavier fluid. The RT instability and its shock-driven analog, the RichtmyerMeshkov $^{2}$ (RM) instability, have been a focus of research in inertial confinement fusion (ICF) for some time. ${ }^{3-19}$ In ICF, the driver-laser light, $x$ rays, or ions--heats the outer layer of the capsule wall, causing it to ionize and expand rapidly. The result is a low-density ablated plasma accelerating the high-density capsule wall ("pusher"). The ablation front is RT unstable, and outer-surface imperfections grow. This growth can seed perturbations at the pusher inner wall, which in turn become RT unstable during deceleration and stagnation. Ultimately, pusher material can mix into the fuel, degrading performance.

The role of the RT instability in ICF can be understood heuristically as follows. The goal of ICF is to maximize the fuel core pressure $P_{\text {core }}$ for a minimum applied (ablation) pressure $P_{\mathrm{a}}$. To see how we might do this, we write 20

$\frac{P_{\text {core }}}{P_{\text {a }}}=10\left(\frac{R_{0}}{\Delta R}+1\right)\left(\frac{R_{0}}{R_{s}}\right)^{0.9}$,

where $R_{0} / \Delta R$ is the capsule aspect ratio (the ratio of initial shell radius to shell thickness) and $R_{0} / R_{s}$ the convergence ratio [the ratio of outer capsule radius to final compressed hot fuel radius (the "hot spot" radius)]. The $\left[\left(R_{0} / \Delta R\right)+1\right]$ factor results from converting the kinetic energy of the pusher into pressure at stagnation, by using Bernouilli's theorem, i.e., $P_{\text {stag }}=P_{\mathrm{a}}+(1 / 2) \rho v^{2}$. The $\left(R_{0} / R_{\mathrm{S}}\right)^{0.9}$ factor results from the pressure multiplication due to the spherically imploding shock wave and is based on the self-similar solutions of Guderley. ${ }^{20}$
From Eq. (1), we immediately see that maximum pressure amplification occurs for high-aspect-ratio capsules with a high convergence ratio.

The RT instability limits the aspect ratio, however, as we see from the following. In the linear regime, perturbation growth is exponential in time,

$\eta=\eta_{0} \int \gamma d t$

where the exponent $\int \gamma d t$ represents growth in terms of perturbation $e$-foldings. A dispersion curve for the RT growth rate $\gamma$ can be written as 8

$\gamma \approx\left(\frac{k g}{1+k L}\right)^{1 / 2}-\beta k v_{\mathrm{a}}$,

where $k=2 \pi / \lambda$ is the perturbation wave number, $g$ is the pusher acceleration, $L=\rho / \Delta \rho$ is the density gradient scalelength at the ablation front, $\beta$ is a multiplier usually set in the range of 1 to $3, v_{\mathrm{a}}=\dot{m} / \rho$ is the ablation velocity, $\dot{m}$ is the mass ablation rate per unit area, and $\rho$ is a characteristic density at the ablation front. If we assume (1) a constant acceleration over a distance $S \approx R_{0} / 2$, (2) that $80 \%$ of the pusher is ablated over this distance $\left(\int v_{a} d t \approx 0.8 \Delta R\right.$ ), and ( 3 ) a density gradient scale length that is 10 to $20 \%$ of the shell thickness ( $L=\alpha \Delta \mathrm{R}$, with $\alpha=0.1-0.2$ ), then we can approximate the perturbation $e$-foldings as

$$
\int \gamma d t \approx\left(\frac{\ell}{1+\alpha \ell \frac{\Delta R}{R}}\right)^{1 / 2}-0.8 \ell \frac{\Delta R}{R} .
$$

Here we have substituted $k=\ell / R$, where $\ell$ is the mode number of a spherical harmonic. We have chosen $\beta \sim 1$ which is typical of indirect drive. When $\Delta R / R$ is small, Eq. (4) shows that the perturbation $e$-folding is large. One can maximize $R_{0} / \Delta R$, and hence the pressure 
amplification, only to the extent that the RT growth will allow.

The situation for a capsule implosion, illustrated in Fig. 1, can be approximated as $G_{T}=G_{1} f G_{2}$, where the total growth factor $G_{\mathrm{T}}$ has been decomposed into growth at the ablation front or outer surface $G_{1}$, fractional feedthrough $f$ to the inner surface or pusher-fuel interface, and growth at the inner surface $G_{2}$. Experiments to measure mix (and hence $G_{T}$ ) directly in implosions are difficult, typically relying on spectroscopic tracer layers or yield degradation to signal the onset of mix. ${ }^{18,19}$ The dominant source for the total perturbation growth and subsequent mixing, however, is the growth $G_{1}$ of outersurface perturbations during the acceleration phase. This can be measured directly with high precision with face-on experiments in planar geometry. The integral effect, namely, outer-surface growth, feedthrough, and inner-surface growth, can be measured with side-on imaging of cylindrical implosions. The HEP2 campaign comprises these two areas-planar and cylindrical RT experiments.

The evolution of a single-mode perturbation at the ablation front of an accelerated planar foil is expected to have three distinct phases. (1) When the drive first turns on, a strong shock is launched through the foil during compression. The shock front will typically be deformed, bearing the imprint of any initial surface imperfections. The behavior of this perturbed (or "rippled") shock front is dynamically similar to that produced by the RM instability. ${ }^{2}$ Material behind the shock develops a lateral velocity component, moving from regions in which the foil was thinner (initial perturbation valley) towards regions in which the foil was thicker (initial perturbation peak), increasing the areal density modulation. The shape of the shock front is not constant, but evolves with time. ${ }^{21,22}$ The areal density modulation may decrease or even reverse phase, if the foil is thick enough with respect to the perturbation wavelength. (2) After the shock breaks out of the back of the foil (the side away from the drive), and a rarefaction wave returns to the ablation front, the compressed foil accelerates as a unit. Perturbation growth continues, now as a result of the RT instability. The linear regime is defined by $k \eta<<1$, where $k=2 \pi / \lambda$ represents the perturbation wave number and $\lambda$ and $\eta$ are the perturbation wavelength and spatial amplitude. In the linear regime, the perturbation grows exponentially as given in Eq. (2), namely, $\eta(t)=\eta_{0} e^{\gamma t}$, where the growth rate $\gamma$ can be written approximately as in Eq. (3) in the form of a dispersion curve,

$$
\gamma \approx\left(\frac{k g}{1+k L}\right)^{1 / 2}-\frac{\beta k \dot{m}}{\rho} \text {. }
$$

(3) After sufficient growth, $k \eta$ is no longer small and the perturbation enters the nonlinear regime. The shape of the perturbation changes from sinusoidal to "bubble and spike," which corresponds in Fourier space to the generation of higher harmonics. ${ }^{7}$ Within the framework of third-order perturbation theory, ${ }^{23}$ the amplitudes of the perturbation fundamental mode (first harmonic) $\eta_{1}$, second harmonic $\eta_{2}$, and third harmonic $\eta_{3}$ can be written as

$$
\begin{aligned}
& \eta_{1} \approx \eta_{\mathrm{L}}\left(1-\frac{1}{4} k^{2} \eta_{\mathrm{L}}^{2}\right), \\
& \eta_{2} \approx \frac{1}{2} k \eta_{\mathrm{L}}^{2}, \\
& \eta_{3} \approx \frac{3}{8} k^{2} \eta_{\mathrm{L}}^{3},
\end{aligned}
$$

(a) Ablation front $\left(G_{1}\right)$

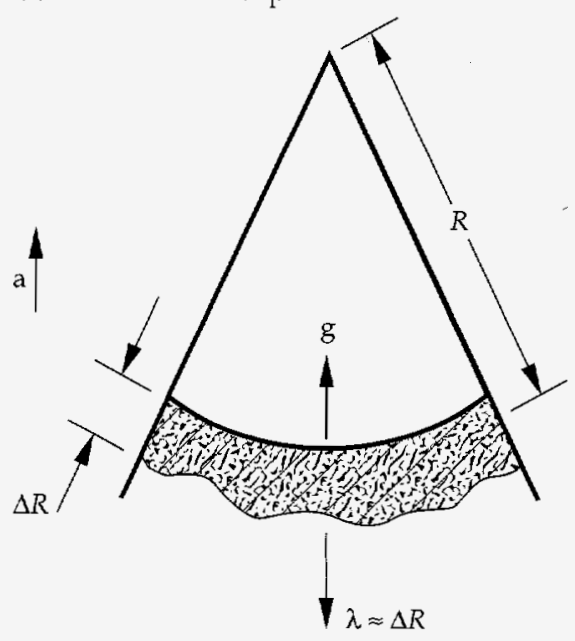

(b) Feedthrough $(f)$

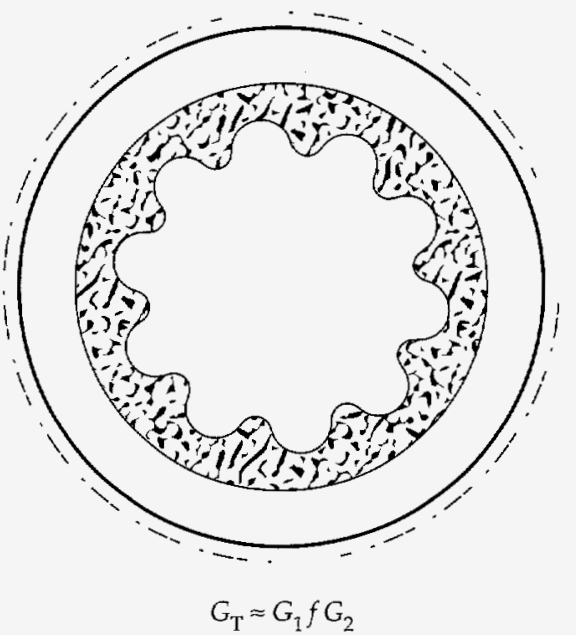

(c) Stagnation $\left(G_{2}\right)$

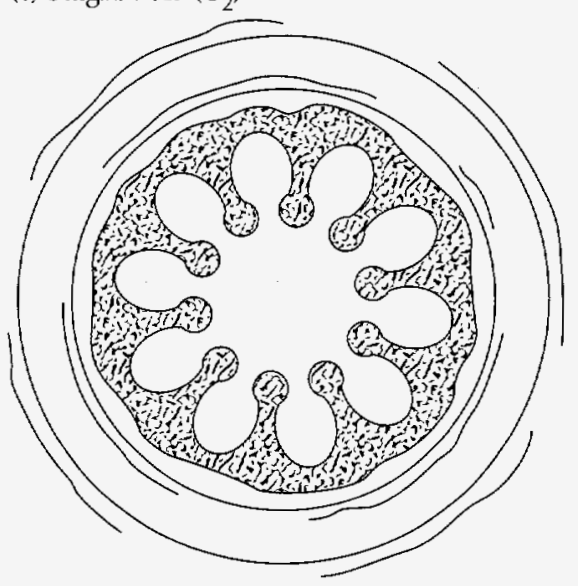

FIGURE 1. Schematic showing how RT perturbation growth affects an implosion. The quantities $G_{\mathrm{T}}, G_{1}, f_{r}$ and $G_{2}$ correspond to the total growth factor, growth factor at the outer surface during acceleration, feedthrough to the inner surface, and growth factor at the inner surface during deceleration. (20-03-1293-4392pb02) 
where $\eta_{L}$ is the linear-regime spatial amplitude given by Eq. (2). The wave numbers of the first three harmonics correspond to $k_{n}=2 \pi n / \lambda, n=1,2,3$. At third order, we see in Eq. (5a) the occurrence of negative feedback to the first harmonic; that is, the growth of the fundamental is decreased. The perturbation growth is said to "saturate." In the asymptotic limit of the nonlinear regime, the bubble amplitude can be written as

$\eta(t)=\int(F g \lambda)^{1 / 2} d t$

which corresponds to a perturbation growing at its terminal bubble velocity, 24

$v_{B}=(F g \lambda)^{1 / 2}$,

where $F=u^{2} / g l$ is the dimensionless Froude number, which depends only on the shape of the perturbation (here $u, g$, and $l$ are characteristic flow velocity, acceleration, and spatial scale, respectively). As derived by Layzer, ${ }^{25} F^{1 / 2}=1 /(6 \pi)^{1 / 2}=0.23$ in two dimensions (2-D) and 0.36 in three dimensions (3-D) for an axisymmetric bubble. If we define the transition into the nonlinear regime as taking place when the growth in the fundamental mode is reduced by $10 \%$, then from Eq. (5a) we have $k^{2} \eta_{\mathrm{L}}^{2}=0.1$, or $\eta_{\mathrm{L}} / \lambda \approx 0.1$, which is a typical and widely used threshold for nonlinearity. This same transition criterion results if one assumes that the onset of nonlinearity occurs when the linear-regime perturbation velocity is equal to the asymptotic nonlinear bubble velocity, namely, $\dot{\eta} \approx v_{\mathrm{B}}$, or $\eta=(\mathrm{kg})^{1 / 2} \eta=(g \lambda / 6 \pi)^{1 / 2}$. Rearranging again leads to $\eta / \lambda \approx 0.1$ at saturation.

In the nonlinear regime, ${ }^{26-31}$ mode coupling leads to the appearance of "beat" modes $k_{i} \pm k_{j}$. To second order, this can be written as

$\eta_{k_{i} \pm k_{j}} \approx \mp \frac{1}{2}\left(k_{i} \pm k_{j}\right) \eta_{k_{i}}^{\mathrm{L}} \eta_{k_{j}}^{\mathrm{L}}$;

Eq. (8) is derived in the Appendix. Mode coupling redistributes a multimode perturbation to longer and shorter wavelengths and affects the saturation of individual modes. If a perturbed interface has a sufficiently dense Fourier composition, it becomes convenient to think of the perturbation in terms of a characteristic wave number $k_{\text {char }}=2 \pi / \lambda_{\text {char }}$ and a characteristic spatial amplitude $\eta_{\text {char. }}$ In these terms, the criterion for the onset of saturation becomes $k_{\text {char }} \eta_{\text {char }}$ no longer being small. Within a continuum model ${ }^{32}$ this leads to individual constituent modes saturating when their amplitudes reach a threshold $S_{k}$ given by

$S_{k}=\frac{v_{2 D}}{L^{1 / 2} k^{3 / 2}}$, in $2-\mathrm{D}$
$S_{k}=\frac{v_{3 D}}{L k^{2}}$, in $3-D$

where $L$ represents the system size and $v$ is a parameter determined by comparison with simulations or data.

We present here the results of an extensive, multiyear experimental and computational study of perturbation growth on planar foils and on imploding cylinders

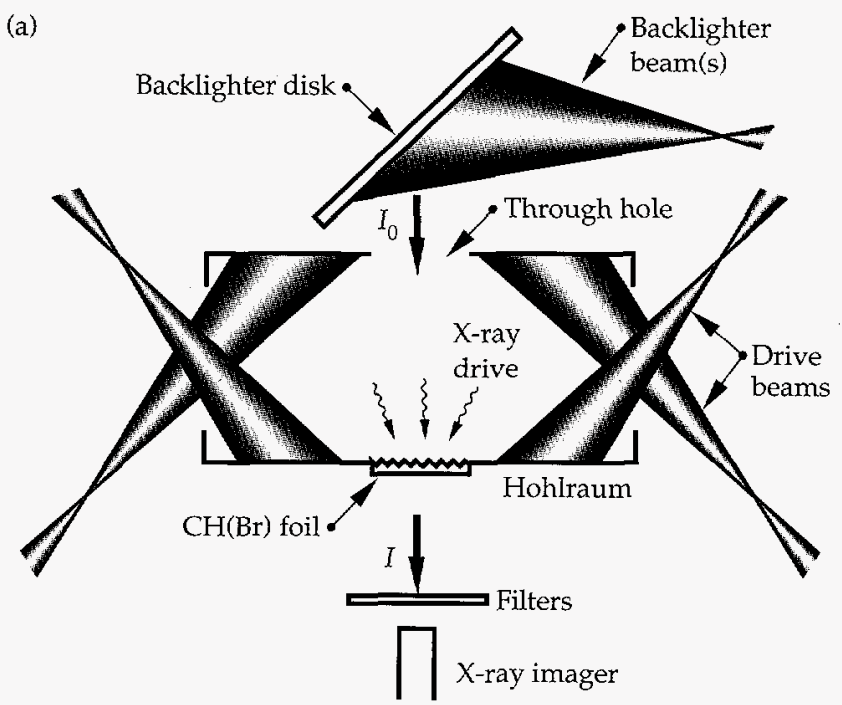

(b)

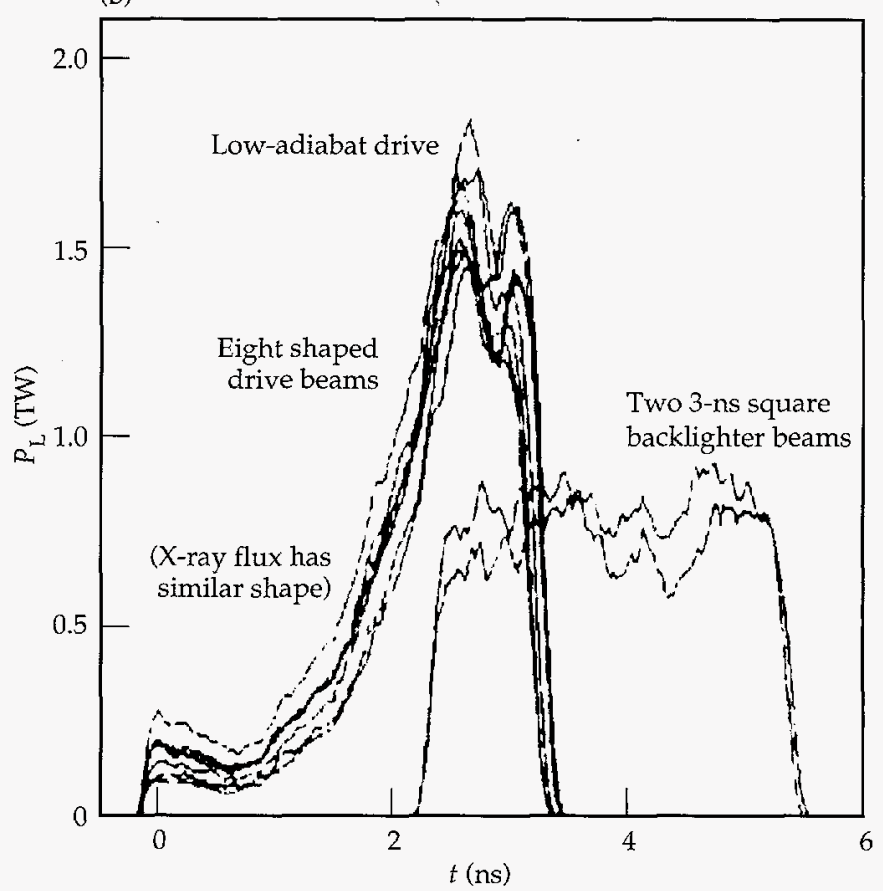

FIGURE 2, (a) The experimental configuration consists of a cylindrical $\mathrm{Au}$ hohlraum ( $3000 \mu \mathrm{m}$ long, $1600 \mu \mathrm{m}$ diam) with the modulated $\mathrm{CH}(\mathrm{Br})$ foil mounted on the wall. (b) In the hohlraum, eight $\lambda=351-\mathrm{nm}$ laser beams are converted to $x$ rays, which ablatively accelerate the foil. Two additional laser beams at $\lambda=528 \mathrm{~nm}$ generate backlighter $x$ rays used for in-flight diagnosis of the foil. $\quad(20-03-1293-4392 \mathrm{pb} 03)$ 
driven with an $x$-ray drive. We investigated initial perturbations of the form

$\eta(x)=\sum_{n=1}^{m} \eta_{n} \cos \left(k_{n} x\right)$,

where $k_{n}=2 \pi n / \lambda$, for $m=1,2$, and 8 . The modes are enumerated as harmonics of the longest repeating pattern. In the next section, we discuss the "Experimental Details," and in "Drive Characterization" we present our drive characterization work. The following sections discuss "Single-Mode Experiments," "Two-Mode Experiments," and "Eight-Mode Experiments." Our 3-D single-mode experiments and simulations are discussed in " $3-\mathrm{D}$ Single-Mode Experiments." The cylindrical experiments are discussed in "The RT Instability in Cylindrical Implosions," and conclusions are given in "Summary." The final section, "Appendix: Amplitude of Coupled Modes," discusses a second-order perturbation model.

\section{Experimental Details}

Figure 2 shows the experimental setup for the planar experiments using a shaped, low-adiabat drive. Sinusoidal surface perturbations are molded onto one side of a planar $750-\mu \mathrm{m}$-diam bromine-doped $\mathrm{CH}$ foil $\left[\mathrm{C}_{50} \mathrm{H}_{47} \mathrm{Br}_{2.7}\right.$, or " $\left.\mathrm{CH}(\mathrm{Br})^{\prime \prime}\right]$ of density $\rho=1.26 \mathrm{~g} / \mathrm{cm}^{3}$. A subset of the experiments were with fluorosilicone $\left(\mathrm{SiOC}_{4} \mathrm{H}_{7} \mathrm{~F}_{3}\right.$ or "FS", at $\left.\rho=1.28 \mathrm{~g} / \mathrm{cm}^{3}\right)$. As shown in Fig. 2(a), the foil is mounted across a hole in the wall of

(a)

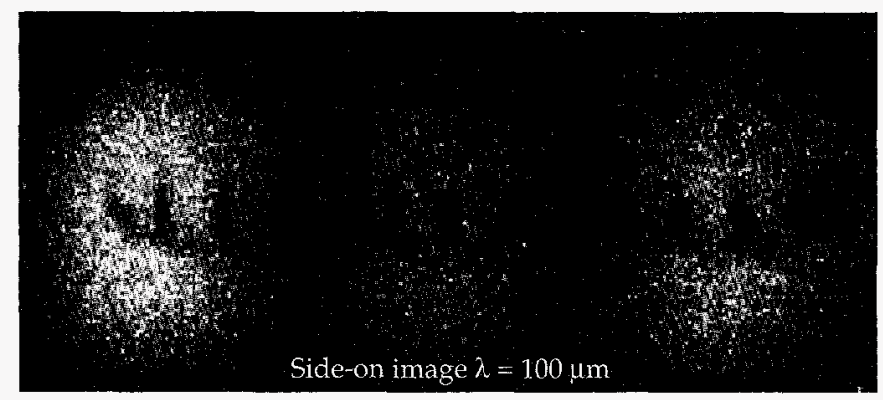

(b)

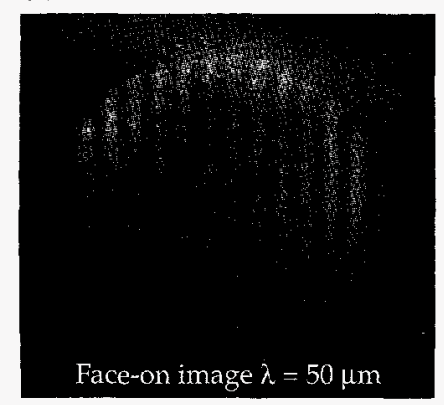

(c)

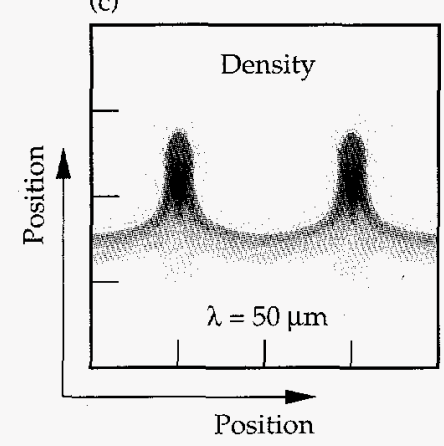

FIGURE 3. Sample images taken (a) in side-on geometry, (b) in faceon geometry, and (c) from 2-D simulations for planar experiments with FS foils. (20-03-1293-4393pb01) a 3000- $\mu \mathrm{m}$-long, 1600- $\mu \mathrm{m}$-diam cylindrical Au hohlraum with the perturbations facing inwards. The foil is diagnosed by back-illumination with an 800 - $\mu$ m-diam spot of $x$ rays created by irradiating a backlighter disk with one or two $\lambda=528$-nm Nova ${ }^{33}$ beams, typically delayed relative to the drive beams, as shown in Fig. 2(b). The modulations in foil areal density cause modulations in the transmitted backlighter x-ray flux, which are recorded as a function of time with gated or streaked $x$-ray imaging diagnostics. This is illustrated in Fig. 3 (taken from Refs. 14 and 15).

Instrumental spatial resolution is most conveniently expressed as the modulation transfer function (MTF), namely, the ratio of observed to actual contrast $[\Delta \ln E$, where $E=$ film exposure]. The MTF for the 22×-magnification grazing-incidence Wölter $x$-ray microscope ${ }^{34}$ used for most of these experiments is given by ${ }^{35-37}$

$M(k)=\frac{1}{1+(k \sigma)^{2}}$,

with $\sigma=6.65 \mu \mathrm{m}$; Fig. 4(a) shows $M$ vs perturbation wavelength. The inverse Fourier transform of $M(k)$ corresponds to an exponential resolution function,

$R(x)=e^{-x / \sigma}$.

Figure 4(a) also shows a curve corresponding to Eq. (10) with $\sigma=8.1 \mu \mathrm{m}$; this is the lowest MTF that is consistent with the data.

Equations (10) and (11) correspond to the "top" sector of the Wölter microscope, which was used for most of the shots in this work. The "west" sector was used in one experiment; its resolution (which is slightly worse than that of the top sector) is given by

$$
R(x)=\frac{1}{1+\alpha}\left[\exp \left(-x / \sigma_{1}\right)+\alpha \exp \left(-x / \sigma_{2}\right)\right]
$$

with $\alpha=0.22, \sigma_{1}=3.5 \mu \mathrm{m}$, and, $\sigma_{2}=18 \mu \mathrm{m}$. Table 1 gives the correspondence between experiment and Wölter sector used. Figure 4(b) shows the MTF for one of the gated x-ray pinhole cameras used for some of these experiments, the FXI ${ }^{38}$ This camera was run at $8 \times$ magnification with $10-\mu \mathrm{m}$ pinholes.

The Wölter microscope is a grazing-incidence $x$-ray optic, so it has a high-energy cutoff in its reflectance. We measured this cutoff on an identical second Wölter optic, ${ }^{36}$ as illustrated in Fig. 5. The solid curve in Fig. 5(a) gives the $x$-ray emission spectrum resulting from electron-beam excitation of a cold $\mathrm{Nb}$ target at $5 \mathrm{kV}$. The dotted curve represents the same spectrum after double reflection off the $\mathrm{x}$-ray optic. The ratio of these two curves (dotted/solid) gives the reflectance, shown in Fig. 5(b). The high-energy cutoff is at $\sim 3 \mathrm{keV}$; the peak reflectance is only about $10 \%$. The smooth dashed curve in Fig. 5(b) is the theoretical double-bounce 
reflectance of an ideal $\mathrm{Ni}$ surface at grazing angles of $1.1^{\circ}$ and $1.2^{\circ}$, multiplied by an overall degradation factor of 0.3 , and represents the reflectance assumed in the post-processing of the simulations for these experiments. The degradation factor is presumably caused by surface roughness and absorption by contaminants that have settled onto the Wölter optic surface.

Figure 6 shows the measured (time-integrated) spectra for $\mathrm{Mo}$, Rh, and Sc, the backlighter materials used in the face-on experiments. On separate shots, the backlighter disks were irradiated with 528-nm light at $I \approx 1 \times 10^{14} \mathrm{~W} / \mathrm{cm}^{2}$. The Mo spectrum [Fig. 6(a)] is dominated by $n=3 \rightarrow 2 \mathrm{~L}-\mathrm{b}$ and emission at $2.4-2.8 \mathrm{keV}$; the $\mathrm{Rh}$ spectrum [Fig. 6(b)] is also dominated by $n=3 \rightarrow 2$ L-band emission, here at $2.8-3.3 \mathrm{keV}$. The Sc spectrum [Fig. 6(c)] is dominated by the $n=2 \rightarrow 1 \mathrm{He}_{\alpha} \mathrm{K}$ lines. These backlighter materials were chosen by considering the total optical depth (OD) of the experimental foils and the response of the recording instruments.
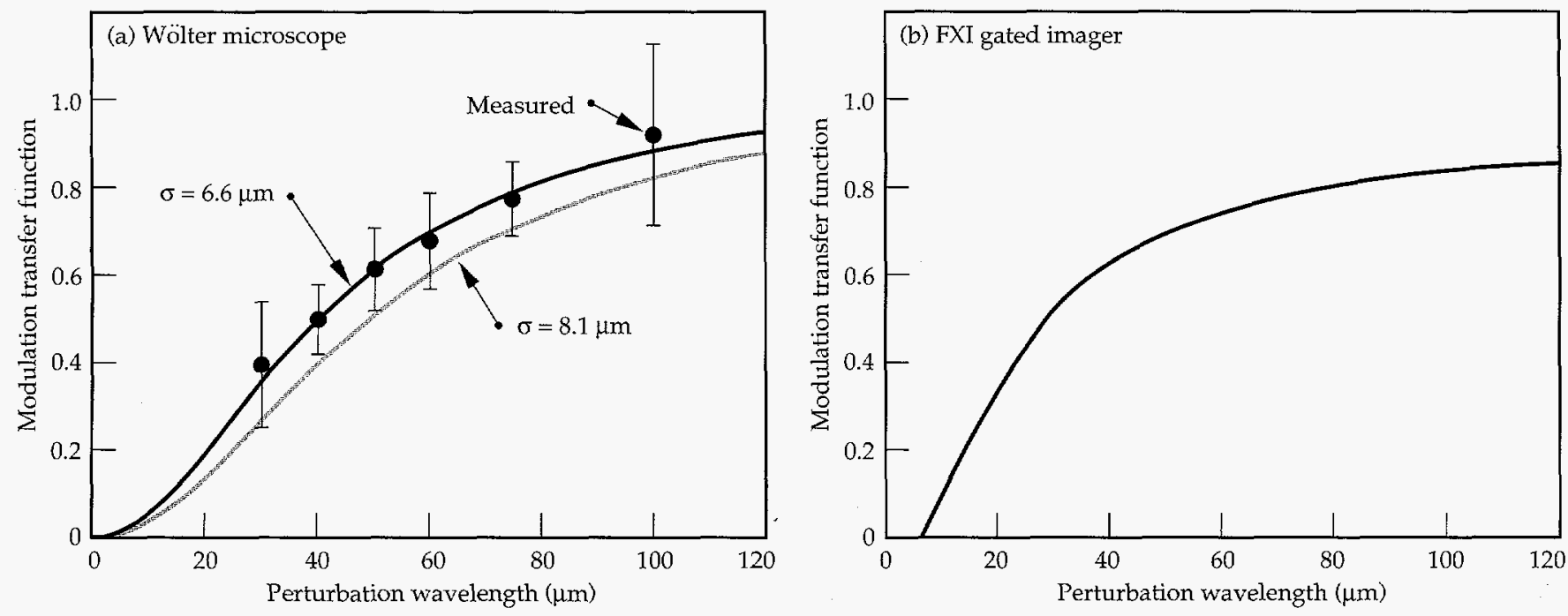

FIGURE 4. (a) Instrument modulation transfer function (MTF) vs perturbation wavelength for the $22 \times$-magnification grazing-incidence Wölter x-ray microscope. The data points represent the measured MTF from the observed $t=0$ contrast from accelerated rippled-foil targets. The data points and error bars for the $\lambda=50 \mu \mathrm{m}$ and $\lambda=75 \mu \mathrm{m}$ perturbations correspond to the means and standard deviations of the deduced MTF from seven and nine separate rippled-foil shots, respectively. The other data points correspond to single shots at each wavelength, and the error bars correspond to the standard deviation assuming that each individual period is independent data. The black curve represents a best fit of the data with the function $M(k)=1 /\left[1+(k \sigma)^{2}\right]$, with $\sigma=6.65 \mu \mathrm{m}$; the gray curved is $M(k)$ for $\sigma=8.1 \mu \mathrm{m}$. (b) MTF for the FXI gated imager at $8 \times$ magnification with 10- $\mu \mathrm{m}$ pinholes and a Sc backlighter. $\quad(20-03-0394-0738 \mathrm{pb03})$

TABLE 1. Compilation of the data for each face-on single-mode shot, using the Wölter $x$-ray microscope. The ten columns in order give (1) foil material, (2) drive pulse shape, (3) perturbation wavelength, (4) the perturbation initial amplitude, (5) the foil thickness, (6) the total laser energy, (7) the sector (top or west) of the Wolter microscope used, (8) the backlighter material used, (9) the observed growth factor, and (10) the predicted growth factor from the LASNEX simulations.

\begin{tabular}{|c|c|c|c|c|c|c|c|c|c|}
\hline Foil & Drive & $\begin{array}{c}\lambda \\
(\mu \mathrm{m})\end{array}$ & $\begin{array}{c}\eta_{0} \\
(\mu \mathrm{m})\end{array}$ & $\begin{array}{r}\text { Thk } \\
(\mu \mathrm{m})\end{array}$ & $\underset{(\mathrm{kJ})}{E_{\text {Laser }}}$ & Sector & Back-lighter & $G_{\text {obs }}$ & $G_{\text {LASNEX }}$ \\
\hline $\mathrm{CH}(\mathrm{Br})$ & Shaped & 100 & 2.4 & 48 & 16.1 & Top & Rh & 3.8 & 5.8 \\
\hline $\mathrm{CH}(\mathrm{Br})$ & Shaped & 70 & 2.4 & 50 & 18.5 & Top & $\mathrm{Rh}$ & 6.5 & 7.3 \\
\hline $\mathrm{CH}(\mathrm{Br})$ & Shaped & 50 & 0.42 & 57 & 17.4 & West & Mo & 19.9 & 24.0 \\
\hline $\mathrm{CH}(\mathrm{Br})$ & Shaped & 30 & 1.5 & 53 & 15.1 & Top & $\mathrm{Rh}$ & 7.2 & 9.7 \\
\hline FS & Shaped & 50 & 4.5 & 57.5 & 19.3 & Top & $\mathrm{Rh}$ & 6 & 4 \\
\hline FS & Shaped & 50 & 0.8 & 56.0 & 16.6 & Top & Rh & 22 & 20 \\
\hline FS & Shaped & 50 & 0.16 & 65.5 & 16.7 & Top & $\mathrm{Rh}$ & 75 & 75 \\
\hline FS & 1 ns sq. & 50 & 2.2 & 34.2 & 13.4 & Top & Dy & 2.9 & 2.8 \\
\hline $\mathrm{CH}$ & $1 \mathrm{~ns} \mathrm{sq}$. & 50 & 2.5 & 59.4 & 13.9 & Top & $\mathrm{U}$ & 1.7 & 1.9 \\
\hline
\end{tabular}




\section{Drive Characterization}

Figure 7 shows the low-adiabat $x$-ray drive used in most of these experiments. ${ }^{14,15,37}$ This drive was generated by focusing eight 351-nm, 2.0-2.4-kJ, 3.3-ns temporally shaped Nova beams into the hohlraum, where they are converted to approximately thermal $x$ rays. The black curve shows the total power of the eight laser beams on a typical shot. The intensity during the first 1.6-1.8 ns of the drive, called the "foot," is about a factor of 10 lower than in the 16-TW peak, which occurs at $2.6 \mathrm{~ns}$. This shaped pulse leads to a lower adiabat and higher compression than if the same total laser energy were delivered in a square pulse. The $\mathrm{x}$-ray drive used in our analysis results from a two- dimensional (2-D) hohlraum simulation ${ }^{39}$ using the experimental laser power $P_{L^{\prime}}$ and is shown as radiation temperature $T_{R}(t)$ by the gray curve in Fig. 7 . This $x$-ray drive has been checked by two independent experimental techniques: (1) shock breakout trajectory through an Al wedge mounted on the hohlraum and viewed with a streaked UV imager, ${ }^{40}$ and (2) acceleratedfoil trajectory using streaked side-on radiography. ${ }^{14,37}$

The resulting $T_{\mathrm{R}}$ profile has a $\sim 95-\mathrm{eV}$ foot increasing to $200 \mathrm{eV}$ in the peak at about $3 \mathrm{~ns}$. This shape mimics, on a short time scale, the early stages of an ignition pulse shape, which typically has a $\sim 90-\mathrm{eV}$ foot followed by a stepped ramp to a $300-\mathrm{eV}$ peak. ${ }^{41}$ (a)

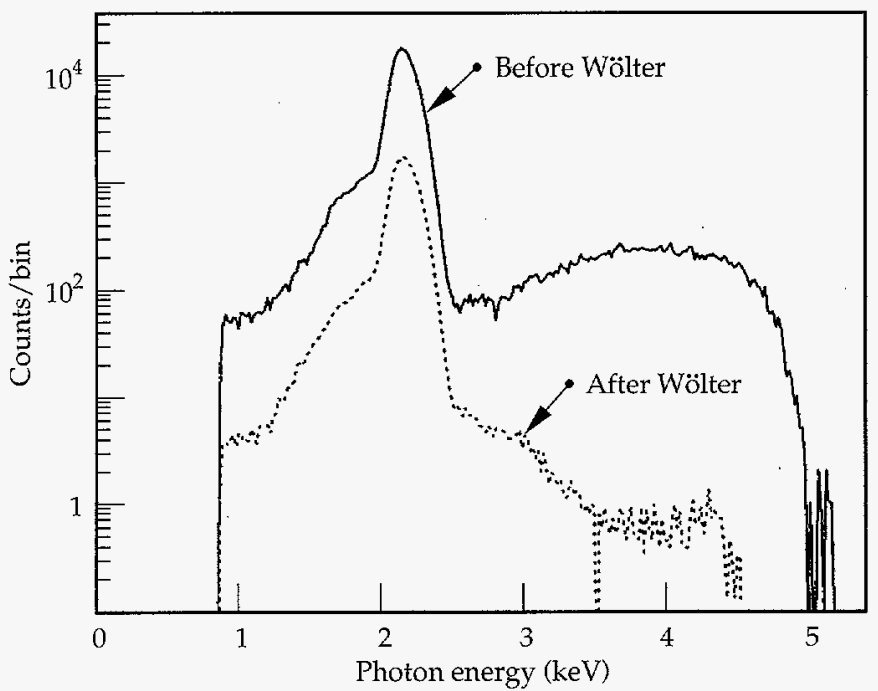

(b)

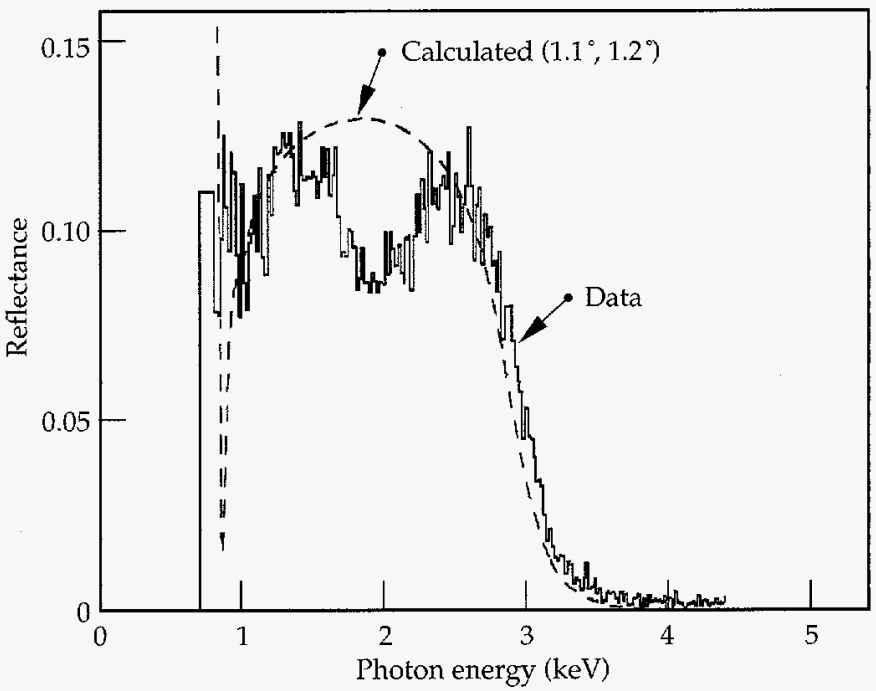

FIGURE 5. Measured Wölter reflectivity vs $\mathrm{x}$-ray energy. In (a) the solid curve corresponds to the x-ray emission spectrum from a $\mathrm{Nb}$ transmission target bombarded by a focused electron beam accelerated across a potential difference of $5 \mathrm{kV}$. The dotted curve is the same except the $x$ rays have undergone two $\sim 1^{\circ}$ grazing-incidence reflections off the Ni surface of the Wölter x-ray optic. (b) The solid histogram gives the ratio (dotted/solid) of the two curves in (a) and corresponds to the Wölter reflectance. The smooth dashed curve represents the calculated reflectance for two ideal Ni surfaces at grazing angles of $1.1^{\circ}$ and $1.2^{\circ}$, with an overall degradation factor of 0.3 . (20-03-0394-0740pb02)

(a) Molybdenum $(Z=42)$

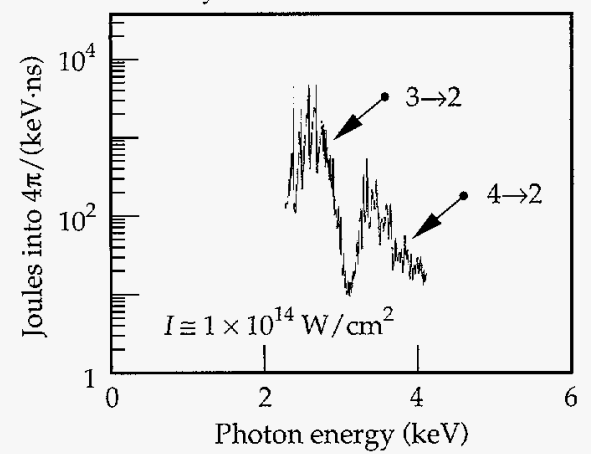

(b) Rhodium $(Z=45)$

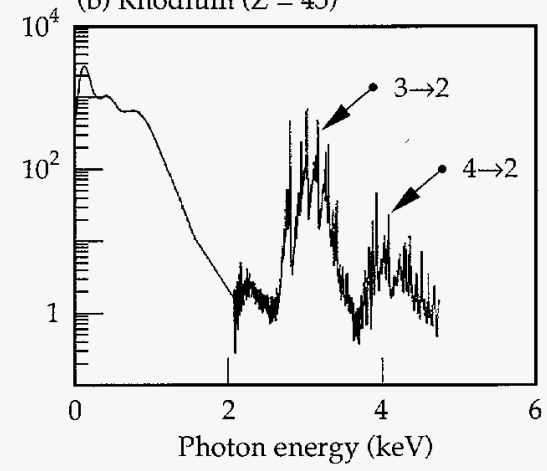

(c) Scandium $(Z=21)$

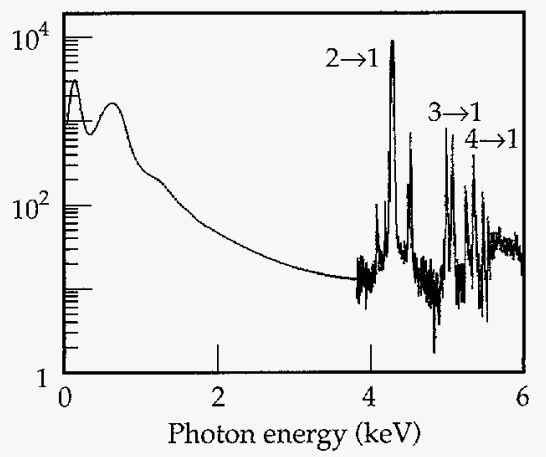

FIGURE 6. Time-integrated spectra for the backlighters used in the face-on experiments. In each case, a single 5 -ns laser beam $(\lambda=528 \mathrm{~nm}$, $I \approx 1 \times 10^{14} \mathrm{~W} / \mathrm{cm}^{2}$ ) was used. The backlighter disks were (a) $\mathrm{Mo},(\mathrm{b}) \mathrm{Rh}$, and (c) Sc. The dominant transitions are marked. The high-energy portion of each spectrum was measured with a static crystal (RAP) spectrometer, and the low-energy portions with a filtered photodiode array. (20-03-0394-0739pb02) 
Figure 8 shows the drive spectrum during the foot and at peak power for the nominal conditions of this investigation. For comparison we also show Planckian spectra corresponding to radiation temperatures $T_{\mathrm{R}}=95 \mathrm{eV}$ during the foot and $T_{R}=200 \mathrm{eV}$ during the peak. The actual drive spectrum used derives from a full hohlraum simulation, ${ }^{39}$ but is not too different from the timedependent combination of a Planckian with the spectrum

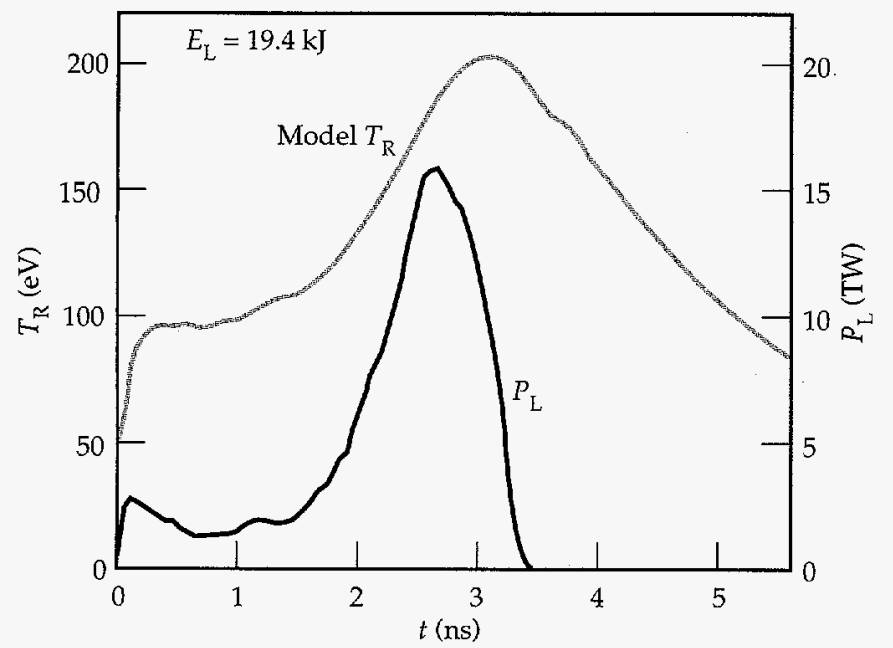

FIGURE 7. Drive expressed in terms of laser power and x-ray radiation temperature. Black curve (scale on right) gives total power vs time of the eight $\lambda=351-\mathrm{nm}$ drive laser beams. The adopted drive temperature $T_{R}(t)$, shown by the gray curve (scale on left), was obtained from a 2-D hohlraum simulation. The first 2 ns represents the "foot"; peak laser power $P_{\mathrm{L}}$ occurs at $2.6 \mathrm{~ns}$; peak $T_{\mathrm{R}}$ occurs at $3 \mathrm{~ns} . \quad(20-03-1293-4397 \mathrm{pb} 02)$

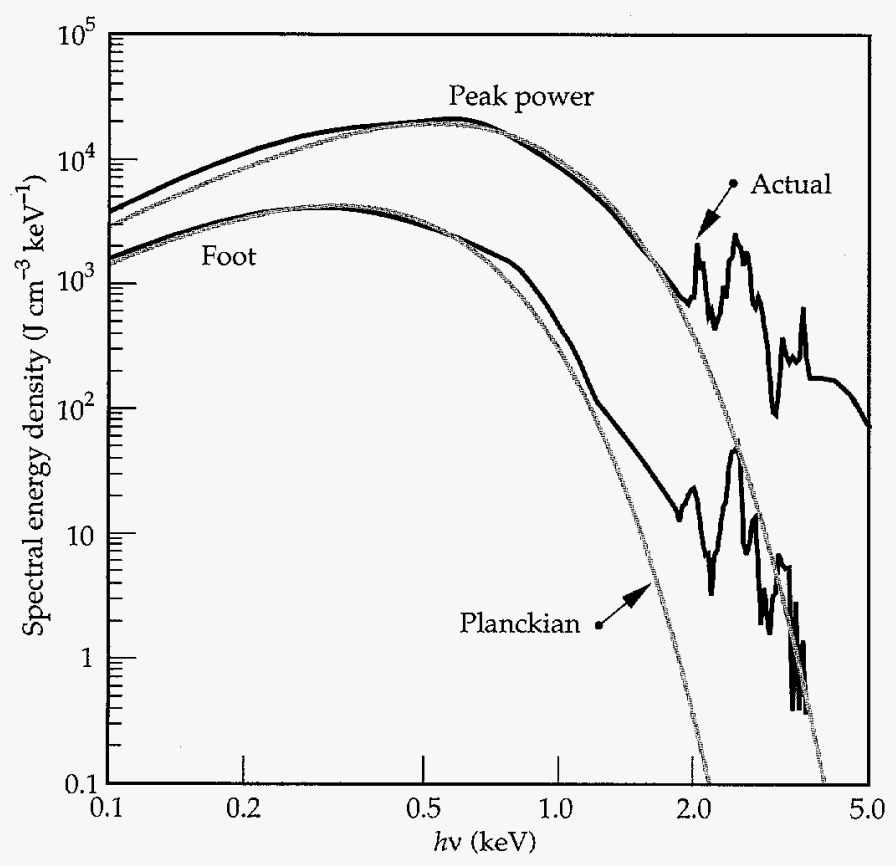

FIGURE 8. Black curves represent nominal drive spectra during the foot and the peak of the drive shown in Fig. 7. Gray curves correspond to Planckian spectra at $T_{\mathrm{R}}=100$ and $200 \mathrm{eV}$. $\quad(20-03-0394-0743 \mathrm{pb02})$ from a planar Au disk illuminated by a shaped $\lambda=351-\mathrm{nm}$ laser pulse. The weighting of the two spectra for this case (shown in Fig. 8) corresponds to the relative solid angles subtended by the foil mounted on the hohlraum wall for the eight laser spots in the hohlraum versus that for the wall area not directly illuminated with laser light. The contribution of the Au M-band emission from the laser spots causes the drive spectrum to be "harder" than a Planckian spectrum.

From the strong-shock relations for ideal fluids, ${ }^{42}$ the shock-front velocity $v_{\mathrm{s}}$ and pressure $P$ behind the shock front are related by $P \approx \rho_{\mathrm{us}} v_{\mathrm{s}}^{2}$, where $\rho_{\mathrm{us}}$ is the density of the unshocked material. From Ref. 43 we can write $P \propto T_{\mathrm{R}}{ }^{7 / 2}$, so we have $T_{\mathrm{R}} \propto P^{2 / 7} \propto v_{\mathrm{s}}{ }^{4 / 7}$. When applied to $\mathrm{Al}$, for example, and after correcting for albedo effects, this becomes ${ }^{40}$

$T_{\mathrm{R}}=\frac{v_{\mathrm{s}}^{0.63}}{80}$

with $T_{\mathrm{R}}$ in $\mathrm{eV}$ and $v_{\mathrm{s}}$ in $\mathrm{cm} / \mathrm{s}$; the numerical factor arises from the equation of state (EOS) of Al. A

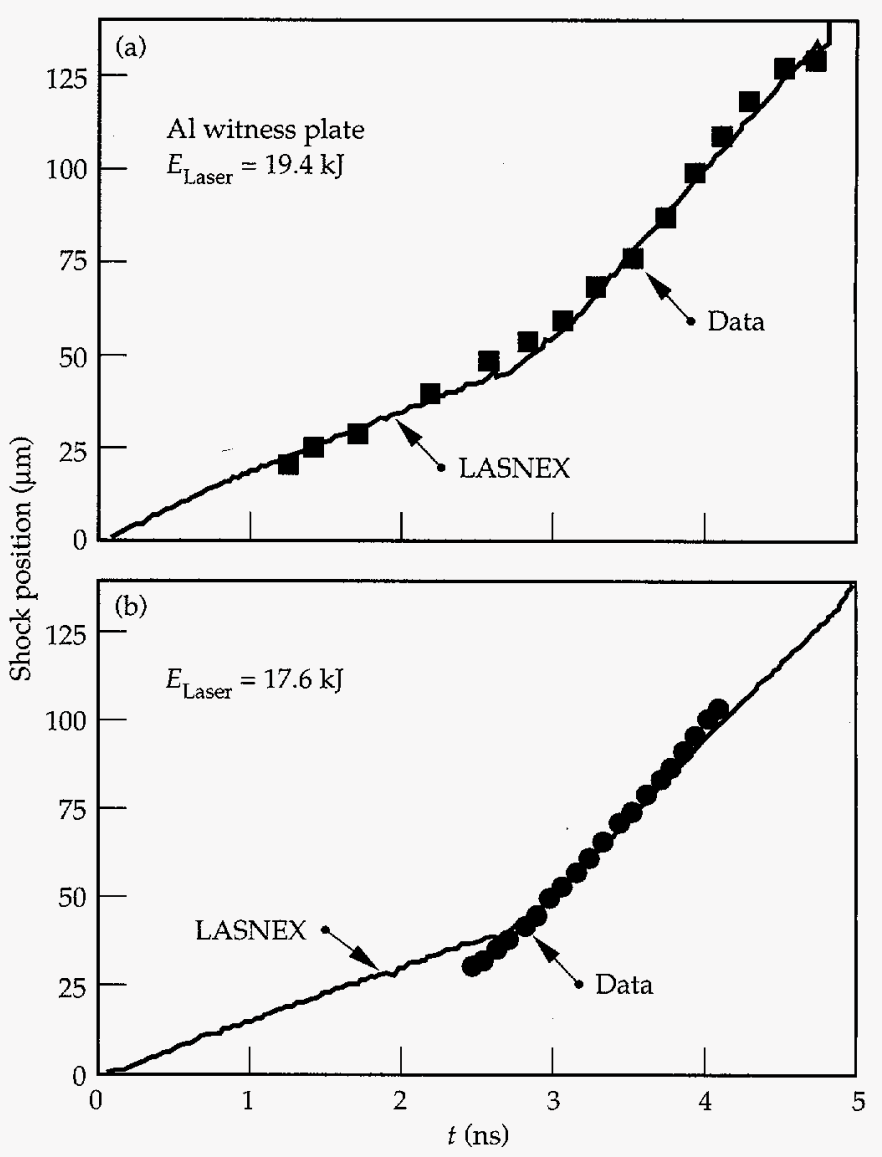

FIGURE 9. The drive is characterized with measurements of shock trajectory, deduced from measuring in face-on geometry the shock breakout time across a variable-thickness Al wedge mounted on the wall of the hohlraum. Corresponding 1-D LASNEX simulations are shown. (a) Results for 19.4-kJ shot discussed in Figs. 7 and 8.

(b) Results for $E_{\text {Laser }}=17.6 \mathrm{~kJ} . \quad(20-07-0394-0744 \mathrm{pb03})$ 
measurement of shock velocity therefore allows us to deduce the ablation pressure and drive temperature.

Figure 9 shows measured shock trajectories for two shots. The measurements were made by viewing a wedged $\mathrm{Al}$ witness plate face-on with a streaked $\mathrm{UV}$ imager. ${ }^{40}$ The shock breakout time is recorded as a function of position across the increasing thickness of the witness plate, allowing the shock trajectory to be reconstructed. Figure 9 shows the experimental trajectories at two laser energies and the corresponding simulations from 1-D LASNEX ${ }^{44}$ using the drive described above (scaled in proportion to the laser power history for the lower-energy shot). The absolute timing of the shock trajectory data relative to drive turn-on $(t=0)$ was experimentally determined in the data shown in Fig. 9(b) but not in Fig. 9(a), where only the relative time was measured. The agreement between the data and simulation is very good. The data for the higher-energy shot [Fig. 9(a)] and the simulations for both shots show a two-component trajectory corresponding to an initial shock launched by the foot, and a delayed second shock coming from the peak of the drive. For the measured two-shock system in Fig. 9(a), the first shock has a velocity $v_{1} \approx 20 \mu \mathrm{m} / \mathrm{ns}$, and the second shock has a peak velocity of $v_{2} \approx 53 \mu \mathrm{m} / \mathrm{ns}$. The simulations give velocities of 16 and $46 \mu \mathrm{m} / \mathrm{ns}$, respectively. Applying Eq. (13) directly to the data gives $T_{R} \approx 120$ and $215 \mathrm{eV}$ for the foot and peak of the drive, as compared to 100 and $200 \mathrm{eV}$ from the simulations.

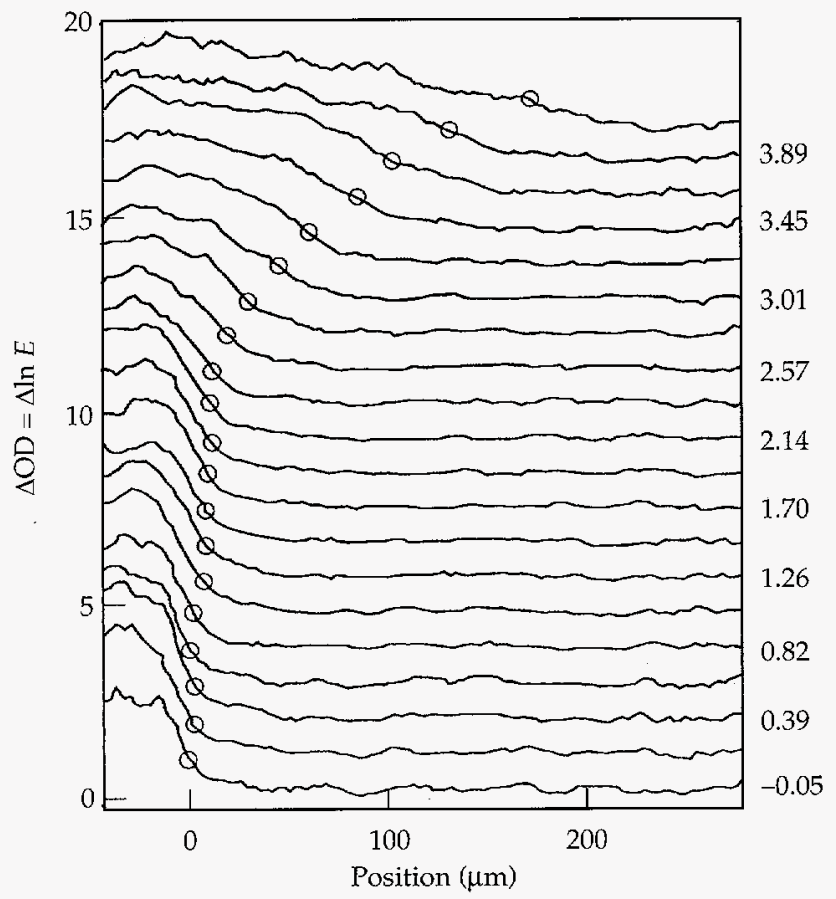

FIGURE 10. Foil trajectory (for a FS foil) in side-on geometry measured with the Wölter $x$-ray microscope. The profiles are of optical depth OD, and are artificially offset vertically by time, as indicated on the righthand vertical axis. The circles represent the position that was taken as the foil rear edge (side away from $x$-ray drive). (20-03-0895-2047pb01)
The foil trajectory, which is a measure of the gross hydrodynamics, was obtained by viewing across the rear edge of the foil in side-on geometry, ${ }^{14,15}$ as shown in Fig. 10. This trajectory is reproduced very well with the 1-D simulation using the drive model described above, as illustrated in Fig. 11 for a $\mathrm{CH}(\mathrm{Br})$ foil. The back edge of the foil does not begin to move until shock breakout at $\sim 2.6 \mathrm{~ns}$. The foil then accelerates during the interval $2.6 \leq t \leq 3.6 \mathrm{~ns}$, after which the drive is turning off and the foil begins to coast. The black curve represents the foil trajectory from the 1-D LASNEX simulation; the gray curve represents the acceleration of the ablation front, defined as the zone of half peak density. The fluctuations in the acceleration at 0.2 and $2.2 \mathrm{~ns}$ are due to the passage of the first and second shocks. The inset in Fig. 11 gives the ablation velocity $v_{\mathrm{a}}=\dot{m} / \rho_{\max }$ and density gradient scalelength $L=\rho / \nabla \rho$, where $\dot{m}$ is the mass ablation rate per unit area, $\rho_{\max }$ is the peak density, and $\rho$ and $\nabla \rho$ are the density and its gradient. (Computationally, the scalelength $L$ is taken as the minimum value of $\left.[\partial(\ln \rho) / \partial z]^{-1}\right)$. Calculations based on this drive give better agreement with experiment than was obtained in preliminary results. ${ }^{45}$

It is instructive to look in more detail at the effect of this shaped drive on the foil. Figures 12(a)-12(c) show results from the 1-D LASNEX simulations for radiation drive temperature, ablation pressure (defined as peak pressure in the simulation), and foil peak density as functions of time. Figures 12(d)-12(f) give the corresponding spatial profiles of electron temperature $T_{\mathrm{e}}(z)$, pressure $P(z)$, and density $\rho(z)$ at five times spanning

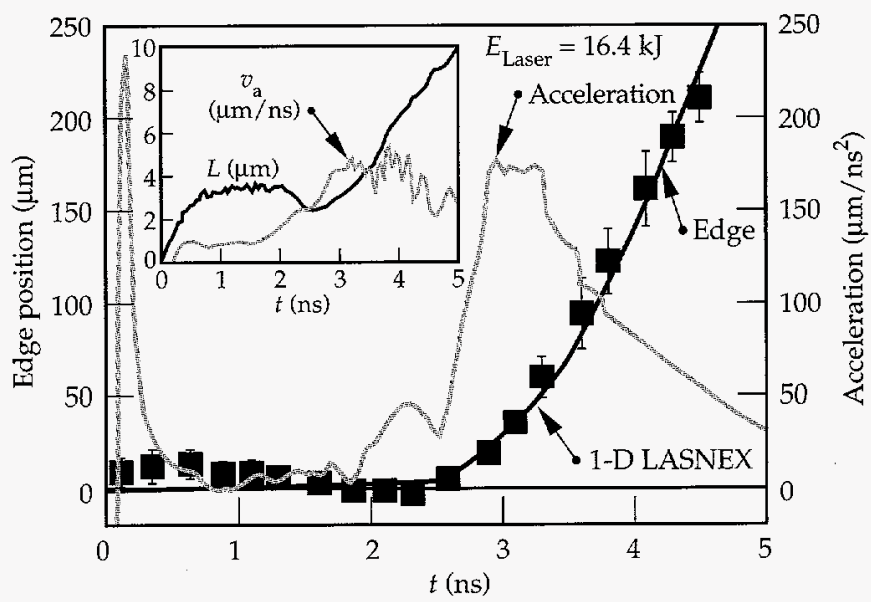

Figure 11. The square plotting symbols represent the measured position of the rear edge (side away from the $x$-ray drive) of the foil as a function of time. These data represent the average of two shots, one using a $50-\mu \mathrm{m}$-thick and the other a 48 - $\mu \mathrm{m}$-thick $\mathrm{CH}(\mathrm{Br})$ foil. Error bars represent uncertainties in defining the edge of the foil. The black curve represents the result of the 1-D LASNEX simulation. The rear edge of the foil does not start to move until after shock breakout at $2.6 \mathrm{~ns}$. The gray curve represents the acceleration of the ablation front (defined as the zone of half peak density). Inset: ablation velocity and density gradient scalelength from the simulation. (20-07-0592-1702Bpb02) 
1.3-2.9 ns. In Fig. 12(a), the drive $T_{\mathrm{R}}(t)$ shows a $\sim 90-\mathrm{eV}$ foot for the first $1.5 \mathrm{~ns}$, an increase to a peak of $190 \mathrm{eV}$ at $3 \mathrm{~ns}$, and a decay to $\sim 100 \mathrm{eV}$ at $5 \mathrm{~ns}$. The resulting ablation pressure in the $\mathrm{CH}(\mathrm{Br})$ foil (Fig. 12b) shows a 3-5 Mbar foot and a peak of $28 \mathrm{Mbar}$ at $3 \mathrm{~ns}$, i.e., at the same time as the peak in $T_{R}$. [This is also the time of peak acceleration and peak ablation velocity (Fig. 11).] The peak density of the foil [Fig. 12(c)] is generated just behind the strongest shock, reaching $6.5 \mathrm{~g} / \mathrm{cm}^{3}$ or a compression of 5.2 at $2.6 \mathrm{~ns}$, when the second shock breaks out of the back of the foil and is at maximum strength. (This is also the time of minimum $L$, shown in the inset in Fig. 11.) The $T_{\mathrm{e}}(z)$ profile at $1.3 \mathrm{~ns}$ [Fig. 12(d)] shows a sharp increase at $z=-20 \mu \mathrm{m}$ because of the passage of the first shock. Notice, however, that a low level of heating precedes the first shock $(z>-20 \mu \mathrm{m})$. This "preheat" results from the deep penetration of the hard component $(h v \gtrsim 1.4 \mathrm{keV})$ of the drive spectrum. By $2.5 \mathrm{~ns}$, the second shock has overtaken the first, and the sharp features in $T_{\mathrm{e}}(z)$ are washed out. The pressure profiles [Fig. 12(e)] convey a similar picture. During the interval 1.3-2.1 ns, the sharp rise in pressure to $4 \mathrm{Mbar}$ due to the first shock is readily apparent. At $2.5 \mathrm{~ns}$, the second shock has overtaken the first, increasing the pressure to $20 \mathrm{Mbar}$, and at $2.9 \mathrm{~ns}$ the pressure reaches its maximum of $28 \mathrm{Mbar}$. Figure 12(f) shows the effect on the foil compression of this staged two-shock drive. The compression at $1.3 \mathrm{~ns}$ just behind the first shock increases the foil density from 1.26 to $3.3 \mathrm{~g} / \mathrm{cm}^{3}$. By the time the second shock has overtaken the first at $2.5 \mathrm{~ns}$, the peak density has reached $6.4 \mathrm{~g} / \mathrm{cm}^{3}$, corresponding to a compression of 5 . This is greater than the maximum possible compression of 4 for a single strong shock (in the ideal-gas limit). The staging of multiple shocks allows a higher compression by maintaining the foil on a lower adiabat.

We illustrate the lower adiabat achievable with this shaped drive by characterizing an adiabat with the ratio $\alpha=P / P_{\mathrm{FD}}$ of pressure at peak density to that of a Fermi-degenerate gas at the same density (which represents the lowest possible internal energy). For $P_{\mathrm{FD}^{\prime}}$ we use the pressure at zero temperature for the EOS of the foil. For the low-adiabat drive shown in Fig. 12, we have $\alpha \approx 2$; for a 1 -ns square drive at the same laser energy ${ }^{13}$ we have $\alpha \approx 9$, a factor of 4.5 higher. Note that the EOS for a real material differs significantly from that of an ideal Fermi fluid for densities only a few times solid density. The value of the parameter $\alpha$ defined above does not uniquely characterize an isentrope but changes with density along one. The value of $\alpha$ goes to infinity at solid density because $P_{\mathrm{FD}} \rightarrow 0$. The value of $\alpha$ does, however, approach a constant along an isentrope in the limit $\rho \rightarrow \infty$. This asymptotic limit of $\alpha$ is about 1.25 for our low-adiabat drive, but it is 2.3 for a 1-ns square drive. Thus, if the foil driven with the shaped pulse were to be compressed to high density without introducing additional entropy, as in a capsule implosion, its compression would be close to that expected in the degenerate limit. (a)

FIGURE 12. Various representations of the $16.4-\mathrm{kJ}$ drive corresponding to results shown in Fig. 11 from 1-D simulations to illustrate the effect of pulse shaping. (a) Laser power $P_{\mathrm{L}}$ and radiation temperature $T_{R}$ vs time. (b) Ablation pressure (defined as the peak pressure in the simulation) vs time. (c) Peak density vs time. (d)-(f) Spatial profiles of (d) electron temperature $T_{\mathrm{e}^{\prime}}$ (e) pressure in the foil, and (f) foil density at $1.3,1.7,2.1$, 2.5 , and 2.9 ns, spanning the foot through the peak of the drive. The 0 - $\mu \mathrm{m}$ position corresponds to the initial position of the back edge of the foil (the side away from the drive). The drive is incident from the left (negative) side. (20-03-0794-2831pb04)

(b)

(c)
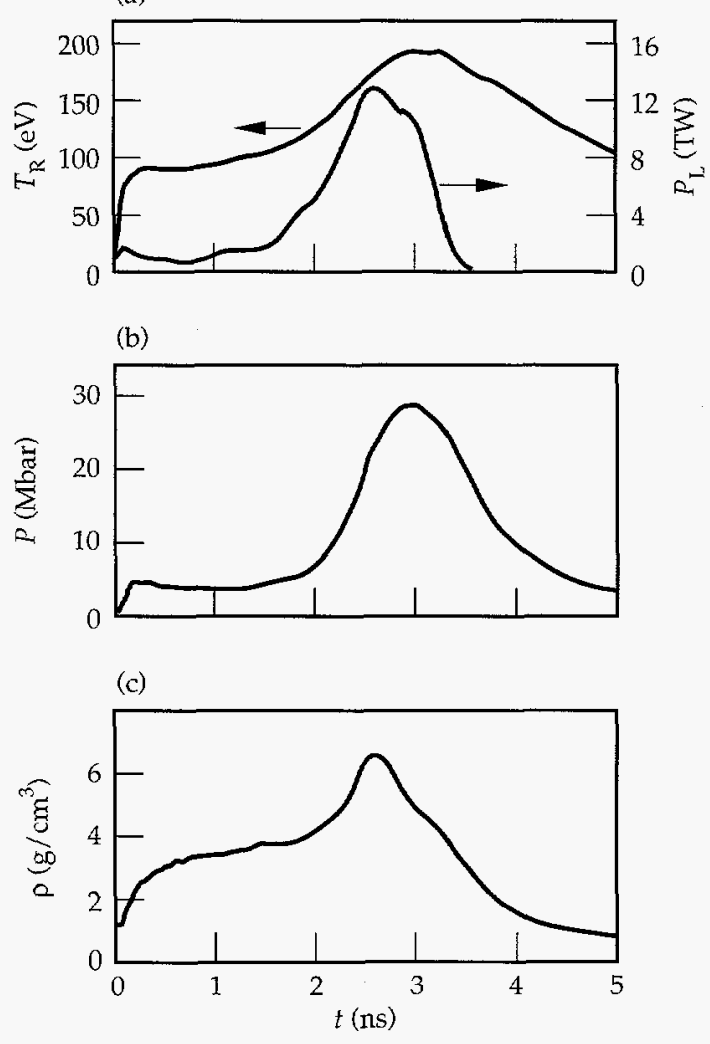

(d)

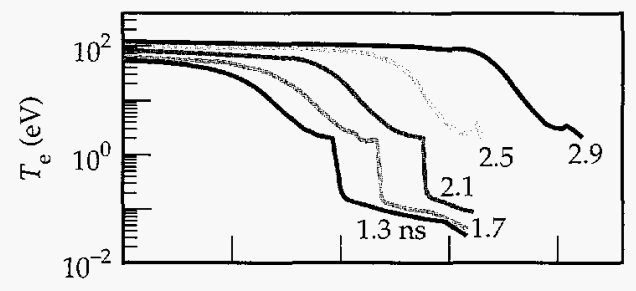

(e)

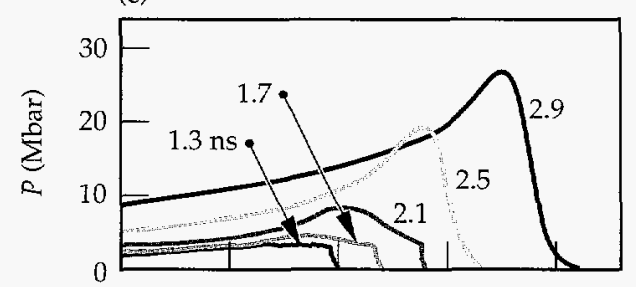

(f)

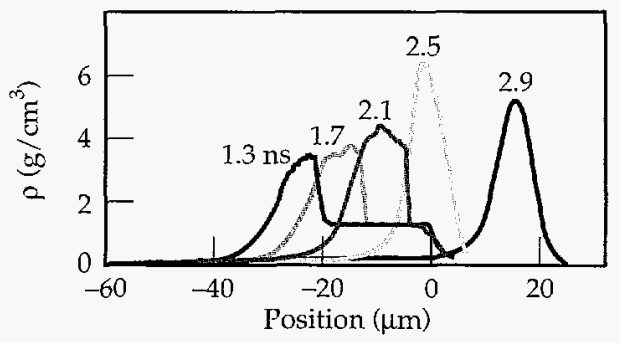




\section{Single-Mode Experiments}

Figure 13 shows the data from a single-mode faceon experiment with a $\lambda=100 \mu \mathrm{m}, \eta_{0}=2.4 \mu \mathrm{m}$ initial perturbation on a $48 \mu \mathrm{m}$ thick $\mathrm{CH}(\mathrm{Br})$ foil. ${ }^{37}$ Figure 13(a) shows the "raw" image; Fig. 13(b) shows profiles of modulations in optical depth $\Delta \mathrm{OD} \approx-\ln E$ at early, intermediate, and late times. The initial perturbation amplitude was large, and we observe clear sinusoidal contrast ( $\triangle \mathrm{OD}$ ) even at the earliest time, 0.2 ns. At $2.2 \mathrm{ns,}$ the contrast is slightly greater and still sinusoidal, indicating that the growth is still in the linear regime. At $4.2 \mathrm{~ns}$, the shape of the perturbation has deviated substantially from sinusoidal, forming sharp spikes and bubbles of high and low $\mathrm{OD}$, respectively; the perturbation has entered the nonlinear regime. The transition from the linear to nonlinear regime is particularly clear in Fourier space; Fig. 13(c) shows the real components of the Fourier transform for the three $\triangle O D$ profiles. At $0.2 \mathrm{~ns}$, only the $\eta_{1}$ fundamental mode (first harmonic) exists, indicating a purely sinusoidal perturbation. At $2.2 \mathrm{~ns}, \eta_{1}$ has grown slightly but is still the only component, indicating a purely sinusoidal shape and linear regime. At $4.2 \mathrm{~ns}$, a whole spectrum of higher Fourier harmonics-up to the fifth-is observed, corresponding to the bubble-and-spike shape of the top lineout in Fig. 13b. The perturbation is fully into the nonlinear regime.

Figure 14 shows the results of our $\lambda$-scaling experiments with single-mode $\mathrm{CH}(\mathrm{Br})$ foils. The data points represent the observations for the fundamental and the second harmonic. The error bars represent the standard deviation of the ensemble formed by treating each individual period of the perturbations as independent data. The solid curves are the corresponding 2-D LASNEX $^{44}$ simulations. The $\lambda=100 \mu \mathrm{m}$ data shown in Fig. 14(a) is the full time evolution of the results shown at three particular times in Fig. 13. The perturbation growth evolves through three stages. Before shock breakout $(t<2.5 \mathrm{~ns})$, the perturbation is growing only slowly because of the rippled shock dynamics. ${ }^{21,22}$ For a brief period after shock breakout ( $2.5 \mathrm{~ns} \leq t \leq 3.2 \mathrm{~ns})$ the perturbation is growing strongly because of the RT instability in the linear regime. Late in time $(t>3.2 \mathrm{~ns})$, the perturbation "saturates." The evolution has entered the nonlinear regime, the second harmonic appears, and the observed contrast rolls over. The growth factor $G$, defined as the ratio of peak to initial contrast, was small here $-G=4$. A similar situation occurs for $\lambda=70 \mu \mathrm{m}, \eta_{0}=2.4 \mu \mathrm{m}$ [Fig. 14(b)]. Here, the overall growth was slightly greater, $G=6$, reflecting the higher growth rate. For $\lambda=50 \mu \mathrm{m}, \eta_{0}=0.4 \mu \mathrm{m}$ [Fig. 14(c)], the situation is qualitatively different. Because the initial amplitude is small, the perturbation evolution remains primarily in the linear regime, achieving the higher growth $G=20$. Figure 14(d) shows the results for $\lambda=30 \mu \mathrm{m}, \eta_{0}=1.5 \mu \mathrm{m}$. Because of the low instrumental MTF at $\lambda=30 \mu \mathrm{m}$ (see Fig. 4), the contrast remains low and no higher harmonics are observed. The observed growth factor for the fundamental mode was $G=7$. Table 1 lists the key parameters for these experiments, including observed and simulated growth factors.

The solid curves in Fig. 14 represent the corresponding results from 2-D LASNEX simulations, after convolution (a)

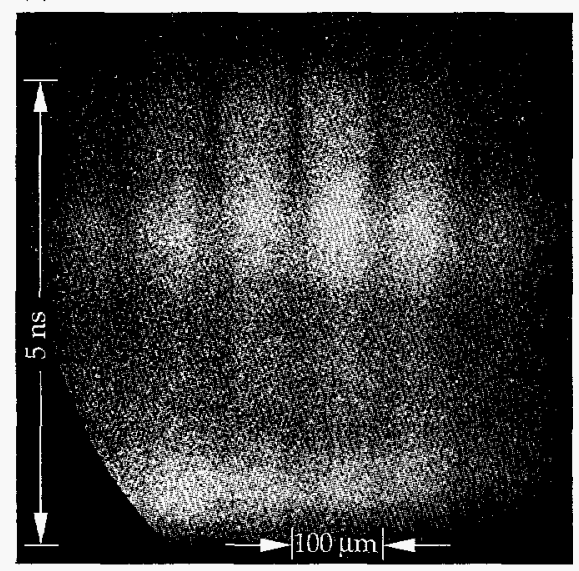

(b)

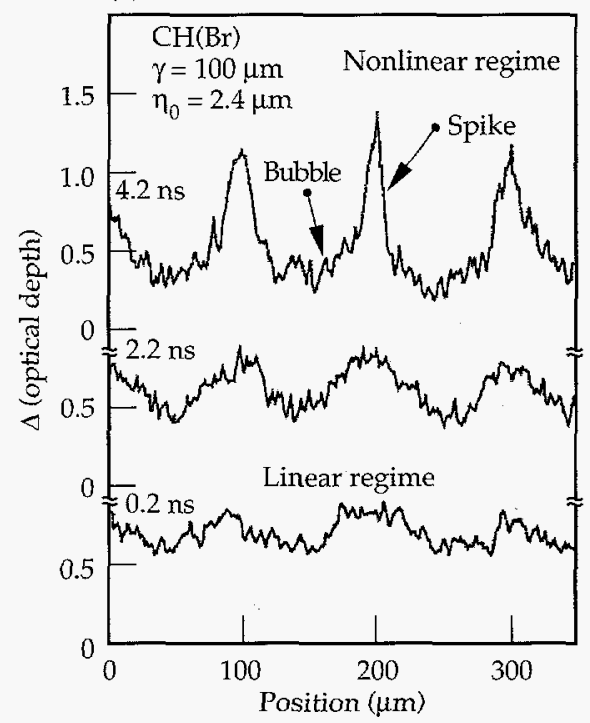

(c)

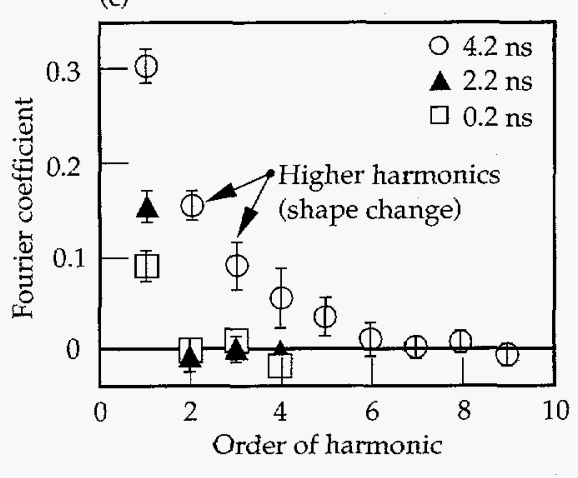

FIGURE 13. Various representations for single-mode face-on data for a $\lambda=100 \mu \mathrm{m}, \eta_{0}=2.4 \mu \mathrm{m}$ perturbation imposed on a $48-\mu \mathrm{m}$-thick $\mathrm{CH}(\mathrm{Br})$ foil. (a) The "raw" streaked image is shown as film density. The film response is removed using a calibrated exposure across a precision P20 optical density wedge. (b) Profiles of $\Delta$ (optical depth) $=-\Delta \ln E$ at $0.2,2.2$, and 4.2 ns. (c) Real components of the Fourier transforms for the profiles in (b). At late time, the perturbation enters the nonlinear regime, and up to the fifth harmonic of the perturbation Fourier composition is observed. (20-03-0394-0748pb02) 
of the simulated image exposure with the instrumental resolution function [Eq. (11)]. (Our modeling is discussed in detail in Ref. 45.) Qualitatively, the simulations agree quite well with the data. There is modest growth dur- ing the shock transit phase, strong growth after shock breakout, and then saturation with the appearance of the second harmonic, indicating entry into the nonlinear regime.
FIGURE 14. Results from single-mode, $\lambda$-scaling series for various values of $\lambda$ and $\eta_{0}$. Data points represent first harmonic (fundamental mode) and second harmonic Fourier coefficients of $\ln E$. Solid curves are corresponding results from 2-D LASNEX simulations. (a) Shows full time dependence for data of Fig. 13. All shots except that in (c) used the "top" sector of the $22 \times$ and a $\mathrm{Rh}$ backlighter; in (c), the west sector was used, and a Mo backlighter was used for slightly improved contrast. (20-03-0394-1063pb02)

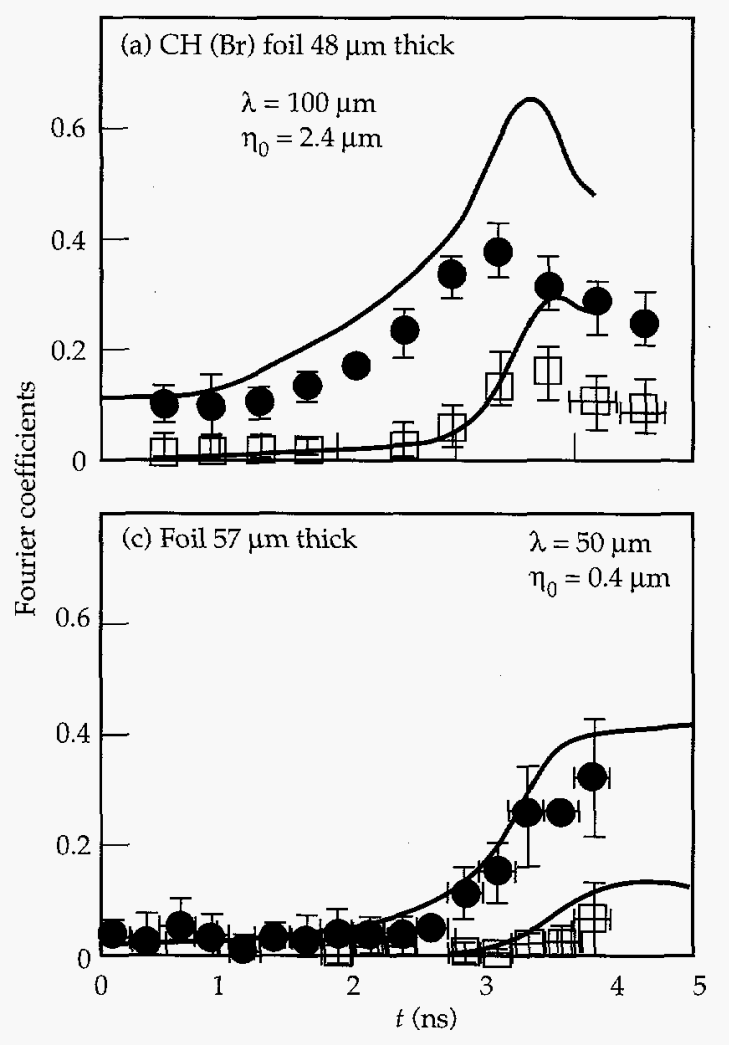

(a) $\lambda=50 \mu \mathrm{m}, \eta_{0}=0.4 \mu \mathrm{m}$

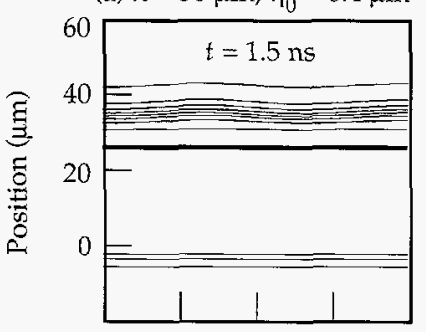

(e) $\lambda=70 \mu \mathrm{m}, \eta_{0}=2.4 \mu \mathrm{m}$

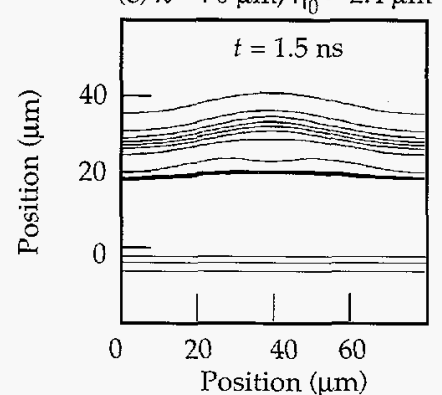

(b)

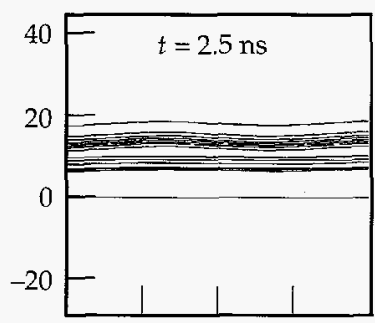

(f)

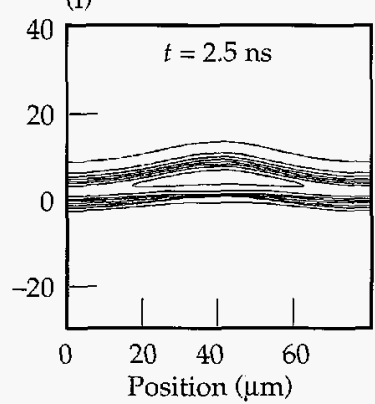

(c)

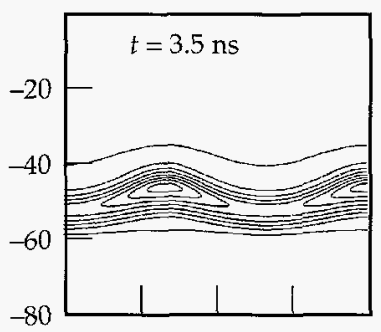

(g)

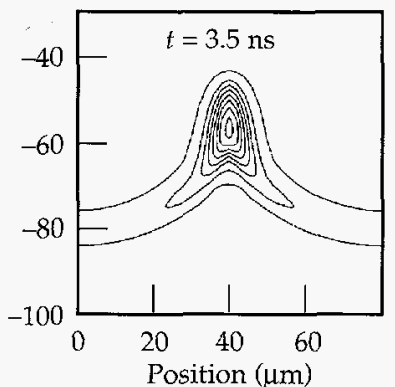

(d)

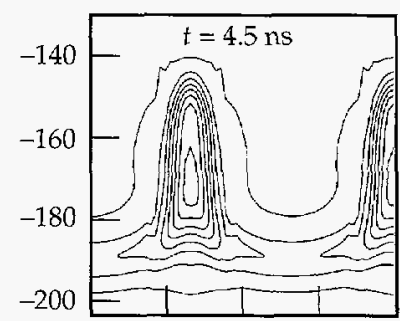

FIGURE 15. Isodensity contour plots from 2-D simulations, in 1-ns steps, for perturbation growth from (a-d) $\lambda=50 \mu \mathrm{m}, \eta_{0}=0.4 \mu \mathrm{m}$ and (e-g) $\lambda=70 \mu \mathrm{m}, \eta_{0}=2.4 \mu \mathrm{m}$, corresponding to the results shown in Figs. 14(c) and 14(b), respectively. The contours correspond to equal density steps and span densities of (a) 0.2177-3.265, (b) 0.4837-7.256, (c) 0.3389-5.084, (d) 0.1548-2.322, (e) 0.2287-3.431, (f) 0.4976-7.464, and (g) $0.5786-8.679 \mathrm{~g} / \mathrm{cm}^{3} . \quad(20-03-0894-3227 \mathrm{pb02})$ 
This basic behavior can be understood in terms of the simple linear and perturbation theories outlined in Eqs. $(2,3,5)$. In the linear regime, $\mathrm{RT}$ growth is exponential. For $\eta / \lambda \gtrsim 0.1$, the evolution enters the nonlinear regime, higher harmonics appear, and at third order, the growth in the fundamental mode is reduced. The late-time rollover in the simulations (and presumably in the data), is partially an instrumental artifact, however. Mass is being concentrated in long, narrow spikes in the nonlinear phase, as illustrated in Fig. 15 with isodensity contour plots from the simulations for $\lambda=50$ and $70 \mu \mathrm{m}$. For example, at $t=3.5 \mathrm{~ns}$ for $\lambda=70 \mu \mathrm{m}$ [Fig. 15(g)], $50 \%$ of the highest density contours lie within a 10- $\mu \mathrm{m}$ region at the center of the spike, which is difficult to resolve with the 10-15- $\mu \mathrm{m}$ resolution of the imaging instrument used (see Fig. 4). The observed contrast is therefore decreasing after $t=3.5 \mathrm{~ns}$. Notice that there is much less concentration of material in the spike at $3.5 \mathrm{~ns}$ for $\lambda=50 \mu \mathrm{m}$ [Fig. 15(c)]. For this case, spatial resolution does not become an issue until very late $(t \approx 4.5 \mathrm{~ns})$. For completeness, Fig. 16 shows the simulations before and after convolution with the instrument resolution function. The contrast is greater before inclusion of the MTF, the difference being greatest for the shortest wavelengths.

The simulations systematically predict slightly more growth than is observed (Fig. 14). The seeds of this discrepancy occur early-by $1.5 \mathrm{~ns}$ for the $\lambda=100$ and $70-\mu \mathrm{m}$ foils, well before the shock has broken out-so the disagreement occurs during the shock transit phase. We consider possible causes for this discrepancy in Fig. 17, using the $\lambda=70 \mu \mathrm{m}$ experiment as a test case.

(a) $\lambda=100 \mu \mathrm{m}, \eta_{0}=2.4 \mu \mathrm{m}$

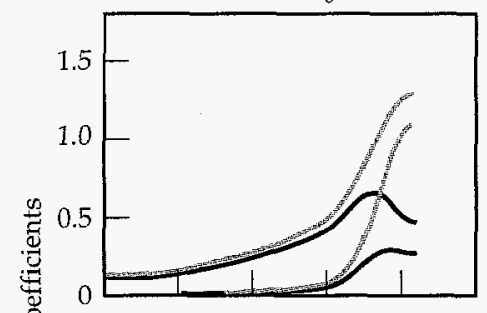

(c) $\lambda=50 \mu \mathrm{m}, \eta_{0}=0.4 \mu \mathrm{m}$

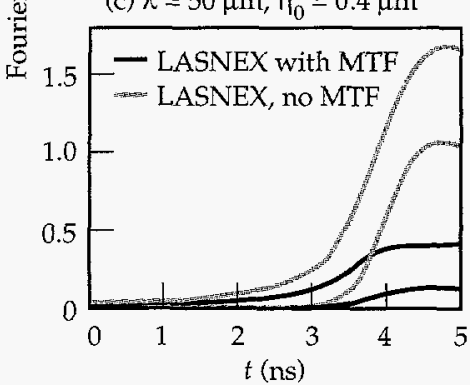

FIGURE 16. Effect of instrument resolution on the results from the simulations. Black curves in (a)-(d), which include the effects of instrumental MTF, are reproduced from Fig. 14. Gray curves represent the simulation results before inclusion of MTF. $\quad(20-03-0394-0745 \mathrm{pb} 03)$
Possible uncertainties in the drive $T_{R}(t)$ could arise from uncertainties in the albedo of the Au hohlraum wall early in time and from stagnation of Au plasma on the hohlraum axis late in time. To assess the sensitivity of perturbation growth to uncertainties in the drive, we compare in Fig. 17(a) the results of simulations in which the foot of the drive was $10 \mathrm{eV}$ higher and the peak $10 \mathrm{eV}$ lower than the nominal drive. This variation in the drive produces very little variation in the predicted overall growth for $\lambda=70 \mu \mathrm{m}$.

Different EOS models can lead to different predicted foil compression, which affects the RM-like growth during shock transit. In Fig. 17(b) we compare the effect of using a tabular EOS library with that of using an in-line QEOS model. ${ }^{46}$ The QEOS model generates a slightly stiffer EOS, which leads to less foil compression and hence to $15 \%$ less perturbation growth.

There is also uncertainty in the exact magnitude of preheat in the drive spectrum early in time. In Fig. 17(c) we assess the sensitivity of perturbation growth to preheat by comparing simulations with a nominal drive spectrum and with a "high preheat" drive, in which the drive spectrum above $h v=1.4 \mathrm{keV}$ is increased by a factor of 10 for the first $2 \mathrm{~ns}$, while keeping the total (a) $\lambda=70 \mu \mathrm{m}, \eta_{0}=2.4 \mu \mathrm{m}$

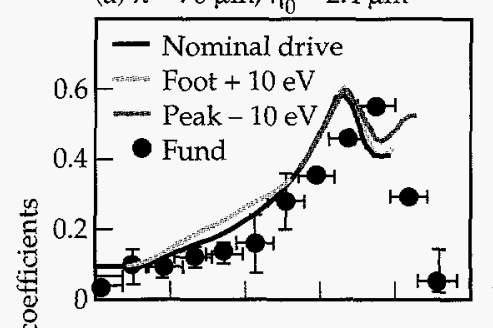

(c)

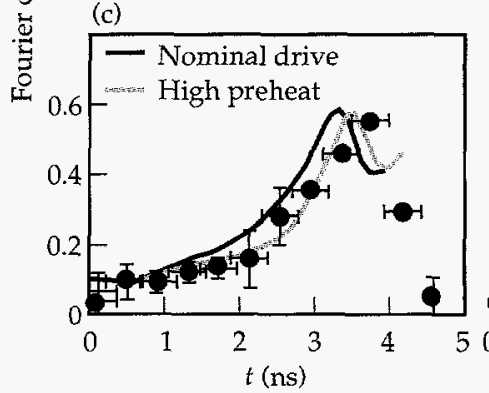

(b)

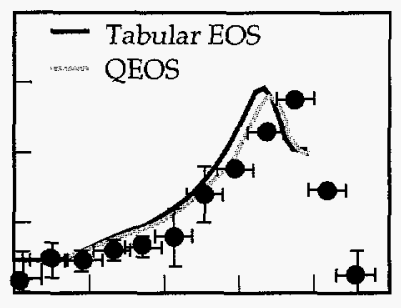

(d)

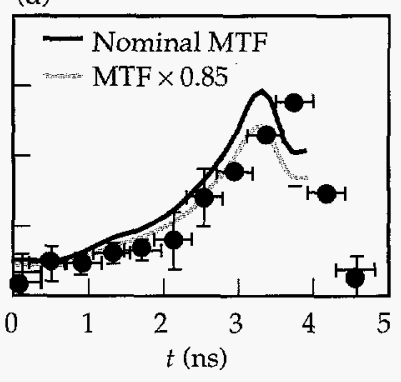

FIGURE 17. Sensitivity of perturbation growth for $\lambda=70 \mu \mathrm{m}, \eta_{0}=2.4 \mu \mathrm{m}$ to (a) drive, (b) EOS, (c) preheat, and (d) MTF. Data and black curves are reproduced from Fig. 14(b). (a) The light gray curve corresponds to a simulation in which the radiation temperature in the foot of the drive was increased by $10 \mathrm{eV}$; the dark gray curve represents a simulation in which the peak of the drive was reduced by $10 \mathrm{eV}$. (b) The nominal simulation (black) uses a tabular EOS library. The gray curve uses an in-line model called QEOS, which results in a slightly "stiffer" EOS. (c) The gray curve corresponds to a simulation in which the preheat in the drive was artificially increased: during the first $2 \mathrm{~ns}$ (the foot), the drive for $h v \geq 1.4 \mathrm{keV}$ was increased by a factor of 10 , and the drive for $h \nu<1.4 \mathrm{keV}$ was decreased to maintain the same total energy. (d) The gray curve corresponds to post-processing the simulation with a resolution function artificially degraded to give a 15\% lower MTF. $\quad(20-03-0894-2839 \mathrm{pb} 03)$ 
drive power constant. Enhancement of the preheat significantly reduces perturbation growth.

We also illustrate the sensitivity of observed growth to uncertainties in the MTF. Decreasing the MTF by $15 \%$, the maximum reduction consistent with the data shown in Fig. 4(a), decreases the predicted growth by 15\%, as shown in Fig. 17(d).

To summarize, the simulations slightly but systematically overpredict perturbation growth during the shock transit phase. A possible cause is higher preheat in the foot of the drive, but a combination of stiffer EOS and degraded MTF may also be involved.

We have also done single-mode 2-D experiments aimed at measuring large RT growth factors. ${ }^{14}$ These experiments differed from those described above only in the use of fluorosilicone $\left(\mathrm{SiOC}_{4} \mathrm{H}_{7} \mathrm{~F}_{3}\right.$, or "FS") as the foil material. We used FS because its admixture of opacities shields the foil from $x$-ray preheat, keeping the foil on a lower adiabat. The density gradient at the ablation front is therefore steeper, and the ablation velocity is lower, leading to higher $\mathrm{RT}$ growth factors.

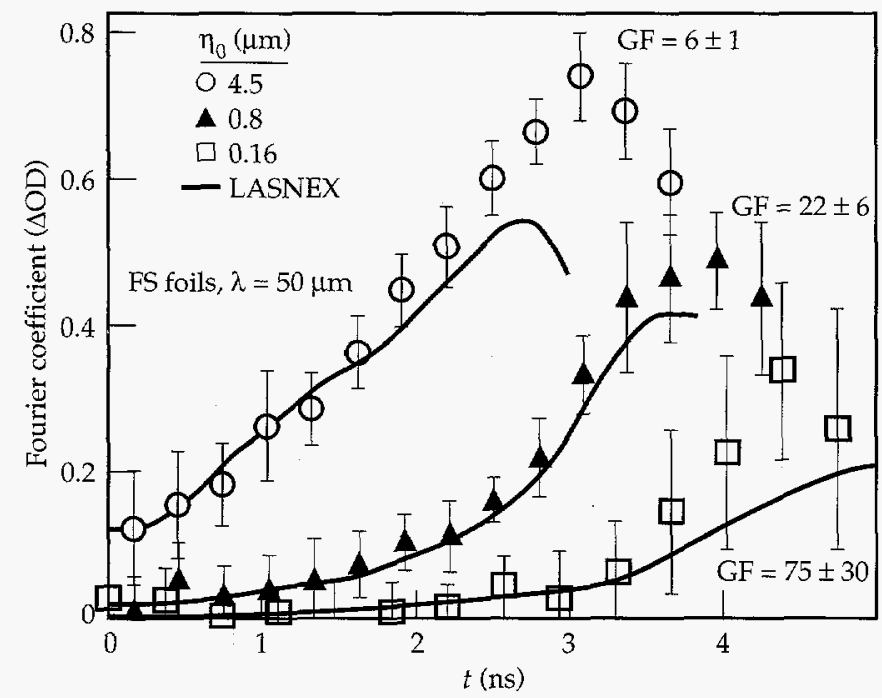

FIGURE 18. Amplitude $\left(\eta_{0}\right)$ scaling results for three fluorosilicone (FS) foils. Perturbations were $\lambda=50 \mu \mathrm{m}, \eta_{0}=0.16,0.8$, and $4.5 \mu \mathrm{m}$; the smallest- $\eta_{0}$ perturbation yielded the highest growth factor $(G=$ 75). (20-03-1293-4395pb01)
We did these experiments in an amplitude-scaling series, starting with large amplitude to see the initial contrast easily. In this case, however, the RT evolution quickly enters the nonlinear regime, higher harmonics are formed, the perturbation takes on the classic bubble-and-spike shape, and the growth slows sharply, ultimately changing from exponential to linear. We then shot an intermediate-amplitude target, which entered the nonlinear regime only towards the end of the acceleration. Finally, to maximize the observed growth, we used a very-small-amplitude perturbation, so that the foil remained in the linear regime throughout the acceleration. Figure 18 shows the results. For the smallest amplitude perturbation, a growth factor of 75 was observed in the fundamental mode. The peak-to-valley amplitude grew by a factor of 80 (that is, $4.4 e$-foldings of growth), in agreement with the simulations.

To better illustrate the differences between the evolution of these three targets, we use the simulations to show in Fig. 19 the actual shape of the perturbations at peak growth. The large- $\eta_{0}$ foil [Fig. 19(a)] shows the classic bubble-and-spike shape of the nonlinear RT

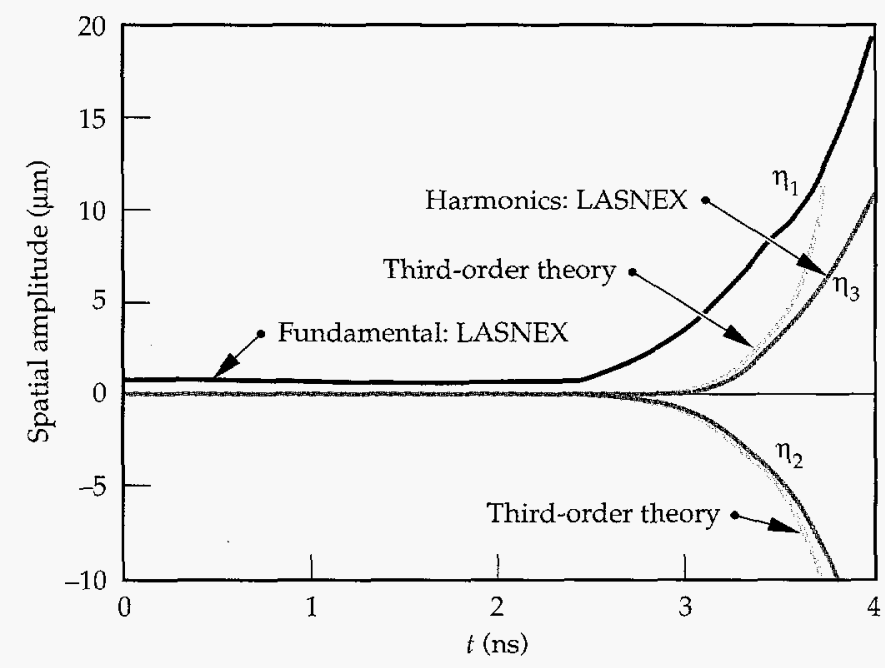

FIGURE 20. LASNEX simulations of the RT evolution for the intermediate- $\eta_{0}$ foil (from Fig. 18), and corresponding results from third-order perturbation theory. (20-03-0895-2046pb01) (a)

FIGURE 19. Shapes of perturbations (from Fig. 18) at peak growth, from 2-D LASNEX simulations. Increased darkness corresponds to higher density. (20-03-1293-4542pb01)

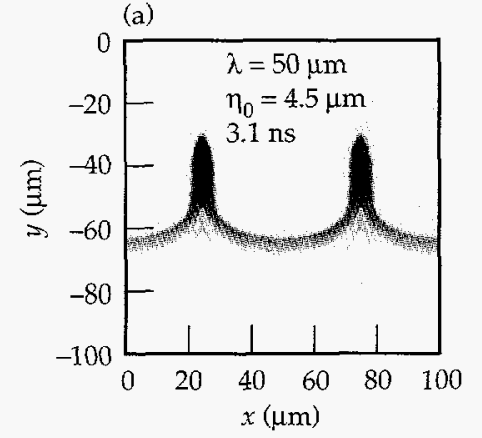

(b)

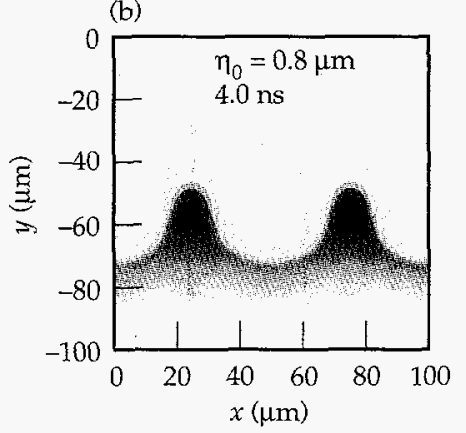

(c)

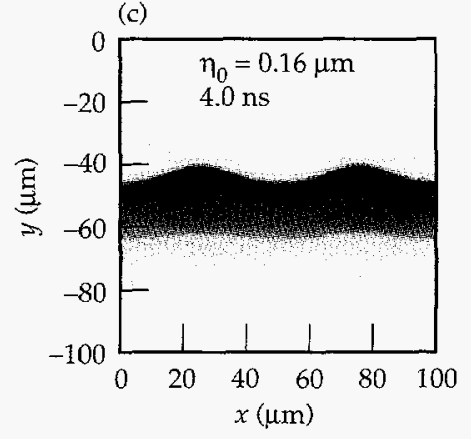


regime; the small- $\eta_{0}$ foil [Fig. 19(c)] still looks largely sinusoidal, indicating linear RT evolution; the intermediate- $\eta_{0}$ foil [Fig. 19(b)] is midway in between.

The transition to the nonlinear regime can be illustrated qualitatively with third-order perturbation theory. ${ }^{14,23}$ We compare the results from the LASNEX simulation for the intermediate- $\eta_{0}$ foil $\left(\eta_{0}=0.8 \mu \mathrm{m}\right)$ with those obtained from perturbation theory [Eq. (5)]. Amplitudes in areal density $\rho R$ are converted to spatial amplitude by dividing by a characteristic density. Figure 20 shows the results. Third-order perturbation theory predicts the entry into the nonlinear regime very well.

The quantity of most interest in RT instability studies is the growth rate $\gamma$ in Eq. (2), which is often parametrized as in Eq. (3) in terms of foil acceleration $g$, density $\rho$, and density gradient scalelength $L$.

Unfortunately, because of the nature of our low-adiabat drive, the foil $g, \rho$, and $L$ are not constant, as shown in Figs. 11 and 12. Hence, our RT growth cannot be characterized in terms of a single value of $\gamma$ over the full duration of the foil acceleration. Nevertheless, from. linear-regime simulations for $\lambda=50 \mu \mathrm{m}$ [Figs. 21(b), 21(c)], we show the dispersion curves in Fig. 21(d) for $\mathrm{CH}(\mathrm{Br})$ and $\mathrm{FS}$, using parameters characteristic of the foils at $t=3.0 \mathrm{~ns}$. Even though the exact quantitative form of Eq. (3) for indirect drive is not settled, the equation appears qualitatively to describe the effect of stabilization at the ablation front. This has recently been demonstrated conclusively by comparing RT growth at the ablation front with that at an embedded interface, away from the ablation front, for this same drive and ablator material. At the ablation front, no growth was observed for wavelengths shorter than $30 \mu \mathrm{m}$, whereas at the embedded interface, strong growth down to wavelengths as short as $10 \mu \mathrm{m}$ was observed. ${ }^{47}$

Earlier experiments were done with a 1-ns drive pulse shape, using FS and $\mathrm{CH}$ foils. ${ }^{13}$ Figure 22 shows the results. This drive puts the foils on a much higher adiabat; the duration of the acceleration was short, and the growth was predominantly due to the rippled shock dynamics. Growth factors were $G \approx 2.5$ and $G=1.5$ for the FS and $\mathrm{CH}$ foils, respectively.

All of our single-mode 2-D experiments can be qualitatively understood by the density profiles in Fig. 23, where we have carried out 1-D LASNEX simulations with the shaped drive, changing only the foil material. The FS foil remains on the lowest adiabat, has the steepest density gradient at the ablation front, and (as we saw above) exhibits the largest RT growth factors. The $\mathrm{CH}$ foil is at the opposite extreme. With no opacity shield against the hard $x$ rays in the drive, the $\mathrm{CH}$ foil jumps to a very high adiabat, the density gradient at (a)

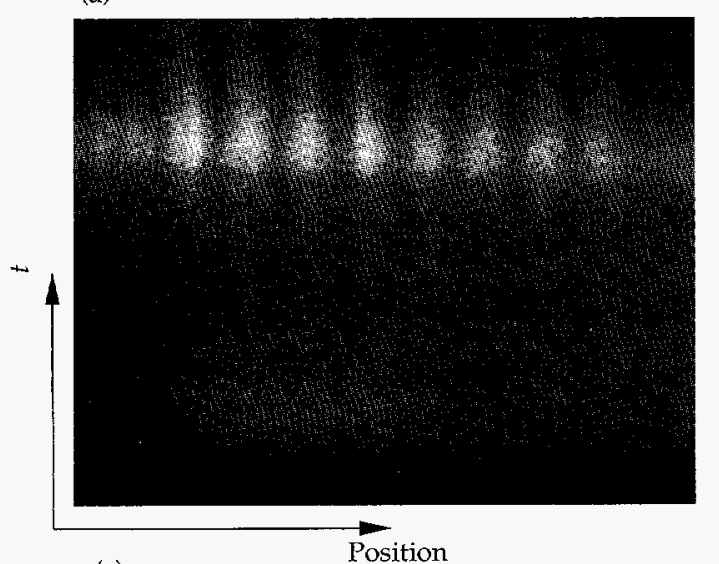

(c)

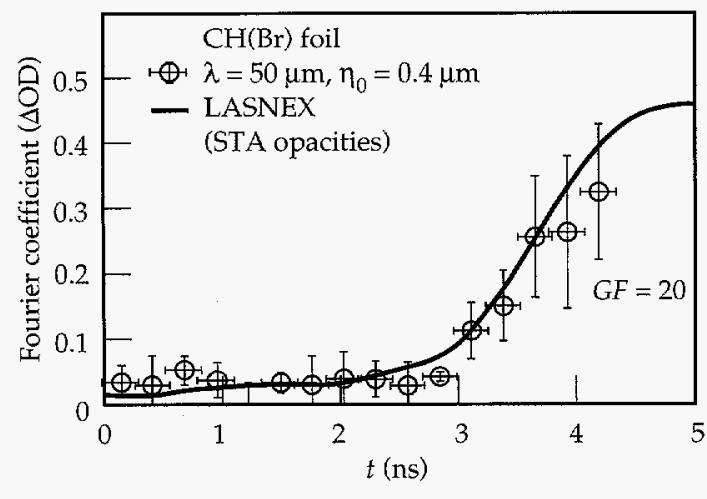

(b)

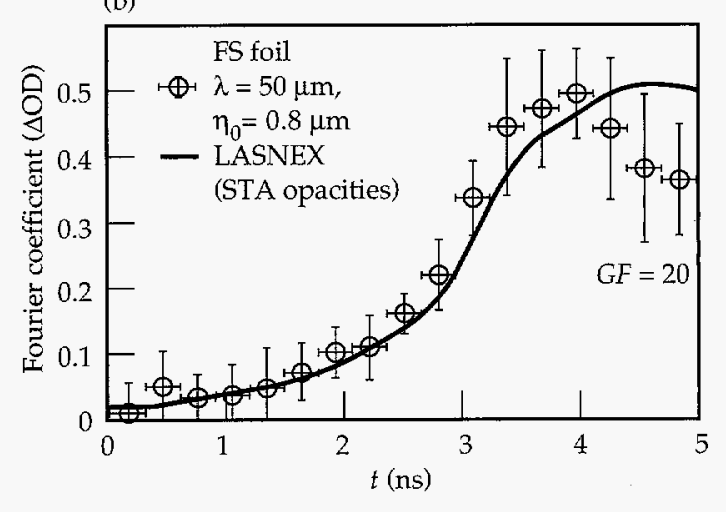

(d)

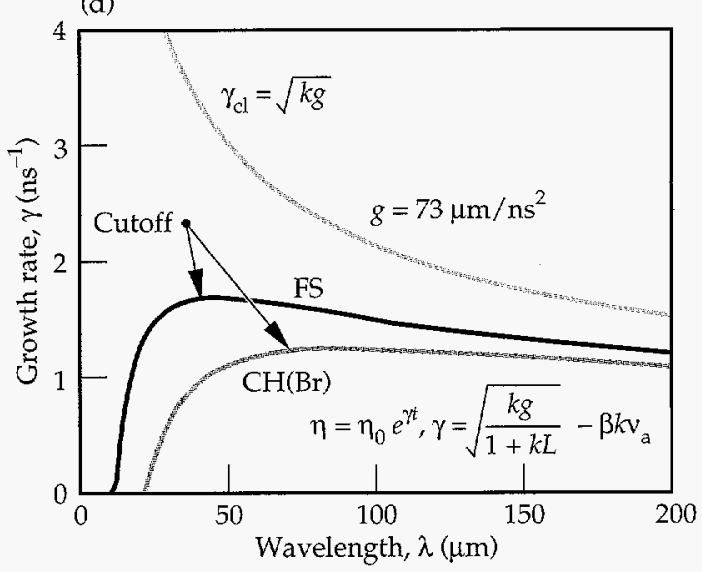

FIGURE 21. Results for intermediate- $\eta_{0}$ foil (from Fig. 18): (a) raw data, (b) Fourier coefficient of fundamental mode vs time. (c) As in (b), but for a $\mathrm{CH}(\mathrm{Br})$ foil (from Fig. 14c).

(d) Dispersion curve for FS and $\mathrm{CH}(\mathrm{Br})$ with $\beta=2.5$ : for $F S, L=1.6 \mu \mathrm{m}$, $v_{\mathrm{a}}=3.2 \mu \mathrm{m} / \mathrm{ns}$; for $\mathrm{CH}(\mathrm{Br}), \mathrm{L}=4.0 \mu \mathrm{m}$, $v_{\mathrm{a}}=3.9 \mu \mathrm{m} / \mathrm{ns}$. (20-03-1293-4398pb01) 
the ablation front has only a very gentle slope, the ablation velocity is very high, the RT growth is practically zero, and the foil burns through quickly. The $\mathrm{CH}(\mathrm{Br})$ foil falls between these two extremes.

\section{Two-Mode Experiments}

We next turn to the two-mode experiments. ${ }^{37,48}$ Figure 24(a) shows the two-mode perturbations we investigated; the upper side of each curve corresponds to the foil. Figure 24(a) shows a large-amplitude twomode perturbation given by $\lambda_{2}=75 \mu \mathrm{m}$ and $\lambda_{3}=50 \mu \mathrm{m}$, with $\eta_{2}=\eta_{3}=2 \mu \mathrm{m}$.

Characterization of the initial perturbations is critical for proper interpretation of the RT growth. The initial perturbations are characterized by three independent techniques-interferometry, contact profilometry, and $x$-ray radiography - and are accurate to $10 \%$ or better. Figure 25 shows examples of characterization by profilometry and radiography for the two-mode foils (and for the eight-mode foils of the next section, "Eight-Mode Experiments"). The agreement between the two techniques is very good except at the shortest wavelengths $(\lambda \leq 25 \mu \mathrm{m})$ because of the finite resolution of our radiography setup. We use the $\eta_{n}$ from contact profilometry as the most accurate initial amplitudes.

Figure 26 shows the results for the large-amplitude two-mode foil shown in Fig. 24(a). Figure 26(a) shows the raw image, and Fig. 26(b) shows a profile of $\ln E$ at 2.7 ns aligned relative to the mold, showing how the phase of the data is established. Figure 26(c) illustrates the analysis technique with a profile of $\ln E$ at $3.3 \mathrm{~ns}$. To remove the long-range structure arising from the backlighter, we fit a low-order polynomial to the profile of $\ln E$, shown by the gray curve. The lower solid curve in
Fig. 26(c) shows the $\ln E$ profile after having subtracted the fit to the backlighter structure. The vertical dotted lines represent the boundaries for the Fourier analysis. The histogram in Fig. 26(d) shows the real component of the Fourier transform of $\ln E$ at $3.3 \mathrm{~ns}$. Because of the cosine symmetry of the perturbation, the imaginary component (not shown) is identically zero except for random noise.

The Fourier modes are enumerated as harmonics of the longest repeating pattern $(150 \mu \mathrm{m})$. Hence, the two pre-existing modes are $k_{2}(\lambda=75 \mu \mathrm{m})$ and $k_{3}(\lambda=50 \mu \mathrm{m})$. Because the initial amplitudes are large, the perturbation

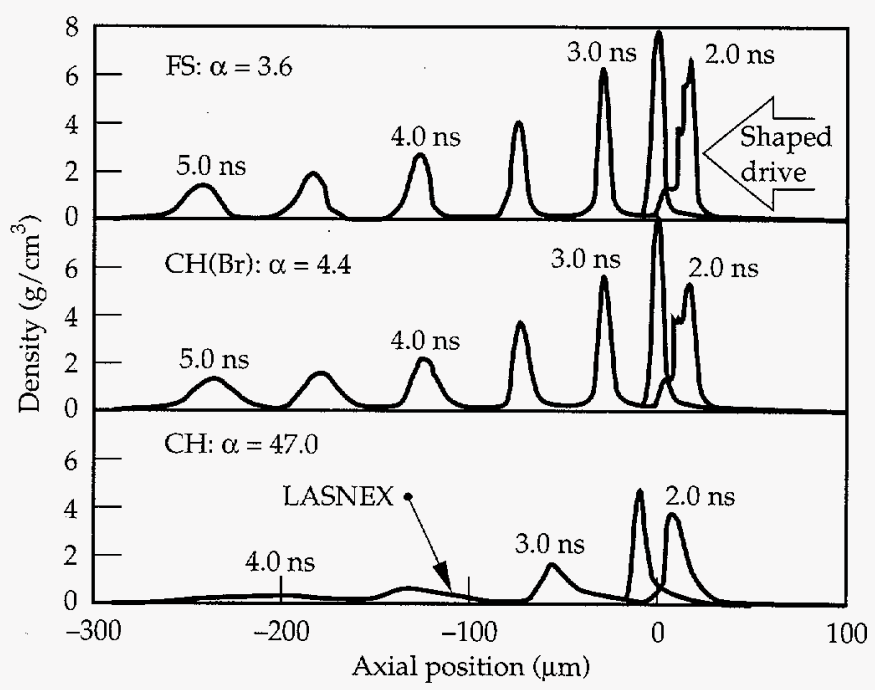

FIGURE 23. Density profiles from 1-D LASNEX simulations for FS, $\mathrm{CH}(\mathrm{Br})$, and $\mathrm{CH}$, assuming the same low-adiabat drive for each. (20-03-1293-4401pb01)
FIGURE 22. Results from the 1-ns square drive pulse shape for (a) FS and (b) CH foils. For this high-adiabat drive, the growth factors were $G<3$. (20-03-1293-4400pb02)
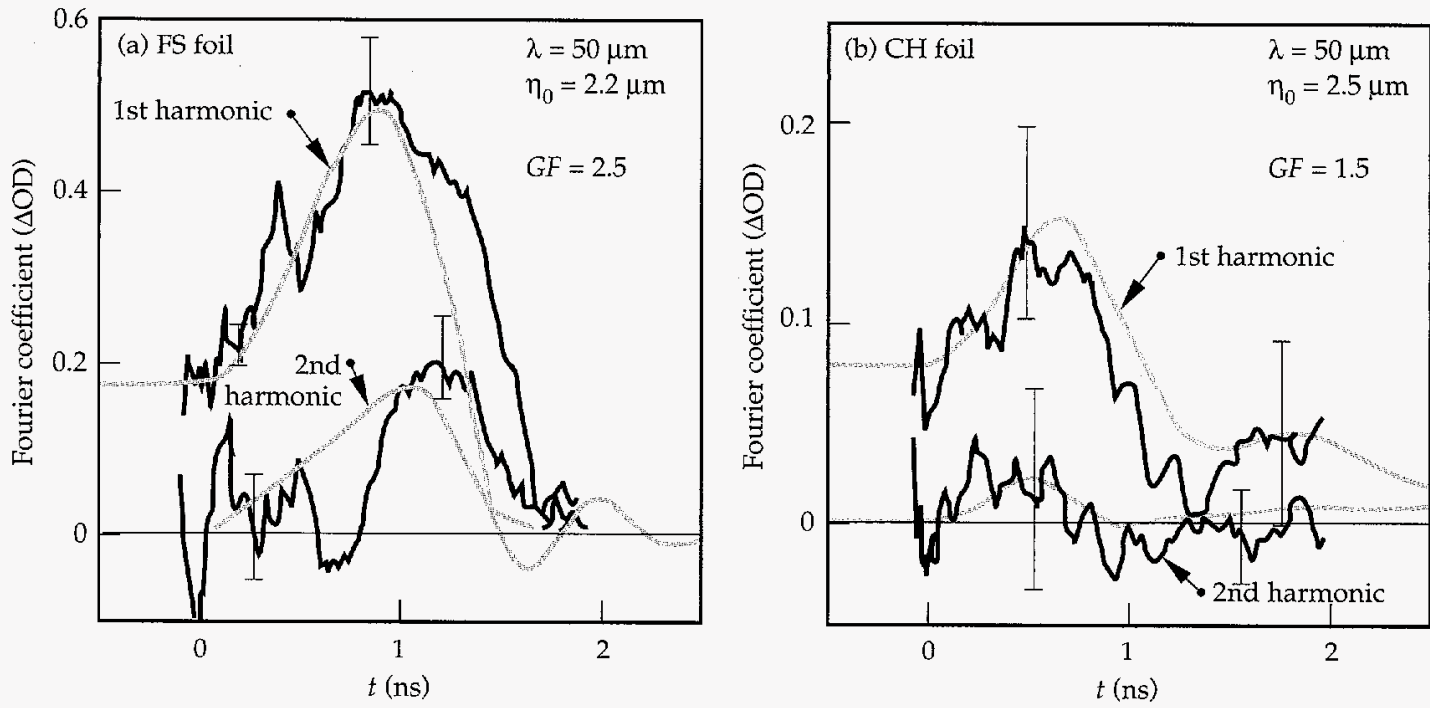

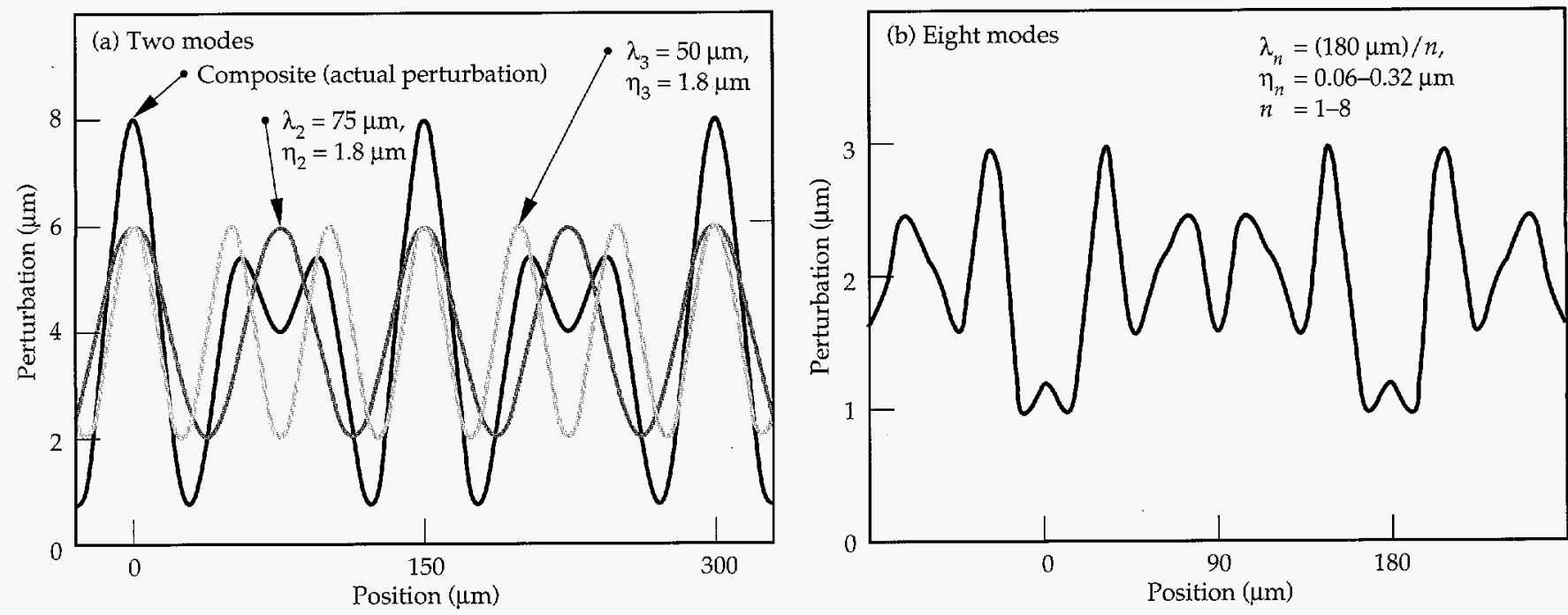

FIGURE 24. Multimode perturbation patterns investigated. (a) Two-mode perturbation: curves correspond to $\lambda_{2}=75 \mu \mathrm{m}, \eta_{2}=1.8 \mu \mathrm{m}$ and $\lambda_{3}=50 \mu \mathrm{m}, \eta_{3}=1.8 \mu \mathrm{m}$. Their superposition represents the actual perturbation. The foil corresponds to the upper side of the curve. (b) Eightmode perturbation corresponds to the sum of wavelengths $\lambda_{n}=(180 \mu \mathrm{m}) / n, n=1-8$; Table 1 gives corresponding amplitudes. (20-03-0394-0741pb03)
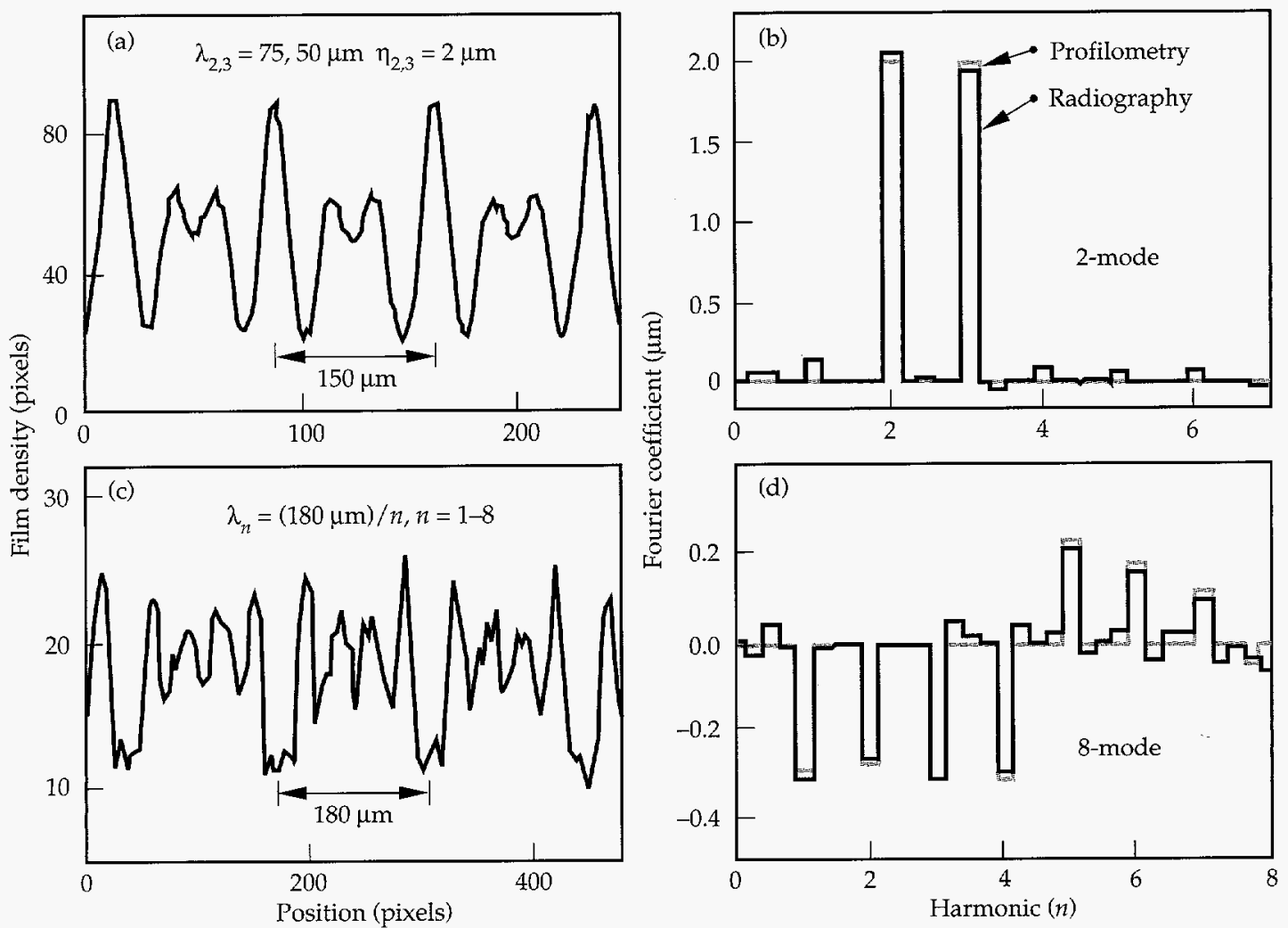

FIGURE 25. Multimode perturbations characterized by contact profilometry and radiography. (a) Lineout from the 2-D radiograph of the large-amplitude two-mode perturbation shown in Fig. 24(a). The contact radiograph corresponds to a 2-hr exposure on Kodak high-resolution glass plate film using a Mo anode at an electron accelerating voltage of $5 \mathrm{kV}$ and a 1-um Ag filter. (A film density of 1024 pixels corresponds to an optical depth of 5.115.) The film response is removed using a simultaneous exposure across a Be step wedge covering the same range in optical depth. (b) The black histogram is the real component of the Fourier transform of the curve shown in (a). The gray histogram represents the Fourier transform of the contact profilometer trace (not shown), and is essentially identical with the radiography result. (c) Same as (a) except for the eight-mode perturbation shown in Fig. 24(b). (d) Same as (b) except for the eight-mode perturbation. (20-03-0394-0742pb03) 
quickly evolves into the nonlinear regime with the appearance at $3.3 \mathrm{~ns}$ of the second harmonic of $k_{2}$, namely, $2 k_{2}$. We also observe very distinct $k_{1}=k_{3}-k_{2}$ and $k_{5}=k_{3}+k_{2}$ coupled modes corresponding to $\lambda=150$ and $30 \mu \mathrm{m}$, respectively. Notably absent is the second harmonic of $k_{3}$, namely, $2 k_{3}$. This is because $3 k_{2}$, the third harmonic of $k_{2}$, has the same magnitude as $2 k_{3}$ but the opposite sign, leading to a cancellation.

The results shown in Fig. 26(d) are rather insensitive to the exact functional form used in fitting the background. The smooth gray curve shown in Fig. 26(c) corresponds to a fifth-order polynomial fit. If we had chosen a second-order polynomial to fit the background, the $k_{1}$ term would have been $5 \%$ larger, and the other modes would have changed by $\sim 1 \%$. With no background subtraction at all, the $k_{1}$ mode would have been only $20 \%$ different, and the other modes would have varied by $\sim 5 \%$ or less. This is illustrated by showing the Fourier composition of the background itself. The gray curve in Fig. 26(d) corresponds to the Fourier transform of the gray curve in Fig. 26(c). Thus there is little sensitivity to the exact details of how we treat the backlighter background subtraction.

It is instructive to view these nonlinear mode coupling effects within the context of perturbation theory. ${ }^{26} \mathrm{We}$ consider here only a qualitative application for the coupling from two pre-existing modes $k_{2}$ and $k_{3}$ using Eq. (8) from the Introduction, namely,

$\eta_{k_{i} \pm k_{j}} \approx \mp \frac{1}{2}\left(k_{i} \pm k_{j}\right) \eta_{k_{i}}^{\mathrm{L}} \eta_{k_{j}}^{\mathrm{L}}$

where $\eta_{k_{n}}^{\mathrm{L}}$ represents the spatial amplitude of mode $k_{n}$ had the growth been entirely in the linear regime.

Notice that $\eta_{k_{3}-k_{2}}$ has the same sign as the product $\eta_{k_{2}}^{L} \eta_{k_{3}}^{L}$, whereas $\eta_{k_{3}+k_{2}}$ has the opposite sign, in agreement with the experimental observation shown in Fig. 26(d). If the boundaries of the Fourier transform are shifted by $75 \mu \mathrm{m}$ (that is, by half of the fundamental $n=1$ period), the $k_{2}$ and $k_{3}$ modes have opposite signs (not shown). The $k_{3}-k_{2}$ mode is then negative, and the $k_{3}+k_{2}$ mode is positive, again in agreement with Eq. (8). Qualitatively at least, the observation of the $k_{3} \pm k_{2}$ coupled terms can be understood from second-order perturbation theory. The modes are too large already by shock breakout to apply second-order theory quantitatively, however

Figure 27 shows the full time dependence of the two-mode data shown in Fig. 26. The plotting symbols represent the data, which again correspond to the real (a)

FIGURE 26. Various representations for the large-amplitude twomode data. (a) Raw streaked image. (b) Latetime $(2.7 \mathrm{~ns})$ profile of $\ln E$ and initial perturbation on the mold, corresponding to the superposition of a $\lambda=75 \mu \mathrm{m}$ and $\lambda=50$ $\mu \mathrm{m}, \eta_{0}=1.8 \mu \mathrm{m}$ perturbation. Data are aligned relative to the mold showing how the perturbation phase is established.

(c) Background-subtraction technique, illustrated on a late-time (3.3 ns) profile of $\ln E$ : "raw" profile, polynomial fit, and result after subtracting background. Vertical dotted lines represent boundaries for Fourier analysis. (d) Real component of Fourier transform of background-subtracted profile in (c), and Fourier transform of background itself. (20-03-0394-0746pb02)

(c)
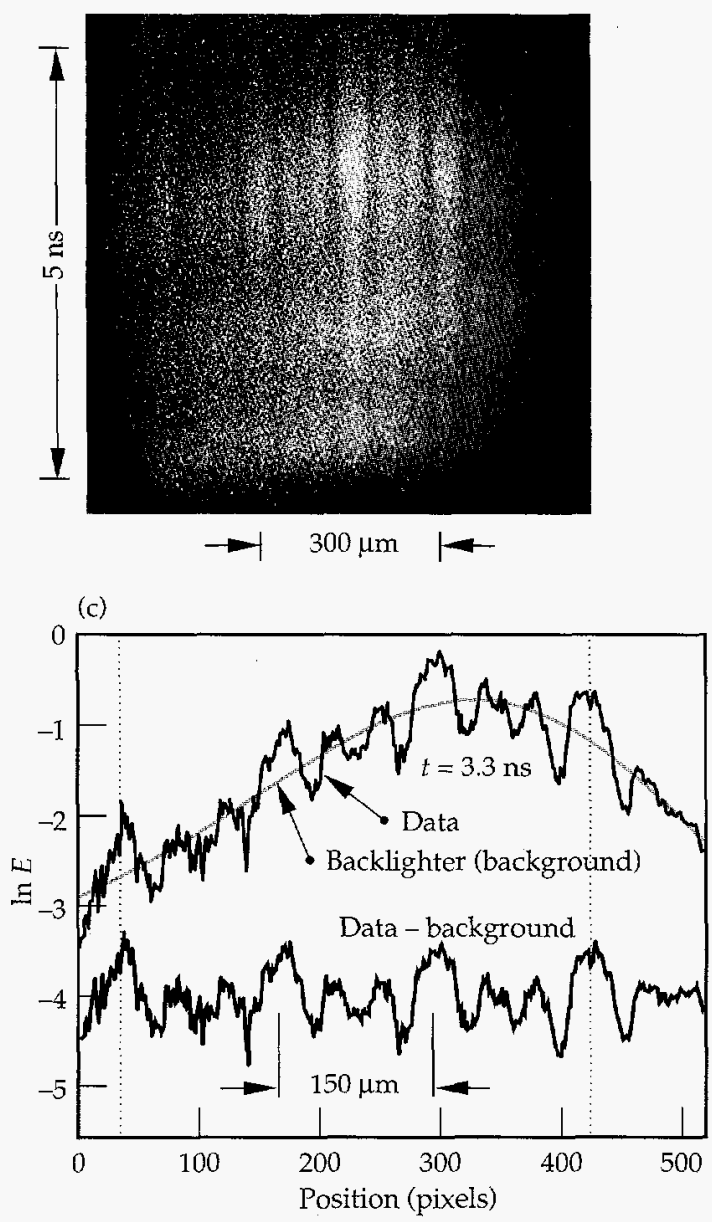

(b)

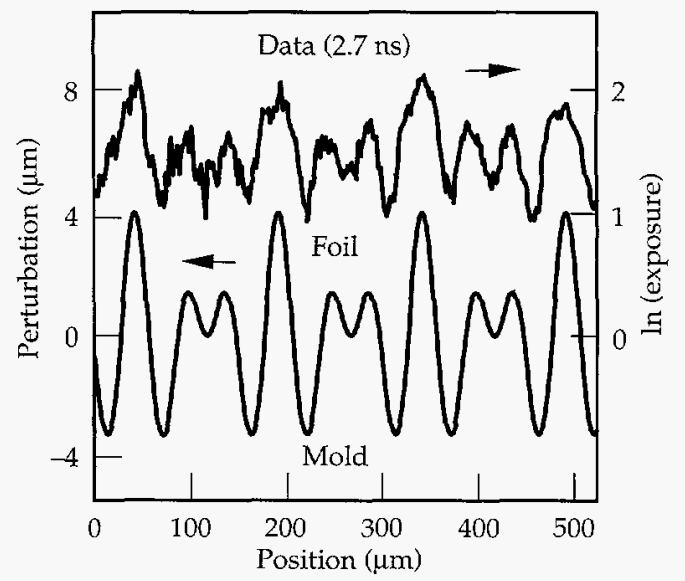

(d)

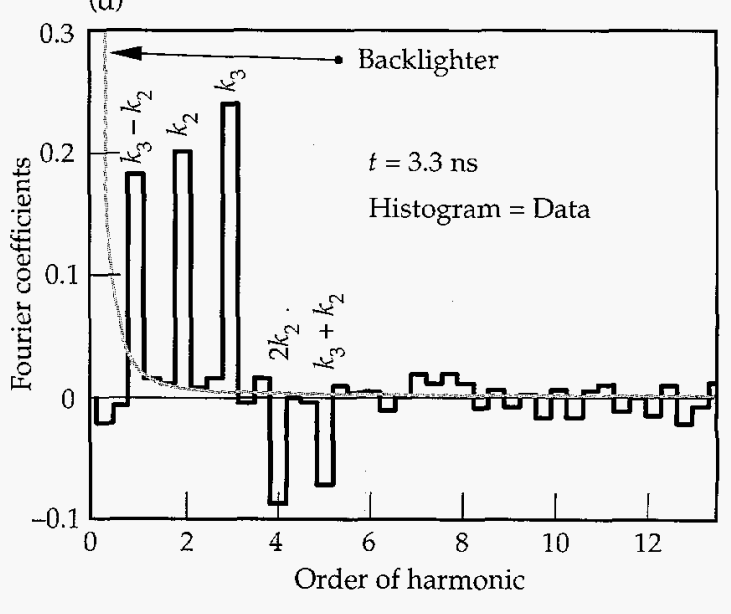


component of the Fourier transform of $\ln E$. Because of symmetry, the imaginary component of the Fourier transform should be zero, and we take its value as a measure of the error for each point. The $k_{3} \pm k_{2}$ coupled terms are not observed until after about $t=2.5 \mathrm{~ns}$, after the perturbation has entered the nonlinear regime. The black curves in Fig. 27 are the corresponding results from 2-D LASNEX simulations, after convolution of the simulated image exposure with the instrumental spatial resolution function [Eq. (11)]. The gray curves in Fig. 27(a) correspond to simulations of the evolution of each mode had it been the only mode initially present. The departure of the black curves from the gray curves for $t \geq 3 \mathrm{~ns}$ coincides with the growth of the coupled terms, as shown in Fig. 27(b). The Fourier composition of the perturbation is redistributed into a broader spectrum because of the $k_{3} \pm k_{2}$ mode coupling. This corresponds in physical space to a change of shape: mode coupling makes the bubbles broader and flatter and the spikes narrower. This is illustrated explicitly in the next section.

\section{Eight-Mode Experiments}

Figure 24(b) shows a small-amplitude eight-mode perturbation given by $\lambda_{n}=(180 \mu \mathrm{m}) / n$. The individual amplitudes $\eta_{n}$ (given in Table 1 ) are about a factor of 10 smaller than those for the two-mode foil. Figure 28(a) shows the raw image of the experimental shot, and Fig. 28(b) gives a late-time profile of $\ln E$ aligned relative to the mold, showing how the phase of the data is established. ${ }^{37,48}$ The vertical dashed lines indicate the boundaries for the Fourier analysis. The black histograms in Figs. 28(c) and 28(d) show the real component of the Fourier transform at an early time ( $3.2 \mathrm{~ns})$ and at late time (4.4 ns). Because of the cosine symmetry, the imaginary component (shown by the gray histograms) oscillates around the baseline as random background and serves as an estimate of the error. Early in time in the linear regime [Fig. 28(c)], only the pre-existing modes grow, in accordance with their initial amplitudes and growth rates. The $k_{3}$ mode $(\lambda=60 \mu \mathrm{m})$ dominates. Late in time [Fig. 28(d)], the perturbation has entered the nonlinear regime and the modes no longer grow independently. The $k_{3}$ mode no longer dominates, its magnitude having been reduced by $k_{3}-k_{2}$ mode coupling to drive up the $k_{1}$ mode. This causes the $k_{1}$ term to reverse phase; the initial sign of the $k_{1}$ mode was negative, as given in Table 1.

Figure 29 shows the full time evolution of the eightmode data. The plotting symbols correspond to the real component of the Fourier transform of the data, and the error bars correspond to the imaginary component. The black curves represent the eight-mode LASNEX simulation after convolving $\ln E$ with the spatial resolution function [Eq. (11)]; the qualitative agreement with the data is good. The light gray curves represent the single-mode simulations, in which it is assumed that each mode existed alone. As a result of mode coupling, modes $k_{2}$ through $k_{5}$ grow less than they would have alone. Growth reduction is least for mode $k_{4}$, which has the largest amplitude at the time of saturation. The presence of multiple modes causes nonlinearity to occur earlier than if the modes had existed alone.

Modes $k_{1}, k_{6}$, and $k_{7}$ reverse phase and grow with the opposite sign. These modes show most prominently the effects of coupling from the dominant modes, $k_{2}$, $k_{3}$, and $k_{4}$. The dark gray curves show the amplitudes predicted for these modes from second-order theory, described in more detail below. The phase reversal for mode $k_{1}$, for example, can be understood from Eq. (8) and considering only the dominant modes $k_{2}, k_{3}$, and $k_{4}$. Both the $k_{3}-k_{2}$ and $k_{4}-k_{3}$ coupled terms are positive, tending to cause a phase reversal in the growth of (a)

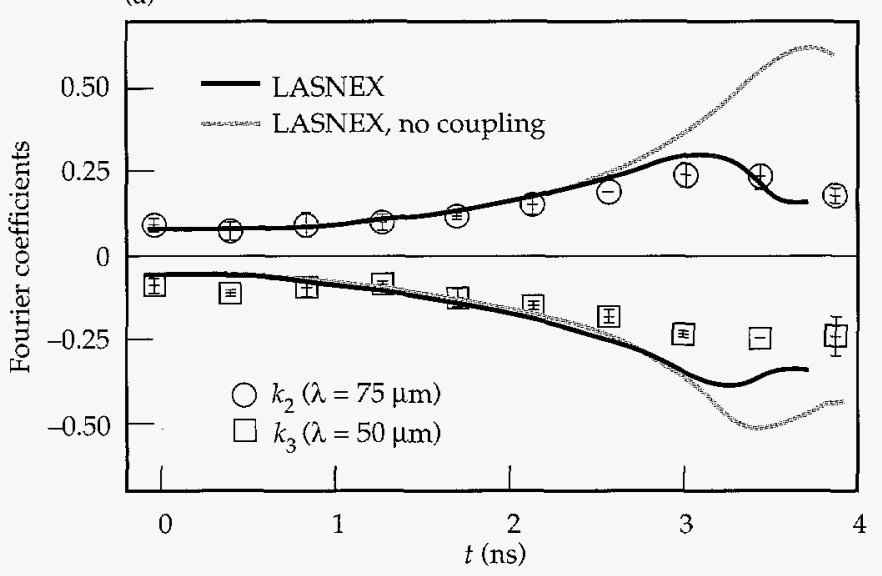

(b)

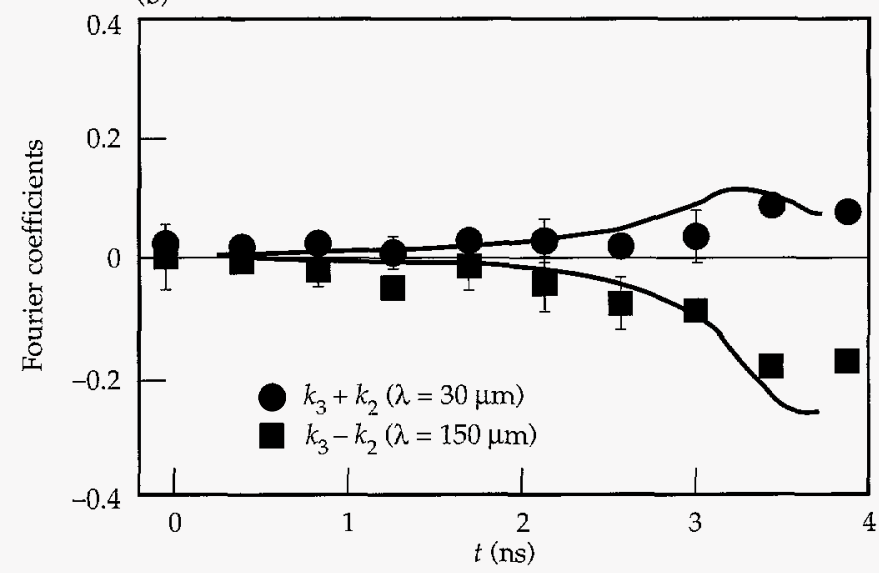

FIGURE 27. Full time dependence for two-mode data of Fig. 26. (a) Results for the $\lambda=75 \mu \mathrm{m}$ component of the pre-existing perturbation and for $k_{3}$ (the $\lambda=50 \mu \mathrm{m}$ component). (b) The $k_{3}+k_{2}, \lambda=30 \mu \mathrm{m}$ and $k_{3}-k_{2}, \lambda=150 \mu \mathrm{m}$ coupled terms and corresponding 2-D LASNEX two-mode and single-mode results. $(20-03-0394-0747 \mathrm{pb} 03)$ 
the $k_{1}$ mode (which was initially negative), as observed. Similarly, the phase reversals of modes $k_{6}$ and $k_{7}$ can also be understood from Eq. (8), considering $k_{i}+k_{j}$ coupling from the dominant modes $k_{2}, k_{3}$, and $k_{4}$. Figure 29(d) also shows (dashed curve) the result for mode $k_{4}$ of an eightmode simulation with enhanced preheat in the drive (factor of 10 increase in drive spectrum for $h v>1.4 \mathrm{keV}$ and $t<2 \mathrm{~ns}$, as discussed in the earlier section "SingleMode Experiments"). As in Fig. 17(c), the enhanced preheat reduces the perturbation growth considerably, bringing the simulation into good agreement with the data. This is true for all eight modes, although we show only the enhanced-preheat result for mode $k_{4}$.

The second-order perturbation model can be quantitatively applied to the eight-mode experiment $26,37,48,49$ using Eq. (A3) from the final section "Amplitude of Coupled Modes,"

$\eta_{k} \approx \eta_{k}^{\mathrm{L}}+\frac{1}{2} k\left(\sum_{k^{\prime}} \eta_{k^{\prime}}^{\mathrm{L}} \eta_{k+k^{\prime}}^{\mathrm{L}}-\frac{1}{2} \sum_{k^{\prime}<k} \eta_{k^{\prime}}^{\mathrm{L}} \eta_{k-k^{\prime}}^{\mathrm{L}}\right)$, by summing over the products of all pairs of modes whose sum or difference equals the $k$ of interest. The $\eta_{k_{n}}$ represent spatial amplitudes, which we define from the LASNEX simulations by dividing the modulations in areal density by the foil peak density, that is,

$\eta(\mu \mathrm{m}) \approx \frac{\delta \int \rho d z}{\rho_{\max }}$.

We apply this model to our experiment as follows. For each $\lambda_{n}$ in the eight-mode foil, 2-D LASNEX simulations are run for perturbations of very small initial amplitude, ensuring that the RT evolution remains in the linear regime. The $\eta_{k_{n}}^{\mathrm{L}}(t)$ are then obtained by scaling by the ratio of actual to the assumed initial amplitude. This technique of generating the $\eta_{k_{n}}^{\mathrm{L}}(t)$ automatically includes the effects of the time-dependent acceleration, compression, density gradient, and ablative stabilization. The dark gray curves in Figs. 29(a), 29(f), and $29 \mathrm{~g}$ ) show the results from this perturbation analysis for $t \leq 3.7 \mathrm{~ns}$. After this time, the central assumption of
FIGURE 28. Eightmode data in various representations. (a) Raw streaked image. (b) Late-time profile of $\ln E$, aligned with the mold as in Fig. 26. Vertical dashed curves give boundaries used in Fourier analysis. (c) Real and imaginary components of Fourier transform of "earlytime ${ }^{\prime \prime}(3.2 \mathrm{~ns})$ profile of $\ln E \approx-\mathrm{OD}$. The imaginary component (which should be zero because of the cosine symmetry) illustrates the level of background noise and can be used for an estimate of the error. The dominant $k_{3}(\lambda=60 \mu \mathrm{m})$ mode is indicated. (d) Same as (c) except at late time (4.4 ns). The dominant term is now $k_{4}(\lambda=45 \mu \mathrm{m})$, and the strength from the $k_{2}$ and $k_{3}$ terms has been redistributed into the $k_{1}=k_{3}-k_{2}$ coupled mode, as indicated. (20-03-1293-4406pb02) (a)

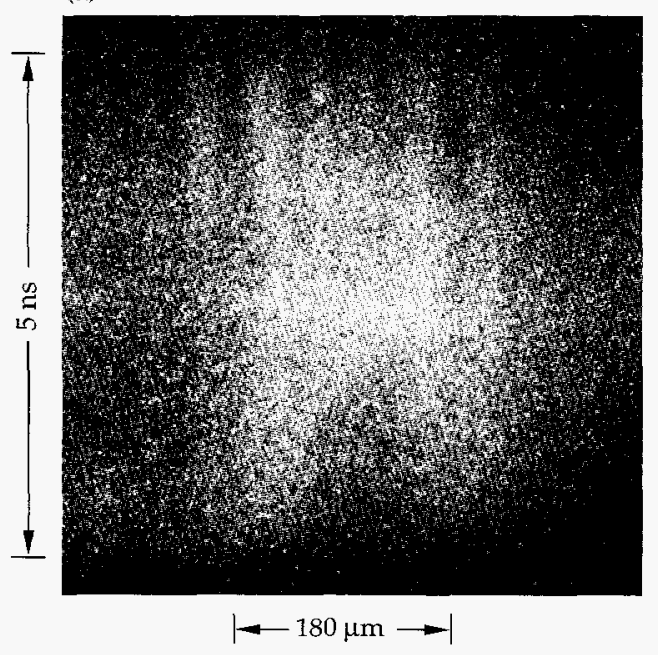

(c)

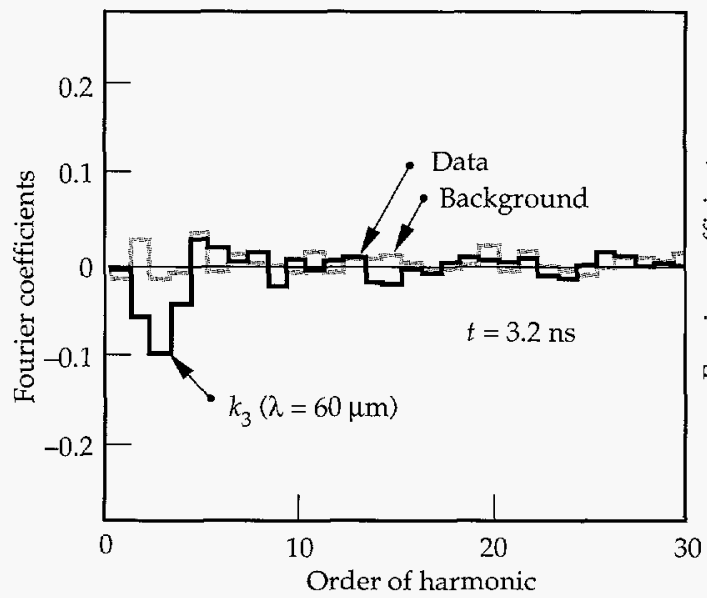

(b)

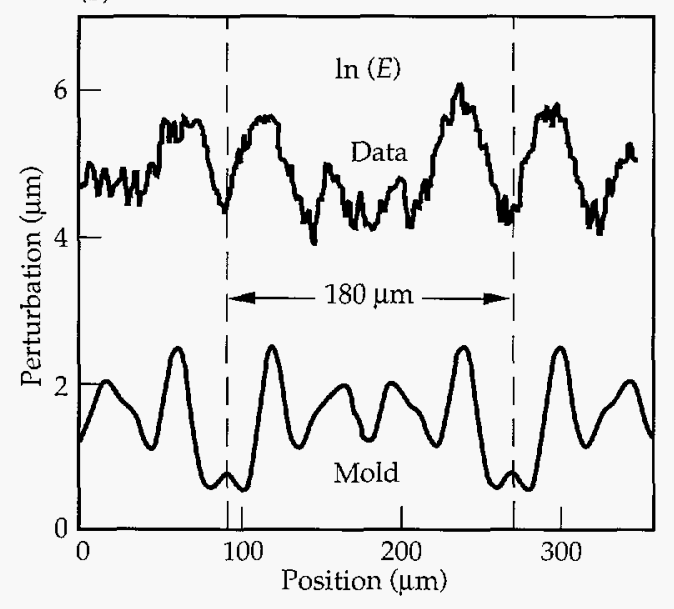

(d)

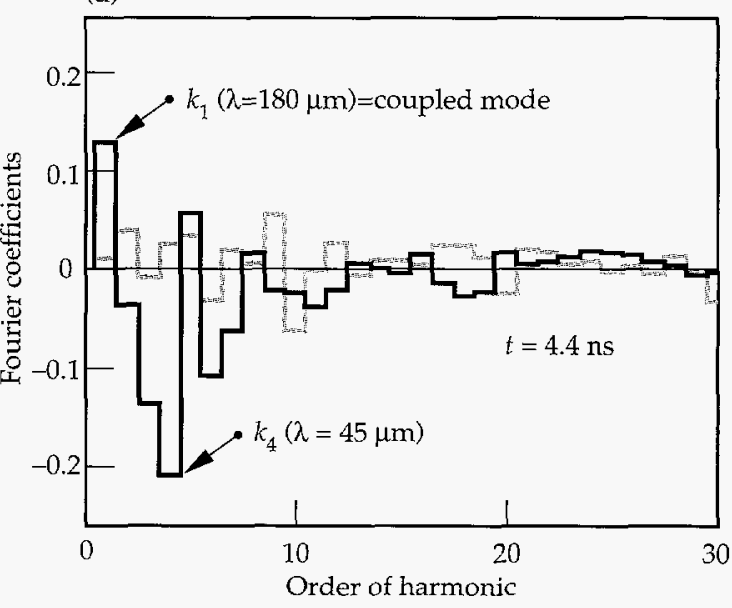


the model (dominant modes not affected by the coupling terms) is violated and the model is no longer applicable. In each case, the phase reversals are well described by second-order perturbation theory.

At late times ( $t \geq 4 \mathrm{~ns})$, the saturation of modes $k_{2}$ and $k_{3}$ results from the redistribution of the Fourier components because of mode coupling. In physical space, this corresponds to a change in shape, as illustrated in Fig. 30 for the two-mode and eight-mode perturbations. The black curves correspond to profiles of $\ln E$ taken from the LASNEX simulations before inclusion of the instrument spatial resolution function. The gray curves represent the sum of the results from the single-mode simulations. Comparing the gray and black curves, we see that the shapes of the perturbations with and without mode coupling differ. With mode coupling, the bubbles are broader and flatter and the spikes are narrower. This shape effect has been observed in other simulations, ${ }^{29}$ but to our knowledge this is the first experimental observation of the effect in ablatively accelerated foils.

The results from our eight-mode experiment can also be compared with results from a saturation model developed for a full continuum of initial modes. ${ }^{32}$ The basic premise of this model is that a perturbation corresponding to a full continuum of modes saturates when the product $k_{\text {char }} \eta_{\text {char }}$ is no longer small, that is, when

$\eta_{\text {char }}=\varepsilon_{1} \lambda_{\text {char }}=\frac{2 \pi \varepsilon_{1}}{k_{\text {char }}}$

where $\eta_{\text {char }}$ and $\lambda_{\text {char }}$ are a characteristic spatial amplitude and wavelength and $\varepsilon_{1}$ is some number less than unity. But $\eta_{\text {char }}$ can be approximated as the quadrature sum of individual modes within a band $\Delta k$ centered around $k_{\text {char }}=2 \pi / \lambda_{\text {char, }}$ namely,

$\eta_{\text {char }}=\left(\sum_{\Delta k} \eta_{k}^{2}\right)^{1 / 2} \approx\left(\Delta k \frac{L}{2 \pi} S_{k}^{2}\right)^{1 / 2} \approx\left(\varepsilon_{2} k \frac{L}{2 \pi} S_{k}^{2}\right)^{1 / 2}$,

where $\Delta k=\varepsilon_{2} k$ for some $\varepsilon_{2}<1, L$ is the system size, $L / 2 \pi$ is the $2-\mathrm{D}$ density of Fourier states, and $S_{k}$ is a

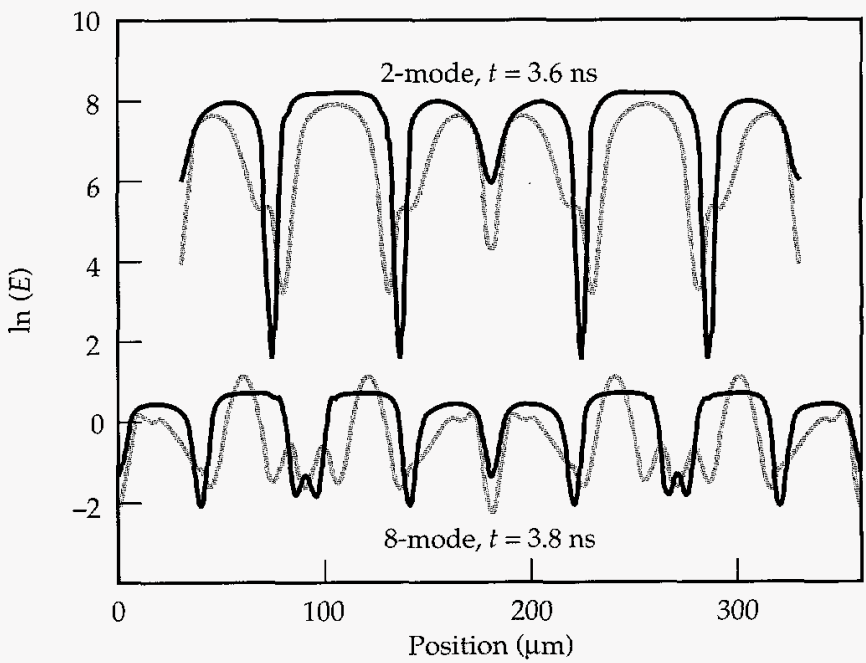

FIGURE 30. Effect of mode coupling on perturbation shape. The upper black curve represents the two-mode simulation at $3.6 \mathrm{~ns}$, and the upper gray curve corresponds to the sum of the simulations for the two individual modes run alone. The lower curves are the same only for the eight-mode perturbation at $3.8 \mathrm{ns.} \mathrm{(20-03-0194-0106pb02)}$ (a) $k_{1}(\lambda=180 \mu \mathrm{m})$

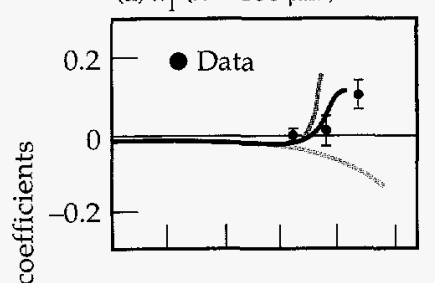

(e) $k_{5}(\lambda=36 \mu \mathrm{m})$

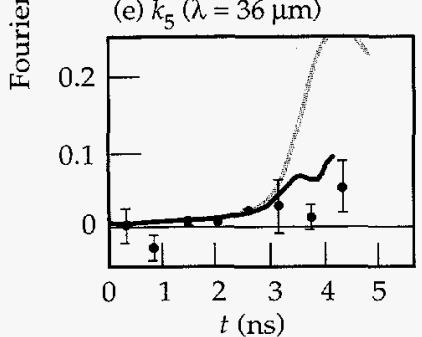

(b) $k_{2}(\lambda=90 \mu \mathrm{m})$

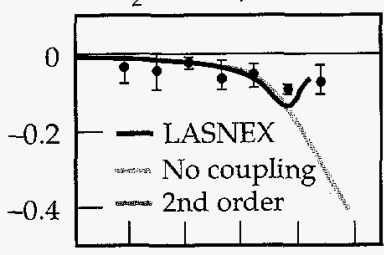

(f) $k_{6}(\lambda=30 \mu \mathrm{m})$

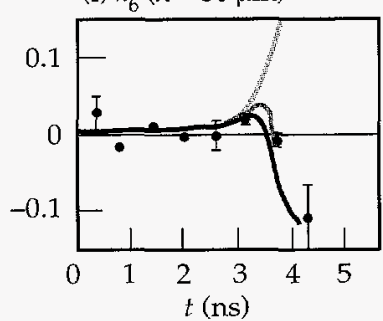

(c) $k_{3}(\lambda=60 \mu \mathrm{m})$

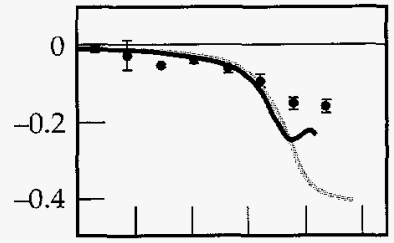

(g) $k_{7}(\lambda=25.7 \mu \mathrm{m})$

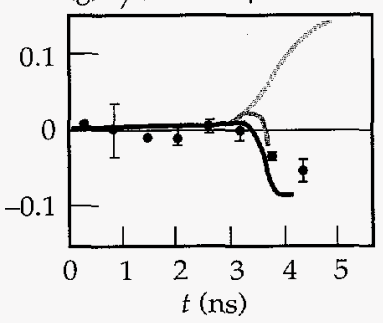

(d) $k_{4}(\lambda=45 \mu \mathrm{m})$

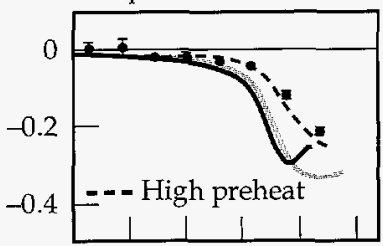

(h) $k_{8}(\lambda=22.5 \mu \mathrm{m})$

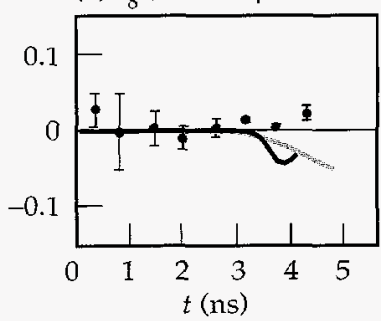

FIGURE 29. Full time dependence for data shown in Fig. 28. (a)-(h) Growth of modes $\lambda_{n}=(180 \mu \mathrm{m}) / n, n=1-8$, vs time. Solid circles represent data; black curves represent corresponding 2-D LASNEX simulations. Light gray curves represent simulations under the assumption that each individual mode existed alone, and the dark gray curves for modes $k_{1}, k_{6}$, and $k_{7}$ correspond to the results of a calculation using weakly nonlinear, second-order perturbation theory. The dashed curve for mode $k_{4}$ corresponds to a simulation with assumed enhanced preheat in the foot of the drive. $\quad(20-03-0394-0749 \mathrm{pb} 03)$ 
typical spatial amplitude of an individual mode within $\Delta k$. Combining Eqs. (15) and (16) gives

$$
\begin{aligned}
& S_{k}=\frac{v_{2 D}}{L^{1 / 2} k^{3 / 2}}, \quad(\text { in 2-D), } \\
& S_{k}=\frac{v_{3 D}}{L k^{2}} \quad \text { (in 3-D), }
\end{aligned}
$$

where $\varepsilon_{1}$ and $\varepsilon_{2}$ have been combined into a single parameter $v$ (set in Ref. 32 for the 2-D case to $v_{2 D}=1.14$ ), and $L$ represents here the longest wavelength in the periodic perturbation, $L=180 \mu \mathrm{m}$. The result for 3-D given in Eq. (17b) is derived the same way, but with $\varepsilon_{2} k$ replaced by $\left(\varepsilon_{2} k\right)^{2}$, and the density of states factor becomes $(L / 2 \pi)^{2}$. The normalization $v_{2 \mathrm{D}}=1.14$ was arrived at by comparison with the classical (incompressible) fluid RT experiments of Read and Youngs. ${ }^{50}$ The above saturation results, namely Eq. (17a) and (17b), can be derived more elegantly as follows. A multimode perturbation in a localized region of space can be thought of as a wavepacket. Over a small but finite spectral range, this wavepacket cannot be distinguished over short distances from a single-mode at wave vector $\mathbf{k}$. We expect RT saturation to occur at roughly the same amplitude in both cases (wavepacket vs single-mode), which means that amplitudes at saturation of the individual components of the wavepacket will be less than the amplitude of the single mode. Based on the criterion of Layzer for a single mode, ${ }^{25}$ saturation is expected to occur when the spacial amplitude $\eta$ reaches $\sim(0.6 / k)$. In the multimode case, an additional factor of $\lambda / L$ enters to account for the number of similar modes about $k$ that can contribute to the saturation of mode $k$. Hence $S_{k} \approx \lambda / k L=2 \pi / k^{2} L$, as given by Eq. $17(\mathrm{~b})$. We apply this saturation model, namely Eq. 17(a), to our eight-mode experiment by calculating with LASNEX the growth of each mode $k_{n}$ in the linear regime until its spatial amplitude defined by Eq. (14) exceeds the $S_{k_{n}}$ given by Eq. (17). At this time we make a smooth transition to bubble growth that is linear in time, corresponding to a terminal bubble velocity equal to the velocity at saturation. This transition to saturated growth is accomplished with the logarithmic construction

$\eta_{k}(t)=S_{k}\left\{1+\ln \left[\eta_{k}^{L}(t) / S_{k}\right]\right\}$,

where $\eta_{k}^{L}(t)$ represents the spatial amplitude had the growth remained in the linear regime. The modes are added in quadrature to produce the predicted rootmean-square (rms) bubble amplitude. We compare this with the rms bubble amplitude from the LASNEX eight-mode simulation. Bubbles are defined in terms of foil areal density $\int \rho d z$ (that is, the foil $\rho r$ ) by considering only those perturbations leading to $\rho r<(\rho r)_{a y}$. Figure 31 shows the result for the nominal $v_{2 D}=1.14$ and for values of $v_{2 D}$ a factor of 2 higher and lower than 1.14. The result corresponding to $v_{2 D}=0.57$ agrees best with the LASNEX eight-mode simulation. This is only a crude test of the model for ablatively accelerated foils, because the density of Fourier modes is low. Future work will involve 3-D experiments with a near continuum of modes and larger growth factors as a better test of this saturation model.

\section{3-D Single-Mode Experiments}

The nonlinear RT growth of a perturbation depends upon its shape. Perturbations of the same magnitude wavenumber $k=\left(k_{x}^{2}+k_{y}^{2}\right)^{1 / 2}$ can have different shape and can therefore evolve differently in the nonlinear regime while having the same linear-regime $R T$ growth rate. In this section we examine how the $3-\mathrm{D}$ shape affects the growth of single-mode perturbations on planar foils. ${ }^{51}$ The $\mathrm{CH}(\mathrm{Br})$ foils were made using a new laser ablation technique to make molds in Kapton or Mylar substrates. ${ }^{52}$ We prepared perturbed foils all with the same magnitude wave vector $k=\left(k_{x}^{2}+k_{y}{ }^{2}\right)^{1 / 2}$ and nominally the same amplitude. The " $2-\mathrm{D}^{\prime \prime}$ foil (1-D wave vector $\mathbf{k}=k_{x}$ ) was a simple $\lambda=50 \mu \mathrm{m}$ sinusoid with initial amplitude $\eta_{0}=2.5 \mu \mathrm{m}$. One of the " $3-\mathrm{D}^{\prime \prime}$ foils [2-D wave vector $k=\left(k_{x}, k_{y}\right)$ ] corresponded to a "stretched" $k_{x}=3 k_{y}$ perturbation, and the other was a square $k_{x}=k_{y}$ mode. The three foils were characterized using contact radiography [Fig. 32(a)-32(c)] and contact profilometry. The radiographs were converted to spatial amplitudes using a $\mathrm{CH}(\mathrm{Br})$ step wedge. Figure 32(d)-32(f)] show corresponding images from Nova shots at $4.3 \mathrm{~ns}$, which is near peak growth. The gated $x$-ray pinhole camera for these images was run at $8 \times$ magnification

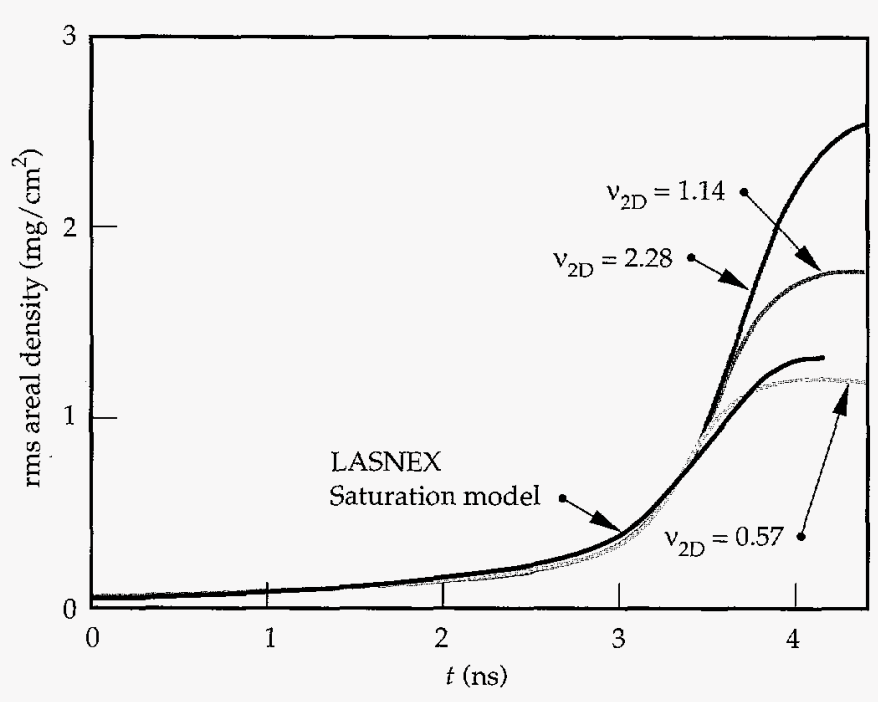

FIGURE 31. Results of eight-mode LASNEX simulation and corresponding results from multimode saturation theory. Black curve corresponds to the rms perturbation in areal density $\Delta \int \rho d z$ from the eight-mode simulation, where we consider only the bubble amplitude. Other curves correspond to results of the saturation model for normalizations [Eq. (17a)] of $v_{2 D}=2.28,1.14$, and 0.57. (20-03-0194-0131pb02) 
with $10-\mu \mathrm{m}$ pinholes and $150-\mu \mathrm{m}$ Be filtering. The backlighter was Sc at $4.3 \mathrm{keV}$.

Each image from the Nova shots [Fig. 32(d)-32(f)] is converted to $\ln E \propto-\mathrm{OD}=-\int \rho \kappa d z$. Hence, modulations in $\ln E$ correspond to modulations in foil areal density. To visualize the 3-D RT evolution better, we focus momentarily on the $k_{x}=k_{y}$ mode. Figure 33(a) gives a 3-D surface perspective of the data shown in Fig. 32(f). Figure 33(b) shows the corresponding simulation with the new 3-D radiation-hydrodynamics code HYDRA. ${ }^{53}$ The height of these surfaces is proportional to -(areal density), and crudely speaking, represents the boundary between the hot, low-density ablated plasma and the dense pusher material ahead of it.

In the reference frame of the accelerating ablation front, one would see a broad, hot bubble of ablated plasma rising up through the pusher and spikes of dense pusher fluid falling essentially freely through the ablated plasma. This canonical shape of the 3-D RT instability can be understood from a simple buoyancyvs-drag equation, 54,55

$$
\rho_{1} V \frac{\partial u}{\partial t}=\left(\rho_{1}-\rho_{2}\right) V g-c_{\mathrm{D}} \rho_{1} u^{2} S,
$$

(a)

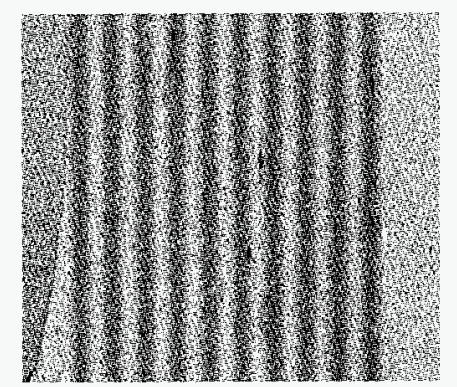

(d)

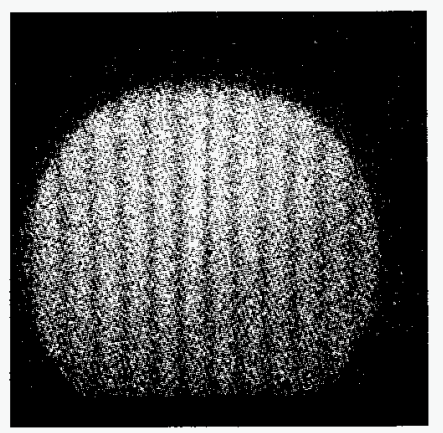

(b)

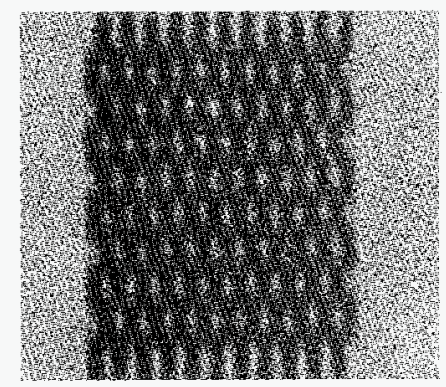

(e)

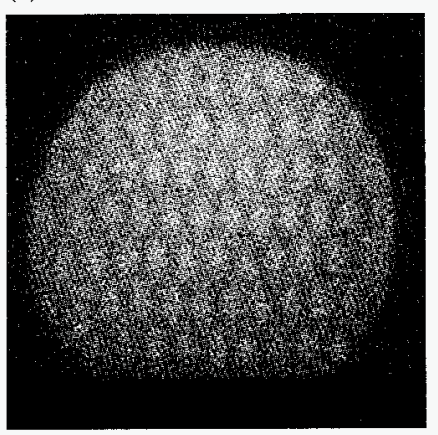

(c)

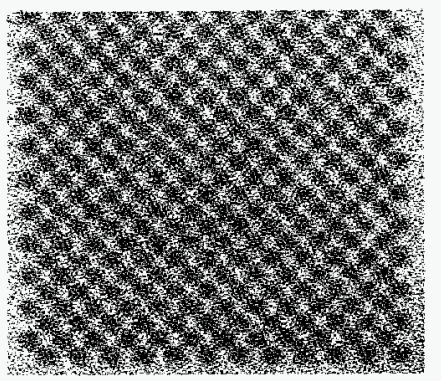

(f)

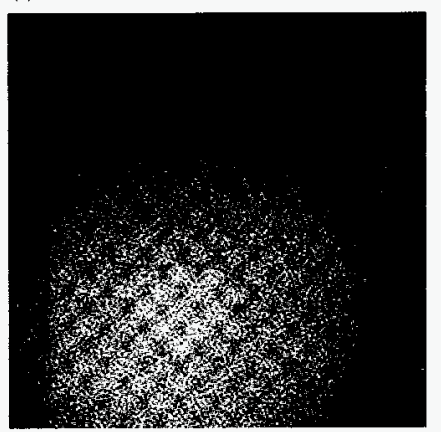

FIGURE 32. (a)-(c)

Contact radiographs of foils identical to those used in the 3-D singlemode Nova experiments. Perturbations correspond to (a) $2-\mathrm{D} \lambda=50 \mu \mathrm{m}$, $\eta_{0}=2.5 \mu \mathrm{m}$; (b) 3-D $k_{x}=3 k_{y}, \lambda_{x}=53 \mu \mathrm{m}$ $\lambda_{y}=158 \mu \mathrm{m}, \eta_{0}=2.4$ $\mu \mathrm{m}$; and (c) 3-D $k_{x}=k_{y^{\prime}}$ $\lambda_{x}=\lambda_{y}=71 \mu \mathrm{m}$, $\eta_{0}=2.7 \mu \mathrm{m}$. (d)-(f) Corresponding images from Nova shots at $4.3 \mathrm{~ns}$. (20-03-0595-1390pb01) (a)

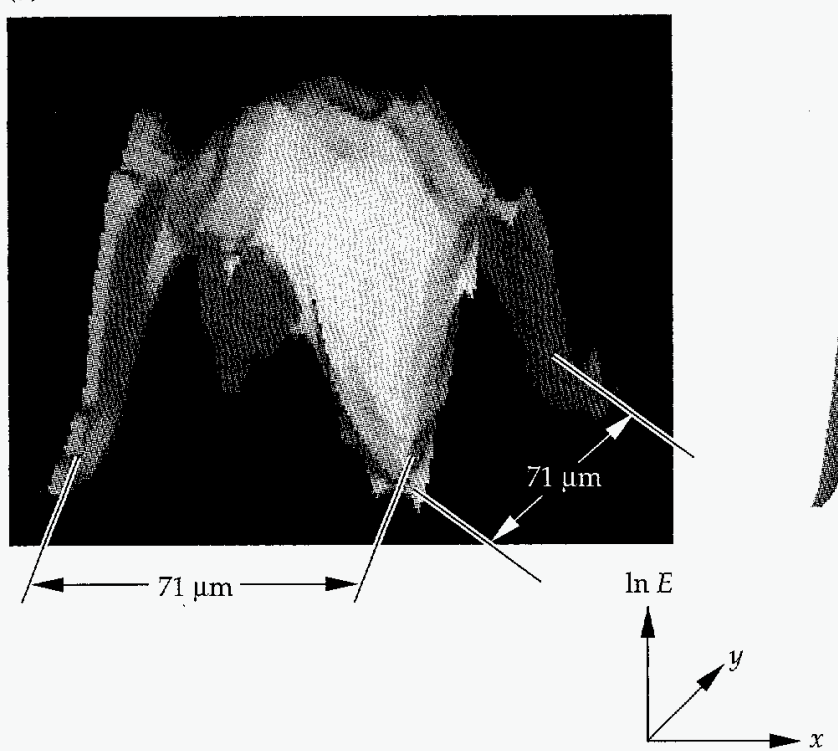

(b)

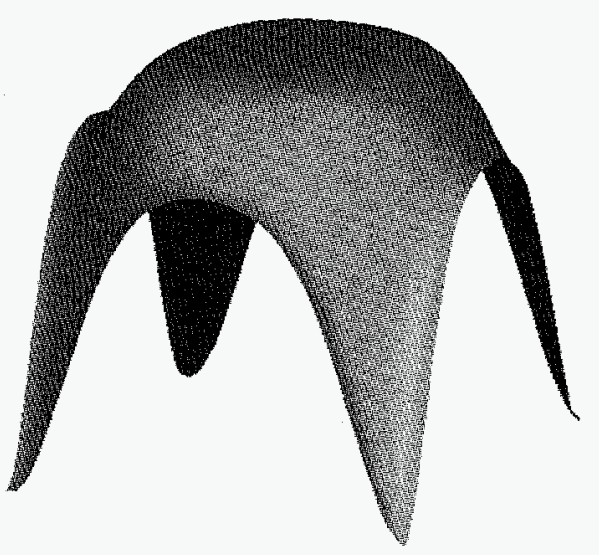

FTGURE 33. 3-D surface perspective of the $k_{\mathrm{x}}=k_{\mathrm{y}}$ case from Fig. 32 as (a) measured on the Nova shot at $4.3 \mathrm{~ns}$ and (b) simulated with the 3 -D radiationhydrodynamics code HYDRA. ${ }^{53}$ The height is proportional to $\ln E$. (50-05-0595-1116pb01) 
where subscripts 1 and 2 refer to the dense pusher fluid and low-density ablation plasma, respectively, $V$ is the volume of fluid pushed aside by the bubble passage, $S$ is the cross-sectional area of the bubble, and $c_{D}$ is the drag coefficient. Equation (19) states that the net force on the mass $\rho_{1} V$ of the heavy fluid equals the buoyancy force minus drag. The bubble tip naturally acquires the shape that minimizes the drag per unit mass, i.e., the bubble evolves towards a shape that minimizes $S / V$, which implies a spherical bubble tip shape, as observed. This is entirely equivalent to the common interpretation.
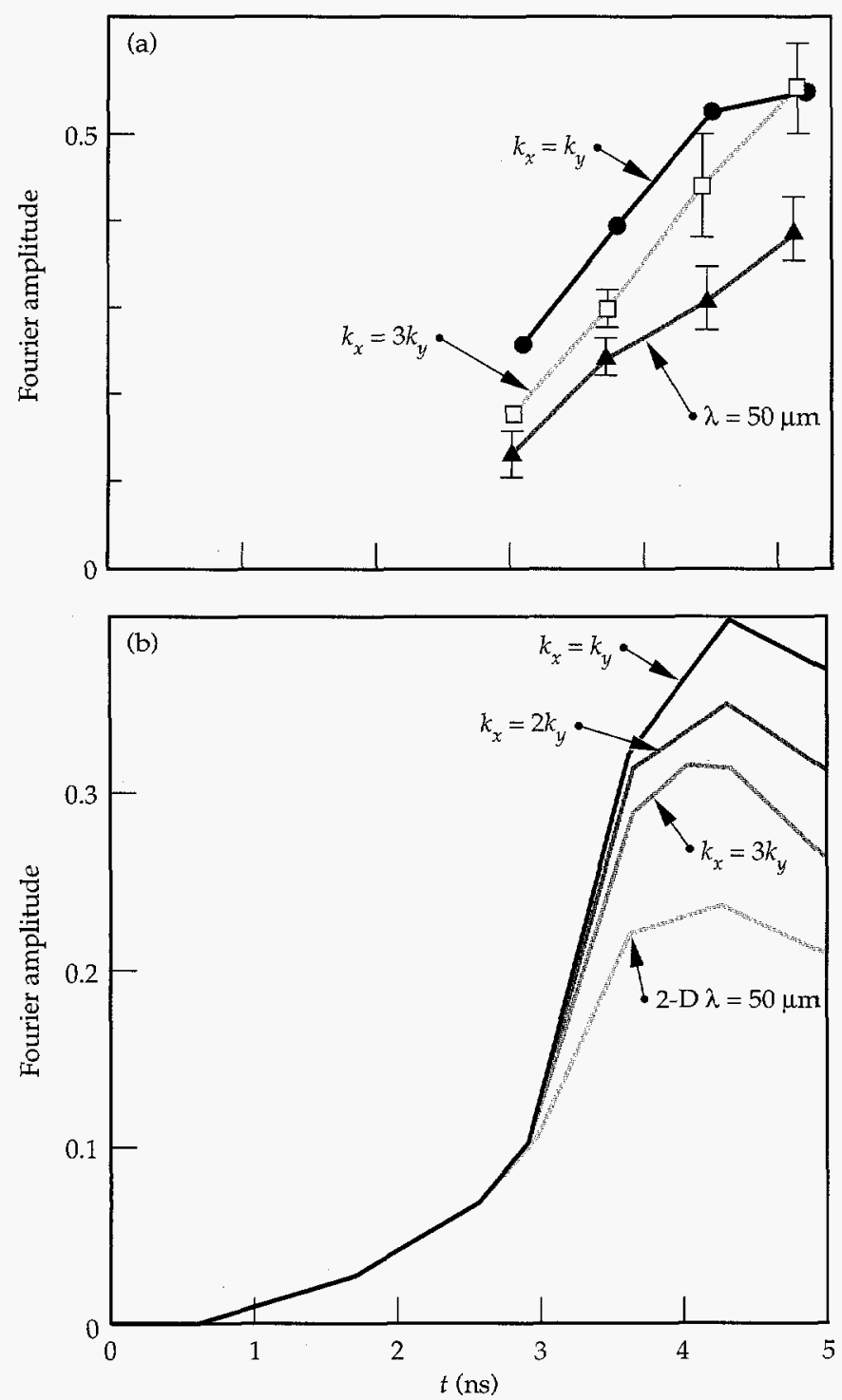

FIGURE 34. (a) Results of the evolution of the fundamental mode Fourier amplitude of $\ln E$ for the 3-D $k_{x}=k_{y}, k_{x}=3 k_{y}$ and 2-D $\lambda=50 \mu \mathrm{m}$ perturbations. Connecting lines are meant only to guide the eye. (b) Predicted Fourier amplitude of $\ln E$ from 3-D HYDRA simulations for the evolution of four perturbation shapes, all with the same wave vector magnitude $k=\left(k_{x}^{2}+k_{y}^{2}\right)^{1 / 2}$, for somewhat different drive histories and foil thicknesses from those of (a). The most symmetric $\left(k_{x}=k_{y}\right)$ mode is predicted to grow the most, the 2-D $\lambda=50 \mu \mathrm{m}$ mode to grow the least, and the 3-D stretched cases fall in between, in agreement with the experiments. $\quad(20-03-0595-1389 \mathrm{pb} 01)$

that for a spherical 3-D bubble the flow can carry material away from the bubble tip on all sides, allowing it to transit more easily into the spike regions. ${ }^{51,56}$ One can also obtain from Eq. (19) the form of the terminal bubble velocity of Eq. (7). When buoyancy is exactly balanced by drag, one has $\left(\rho_{1}-\rho_{2}\right) V g=c_{\mathrm{D}} \rho_{1} u^{2} S$. If we let $V \propto \lambda^{3}$, $S \propto \lambda^{2}$, and $\rho_{2} \approx 0$, we then have $\rho_{1} \lambda^{3} g \propto c_{D} \rho_{1} u^{2} \lambda^{2}$; this yields $u^{2} \propto g \lambda$, as in Eq. (7). Also note that if $g=0$, as in the Richtmyer-Meshkov instability (long after shock passage), we obtain $u_{\mathrm{RM}} \sim \lambda / t$, as pointed out by Alon et al. ${ }^{55}$ and as was recently observed. ${ }^{57}$

The images are Fourier analyzed, and the amplitudes corresponding to the fundamental mode are extracted. For an experimental demonstration of the effects of dimensionality on perturbation growth, we conducted shots for three targets (2-D $\lambda=50 \mu \mathrm{m}, 3-\mathrm{D} k_{x}=k_{y}$ and $k_{x}=3 k_{y}$ ). The total laser energy for these shots was similar, and the timing and filtering of the diagnostic were identical. Figure 34(a) shows the results for the evolution of the fundamental mode. The $k_{x}=k_{y}$ square 3 -D mode grows the most, the $\lambda=50 \mu \mathrm{m} 2-\mathrm{D}$ mode

(a) Rough foil
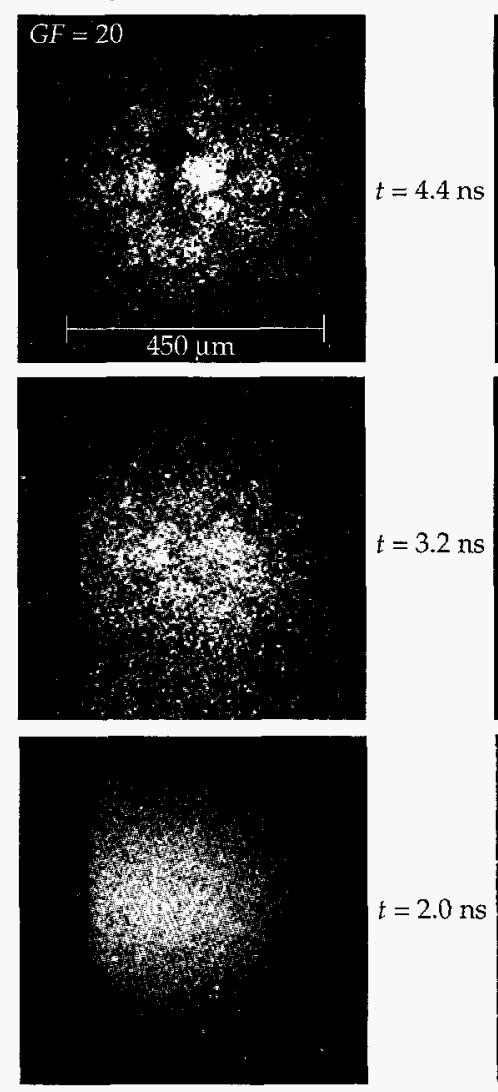

FIGURE 35. Typical 2-D images from surface-finish experiments using $\mathrm{CH}(\mathrm{Br})$ foils with two surface finishes, (a) rough $\left(\sigma_{\mathrm{rms}}=1.7 \mu \mathrm{m}\right)$ and $(\mathrm{b})$ smooth $\left(\sigma_{\mathrm{rms}}=10 \mathrm{~nm}\right)$. The diagnostic was a gated $x$-ray framing camera filtered with $381 \mu \mathrm{m}$ Be; foil was back-illuminated with $x$ rays generated by a $\sim 500-\mu \mathrm{m}$-diam random phase plate (RPP)-smoothed laser beam incident on a Sc backlighter disk. (20-03-1293-4408pb01) 
grows the least, and the $k_{x}=3 k_{y}$ stretched 3-D mode falls in between.

Figure 34(b) shows results from HYDRA simulations which were performed to help select parameters for the experiments. The foil thicknesses and drive histories in the experiments were somewhat different than those used in these simulations. The perturbations, in order of decreasing peak growth, correspond to $k_{x}=k_{y^{\prime}} k_{x}=2 k_{y^{\prime}}$
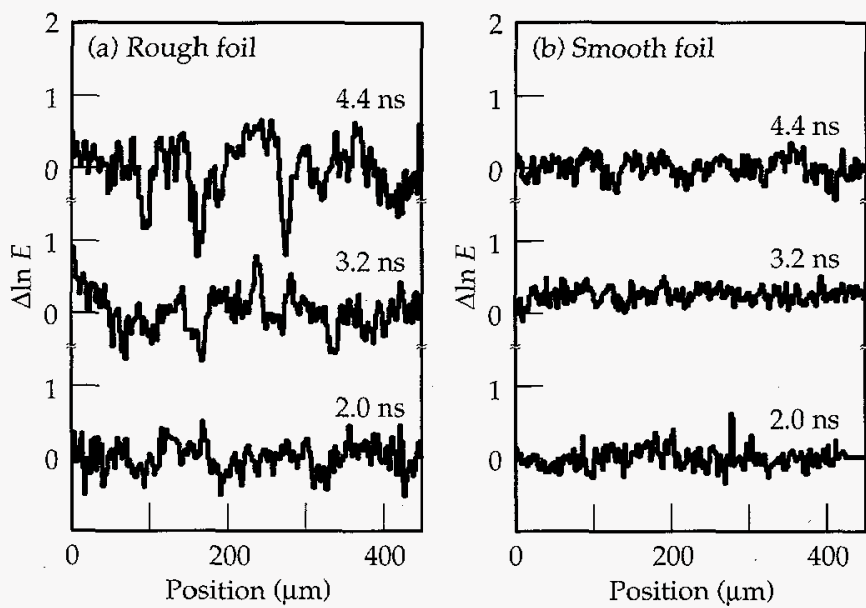

FIGURE 36. Corresponding $\Delta \ln E$ lineouts from Fig. 35 for (a) rough foil, (b) smooth foil. (20-03-0293-0379pb01) $k_{x}=3 k_{y^{\prime}}$ and 2-D $\lambda=50 \mu \mathrm{m}$. Our simulations clearly show that the most symmetric perturbations grow the most, as reported by others. ${ }^{23,56,58,59}$ This agrees qualitatively with our experimental observations; quantitative comparisons are under way.

Ultimately we are interested in the fully 3-D evolution at the ablation front of a perturbation consisting of a full continuum of modes. Recent progress has been made in developing 3-D modeling capability. 5,51,53,56,58-61 We have therefore begun a series of surface-finish experiments to compare the perturbation growth from a randomly roughened surface with that from a smooth surface. We generated the rough surface by sand blasting a glass mold with $50-\mu \mathrm{m} \mathrm{Al}_{2} \mathrm{O}_{3}$ pellets. The typical deviation from the average for the smooth foil is $\sigma_{\mathrm{rms}}=10 \mathrm{~nm}$, whereas $\sigma_{\mathrm{rms}}=1.7 \mu \mathrm{m}$ for the rough foil.

Figure 35 shows gated images taken at 2.0,3.2, and $4.4 \mathrm{~ns}$ when such foils were accelerated. Late in time, the surface of the rough foil has evolved into large, roughly hexagonal bubbles of transverse size $\sim 100 \mu \mathrm{m}$. Figure 36(a) shows this more quantitatively in $\Delta \ln E \approx-\Delta \mathrm{OD}$ profiles. These horizontal lineouts represent the central region of each image shown in Fig. 35; the late-time lineout contains the prominent central bubble at $4.4 \mathrm{~ns}$. Figure 36 (b) shows similar profiles for a standard smooth foil; there is no obvious perturbation growth into bubbles and spikes. Figure 37 shows the (a) Rough foil

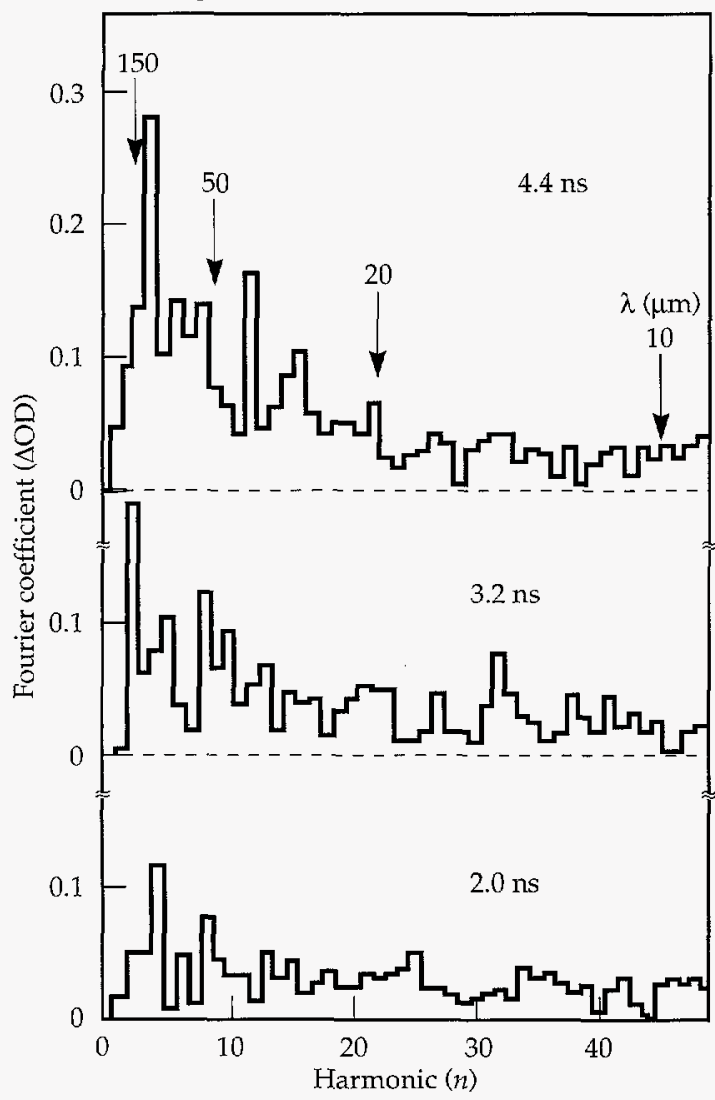

(b) Smooth foil

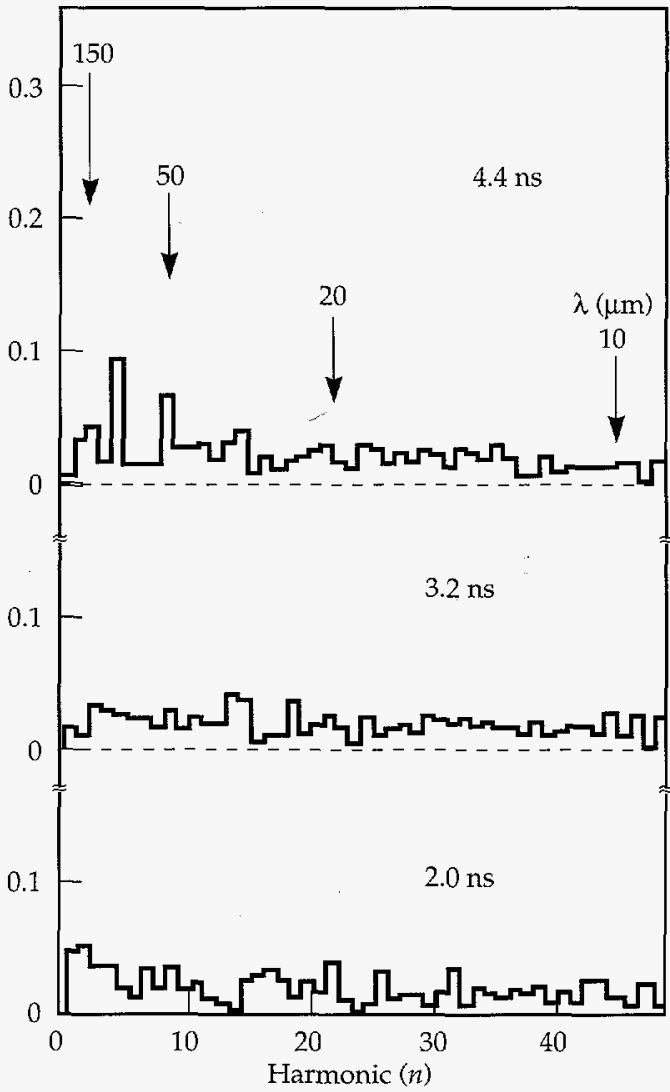

FIGURE 37. Absolute value of the real component of the Fourier transform of the $\Delta \ln E$ profiles shown in Fig. 36. Harmonic $n$ and wavelength $\lambda_{\eta}$ are related by $\lambda_{n}=(450 \mu \mathrm{m}) / n$, as indicated for a few cases by arrows. (20-03-1293-4409pb01) 
corresponding Fourier transforms. The smooth foil again shows no significant growth. For the rough foil, the growth late in time is dominated by harmonics $n=3-8$, corresponding to $\lambda=150-56 \mu \mathrm{m}$. This is reasonable, because perturbations with $\lambda>150 \mu \mathrm{m}$ grow too slowly, and perturbations with $\lambda<56 \mu \mathrm{m}$ are more strongly ablatively stabilized [see Fig. 21(d)].

Late in time, the Fourier spectrum for the rough surface is dominated by the fourth harmonic, with peak amplitude $\eta=0.28$. This fourth harmonic results from the prominent 112- $\mu \mathrm{m}$-diam bubble shown in the center of the image at $4.4 \mathrm{~ns}$ in Figs. 35(a) and 36(a). We use this amplitude to make a crude estimate of growth factor in optical depth, $G_{O D}$ for the dominant mode. From the surface profile of a similar rough foil, we estimate $\eta_{0}(\lambda=100 \mu \mathrm{m}) \approx 0.3 \mu \mathrm{m}$. The initial contrast for a $\mathrm{CH}(\mathrm{Br})$ foil with a $\lambda=100 \mu \mathrm{m}, \eta_{0}=5 \mu \mathrm{m}$ pure sinusoidal initial perturbation was measured on a separate shot (using the same diagnostic, backlighter, and filtering) to be $\Delta \mathrm{OD}_{0}=0.14$. We therefore estimate the growth factor for the dominant mode in Fig. 37(a) to be $G_{\mathrm{OD}}=\eta / \eta_{0} \approx 0.28 /[(0.14)(0.3 \mu \mathrm{m}) /(5.0 \mu \mathrm{m})]=33$. This is considerably less than expected in a typical implosion, but it represents a first step towards experimentally addressing the question of 3-D growth from a random initial surface finish. An analysis based on 2-D LASNEX simulations and Haan's 3-D saturation theory ${ }^{32}$ has been done for this rough surface experiment. The results, shown in Fig. 38, suggest that the multimode RT evolution was just entering the saturation regime, according to Haan's criterion. We are therefore designing a drive to produce a higher growth factor and having a longer acceleration interval, to allow a more discriminating test of multimode saturation physics.

\section{The RT Instability in Cylindrical Implosions}

The RT growth during ablative acceleration has been measured in many direct- and indirect-drive experiments. These measurements have verified the predicted stabilizing effect of mass ablation and density gradients. But few of these experiments have examined the role of feedthrough or of the deceleration phase, during which the growing perturbations may enhance thermal losses from the "hot spot" or reduce the efficiency of compressional work done by the shell on the fuel. Qualitatively, RT growth on the inner surface during deceleration differs from growth on the outer surface during acceleration by the lack of ablative stabilization. The RT growth at the inner surface is moderated, however, by the density gradient. In addition, convergent effects are important during these three phases. During ablative acceleration, convergence introduces a different threshold for nonlinear effects because of a decrease of perturbation wavelength in time, ${ }^{32}$ thin-shell effects, $7,29,62$ and a change in perturbation amplitude arising from the combination of convergence and compressibility. ${ }^{63}$ Feedthrough is decreased because the pusher shell thickens during convergence. Convergence effects are magnified during deceleration. $5,32,61,63$

Current designs for indirectly driven ignition capsules ${ }^{41}$ operate near the edge of present capabilities to fabricate smooth surfaces. It is important to assess the magnitude of feedthrough in ICF implosions if we are to correctly model the relative roles of perturbations on the outer and inner surfaces of capsules for these capsule designs. The effect of convergence on feedthrough is an integral part of the problem, so experiments in (a)

FIGURE 38. Saturation analysis based on 2-D LASNEX simulations and the Haan 3-D saturation theory ${ }^{32}$ for the rough surface foils, shown in terms of (a) $\sigma_{\mathrm{rms}}$ vs time and (b) Fourier spectrum at peak growth. (20-03-1293-4501pb02)

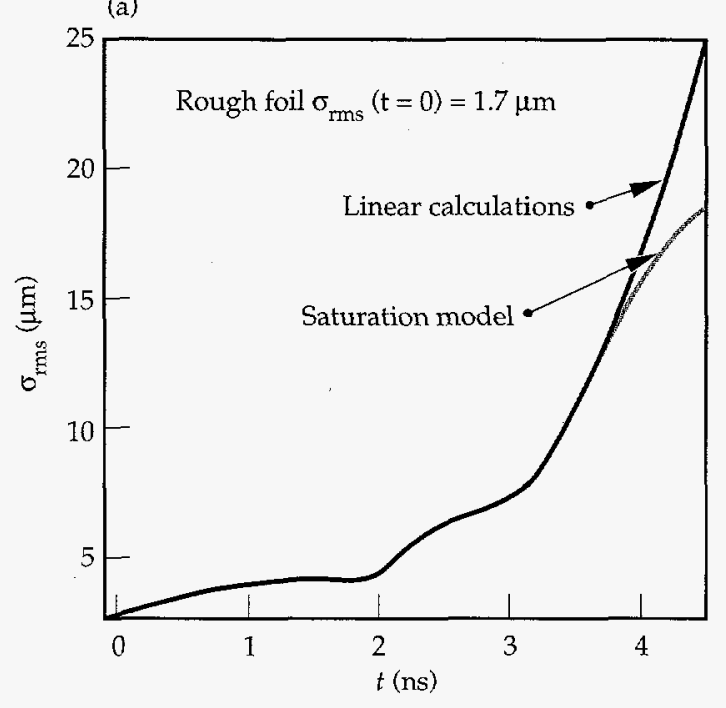

(b)

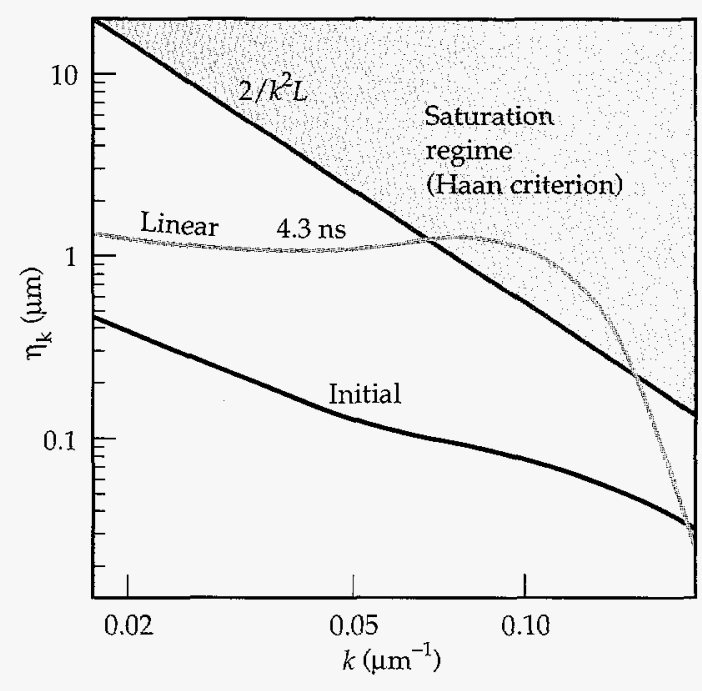


convergent geometry are desirable. Few experiments to study convergent RT instability have been performed ${ }^{64}$ because of the difficulty of diagnosis. Perturbations and mixing-layer widths are difficult to measure in spherical geometry because of the lack of a direct line of sight and because of errors associated with Abel inversions in spherical geometry near peak convergence. Although perturbation growth can be indirectly inferred from time-dependent $x$-ray spectral line ratios in spherical implosions, $18,19,65$ the results depend on the details of difficult atomic physics and radiative transport calculations. We have used an indirectly driven cylindrical configuration to allow diagnostic access and superior control of the shell's inner surface during target fabrication, although questions arise concerning edge effects and implosion symmetry. We chose a feedthrough experiment to demonstrate that quantitative RT experiments can be performed in cylindrical geometry and to measure feedthrough in a radiation-driven target for the first time.

Figure 39 shows the experiment geometry. The cylindrical polystyrene shell was mounted orthogonal to the hohlraum axis, to allow a direct line of sight to the diagnostics, to avoid interference with laser beams, and to avoid radiation flow into the ends of the cylinder (as may occur in a coaxial configuration). Eight 351-nm, 2.5-kJ, 2.2-ns Nova drive beams are pointed symmetrically about the cylinder. A low-adiabat drive was used, consisting of a low-power foot followed by a ramp to higher power, with a peak-to-foot ratio of about 3 . A separate $528-\mathrm{nm}, 2$-ns beam was used to irradiate a 2-mm-diam Ag disk to create an x-ray backlighter of photon energy $\sim 3-3.6 \mathrm{keV}$. An RPP 66 was used to smooth the beam intensity onto the Ag disk, and the

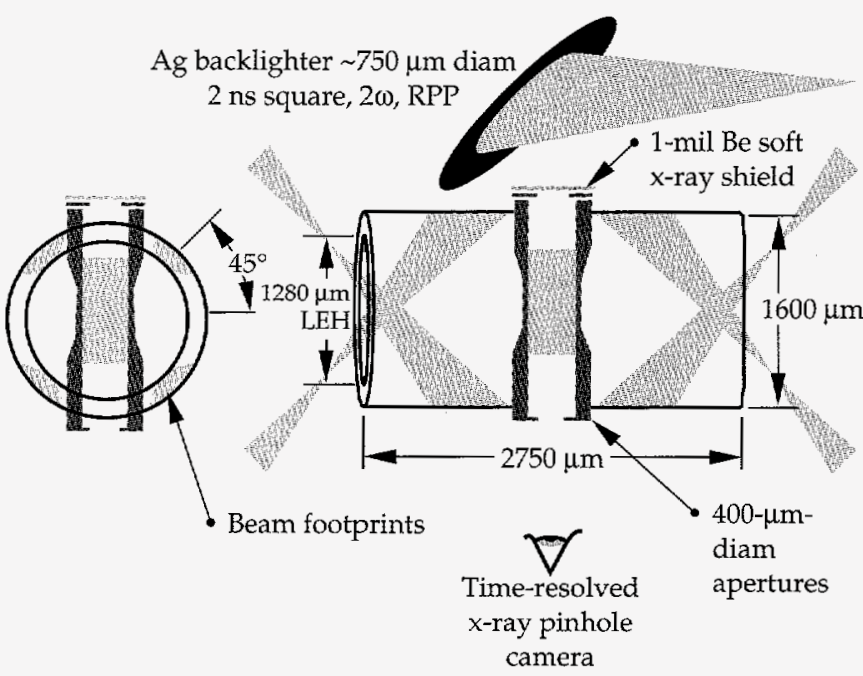

FIGURE 39. Side and transverse views of experimental geometry for the cylindrical implosion Rayleigh-Taylor experiment.

(20-03-0895-2048pb01) resultant laser spot was $\sim 750 \mu \mathrm{m}$ in diameter. The disk was located $\sim 3.5 \mathrm{~mm}$ away from the center of the cylinder and was oriented so that the disk normal bisected the angle between the cylinder axis and the backlighter laser direction. A 1-mil Be foil was placed between the backlighter and cylinder to filter out soft $x$ rays and to keep reflected $2 \omega$ light from the backlighter from striking the inside of the cylinder. There should be no $2 \omega$ unconverted light from the eight Nova drive beams. with a line of sight to the cylinder, so a Be shield on the other side of the cylinder is unnecessary.

Figure 40 shows the polystyrene cylinder in detail. The cylinder has an outer diameter of $630 \mu \mathrm{m}$, an inner diameter of $430 \mu \mathrm{m}$, and length of $1800 \mu \mathrm{m}$. The outer diameter is tapered toward the center of the cylinder, allowing the central region of the cylinder to implode before the ends and minimizing edge effects.

Perturbations were machined onto the outer surface of the central $400-\mu \mathrm{m}$-long region of the cylinder in a dodecagon shape (fundamental mode number $m=12$ ). (A sine wave perturbation would be preferable, because it contains no harmonics, but a sine wave is much more difficult to fabricate than a dodecagon.) A 4- $\mu$ m-thick, $160-\mu \mathrm{m}$-long dichlorostyrene $\left(\mathrm{C}_{8} \mathrm{H}_{6} \mathrm{Cl}_{2}\right)$ belt was placed around the center of the cylindrical shell, flush with the shell's inner surface. The belt served as a marker layer, because it is opaque to the x-ray backlighter, whereas the polystyrene cylinder is relatively transparent. The time-resolved pinhole camera diagnostic images the marker layer; late in time, it also images some of the surrounding compressed material. The marker layer is on the inner surface of the cylinder and has no initial perturbations, so any perturbations observed indicate feedthrough of the initial outer-surface perturbations to the inner surface. The marker

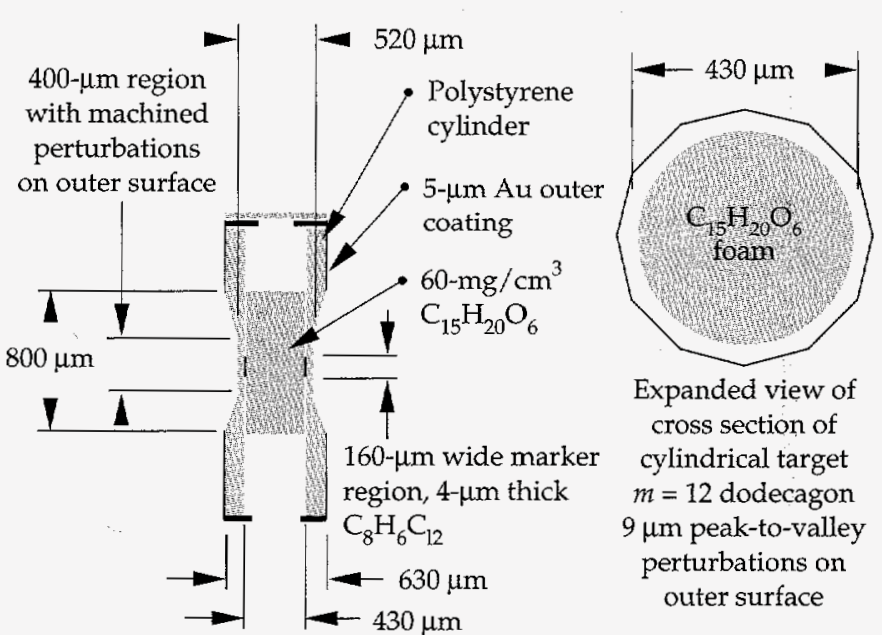

FIGURE 40 . Side and end views of target of Fig. 39. (20-03-0895-2049pb01) 
layer has a density close to that of the unchlorinated polystyrene $\left(1.4 \mathrm{~g} / \mathrm{cm}^{3}\right.$ vs $\left.1.0 \mathrm{~g} / \mathrm{cm}^{3}\right)$; calculations show that this slight density mismatch does not cause significant RT growth. A $60-\mathrm{mg} / \mathrm{cm}^{3}$ microcellular triacrylate foam $\left(\mathrm{C}_{15} \mathrm{H}_{20} \mathrm{O}_{6}\right)$ is placed inside the shell to provide a back-pressure as the cylinder implodes. The foam has a cell size $\sim 1-3 \mu \mathrm{m}$. The foam is shorter than the cylinder to minimize opacity to the backlighter. The cylinder is fabricated by coating the marker layer on a mandrel, machining the marker layer to size, coating the other polymer layers onto the mandrel and marker layer, machining the coatings, leaching the mandrel out, inserting the cylinder inside the hohlraum, and inserting the foam inside the cylinder. On each end of the cylinder, a 400- $\mu \mathrm{m}$-diam circular aperture made of $25-\mu \mathrm{m}$-thick gold was placed concentric with the cylinder axis. These apertures prevented any $x$ rays emitted by the walls of the cylinder from entering the pinhole camera diagnostic, provided an alignment and parallax diagnostic on the shot, and provided a centering fiducial for each frame on the pinhole camera. The fiducial is crucial for quantitative Fourier analysis of the data.

The cylinder was viewed along its axis with a timeresolved, gated $x$-ray pinhole camera (GXI). ${ }^{67} \mathrm{~A} 4 \times 4$ pinhole array with pinhole diameters of $\sim 7 \mu \mathrm{m}$ allowed 16 images spaced $\sim 55 \mathrm{ps}$ apart to be projected onto four microchannel-plate strip lines with a magnification of 12. The filters used were $12.7-\mu \mathrm{m}$ Ti and $150-\mu \mathrm{m} \mathrm{Be}$. The Ti was chosen to block $x$ rays with energies above $4.75 \mathrm{keV}$ and to allow the silver L-shell backlighter radiation through.

To determine the contrast ratio expected between the tracer layer and the central foam region, a backlit nonimplosion shot was taken with a $40-\mu \mathrm{m}$-thick piece of chlorinated polystyrene on top of part of the foam.

The contrast in exposure was measured to be 16:1 after a density-to-exposure correction of the film. A streaked $x$-ray crystal (KAP) spectrometer viewed the Ag backlighter disk and provided the backlighter spectrum as a function of time. Figure 41 shows a measured backlighter spectrum on an implosion shot. The opacity of cold chlorinated polystyrene and the gold photocathode GXI response are overlaid. The chlorinated polystyrene absorbs virtually all the Ag L-shell radiation, and the GXI detects most of the backlighter where there is no chlorinated polystyrene. The transmission of $3.5-\mathrm{keV}$ $x$ rays through $100 \mu \mathrm{m}$ of cold polystyrene is $\sim 0.6$. Figure 42 shows the total $3 \omega$ laser power into the hohlraum and the spectrally integrated $x$-ray backlighter flux as a function of time. The GXI is timed to measure during the peak $x$-ray backlighter fluence. The hohlraum was viewed along its axis with the west axial $x$-ray imager (WAX) ${ }^{68}$, another time-resolved pinhole camera with a serpentine microchannel plate strip. This was used to verify that there was no beam clipping on the laser entrance holes.

One purpose of a narrow $160-\mu \mathrm{m}$ tracer region is to minimize the effect of misalignment on the pinhole camera measurement of the interface location. For example, a $1^{\circ}$ tilt in the cylinder with respect to a point on the GXI would result in a lateral spread of $2.8 \mu \mathrm{m}$ in an interface. Since the pinhole array also has a lateral spread in location, there is parallax between images. For a perfectly aligned cylinder, the four center pinholes have a parallax of $0.9^{\circ}$, the next eight surrounding pinholes have a parallax of $2^{\circ}$, and the four corner pinholes have a parallax of $2.7^{\circ}$. The alignment of the cylinder relative to the GXI, measured on a shot with fiducial wires across the front and back faces of the cylinder, was $0.4^{\circ} \pm 0.6^{\circ}$. Thus the lateral spread in an image is primarily due to parallax.

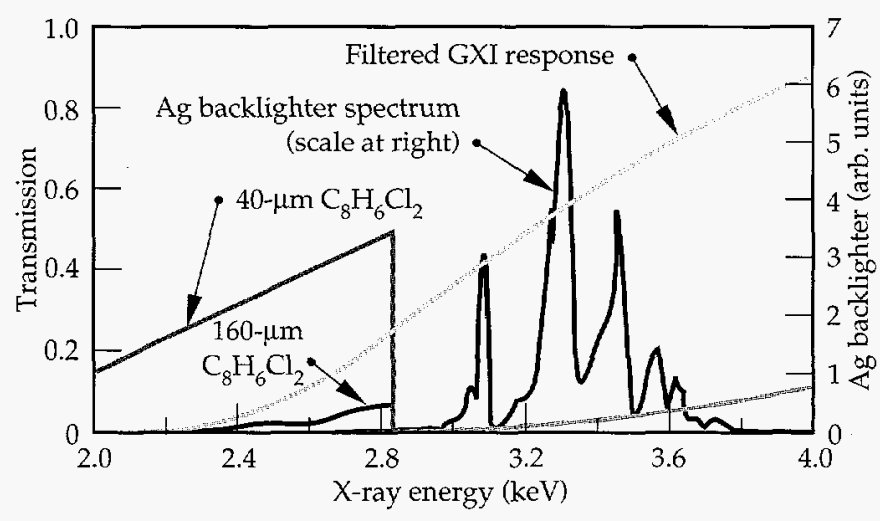

FIGURE 41. The measured $x$-ray spectrum of the Ag backlighter compared with the gated $x$-ray imager spectral response and the transmission assuming cold opacities of the tracer layer. $160 \mu \mathrm{m}$ is the initial length of the marker layer, and $40 \mu \mathrm{m}$ represents an expansion of the marker layer by a factor of 4 . $\quad(20-03-1095-2284 \mathrm{pb} 01)$

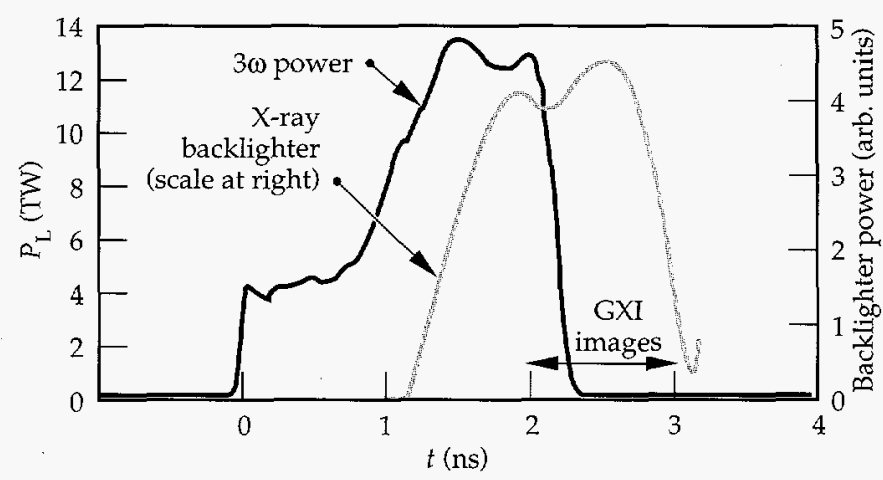

FIGURE 42. The $3 \omega(\lambda=351 \mathrm{~nm})$ power of eight Nova laser beams (left scale) and $x$-ray backlighter power (right scale). The $x$-ray images are taken between 2 and 3 ns, near the peak of the backlighter emission. (20-03-1095-2285pb01) 
Figure 43 shows 12 frames from an implosion with the initially perturbed surface described above. The $m=12$ perturbation is clearly visible. The perturbation amplitude grows in time, and the wavelength decreases as the radius decreases. At $t=2.11 \mathrm{~ns}$ (first image), calculations indicate that the ablation front has not burned through to the marker layer, so the observed $m=12$ feature is the result of feedthrough of the initial perturbation to the marker layer. The tips of the dodecagon have grown into spikes at the ablation front. Figure 44(a), an image of an unperturbed cylindrical implosion under identical conditions to a perturbed implosion [Fig. 44(b)], shows no $m=12$ perturbations. The absence of any $m=12$ feature in this case verifies that the initial perturbations caused the observed feedthrough. (We attribute the small bump at the upper left of the unperturbed shell to a target defect).

In the perturbed image [Fig. 44(b)], we identify a contour $r(\theta)$ at the outer edge at about the $50 \%$ exposure level and draw it in [Fig. 45(a)]. The contour can then be fitted with a Fourier series according to the usual prescription $r(\theta)=\frac{a_{0}}{2}+\sum_{m=1}^{\infty}\left(a_{m} \cos m \theta+b_{m} \sin m \theta\right)$,

where

$a_{m}=\frac{1}{\pi} \int_{-\pi}^{\pi} r(\theta) \cos m \theta d \theta$

and

$b_{m}=\frac{1}{\pi} \int_{-\pi}^{\pi} r(\theta) \sin m \theta d \theta$.

The results of the fit are shown in Fig. 45(b) and the Fourier composition is shown in Fig. 46, where the modal amplitude $\left(a_{m}^{2}+b_{m}^{2}\right)^{1 / 2}$ is plotted vs mode number $m$. The term $a_{0}$ represents the average diameter of the contour, and $a_{1}$ and $b_{1}$ represent the offsets of the center of the contour from the axes of symmetry. The coefficients depend on the choice of the center of the contour. We examined several methods to choose the center of the contour: a least-squares fit to a circle, a minimization of $a_{1}$ and $b_{1}$, and a center based on the measurement of the center of the defining aperture. Of these methods,

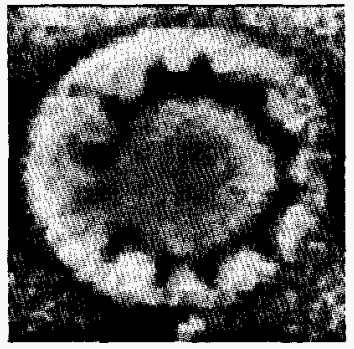

$t=2.11 \mathrm{~ns}$

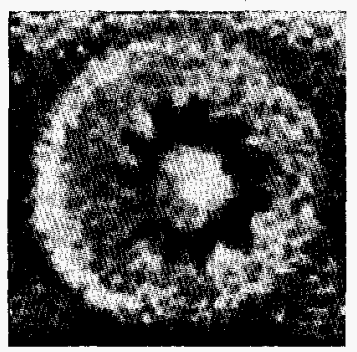

$t=2.36 \mathrm{~ns}$

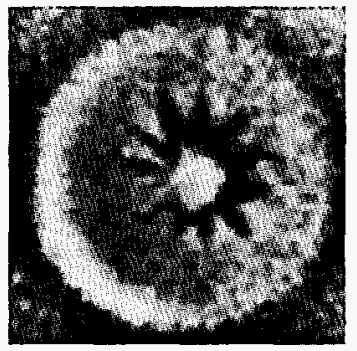

$t=2.61 \mathrm{~ns}$

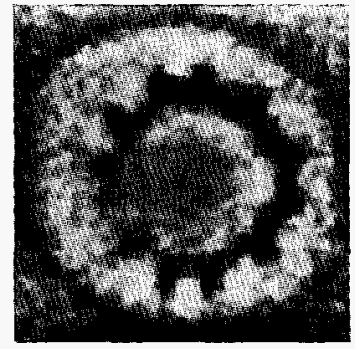

$t=2.16 \mathrm{~ns}$

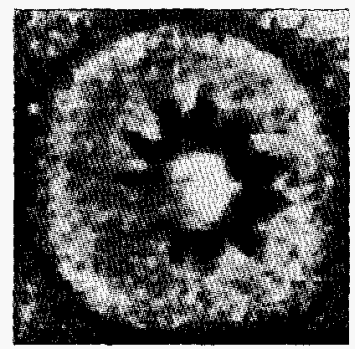

$t=2.41 \mathrm{~ns}$

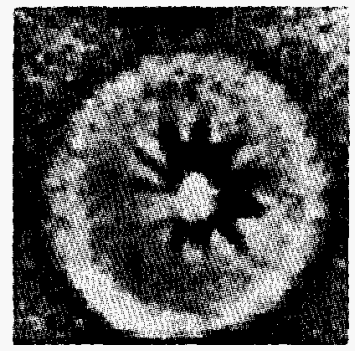

$t=2.66 \mathrm{~ns}$

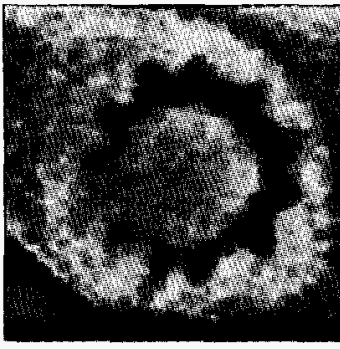

$t=2.22 \mathrm{~ns}$

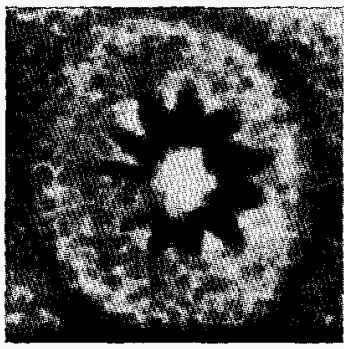

$t=2.47 \mathrm{~ns}$

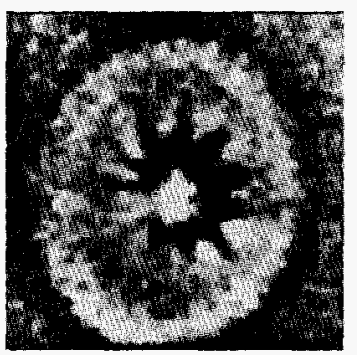

$t=2.72 \mathrm{~ns}$

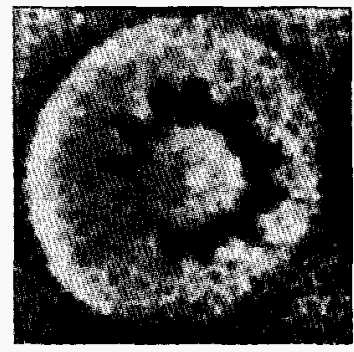

$t=2.30 \mathrm{~ns}$

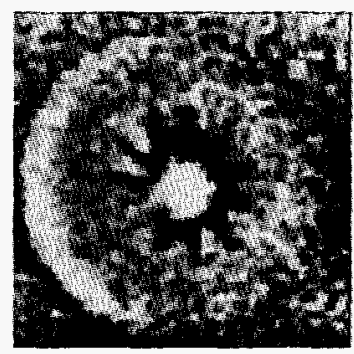

$t=2.55 \mathrm{~ns}$

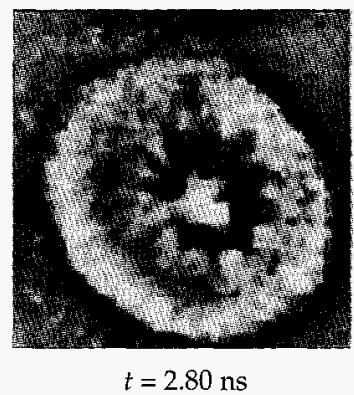

FIGURE 43. A sequence of gated $x$-ray images of the cylindrical backlit implosion from one experiment ( $t=0$ corresponds to the start of the laser pulse). Shock emission appears on center at $2.30 \mathrm{~ns}$. The backlighter spatial extent is limited by the circular apertures at each end of the cylinder, and the deviation from circularity is a measure of the effect of parallax. (20-03-0895-2050pb01) 
only the latter proved reliable and resulted in mode number conservation during the implosion. Figure 47 shows images of the perturbed implosion later in time. One side of the target has imploded closer to the center than the other side. This is probably due to a slight manufacturing imperfection in the target, as if the marker layer were thinner on one side than on the other. The apertures also indicate, on each image, the parallax due to the offset of each pinhole from the axis of the cylindrical shell; the images can be corrected for this effect.

At $2.11 \mathrm{~ns}$, there are significant components at $m=1,4$, 8 , and 12. Mode 1 exists because one side of the cylinder implodes faster than the other. Mode 4 is expected because of the discrete number of beams illuminating the hohlraum. Mode 2 was minimized by the proper choice of beam pointing. Mode 12 has an amplitude of $\eta=10 \mu \mathrm{m}$. Since the instrumental resolution is insufficient to distinguish between mode 12 and modes 11 and 13 , it is reasonable to expect that the effective amplitude of the perturbation at mode 12 is the quadrature sum of modes 11,12 and 13, resulting in an amplitude of $12 \mu \mathrm{m}$. Other methods of analysis, including curve fits to the functional form

$$
r(\theta)=\frac{a_{0}}{2}+\sum_{n=1}^{4}\left(a_{n} \cos 12 n x+b_{n} \sin 12 n x\right)
$$

and variations in the choice of a center, result in a mode12 amplitude of 9-11 $\mu \mathrm{m}$; we assign the value $10 \pm 2 \mu \mathrm{m}$ as the amplitude of mode 12. A Fourier analysis of a dodecagon inscribed in a 260- $\mu \mathrm{m}$-radius circle gives amplitudes of $3.5,0.85$, and $0.34 \mu \mathrm{m}$ for modes 12,24 , and 36 respectively. Thus at $t=2.11 \mathrm{~ns}$, the fundamental has grown by a factor of $2.9 \pm 0.6$ with respect to its initial value.

To test the sensitivity of the analysis to the choice of $50 \%$ isodensity contour, we analyzed several images with isodensity contours ranging from $40 \%$ to $60 \%$. (a)

FIGURE 44. (a) Gated $x$-ray image of an implosion taken with a target with no initial perturbations and (b) an otherwise identical implosion taken with a dodecagon initial perturbation. Images taken $t=2.16 \mathrm{~ns}$. (20-03-0895-2053pb01)

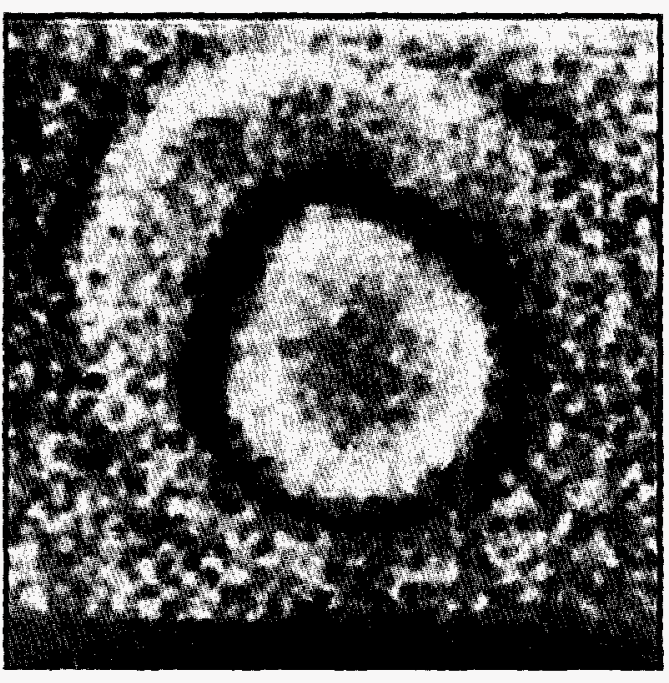

(b)

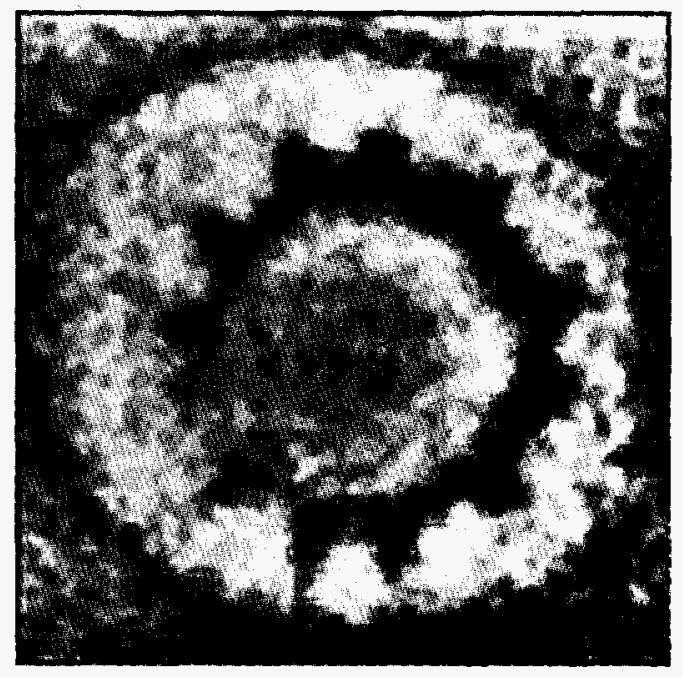

(a)

FIGURE 45. (a) An outer contour taken at $50 \%$ peak exposure, superposed over the initial $x$-ray image taken at $2.11 \mathrm{~ns}$. (b) Results of a Fourier series fitted to the contour shown in (a). The horizontal and vertical axes are equal, each covering a range of $300 \mu \mathrm{m}$. (20-03-0895-2053pb02)

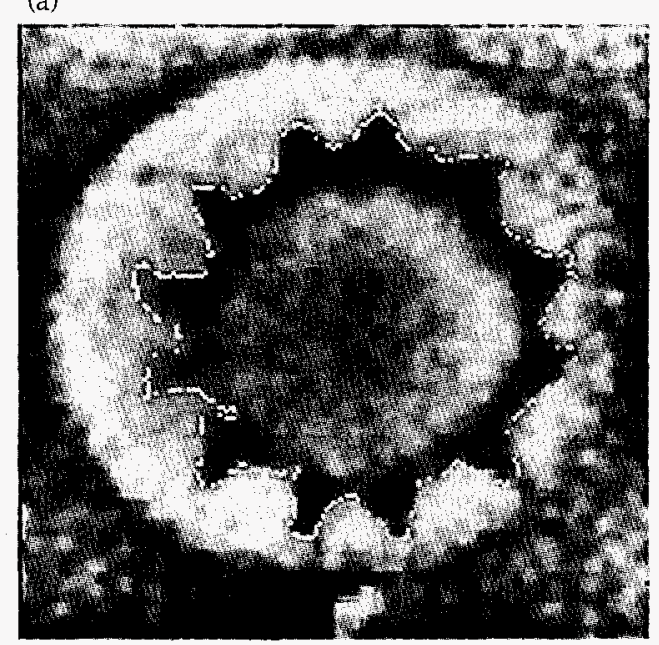

(b)

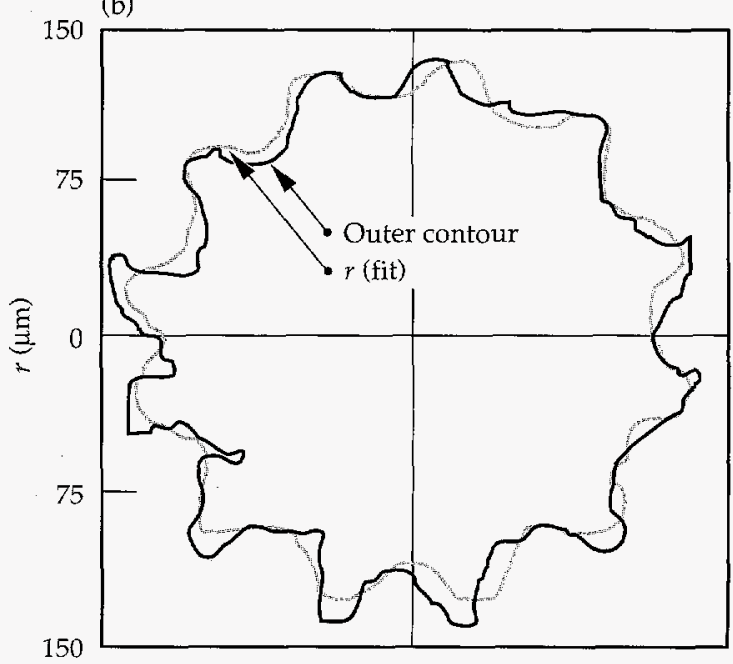


The results were insensitive to choice of isodensity contour. For isodensity values outside this range, the background noise from the microchannel plate occasionally affected the contour, with unphysical contours appearing because of noise spikes.

For each image, we Fourier analyzed the contours for the inner edge of the marker layer (the interface between the marker layer and the TPX foam) and the outer edge (the interface between the marker layer and surrounding polystyrene cylinder). Figure 48 shows the time dependence of $a_{0} / 2$, which represents the average radius. The implosion trajectory is consistent with a $195-\mathrm{eV}$ peak drive temperature in a calculation with the 1-D radiation-hydrodynamic code HYADES. ${ }^{69}$

The perturbation was initialized in its linear regime. At $t=0$, the wavelength of mode $m=12$ is $\lambda=2 \pi R / m=136 \mu \mathrm{m}$, where $R=260 \mu \mathrm{m}$. The amplitude of mode $m=12$ is $\eta_{0}=3.50 \mu \mathrm{m}$ initially, so that

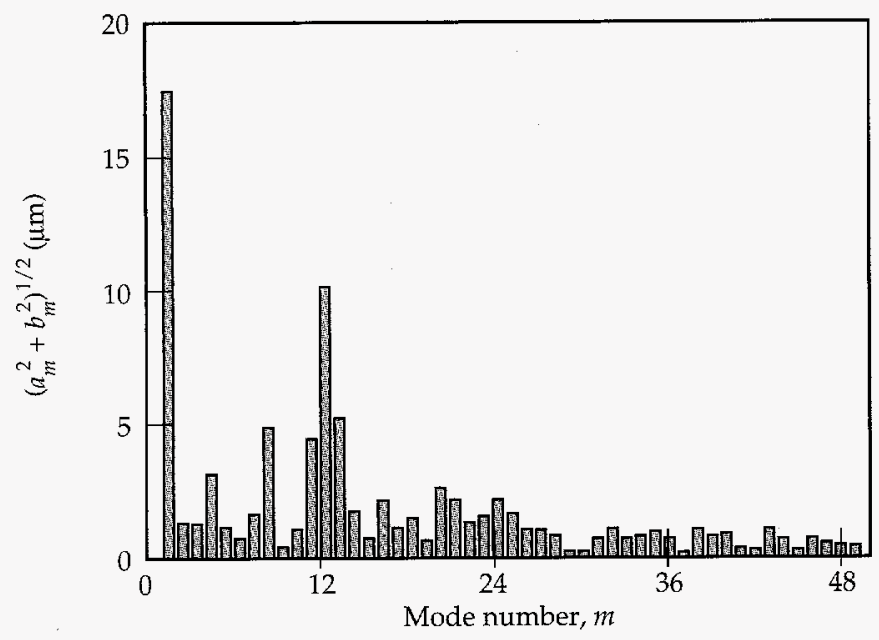

FIGURE 46. Fourier amplitudes vs mode number $m$ for a $50 \%$ outer contour of the gated $x$-ray image at $t=2.11 \mathrm{~ns}$. The largest physically significant amplitude corresponds to $m=12$. (20-03-1095-2286pb01) $\eta / \lambda=0.026$. At $2.11 \mathrm{~ns}$, the radius of the shell is $R=122 \mu \mathrm{m}$, so that $\lambda=2 \pi R / m=64 \mu \mathrm{m}$. The amplitude of mode $m=12$ is $\eta=10 \mu \mathrm{m}$, so $\eta / \lambda=0.16$. The perturbation at the marker layer has exceeded the nominal threshold for nonlinearity $\eta / \lambda \approx 0.1$ at $2.11 \mathrm{~ns}$.

Growth of perturbations on the marker layer result both from shock imprinting and from feedthrough from RT growth at the ablation front. Simple estimates suggest that the latter dominates. To illustrate this, we estimate the marker layer amplitude that would result from shock imprinting alone. The shock is launched from a rippled surface at the ablation front, and hence is itself rippled. A rough estimate of the amplitude of the ripple imparted to the marker layer upon passage of the rippled shock is given by

$\eta_{m}=\eta_{0}\left(1-\Delta R_{\mathrm{o}} / \lambda_{\mathrm{o}}\right) u_{p} / v_{\mathrm{s}}$

Here $\eta_{m}$ is the amplitude of the imprinted ripple on the marker layer, $\eta_{0}$ is the ablation front surface

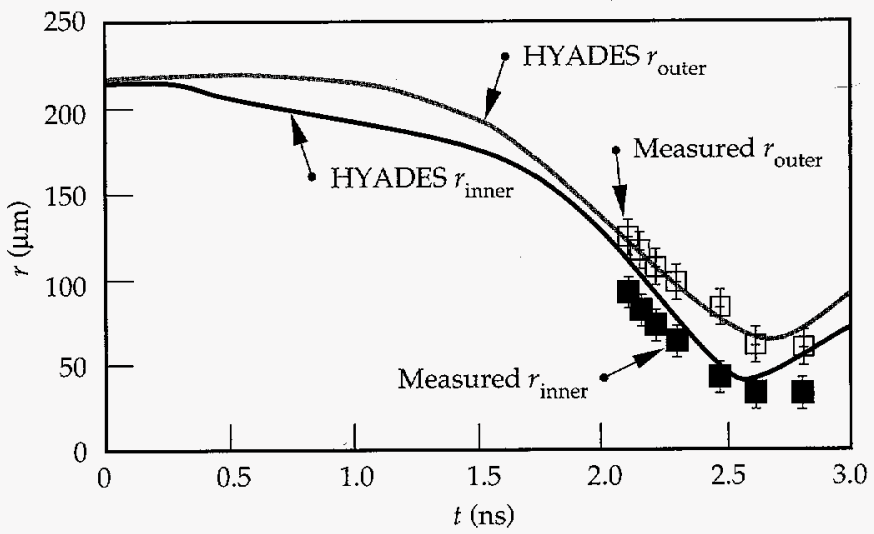

FIGURE 48. Measured outer and inner diameters of the tracer layer vs time, and corresponding trajectories from a HYADES calculation. (20-03-0895-2052pb01)

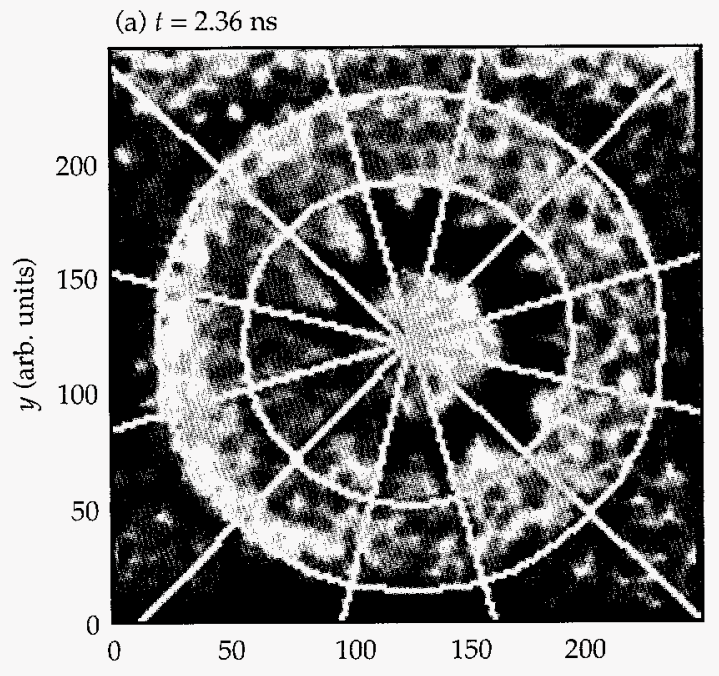

UCRL-LR-105821-95-4 (b) $t=2.61 \mathrm{~ns}$

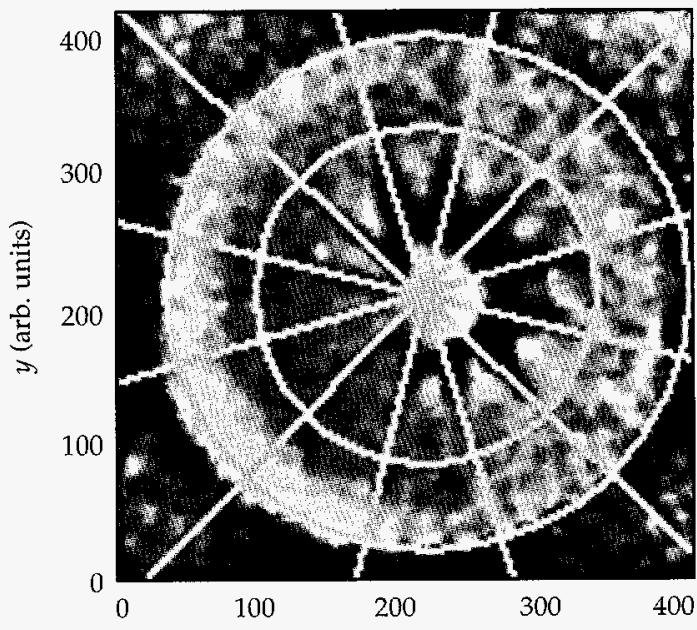

FIGURE 47 . Gated $x$-ray images at (a) 2.36 and (b) $2.61 \mathrm{~ns}$, with a mode 12 uniform in angle grid superposed. Outermost circular contour is a fit to the 400- $\mu$ m-diam Au defining aperture in both images. One side of the target has imploded faster than the other yet the mode number is preserved using a center corresponding to the original cylinder axis. (20-03-1095-2295pb01) 
perturbation initial amplitude, $\Delta R_{\mathrm{o}}$ is the initial distance from the ablation front to the marker layer, $\lambda_{\mathrm{o}}$ is the initial perturbation wavelength, $u_{p}$ the particle velocity behind the shock front, and $v_{s}$ the shock velocity. The factor $\eta_{0}\left(1-\Delta R_{0} / \lambda_{0}\right)$ is an estimate, based on the shock oscillation data of Endo et al., 22 of the amplitude of the shock-front ripple, assuming that it reverses phase after having traveled a distance of $\lambda_{0}$. Since $\Delta R_{\mathrm{o}} \approx 40 \mu \mathrm{m}$ and $\lambda_{\mathrm{o}} \approx 100 \mu \mathrm{m}$, the rippled shock has not reversed phase by the time it reaches the marker layer, but its amplitude is $\sim 40 \%$ lower than $\eta_{0}$. Assuming that this marker layer perturbation then grows linearly in time via the Richtmyer-Meshkov instability, we write

$\eta_{12}(t)=\eta_{m}+\eta_{m} A^{*} k u_{p} \Delta t$,

where $\eta_{12}(t)$ is the amplitude of the fundamental mode at time $t, \mathrm{~A}^{*}$ is the post-shock Atwood number, and $\Delta t=t-t_{\mathrm{s}}$, where $t_{\mathrm{s}}=0.4 \mathrm{~ns}$ is the time when the first shock reaches the marker layer. Using reasonable estimates for the various parameters in this equation, we estimate that $\eta_{12}(2.11 \mathrm{~ns}) \approx 2.5 \mu \mathrm{m}$. Allowing for some additional growth due to the higher $u_{p}$ after the second shock reaches the marker layer at $1.6 \mathrm{~ns}$, we get $\eta_{12}(2.11 \mathrm{~ns}) \approx 3 \mu \mathrm{m}$. The measured amplitude of $10 \mu \mathrm{m}$ at 2.11 ns is considerably larger, suggesting that feedthrough from RT growth at the ablation front is the dominant source of the observed growth.

During deceleration, the inner surface becomes RT unstable and perturbations fed through to the inside surface grow without ablative stabilization. At 2.72 and $2.80 \mathrm{~ns}$ (Fig. 43), visible spikes protrude into the core. The spikes on the inner edge of the marker layer correspond radially to bubbles on the outer surface, such as can be seen in Fig. 43 at 2.30 ns and 2.47 ns. This suggests that ablation-front bubble growth is the dominant feed-through mechanism.

\section{Summary}

We have conducted an extensive series of experiments and simulations to examine the growth of single modes over a range of wavelengths and to examine the effect of multiple modes on perturbation growth. For single modes, the perturbation evolves before shock breakout because of the rippled shock dynamics. After shock breakout, the perturbations grow rapidly in the linear regime and saturate in the nonlinear regime, with the appearance of higher harmonics. In multimode foils, the individual modes grow independently in the linear regime. In the nonlinear regime, the modes become coupled and $k_{i} \pm k_{j}$ terms are clearly observed, in agreement with simulations and second-order perturbation theory. Mode coupling redistributes the perturbation in Fourier space, which in physical space corresponds to a change in perturbation shape. The bubbles become broader and flatter and the spikes narrower. In terms of a continuum model, the individual modes of the 2-D perturbation saturate when they exceed $S_{k}=v_{2 D} / k^{3 / 2} L^{1 / 2}$ in amplitude. The simulations systematically predict slightly more growth than is observed. This could be caused by greater than expected preheat in the foot of the drive, a stiffer EOS for $\mathrm{CH}(\mathrm{Br})$, or a degraded instrumental MTF.

Single-mode experiments very clearly indicate the differences between 2-D and 3-D perturbation shape. Axisymmetric 3-D bubbles grow the largest in the nonlinear regime, consistent with a simple buoyancy-vs-drag argument, third-order perturbation theory, and with full 3-D radiation-hydrodynamics simulations. The obvious next step is to measure the full multimode 3-D perturbation evolution and to compare the results with 3-D simulations and with the predictions of Haan's saturation theory.

In radiation-driven cylindrical implosions, we have observed the RT instability seeded by feedthrough from the outer surface to the inner surface. The mode number was conserved during the implosion. This proof-ofprinciple cylindrical experiment shows the potential for new studies of RT instability in convergent geometry. Ablation-front growth and feedthrough were measured. With higher resolution, studies of the stagnation phase and inner surface breakup may be possible.

\section{Appendix: Amplitude of Coupled Modes}

Following Ref. 26, a solution to a second-order perturbation expansion of the 3-D hydrodynamic equations for inviscid, incompressible fluids can be written as

$\eta_{\mathbf{k}}(t)=\eta_{\mathbf{k}}^{\mathrm{L}}(t)+2 A k \sum_{k_{2}} \eta_{k_{2}}^{\mathrm{L}}(t) \eta_{k_{2}^{\prime}}^{\mathrm{L}}(t) G\left(\mathbf{k}, \mathbf{k}_{2}\right) H\left(\mathbf{k}, \mathbf{k}_{2}, t\right)$,

where $\mathbf{k}_{2}^{\prime}=\mathbf{k}-\mathbf{k}_{2}$ and where superscript $\mathrm{L}$ designates results in the linear regime. The time-independent part of the kernel $G\left(\mathbf{k}, \mathbf{k}_{2}\right)$ is given by

$G\left(\mathbf{k}, \mathbf{k}_{2}\right)=\frac{1}{2} \frac{\gamma_{2}^{2}\left(1-\hat{\mathbf{k}}_{2} \cdot \hat{\mathbf{k}}\right)+\frac{1}{2} \gamma_{2} \gamma_{2}^{\prime}\left(1-\hat{\mathbf{k}}_{2} \cdot \hat{\mathbf{k}}_{2}^{\prime}-2 \hat{\mathbf{k}}_{2} \cdot \hat{\mathbf{k}}\right)}{\left(\gamma_{2}+\gamma_{2}^{\prime}\right)^{2}-\gamma^{2}(k)}$,

where $\mathbf{k}=\left(k_{x}, k_{y}\right)$ is the perturbation wave vector, $\hat{\mathbf{k}}=\mathbf{k} / k$ is the unit vector, and $\gamma(\mathbf{k}), \gamma_{2}$, and $\gamma_{2}$ are the linear growth rates for perturbations with wave vectors $\mathbf{k}, \mathbf{k}_{2}$, and $\mathbf{k}_{2}^{\prime}$, respectively. This weakly nonlinear theory is valid only so long as the dominant modes are not being changed significantly by the nonlinear terms. The full expression for $H\left(\mathbf{k}_{,}, \mathbf{k}_{2}, t\right)$ is complicated, but for the regime considered here we have $H \approx 1$.

Considerable simplification occurs for 2-D cosine perturbations with Atwood number $A=\left(\rho_{1}-\rho_{2}\right) /\left(\rho_{1}+\rho_{2}\right) \approx 1$. If we assume that $\gamma_{k} \propto k^{1 / 2}$, then Eq. (A1) reduces to 
$\eta_{k} \approx \eta_{k}^{\mathrm{L}}+\frac{1}{2} k\left(\sum_{k^{\prime}} \eta_{k^{\prime}}^{\mathrm{L}} \eta_{k+k^{\prime}}^{\mathrm{L}}-\frac{1}{2} \sum_{k^{\prime}<k} \eta_{k^{\prime}}^{\mathrm{L}} \eta_{k-k^{\prime}}^{\mathrm{L}}\right)$

where $k, k^{\prime}>0$, and the time dependence has been dropped from the notation for simplicity. For example, Eq. (5b) for the second harmonic of a single-mode perturbation, $\eta_{2} \approx \frac{1}{2} k_{1} \eta_{\mathrm{L}}^{2}$, comes from the second term in the summation in Eq. (A3), where $k=2 k_{1}$ and $k^{\prime}=k_{1}$.

Another simple case of general interest arises for $k=k_{3} \pm k_{2}$. Here, Eq. (A3) reduces to

$$
\eta_{k_{3} \pm k_{2}} \approx \mp\left(k_{3} \pm k_{2}\right) \eta_{k_{2}}^{L} \eta_{k_{3}}^{L}
$$

where $\eta_{k}^{\mathrm{L}}$ represents the spatial amplitude attained by mode $k_{n}$ had the growth been entirely in the linear regime.

\section{Notes and References}

1. S. Chandrasekhar, Hydrodynamic and Hydromagnetic Stability (Oxford U. P., London, 1968), Ch. 10; Lord Rayleigh, Scientific Papers, Vol. II (Cambridge U. P., Cambridge, 1900), p. 200; G. I. Taylor, Proc. Roy. Soc. A201, 192 (1950).

2. R. D. Richtmyer, Commun. Pure Appl. Math. 13, 297 (1960); E. E. Meshkov, Izv. Akad. Nauk. SSSR, Mekh. Zhidk. Gaz. 5, 151 (1969) (translation, NASA TTF-13-074, 1970).

3. J. D. Lindl and W. C. Mead, Phys. Rev. Lett. 34, 1273 (1975).

4. E. Gamaly, "Hydrodynamic Instability of Target Implosions in ICF," in Nuclear Fusion by Inertial Confinement: A Comprehensive Treatise, G. Velarde, Y. Ronen, and J. M. Martinez-Val, Eds. (CRC Press, Boca Raton, 1993), p. 321.

5. H. Sakagami and K. Nishihara, Phys. Rev. Lett. 65, 432 (1990); Phys. Fluids B 2, 2715 (1990).

6. S. E. Bodner, Phys. Rev. Lett. 33, 761 (1974).

7. R. L. McCrory, L. Montierth, R. L. Morse, and C. P. Verdon, Phys. Rev. Lett. 46, 336 (1981).

8. J. D. Lindl, LLNL Laser Program Annual Report, 2-40 to 2-46, UCRL-56055-83, Lawrence Livermore National Laboratory, Livermore, California (SRD, 1983); H. Takabe, K. Mima, L. Montierth, and R. L. Morse, Phys. Fluids 28, 3676 (1985); D. H. Munro, Phys. Rev. A 38, 1433 (1988); M. Tabak, D. H. Munro, and J. D. Lindl, Phys. Fluids B 2, 1007 (1990).

9. A. Caruso, V. A. Pais, and A. Parodi, Laser Part. Beams 10, 447 (1992).

10. J. Grun et al., Phys. Rev. Lett. 58, 2672 (1987).

11. M. Desselberger, O. Willi, M. Savage, and M. Lamb, Phys. Rev. Lett. 65, 2997 (1990); M. Desselberger, O. Willi, M. Savage, and M. Lamb, Phys. Fluids B 5, 896 (1993).

12. S. G. Glendinning et al., Phys. Rev. Lett. 69, 1201 (1992).

13. J. D. Kilkenny, Phys. Fluids B 2, 1400 (1990).

14. B. A. Remington et al., Phys. Rev. Lett. 67, 3259 (1991); B. A. Remington, S. W. Haan, S. G. Glendinning, J. D. Kilkenny, D. H. Munro, and R. J. Wallace, Phys. Fluids B 4, 967 (1992).

15. B. A. Remington et al., Phys. Fluids B 5, 2589 (1993).

16. Guy Dimonte and Bruce Remington, Phys. Rev. Lett. 70, 1806 (1993).

17. B. A. Hammel et al., Phys. Fluids B 5, 2259 (1993); B.A. Hammel, J.D. Kilkenny, D. Munro, B. A. Remington, H. N. Kornblum, T. S. Perry, D. W. Phillion, and R. J. Wallace, Phys. Plasmas 1, 1662 (1994)

18. B. A. Hammel et al., J. Quant. Spectrosc. Radiat. Transfer 51, 113 (1994).
19. J. Delettrez, D. K. Bradley, P. A. Jaanimagi, and C. P. Verdon, Phys. Rev. A 41, 5583 (1990); D. K. Bradley, J. A. Delettrez, and C. P. Verdon, Phys. Rev. Lett. 68, 2774 (1992).

20. R. G. Evans, Can. J. Phys. 64, 893 (1986); J. Meyer-Ter-Vehn, Nucl. Fusion 22, 561 (1982); G. I. Guderley, Luftfahrtforschung 19, 3032 (1942).

21. D. H. Munro, Phys. Fluids B 1, 134 (1989).

22. T. Endo et al., Phys. Rev. Lett. 74, 3608 (1995).

23. J. W. Jacobs and I. Catton, J. Fluid Mech. 187, 329 (1988);

J. W. Jacobs and I. Catton, J. Fluid Mech. 187, 353 (1988).

24. R. M. Davies and G. I. Taylor, Proc. Roy. Soc. A 200, 375 (1950); G. R. Baker, D. I. Meiron, and S. A. Orszag, Phys. Fluids 23, 1485 (1980).

25. D. Layzer, Astrophys. J. 122, 1 (1955).

26. S. W. Haan, Phys. Fluids B 3, 2349 (1991).

27. D. Ofer, D. Shvartz, Z. Zinamon, and S. A. Orszag, Phys. Fluids $B$ 4, 3549 (1992).

28. E. G. Gamaly et al., Laser Part. Beams 8, 173 (1990).

29. C. P. Verdon et al., Phys. Fluids 25, 1653 (1982).

30. G. R. Baker, D. I. Meiron, and S. A. Orszag, Phys. Fluids 23, 1485 (1980).

31. J. P. Dahlburg, J. H. Gardner, and M. H. Emery, Bull. Am. Phys. Soc. 35, 1969 (1990).

32. S. W. Haan, Phys. Rev. A 39, 5812 (1989).

33. E. M. Campbell, Laser Part. Beams 9, 209 (1991).

34. R. J. Ellis et al., Rev. Sci. Instrum. 61, 2759 (1990).

35. B. A. Remington, S. G. Glendinning, R. J. Wallace, S. Rothman, and R. Morales, Rev. Sci. Instrum. 63, 5080 (1992).

36. R. Morales, B. A. Remington, T. Schwinn, Rev. Sci. Instrum. 66, 700 (1995); B. A. Remington and R. I. Morales, Rev. Sci. Instrum. 66, 703 (1995).

37. B. A. Remington et al., Phys. Plasmas 2, 241 (1995).

38. K. S. Budil et al., "The Flexible X-Ray Imager," Lawrence Livermore National Laboratory, Livermore, CA, UCRL-JC120837; in press, Rev. Sci. Instrum. (1996).

39. L. J. Suter et al., Phys. Rev. Lett. 73, 2328 (1994).

40. R. L. Kauffman et al., Phys. Rev. Lett. 73, 2320 (1994).

41. S. W. Haan et al., Phys. Plasmas 2, 2480 (1995).

42. Ya. B. Zel'dovich and Yu. P. Raizer, Physics of Shock Waves and High-Temperature Phenomena (Academic, New York, 1966), Vol. I, p. 52 .

43. S. P. Hatchett, Lawrence Livermore National Laboratory, Livermore, CA, UCRL-JC-108348 (1991).

44. G. B. Zimmerman and W. L. Kruer, Comm. Plasma Phys. Controlled Fusion 2, 51 (1975).

45. S. V. Weber, B. A. Remington, S. W. Haan, B. G. Wilson, and J. K. Nash, Phys. Plasmas 1, 3652 (1994).

46. R. M. More, K. H. Warren, D. A. Young, and G. B. Zimmerman, Phys. Fluids 31, 3059 (1988).

47. K. S. Budil et al., Lawrence Livermore National Laboratory, Livermore, CA, UCRL-JC-121565 (1995); to be submitted to Phys. Rev. Lett.

48. B. A. Remington et al., Phys. Rev. Lett. 73, 545 (1994).

49. M. J. Dunning and S. W. Haan, Phys. Plasmas 2, 1669 (1995).

50. D. L. Youngs, Physica 12D, 32 (1984); K. I. Read, Physica 12D, 45 (1984).

51. M. M. Marinak et al., Phys. Rev. Lett. 75, 3677 (1995).

52. R. J. Wallace, R. L. McEachern, and W. W. Wilcox, ICF Quarterly Report 4(3), 79, Lawrence Livermore National Laboratory, Livermore, CA, UCRL-LR-105821-94-3 (1994).

53. M. M. Marinak, R. E. Tipton, B. A. Remington, et al., ICF Quarterly Report 5(3), 168, Lawrence Livermore National Laboratory, Livermore, CA, UCRL-LR-105821-95-3 (1995); M. M. Marinak et al., Phys. Plasmas, in press (1996).

54. L. D. Landau and E. M. Lifshitz, Fluid Mechanics, 2nd ed. (Pergamon Press, New York, 1987), p. 180.

55. U. Alon, J. Hecht, D. Ofer, and D. Shvarts, Phys. Rev. Lett. 74, 534 (1995). 
56. J. P. Dahlburg et al., Phys. Fluids B 5, 571 (1993).

57. T. A. Peyser et al., Phys. Rev. Lett. 75, 2332 (1995); G. Dimonte et al., Phys. Rev. Lett. 74, 4855 (1995).

58. J. Hecht, U. Alon, and D. Shvarts, Phys. Fluids 6, 4019 (1994).

59. D. Shvarts, U. Alon, D. Ofer, R. L. McCrory, and C. P. Verdon, Phys. Plasmas 2, 2465 (1995).

60. D. L. Youngs, Phys. Fluids A 3, 1312 (1991); Laser Part. Beams 12 , 725 (1994).

61. R. P. J. Town, A. R. Bell, Phys. Rev. Lett. 67, 1863 (1991).

62. G. R. Baker, R. L. McCrory, C. P. Verdon et al., J. Fluid Mech. 178, 161 (1987).

63. F. Hattori, H. Takabe, and K. Mima, Phys. Fluids 29 (5), 1719 (1986).

64. M. A. Sweeney, F. C. Perry, J. Appl. Phys. 52, 4487 (1981); J. S. Wark, J. D. Kilkenny, A. J. Cole, M. H. Key, P. T. Rumsby, Appl. Phys. Lett. 48, 969 (1986); H. Nishimura, et al., Phys. Fluids 31, 2875 (1988).
65. T. R. Dittrich et al., Phys. Rev. Lett., 73, 2324 (1994).

66. Y. Kato and K. Mima, Phys. Rev. Lett. 53, 1057 (1984).

67. J. D. Kilkenny et al., Rev. Sci. Instrum. 59, 1793 (1988);

O. L. Landen et al., "Gain Uniformity, Linearity, Saturation and Depletion in Gated Microchannel-Plate X-Ray Framing Cameras," Ultrahigh-and High-Speed Photography, Videography, and Photonics '93, P. W. Roehrenbecks, Ed. (SPIE, San Diego, California, 1993), Vol. 2002, pp. 2-12.

68. P. M. Bell, J. D. Kilkenny, G. Power, R. Bonner, and D. K. Bradley, in Ultrahigh Speed and High Speed Photography, Photonics, and Videography'89, SPIE Vol. 1155 (SPIE, Bellingham, WA, 1989), pp. $430-444$.

69. J. T. Larsen, "HYADES-A Radiation Hydrodynamics Code for Dense Plasma Studies," 4th International Workshop on Radiative Properties of Hot Dense Matter, W. Goldstein, C. Hooper, J. Gauthier, J. Seely, R. Lee, Eds. (World Scientific, Sarasota, Florida, 1990), p. 321 . 


\title{
Diagnosis of Pusher-Fuel Mix IN INDIRECTLY DRIVEN NOVA IMPLOSIONS (HEP3)
}

\author{
T. R. Dittrich \\ R. E. Turner \\ B. A. Hammel \\ S. W. Haan \\ C. J. Keane \\ L. J. Suter \\ R. McEachern
}

\section{Introduction}

A key issue for inertial confinement fusion (ICF) is the hydrodynamic stability of the imploding capsule. Imperfections on the capsule surface can grow into large perturbations that degrade capsule performance. Understanding this process is crucial if we are to successfully predict requirements for future high-gain ICF capsules. Experiments on the Nova laser at Lawrence Livermore National Laboratory have directly measured perturbation growth on planar foils, ${ }^{1}$ and three experimental groups have investigated backlit perturbation growth using imploding spheres. ${ }^{2-4}$ In addition to these efforts, which concentrate on indirectly driven implosions, is work investigating the hydrodynamic stability of directly driven ICF capsules. 5,6 In these direct-drive experiments the laser light shines directly on the capsules, causing the implosion and providing the seed for perturbation growth.

This article reports measurement, via emission from spectroscopic tracers, ${ }^{7}$ of the full process of perturbation growth leading to pusher-fuel mix in spherical implosions, and shows perturbation growth dependence on initial perturbation amplitude and wavelength. In contrast to the cited direct-drive work, we have in this experiment separated the drive from the perturbation seed. (For a review of x-ray spectroscopy of ICF plasmas see Refs. 8 and 9.)

The purpose of the experiments described here was to study, in a controlled manner, the effects of the Rayleigh-Taylor (RT) instability on capsule implosion performance. The mechanism by which RT growth degrades capsule performance can be summarized as follows: As the ablation phase of the implosion proceeds, surface imperfections grow via the RT instability as low-density ablated material pushes on the high-density shell. This growth causes the imploding shock to deviate from spherical, carrying the perturbation information through the shell and rippling the interface between pusher and fuel. When the fuel is compressed later in time, the pusher-fuel interface becomes RT unstable, which causes this rippling to grow and produce a region of mixed pusher and fuel material. Increasing the initial outer surface perturbation increases the degree of pusher-fuel mixing.

\section{Experiment}

These Nova experiments use plastic-shelled capsules filled with deuterium $\left(D_{2}\right)$. A typical capsule shell in the experiment had a $420-\mu \mathrm{m}$ inside diameter and a 55- $\mu \mathrm{m}$-thick wall, and consisted of three layers. The inner layer, the pusher, was $\sim 3 \mu \mathrm{m}$ of polystyrene doped with $1.0 \%$ (atomic) $\mathrm{Cl}$. The middle layer was a 3 - $\mu \mathrm{m}$-thick permeation barrier made of polyvinylalcohol (PVA), which sealed in the fuel gas. An outer layer of plasma polymer $\left(\mathrm{CH}_{1.3}\right)$ was deposited over the inner layers, ${ }^{10}$ forming the ablator. The capsules were filled with $50 \mathrm{~atm} \mathrm{D}_{2}$ gas and $0.1 \mathrm{~atm}$ Ar.

The Nova laser indirectly drove the implosion of these capsules. A square pulse of laser light with a duration of $1 \mathrm{~ns}$ heated a cylindrical gold case, or hohlraum, with (typically) $17 \mathrm{~kJ}$ of laser energy. The hohlraum, reaching a peak radiation temperature of $230 \mathrm{eV}$, then emitted $x$ rays that ablated the plastic and caused the implosion.

The capsules had relatively low convergence $(\sim 8)$ and had considerably less sensitivity to growth of surface perturbations compared to that predicted for current high-gain ICF capsule designs. We chose capsule implosions with low convergence so that asymmetries in the $x$-ray drive would have little effect on the implosions and would, therefore, not complicate the perturbation growth effects.

To make capsules with various degrees of surface roughness, many polystyrene beads, ranging in diameter from 0.6 to $7 \mu \mathrm{m}$, were embedded in the PVA layer. When the ablator layer was deposited onto this rough PVA surface, the perturbations were imprinted on the 
outer surface. This method of using a capsule with a controllably rough outer surface as seed for RT growth during implosion contrasts with the method used in Refs. 5 and 6, where direct-drive laser illumination nonuniformity was assumed to be the dominant source of initial amplitude seeds to the RT instability and to be representable by a semi-empirical analytic expression.

After shooting these capsules, we developed the capability of characterizing shell surfaces using an equatorially tracing atomic force microscope (AFM). Using this AFM, we took equatorial traces of capsules from the same production runs as capsules that were shot. (To verify surface similarity, we compared scanning electron microscope images of these traced capsules with images of the shot capsules.) We converted the equatorial traces to power spectra and combined them to form ensemble averages. By assuming that the surface bumpiness is isotropic, we transformed these onedimensional (1-D) average power spectra into 2-D (spherical surface) power spectra: ${ }^{11}$

$P_{2 \mathrm{D}}(l)=\left(l+\frac{1}{2}\right) \sum_{n=l, l+2}^{\infty}\left[P_{1 \mathrm{D}}(n)-P_{1 \mathrm{D}}(n+2)\right]$

$\times \frac{(n+l) ! !(n-l-1) ! !}{(n-l) ! !(n+l+1) ! !}$

where $l$ is the perturbation mode number, $P_{1 \mathrm{D}}$ is the $1-\mathrm{D}$ power spectrum and $P_{2 \mathrm{D}}$ is the 2-D power spectrum. Figure 1 shows 2-D (spherical surface) power spectra of capsules with rms $=0.03,0.31$, and $1.75 \mu \mathrm{m}$.

We diagnosed enhanced pusher-fuel mix due to these surface perturbations in two ways. First, we monitored the variation in capsule DD neutron yield with roughness; this we expected to decrease with increasing surface roughness as cold dense pusher material increasingly poisoned the fuel. Second, we monitored the variation in the $x$-ray self-emission of included trace elements, or dopants, 7,12 with roughness. We expected the $x$-ray emission of the pusher dopant, $\mathrm{Cl}$, to increase relative to the $\mathrm{x}$-ray emission of the fuel dopant, $\mathrm{Ar}$, as the surface roughness was increased. In the temperature and density regime accessed in these experiments, the variation of $x$-ray line radiation from these dopants depends strongly on temperature. During the implosion, PdV work heated the fuel and the Ar, but conduction and convection, due to mix, heated the $\mathrm{Cl}$. At peak x-ray emission the imploded capsule had steep gradients through the mix region in both electron temperature and density as a function of radius. This made a simple one-temperature, 1-D understanding of this process difficult. We therefore observed the $x$-ray emission by means of a crystal spectrometer coupled to an x-ray streak camera, which had temporal resolution of $\sim 30$ ps and a spectral resolving power $(\lambda / \delta \lambda)-700 .^{13}$

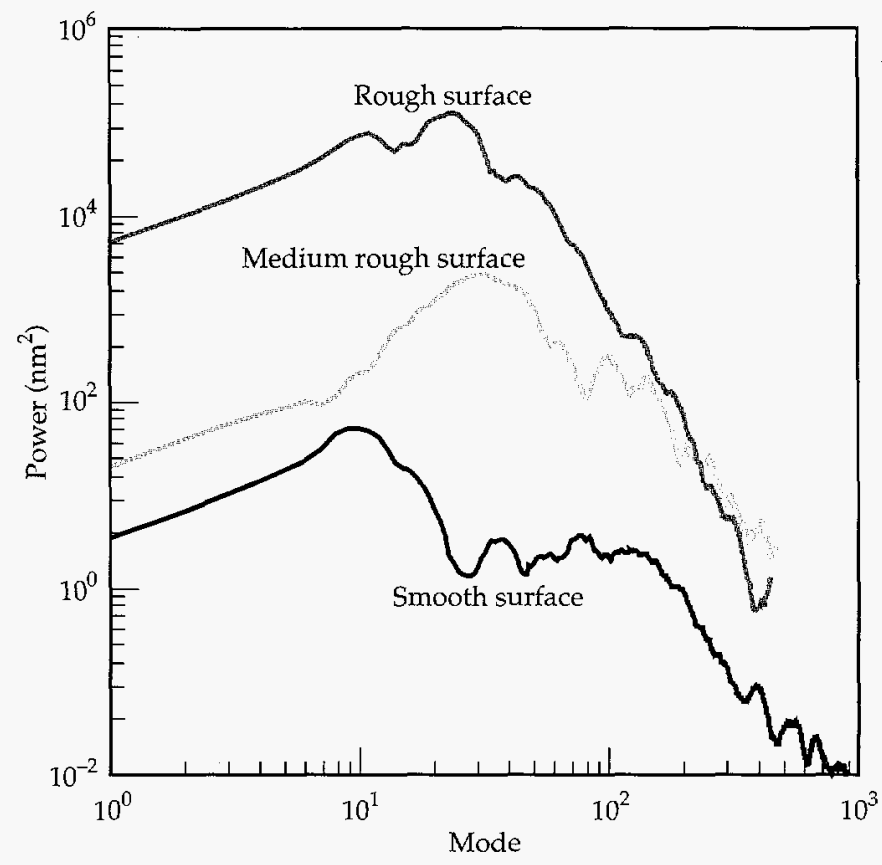

FIGURE 1. The 2-D (spherical surface) power spectra characterizing the outer surface of three representative capsules with rms $=0.03$, 0.31 , and $1.70 \mu \mathrm{m} . \quad(20-03-0795-1848 \mathrm{pb} 01)$

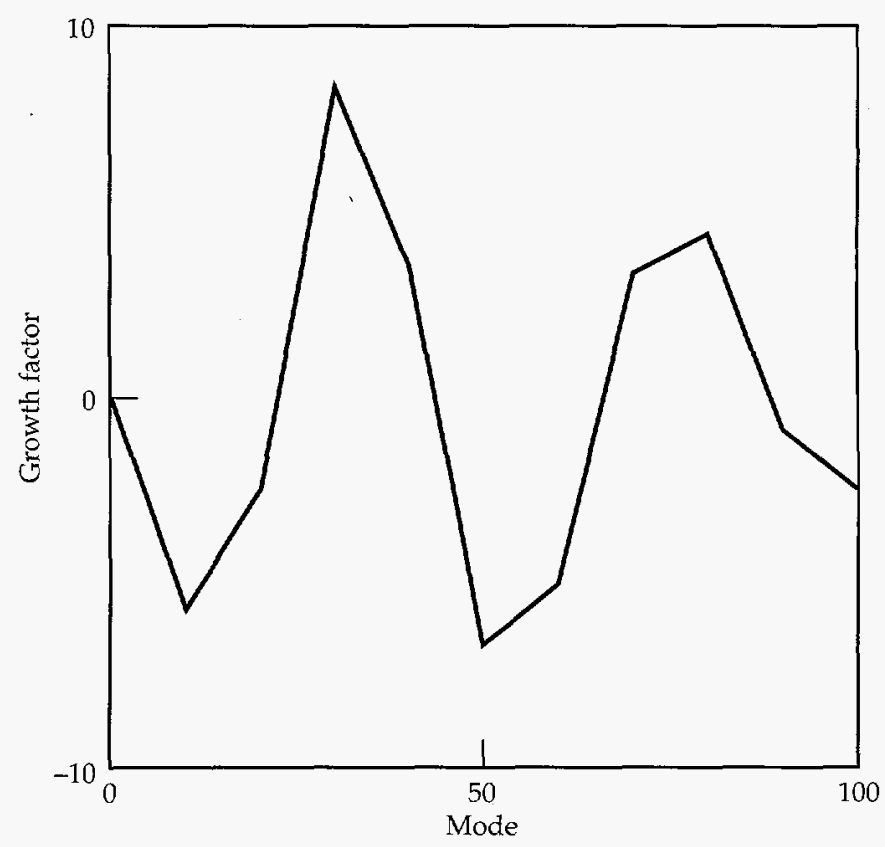

FIGURE 2. Growth factor at pusher-fuel interface vs mode number for perturbations initially on the capsule outer surface. This is a snapshot at time of peak neutron production. Negative values indicate phase change of the perturbation. (20-03-0795-1849pb01) 


\section{Analysis Method}

Simulating the implosion of these capsules was a multistep process. First, we estimated the capsule's sensitivity to surface perturbations. Second, we combined the surface roughness with the perturbation growth factors. Third, we ran implosion simulations to generate emission spectra. This section describes the three steps.

First, we estimated the capsule's sensitivity to surface perturbations using several 2-D LASNEX ${ }^{14}$ simulations, from which we estimated linear growth for several single modes. The initial perturbations in these simulations were small to ensure that only linear growth would occur. This perturbation growth included the effects of stabilization at the ablation surface, feed-through between the interfaces, and RT/Richtmyer-Meshkov instability growth at the pusher-fuel interface. Figure 2 plots these linear growth factors at the pusher-fuel interface vs perturbation mode number. This growth factor is the amplification of a perturbation initially on the outer surface as it imprints on the inner capsule surface. It is typically quoted at peak neutron emission. Negative growth factor values indicate phase change of the perturbation. This linear perturbation growth is small relative to high-gain ICF capsules because of favorable stabilization mechanisms at the ablation surface.

Next, we combined the surface roughness, $P_{2 \mathrm{D}}(l)$, with the linear perturbation growth factors per standard linear analysis. We estimated nonlinear saturation with Haan's criterion, ${ }^{15}$ which states that saturation occurs on a spherical surface of radius $R$ when amplitudes become larger than $4 R / l^{2}$. These saturated amplitudes then grow at a constant rate, rather than exponentially. This procedure predicted the pusher-fuel mixing vs time, estimated from the calculated rms deviation, $\sigma$, of the pusher-fuel surface from spherical. The limit of the bubble tips outward was taken to be $\sqrt{2} \sigma$, and the extent of spike tips inward to be $(1+A) \sqrt{2} \sigma$,

TABLE 1. Nova shots in the $\mathrm{Ar} / \mathrm{Cl}$ implosion series.

\begin{tabular}{ccccc} 
Shot & $\begin{array}{c}\text { Imbedded } \\
\text { bead diameter } \\
(\mu \mathrm{m})\end{array}$ & $\begin{array}{c}\text { Surface } \\
\text { rms } \\
(\mu \mathrm{m})\end{array}$ & $\begin{array}{c}\text { Observed } \\
\text { yield } \\
\left(10^{9} \text { neutrons }\right)\end{array}$ & $\begin{array}{c}\text { Observed yield f } \\
\text { clean (no mix }) \\
\text { yield }\end{array}$ \\
\hline 1 & None & 0.031 & 1.21 & 0.72 \\
2 & None & 0.031 & 1.26 & 0.83 \\
3 & None & 0.064 & 0.53 & 0.33 \\
4 & None & 0.065 & 1.01 & 0.60 \\
5 & 0.6 & 0.307 & 0.60 & 0.35 \\
6 & 2.0 & 0.308 & 1.22 & 0.49 \\
7 & 2.0 & 0.308 & 0.65 & 0.44 \\
8 & $3.9-7.0$ & 1.70 & 0.66 & 0.20 \\
9 & $3.9-7.0$ & 1.70 & 0.70 & 0.20 \\
\hline
\end{tabular}

where $A$ is the Atwood number. This mix-region size estimate also included contributions from both initial pusher-fuel surface imperfections and effects of embedding microspheres in the PVA layer of some of the capsules. Atomic mixing of the pusher and fuel was assumed throughout the mixed region. This modeling distributed the materials within this region so as to maintain a constant concentration while conserving individual material amounts.

Finally, LASNEX implosion simulations used this time-dependent mix region in a self-consistent manner (i.e., the mixing affected the hydrodynamic evolution) and generated emission spectra by means of Detailed Configuration Accounting (DCA). ${ }^{16,17}$ These 1-D simulations used detailed atomic models for both $\mathrm{Ar}$ and $\mathrm{Cl}$. The DCA simulated spectra were calculated using 69and 70-level atomic models for $\mathrm{Cl}$ and $\mathrm{Ar}$, respectively. We produced the models with the DSP ${ }^{18}$ code, which contains atomic physics identical to that used in the RATION code. ${ }^{19}$

\section{Data Analysis}

Table 1 lists capsule surface conditions and observed and simulated yields for the nine Nova shots that comprise this experimental series. We have chosen three of these shots to illustrate the variation in $x$-ray spectral output during implosion with initial capsule surface roughness.

Figure 3 shows spectra at peak $x$-ray emission for the implosion of a smooth (0.03- $\mu \mathrm{m}$-rms) capsule. Figure 3(a) shows the spectrum observed with the spectrometer and Fig. 3(b) shows the analogous DCA simulated spectrum. Very little $\mathrm{Cl} \mathrm{Ly}-\alpha$ emission, relative to $\mathrm{Ar} \mathrm{Ly}-\alpha$, is evident in either of these spectra. The simulations indicate that $6 \%$ of the total $\mathrm{Cl}$ mass has reached at least $600 \mathrm{eV}$. The simulated Ly- $\alpha$ satellite line strengths, on the low-energy side of the Ly- $\alpha$ lines, differ from those observed; the large absorption feature evident in Fig. 3(b) at $2.75-2.80 \mathrm{keV}$ is probably due to errors in calculating the opacity of the $\mathrm{Cl}$ He- $\alpha$ line in the colder plastic away from the pusher-fuel interface. Figure 4 shows the spectra from an intermediately rough $(0.31-\mu \mathrm{m}-\mathrm{rms})$ capsule. In this case the Ly- $\alpha$ emissions from both the $\mathrm{Cl}$ and $\mathrm{Ar}$ are comparable in strength, and $10 \%$ of the total $\mathrm{Cl}$ mass (according to simulation) has reached at least $600 \mathrm{eV}$. Figure 5 shows the spectra from a very rough $(1.75-\mu \mathrm{m}-\mathrm{rms})$ capsule. In this case the $\mathrm{Cl} \mathrm{Ly}-\alpha$ emission is stronger than the Ar Ly- $\alpha$ emission, and $15 \%$ of the total $\mathrm{Cl}$ mass (according to simulation) has reached at least $600 \mathrm{eV}$. The simulations show that from smooth to rough capsules the $\mathrm{Cl} \mathrm{Ly}-\alpha$ emission increased by $350 \%$ but the Ar Ly- $\alpha$ emission decreased by $30 \%$. 
FIGURE 3. Spectra at peak x-ray emission from the implosion of a smooth surface (rms $=0.03 \mu \mathrm{m}$ ) capsule. (a) is as observed by the streaked crystal spectrometer. (b) is the 1-D DCA simulation of this shot. Relevant emission lines of $\mathrm{Cl}$ and Ar are labeled.

(20-03-0795-1850pb02)
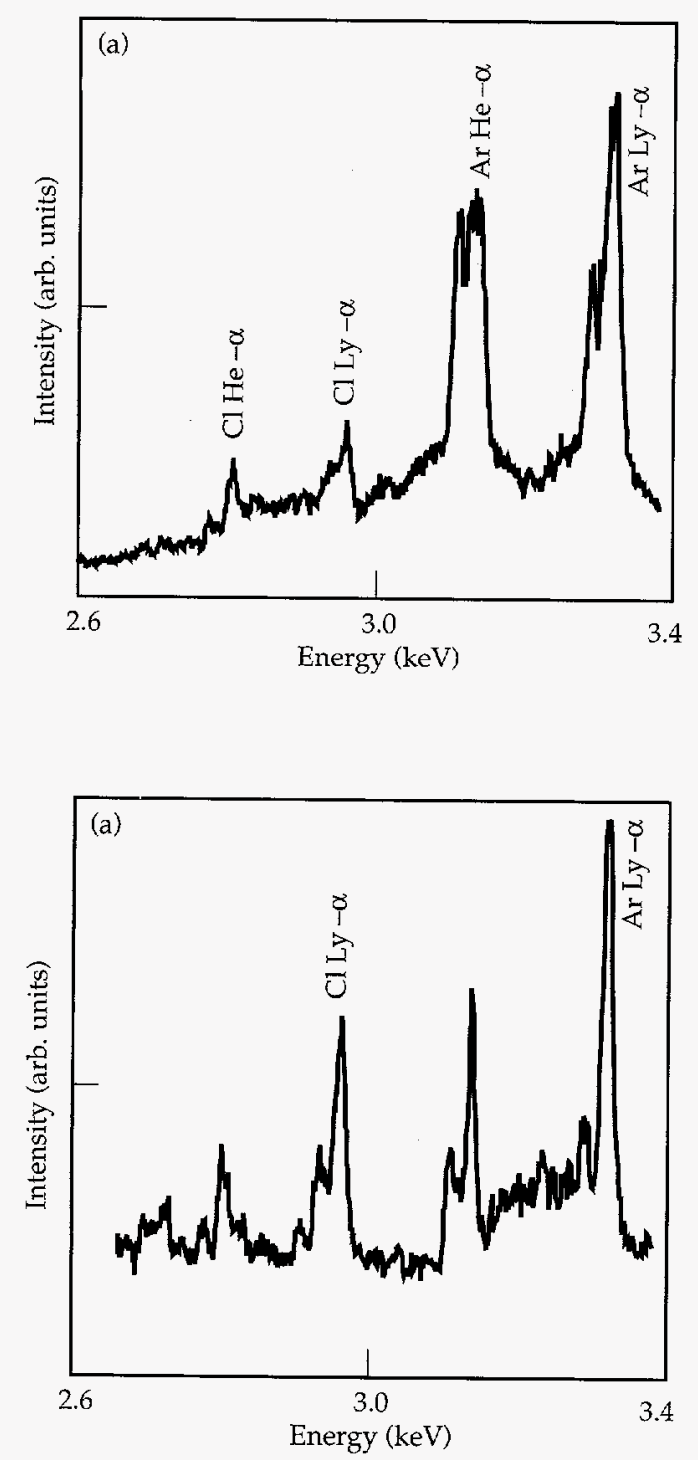

FIGURE 4. Same as Fig. 3, for an intermediate roughness

( $\mathrm{rms}=0.3 \mu \mathrm{m}$ ) capsule (20-03-0795-1851pb02)
FIGURE 5. Same as Fig. 3 , for a very rough ( $\mathrm{rms}=1.7 \mu \mathrm{m})$ capsule. (20-03-0795-1852pb02)

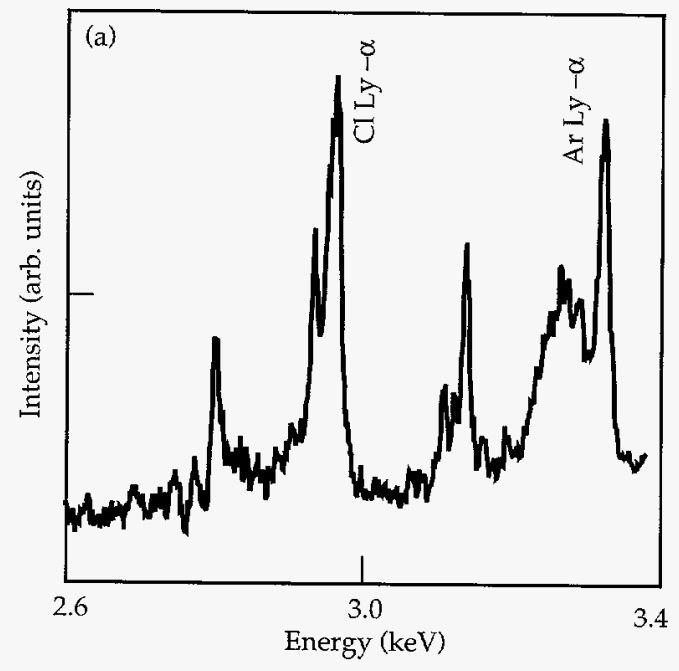

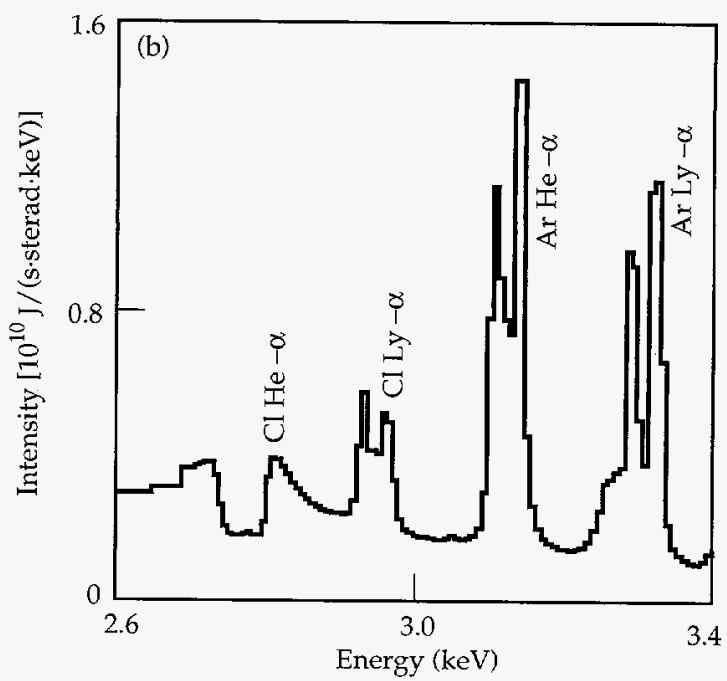
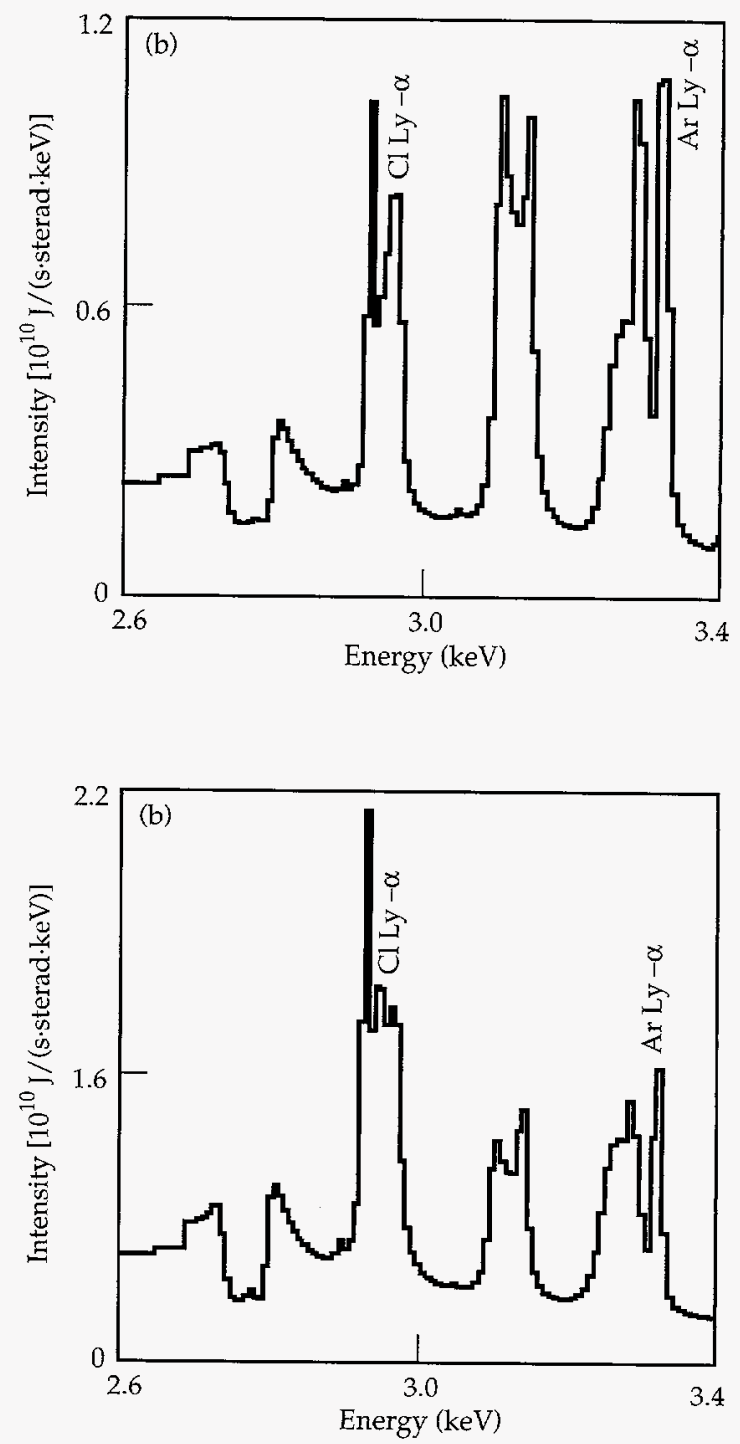


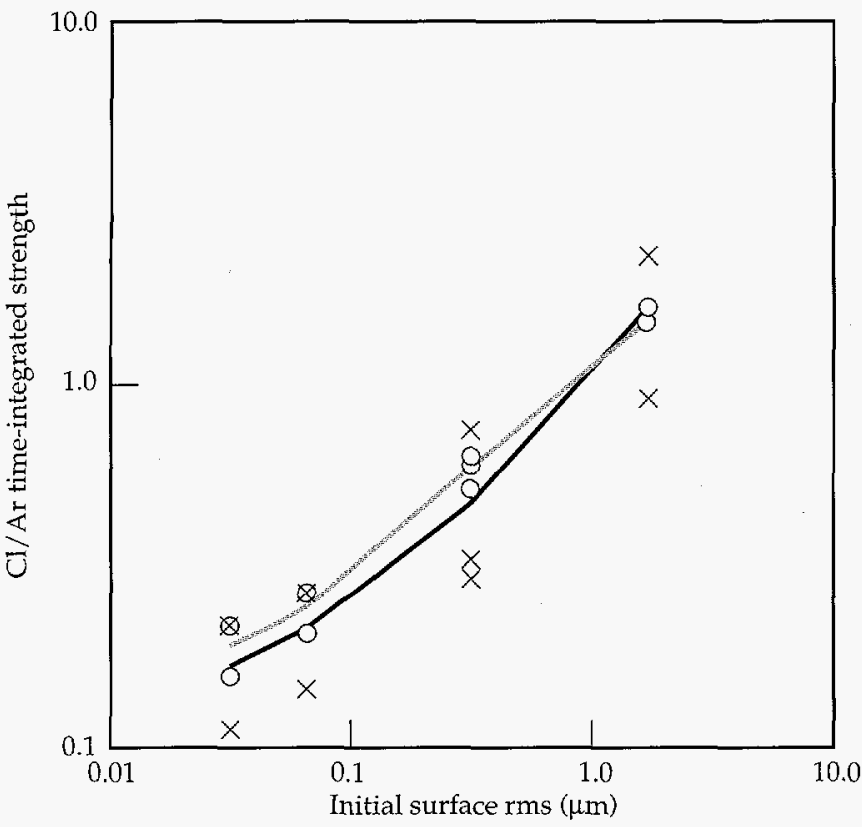

FIGURE 6. Comparison of simulated and observed emission spectra from the nine shots in the series. The ratio of time-integrated $\mathrm{Cl}$ and Ar Ly- $\alpha$ emission is plotted vs surface finish. $X$ marks experimental points; $O$ marks simulation points. The black and gray lines are the results of averaging the experimental and simulation values, respectively, at each distinct rms value. (20-03-0795-1853pb01)

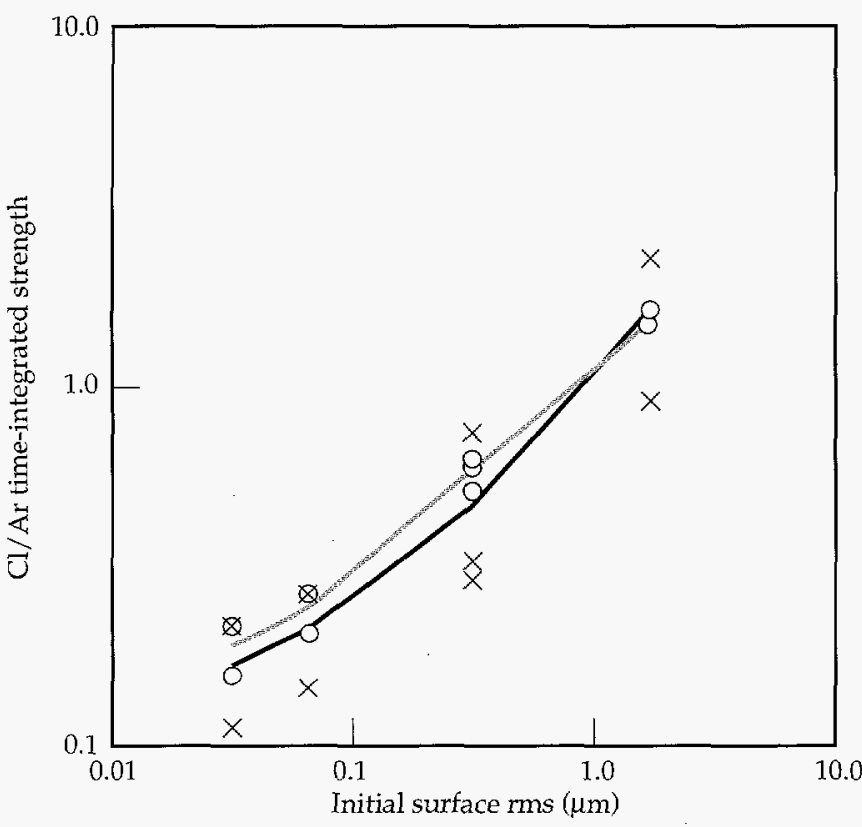

FIGURE 7. Observed and simulated mixed neutron yield over clean yield, vs surface finish for the same nine-shot data set. The black and gray lines are the results of averaging the experimental and simulation values, respectively, at each distinct rms value. $\quad(20-03-0795-1854 \mathrm{pb} 01)$
To quantitatively compare the observed and simulated spectra, we ratio the time-integrated Ly- $\alpha$ emission from the two dopants. (At each time we estimated this line emission by subtracting the continuum and background and deconvolving the result into distinct Gaussianshaped peaks. In this manner we eliminated contributions from the strong satellite lines of the Ly- $\alpha$ transitions and the $\mathrm{Cl} \mathrm{He}-\beta$ line at $3.27 \mathrm{keV}$.) The Ar Ly- $\alpha$ strength effectively normalizes the $\mathrm{Cl} \mathrm{Ly}$ - $\alpha$ strength to the specifics of capsule performance such as capsule size, laser drive, and diagnostic calibration.

Figure 6 shows this comparison of observed and simulated emission spectra by means of the ratio of time-integrated Ly- $\alpha$ lines for all nine shots. The ratio of emission strength smoothly changed a factor of $\sim 9$ for a surface rms change of $\sim 50$. Figure 7 shows observed and simulated mixed neutron yield over clean yield vs rms surface finish for the same nine-shot data set.

To test the importance of the saturation modeling, we also estimated the perturbation growth with unmodified linear analysis. The modification due to saturation is quite small, and given the large spread in experimental results, no conclusion can be made regarding the correctness of the saturation modeling procedure.

\section{Conclusion}

Both the observed x-ray emission and neutron yield from the 1-ns-drive Nova implosions show significant variation as a function of initial capsule surface finish. Furthermore, we can successfully interpret this variation as a dependence of pusher-fuel mix on initial surface roughness. This interpretation derives from modeling based on linear analysis using multiple 2-D LASNEX simulations and 1-D mixed implosion modeling using LASNEX and DCA to simulate neutron output and x-ray emission of included dopants.

\section{Acknowledgments}

The authors wish to thank Craig Moore for his efforts in capsule characterization, Ravi Upadhye and Blanca Haendler for their early work in rough-surfaced capsule fabrication, and Steve Langer, Steve Hatchett, and Yim Lee for assistance in simulation details. 


\section{Notes and References}

1. B. A. Remington, S. V. Weber, S. W. Haan, J. D. Kilkenny, et al., Phys. Fluids B 5, 2589-2595 (1993).

2. J. S. Wark, J. D. Kilkenny, A. J. Cole, M. H. Key, and P. T. Rumsby, Appl. Phys. Lett. 48, 969-971 (1986).

3. H. Nishimura, H. Takabe, K. Mima, F. Hattori, et al., Phys. Fluids 31, 2875-2883 (1988).

4. W. Mead, A. Hauer, Los Alamos National Laboratory, Los Alamos, NM, private communication, 1988.

5. D. K. Bradley, J. A. Delettrez, and C. P. Verdon, Phys. Rev. Lett. 68, 2774-2777 (1992).

6. J. Delettrez, D. K. Bradley, P. A. Jaanimagi, and C. P. Verdon, Phys. Rev. A 41, 5583-5593 (1990).

7. B. A. Hammel, C. J. Keane, T. R. Dittrich, D. R. Kania, et al., I. Quant. Spectrosc. Radiat. Transfer 51, 113-124, (1994).

8. A. A. Hauer, N. D. Delamater, and Z. M. Koenig, Laser and Particle Beams 9, 3-48 (1991).

9. C. J. Keane, B. A. Hammel, D. R. Kania, J. D. Kilkenny, et al., Phys. Fluids B 5, 3328-3336 (1993).
10. S. A. Letts, D. W. Meyers, and L. A. Witt, J. Vac. Sci. Technol. 19, 739-742 (1981).

11. S. Pollaine, S. P. Hatchett, S. H. Langer, ICF Quarterly Report 4(3), 87-89, Lawrence Livermore National Laboratory, Livermore, CA, UCRL-LR-105820-94-3 (1994).

12. B. A. Hammel, C. J. Keane, M. D. Cable, D. R. Kania, et al., Phys. Rev. Lett. 70, 1263-1266 (1993).

13. B. A. Hammel, P. Bell, C. J. Keane, R. W. Lee, Rev. Sci. Instrum. 61, 2774-2776 (1990).

14. G. B. Zimmerman, W. L. Kruer, Comments on Plasma Physics and Controlled Fusion 2, 51-61 (1975).

15. S. W. Haan, Phys. Rev. A 39, 5812 (1989).

16. Y. T. Lee, D. S. Bailey, and G. B. Zimmerman, Laser Program Annual Report 1985, UCRL-50021-85, 2.81-2.86 (1985).

17. Y. T. Lee, J. Quant. Spectrosc. Radiat. Transfer 38, 131-145 (1987).

18. C. J. Keane, R. W. Lee, and J. P. Grandy, in Proceedings of the 4 th International Workshop on the Radiative Properties of Hot Dense Matter, Eds., W. Goldstein, C. Hooper, J. Gauthier, J. Seely, and R. W. Lee (World Scientific, Singapore, 1991), p. 233.

19. R. W. Lee, B. L. Whitten, and J. E. Strout, III, J. Quant. Spectrosc. Radiat. Transfer 32, 91 (1984). 


\section{High-GROWTH-FACTOR IMPLOSIONS (HEP4)}

\author{
O. L. Landen \\ C. J. Keane \\ B. A. Hammel \\ W. K. Levedahl \\ R. E. Chrien*
}

N. D. Hoffman*

J. D. Colvin ${ }^{*}$

P. A. Amendt

S. P. Hatchett

M. M. Marinak

L. J. Suter
M. D. Cable

R. Cook

T. R. Dittrich

S. W. Haan

R. G. Hay

R. J. Wallace

\author{
H. N. Kornblum
}

R. A. Lerche

R. L. McEachern

T. J. Murphy

M. B. Nelson

\section{Introduction}

In inertial confinement fusion (ICF), ${ }^{1,2}$ the kinetic energy of an ablating, inward-driven, solid spherical shell is used to compressionally heat the low-density fuel inside. For a given drive, the maximum achievable compressed fuel density and temperature-and hence the maximum neutron production rate-depend on the degree of shell isentropy ${ }^{2,3}$ and integrity maintained during the compression. Shell integrity will be degraded by hydrodynamic instability growth ${ }^{2,4-7}$ of areal density imperfections in the capsule. Surface imperfections on the shell grow as a result of the Richtmyer-Meshkov ${ }^{8}$ and Rayleigh-Taylor' (RT) instabilities when the shell is accelerated by the ablating lower-density plasma. Perturbations at the outer capsule surface are transferred hydrodynamically to the inner surface, where deceleration of the shell by the lower-density fuel gives rise to further RT growth at the pusher-fuel interface. A widely used dispersion relation 5,10 for the RT growth rate $\gamma$ in the presence of a density scalelength $L$ and a mass ablation rate $d m / d t$ is

$\gamma=\left[\frac{k g}{(1+k L)}\right]^{1 / 2}-\frac{\beta k}{\rho} \frac{d m}{d t}$

where $k$ is the wave number of the seeding perturbation, $g$ is the acceleration or deceleration, $\beta$ is a constant between 1 and 3 determined empirically, and $\rho$ is the peak shell density. Equation (1) indicates that $\gamma$ increases as interfaces become sharper (smaller $L$ ), as peak shell densities increase, and as mass ablation rates decrease.

\footnotetext{
*os Alamos National Laboratory, Los Alamos, New Mexico
}

\section{Implosion Design}

In the HEP3 implosion campaign, ${ }^{6}$ the unsaturated to weakly nonlinear low-growth regime was studied. See the previous article, "Diagnosis of Pusher-Fuel Mix in Indirectly Driven Nova Implosions (HEP3)," p. 265 for a discussion. Unshaped drive pulses and lowopacity plastic capsules were used, which allowed strong shock and hard x-ray preheating. This led to shallower ablation-front gradients and lower shell densities during the implosion phase. The goal of the HEP4 campaign was to increase susceptibility to RT growth in a more isentropic implosion. HEP4 designs ${ }^{11}$ have used shaped drive, and $x$-ray preheat shielding, ${ }^{12}$ by adding mid- $Z$ dopants in the capsule ablator for reducing preheat.

\section{Role of Doped Ablators}

Use of a mid-Z dopant in the ablator has three principal effects. First, these dopants are chosen to preferentially absorb the $1-3-\mathrm{keV} x$ rays (arising from the Au hohlraum laser plasmas) that volumetrically preheat Nova-scale plastic shells most efficiently. A cooler shell will expand less quickly, thereby maintaining a higher shell density $\rho$ and steeper interface density gradients for a longer time. ${ }^{13}$ Second, doping reduces the ablation scalelength by reducing the distance over which the soft drive $x$ rays $^{14}$ are absorbed. Third, the increase in shell density leads to a thinner shell during compression and thus gives rise to more efficient feedthrough of surface perturbations with skin depths $\approx 1 / k$. These effects combine to increase predicted maximum linear growth factors GF (ratios of final pusherfuel to initial outside surface perturbation amplitudes) from 10 to 110 as the doping is increased from 0 with no pulse shaping (HEP3 conditions) ${ }^{6}$ to 2 at. $\%$ Ge or $\mathrm{Br}$ 
with a two-step pulse (HEP4 conditions). ${ }^{13}$ Mid-Z dopants, in particular $\mathrm{Br}$, were used extensively to increase growth factors ${ }^{15}$ in planar RT experiments ${ }^{16}$ conducted in the HEP2 campaign.

A linear GF of 100 , for example means that, in the absence of saturation, an initial perturbation amplitude of $0.1 \mu \mathrm{m}$ with mode number $l=k r(t)$ on the surface of a capsule of radius $r$ would lead to a $10-\mu \mathrm{m}$ perturbation of the same mode number at the pusher-fuel interface at peak neutron production time. In general, a full spectrum of randomly phased modes will initially be present. Each mode number will have a different predicted GF through the dependence on wave number $k$ in Eq. (1). Growth of high-mode-number perturbations (large $k$ ) is reduced by ablation, density gradients, inefficient feedthrough, and (when amplitudes become comparable to the wavelength) by the onset of saturation. Long wavelengths (small $k$ ) have low growth rates from the outset. One can therefore expect a bellshaped growth factor spectrum that peaks near some intermediate mode number. ${ }^{13}$

\section{Instability Growth Modeling}

Until recently, three-dimensional (3-D) codes handling multimode growth up to and beyond saturation have not been available or practicable. In their place, a multimode mix model ${ }^{4,6}$ has been used extensively, as described more fully in "Diagnosis of Pusher-Fuel Mix in Indirectly Driven Nova Implosions (HEP3)" on p. 265 of this Quarterly. Briefly, a series of linear single-mode growth-rate simulations is used to calculate the amplitude evolution of each mode initially present on the capsule surface, starting with amplitudes small enough to ensure that the growth remains linear throughout the implosion. The time dependence of the linear mode amplitudes are then obtained by multiplying these growth factors by the initial amplitude spectrum. If the individual amplitudes grow large enough $\left(\geq 2 r / l^{2}\right)$, they are corrected for saturation in the presence of a full spectrum of modes. ${ }^{4}$ The quadrature sum of the perturbation amplitudes fed through to the pusher-fuel interface is then used to set the annular width for a one-dimensional (1-D) model of atomically mixed shell and fuel located at the shell-fuel interface. A 1-D implosion simulation incorporating this evolving mix layer is then used to predict observables such as neutron yield. The yield drops as the calculated mix width becomes a larger fraction of the converged fuel radius, principally because of enhanced conduction cooling by shell material penetrating closer to the central, hottest fuel region, which provides most of the fusion reactions.

Figure 1 shows the calculated mix amplitudes (normalized to converged fuel radius at peak neutron emission time and defined as approximately one-third the mix width) vs initial outside surface roughness for the low-growth HEP3 implosions, the present HEP4 implosions, and the proposed National Ignition Facility (NIF) conditions. ${ }^{17}$ The gray lines with unit slope correspond to assuming no growth saturation. The departure from unit slope for each black curve signifies the predicted onset of growth saturation. A typical intrinsic surface roughness power spectrum was assumed for these calculations. The large fractional mix widths calculated for the NIF implosions are a consequence of the more elaborate pulse shaping used to keep NIF implosions nearly isentropic. Ignition for NIF will require that the final mix amplitude not exceed about one-fifth of the converged capsule radius. ${ }^{17}$ This criterion is equivalent to a factor of $\sim 2$ yield reduction for nonigniting target designs such as those used in HEP3 and HEP4. The present HEP4 experiments were designed to span both sides of this threshold by varying initial surface roughnesses from $0.01 \mu \mathrm{m} \mathrm{rms}$ upward, while approaching the growth factors expected of the NIF targets.

Figure 1 shows that a necessary condition for ignition sets the upper limit on initial capsule outside surface finish at a currently achievable roughness of $0.03 \mu \mathrm{m}$ rms. If there were no growth saturation, NIF capsules would need to be considerably smoother (by a factor of 2), which is at the limit of current technology. A specific goal of the HEP4 campaign was therefore to test the validity of the growth saturation model.

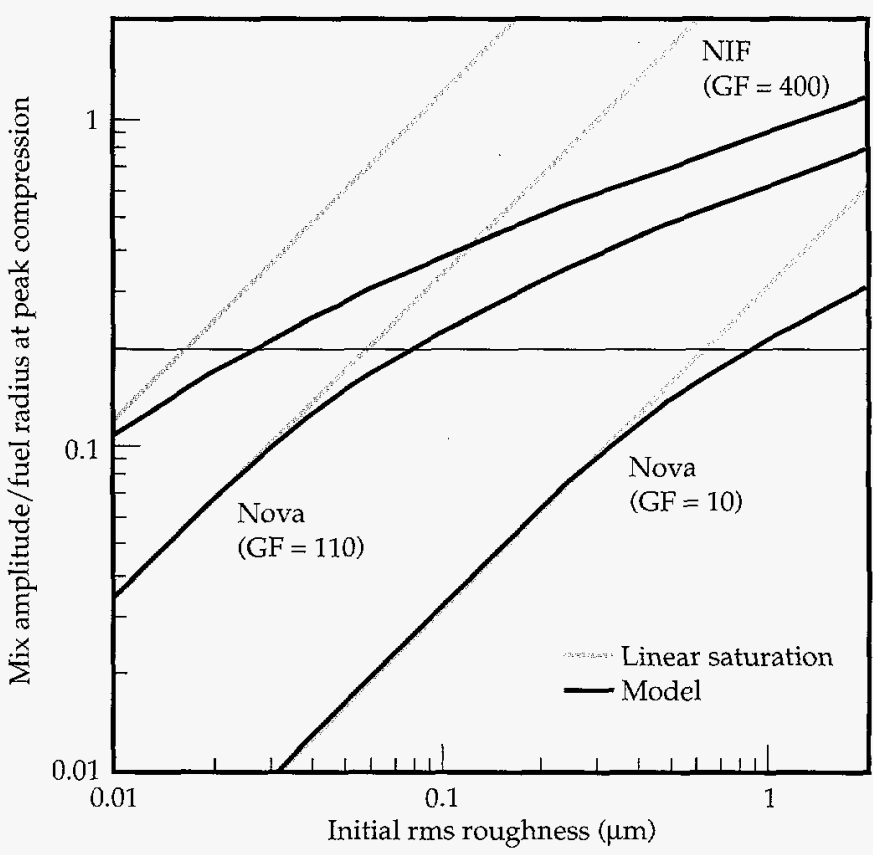

FIGURE 1. Calculated mix amplitude (normalized to fuel radius at peak neutron emission time) vs initial surface roughness. Starting from the right, the three curves correspond to the low-growth HEP3 design, the present higher-growth HEP4 design, and the NIF design. Black and gray curves correspond to calculations with and without growth saturation, respectively. The section of the NIF curve above a normalized mix amplitude of 0.2 corresponds to loss of ignition. (20-03-0995-2096pb01) 


\section{Capsule Design}

Figure 2 shows the cross section of a typical capsule. The ablator, a $1.1-1.3 \mathrm{~g} / \mathrm{cm}^{3}$ plasma-polymerized plastic $\left(\mathrm{CH}_{1.3}\right)$, typically $39 \mu \mathrm{m}$ thick, is doped ${ }^{18}$ with up to 3 at.\% $\mathrm{Br}$ or $\mathrm{Ge}$. The early experiments used $\mathrm{Br}$ doping; the later experiments switched to $\mathrm{Ge}$, which was more robust and easier to fabricate. A 3- $\mu \mathrm{m}$ polyvinyl alcohol (PVA) intermediate layer serves to confine the fuel. In early experiments, the inner polystyrene shell $(\sim 440 \mu \mathrm{m}$ i.d. and $3 \mu \mathrm{m}$ thick) was doped with 0.07 at.\% Ti. The fuel consists of $25 \mathrm{~atm}$ each of $\mathrm{D}_{2}$ and $\mathrm{H}_{2}$, doped with

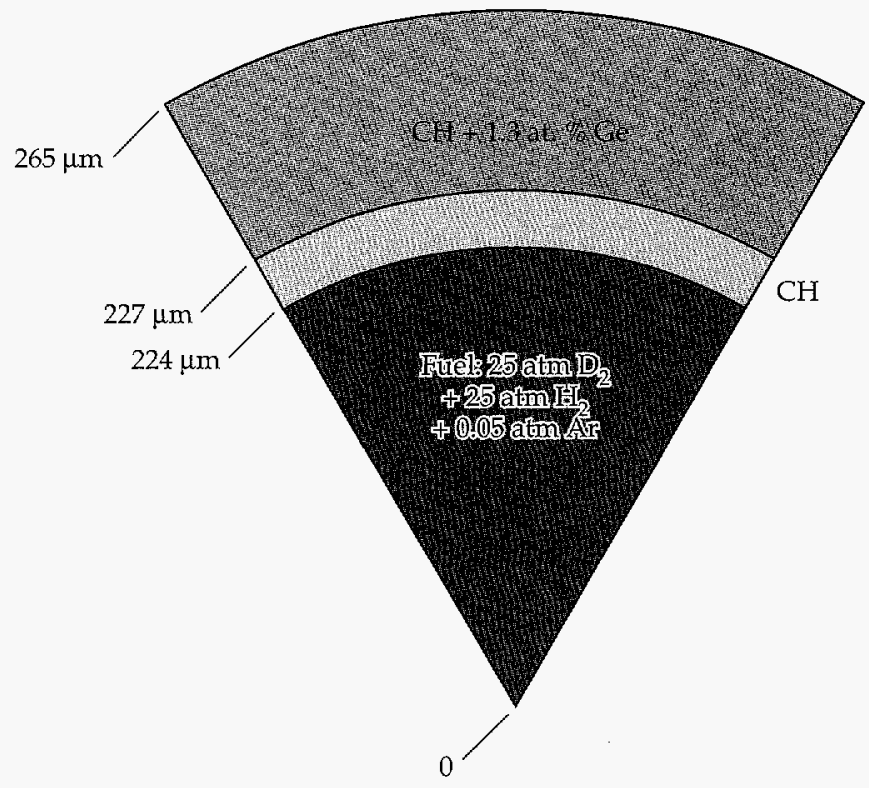

FIGURE 2. Cross section of a typical deuterated-fuel capsule design. (20-03-0995-2107pb03)

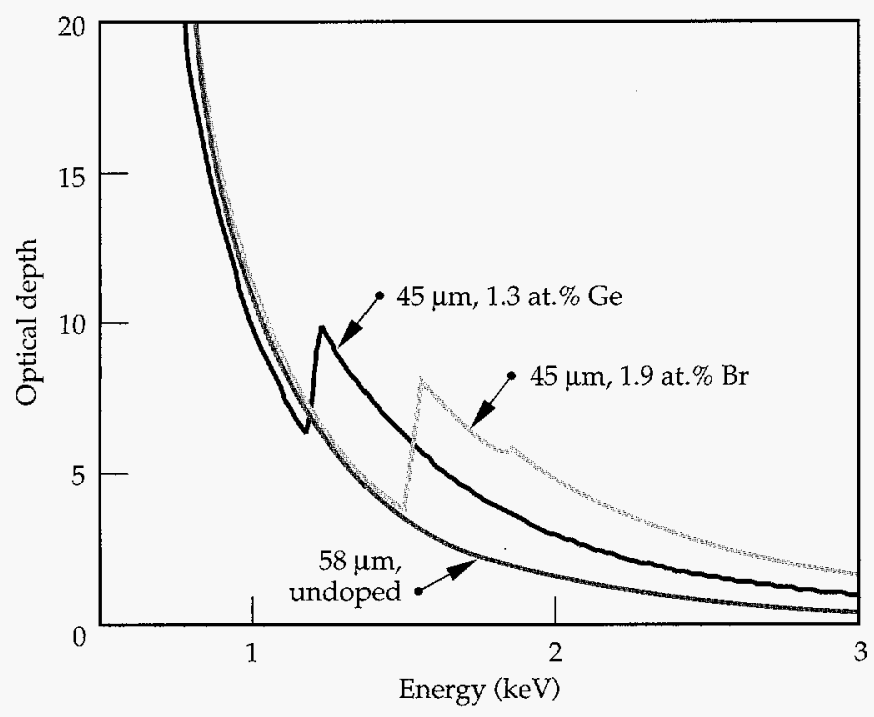

FIGURE 3. Initial capsule ablator opacity vs photon energy for 58 - $\mu$ m-thick undoped plastic ablators and for $45-\mu \mathrm{m}$-thick 1.3 at. $\%$ Ge and 1.9 at.\% Br-doped plastic ablators. (20-03-0995-2099pb01)
0.05 at.\% Ar. The dilution of the $\mathrm{D}$ with $\mathrm{H}$ was necessary to avoid saturation in the secondary-neutron detector. The Ar and Ti serve as noninvasive emission diagnostics of electron temperature and electron density and of the spatial profile of the fuel and shell during the burn phase. Figure 3 shows the initial shell optical depth vs photon energy and shows, for example, that 1.3 at.\% Ge doubles the initial capsule optical depth above the Ge $(n=2)$ bound-free absorption edge at $1.2 \mathrm{keV}$. Simulations using the measured photon flux above $1.2 \mathrm{keV}$ indicate that such shielding reduces the entropy of the inner shell surface relative to the undoped case by $20 \%$ before the first shock arrives at $1 \mathrm{~ns}$.

The RT seeding is provided by pre-roughening the capsule surface by ultraviolet (UV) laser ablation ${ }^{19}$ of 200 randomly distributed $75-\mu \mathrm{m}$-diam pits of equal depths, which yields a continuous distribution of perturbation wave numbers. The surface roughness is quantified by averaging a series of circumferential depth profiles obtained by atomic force microscopy. ${ }^{20}$ The profiles are Fourier-transformed to yield 1-D power spectra, which are found to be in excellent agreement with spectra predicted by a model assuming randomly located pits of the measured shape. By assuming isotropy, 1-D spectra can be converted ${ }^{21}$ to $2-D$ power spectra, which serve as input to simulations. A simple measure of the surface roughness is taken to be the square root of the summed power spectra, expressed as a rms roughness. For the purposes of defining roughness, the lowest order modes $(l<10)$, which grow the least according to Eq. (1), are not included. By varying the pit depths, rms roughnesses so defined covered the range between 0.01 and $2 \mu \mathrm{m}$.

\section{Hohlraum Drive}

The capsules are mounted in the center of a $2400-\mu \mathrm{m}$ long, $1600-\mu \mathrm{m}$-diam Au hohlraum with 1200- $\mu \mathrm{m}$-diam laser entrance holes on each end. A hohlraum of pentagonal cross section was used to avoid line focusing of reflected laser light onto the capsule surface, as is observed with cylindrical hohlraums. X-ray and optical measurements indicate that such line foci reach irradiances of $10^{14} \mathrm{~W} / \mathrm{cm}^{2}$ over the first $200 \mathrm{ps}$, which could seed RT-unstable perturbations of similar magnitude to some of the smaller amplitude ablated pits.

The soft $x$-ray drive was generated by irradiating the inner hohlraum walls with ten accurately synchronized (10 ps rms fluctuation), precision-pointed ( $30 \mu \mathrm{m}$ rms fluctuation), power-balanced ( $<10 \%$ rms fluctuation), ${ }^{22}$ 0.35- jum, 2.2-ns-long, 3-kJ Nova pulses. Figure 4(a) shows the absorbed power from a ramped pulse shape, called PS26, chosen to provide reduced shock preheating 23 and to more closely approximate ignition-scale drive. The average $x$-ray flux at the capsule, plotted in Fig. 4(a) as a blackbody flux temperature, was inferred from filtered, time-resolved, multichannel (Dante) 
measurements ${ }^{24}$ of $x$-ray re-emission from the hohlraum walls. Measurements were performed on both laserirradiated and unirradiated walls. ${ }^{25}$ The drive was independently inferred from simultaneous UV shock breakout measurements ${ }^{26}$ using $\mathrm{Al}$ wedges [Fig. 4(b)]; the results are in good agreement with simulations based on the measured drive shown in Fig. 4(a). The peak drive temperature was $237 \pm 7 \mathrm{eV}$; the uncertainty corresponds to a factor of 2 uncertainty in calculated neutron yield.

Harder $x$ rays emanating from the high-temperature, low-density Au laser plasmas (principally $\mathrm{Au} n=4$ to $n=3$ and $n=5$ to $n=3$ transitions between 2 and $4 \mathrm{keV}$ ) are also present. Figure 3 shows that the ablator optical depth is only 1 to 2 for these $x$ rays, making them an important source of preheating of the inner shell. Their fractional contribution to the total drive at the capsule (shown in Fig. 5) was determined from a solid-angle average formed by combining the Dante localized absolute flux measurements with 2-D spatially resolved $x$-ray images of the hohlraum wall. We attribute the initial spike in the hard $\mathrm{x}$-ray fraction to reflected laser light efficiently illuminating much of the hohlraum walls; $0.1 \mathrm{~ns}$ later, plasma expansion has greatly diminished the reflectivity and localized the hard $\mathrm{x}$-ray production at the first-hit locations of the laser. The error bars represent only Dante uncertainties; the assumption of an optically thick Lambertian source for the harder $x$ rays may result in an additional $2 \times$ underestimate of their fraction. However, simulations show that admitting a total factor of 3 underestimate in hard x-ray fraction will decrease yields for 1.3 at. \% Ge-doped capsules by only $30 \%$.

\section{Deuterated Fuel Capsules}

The implosions are diagnosed by primary and secondary neutron yields, ${ }^{27}$ neutron production times, ${ }^{28}$ time-resolved $x$-ray imaging, ${ }^{29}$ and time-resolved $x$-ray spectroscopy of tracer dopants in the shell and fuel. ${ }^{13,23,30,31}$ The results of an early campaign with Brdoped capsules have been published elsewhere. ${ }^{30}$ The newer Ge-doped capsule implosion results described here have been more thoroughly characterized and modeled. Results are compared with predictions made using the 2-D radiation hydrodynamics code LASNEX. ${ }^{32}$ A new 3-D radiation hydrodynamics code, HYDRA, ${ }^{33}$ is also being used to simulate these highgrowth-factor implosions.

\section{Performance vs Preheat Shielding}

The first HEP4 implosions served to test our understanding of the behavior of the smoothest available plastic capsules as ablator doping was increased. In the limit of negligible RT growth, one would expect that the increase in in-flight shell density $\rho$ due to preheat shielding provided by the mid- $Z$ dopant would improve capsule performance. Specifically, for a fixed implosion velocity $v$, the compressional pressure ${ }^{2,3,10} \rho v^{2}$ that determines the final fuel areal density and yield achievable should increase with $\rho$.

Figure 6 shows that the measured yield does indeed increase with Ge doping, with a slope consistent with that of the corresponding 1-D simulations. Peak neutron production times $(2.2 \pm 0.1 \mathrm{~ns})$ and hence implosion velocities were kept fixed by varying the initial ablator thicknesses (from $44 \mu \mathrm{m}$ at 2 at. $\%$ Ge to $58 \mu \mathrm{m}$ for undoped ablators) to compensate for changes in initial shell density and opacity when incorporating Ge in the ablator. Capsules were selected for best surface finish
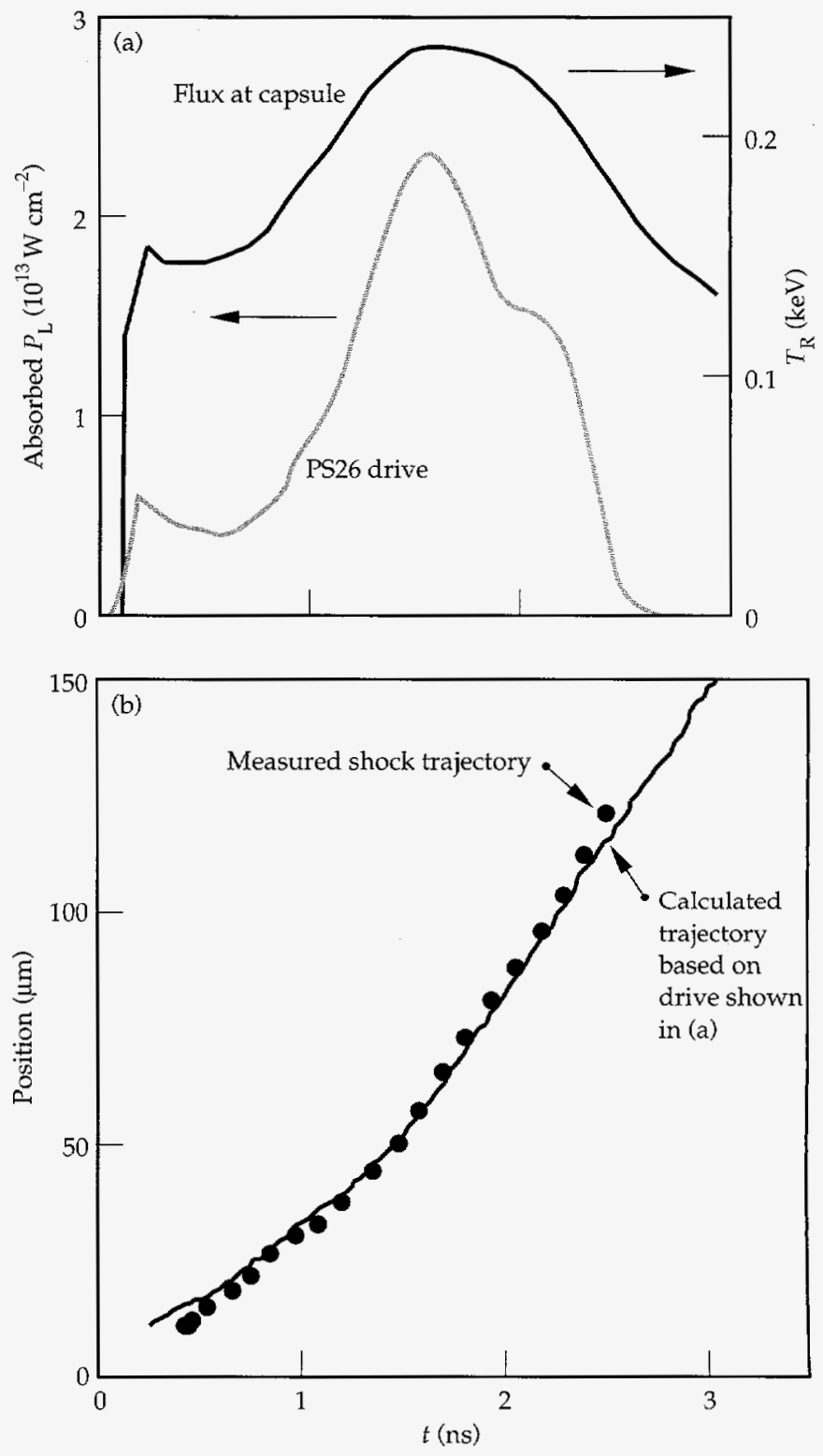

FIGURE 4. (a) Measured absorbed laser power and measured soft $x$-ray flux (plotted as a blackbody flux temperature) from absolutely calibrated filtered diode array (Dante). (b) Measured shock trajectory in Al wedge and corresponding predicted trajectory based on the $\mathrm{x}$-ray drive in (a). (20-03-0995-2100pb01) 
( $<0.03 \mu \mathrm{m} \mathrm{rms}$ roughness). The simulations used the measured drive flux and spectrum at each time as baseline input, with slight modifications for $(<10 \%)$ shot-to-shot variations in laser energy and capsule dimensions. The systematic factor of 3-4 discrepancy between simulated and measured yields for smooth capsules is not completely understood, but 3-D calculations which include long wavelength shell thickness variations significantly reduce this discrepancy. These calculations are described in the section "Recent

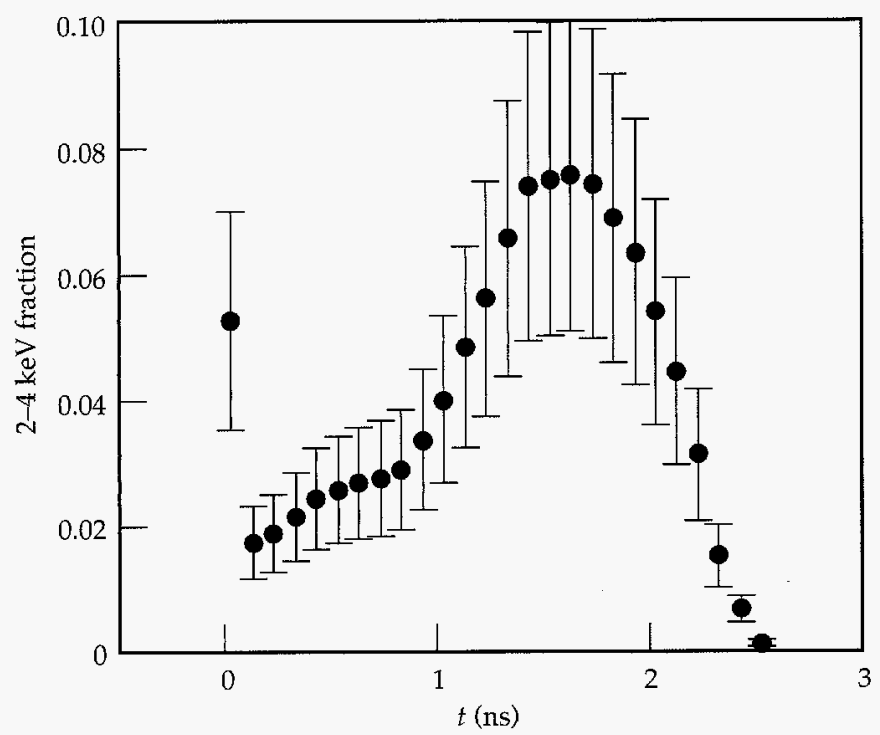

FIGURE 5. Measured 2-4-keV hohlraum power as a fraction of total $x$-ray power. Error bars represent uncertainty in Dante calibration and unfold only. (20-03-0995-2101pb01)

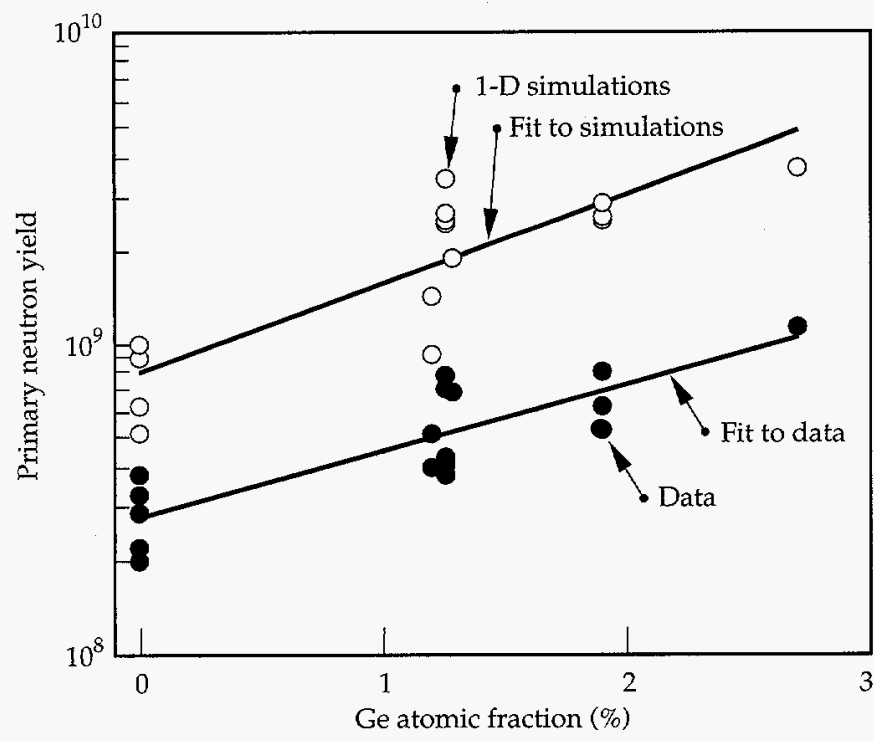

FIGURE 6. Measured and calculated primary (DD) neutron yields vs doping for smooth capsules. Solid lines are linear fits to data and simulations. $\quad(20-03-0995-2103 \mathrm{pb} 01)$
Modeling Advances." Nevertheless, the increase in yield as preheat shielding is increased for best surface finish capsules is encouraging for NIF, because it demonstrates a hydrodynamically similar implosion for which the beneficial effects of a more isentropic compression outweigh the detrimental effects of increased susceptibility to RT growth.

Figure 7 displays $4-\mathrm{keV}$ x-ray snapshots showing a reduction in imploded core image size as the ablator doping is increased from zero to 1.3 to 2.7 at. \% Ge. The core images, captured with $7-\mu \mathrm{m}$ and 80 -ps resolution by gated pinhole cameras, are dominated by Ar bound-free emission from the doped fuel. Figure 7(d) shows the azimuthally averaged $50 \%$ contour diameters extracted from such images. The average measured $x$-ray radii decrease monotonically with increasing $\mathrm{Ge}$ doping, with a slope consistent with post-processed 2-D integrated hohlraum and capsule simulations of image size, but with an overall $30 \%$ size reduction.
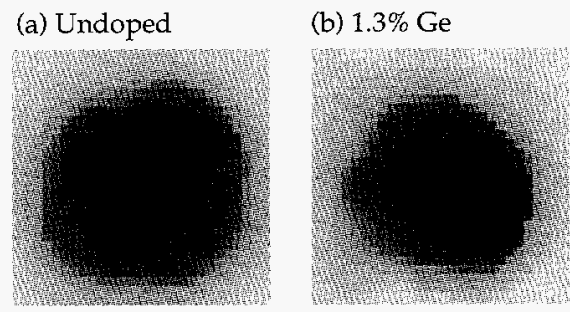

(c) $2.7 \% \mathrm{Ge}$

(d) FWHM vs Ge atomic fraction

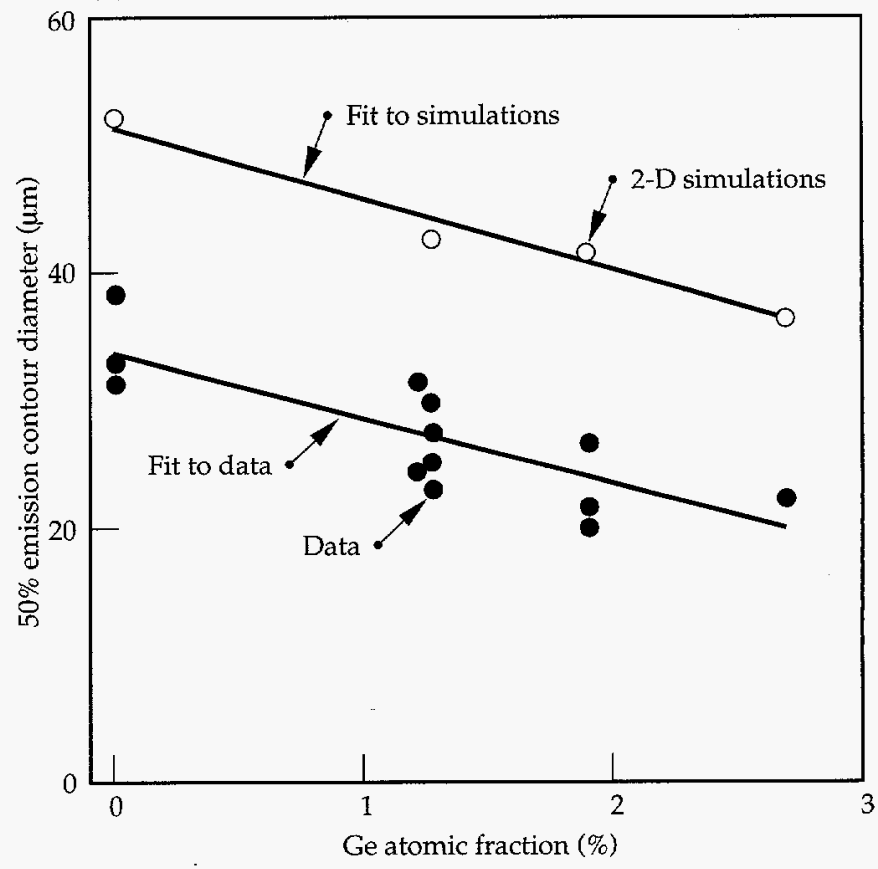

FIGURE 7. X-ray images at $4 \mathrm{keV}$ of imploded cores from smooth capsules at peak emission time for (a) no doping, (b) 1.3 at.\% Ge, and (c) 2.7 at.\% Ge. (d) Measured (solid circles) and calculated (open circles) azimuthally averaged diameters of $50 \%$ x-ray emission contours vs Ge doping. Solid lines are linear fits to data and simulations. (20-03-0995-2102pb01) 
Fuel areal densities and (by invoking particle conservation) fuel convergences were also inferred from the fraction of secondary DT reactions and the resultant secondary-neutron energy spectrum ${ }^{27}$ measured by a large neutron scintillator array (LaNSA). This diagnostic technique, used extensively in the HEP1 campaign, ${ }^{25}$ works on the principle that the triton produced in the primary DD reaction undergoes secondary DT reactions with a probability that depends on the areal density of deuterons seen by the escaping triton. At all Ge dopings, the inferred fuel convergences are within $10 \%$ of the values obtained from simulations. To reconcile the $x$-ray size discrepancy with the agreement in convergence, we note that emissivities for kilovolt $x$-ray photons are sensitive to sub-kilovolt variations in plasma temperature, while the secondary reaction between $\mathrm{MeV}$ tritons and deuterons is not. Hence the $30 \%$ overprediction in $x$-ray core image size shown in Fig. 7(d) may be evidence that the simulations, while correctly predicting the final fuel radius, overpredict the plasma temperature in the outer regions of the compressed fuel. Such increased cooling is seen in 3-D calculations which include long wavelength variations in capsule wall thickness.

Spatially integrated, time-resolved spectra of Ar and Ti line and continuum emission were also recorded during the fusion phase. ${ }^{30}$ The emission lines of interest are those of He-like Ti $\left(1 \mathrm{~s} 2 \mathrm{p}-1 \mathrm{~s}^{2}\right)$ and of optically thin He-like $\operatorname{Ar}\left(1 \mathrm{~s} 3 \mathrm{p}-1 \mathrm{~s}^{2}\right)$ and H-like Ar (3p-1s). The measured Ar and Ti line durations for 1.9 at.\% Br-doped capsules were 150 and 80 ps FWHM, respectively, half the corresponding durations for undoped capsules and in good agreement with simulations. Predicted H-like emission from the Ti dopant in the inner portion of the shell was not observed, again suggesting that the volume comprising the outer regions of the fuel and the inside of the shell is cooler than expected.

The agreement between measured and predicted trends in capsule performance as preheat shielding is increased indicates that the goal of mimicking more isentropic NIF-like implosion conditions has been attained. Specifically, smaller cores, shorter burn phases, and higher neutron production rates are observed as preheat shielding is increased.

\section{Performance vs Surface Roughness}

Figure 8 shows primary neutron yield for undoped and doped capsules vs initial surface roughness. Between best surface finish $(<0.03 \mu \mathrm{m} \mathrm{rms})$ and $1 \mu \mathrm{m}$ rms roughness, the yields of undoped capsules drop by a factor of only 1.5 , while those of 1.3 at.\% Ge-doped capsules drop by a factor of 6 . This finding is qualitatively consistent with the transition from low- (GF 10) to high-growthfactor $(\mathrm{GF}=110)$ behavior expected with doping.

Moreover, we observe a statistically significant factor-of-2 yield degradation between doped capsules with best surface finish and doped capsules with $0.1-\mu \mathrm{m}$ rms roughness; from Fig. 1, the latter correspond closely to the $20 \%$ mix fraction growth that determines the NIF ignition threshold.

Figure 9 compares the averaged doped capsule yields vs surface finish with various simulation results. The lowest curve, which represents the atomic mix model with no saturation, severely overestimates the yield degradation for large initial surface roughnesses. The other curves represent the same model corrected for the different saturation behavior predicted for 3-D or 2-D multimode growth. ${ }^{34}$ The atomic mix models are in fairly good agreement with data at the rough end. As discussed in the section on 3-D modeling advances, the factor of 3-4 yield discrepancy, which remains at the smooth capsule end can be largely accounted for by long wavelength capsule wall thickness variations. The larger yield degradation calculated for the 3-D saturation model is a consequence of the later onset of saturation ${ }^{4}$ and of the higher terminal velocity of low-density 3-D fuel bubbles rising into the shell. The atomic mix model with the 2-D saturation prescription, although not strictly comparable with the 3-D nature of perturbation growth in the experiment, is included to show good agreement with 2-D finely zoned multimode simulations (shown as triangles).

These multimode simulations, which follow perturbation growth past saturation and do not need to combine modes into a 1-D mix description, were made possible by using only a few photon groups. ${ }^{33}$ This method is

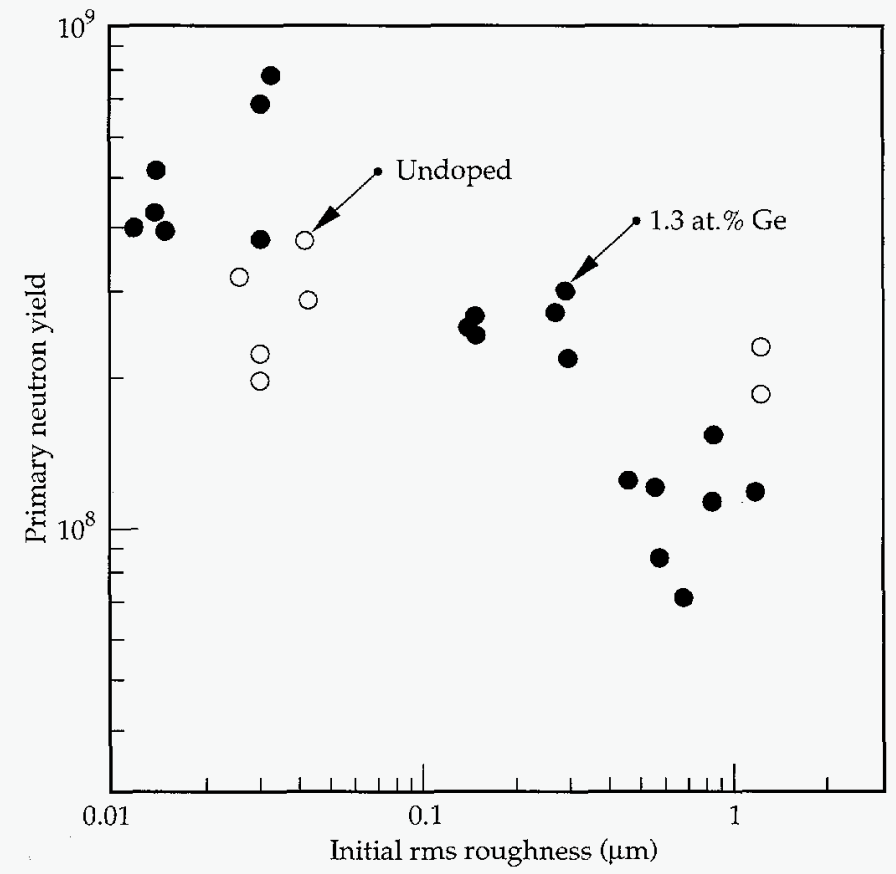

FIGURE 8. Measured primary neutron yields for 1.3 at.\% Ge-doped (solid circles) and undoped (open circles) capsules vs initial rms surface roughness. (20-03-0995-2104pb01) 
only applicable when diffusive radiation transport is used, speeding up computer simulations. The 3-D nature of the perturbations is accounted for by adjusting

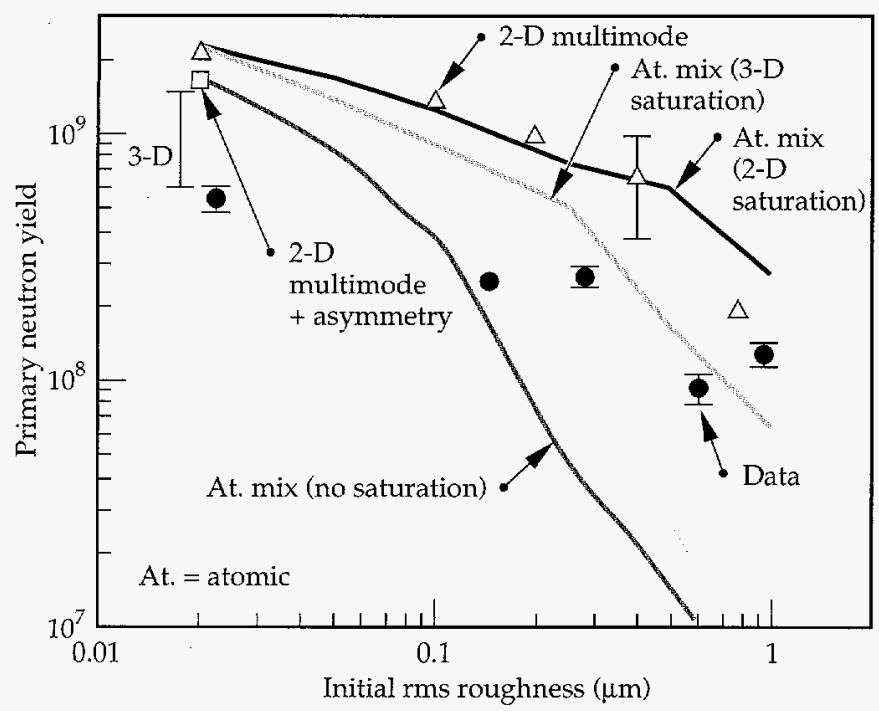

FIGURE 9. Measured and calculated primary neutron yields for 1.3 at.\% Ge-doped capsules vs initial surface roughness. Solid circles are averaged data points from Fig. 8. Solid curves are predictions from the atomic mix model with no saturation, with a 2-D saturation, and with a 3-D saturation prescription. The square and the triangles are predictions from 2-D multimode simulations with and without flux nonuniformities. The vertical line at the upper left represents the range of yields calculated by the 3-D HYDRA code for a smooth capsule by varying the relative orientation between low-order capsule and radiation flux nonuniformities. (20-03-0995-2105pb01)

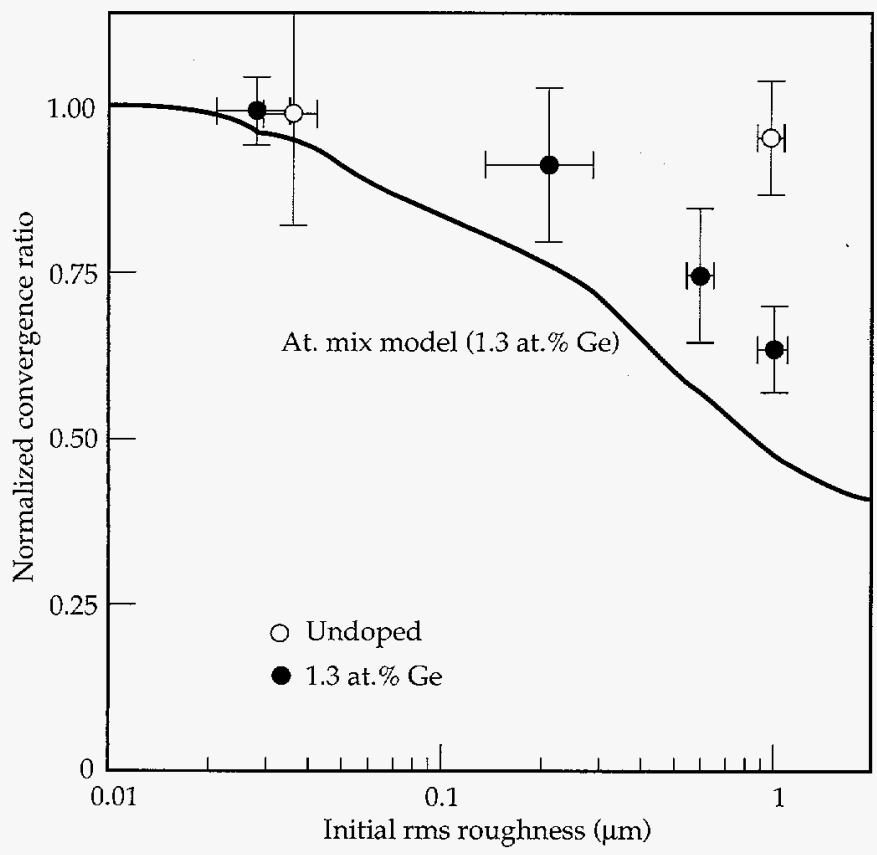

FIGURE 10. Relative capsule convergence (inferred from secondary neutron yields and spectra) vs initial surface roughness for undoped and 1.3 at.\% Ge-doped capsules. The curve is a prediction for 1.3 at.\% Ge doping, using the atomic mix model with 3-D saturation.

Convergences are normalized to calculated convergence for smooth, doped capsules. (20-03-0995-2106pb01) the 2-D surface power spectra so that each mode makes the same relative contribution to the total rms roughness as in three dimensions. As 2-D simulations are carried out on a $90^{\circ}$ quadrant, power in odd modes is "aliased" into the adjacent even modes. At peak neutron emission time, the 2-D simulations show classic "bubble and spike" saturated RT growth for the dominant modes at the pusher-fuel interface for initial surface roughnesses greater than $0.1 \mu \mathrm{m}$. The effects of low-mode drive nonuniformities, which distort the pusher-fuel interface, were included in some 2-D multimode calculations. For example, the predicted extra yield degradation due to flux nonuniformities for a smooth, doped capsule is $\sim 30 \%$, as shown by the square in Fig. 9. More appropriate 3-D multimode simulations treating such flux asymmetries in their exact 3-D orientations are described under Recent Modeling Advances, below. In particular, the effects of synergism between growth of low-order surface perturbations and flux asymmetry-seeded perturbations are quantified there.

Figure 10 shows relative capsule convergences inferred from secondary-neutron yields and spectra from doped and undoped capsules vs initial surface roughness. The data points are averaged over capsules of similar roughness, and standard errors are shown. As expected, only the convergence of the high-growth, doped capsule drops significantly as capsule surface roughness is increased to $1 \mu \mathrm{m} \mathrm{rms}$. The solid line is in fair agreement with the data. It represents post-processed predictions of the inferred convergence for 1.3 at. $\% \mathrm{Ge}$ doping based on following the triton trajectory through both the pure fuel and the 1-D atomically mixed layer.

Figures 9 and 10 show that the primary and secondary gas yields are best suited to inferring large mix fractions. This is because the gas yield for nonigniting capsules is dominated by the hottest (central) region, which is farthest from the pusher-fuel interface and least affected by conduction cooling. By contrast, shell conditions can be most sensitive to small amounts of mix as penetrating spikes of shell material enter a region of steeply rising temperature. ${ }^{6,13}$ For the lower-convergence HEP3 targets, moderate shell-gas mix was inferred from ratios of shell to gas dopant $x$-ray line emissivities. ${ }^{6}$ Analogous measurements ${ }^{23,30}$ attempted for $\mathrm{HEP} 4$ are sensitive to the significant $x$-ray reabsorption by the more converged and compressed shells. For example, for the Ar 3-1 lines, the shell optical depth at peak neutron production time is $\sim 5 .^{13}$

\section{Deuterated-Shell Implosions}

One technique for circumventing the problem of high shell x-ray opacity in inferring shell mix is to measure the neutron yields from capsules with deuterated shells. ${ }^{35}$ Figure 11 shows a cross section of the capsule design. The only differences with respect to the usual capsule (Fig. 2) are a 75-atm H fill (to provide 
an inert fill with the same convergence as the 50-atm fill shown in Fig. 2) and a 4- $\mu$ m-thick deuterated polystyrene inner shell. Peak growth factors are a factor of 2 higher, principally because the shell yield (which depends on thermal conduction) is delayed with respect to the fuel yield, allowing more time for perturbation growth. Small amounts of mix should now act to increase the yield by introducing $\mathrm{D}$ into the hotter central gas region. For example, the fusion rate at the $\sim 1-\mathrm{keV}$ temperature of interest and for an ion density $n$ scales as $n^{2} T^{7}$, and hence as $T^{5}$ for a profile with constant pressure $n T$. For larger mix fractions, compression is reduced (see Fig. 10), reducing temperatures throughout the capsule. Therefore, after an initial rise in yield with increasing mix fraction, a drop in shell yield might be expected.

Figure 12 shows the observed dependence of shell primary yield on surface roughness. The implosion conditions were identical to those in the deuteratedfuel implosions except for a $7 \%$ lower drive designed to reduce ultra-hard x-ray contamination of the neutron diagnostics measuring the low yields. The yield remains nearly constant with increasing surface roughness up to $0.5 \mu \mathrm{m}$ and finally falls for rougher surfaces. The standard atomic mix model with 3-D saturation, shown as the middle curve in Fig. 12, predicts only a slight increase in yield with surface roughness, in fair agreement with the data. The explanation for this behavior is that the shell yields are sensitive to an additional ingredient in the model that does not affect gas yields: an enhanced heat diffusivity term scaling as $\alpha L(d L / d t)$ over the 1-D mix layer of width $L$, where $\alpha$ is a heat diffusivity multiplier. ${ }^{13}$ This extra heat flow is used to mimic the heat dilution that occurs as the surface area of a more realistic RT-modulated interface grows. The middle curve in Fig. 12 uses $\alpha=1$, but the result changes little for $\alpha$ between 0.5 and 2. If $\alpha$ is set to 0 , the top curve results, in significant disagreement with the data. The reduction in yield with enhanced heat diffusivity is a consequence of reducing temperature gradients in the mix region, thereby dropping peak temperatures in the innermost shell region, which dominates the yield. For NIF 1-D capsule designs, such a heat flux term is essential in the 1-D atomic mix model to correctly account for heat transfer from the inner hot DT gas to the outer cold DT pusher. The absence of $\mathrm{H}$-like Ti shell emission in earlier experiments is also more consistent with a reduction in shell temperature gradients. By contrast, gas yields are factors of 5 less sensitive than shell yields to the heat diffusivity term; in the atomic mix predictions of gas yields shown in Fig. 9, we used $\alpha=1$.

Figure 12 also shows predictions of $2-\mathrm{D}$ multimode $(l=2,4,6, \ldots, 48)$ and single-mode $(l=24)$ calculations, which are in good agreement with the data and with the atomic mix model, including enhanced heat diffusion.
The simpler single-mode simulations assume that all the roughness is concentrated in a dominant mode. The predicted yields fall rapidly at about $0.4 \mu \mathrm{m}$ rms roughness, for which the increased heat losses to the deuterated layer are insufficient to raise or even maintain the $\mathrm{D}$ temperature because of the reduced compressional

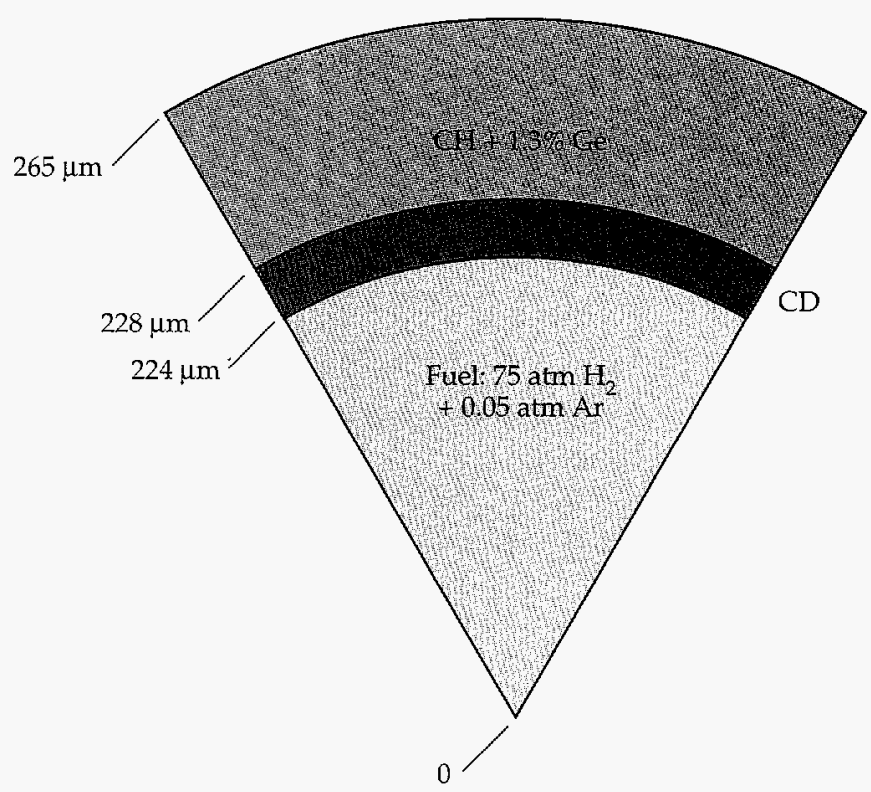

FIGURE 11. Cross section of a typical deuterated-shell capsule design. (20-03-0995-2107pb02)

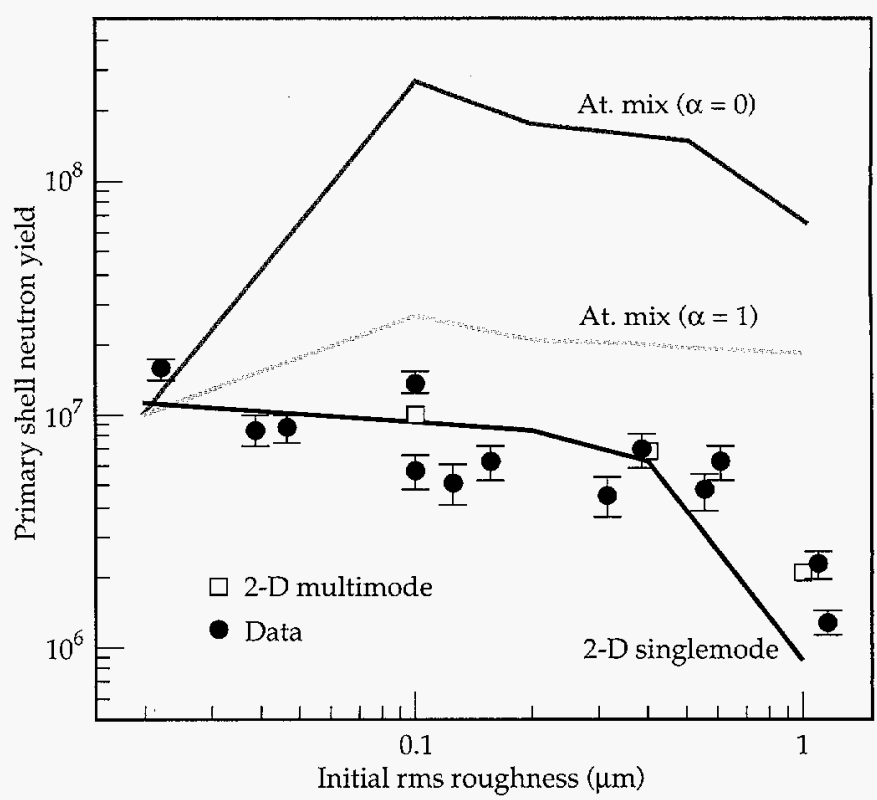

FIGURE 12. Measured and calculated primary yields for 1.3 at. $\%$ Ge-doped deuterated-shell capsules vs initial surface roughness. Solid circles are the data with experimental uncertainties. The top and middle curves are predictions from the atomic mix model without $(\alpha=0)$ and with $(\alpha=1)$ enhanced heat diffusion. The lower curve and squares are predictions from 2-D single-mode $(l=24)$ and multimode $(l=2,4,6, \ldots, 48)$ simulations. $\quad(20-03-0995-2107 \mathrm{pb} 01)$ 
heating of the gas. For example, the peak compressional heating power in the implosion of a capsule with $1 \mu \mathrm{m}$ rms roughness is only $72 \%$ of the unperturbed value. The simulations indicate shell breakup for capsules above $0.4 \mu \mathrm{m}$ rms roughness.

\section{Recent Modeling Advances}

In current HEP4 work, we have advanced to 3-D modeling using the new HYDRA code. ${ }^{33}$ Simulations in progress include modeling of both multimode and single-mode perturbation growth; additional distortion from radiation flux asymmetries can be included. The code uses multigroup radiation diffusion.

Figure 13 shows a snapshot of the ablation front and pusher-fuel interface for a typical single-mode growth simulation $(l=18)$ of a $0.25-\mu \mathrm{m}$ rms perturbation. The interfaces shown represent an isodensity surface at $17.2 \mathrm{~g} / \mathrm{cm}^{3}, 40$ ps before peak neutron production time. The white contour lines show the $90^{\circ} \times 36^{\circ}$ repeating sector used in the simulation. The 92 pits on the outside surface (modeled as hyperGaussians) have fed through to form bubbles of fuel rising into the shell and parabolic ridges of shell material penetrating the fuel. A Kelvin-Helmholz instability at the fuel-pusher interface causes significant roll-up in the bubble tips. The calculated yield was $82 \%$ of the yield for a perfectly smooth capsule surface.

Recently, the measured lowest-order capsule imperfections have been added to the input of a 3-D

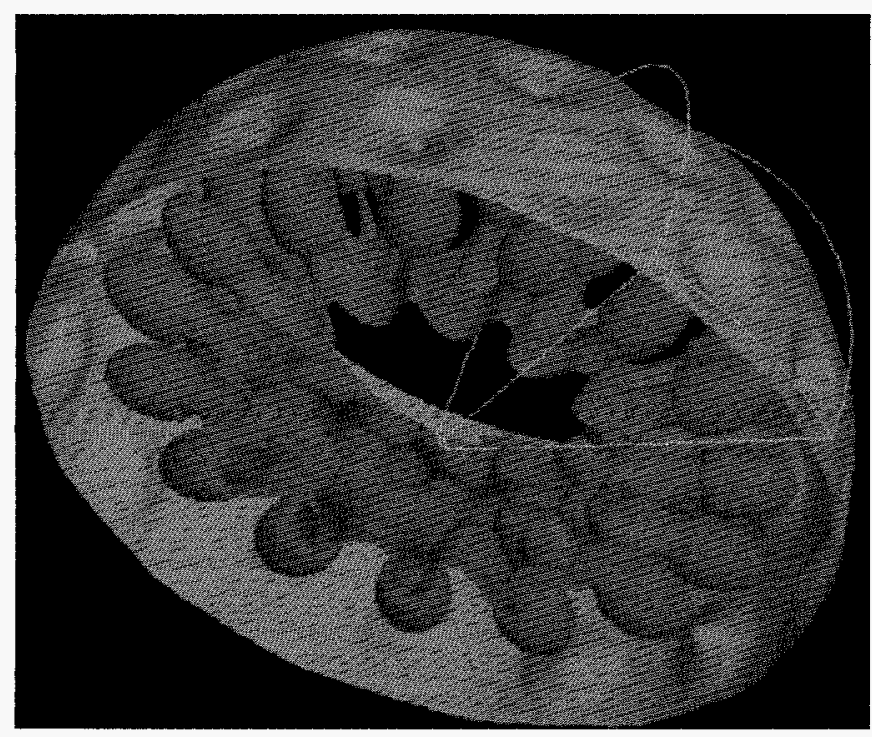

FIGURE 13. Three-dimensional simulation of isodensity $\left(17.2 \mathrm{~g} / \mathrm{cm}^{3}\right)$ surface contour of imploded capsule, 40 ps before peak neutron emission time. Outside surface ( $70 \mu \mathrm{m}$ diam) shows ablation front growth. Initial perturbation was a $0.25-\mu \mathrm{m}$ rms single-mode pattern $(l=18)$ created by 92 pits on 1.3 at.\% Ge-doped capsules. White contour lines define the $90^{\circ} \times 36^{\circ}$ repeating sector used in the simulation (08-00-0695-1495pb01) multimode simulation, which included radiation flux asymmetries. In particular, the importance of a worstcase 5- $\mu \mathrm{m}$ variation in capsule shell thickness from pole to equator has been studied. By aligning the capsule so that the thinnest part of the shell faces the hohlraum midplane, where a combination of $P_{2}$ and $m=5$ radiation flux asymmetries already act coherently, 3-D HYDRA simulations predict a 60\% drop in yield, from $1.44 \times 10^{9}$ to $6 \times 10^{8}$ (see Fig. 9). The yield drops sharply because the thinner sections of the shell converge faster and allow spikes of shell material from RT growth of $m=5$ seeded perturbations to meet at peak neutron emission time. Hence, the scatter in the smooth capsule yields may be due to the arbitrary capsule-to-hohlraum orientation inherent during target assembly and the variable amplitude of lowest-order fluctuations in capsule shell thickness.

\section{Conclusion}

Low-entropy Nova implosions using $x$-ray preheat shielded, doped plastic capsules with reproducible, well-characterized pre-roughened surface finishes have demonstrated large hydrodynamic instability growth similar to that expected in ignition-scale targets. The expected transition to lower entropy and higher instability growth, and hence to ignition-scalable behavior, was experimentally demonstrated by comparing the performance of doped and undoped capsules inferred from x-ray and neutron measurements. To avoid difficulties with high shell $x$-ray opacities, pusher-fuel mix was inferred from neutron yields rather than from the dopant $x$-ray line ratios described in HEP3. The large scatter in the yields of the smoothest capsules may be related to the arbitrary orientation of low-order capsule-shell-thickness nonuniformities with respect to radiation-seeded asymmetries, which could lead to large differences in imploded capsule shape. Average neutron yields for the smoothest capsules remain lower than expected for both undoped and doped capsules, although 3-D calculations which include the low order capsule, wall thickness variations are expected to significantly reduce this discrepancy. Neutron yields from roughened capsules suggest that there is similar or less growth than predicted by models including growth saturation, thereby validating current capsule surface finish requirements for ignition designs. Yields from complementary deuterated-shell experiments agreed with the models and clearly show that enhanced heat diffusion must be included in the traditional atomic mix model. 


\section{Acknowledgments}

We thank B. A. Remington, S. V. Weber, J. D. Kilkenny, and J. D. Lindl for their useful input. The authors are also indebted to the LLNL (and LANL) staff for providing high-quality targets, diagnostic maintenance, and laser shots.

\section{Notes and References}

1. J. H. Nuckolls, L. Wood, A. Thiessen, and G. B. Zimmerman, Nature 239, 139 (1972).

2. J. D. Lindl, R. L. McCrory, and E. M. Campbell, Phys. Today 45, 32 (1992).

3. J. Meyer-ter-Vehn, Nucl. Fusion 22, 561 (1982).

4. S. W. Haan, Phys. Fluids B 3, 2349 (1991); S. W. Haan, Phys. Rev. A 39, 5812 (1989).

5. J. D. Kilkenny, S. G. Glendinning, S. W. Haan, B. A. Hammel, et al., Phys. Plasmas 1, 1379 (1994).

6. T. R. Dittrich, B. A. Hammel, C. J. Keane, R. McEachern, et al., Phys. Rev. Lett. 73, 2324 (1994).

7. J. D. Lindl and W. C. Mead, Phys. Rev. Lett. 34, 1273 (1975); S. E. Bodner, Phys. Rev. Lett. 33, (1974); H. Sakagami and K. Nishihara, Phys. Fluids B 2, 2715 (1990).

8. R. D. Richtmyer, Commun. Pure Appl. Math. 13, 297 (1960); E. E. Meshkov, Izv. Akad. Nauk. SSSR, Mekh. Zhidk. Gaz. 5, 151 (1969) (NASA TTF-13-074, 1970).

9. Lord Rayleigh, Scientific Papers (Cambridge Univ. Press, Cambridge, 1900), Vol. II, p. 200.

10. J. D. Lindl, LLNL Laser Program Annual Report, 2-40 to 2-46, Lawrence Livermore National Laboratory, Livermore, CA, UCRL-56055-83 (SRD, 1983); H. Takabe, K. Mima, L. Montierth, and R. L. Morse, Phys. Fluids 28 (1985); D. H. Munro, Phys. Rev. A 38, 1433 91988); M. Tabak, D. H. Munro, and J. D. Lindl, Phys. Fluids B 2, 1007 (1990).

11. W. K. Levedahl and B. A. Hammel, ICF Program Annual Report 1988/89 (U) , 86, Lawrence Livermore National Laboratory, Livermore, CA, UCRL-LR-116901-88/89 (report SRD).

12. J. H. Gardner and S. E. Bodner, Phys. Rev. Lett. 47, 1137 (1981); B. Yaakobi, J. Delettrez, L. M. Goldman, R. L. McCrory, et al., Opt. Commun. 41, 355 (1982);

H. Nishimura, T. Kiso, H. Shiraga, T. Endo, et al., Phys. Plasmas 2, 1 (1995).

13. C. J. Keane, R. C. Cook, T. R. Dittrich, B. A. Hammel, et al., Rev Sci. Instrum. 66, 689 (1995); C. J. Keane, G. W. Pollak, R. C. Cook, T. R. Dittrich, et al., J. Quant. Spectrosc. Radiat. Transfer 54, 207 (1995).

14. R. Pakula and R. Sigel, Phys. Fluids 28, 232 (1985); M. Murakami and J. Meyer-ter-Vehn, Nucl. Fusion 31, 1315 (1991);

R. L Kauffman, L. J. Suter, C. B. Darrow, J. D. Kilkenny, et al., Phys. Rev. Lett. 73, 2320 (1994).

15. S. V. Weber, B. A. Remington, S. W. Haan, B. G. Wilson, and J. K. Nash, Phys. Plasmas 1, 3652 (1994).
16. B. A. Remington, S. W. Haan, S. G. Glendinning, J. D. Kilkenny, et al., Phys. Rev. Lett. 67, 3259 (1991); B. A. Remington, S. V. Weber, S. W. Haan, I. D. Kilkenny, et al., Phys. Fluids B 5, 2589 (1993).

17. S. W. Haan et al., Phys. Plasmas 2, 2480 (1995).

18. R. Cook, G. E. Overturf, S. R. Buckley, and R. McEachern, J. Vac. Sci. Technol. A 12, 1275 (1994).

19. R. J. Wallace, R. L. McEachern, and W. W. Wilcox, ICF Quarterly Report 4(3), 79, Lawrence Livermore National Laboratory, Livermore, CA, UCRL-LR-105821-94-3 (1994).

20. R. L. McEachern, C. Moore, G. E. Overturf, III, S. R. Buckley, and R. Cook, ICF Quarterly Report 4(1), 25 Lawrence Livermore National Laboratory, Livermore, CA, UCRL-LR-105821-94-1 (1994).

21. S. M. Pollaine, S. P. Hatchett, and S. H. Langer, ICF Quarterly Report 4(3), 87, Lawrence Livermore National Laboratory, Livermore, CA, UCRL-LR-105820-94-3 (1994).

22. C. W. Laumann, J. A. Caird, J. E. Murray, R. E. McEachern, et al., ICF Quarterly Report 4(1), 1-30, Lawrence Livermore National Laboratory, Livermore, CA, UCRL-LR-105820-94-1 (1994).

23. E. M. Campbell, Laser Part. Beams 9, 209 (1991); B. A. Hammel, C. J. Keane, T. R. Dittrich, D. R. Kania, et al., J. Quant. Spectrosc. Radiat. Transfer 51, 113 (1994).

24. H. N. Kornblum, R. L. Kauffman, and J. A. Smith, Reo. Sci. Instrum. 57, 2179 (1986).

25. M. D. Cable, S. P. Hatchett, J. A. Caird, J. D. Kilkenny, et al., Phys. Rev. Lett. 73, 2316 (1994).

26. R. L. Kauffman, H. N. Kornblum, D. W. Phillion, C. B. Darrow, et al., Rev. Sci. Instrum. 66, 678 (1995).

27. M. D. Cable and S. P. Hatchett, J. Appl. Phys. 62, 2223 (1987).

28. R. A. Lerche, D. W. Phillion, and G. L. Tietbohl, Rev. Sci. Instrum. 66, 933 (1995).

29. J. D. Kilkenny, Laser and Part. Beams 9, 49 (1991).

30. O. L. Landen, C. J. Keane, B. A. Hammel, M. D. Cable, et al., J. Quant. Spectrosc. Radiat. Transfer 54, 245 (1995).

31. C. J. Keane, B. A. Hammel, D. R. Kania, J. D. Kilkenny, et al., Phys. Fluids B 5, 3328 (1993); C. J. Keane, B. A. Hammel, A. L. Osterheld, and D. R. Kania, Phys. Rev. Lett. 72, 3029 (1994).

32. G. B. Zimmerman and W. L. Kruer, Comm. Plasma Phys. Controlled Fusion 11, 51 (1975).

33. M. M. Marinak, B. A. Remington, S. V. Weber, R. E. Tipton, et al., "Three-Dimensional Single Mode Rayleigh-Taylor Experiments on Nova," Lawrence Livermore National Laboratory, Livermore, CA, UCRL-JC-120191 (1995); submitted to Phys. Rev. Lett.; M. M. Marinak, R. E. Tipton, B. A. Remington, S. W. Haan, and S. V. Weber, ICF Quarterly Report 5(3), p. 168, Lawrence Livermore National Laboratory, Livermore, CA, UCRL-LR105821-95-3 (1995).

34. C. J. Keane, O. L. Landen, B. A. Hammel, P. Amendt, et al., "Observation of Large-Growth Factor Behavior in Indirectly Driven Spherical Implosions," Lawrence Livermore National Laboratory, Livermore, CA, UCRL-JC-123022 ABS (1995).

35. R. E. Chrien, N. M. Hoffman, and J. D. Colvin, "Fusion Neutrons from the Gas-Pusher Interface in Deuterated-Shell ICF Implosions," Los Alamos National Laboratory, Los Alamos, NM, LA-UR-95-2307 (1995). 


\section{ENERGY COUPLING IN LINED HOHLRAUMS (HLP1, HLP2, AND HLP7)}
R. L. Kauffman
C. W. Hatcher
D. W. Phillion
R. J. Wallace
L.J. Suter
H. N. Kornblum
J. L. Porter ${ }^{*}$
P. E. Young
R. L. Berger
E. F. Lindsey
M.D. Rosen
F. $\mathrm{Ze}$
C. B. Darrow
D. S. Montgomery
R. Thiessen
S. N. Dixit
T. E. Orzechowski
R. E. Turner
J. C. Fernandez
J. A. Cobble

\section{Introduction}

Indirect-drive inertial confinement fusion (ICF) uses high- $Z$ cavities, or hohlraums, to confine $x$ rays for compressing and igniting deuterium-tritium fuel contained in spherical capsules. ${ }^{1,2}$ For laser-driven ICF, the intense laser beams enter the hohlraum through small laser entrance holes (LEHs), heating the high- $Z$ hohlraum walls. The laser-produced radiation heats the unirradiated high- $Z$ walls producing a nearly isotropic radiation environment for spherically compressing the ICF capsule. ${ }^{2}$ The radiation flux on the capsule is not completely isotropic, however, because the laser-irradiated area is generally brighter than the surrounding $x$-ray heated walls and the LEHs do not radiate. Furthermore, the angular distribution of flux on the capsule is time dependent because the unilluminated walls become hotter and more emissive as a function of time, and plasma dynamics cause the laserirradiated area to move. Symmetric implosions are obtained by dynamically balancing the effects of the LEHs, wall heating, and laser-spot motion. ${ }^{3}$

The laser absorption and thus radiation symmetry depend on the dynamics of the hohlraum plasma. For long pulses, such as those required for ignition targets on the proposed National Ignition Facility (NIF), a large volume of the hohlraum can fill to plasma electron densities of $n_{\mathrm{e}} \approx 10^{21} \mathrm{~cm}^{-3}$ or greater, which can cause significant movement of the absorption region. In NIF hohlraum designs, a low- $Z$ plasma replaces the low-density, high- $Z$ blowoff to better control the laserspot motion.

Inverse bremsstrahlung is lower in the low- $Z$ plasma compared with a high- $Z$ plasma at the same density, allowing the laser to deposit its energy in higher-density, high- $Z$ plasma nearer the initial wall position. ${ }^{2,4}$ Calculations indicate that the low- $Z$ plasma reduces the movement of the laser deposition region, making symmetry more easily obtainable. For initial NIF target designs, the low- $Z$ underdense plasma was produced by initially coating the high $Z$ wall with low $-Z$ material. The laser and radiation ablate the thin low- $Z$ coating from the wall, filling the hohlraum with low- $Z$ plasma. For more recent designs, the hohlraum is initially filled with low- $Z$ gas which, when ionized, produces the underdense low- $Z$ plasma. Gas-filled hohlraums avoid problems with plasma stagnation and jetting, which computationally degrade capsule symmetry in the lined-hohlraum designs.

NIF designs using low-Z plasma to control spot motion had not previously been tested. Many of the Hohlraum and Laser Physics (HLP) tasks for the Nova Technical Contract (NTC) were developed to experimentally test the physics for low- $Z$ lined hohlraums. Specifically, HLP1 and HLP2 are tasks to test the energetics of lined hohlraums and to understand the scaling to NIF hohlraums within the energy and power constraints of Nova. HLP1 experiments investigate drive using shaped pulses where the underdense plasma evolution better approximates the NIF plasma but at lower powers than attainable on the NIF. The goal of HLP1 is to demonstrate acceptable laser coupling in hohlraums with a shaped drive pulse producing radiation temperatures $T_{R}$ in the range of $\sim 100$ to $\sim 210 \mathrm{eV}$. HLP2 experiments investigate drive in lined hohlraums at high powers to understand radiation drive scaling to NIF peak drive regimes. HLP2 goals are to demonstrate acceptable coupling in hohlraums with $T_{R}$ of $\geq 270 \mathrm{eV}$. For both tasks, acceptable coupling is defined as absorption fraction $f_{\text {abs }}>90 \%$; stimulated Brillouin scattering (SBS) fraction $f_{\text {SBS }}<5-10 \%$; hot electron fraction $f_{\text {hot }}<5 \%$ at a hot electron temperature, $T_{\text {hot }} \geq 50 \mathrm{keV}$, and stimulated Raman scattering (SRS) fraction $f_{\text {SRS }}<5 \%$.

\footnotetext{
${ }^{*}$ Sandia National Laboratories, Albuquerque, New Mexico

** Los Alamos National Laboratory, Los Alamos, New Mexico
} 
Results from both HLP1 and HLP2 experiments successfully attain the drive goals defined in the NTC, although the liner does reduce the peak drive compared with experiments using unlined hohlraums. Calculations partly predict this reduction in drive as being due to enhanced hydrodynamic losses to the underdense plasma. Although the diagnostics were not optimal, measurements of SBS using subaperture sampling and SRS using $x$-ray bremsstrahlung from fast-electron production suggest that enhanced plasma instability production in the lined hohlraums also contributes to the observed reduced drive. The drive experiments performed for HLP1 and HLP2 use unsmoothed laser beams. Recent experiments demonstrate that stimulated scattering levels are reduced to about the $1 \%$ level using beam-smoothing techniques. These experiments are described in "Laser-Plasma Interactions in NIF-Scale Plasmas (HLP5 and HLP6)" on p. 305 of this Quarterly. For the same laser intensity and pulse length, $x$-ray conversion efficiency inferred in hohlraums is significantly higher than that obtained from isolated flat foils. The HLP task is focused on experiments to understand this difference.

\section{Experiments}

Experiments on Nova use hohlraums whose shape is a right circular cylinder, shown schematically in Fig. 1(a). Nova scale-1 hohlraums are Au and are $1.6 \mathrm{~mm}$ diam $\times 2.55-2.75 \mathrm{~mm}$ long. Typically, the hohlraum wall thickness is $25 \mu \mathrm{m}$, and in some experiments is thinned to $\sim 2 \mu \mathrm{m}$ to image kilo-electron-volt $x$ rays through the wall. Different hohlraum lengths are sometimes used for symmetry or for satisfying other experimental constraints. Different hohlraum sizes are scaled from the scale- 1 size by the ratio of their dimensions to those of a scale- 1 size. For example, a 0.75 -scale hohlraum is $1.2 \mathrm{~mm}$ diam $\times 2 \mathrm{~mm}$ long. The LEH is varied, depending on the experiment, from 50 to $100 \%$ of the hohlraum diameter. For the energetics scaling, the results are scaled to $50 \% \mathrm{LEH}$ for the square pulses and $75 \%$ for the shaped pulses, unless otherwise noted. For all of the energetics studies, the hohlraums are empty, not containing a fuel capsule.

The lined hohlraums are coated with either $\mathrm{Ni}$ or $\mathrm{CH}$ in the form of parylene. The parylene coating is done using vacuum deposition and by allowing the parylene to enter through the LEHs and randomly collide with the wall until it sticks. Since parylene has a low probability of sticking when colliding with a solid surface, it statistically has many collisions with the wall before it sticks. This method can produce uniform surfaces even on the inside hohlraum wall. Typically, the coating thickness is $0.75 \mu \mathrm{m}$ with a density $\rho \approx 1.10 \mathrm{~g} / \mathrm{cm}^{3}$ unless otherwise stated. When fully ionized, this layer would fill the initial volume to a density of $\sim 10^{21}$ electrons $/ \mathrm{cm}^{3}$. The coating thickness is characterized by measuring the Au x-ray fluorescence from a scanning electron microscope (SEM). The SEM signal is calibrated using a series of flat $\mathrm{Au}$ targets overcoated with various thicknesses of parylene. The coating thicknesses on the flat targets are characterized using optical interferometry and a stylus profilometer. In this way, coating thickness for the hohlraums can be measured to an accuracy of $\pm 20 \%$. Uniformity along the inside hohlraum wall is checked using the SEM by cutting open test hohlraums. Also, coating thicknesses on a target used for an experiment could be checked by measuring the thickness on the outside wall.

$\mathrm{Ni}$-coated hohlraums are made using electrodeless Ni plating. The coating is $88 \% \mathrm{Ni}$ by weight with the remaining $12 \%$ by weight being $P$. Its average density is $8.2 \mathrm{~g} / \mathrm{cm}^{3}$, with the nominal coating thickness of $0.15 \mu \mathrm{m}$. Thicknesses on hohlraums are also characterized
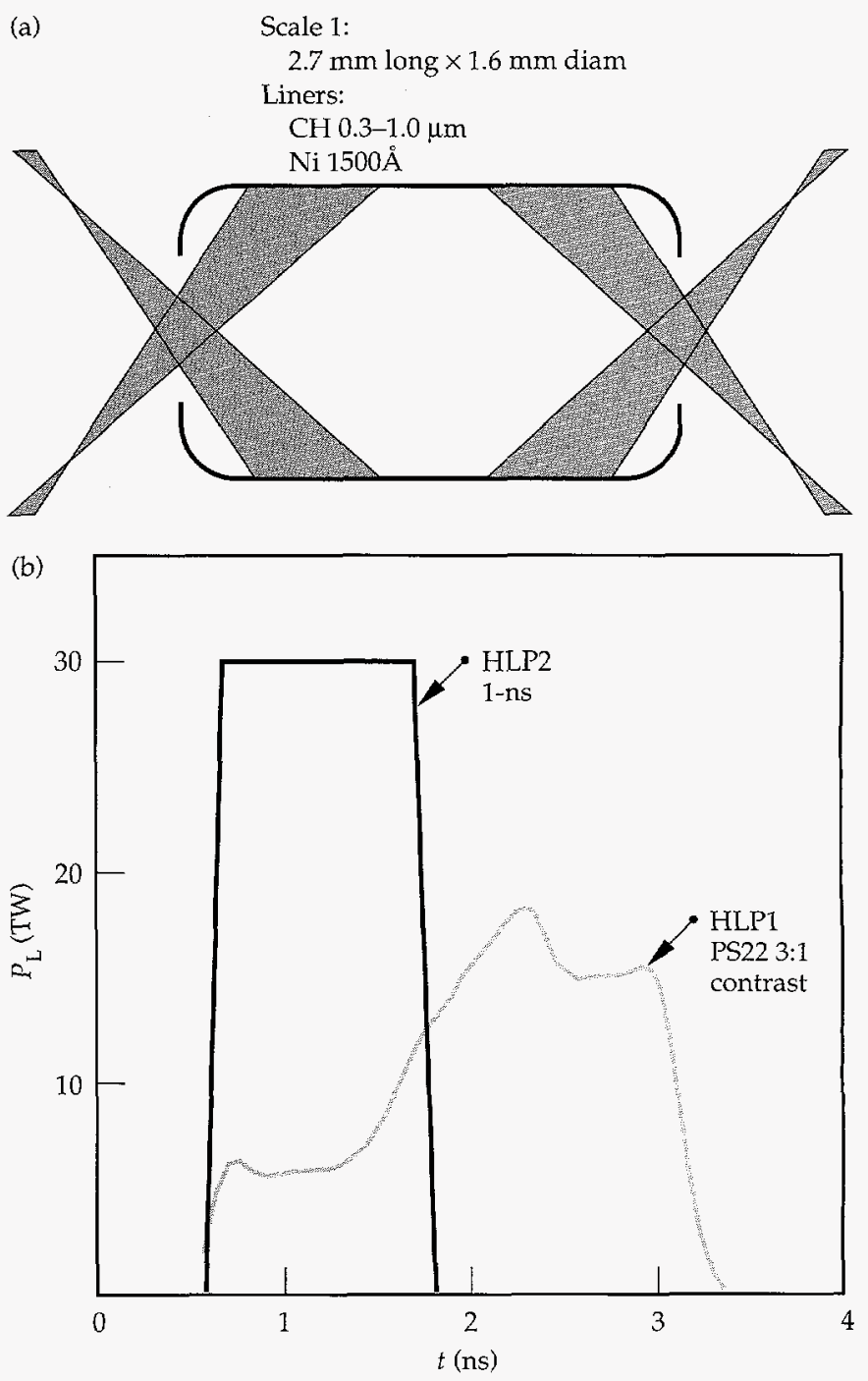

FIGURE 1. Schematic of hohlraum drive experiments. (a) The hohlraum and irradiation geometry. (b) Representative pulse shapes of the incident laser pulses at $0.35 \mu \mathrm{m}$ wavelength. PS22 is a 3:1 contrast shaped pulse for HLP1 and HLP2, using a 1-ns square pulse with a maximum power of $30 \mathrm{TW}$. (20-05-0995-2111pb01) 
using the SEM, measuring the relative $x$-ray fluorescence signal of Ni compared with Au. Again, the system is calibrated using coated Au flats. Errors in thickness are estimated to be $\pm 20 \%$.

Hohlraums are irradiated using the 10 Nova laser beams, 5 per side. Figure 1(b) shows the laser pulses. The pulse shape for HLP1, designated PS22, is a 2.2-nslong pulse with a $3: 1$ contrast between the peak intensity and the foot intensity. This is the same pulse shape used for the bulk of the pulse-shaped symmetry experiments described in the next article, "Nova Symmetry: Experiments, Modeling, and Interpretation (HLP3 and HLP4)," on p. 293. Pulse shapes for HLP2 are 1-ns-long approximately square pulses with total powers up to 30 TW (maximum power available on Nova). The beams are pointed through the center of the LEH and defocused to reduce the intensity of the laser on the wall, shown schematically in Fig. 1(a). For a scale-1 target, the defocusing is $\sim 1 \mathrm{~mm}$, in the diverging direction from best focus, at the LEH. This allows $\sim 100 \mu \mathrm{m}$ clearance of the beam for a $50 \% \mathrm{LEH}$, assuming geometrical optics for an $\mathrm{f} / 4$ beam. The beam irradiates the wall of the hohlraum at an angle of $40^{\circ}$ with respect to the normal of the wall and has a first bounce intensity of $\sim 8 \times 10^{14} \mathrm{~W} / \mathrm{cm}^{2}$ for 2 TW of laser power $P_{\mathrm{L}}$ per beam.

\section{Diagnostics}

$X$-ray drive is measured using two complementary techniques. ${ }^{5}$ One technique measures the shock wave generated by the absorbed $\mathrm{x}$-ray flux in an $\mathrm{Al}$ witness plate. The Al witness plate is a piece of $\mathrm{Al}$ whose thickness continuously varies from one end to the other, or has discrete steps of known thickness, placed over a hole in the hohlraum wall. The shock front is measured by observing the optical emission produced by the emerging shock at the rear of the $\mathrm{Al}$ plate. The optical emission is measured using an ultraviolet (UV) Cassegrain telescope coupled to an optical streak camera. X-ray drive is derived by comparing the measured shock velocity with hydrodynamic calculations or semi-empirical models as described in the article "Planar and Cylindrical Rayleigh-Taylor Experiments on Nova (HEP2)," p. 232. The estimated error for measuring drive is $\pm 5 \mathrm{eV}$, which includes the accuracy of the measurement and the uncertainty in the comparison with the calculations. The other technique measures the $x$-ray flux irradiated from the hohlraum wall using an array of $\mathrm{X}$-ray diodes (XRD). ${ }^{6} \mathrm{~A}$ number of broadband channels are defined in the range from 0.1 to $1.8 \mathrm{keV}$ using thin absorption filters and, for some channels, grazing incident $\mathrm{x}$-ray mirrors. The XRD array measures the hohlraum drive by measuring the flux from an opposite wall in the hohlraum through a hole in the hohlraum wall. The hole is lined with Be to prevent high-Z plasma from occluding the line of sight into the hohlraum. Time-resolved spectra are unfolded from the signals using calibrated channel response. The time resolution is on the order of 150 ps limited by the bandwidth of the detectors and oscilloscopes and the correlation of the timing among the detectors. The spectrally integrated flux is measured to an accuracy of $-20 \%$ including calibration accuracies and unfolding uncertainties resulting in a $\pm 5 \%$ uncertainty when converted to an equivalent radiation drive temperature.

The two measurement techniques are complementary since the shock velocity measures the flux incident on the wall while the XRD array measures the reradiated flux. The two are related by the wall albedo, ${ }^{2}$ or effective reflectivity. The shock velocity is best suited for measuring peak drive and can provide only coarse time-dependent measurements. The XRD array provides much better time-dependent reradiated flux measurements, but the data must be corrected for albedo, which is time dependent, to obtain incident $x$-ray flux. For both techniques, the measurement is usually made in the midplane of the hohlraum between the two sets of beam cones where it is not directly irradiated by the laser. The flux on the ICF capsule, or other areas of the hohlraum, must be derived using detailed radiation hydrodynamic calculations or semi-empirical view factor estimates.

Laser coupling is also studied by measuring the scattered light and $x$-ray bremsstrahlung produced by superthermal electrons. SBS light into the lens is measured using a subaperture sampling on one of Nova's ten beamlines, beamline six (BL6). ${ }^{7}$ The light from approximately $5 \%$ of the beam area is extracted from the reflected beam using an uncoated piece of fused silica. It is then optically relayed to a diagnostic table equipped with an optical calorimeter, a fast-photodiode coupled to a Tektronix 7912 transient digitizer, and a time-resolved optical spectrometer. The calorimeter and photodiode are absolutely calibrated by placing a partially reflecting retroreflector in front of the calorimeter mounted opposite BL6. The retroreflector reflects $7.6 \%$ of the incident energy, and the data is reduced assuming the incident light and backscattered light uniformly fill the lens aperture. More recent experiments with improved diagnostics developed for HLP5 [as discussed in "Laser-Plasma Interactions in NIF-Scale Plasmas (HLP5 and HLP6)" on p. 305] have shown that there can be significant variations in the near-field distribution of the backscattered light in the lens aperture as well as some light being scattered outside of the lens. ${ }^{8}$ The scattered light data presented here have large uncertainties due to the subaperture sampling, but the relative trends in the data among different target types should be qualitatively valid.

Fast-electron production from SRS is studied by measuring the $x$-ray bremsstrahlung from the target in the $20-200-\mathrm{keV}$ spectral range. ${ }^{9}$ The $x$ rays are produced when the fast electrons deposit their energy in the high- $Z$ case. For most of the measurements, 
hohlraums with thin walls $(\sim 2-3 \mu \mathrm{m})$ are used to minimize x-ray absorption in the hohlraum walls. This measurement does not include fast electrons that escape the target or that lose their energy in the plasma before reaching the wall. In addition, time-resolved SRS light spectra are measured $28^{\circ}$ from an incident beamline. Also, a photodiode array measures the angular distribution of the SRS light. SRS light was not measured inside of the lens cone. Recent experiments have shown that a significant amount of SRS light can be scattered into the lens cone. ${ }^{8}$

\section{Results}

Lined hohlraums reduce the amount of high- $Z$ material filling the interior of the hohlraum. The effect of liners on plasma filling can be seen qualitatively in Fig. 2, which shows time-resolved images of kilovolt $x$ rays viewing along the axis of Nova hohlraums. ${ }^{10}$ Figure 2(a) is an image of an unlined hohlraum, and Fig. 2(b) shows a hohlraum lined with $0.75 \mu \mathrm{m}$ of $\mathrm{CH}$. Both images are taken at around $1.5 \mathrm{~ns}$ after the beginning of PS22, which is near the peak of the laser intensity. Both images show five bright spots placed nearly equally inside of the hohlraum. These spots are Au blowoff from the laser beams irradiating the hohlraum walls. The hohlraum wall itself is masked by the LEH, which defines the viewing area. For the unlined hohlraum, the figure also shows five spokes and a central bright spot. These are produced by stagnation of the high- $Z$ plasma from the wall blowoff, indicating that by this time the high- $Z$ plasma has filled the laser irradiation part of the hohlraum. For the lined hohlraum, the bright spokes and central region are replaced by an absence of emission. This region is filled by low- $Z \mathrm{CH}$ plasma, which is a much poorer radiator than high- $Z$ plasma. These images indicate that qualitatively, at least, low- $Z$ plasma does reduce the filling of the hohlraum by high- $Z$ plasma.

Drive at high power has been investigated using 1-ns square pulses for HLP2. Figure 3 shows peak $T_{R}$ measured using shock breakout for both $\mathrm{CH}$ - and $\mathrm{Ni}$-lined hohlraums. Data are included for both scale- 1 and scale- 0.75 hohlraums. Most of the data are taken with 2.55-mm-long hohlraums. Some of the data are from longer hohlraums and have been corrected $(<5 \mathrm{eV})$ to account for this. The data is plotted as a function of $P_{\mathrm{L}}$ / wall area where the wall area is the total area of the hohlraum not corrected for LEH or diagnostic holes. Drive from unlined hohlraums, reported previously, is also plotted for comparison. ${ }^{11}$ Drive from lined hohlraums is below the measured drive for unlined hohlraums. Table 1 summarizes the average reduction in drive for lined hohlraums compared with unlined hohlraums. The uncertainties listed in Table 1 are the standard deviations of the data set. For scale- 1 targets, the reduction is $16-18 \mathrm{eV}$, representing $\sim 25-30 \%$ decrease in available $x$-ray drive for both $\mathrm{CH}$ - and Ni-lined hohlraums.

Figure 4 shows drive from lined hohlraums heated with shaped pulses. Figure 4(a) shows the time-resolved drive derived from the XRD array for both $\mathrm{CH}$ - and $\mathrm{Ni}$ lined hohlraums compared with drive measured from

\section{(a) Unlined hohlraum}

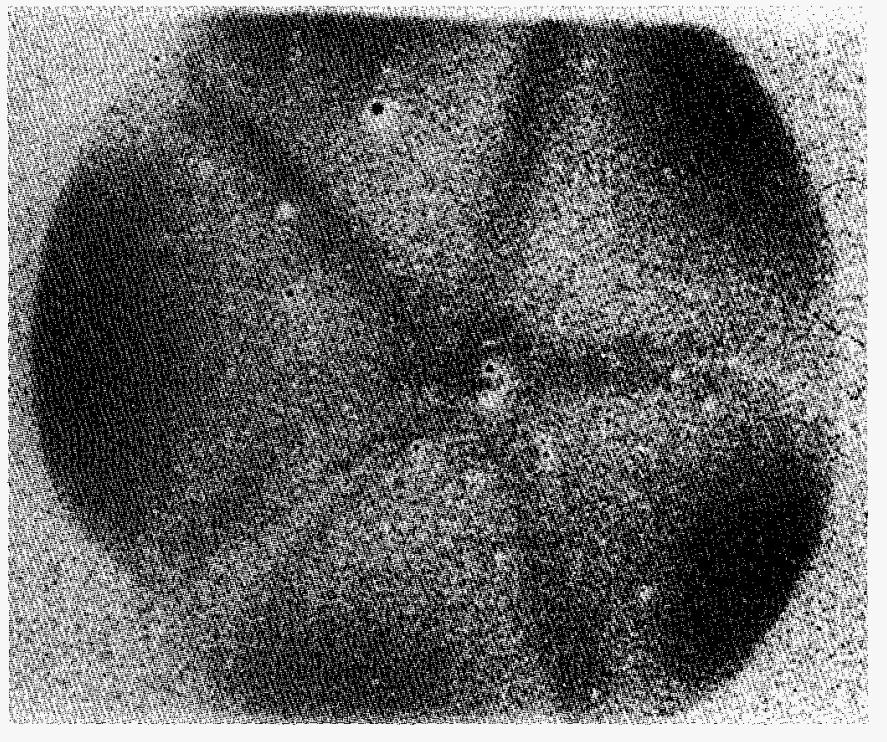

(b) Lined hohlraum

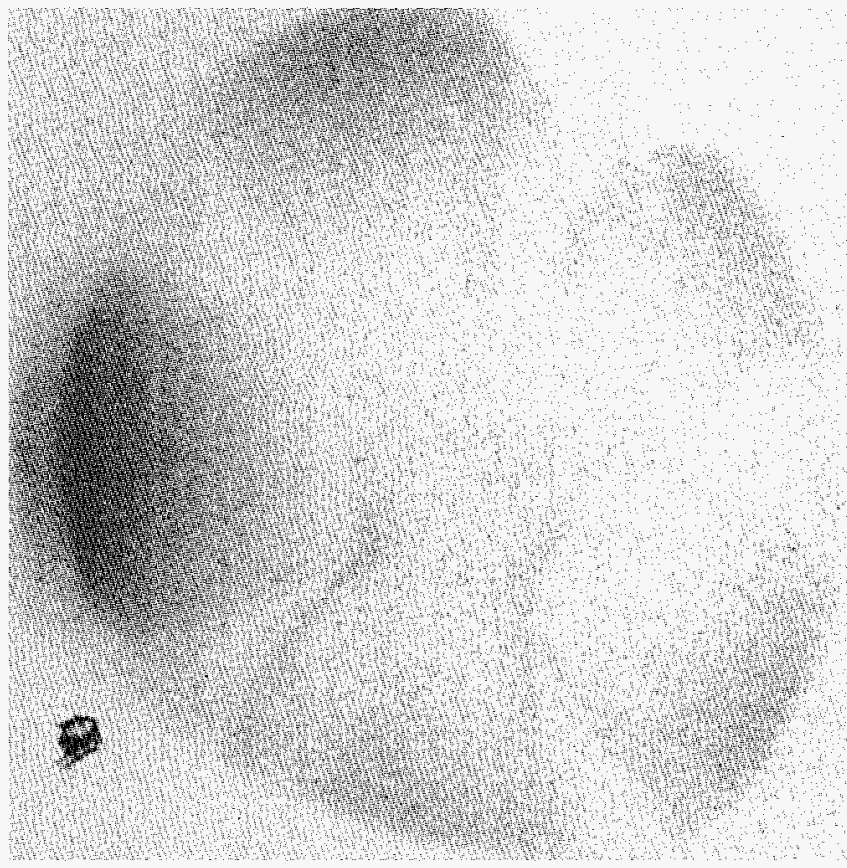

FIGURE 2. X-ray images of the hohlraum heated with PS22 viewing along the axis of the cylinder. The time-resolved images are taken at approximately $1.5 \mathrm{~ns}$, which is about the peak of the laser pulse. The image in (a) is from an unlined hohlraum and (b) is from a hohlraum lined with $0.75 \mu \mathrm{m} \mathrm{CH} . \quad(20-05-0995-2112 \mathrm{pb01})$ 


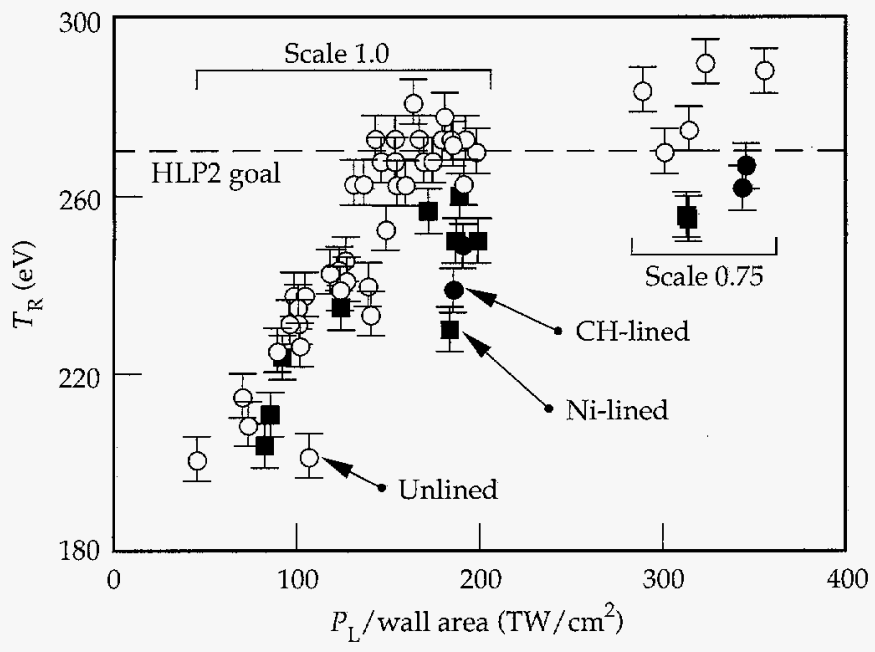

FIGURE 3. Peak radiation temperature $T_{\mathrm{R}}$ measured in scale- 1 and scale-0.75 hohlraums heated with 1-ns square pulses of $0.35-\mu \mathrm{m}$ light. Measurements are made using the shock breakout technique in Al witness plates. Open circles are from unlined Au hohlraums and closed circles and closed squares are from $\mathrm{CH}$ - and Ni-lined hohlraums, respectively. The HLP2 goal of $270 \mathrm{eV}$ is shown as a dashed line: (20-05-1093-3696pb01)

TABLE 1. Reduction in drive of lined hohlraums compared with unlined hohlraums.

\begin{tabular}{|c|c|c|}
\hline Hohlraums & $\begin{array}{c}\Delta T_{R}(e V) \\
\text { experiment }\end{array}$ & $\begin{array}{l}\Delta T_{\mathrm{R}}(\mathrm{eV}) \\
\text { LASNEX }\end{array}$ \\
\hline \multicolumn{3}{|l|}{ Scale 0.75} \\
\hline I ns, $1500 \AA ̊ \mathrm{Ni}, 25 \mathrm{TW}$ & $26 \pm 9$ & - \\
\hline $1 \mathrm{~ns}, 7500 \AA \mathrm{CH}, 25 \mathrm{TW}$ & $17 \pm 9$ & - \\
\hline \multicolumn{3}{|l|}{ Scale 1.0} \\
\hline $1 \mathrm{~ns}, 1500 \AA \mathrm{Ni}, 12 \mathrm{TW}$ & $16 \pm 3$ & 14 \\
\hline $1 \mathrm{~ns}, 1500 \AA \mathrm{Ni}, 25 \mathrm{TW}$ & $18 \pm 6$ & 14 \\
\hline $\mathrm{PS} 22,1500 \AA \mathrm{Ni}$ & $18 \pm 5$ & 14 \\
\hline $\mathrm{PS} 22,4000 \AA \mathrm{CH}$ & $6 \pm 3$ & 9 \\
\hline $\mathrm{PS} 22,7500 \AA \mathrm{CH}$ & $11 \pm 9$ & 12 \\
\hline
\end{tabular}

unlined hohlraums. The main differences in the drive between the lined and unlined hohlraums are that the beginning of the drive is delayed in the initial foot of the pulse and that the peak drive is less for lined hohlraums. Figure 4(b) plots the peak drive as measured from the shock breakout, which also confirms the reduction in peak drive for lined hohlraums. The difference in peak drives for Figs. 4(a) and (b) is the correction for wall albedo. The delay in the beginning of the drive can be understood qualitatively as the reduction in $x$-ray flux initially as the laser burns through the liner before heating the Au walls. As summarized in Table 1, the reduction in peak drive for lined hohlraums is $\sim 20-30 \%$ of the $x$-ray flux for unlined hohlraums. Drive data from unlined hohlraums have been empirically fit with a simple power balance model. ${ }^{11}$ This simple model balances the heating sources with the heat losses. The heating source is the $x$-ray energy produced by the incident laser drive heating the hohlraum walls. The heat losses are the energy absorbed by the hohlraum walls and energy radiated through the laser entrance and diagnostic holes. ${ }^{12,13}$ The hohlraum wall loss is modeled using a Marshak scaling ${ }^{14}$ for wall loss. The power balance equation is

$\eta_{\mathrm{HOHL}} P_{\mathrm{L}}=\sigma T_{\mathrm{R}}{ }^{4} A\left[1-\alpha\left(1-f_{\mathrm{H}}\right)\right]$.

The source term on the left is the incident $P_{\mathrm{L}}$ multiplied by the effective efficiency for converting laser light to drive energy, $\eta_{\mathrm{HOHL}}$. The loss terms are on the right. $\sigma$ is the Stefan-Boltzman constant, $A$ is the hohlraum area, $f_{\mathrm{H}}$ is the fraction of the hohlraum area that is holes, and $\alpha$ is the wall albedo. All of the parameters in the equation can be determined independently by the experimental geometry and the measurement except for $\eta_{\mathrm{HOHL}}$ and $\alpha . \alpha$ is both time and temperature dependent. It can be calculated from LASNEX or simple

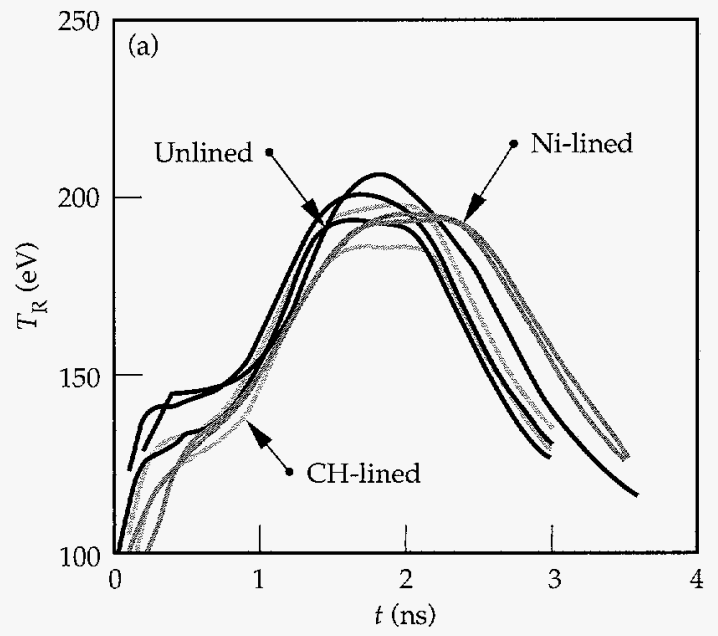

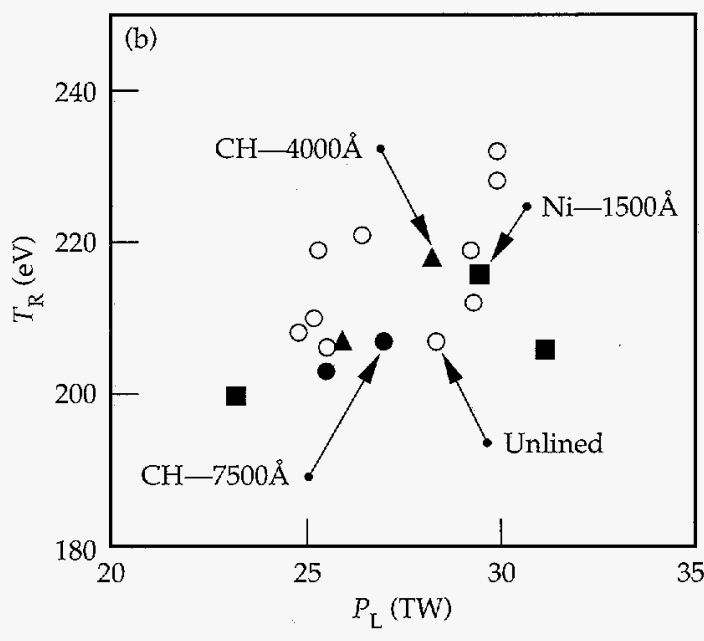

FIGURE 4. Radiation drive measured from scale- 1 hohlraums heated with $0.35-\mu \mathrm{m}$ light using PS22. (a) Compares timeresolved Dante measurements of the drive from unlined and $\mathrm{CH}$ and Ni-lined hohlraums. (b) Shows peak drive measured using the shock breakout technique in $\mathrm{Al}$ witness plates from the different targets. (20-05-1093-3696pb01) 
models of radiation wave scaling and depends on the opacity and equation of state for the material.

Figure 5 shows the data fit with Eq. (1) from the scale- 1 hohlraums heated with square pulses. The fits use power-law scaling of $\alpha$ derived from self-similar solutions to Marshak's wave heating of materials with power-law dependencies for opacity and equation of state. ${ }^{12,13} \eta_{\mathrm{HOHL}}$ is treated as a fitting parameter. Results of the fits are shown Fig. 5. For unlined hohlraums, $\eta_{\mathrm{HOHL}} \approx \sim 0.75$ while $\eta_{\mathrm{HOHL}} \approx 0.53$ to 0.64 for the lined hohlraum data. This simple model suggests that the effective coupling is reduced by $15-30 \%$. The data set is much sparser for the scale- 0.75 data, but it is consistent with similar reduction in effective coupling. The pulse-shaped data cannot be fit easily with an analytic model because of the dependence of $\alpha$ on time and temperature, but the decrease in drive is approximately similar to the 1-ns square results, and therefore the reduction in coupling is expected to be similar.

The reduced coupling for lined hohlraums can be due to several effects. Some reduction is expected, as discussed in the next section, because of the energy expended to heating the liner and to differences in coupling to hydrodynamic motion. Other potential differences are decreased absorption due to higher levels of reflected light, SBS, and SRS.

The absorption is studied by measuring both the scattered SBS and SRS light and fast electrons produced by SRS. Table 2 shows the fraction of SBS light scattered into the lens for the various targets for both $\mathrm{CH}$ - and $\mathrm{Ni}$-lined hohlraums. The data are taken using the subaperture sampling, as discussed earlier. The quoted errors are the standard deviations of the data scatter and are not intended to represent the total error in the experiment. The $\mathrm{CH}$ - and Ni-lined hohlraum experiments were done at different periods on the laser, and the unlined data acquired during the two periods are listed separately for meaningful comparison. The two sets of unlined data show that the measurements are reproducible.

Within the data scatter, no increase in SBS from $\mathrm{CH}$ lined hohlraums is observed compared with SBS from unlined hohlraums. For Ni-lined hohlraums, higher SBS levels are observed for both scale- 0.75 hohlraums heated with 1-ns square pulses and scale-1 hohlraums heated with PS22. The scale- 0.75 hohlraum data during the Ni-lined experiments consist of only two data points for the Ni-lined hohlraums and one data point for the unlined hohlraums. This increase is therefore based on a sparse data set, but it is apparently real. For the $\mathrm{CH}$-lined scale- 0.75 data set, there are three data points for the unlined hohlraums and five data points for the $\mathrm{CH}$-lined hohlraums. There are many shots for the PS22 data, and the increase in scattering for the Nilined hohlraums is apparently real. Some of the large scatter in the data may be due to changes in experi- mental conditions such as changes in the focusing. Some of the data were taken during symmetry studies where the crossing point of the beams changed with respect to the LEH. Some correlations in the data could be observed with the change in focusing. ${ }^{3}$ When scattering data from unlined and $\mathrm{Ni}$-lined hohlraums are compared with similar targeting geometries, scattering levels from $\mathrm{Ni}$-lined hohlraums are consistently higher, although quantitative levels differ.

A significant limitation to these measurements is due to the subaperture sampling and the assumption that it represents the average over the entire beam cone. A full-aperture backscattering station (FABS) as well as a near backscatter imaging (NBI) system have been implemented on Nova since these experiments were done. ${ }^{8}$ These diagnostics can make near-field measurements of the SBS up to angles of $14^{\circ}$ from direct backscatter around beamline 7 (BL7). They have shown that SBS from hohlraums is well collimated but can be shifted with respect to direct backscatter for some targets. ${ }^{15}$ Although no experiments have been done with lined targets since the FABS and NBI have been fielded, results from the FABS and NBI can be

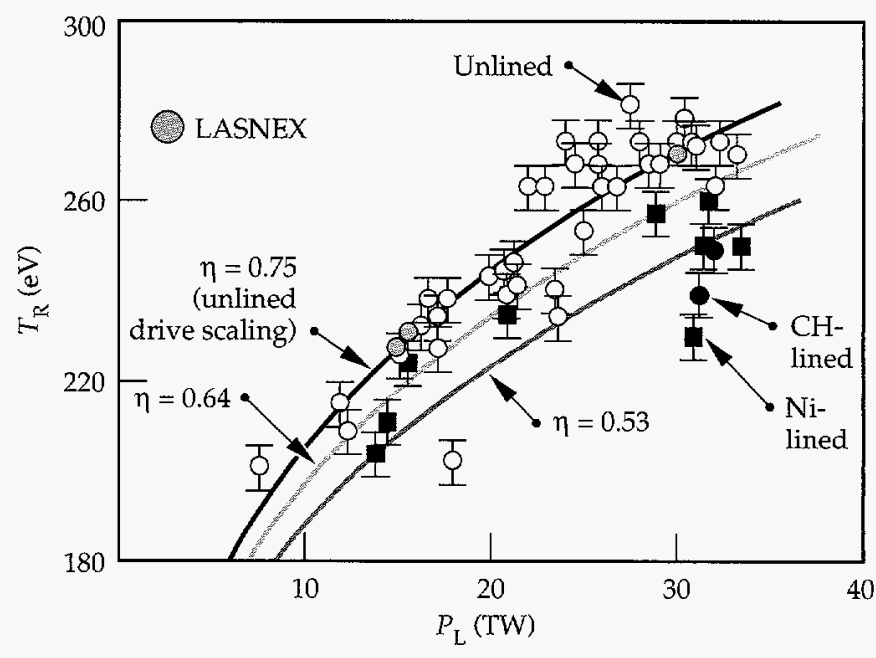

FIGURE 5. Drive scaling for scale-1 hohlraums heated with 1-ns pulses. The data is the same as presented in Fig. 1. The curved lines are fits to the power balance model with different coupling efficiencies. Results of 2-D LASNEX modeling for unlined hohlraums are also shown. $\quad(20-05-1093-3697 \mathrm{pb} 01)$

TABLE 2. SBS reflectivity from lined hohlraums.

\begin{tabular}{lcccc} 
& \multicolumn{2}{c}{ CH data } & \multicolumn{2}{c}{ Ni data } \\
& Unlined & CH-lined & Unlined & Ni-lined \\
\hline 1 ns scale 1 & $1.5 \pm 1 \%$ & $2.8 \pm 0.6 \%$ & $1.6 \pm 1 \%$ & $1.7 \pm 1.2 \%$ \\
1 ns scale 0.75 & $4.6 \pm 1.3 \%$ & $4.5 \pm 1.6 \%$ & $3.5 \% \%$ & $8.7 \pm 0.3 \%$ \\
PS22 scale 1 & $5.8 \pm 2.9 \%$ & $4.5 \pm 2.9 \%$ & $7.3 \pm 4.7 \%$ & $15.1 \pm 5.3 \%$ \\
\hline
\end{tabular}

*Value represents only one experiment. 
compared with the subaperture results for unlined hohlraums. In all cases, the SBS into the lens measured by FABS alone is about half of the results listed in Table 2 for unlined hohlraums. Generally, SBS levels measured with NBI are comparable to those measured by FABS so that the total SBS from unlined hohlraums is comparable to the levels listed in Table 2. This may be purely coincidental, and there is no guarantee that it applies to the lined hohlraum results.

Time-resolved SBS spectra are measured using a grating spectrometer coupled to an optical streak camera. Figure 6 shows an example of the data, displaying isointensity contours of the two-dimensional (2-D) image. The spectral and time resolution are of $3 \AA$ and 30-ps, respectively. Spectra from Ni-lined hohlraums for both 1-ns square and PS22 pulses are red-shifted by $7-8 \AA$ relative to the incident light wavelength.

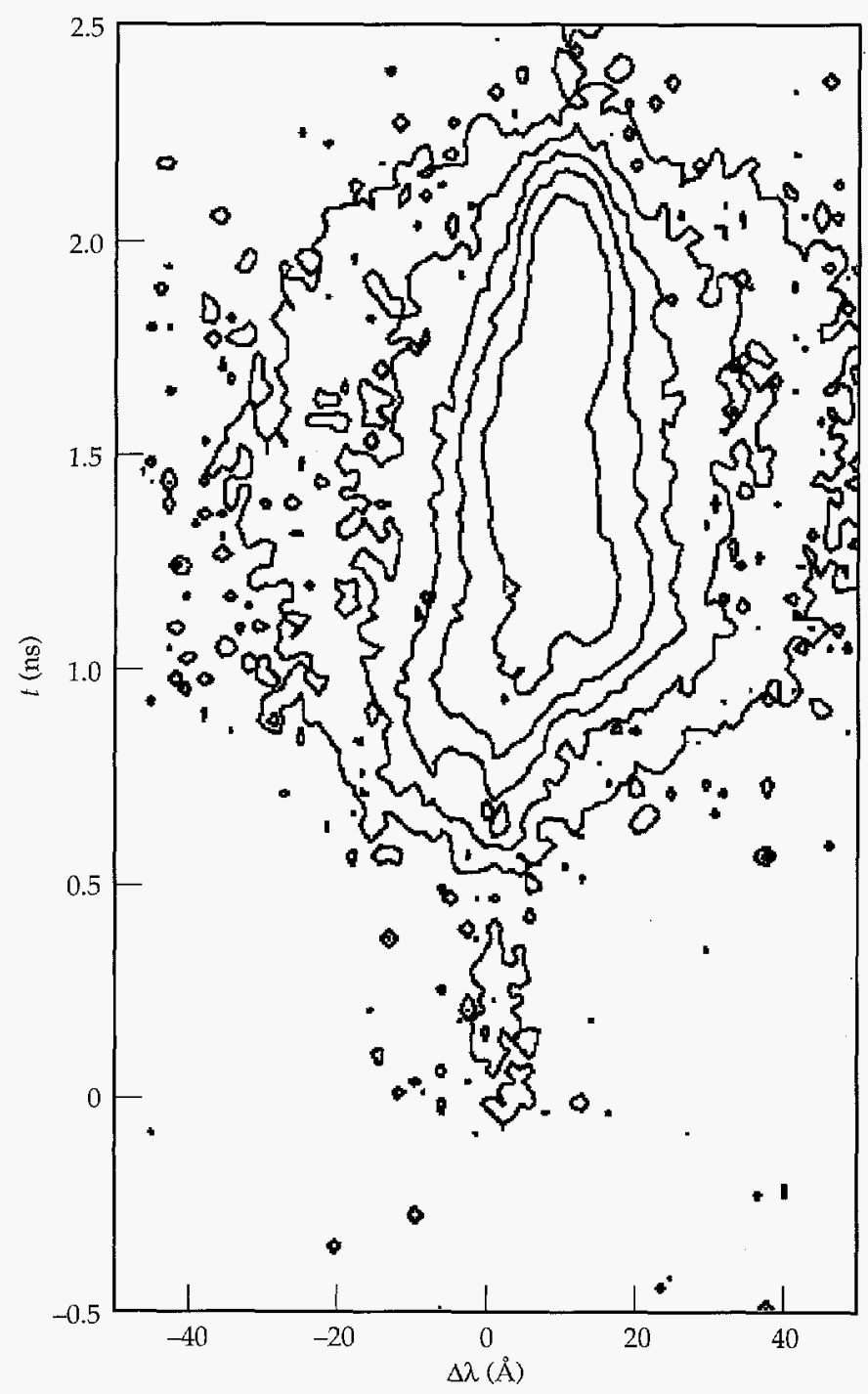

FIGURE 6. Spectrum vs time contour plot of SBS light from a Nilined hohlraum. The spectral shift is measured from the incident 0.35- $\mu \mathrm{m}$ wavelength light. $\quad(20-05-1095-2364 \mathrm{pb} 01)$
Typically, the spectra from unlined hohlraums show little or no shift. This is qualitatively similar to disk targets where the SBS spectrum is shifted more to the blue for high- $Z$ targets compared with low- $Z$ targets. SBS spectra from hohlraums are more red shifted than SBS spectra from disks at similar irradiances. This is presumably due to less Doppler shift, because the plasma flow in the backscatter direction is reduced by the hohlraum, confining the plasma.

As shown in Fig. 7, the spectral data can be integrated over wavelength to obtain the time history of SBS. The time history has been normalized to the calorimeter data for this shot to obtain time-resolved reflectivity. Figure 7 also shows the incident laser pulse. For PS22, the intense part of the SBS signal begins around $1.3 \mathrm{~ns}$, when the incident laser pulse power begins to increase and lasts during the entire high-intensity part of the pulse. A small signal is seen at the beginning of the pulse, but it is a factor of 100 lower. Time history of SBS from 1-ns pulses heating scale-1 hohlraums are qualitatively similar. A signal is seen during the first $300 \mathrm{ps}$ and then it goes away. A second feature turns on at around $700 \mathrm{ps}$, lasting the rest of the pulse. The intensity of the initial feature is higher because the incident beam intensity is higher for 1-ns pulses compared with PS22.

Fast-electron production is studied by measuring the $x$-ray bremsstrahlung from electrons depositing their energy in the hohlraum walls. Figure 8 shows spectra from experiments using both PS22 and 1-ns pulses. For PS22, the superthermal electron production is quite low. Using the expression derived from thick target bremsstrahlung for converting $\mathrm{x}$ rays to electron flux, ${ }^{16}$ the superthermal electron levels for PS22 are less than $0.1 \%$ for all of the targets. For comparison, the

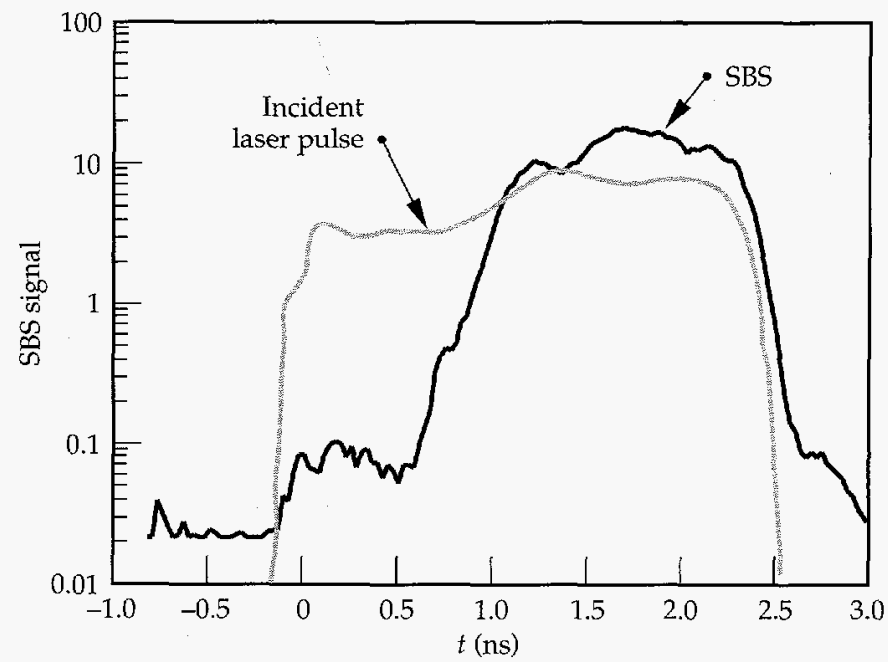

FIGURE 7. Time history of the backscattered light into the BL6 lens. The time history is derived from integrating in wavelength the timeresolved spectrum shown in Fig. 6 and normalizing the time history to the total backscattered light. (20-05-1095-2366 pb01) 
black line in Fig. 8 is the $\mathrm{x}$-ray spectrum calculated assuming $1 \%$ of the incident laser energy is converted to a $50 \mathrm{keV}$ superthermal electron tail. The calculated spectrum does not include self absorption in the target. The data for both lined and unlined hohlraums are an order of magnitude lower. The hot-electron temperatures for the Ni-lined and unlined hohlraums are $\sim 50 \mathrm{keV}$ while the $\mathrm{CH}$-lined hohlraums have slightly lower hotelectron temperatures $(\sim 40 \mathrm{keV})$. A limited amount of data was taken measuring the SRS light levels using diodes positioned around the chamber. The integrated SRS is $\sim 0.2-0.4 \%$, which is slightly higher than levels that would be inferred from fast-electron levels.

The superthermal electron production is higher for both $\mathrm{CH}$-lined and unlined hohlraums heated with 1-ns pulses at high powers. Figure $8(\mathrm{~b})$ shows experiments using 30-TW pulses. For comparison, the black line denotes a fast-electron fraction of $5 \%$ with a $50-\mathrm{keV}$ hot-electron temperature. For the scale- 1 hohlraums, fast-electron fractions are 0.4 and $1.8 \%$ for unlined and $\mathrm{CH}$-lined hohlraums, respectively. For scale- 0.75 hohlraums, the fast-electron fractions are $3 \%$ and $3.8 \%$, for unlined and $\mathrm{CH}$-lined hohlraums, respectively. The fast-electron production levels in unlined hohlraums qualitatively agree with levels measured in disk experiments for similar laser irradiances on the hohlraum wall. ${ }^{17}$ Irradiances on the hohlraum wall are $\sim 1.3 \times 10^{15} \mathrm{~W} / \mathrm{cm}^{2}$ and $\sim 2.3 \times 10^{15} \mathrm{~W} / \mathrm{cm}^{2}$ for scale- 1 and scale- 0.75 hohlraums, respectively. Fast-electron production in disk experiments are $1-2 \%$ with irradiances above $10^{15} \mathrm{~W} / \mathrm{cm}^{2}$. The higher levels inferred in hohlraums may be due to less loss of fast-electron energy to hydrodynamic expansion and the hohlraum walls absorbing a greater fraction of the electrons. The underdense profiles in hohlraums may also be altered with increasing scalelengths at, or below, quarter critical density due to plasma confinement, which also could enhance SRS production. Levels for $\mathrm{CH}$-lined hohlraums are slightly above unlined hohlraums but are still only a small part of the overall energy budget. The increase in fast-electron levels may be due to less collisional damping in the low- $Z$ underdense plasma for the $\mathrm{CH}$-lined hohlraums. Simulations, as discussed below, suggest that SRS is produced in the underdense plasma near the transition region between the $\mathrm{Au}$ and $\mathrm{CH}$. The observations are consistent with these results. SRS light measurements were not made in these experiments.

Recent experiments using the FABS and NBI indicate that SRS light is highly collimated in the backward direction and that total levels can be much higher than inferred from the scattered light diodes or from hard $\mathrm{x}$-ray bremsstrahlung measurements. ${ }^{8}$ SRS levels of $13 \%$ have been observed for one shot in a scale- 0.75 unlined hohlraum heated with $\sim 25 \mathrm{~kJ}$ in a 1-ns square pulse, which is significantly higher than the $3 \%$ inferred from $x$-ray bremsstrahlung. For PS22, SRS levels from FABS and NBI are $2-3 \%$ compared with $\sim 0.1 \%$ levels inferred from $x$-ray bremsstrahlung. Conversely, SRS levels are $\sim 1 \%$ for a scale- 1 unlined hohlraum heated with a 1-ns square pulse, which is comparable to levels inferred from $x$-ray bremsstrahlung. No FABS and NBI data have been taken for lined hohlraums, so the SRS levels scattered into the lens are not known.

Despite the uncertainties in scattered light measurements, lined hohlraums do not show significantly larger scattering losses for most targets compared with unlined hohlraums. For scale-1 targets heated with 1-ns pulses, SBS from CH-lined hohlraums is $\sim 2-3 \%$ comparable to unlined hohlraums, and $\mathrm{f}_{\text {hot }}$ from $\mathrm{CH}$-lined
FIGURE 8 . The x-ray bremsstrahlung spectra from hohlraums heated with (a) PS22 and (b) 1 -ns square pulses. The data and fit from unlined (lined) hohlraums are the open (closed) symbols and dark gray (light gray) lines, respectively. The black lines are calculated spectra for $f_{\text {hot }}$ of $1 \%$ (5\%) for PS22 (1-ns square) laser pulse with a $T_{\text {hot }}$ of $50 \mathrm{keV}$. (20-05-1093-3874pb01) (a) PS22

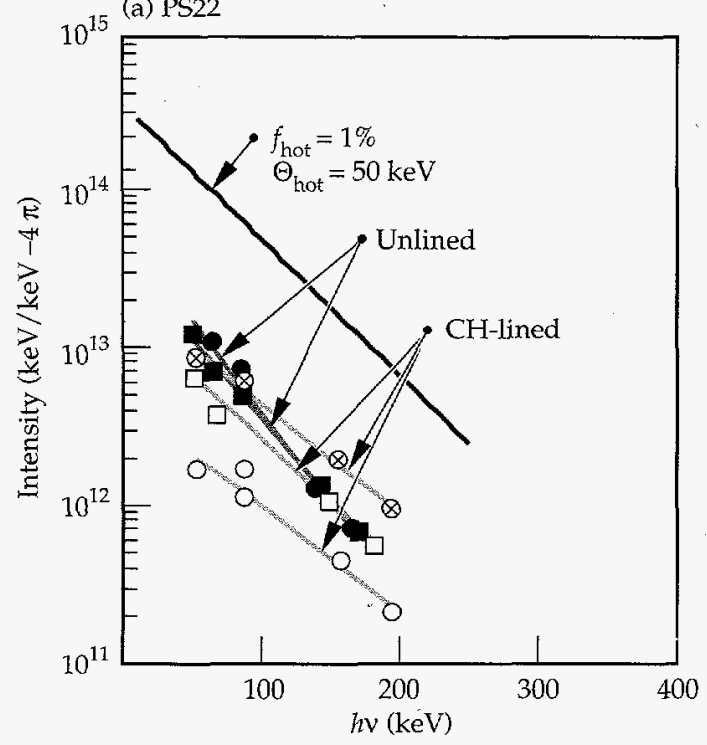

(b) $1 \mathrm{~ns}$

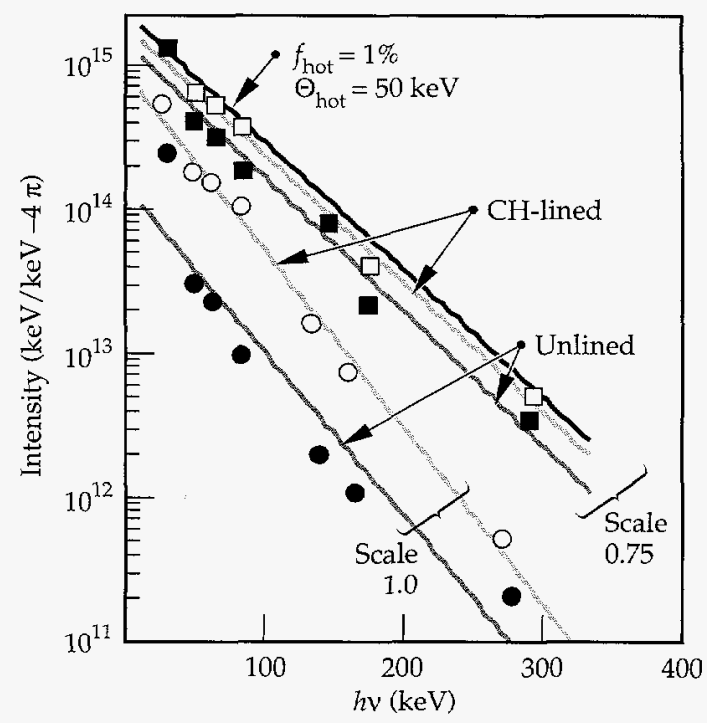


hohlraums is $\sim 2 \%$, a factor of two higher than unlined hohlraums. Even correcting for more recent scattering data, the targets still have $f_{\text {abs }}>90 \%$. For scale- 0.75 hohlraums with 1-ns pulses, recent measurements from unlined hohlraums show higher levels of $f_{S R S}$ than set by the HLP goals consistent with the lower $\eta_{\mathrm{HOHL}}$. Beam smoothing techniques have not been applied to lined hohlraums. The lined hohlraums show slightly increased levels of $f_{\text {hot }}$ but not enough to explain the decreased drive. For shaped pulses, $f_{\mathrm{SBS}}$ is $\sim 15 \%$ for $\mathrm{Ni}$-lined hohlraums compared with $5-7 \%$ for unlined or $\mathrm{CH}$-lined hohlraums. A $10 \%$ reduction in coupling for PS22 would result in about a 5-eV-lower $T_{R}$ consistent with the difference in drive between $\mathrm{CH}$ and Ni-lined hohlraums. Although scattering losses can explain some of the differences in observed coupling, much of the reduction in drive for lined hohlraums is probably due to the difference in energy partition, as discussed in the "LASNEX Modeling" section of this article. All these results are for unsmoothed laser beams. Beam smoothing has been effective in reducing stimulated scattering levels in recent gas-filled hohlraum experiments. 8,15

\section{Semi-Empirical Modeling}

The power balance model described in Eq. (1) provides a reasonable fit to the data for unlined hohlraums. Although the data set is not as extensive for lined hohlraums, the model also can explain the data, but with a reduced coupling efficiency. The model depends

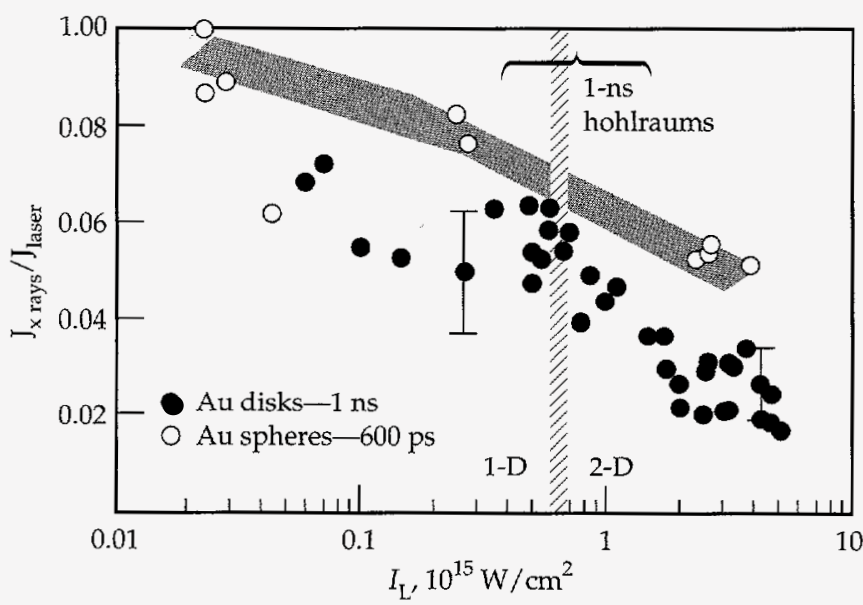

FIGURE 9. Conversion efficiency of converting laser energy into $x$ rays from $0.1-4 \mathrm{keV}$ vs laser intensity $I_{\mathrm{L}}$. The solid circles are data from single-beam irradiation of Au disks with 1-ns pulses of $0.35-\mu \mathrm{m}$ light. The open circles are data from $A u$ spheres uniformly irradiated. The bracket denotes the range of intensities on the wall of a scale-1 Nova hohlraum whose data is shown in Fig. 5. The shaded bar shows the division between the regions where the underdense plasma expansion is quasi-1-D vs 2-D. (20-05-1292-3811pb01) on two parameters: $\alpha$ and $\eta_{\mathrm{HOHL}}$. The other parameters depend on hohlraum geometry and are usually assumed to be defined by the target geometry although, in principle, they could vary during the experiment due to plasma motion. Parts of NTC tasks are to study the wall albedo and laser coupling efficiency. The wall albedo experiments use burn-through patches measuring the time for the radiation wave to heat a known thickness of $\mathrm{Au}$. These experiments show that present opacity and radiation wave models correctly predict burnthrough times for $\mathrm{x}$-ray drive above $200 \mathrm{eV}$. Details of these experiments have been reported elsewhere. ${ }^{18}$

Two sets of experiments have investigated the effective efficiency for converting laser light to drive energy. In one set of experiments defined in HLP7, the conversion efficiency of laser light to $x$ rays using planar disk targets $\eta_{\text {DISK }}$ is studied. The other experiment investigates the difference in $x$-ray production between disk targets and hohlraums.

Scaling of $x$-ray conversion efficiency, $\eta_{\text {DISK }}$, has been studied as a function of laser intensity $I_{L}$, using 1-ns square laser pulses of $0.35-\mu \mathrm{m}$ light. Figure 9 shows the results. The laser energy for most of the shots is $\sim 1.5 \mathrm{~kJ}$ using a single beam of Nova. Intensity is varied by changing the spot size on the target. The $x$-ray intensity includes the spectrum from 0.1 to $\sim 4 \mathrm{keV}$. The intensity from 0.1 to $1.5 \mathrm{keV}$ is measured using the $\mathrm{XRD}$ array and the intensity in the $2-4 \mathrm{keV}$ range is measured using absolutely calibrated $x$-ray spectrographs. For disk experiments shown in Fig. $9, \eta_{\text {DISK }}$ varies from 0.6 to 0.4 for intensities in the range of $\sim 4 \times 10^{14} \mathrm{~W} / \mathrm{cm}^{2}$ to $1.3 \times 10^{15} \mathrm{~W} / \mathrm{cm}^{2}$. This is the intensity range of the initial wall irradiances for scale-1 hohlraums in Fig. 5 where $\eta_{\mathrm{HOHL}}$ is $\sim 0.75$. For scale- 0.75 hohlraums, the initial wall irradiance is $\sim 2.3 \times 10^{15} \mathrm{~W} / \mathrm{cm}^{2}$ and $\eta_{\text {HOHL }}$ is $\sim 0.65$ while $\eta_{\text {DISK }}$ is $\sim 0.25$ at that intensity. Some of the lower conversion efficiency for a disk may be due to 2-D effects, especially at the higher intensity where the spot size is smaller than the expanding plasma. This transition is noted by the shaded line in Fig. 9 that denotes the estimated transition region between 1-D and 2-D corona expansion. For comparison, conversion efficiency from Au spheres, which are more uniformly irradiated, indicate a higher conversion efficiency (as shown in Fig. 9). Higher efficiencies are also obtained using longer pulses. Instantaneous conversion efficiencies increase from $50 \%$ after $1 \mathrm{~ns}$ to $\sim 70 \%$ at $3 \mathrm{~ns}$ for an intensity of $\sim 4 \times 10^{14} \mathrm{~W} / \mathrm{cm}^{2} .{ }^{19}$

As discussed below, the hydrodynamic losses are reduced in a hohlraum as the hohlraum walls confine the underdense plasma expansion and more energy couples into $x$-ray heating. To test this modeling, experiments compared the brightness of the laser irradiated spot inside of the hohlraum with the brightness of the spot on a laser-irradiated disk. The results show that the peak brightness for both the hohlraum and the 
disk is similar, but the extent of the bright spot in the hohlraum is greater and consistent with the enhanced conversion efficiency in the hohlraum. The increase in size of the hot spot in a hohlraum, as a function of time, is shown in Fig. 10 for a 1-ns square pulse experiment. The increase in width of the emission spot is plotted for three shots as a function of time for emission at $450 \mathrm{eV}$ in Fig. 10(a) and $1200 \mathrm{eV}$ in Fig. 10(b), defined by broadband filters and grazing incident $x$-ray mirrors. ${ }^{20}$ The initial width is consistent with the size of the laser beam on the hohlraum wall. The increase in spot size is consistent with LASNEX predictions of the spot width, which are also shown in Fig. 10 (see the shaded circles).

Limited theoretical and experimental work has been done on extending the power balance model to lined hohlraums. The effect of the liner should be minimal on the dynamics of $x$-ray heating on the wall. The liner thicknesses used are typically optically thin to the radiation. Assuming cold opacities, ${ }^{21}$ the $7500-\AA \mathrm{CH}$ and

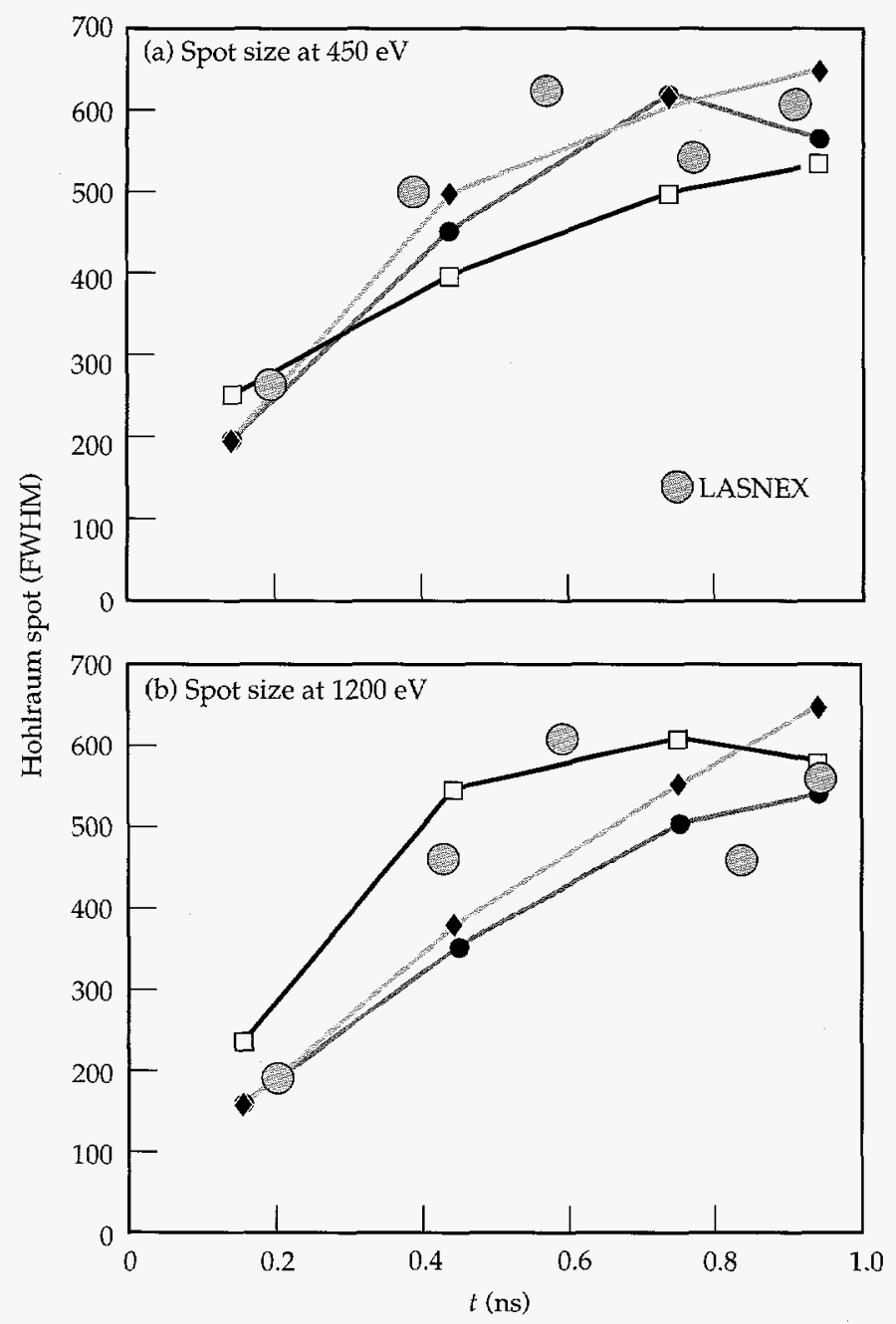

FIGURE 10. The full width at half maximum (FWHM) of the hot spot in a hohlraum produced by the laser spot vs time. The data is taken from three shots for $x$ rays at (a) $450 \mathrm{eV}$ and (b) $1200 \mathrm{eV}$. (20-05-1292-3806pb01)
$1500-\AA$ Ni liners are $~ 1.5$ and 1 Planck mean-free paths thick, respectively, to a $200-\mathrm{eV}$ blackbody radiator. These decrease significantly to much less than one optical depth when the plasma is heated to $100 \mathrm{eV}^{22}$ The low-Z plasma therefore should not significantly modify the radiation heating dynamics of the wall (verified by LASNEX calculations).

To test the effects of the thin overcoat on laser hohlraum coupling, $x$-ray production from $\mathrm{Ni}$-coated Au disks has been measured in a limited number of disk experiments. No experiments have been done on $\mathrm{CH}$-coated disks. Figure 11 compares the conversion efficiency from the overcoated disks with that of pure Au disks as a function of time. Two experiments are done at $\sim 4 \times 10^{14} \mathrm{~W} / \mathrm{cm}^{2}$ with 2 -ns square pulses and one experiment is done at $\sim 1 \times 10^{15} \mathrm{~W} / \mathrm{cm}^{2}$ with a 1 -ns pulse. The data show a slight delay in $x$-ray production ( 200 ps) for a Ni overcoat compared with a Au disk. The time required to burn through the Ni overcoat is estimated to be $\sim 100-300$ ps depending on the model and laser intensity. ${ }^{23}$ After the initial burn-through of the $\mathrm{Ni}, \mathrm{x}$-ray production is nearly equal to that of $\mathrm{Au}$ for $4 \times 10^{14} \mathrm{~W} / \mathrm{cm}^{2}$ but is only $50 \%$ of the pure $\mathrm{Au}$ conversion efficiency for $1 \times 10^{15} \mathrm{~W} / \mathrm{cm}^{2}$. The effect of the overcoat on $\mathrm{x}$-ray production at high intensity is not presently understood. At high intensity the laser spot is smaller than corona expansion scales and is in the 2-D regime. Possible effects of lateral transport and conduction could be affecting the conversion efficiency.

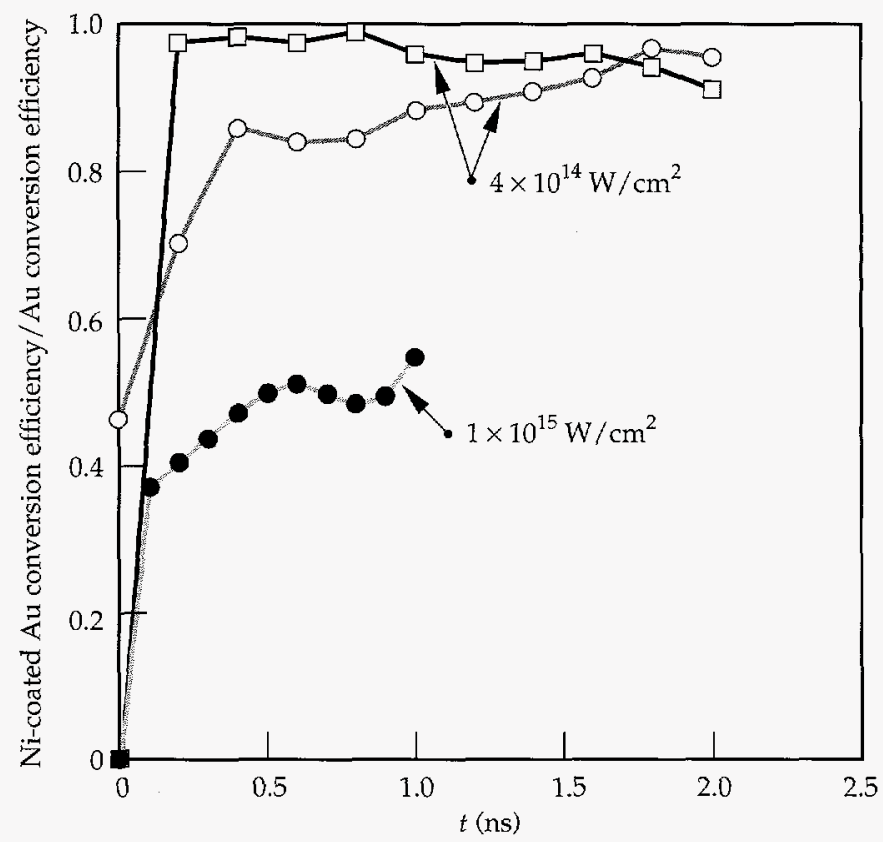

FIGURE 11. The ratio of $x$-ray conversion efficiency from a $1500-\AA$ $\mathrm{Ni}$ coated Au disk to an uncoated Au disk vs time. Data are from a 0.35 - $\mu \mathrm{m}$ laser irradiation of $4 \times 10^{14} \mathrm{~W} / \mathrm{cm}^{2}$ in a 2-ns square pulse and $1 \times 10^{15} \mathrm{~W} / \mathrm{cm}^{2}$ in a 1-ns square pulse. $\quad(20-05-1095-2365 \mathrm{pb01})$ 


\section{LASNEX Modeling}

Using LASNEX, extensive work has been done modeling the hohlraums. ${ }^{24}$ Calculations are fully "integrated" and 2-D. The laser deposition is modeled by using a 2-D representation of the 3-D Nova laser irradiation geometry in a statistical ray generation method. To extract radiation drive from the simulation, a small "virtual" sphere is placed at the center of the hohlraum simulation, and incident flux on the sphere is calculated. The simulations reproduce the $x$-ray drive measured in unlined hohlraums quite well. Figure 5, shown earlier, compares the peak drive calculated for 1 -ns laser pulses with shock velocity measurements. The simulations assume $100 \%$ absorption consistent with experiments for scale- 1 unlined hohlraums where absorption is greater than $95 \%$. Simulations also reproduce drive in shaped-pulse experiments. LASNEX calculates a peak drive of $215 \mathrm{eV}$ for PS22 when timedependent SRS and SBS scattering and diagnostic hole losses are included.

Simulations have also been done for several of the lined hohlraum experiments. Generally, the peak drive is calculated to be lower for lined hohlraums compared with unlined hohlraums. Table 1 lists the decrease in calculated peak drive and compares it with experimental decrements. The lower peak drive agrees with the measurements within the statistical uncertainty of the data. The one data set that has the largest difference is the PS22 Ni-lined data. The calculations assume no change in absorption between lined and unlined targets. When the Ni-lined data is reduced by $\sim 10 \%$ for the increased $f_{\mathrm{SBS}}$ of $\sim 10 \%$, the calculations and data show even better correlation.

Lined hohlraums are calculated to be cooler because more energy couples into plasma blowoff compared with unlined hohlraums. Figure 12 shows calculations of the energy partitioning in an unlined Au hohlraum and a $2150-\AA$ Ni-lined hohlraum. The energy in wall blowoff, defined as the energy contained in the underdense plasma filling the hohlraum, increases by $\sim 1.5 \mathrm{~kJ}$ for the lined hohlraum. This produces a lower $T_{\mathrm{R}}$ because it leaves less energy to heat the walls and, self-consistently, to reradiate out the holes. The cause of the increased blowoff energy is due to the higher specific heat for the low- $Z$ blowoff compared with the high- $Z$ blowoff. The specific heat scales

$\left[\frac{(\mathrm{Z}+1)}{\mathrm{A}}\right]+E_{\mathrm{ion}}$

where $Z$ is the average ion charge and $A$ is the atomic number. For $A u$ irradiated by the laser, $Z$ is $\sim 55$ while $\mathrm{CH}$ and $\mathrm{Ni}$ are nearly fully ionized. The specific heat for $\mathrm{Ni}$ and $\mathrm{CH}$ are factors of 2 to 2.5 times as high as Au from similar plasma conditions.

\section{Summary}

The drive goals for HLP1 and HLP2 are met with over $200 \mathrm{eV}$ obtained for PS22 and $270 \mathrm{eV}$ obtained for a scale- 0.75 hohlraum with 30 TW in a 1-ns pulse. The drive is lower for lined hohlraums compared with unlined hohlraums and is generally explained by higher coupling to plasma blowoff in the hohlraums. Although the diagnostics were not optimal for the experiments, the HLP1 coupling goals for $f_{\text {abs }} f_{\text {SBS }} f_{\text {hot' }}$ and $f_{\mathrm{SRS}}$ appear to have been met for the PS22 CHlined experiments, although for $\mathrm{Ni}$-lined hohlraums $f_{\mathrm{SBS}} \approx 15 \%$. To verify this, more experiments need to be done with the improved Nova diagnostics. The higher levels in Ni-lined hohlraums, compared with $\mathrm{CH}$-lined hohlraums, are consistent with SBS in CH-lined hohlraums being Landau damped by the $\mathrm{H}$ and $\mathrm{C}$
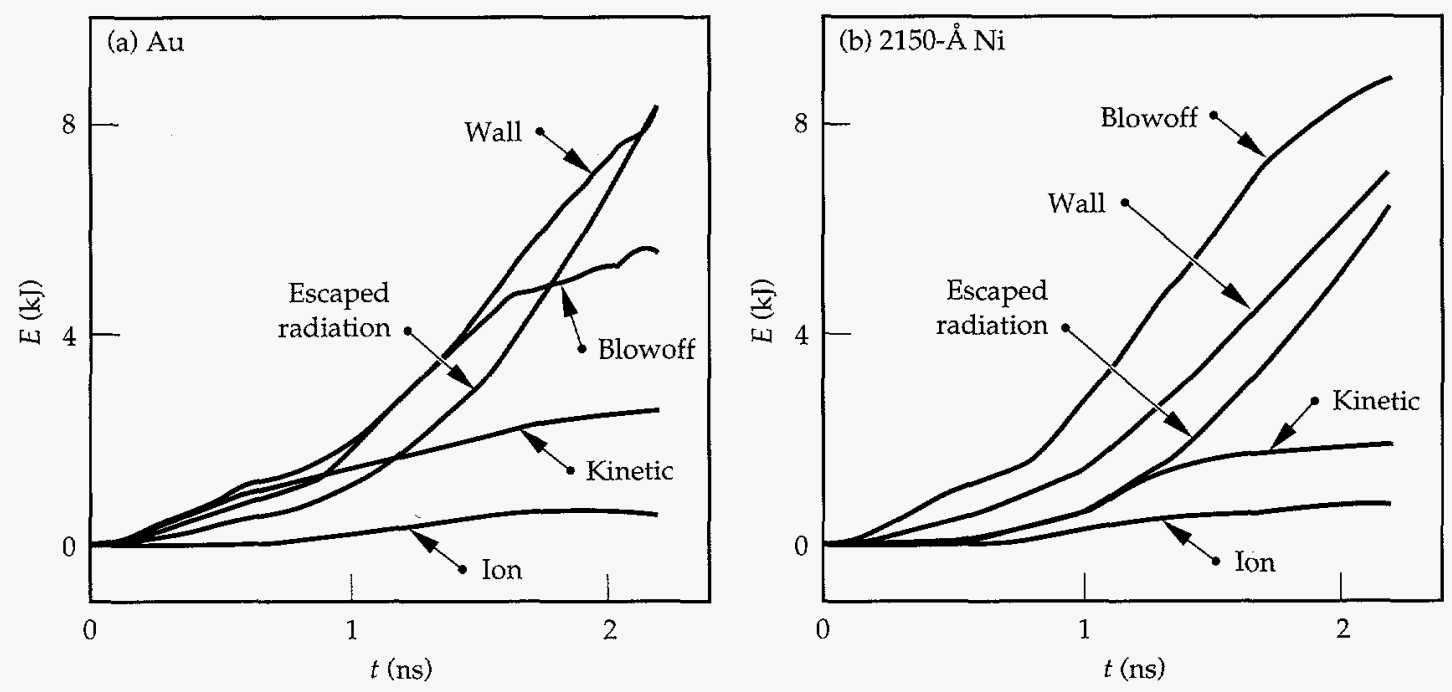

FIGURE 12. Partition of energy $E$ as a function of time from a LASNEX simulation for (a) a pure $\mathrm{Au}$ hohlraum and (b) a hohlraum lined with 2150-Å Ni. Both hohlraums are heated with $0.35-\mu \mathrm{m}$ light using PS22. (50-04-1093-3913pb01) 
mixed species plasma. ${ }^{25}$ By producing a $\mathrm{Ni}$ and $\mathrm{H}$ mixed liner, Landau damping could also be effective for reducing SBS in the Ni-lined plasmas. For HLP2, the scale-1 hohlraums appear to meet the coupling goals, but $f_{\mathrm{SRS}}$ for scale- 0.75 hohlraums appear to be higher than $5 \%$. The most recent measurements for unlined scale- 0.75 hohlraums is $\sim 13 \%$. SRS from lined hohIraums have not been measured, but x-ray bremsstrahlung measurements suggest that $f_{\mathrm{SRS}}$ for lined hohlraums may be only slightly higher. The lower $\eta_{\mathrm{HOHL}}$ for scale- 0.75 unlined hohlraums is consistent with the measured $f_{\text {SRS }}$ and with hohlraum modeling, which includes the scattering models. Future experiments can explore reducing SRS by the use of beam conditioning, as demonstrated in gas-filled hohlraums. ${ }^{15}$

\section{Notes and References}

1. J. H. Nuckolls, Phys. Today 35(9) 24 (1982).

2. J. D. Lindl, Phys. Plasmas 2(11), 3933-4024 (1995).

3. L. J. Suter, A. A. Hauer, L. V. Powers, D. B. Ress, et al., Phys. Rev. Lett. 73, 2328 (1994).

4. S. W. Haan, S. M. Pollaine, J. D. Lindl, L. J. Suter, et al., Phys. Plasmas 2, 2480 (1995).

5. R. L. Kauffman, H. N. Kornblum, D. W. Phillion, C. B. Darrow, et al., Rev. Sci. Instrum. 66, 678 (1995).

6. H. N. Kornblum, R. L. Kauffman, and J. A. Smith, Rev. Sci. Instrum. 57 2179-81 (1986).

7. P. E.. Young, K. G. Estabrook, W. L. Kruer, E. A. Williams, et al., Phys. Fluids B 2, 1907-1917 (1990).

8. R. K. Kirkwood C. A. Back, K. S. Bradley, D. H. Kalantar, et al., Bull. APS 39, 1753 (1994); B. J. Macgowan et al., "Laser-Plasma Interactions in NIF-Scale Plasmas (HLP5 and HLP6)," p. 305 of this Quarterly.
9. Laser Program Annual Report-1978, 6-8, Lawrence Livermore National Laboratory, Livermore, CA, UCRL-50021-78 (1979).

10. P. M. Bell, J. D. Kilkenny, G. Power, R. Bonner, and D. K. Bradley, Ultrahigh Speed and High Speed Photography, Photonics, and Videography' 89 (SPIE, Bellingham, WA, 1989; Proc. SPIE, 1155) pp. $430-444$.

11. R. L. Kauffman, L. J. Suter, C. B. Darrow, J. D. Kilkenny, et al., Phys. Rev. Lett. 73, 2320 (1994).

12. R. Sigel, R. Pakula, S. Sakabe, and G. D. Tsakiris, Phys. Rev. A 38, 5779 (1988).

13. M. D. Rosen, Lawrence Livermore National Laboratory, Livermore, CA, private communication (1995).

14. R. E. Marshak, Phys. Fluids 1, 24 (1958).

15. B. J. MacGowan, R. K. Kirkwood, and D. S. Montgomery, Lawrence Livermore National Laboratory, Livermore, CA, private communication (1995).

16. W. C. Mead, E. M. Campbell, K. G. Estabrook, R. E. Turner, et al., Phys. Fluids 26, 2316 (1983).

17. Laser Program Annual Report-1985, 4-4, Lawrence Livermore National Laboratory, Livermore, CA, UCRL-50021-85 (1986).

18. J. L. Porter, T. J. Orzechowski, M. D. Rosen, A. R. Thiessen, et al., 1994 ICF Annual Report, 125, Lawrence Livermore National Laboratory, Livermore, CA, UCRL-LR-105820-94 (1995).

19. F. Ze, D. R. Kania, S. H. Langer, H. Kornblum, et al., J. Appl. Phys. 66, 1935-39 (1989).

20. F. Ze, R. L. Kauffman, J. D. Kilkenny, J. Wiedwald, et al., Rev. Sci. Instrum. 63, 5124-6 (1992).

21. B. L. Henke, E. M. Gullickson, J. C. Davis, Atomic Data and Nuclear Data Tables 54, 181 (1993).

22. WorkOp-III: 94 Final Report, Eds., A. Rickert, K. Eidmann, J. Meyer-ter-Vehn, F. J. D. Serduke, and C. A. Iglesias, Max-Planck-Institut für Quantenoptik (Report MPQ 204), Hans-Kopferman-Strasse 1, 85748 Garching, Germany (1995).

23. R. Fabbro, C. Max, and E. Fabre, Phys. Fluids 28, 1463 (1985).

24. G. B. Zimmerman and W. L. Kruer, Comments Plasma Phys. Controlled Fusion 2, 51 (1975).

25. E. A. Williams, R. L. Berger, R. P. Drake, A. M. Rubenchik, et al., Phys. Plasmas 2, 129 (1995). 


\title{
NOVA SYMMETRY: EXPERIMENTS, MODELING, AND INTERPRETATION (HLP3 AND HLP4)
}

\author{
L. J. Suter \\ N. Delameter \\ E. K. Lindman* \\ A. A. Hauer" \\ S. G. Glendinning \\ A. R. Thiessen \\ L. V. Powers \\ W. W. Hsing" \\ R. E. Turner \\ D. B. Ress \\ O. L. Landen
}

\section{Introduction}

Understanding and controlling capsule implosion symmetry is a key requirement for inertial confinement fusion (ICF). Symmetry was specifically called out in the Nova Technical Contract (NTC) as the HLP4 task. Later, elements of HLP3 were expanded to include symmetry work. For nearly a decade and a half it has been recognized that the fundamental asymmetry in a laser-heated hohlraum is a long-wavelength pole-waist radiation flux variation ${ }^{1}$ that varies like the $P_{2}$ Legendre polynomial. ${ }^{2}$ It has also been recognized that we can control this asymmetry and achieve nearly symmetric implosions by appropriately pointing the laser beams. ${ }^{3}$

\section{Review of Symmetry in Hohlraums}

To understand symmetry in hohlraums, consider a cylindrically shaped, Nova-like hohlraum with beams aimed as shown in Fig. 1(a). An observer at the capsule location would see a collimated source flux vs angle approximately as shown in the flux vs polar angle plot. The laser-produced hot spot causes a peak in this source at $\sim 60^{\circ}$ polar angle. The cold, nonemitting laser entrance hole (LEH) provides zero flux at low polar angle. If we resolve this source flux vs angle into its Legendre polynomial coefficients, we find a $P_{0}$ and $P_{4}$ component of order unity and a substantial, negative $P_{2}$ component. However, because each point on the capsule's ablation surface integrates radiation flux over its $2 \pi \mathrm{sky}$, the absorbed flux vs angle will be different from the source. For a small capsule, Green ${ }^{2}$ showed that this integration causes the $P_{2}$ component to be attenuated by $\sim 4$, the $P_{4}$ component by 24 , and higher modes by even more. Consequently, the major asymmetry in the absorbed flux is the $P_{2}$ asymmetry. For the capsule in the hohlraum of Fig. 1(a), that asymmetry has a negative $P_{2}$ component which, ultimately, produces a prolate implosion.

We can control the $P_{2}$ asymmetry by changing the pointing/aiming of the laser beams. ${ }^{3}$ Figure 1(b) illustrates a situation where the beams are aimed to form. hot spots much farther apart. Then, the collimated source flux has a substantial, positive $P_{2}$ component as well as $P_{0}$ and $P_{4}$ components of order unity. The $2 \pi$ integration at the ablation surface causes the capsule absorbed flux to be dominated by a $P_{0}$ and positive $P_{2}$ component only, which ultimately produces an oblate implosion. Somewhere in between the situations shown in Figs. 1(a) and 1(b) is a beam pointing where the $P_{2}$ component of the source asymmetry vanishes [(Fig. 1(c)]. There, the ablation flux is dominated by the $P_{0}$ component and small, higher-order modes to produce an implosion that is substantially spherical.

Green's work on the attenuation of the $P_{2}, p_{4}$, and higher components was done for a small capsule inside a spherical hohlraum. Finite-size capsules and cylindrical hohlraums cause quantitative changes ${ }^{4-6}$ but no significant qualitative changes.

\section{Summary of Symmetry Experiments}

Between 1987 and 1993, we performed a number of scaling experiments to examine our ability to understand the time-integrated $P_{2}$ asymmetry and to control it with beam pointing. In the most mature method of assessing the asymmetry, a pure-plastic capsule ${ }^{7}$ filled with $D_{2}$ and a trace of Ar gas is placed in the center of a Nova hohlraum and is imploded by $\mathrm{x}$-ray drive. Following the implosion is a bright flash of $x$ rays produced by the hot, compressed fuel. At that time, we take pictures of the images formed by $x$ rays viewed $90^{\circ}$ off the polar axis through a hole in the side of the

${ }^{*}$ Los Alamos National Laboratory, Los Alamos, New Mexico 
hohlraum. The resulting images show emission that is round, oblate, or prolate, depending on the beam pointing. (We discuss beam pointing later; also see Fig. 4 for an illustration.) Figure 2 shows some of our earliest images, from pure Au hohlraums irradiated by 1-ns flat-top pulses.

These first experiments demonstrated our ability to produce round, relatively symmetric implosions and confirmed that the long-wavelength mode dominated the hohlraum asymmetry. They also showed that we could control implosion asymmetry with beam pointing. ${ }^{8}$ These experiments were done in early versions of our scale 1.0 hohlraums (1600- $\mu \mathrm{m}$-i.d., 2700- $\mu \mathrm{m}$-long, $800-\mu \mathrm{m}$ LEH diam) and used what has become our standard symmetry capsule - nominal dimensions of 440- $\mu \mathrm{m}$-i.d., $55-\mu \mathrm{m}$-thick $\mathrm{CH}$ ablator/pusher, filled with $50 \mathrm{~atm} \mathrm{D}_{2}$ and $0.1 \%$ (at.\%) Ar.
We have produced x-ray images of implosions using a number of gated and time-integrated diagnostics that have evolved over the years. ${ }^{8-12}$ Numerical simulations of our standard symmetry capsule show good sensitivity to asymmetry. For pulse-shaped, convergence- 10 implosions, we estimate that a $7 \%$, fixed-in-time, poleto-waist flux asymmetry will produce a $2: 1$ distortion of the $x$-ray image. We can measure distortions much closer to 1:1; therefore, we believe that the accuracy of our diagnostic technique is about $1 \%$. That is, we can resolve equivalent, fixed-in-time asymmetries of $\sim 1 \%$ pole-to-waist flux variation. A large body of evidence ${ }^{13}$ indicates that these capsules, which operate at a pusher convergence of 7 to 10 depending on the pulse shape, do perform approximately as our model. There is good agreement between simulated and experimental capsule neutron yields, time-of-neutron production, and

\begin{tabular}{|c|c|c|c|}
\hline Hohlraum & $\begin{array}{l}\text { Collimated source } \\
\text { flux vs angle }\end{array}$ & $\begin{array}{l}\text { Collimated } \\
\text { source } \\
\text { flux }\end{array}$ & $\begin{array}{c}\text { Flux capsule } \\
\text { ablation } \\
\text { surface }\end{array}$ \\
\hline
\end{tabular}

(a)
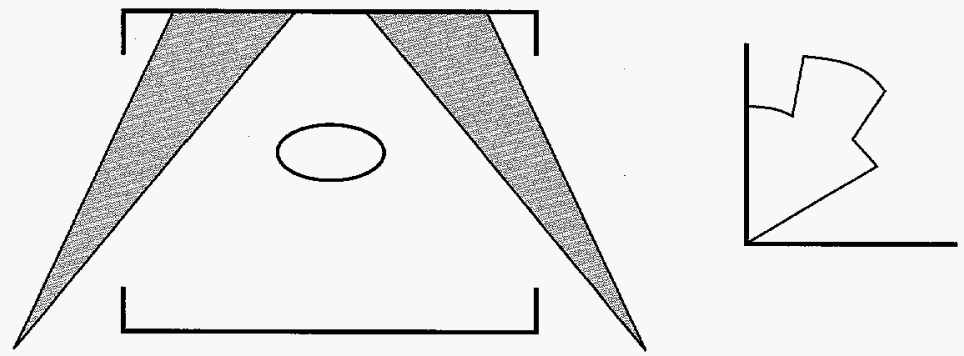

$$
P_{0}-0.4 P_{2}+O(1) P_{4}+\ldots \quad P_{0}-0.1 P_{2}+O(0.01) P_{4}
$$

(b)

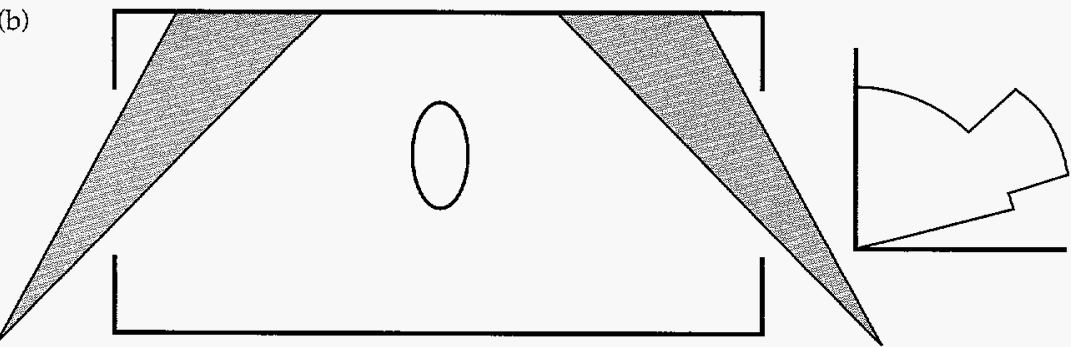

$P_{0}+0.4 P_{2}+\mathrm{O}(1) P_{4}$

$P_{0}+0.1 P_{2}+O(0.01) P_{4}$

(c)

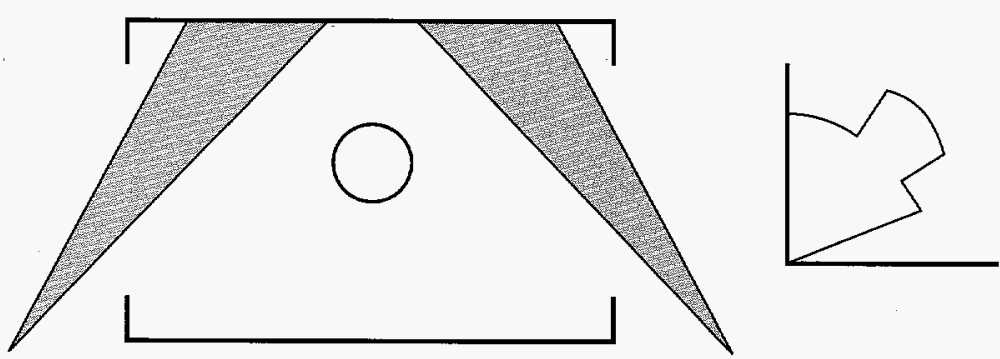

$$
P_{0}+O(0) P_{2}+O(1) P_{4} \quad P_{0}+O(0.01) P_{4}
$$

FIGURE 1. The collimated source flux vs angle viewed by a capsule in a laser-heated hohlraum has a substantial $P_{2}, P_{4}$, etc., component in addition to the $P_{0}$ component. Since each point on the ablation surface integrates over its $2 \pi$ sky, the $P_{4}$, etc., components of drive asymmetry are heavily damped, leaving only the long-wavelength $P_{2}$ component as an asymmetry. This asymmetry can be controlled by beam pointing (see variations in $\mathrm{a}, \mathrm{b}$, and $\mathrm{c}$ for comparison). (20-03-0995-2123pb01) 
image sizes. Spectroscopy confirms theoretical predictions that the $x$ rays are principally produced by the $\mathrm{Ar}$ and that we are, in fact, imaging the fuel volume. Analysis of spectroscopy data and neutron data confirms calculated convergences. Reference 14 provides a comprehensive review of current drive asymmetry measurement techniques. Reference 15 provides a review of symmetry analysis.

The earliest experiments confirmed that the longwavelength mode dominated and that it could be varied from shot to shot by changing the beam pointing in our out, as demonstrated in Fig. $2 .{ }^{16}$ These results were largely qualitative. In 1990, we began producing systematic, quantitative symmetry studies. ${ }^{14}$ The basic procedure of these experiments has been the samefor a given pulse shape and hohlraum type, we produce a symmetry scaling by varying the beam pointing while observing the resulting shapes of the capsules in self emission. Moving the beam pointing in tends to make a more prolate implosion; moving it out makes a more oblate implosion.

Between 1990 and 1993, we produced nine different symmetry scaling databases with the Nova laser using the three pulse shapes illustrated in Fig. 3. We have done three scalings with 1-ns flat-top pulses, five scalings with our 26-kJ, 2.2-ns, 3:1 contrast ratio pulse shape, called PS22, and one scaling with an 8:1 contrast ratio, 3.2-ns, 27-kJ pulse shape, called PS23. We have used these pulse shapes to irradiate both pure $\mathrm{Au}$ hohlraums and lined hohlraums as specified in the NTC. Lined hohlraums are Au hohlraums lined on the inside with a thin layer of either low- $Z$ material (e.g., $\mathrm{CH}$ ) or mid- $\mathrm{Z}$ material (e.g., Ni). We investigated lined hohlraums because we believe something like a liner to be necessary at larger, ignition scales. ${ }^{17}$ For ignition hohlraums, we calculate that pure Au designs will fill with high- $Z$ plasma. This causes the laser absorption region to move almost to the LEH, producing an unacceptably large radiation flux asymmetry on the capsule. Liners are one way to mitigate this effect by replacing the high- $Z$ blowoff with low- or mid-Z blowoff.

With 1-ns flat-tops, we shot both pure $\mathrm{Au}$ and Nilined (1500- $\AA$ ) Au hohlraums fixed in length at $2700 \mu \mathrm{m}$. We have also shot pure Au hohlraums where we varied the length of the hohlraum with the pointing so that the beams always cross in the plane of the LEH. We have done five scalings with PS22: fixed-length $\mathrm{Au}, \mathrm{Ni}-$ lined $\mathrm{Au}$, variable-length pure $\mathrm{Au}, \mathrm{Ni}$-lined $\mathrm{Au}$, and $\mathrm{CH}$-lined $(0.75-\mu \mathrm{m}) \mathrm{Au}$ hohlraums. Our PS23 series used pure Au hohlraums that were open cylinders. As mentioned in the Introduction and in "Ignition Target

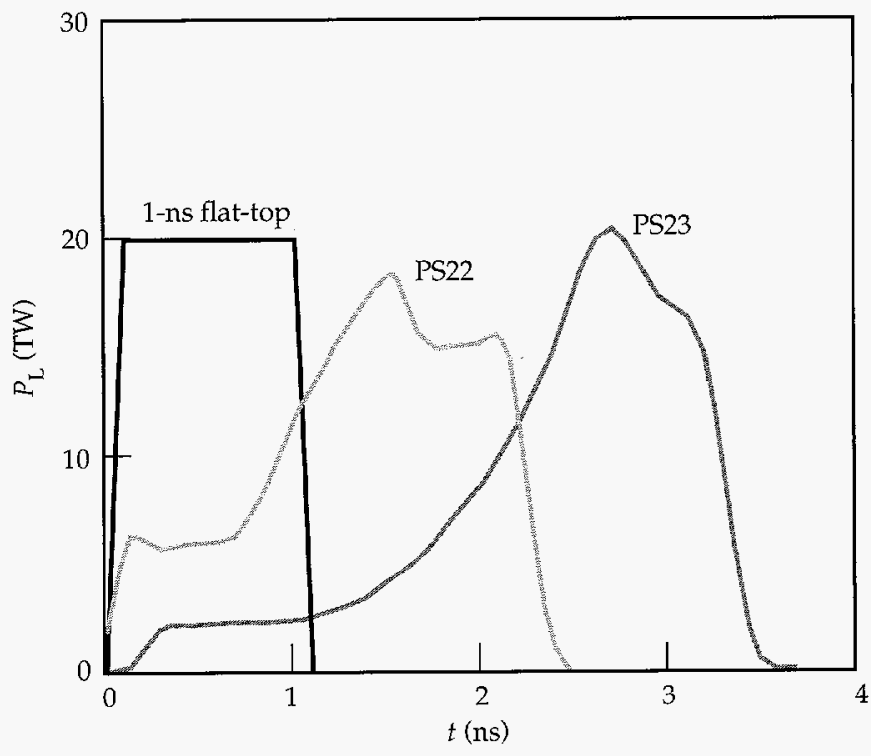

FIGURE 3. Symmetry scaling experiments using three different pulse shapes. (20-03-0995-2124pb01) (a) Inward pointing shifts

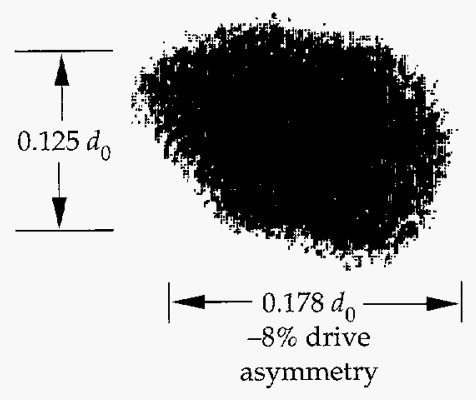

(b) Symmetric

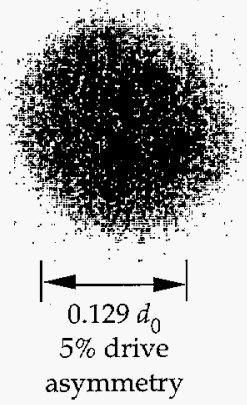

(c) Outward pointing shifts

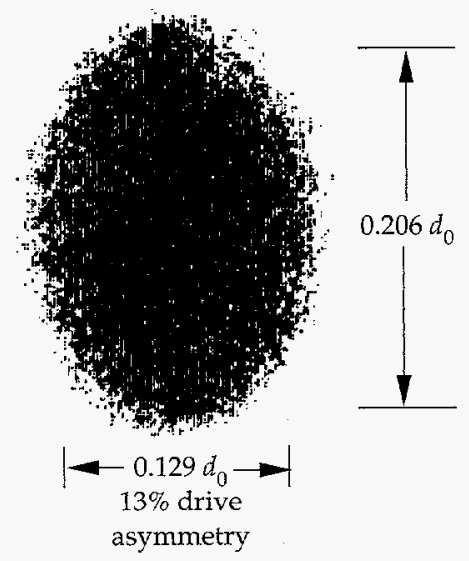

FIGURE 2. Capsule self-emission $x$-ray images from 1-ns experiments at three different beam pointing angles: (a) inward pointing, (b) symmetric, and (c) outward point ing. This illustration demonstrates that the fundamental hohlraum asymmetry is the long-wavelength $P_{2}$ asymmetry and can be controlled with beam pointing. (20-03-0995-2125pb01) 
Design for the National Ignition Facility" on p. 215, the NIF baseline target now uses an initial gas fill to displace the Au blowoff. Nova experiments with gas-filled hohlraums have also demonstrated control of symmetry. However, these experiments are still in progress and therefore will be reported at a later time.

\section{Modeling the Experiments}

We use our 2-D LASNEX computer $\operatorname{code}^{18}$ to perform detailed modeling of these experiments. Figure 4 is a cut-away, at $t=0$, from a simulation of a hohlraum containing a pure-plastic capsule that is irradiated by a "realistic" 2-D representation of a three-dimensional (3-D) Nova laser beam. ${ }^{19}$ The wall materials, laser power vs time, etc., of a given simulation are our best estimate of what was used in the experiments we are trying to model. To model a given symmetry scaling, we performed a number of simulations with different beam. pointings. At stagnation, our simulated capsules, like real capsules, produce a burst of $x$ rays that can be imaged. A post-processor simulates the actual x-ray diagnostics, producing synthetic images that vary with pointing from oblate or prolate (like the experiment). The ratio of the image's full-width at half maximum (FWHM), perpendicular to the polar axis to the FWHM along the polar axis is the "distortion," the quantity we vary with pointing and compare with experiment.

Our calculations start off fully Lagrangian (matter is fixed in the zones of a moving mesh). Later in time, after a considerable amount of blowoff has filled the hohlraum, we perform a major rezone and change our numerical scheme. The main part of the hohlraum becomes Eulerian (matter flows through a fixed mesh), allowing the calculation to run in spite of the large sheer flows. Most of the capsule, however, remains Lagrangian - the accepted procedure for modeling nearly spherical implosions. We interface the Eulerian and Lagrangian regions with a stretching region that maintains equal-ratio zoning-a hybrid mesh that has matter flowing through it while moving slowly. Using three numerical schemes in the same calculation allows us to simulate both the main hohlraum and the capsule with the most appropriate numerical technique.

To provide an example of how we model a given scaling, consider a series of shots with Au hohlraums (1600- $\mu$ m-diam with 1200- $\mu$ m-diam LEHs lined with $1500-\AA \mathrm{Ni}$ ). In this series, to keep LEH effects approximately the same for all pointings we varied the hohlraum length with pointing so that the beams always crossed in the plane of the LEH. We used the nominal capsules, defined earlier, and irradiated these targets with PS22. The self-emission $x$-ray images from the imploded capsule were the key observations made on this (and all other) scaling series. Our x-ray diagnostics were time-resolved ( 100-ps frame time) and time-integrated cameras filtered to measure emission $>3 \mathrm{keV}$ from the Ar fuel dopant.

Figure 5 displays the results of the PS22 series experimental scaling and compares it with our model. The solid circles are distortions from our experiments as a function of beam pointing. The horizontal error bar shows an estimated \pm 50 - $\mu \mathrm{m}$ systematic uncertainty in the absolute pointing of the beams (the relative shotto-shot pointing jitter is believed to be considerably smaller than this). The open circles are modeling distortions. Both experiment and modeling agree that we can control Nova symmetry by varying the beam pointing. Also, they both produce about the same

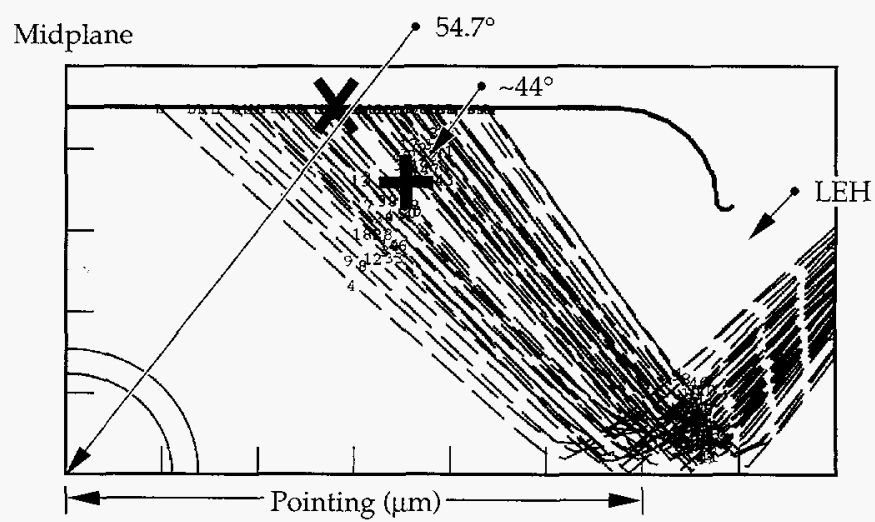

FIGURE 4. Cutaway at $t=0$ from a 2-D simulation of a hohlraum containing a pure-plastic capsule with a realistic representation of Nova's beams. The calculation is cylindrically symmetric around the horizontal axis and left-right symmetric across the midplane. The beams enter through LEHs at the ends of the hohlraum. Pointing is the distance between the midplane and where the beams cross (i.e., the reflection off the horizontal, rotational axis of symmetry). The " $X$ " and " + " show the center of emissivity early in time at $1.4 \mathrm{~ns}$, respectively. (20-03-0995-2126pb01)

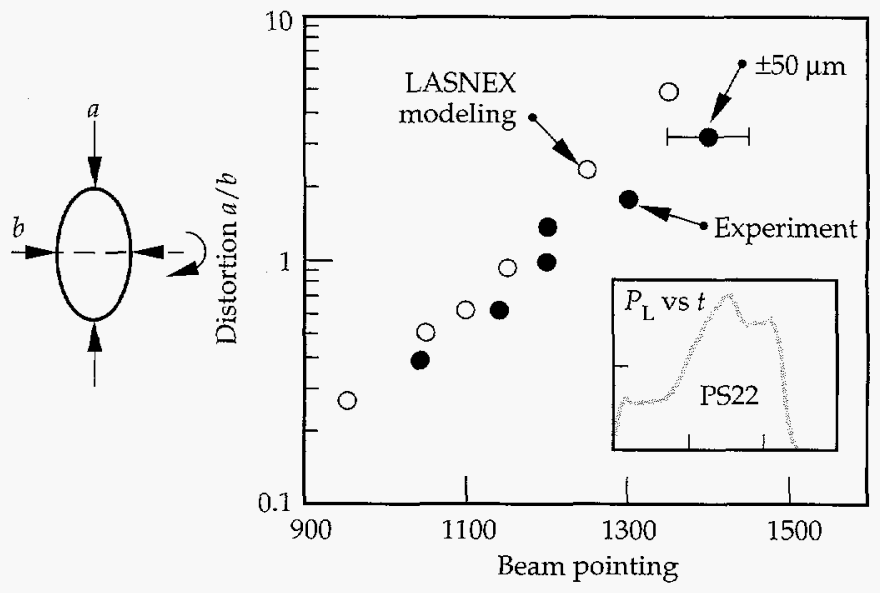

FIGURE 5. Capsule image distortion vs pointing from a series using Ni-lined hohlraums irradiated by PS22. (Inset: Relative laser power vs time for PS22.). Experimental uncertainty is $\pm 50 \mu \mathrm{m}$. (20-03-0995-2127pb01) 
pointing of best symmetry $(\sim 1200 \pm 50 \mu \mathrm{m}$ in experiment and $\sim 1150 \mu \mathrm{m}$ in modeling).

The pointing of best symmetry changes as we vary the pulse shape. For example, another scaling series used fixed length (2700- $\mu \mathrm{m})$, Ni-lined Au hohlraums irradiated with 1-ns flat-top pulses. In this series, both modeling and experiment also verified that we can control Nova symmetry by varying the beam pointing. However, for this pulse shape, the pointing of best symmetry is about $100 \mu \mathrm{m}$ outward from the best pointing found in the PS22 series. For this 1-ns experiment, the pointing of best symmetry is $\sim 1320 \pm 50 \mu \mathrm{m}$ in experiment and $\sim 1250 \mu \mathrm{m}$ in modeling.

\section{Scaling of the Pointing of Best Symmetry}

Figure 6 summarizes our ability to estimate the pointing of best symmetry over our nine symmetry scaling databases. It plots the pointing of best symmetry inferred from experiment against that of our integrated LASNEX simulations. Overall, we find the agreement to be very satisfactory. The vertical error bars in this plot indicate only the uncertainty in the pointing of best symmetry extracted from each experimental dataset, using the nominal pointing. The error bars do not include the systematic uncertainty in Nova's absolute pointing ( $50 \mu \mathrm{m})$, which would allow all the points to be moved as a group, either up (toward poorer agreement) or

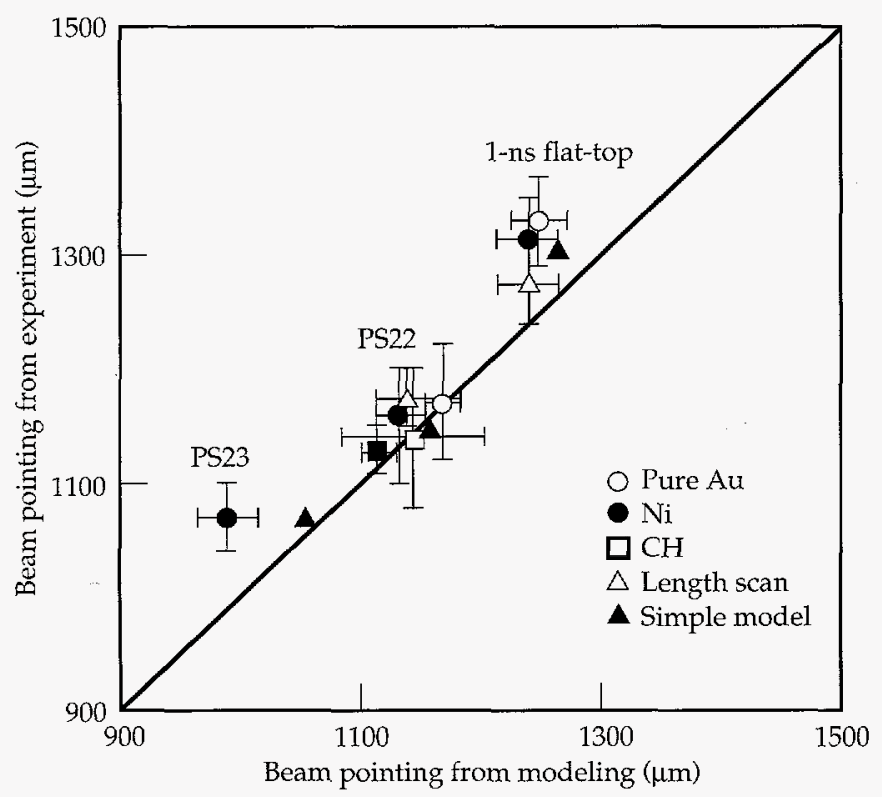

FIGURE 6. Pointing of best symmetry in our experiment vs pointing of best symmetry from our modeling. The longer the pulse shape, the farther in the beams need to be moved to get good symmetry. The solid triangles plot the pointing to compare with Eq. (2). (20-03-0995-2128pb01) down (better agreement). Regardless of systematic differences, the most significant and apparent feature of the plot is this: the longer the pulse shape, the farther in we must point the beams to get good symmetry.

The reason for this is found in the basic principles of symmetry scaling in Nova-type hohlraums. First, there are hot, laser-produced emission rings that migrate toward a smaller polar angle (when viewed from the capsule position) because of bulk plasma evolution. We refer to this migration as "spot motion." Second, there is also an "optimal" polar angle for the rings where time-integrated pole-flux equals equator flux ( $\sim 48^{\circ}$ for these hohlraums). Third, to get good symmetry we must point the beams so the emission rings pass through $48^{\circ}$ when we deliver $\sim 50 \%$ of a shape's useful energy. Since spot velocity is weakly dependent on laser intensity, we move the beams farther inward with longer pulses.

In our simulations, there are three components to spot motion. First, dense plasma evolution from the cylindrical walls causes the laser deposition region to move inward and, because of the beam geometry, toward the LEH. Second, there is a refractive component off plasma that accumulates on axis. Third, there can be a low-intensity volume emission when $\mathrm{Au}$ blowoff fills the hohlraum, pulling the average center of emissivity farther down from the walls. In our simulations, which use a nonLTE, average-atom atomic physics model, ${ }^{20}$ the Au blowoff is optically thin to thermal radiation. Consequently, volume absorption does not play a significant role in determining the capsule flux.

To see how spot motion can cause the scaling shown in Fig. 6, first recall that the fundamental asymmetry in a left-right symmetric, Nova-like hohlraum is a longwavelength, pole-to-waist flux variation that varies like the $P_{2}$ Legendre polynomial. ${ }^{2}$ Whether the asymmetry is pole high or equator high depends on the polar angle to the center of the laser-produced, x-ray emission ring. In an idealized hohlraum without LEHs and with otherwise uniform walls, the drive asymmetry will clearly be pole high when the emission ring is at a very small polar angle and equator high when the emission ring is near the midplane of the hohlraum. Somewhere in between, the pole and equator fluxes will be equal. In a spherical hohlraum, the $P_{2}$ component of capsule flux vanishes when the $P_{2}$ component of the source flux is zero. ${ }^{2}$ This occurs when the "center of emissivity" of the emission ring is at the polar angle where $P_{2}$ is zero, $54.7^{\circ}$. For larger angles, the flux onto the capsule will be equator high, and for smaller angles, pole high.

An LEH modifies this description quantitatively, but not qualitatively. To compensate for the lack of wall radiation from the $\mathrm{LEH}$, the angle to the center of emissivity in a spherical hohlraum must be smaller 
than $54.7^{\circ}$ for the net $P_{2}$ component to vanish. Exactly how much smaller than $54.7^{\circ}$ is a function of both the emission-ring to background-wall intensity ratio and the LEH size. For example, the angle where the $P_{2}$ component of the source vanishes is about $44^{\circ}$ in an idealized spherical hohlraum with wall and LEH areas the same as ours and a wall albedo (which determines the emission-ring to background-wall intensity ratio) of $\sim 0.7$. This albedo is typical of a rising, nanosecondscale pulse shape. In our more detailed LASNEX simulations, we find the pole:waist fluxes are balanced when the center of emissivity is at $\sim 48^{\circ}$. These simulations include higher $l$-mode components, volume emission, and mode-coupling due to having a sphere inside a cylinder.

With this background, we can simply interpret features found in our LASNEX modeling. Figure 7 plots ratios of capsule ablation pressure at the pole to that at the equator vs time in pure Au hohlraums near the pointing of best symmetry for each pulse shape. Early in time, the ablation pressure is equator high. Later in time, the pressure is pole high. Analysis of our simulations indicates that this time-dependent asymmetry is produced mainly by spot motion. As shown in Fig. 4, the angle to the center of the beam at $t=0$ for PS22 is $>54.7^{\circ}$. Quantitative analysis shows the center of emissivity to be located at the " $X$ " in Fig. 4 at $\sim 57^{\circ}$. The simple symmetry arguments lead us to expect the drive to be equator high, as Fig. 7 shows in the early PS22 curve. By $1.4 \mathrm{~ns}$, spot motion has caused the center of emissivity to move to the " + " in Fig. 4 to $\sim 44^{\circ}$.

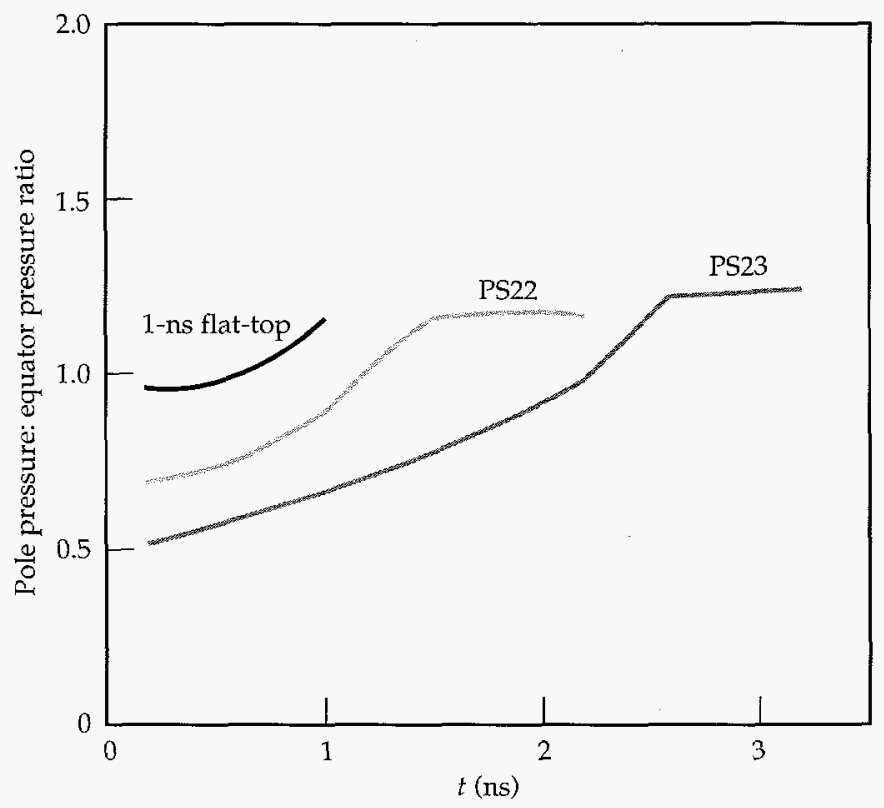

FIGURE 7. Ratio of capsule ablation pressure at the pole to the ablation pressure at the equator vs time for our three pulse shapes. (20-03-0995-2129pb01)
There, the ablation pressure ratio has become about $10 \%$ pole high.

Spot motion, the migration of the radiation production region to smaller polar angles, causes a simulated Nova-type hohlraum to have the characteristic equatorhigh to pole-high asymmetry swing shown in Fig. 7.

For all three pulse shapes, we find that near the pointing of best symmetry the center of emissivity sweeps through the "optimal angle" (where pole pressure $=$ equator pressure, $\sim 48^{\circ}$ for these hohlraums) when $\sim 50 \%$ of a shape's useful energy has been delivered. This $50 \%$ value makes sense. If the pressures were equal when a very different fraction of energy was delivered, the implosion would be dominated by either pole-high or equator-high flux and would be obviously distorted.

Therefore, since spot velocity depends weakly on laser power $P_{L}$, longer pulses need more inward pointing for best symmetry because $t_{50 \%}$, the time to deliver $\sim 50 \%$ of the energy, is longer, leading to more spot motion.

LASNEX simulations show spot-angular velocity to be very weakly dependent on $P_{\mathrm{L}}$. Figure 8 is a plot of the angle to the center of emissivity vs time for our three pulse shapes (refer to Fig. 3). Over the period when the first $50 \%$ of the laser energy is delivered, the angular velocity $d \theta / d t$ of the center of emissivity in our simulations increases only as the logarithm of $P_{\mathrm{L}}$ (measured in TW) closely following

$\frac{d \theta}{d t}=\frac{6^{\circ}}{\mathrm{ns}}+\frac{3 \cdot 9^{\circ}}{\mathrm{ns}}\left(\log _{10} P_{\mathrm{L}}\right)$

We can couple this expression for spot motion with the need to have the center of emissivity at $\sim 48^{\circ}$ when $\sim 50 \%$ of the laser energy has been delivered to produce a simple expression for the pointing of best symmetry

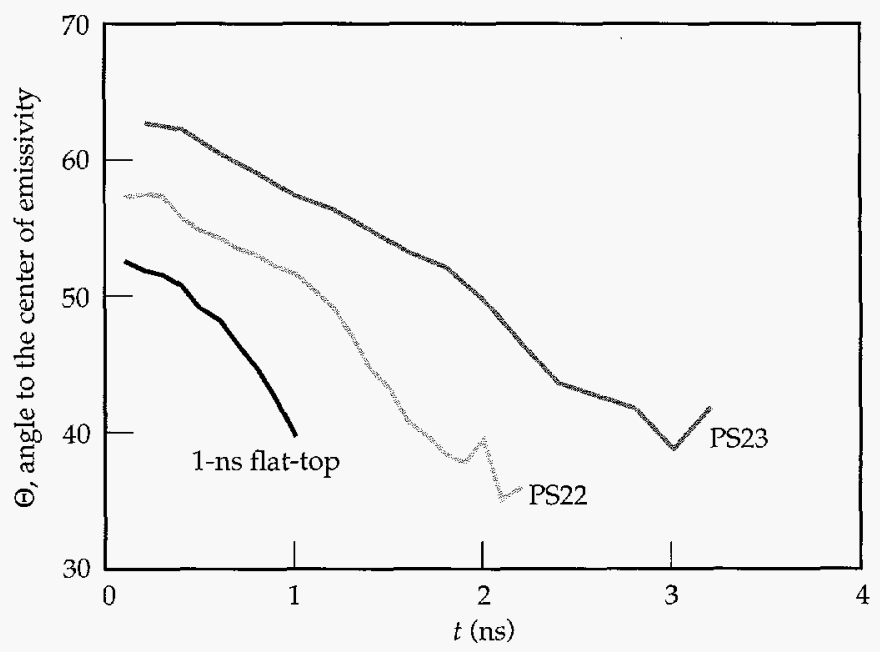

FIGURE 8. Angle to the center of emissivity vs time in our LASNEX simulations of the three pulse shapes. The angular velocity during the period when the first half of a pulse shape's energy is delivered varies slowly with laser intensity. (20-03-0995-2130pb01) 
best pointing $=671 \mu \mathrm{m}+\left[\frac{800 \mu \mathrm{m}}{\tan \left(48^{\circ}+\frac{d \theta}{d t} \times t_{50 \%}\right)}\right]$.

This expression is for our standard $800-\mu \mathrm{m}$ radius hohlraums and Nova's $50^{\circ}$ half-cone angle. The results of this very simple model are plotted as the filled triangles in Fig. 6 and also agree well with our database.

\section{Spot Motion}

Since 1986, we have performed separate experiments to observe laser-produced $x$-ray emission spots and their migration..$^{21}$ We cut an $800 \mu \mathrm{m} \times 1200 \mu \mathrm{m}$ rectangular observation port into the side of a hohlraum through which we take time-resolved $x$-ray images of the opposite wall with a three-channel $x$-ray framing camera that takes snapshots at four different times. ${ }^{22}$ Our field of view includes the initial spot of a beam as well as some of the surrounding region. Figure 9 shows the images from the $450-\mathrm{eV}$ channel for a pure Au PS22 experiment.
Qualitatively, these images verify that there are spots and that they do migrate toward the LEH. Quantitatively, we analyze these images to find the center of emission, within the port's field of view, at various times. For comparison, we quantitatively analyze LASNEX simulations of the experiment with a postprocessor that mimics the imaging diagnostic and the analysis. Figure 10 shows such a comparison, plotting the position of the center of emission for the $450-\mathrm{eV}$ channel from pure Au PS22 simulations and experiments. The good comparison with LASNEX shows that we can calculate the component of spot motion observed-the component along the hohlraum wall. The data from four different shots show that the motion is reproducible. Reproducible spot motion has also been indirectly corroborated by Precision Nova shots that produced nearly identical capsule shapes and performances for five PS22 shots at one pointing and four shots at another (see Fig. 11).

\section{Time-Dependent Asymmetry}

An essential feature of theory and simulation is that the asymmetry in a Nova-like hohlraum will vary over

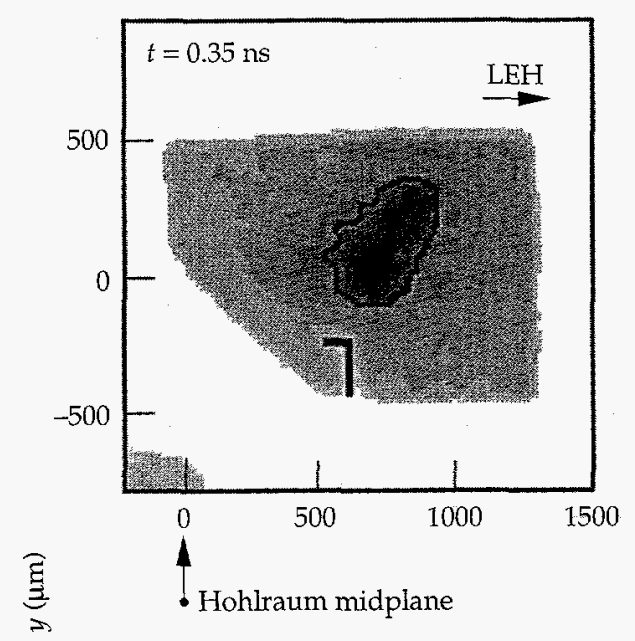

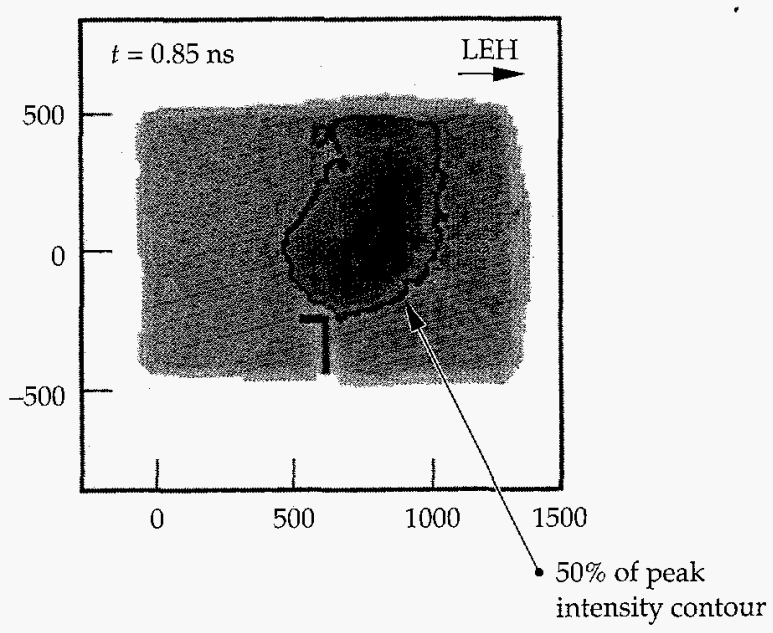

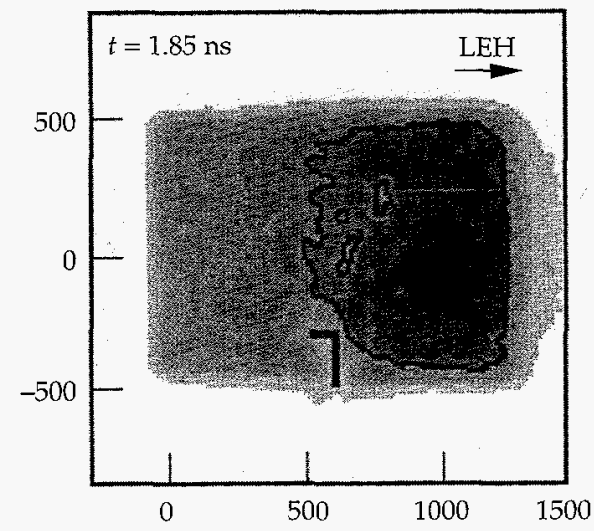

FIGURE 9. 450-eV images of spots inside a pure Au hohlraum during a PS22 experiment. The black " $7 "$ is a spatial fiducial. These images show a well defined soft $x$-ray spot that moves toward the LEH.

(20-03-0995-2131pb01)

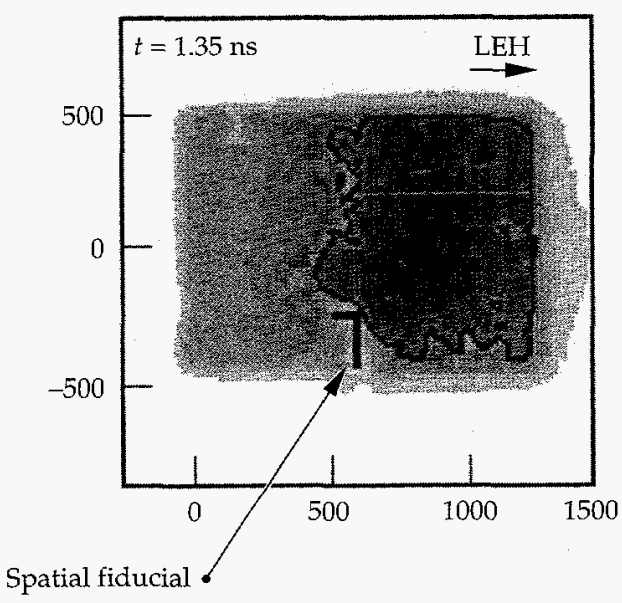

$z(\mu \mathrm{m})$ 
time. This causes the variation in the pole-to-equator ablation pressure ratio shown in Fig. 7. Time-dependent asymmetry results both from changing hot spot to background-wall ratio and from spot motion.

Experiments provide persuasive evidence that the time-dependent asymmetry in Nova hohlraums is about as we expect. The hot spot to background-wall ratio is largely determined by the albedo of the Au wall. Separate measurements of Au wall losses ${ }^{23}$ indicate that LASNEX accurately estimates Au albedo at standard Nova temperatures $(-220 \mathrm{eV})$. The spot-motion experiments described earlier are qualitatively and quantitatively close to what we expect. Finally, the changes in the pointing of best symmetry (Fig. 6) with pulse shape indicate that the variation in flux at the capsule is qualitatively what we expect-the asymmetry goes from waist high early in time to pole high later in time.

To understand this final point, consider PS22 at its experimental pointing of best symmetry, $1200 \mu \mathrm{m}$. According to Fig. 7, the first 1 ns of PS22 should provide a waist-high radiation flux that drives a prolate implosion. However, our 1-ns implosions at $1200 \mu \mathrm{m}$ are prolate, showing that at $\sim 20$ TW the first $1 \mathrm{~ns}$ of drive at this pointing is predominantly waist high. Because the first nanosecond of PS22 is actually at much lower power, $\sim 6 \mathrm{TW}$, we expect it to also be predominantly waist high since there is (slightly) less spot motion at lower power, and spot motion is needed to get into the pole-high regime. Moreover, we argue that the entire first nanosecond should be waist high. If the PS22 flux did change to pole high before $1 \mathrm{~ns}$, so much

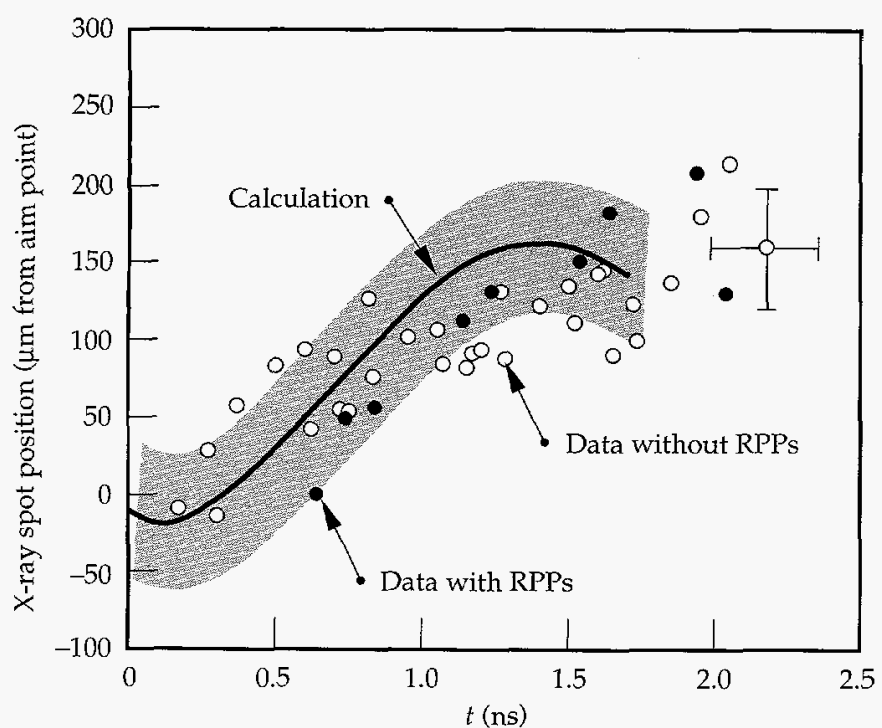

FIGURE 10. Distance from the hohlraum midplane to the center of emission at $h v \approx 450 \mathrm{eV}$, vs time in a pure Au hohlraum irradiated by PS22. We observe spots that migrate about as expected from simulations. (20-03-0995-2132pb01) of the PS22 energy would be generating pole-high flux that $1200 \mu \mathrm{m}$ would not be PS22's pointing of best symmetry. Continuing the argument, since the early part of PS22 is waist high, some latter part of PS22 must be pole-high to compensate for this early-time asymmetry. Consequently, the scaling of the pointing of best symmetry with pulse length (shown in Fig. 6) is evidence that the time-dependent asymmetry goes from waisthigh early in time to pole-high later in time, therefore corroborating the qualitative behavior of Fig. 7 .

Other experiments are ongoing to more directly measure the time-dependent asymmetry in Nova hohlraums. One approach, proposed by Wilson, ${ }^{24}$ uses the full pulse with a series of capsules designed to implode at different times during the pulse. This is accomplished

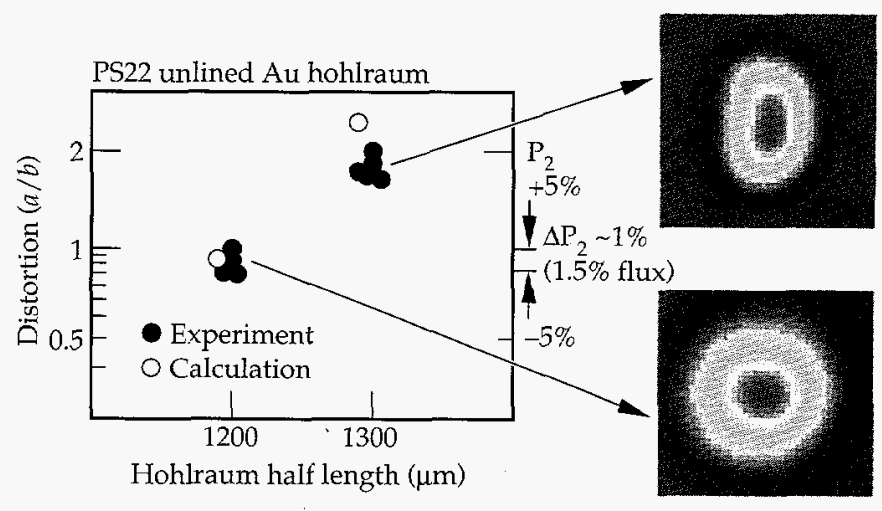

FIGURE 11. Precision Nova implosions have shown excellent reproducibility of symmetry. (08-00-1293-4416pb01) (a)
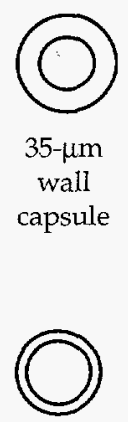

$20-\mu \mathrm{m}$

wall

capsule (b)
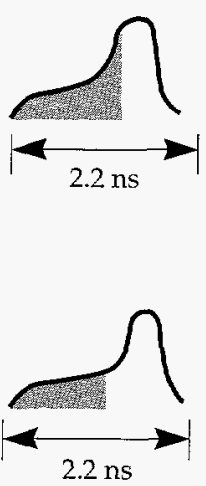

(c)
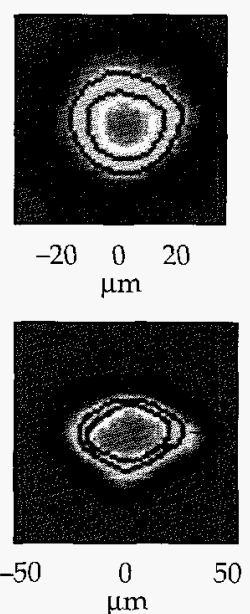

(d)

$(a / b)_{\text {expt. }}=0.92$ $(a / b)_{\text {theo. }}=0.96$

$(a / b)_{\text {expt. }}=0.68$ $(a / b)_{\text {theo. }}=0.71$

FIGURE 12. Data from PS22 using the variation in implosion time of capsules with different wall thickness: (a) initial capsule configuration; (b) shaded portion of the drive represents the effective sampling interval for the implosion for the two cases; (c) implosion image data taken orthogonal to the hohlraum axis; (d) comparison of the measured capsule eccentricity with the calculated value. (02-08-1094-3596pb03) 
by keeping the capsule's i.d. approximately fixed and thinning the ablator to significantly less than its standard $55-\mu \mathrm{m}$ thickness. The thinnest capsules view only the early-time asymmetry, and a record of the evolution of asymmetry can be obtained. Figure 12 shows results of this technique for PS22 and Au hohlraums. ${ }^{25}$ Another technique replaces the capsule with a uniform sphere of material. The $x$-ray flux in the hohlraum will drive a shock into this material, which can be imaged by $x$-ray backlighting (see Fig. 13). Distortion of the shock front is approximately related to the drive pressure nonuniformity by

$\frac{d}{d t}\left(r_{\text {equator }}-r_{\text {pole }}\right) \approx \frac{1}{2} \sqrt{\frac{\bar{P}}{\rho}}\left(\frac{P_{\text {pole }}}{P_{\text {equator }}}-1\right)$.
For PS22, Fig. 13 shows the calculated ratio of $P_{\text {pole }} / P_{\text {equator }}$, which can then be compared with the measurement. The average pressure $\bar{P}$ can be obtained from the shock velocity. Figure 14 shows the results from a Nova experiment. ${ }^{26}$ In this figure, $A_{2}$ is the second Legendre coefficient of the position of the shock trajectory, and $A_{0}$ is the average distance moved by the shock. The numerical calculation and the data are in agreement for this experiment. Calculations ${ }^{27}$ show that, with the current resolution of about $2 \mu \mathrm{m}$ in the shock position, time variations in NIF target fluxes can be obtained to about $2 \%$.

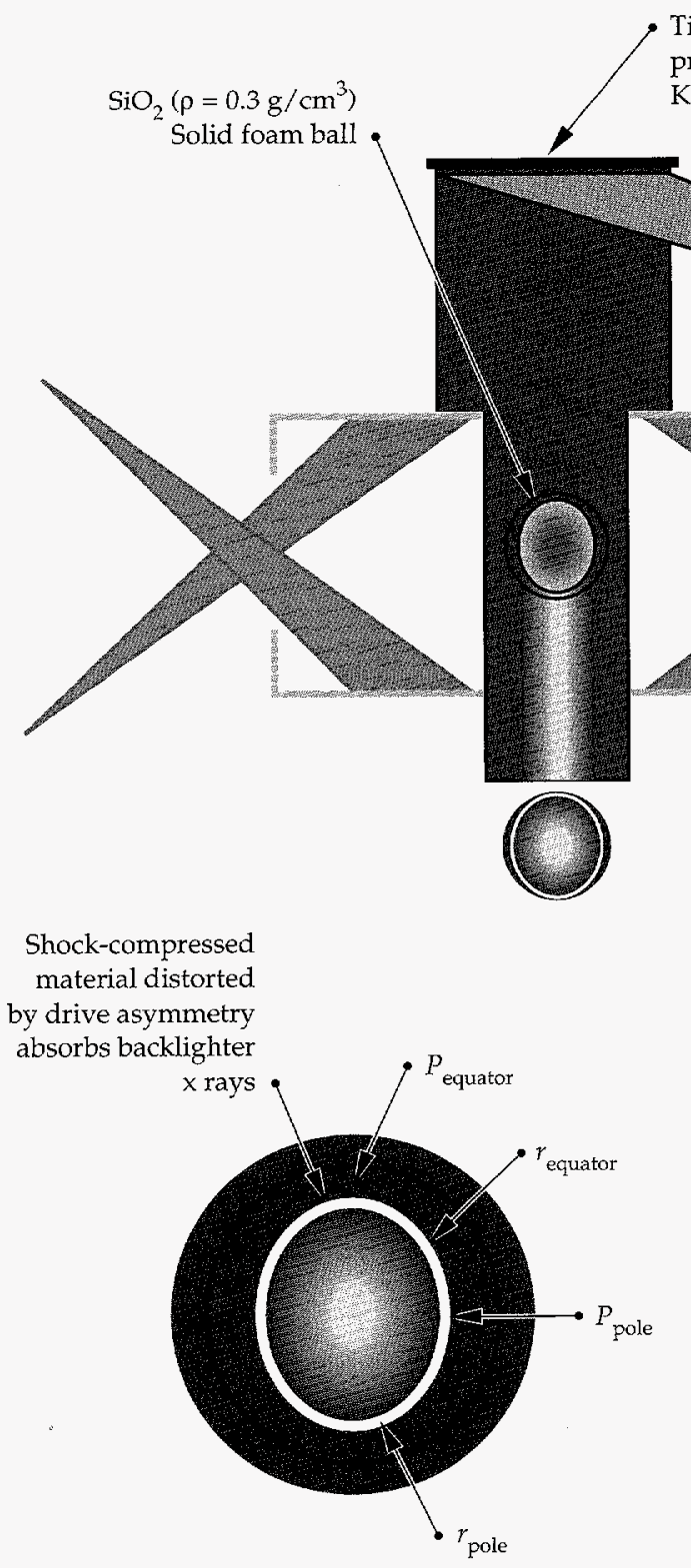

Ti backlighter disk producing $4.7-\mathrm{keV}$ K-shell emission
Backlighter beams:

$8.0 \mathrm{~kJ}, 2$ ns pulse at

$2 \omega$ delayed $\sim 0.5 \mathrm{~ns}$
FIGURE 13. X-ray radiography can be used to infer timedependent asymmetry from imaged shock distortion. (50-04-1093-3882Apb03)

$$
\frac{d}{d t}\left(r_{\text {equator }}-r_{\text {pole }}\right) \sim \frac{\bar{P}^{1 / 2}}{2}\left[\frac{P_{\text {pole }}}{P_{\text {equator }}}-1\right]
$$

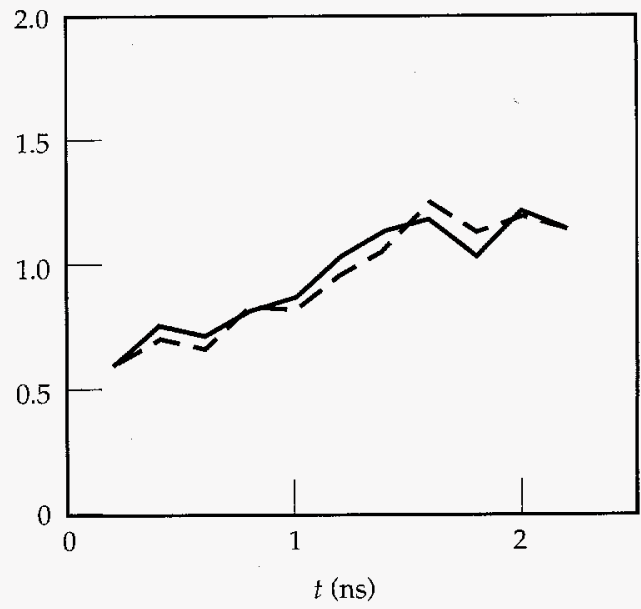




\section{The Role of the LEH}

Figure 15 summarizes the main effect an LEH can have on simulated beam propagation. It shows rays and electron density contours 2 ns into PS22. For nominal pointing shown in Fig. 15(a), when Nova's beams initially cross in the plane of the LEH, all rays are far from the blowoff that expands from the lip. Consequently, they are unaffected by LEH blowoff. However, Fig. 15(b) shows what can happen if we move the beams farther inward, close to the LEH. The part of the beam closest to the LEH intersects blowoff dense enough to refract those rays downward. Since these rays otherwise strike closest to the midplane of the hohlraum, the effect of the refraction is to shift the center of deposition toward the LEH. This shifts the asymmetry back in the pole-high direction. Note, however, that even when there is considerable interaction between the rays and the lip blowoff, the density distributions shown in Fig. 15 are very similar. This implies that there is no significant amount of additional blowoff produced by the interaction. These LEH results basically reproduce earlier findings of Lasinski, ${ }^{28}$ who modelled an isolated LEH as a thin bracelet of material subjected to both a radiation source and a laser source. Her modeling agrees quite well with available data on radiation-driven LEH hydrodynamics. 28,29

Modeling and experiment show that bringing the beam too close to the LEH can cause different symmetry behavior, as shown in Fig. 15(b). Consider a case

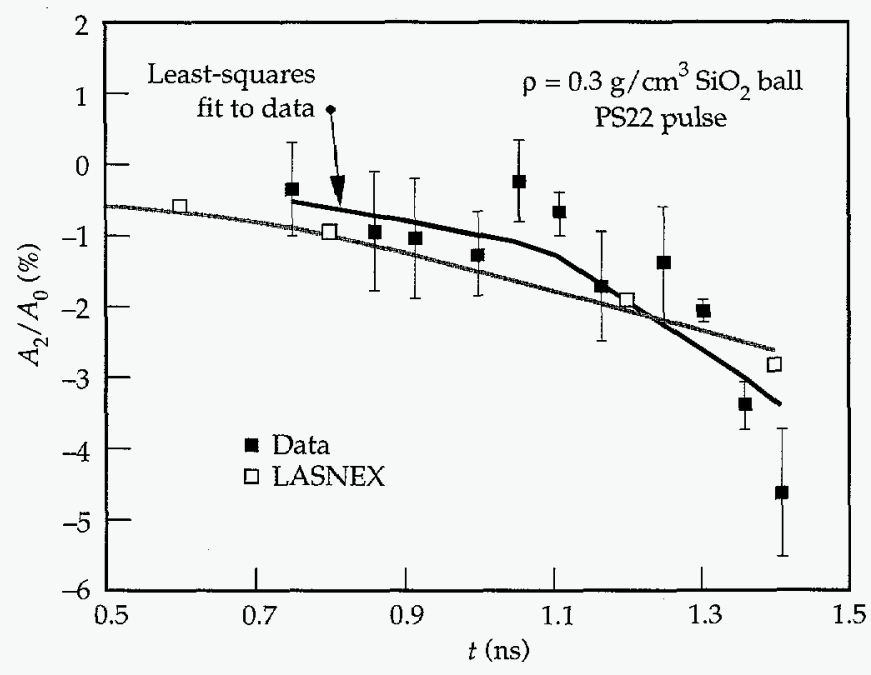

FIGURE 14. The foam witness ball technique can measure pressure asymmetries with nearly $10 \%$ accuracy on Nova. $A_{2}$ is the second Legendre coefficient of the shock trajectory. $A_{0}$ is the average shock position. (50-04-1093-3891 Apb02) where the LEH should have no effect- the distortion vs pointing scaling shown earlier in Fig. 5 . This is the Ni-lined, PS22 scaling where the hohlraum length changed with pointing. In the experiments of Fig. 5, we expect the laser-LEH interaction to always be the nominal situation shown in Fig. 15(a). The straight-line scaling of Fig. 5 indicates there is no obviously "different" symmetry regime at inner pointing. (a) Nominal pointing

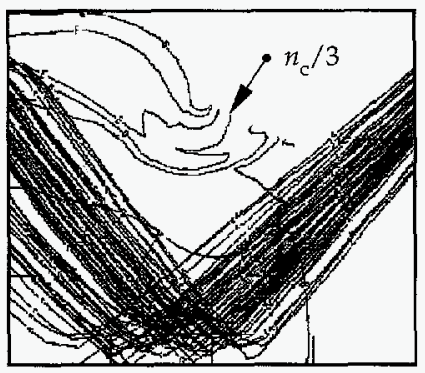

(b) Beam farther inward

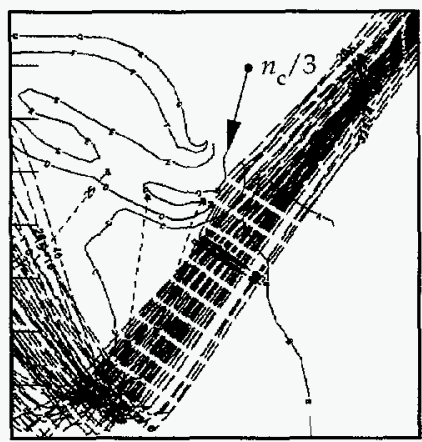

FIGURE 15. Simulated laser rays and density contours 2 ns into PS22 in a Ni-lined hohlraum. (a) With nominal pointing the laser is sufficiently far from the cylindrically expanding blowoff from the LEH lip that there is no significant interaction. (b) If the beam is moved too far inward, it can intercept relatively dense plasma and be partially refracted. (20-03-0995-2133pb01))

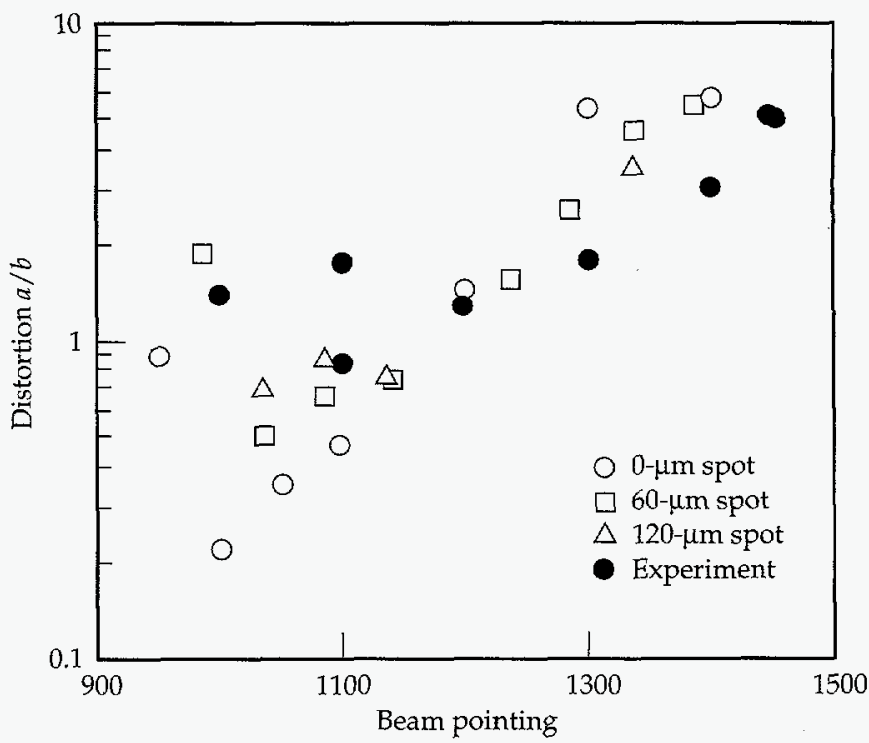

FIGURE 16. Symmetry scaling experiments (solid circles) in fixed length hohlraums show a "different" behavior at inner pointing than experiments where we vary the length with the pointing (see Fig. 5). This trend is reproduced in our simulations where we see it is due to refraction such as shown in Fig. 11(b). (20-03-0995-2134pb01)) 
Figure 16 plots simulations and experiments where the symmetry at inner pointing is different, showing a $\mathrm{Ni}$-lined, PS22 scaling performed with fixed-length $(2700-\mu \mathrm{m})$ hohlraums. At inner pointing, the beams approach the LEH and can be refracted. Comparison with Fig. 5 shows a different behavior at inner pointing.

Similar to the experiment, the LASNEX modeling shows a different symmetry regime can be found at inner pointing with fixed-length hohlraums. This is due to refraction by LEH blowoff. The size of the effect depends on the details of the beams. We studied this effect with simulations of three different realizations of Nova's beams, all of them using the 3-D geometric optics raytrace algorithm devised by Friedman. ${ }^{19}$ The nominal beam representation has, at best focus, a $60-\mu \mathrm{m}$-diam, 1 -sigma circle of confusion. This produces the rays shown in Figs. 4 and 15 and is a best-guess lower bound on the wings of Nova's beams. This beam representation produces the open squares of Fig. 16.

In addition to the $60-\mu \mathrm{m}$ circle of confusion, we simulated beams with $120-\mu \mathrm{m}$-diam circles of confusion (upper bound to Nova) and 0- $\mu \mathrm{m}$-diam circles of confusion (better than diffraction limited). The $0-\mu \mathrm{m}$ circle of confusion beams (open circles of Fig. 16) cause no break in the distortion vs pointing scaling until the beam is so far in that it strikes the LEH. The $120-\mu \mathrm{m}$ circle of confusion (open triangles of Fig. 16) representation shows somewhat more of an inner pointing refractive effect than the nominal beam representation.

The scalings of Fig. 16, together with those of Fig. 5, are evidence that the LEH can have an effect on symmetry. They also indicate that LASNEX can estimate when, and roughly how much, an LEH will have a major effect on hohlraum symmetry.

\section{Summary}

Our 2-D LASNEX simulations of Nova's nine symmetry scaling databases reproduce the fundamental features seen in the experiments. In particular, we predict how we must change Nova's beam pointing to achieve best symmetry with various pulse shapes. Analysis indicates that the need to change pointing with different pulse shapes is a result of spot motion. Complementing direct-symmetry measurements, we have also observed and modelled hot spots in hohlraums. We find hot-spot motion to be real, reproducible, and very close to what we expect from LASNEX. These measurements also indicate that time-dependent asymmetry in the Nova hohlraums behave close to our modeling. Experiments and modeling also indicate that we estimate when, and roughly how much effect, an LEH will have on hohlraum symmetry.

\section{Notes and References}

1. Laser Program Annual Report-1979, 2-77 to 2-88, Lawrence Livermore National Laboratory, Livermore, CA, UCRL-50055-79 (1979, report SRD); Laser Program Annual Report-1980, 2-10 to 2-22, Lawrence Livermore National Laboratory, Livermore, CA, UCRL-50055-80 (1980, report SRD).

2. J. Green, Research and Development Associates, private communication (1980); M. Murakami and K. Nishihara, Jpn. J. Appl. Phys. 25, 242 (1986); A. Caruso, Internal Confinement Fusion (Proc. Course and Workshop, Varenna, 1988), Casa Editrice Compositori, Bologna (1988) 139.

3. Laser Program Annual Report-1984, 2-13, Lawrence Livermore National Laboratory, Livermore, CA, UCRL-50055-84, M-3679, C-72 (December 1985, report SRD).

4. S. W. Haan, "Radiation Transport Between Concentric Spheres," Lawrence Livermore National Laboratory, Livermore, CA, UCRL-ID-118152 (1994); J. Green, Research and Development Associates, private communication (1983).

5. Laser Program Annual Report-1983, 2-21, Lawrence Livermore National Laboratory, Livermore, CA, UCRL-50055-83 M3679, C-72 (September 1984, report SRD).

6. Laser Program Annual Report-1985, 30-31, Lawrence Livermore National Laboratory, Livermore, CA, UCRL-50055-85 M3679, C-72 (September 1986, report SRD).

7. A. Burnham, J. Grenz, and E. Lilley, J. Vac. Sci. Technol. A 5 (6), 3417 (1987).

8. Laser Program Annual Report 1986/87, 2-50 to 2-54 and 4-25 to 4-34, Lawrence Livermore National Laboratory, Livermore, CA, UCRL-50055-86/87 (1988, Title U, report SRD); Laser Program Annual Report: Target Design 1988, 51-75, LLNL, XDIV-90-0054 (Title U, report SRD); ICF Program Annual Report 1988/89 (U), 192, LLNL, UCRL-LR-116901-88/89 (report SRD).

9. D. B. Ress, Lawrence Livermore National Laboratory, Livermore, CA, private communication (1995).

10. A. Hauer, Los Alamos National Laboratory, Los Alamos, NM, private communication (1992).

11. J. D. Kilkenny P. Bell, R. Hanks, G. Power, R. E. Turner, and J. Wiedwald, Rev. Sc. Instrum. 591793 (1988).

12. A. Hauer, Los Alamos National Laboratory, Los Alamos, NM, private communication (1995).

13. ICF Program Annual Report 1988/89 (U), 9, Lawrence Livermore National Laboratory, Livermore, CA, UCRL-LR-116901-88/89 (report SRD).

14. A. Hauer, et. al., Rev. Sci. Instrum 66(1), 672 (1995).

15. L. J. Suter, A. A. Hauer, L. V. Powers, D. B. Ress, et al., Phys. Rev. Lett. 73(17), 2328 (1994).

16. R. E. Turner and L. J. Suter, Bull. Am. Phys. Soc. 1 (1988); Y. Kato, et. al., Proc. 13th Int'l Conf. Plasma Physics and Controlled Nuclear Fusion Research, Washington, DC, 1990.

17. Laser Program Annual Report: Target Design 1988 (U), 223, Lawrence Livermore National Laboratory, Livermore, CA, XDIV-90-0054 (May 1990, report SRD).

18. G. Zimmerman and W. Kruer, Comments Plasma Phys. Controlled Fusion 2, 85 (1975).

19. A. Friedman, Laser Program Annual Report 83, 3-51, Lawrence Livermore National Laboratory, Livermore, CA, UCRL-50021-83 (1983).

20. D. E. Post, R. V. Jensen, C. B. Tarter, W. H. Grasberger, and W. A. Lokke, Atomic Data and Nuclear Tables 20(5) (Nov 1977); G. B. Zimmerman and R. M. More, JQSRT 23, 517 (1980).

21. D. Ress, L. V. Powers, L. J. Suter, R. J. Wallace, and F. Ze, Bull. 
Am. Phys. Soc. 38, 1885 (1993); L. V. Powers, D. B. Ress, L. J. Suter and F. Ze, Defense Research Review 6(10), (1994, report SRD).

22. G. Glendinning, Lawrence Livermore National Laboratory, Livermore, CA, private communication (1995).

23. J. L. Porter, T. J. Orzechowski, M. D. Rosen, A. R. Thiessen, et al., ICF Quarterly Report 4(4), 125-131, Lawrence Livermore National Laboratory, Livermore, CA UCRL-LR-105821-94-4 (1994).

24. D. Wilson, Los Alamos National Laboratory, Los Alamos, NM, private communication (1992).

25. A. A. Hauer, "X-Ray Driven Implosions in Laser Heated
HohIraums," Laser Plasma Interactions, Eds., M. Hooper and A. Cairns (Redwood Burn, Trowbridge, England, 1995), vol, 5, pp. 5-24.

26. P. Amendt, S. G. Glendinning, B. A. Hammel, R. G. Hay, and L. G. Suter, Rev. Sci. Instrum. 66(1), 785 (1995).

27. P. Amendt, Lawrence Livermore National Laboratory, Livermore, CA, private communication (1995).

28. B. F. Lasinski, Lawrence Livermore National Laboratory, Livermore, CA, private communication (1990).

29. C. Gomez, Los Alamos National Laboratory, Los Alamos, NM, private communication (1991). 


\section{LASER-Plasma INTERACTIONS IN NIF-ScAlE Plasmas (HLP5 AND HLP6)}

$\begin{array}{lllll}\text { B. MacGowan } & \text { M. Casanova* } & \text { K. Estabrook } & \text { R. Kirkwood } & \text { J. Moody } \\ \text { R. Berger } & \text { B. Cohen } & \text { S. Glenzer } & \text { W. Kruer } & \text { D. Munro } \\ \text { J. Fernandez** } & \text { D. Desenne* } & \text { D. Hinkel } & \text { A. Langdon } & \text { L. Powers } \\ \text { B. Afeyan } & \text { D. Dubois** } & \text { D. Kalantar } & \text { B. Lasinski } & \text { H. Rose* } \\ \text { C. Back } & \text { A. Dulieu* } & \text { R. Kauffman } & \text { D. Montgomery } & \text { C. Rousseaux* } \\ \text { G. Bonnaud* } & \text { R. Turner } & \text { B. Wilde** } & \text { S. Wilks } & \text { E. Williams }\end{array}$

\section{Introduction}

The understanding of laser-plasma interactions in ignition-scale inertial confinement fusion (ICF)

hohlraum targets is important for the success of the proposed National Ignition Facility (NIF). The success of an indirect-drive ICF ignition experiment depends on the ability to predict and control the history and spatial distribution of the $x$-radiation produced by the laser beams that are absorbed by the inside of the hohlraum wall. Only by controlling the symmetry of this x-ray drive is it possible to obtain the implosion symmetry in the fusion pellet necessary for ignition. ${ }^{1}$ The larger hohlraums and longer time scales required for ignition-scale targets result in the presence of several millimeters of plasma (electron density $n_{\mathrm{e}} \approx 0.1 n_{\mathrm{c}} \approx 10^{21} \mathrm{~cm}^{-3}$ ), through which the $3 \omega(351-\mathrm{nm})$ laser beams must propagate before they are absorbed at the hohlraum wall. Hydrodynamic simulations show this plasma to be very uniform [density-gradient scalelength $\left.L_{n}=n_{\mathrm{e}}\left(d n_{\mathrm{e}} / d x\right)^{-1} \approx 2 \mathrm{~mm}\right]$ and to exhibit low velocity gradients [velocity-gradient scalelength $\left.L_{v}=\mathcal{C}_{\mathrm{s}}(d v / d x)^{-1}>6 \mathrm{~mm}\right]^{2}$

The propagation of the beams to the hohlraum wall can be affected by various scattering and laser selffocusing (filamentation) processes within the low-density plasma inside the hohlraum. For example, while traversing such a plasma, the incoming light wave can resonantly decay into a backscattered light wave and either an ion sound wave or an electron plasma wave. The backscattered light wave can beat with the incident light wave at a frequency that pumps the plasma wave; this process can increase the amplitude of the plasma wave, increasing its scattering efficiency. Hence

\footnotetext{
* Centre D'Etudes de Limeil-Valenton, France

* Los Alamos National Laboratory, Los Alamos, New Mexico
}

an unstable feedback loop is formed that can cause the amplitudes of the plasma and scattered light waves to grow exponentially on time scales of 0.1 to 10 ps. For scattering from ion sound waves, these parametric scattering instabilities are called stimulated Brillouin scattering (SBS); for scattering from electron plasma waves, they are called stimulated Raman scattering (SRS). ${ }^{3}$ Both of these instabilities can lead to undesirable effects, including significant amounts of light reflecting from the plasma or shining directly onto the capsule, spoiling the illumination symmetry.

Another instability that can affect laser beam propagation is beam filamentation, ${ }^{4}$ which occurs when individual speckles ("hot spots") within the beam selffocus as a result of refractive index changes caused by changes in the local plasma density produced by the laser field through transverse ponderomotive pressure. This effect can result in a local increase in intensity and eventual beam breakup. Filamentation is of particular concern for NIF hohlraums, because the intensity threshold for filamentation is affected by the length and transverse scale of the hot spot. For a beam smoothed with a random phase plate (RPP), these scales are determined by the beam $f /$ number. The $f / 8$ focusing geometry planned for the NIF will have speckles $180 \mu \mathrm{m}$ long for 351-nm light, contrasting with 50-um speckles for the f/4.3 optics used at the LLNL Nova laser. Calculations indicate that at the expected $2 \times 10^{15} \mathrm{~W} \mathrm{~cm}^{-2}$ irradiance planned for the NIF, a significant fraction of an $f / 8$ beam would be above the intensity threshold for filamentation, in the absence of any beam smoothing beyond use of a RPP. ${ }^{4}$

In most laser-produced plasmas, SBS and SRS are limited by plasma inhomogeneity ${ }^{3}$; in the plasmas expected in the NIF hohlraums, however, wave damping, 
pump depletion, or some other nonlinear saturation mechanism would be the only limitations on instability growth. Some of our interaction experiments in homogeneous low-density plasmas have shown as much as $35 \%$ of the incident laser energy backscattered as SBS; in other experiments, SRS reflected fractions as high as $25 \%$ have been observed. Although these are extreme cases that represent scattering from plasmas that have less damping of instabilities than we expect in the NIF plasma, they illustrate the potential of parametric instabilities to cause problems. This amount of backscattering would obviously reduce coupling to the hohlraum wall, reducing the $x$-ray drive and increasing the incident laser energy required to drive a target to ignition. (SRS could cause additional problems, because the process not only transfers energy into backscattered light and electron plasma waves but also produces suprathermal electrons with tens of keV energy. These "hot" electrons can preheat the DT fuel in the capsule, reducing the efficiency of the subsequent implosion.) Significant energy scattering due to instabilities such as SBS and SRS would also make it more difficult to meet the NIF power-balance specification of $<8 \%$ variation in power between beams, which is needed to meet the symmetry requirements for a high-convergence implosion. ${ }^{5}$ SRS and SBS exacerbated by beam filamentation are therefore a concern for indirect-drive ICF, especially in the larger and more uniform plasmas expected in an ignition-scale hohlraum.

The basic design for the NIF hohlraum [Fig. 1(a)] is an Au cylinder about $9 \mathrm{~mm}$ long containing a low- $Z$ gas (a mixture of $\mathrm{He}$ and $\mathrm{H}_{2}$ ). The low- $Z$ gas slows the expansion of the Au hohlraum wall and provides a medium (other than low-density Au ablated from the wall) through which the beams can propagate without significant loss of energy from inverse bremsstrahlung absorption. The current NIF design has two cones of beams (called the inner and outer cones) on each side of the hohlraum. Figure 1(b) shows the history of the NIF laser pulse; Fig. 1(c) shows the density and beam intensity along the beam path for the time of peak calculated SRS and SBS gain (also the time of peak power, $13.5 \mathrm{~ns})$. Figure 1(d) shows the calculated temperature profiles at the same time. The profiles in Figs. 1(c) and (d) represent the beam intensity, electron density, and electron temperature encountered along the inner and outer cone beam paths. (a) NIF baseline hohlraum

FIGURE 1. (a) NIF baseline hohlraum, showing inner and outer beam cones; (b) power history for one NIF beam; (c) electron density (as a fraction of critical density $n_{c}=9 \times 10^{21} \mathrm{~cm}^{-3}$ ) and laser beam intensity vs position in NIF hohlraum at time of peak laser power. Laser beam incident from left. Dashed and solid curves represent parameters for outer and inner beam cones, respectively. Lighter portions of each curve indicate where the laser is interacting with $\mathrm{Au}$ plasma; other regions correspond to low- $Z$ (a $\mathrm{He} / \mathrm{H}$ mix) fill gas plasma. (d) Electron temperature for inner and outer beams. (20-07-1095-2434pb01)

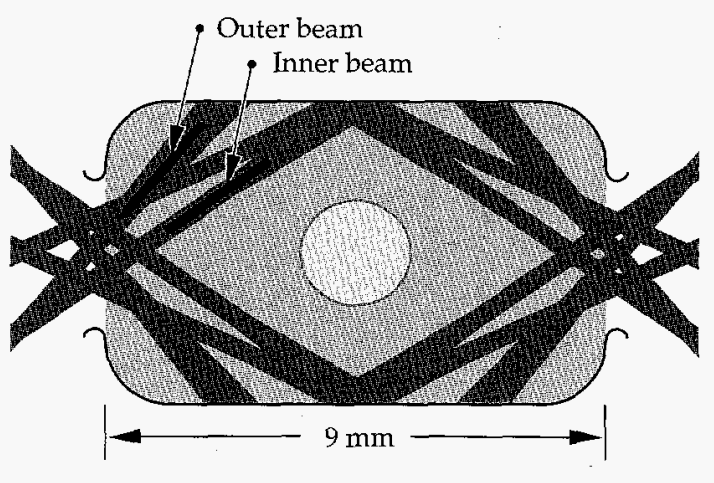

(b) NIF inner beam laser pulse
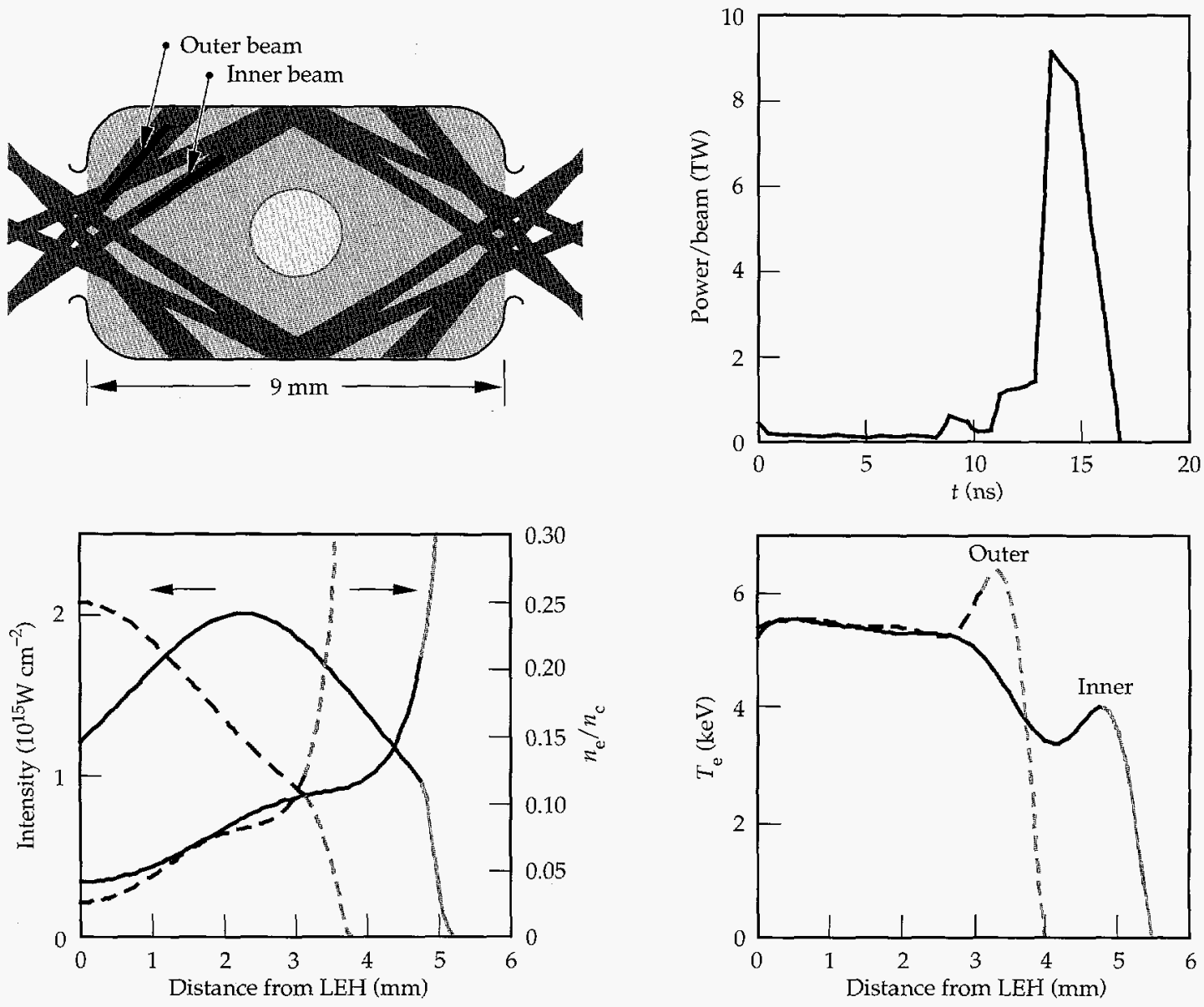
The output from the 2-D hydrodynamics simulations of the NIF target can be post-processed with the Laser Interactions Post-processor (LIP) 6 to obtain the total gain exponent for SBS and SRS as a function of time and scattered-light wavelength. The calculated peak gain exponents along the path of a $2 \times 10^{15} \mathrm{~W} \mathrm{~cm}^{-2}$ NIF beam are 25 for SRS and 30 for SBS. Figure 2 shows the post-processed linear gain exponents for SBS and SRS, as functions of scattered-light wavelength and of time throughout the NIF pulse, for the inner and outer beam cones. The plots show gray-scale contours of equal calculated gain exponent; darker regions correspond to higher gain exponent.

Figures 2(a) and (b) show the history of the SBS for the inner and outer beams, respectively. Some of the spectral features of the SBS gain calculations and differences between the inner-beam and outer-beam cases can be understood in terms of the plasma conditions under which the scattering would occur. For instance, Fig. 1(c) indicates that the inner beams traverse a longer length of low-density fill plasma than the outer beams. The SBS gain peaks at a wavelength of $\Delta \lambda \approx 14 \AA$ for the inner-beam case; for the outer beam, which has less path length in the low- $Z$ plasma, the main spectral feature is a narrow gain spike at $\Delta \lambda \approx 7 \AA$.

To understand these observations, consider that the wave-number-matching condition for SBS requires that

$\mathbf{k}_{0}=\mathbf{k}_{s}+\mathbf{k}_{i a}$, (a) SBS, inner cone

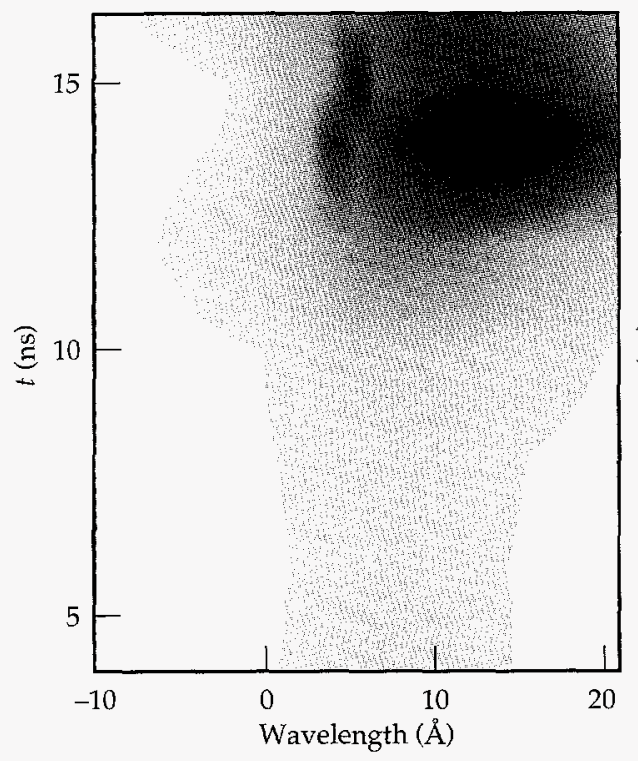

(c) SRS, inner cone

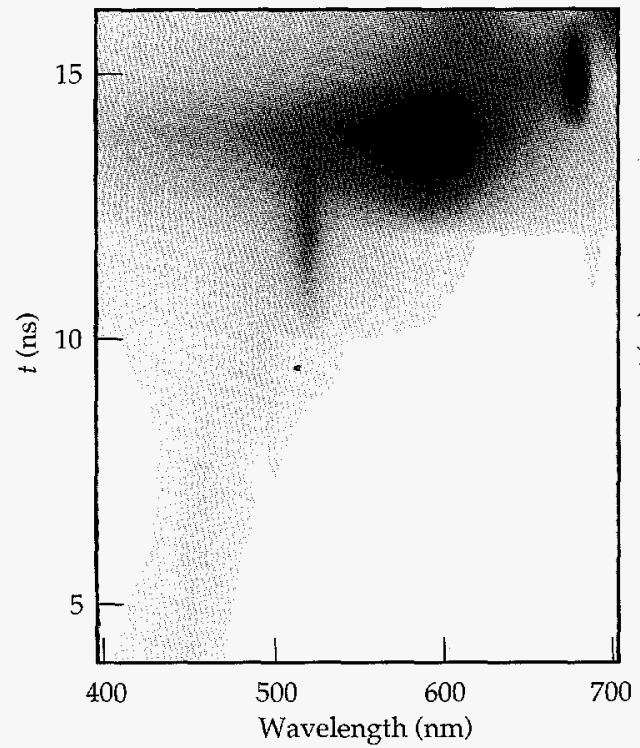

(b) SBS, outer cone

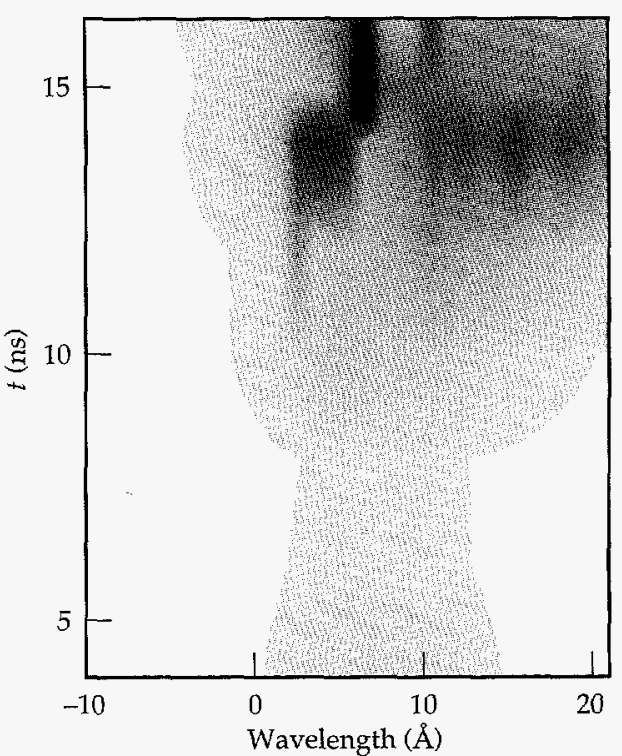

(d) SRS, outer cone

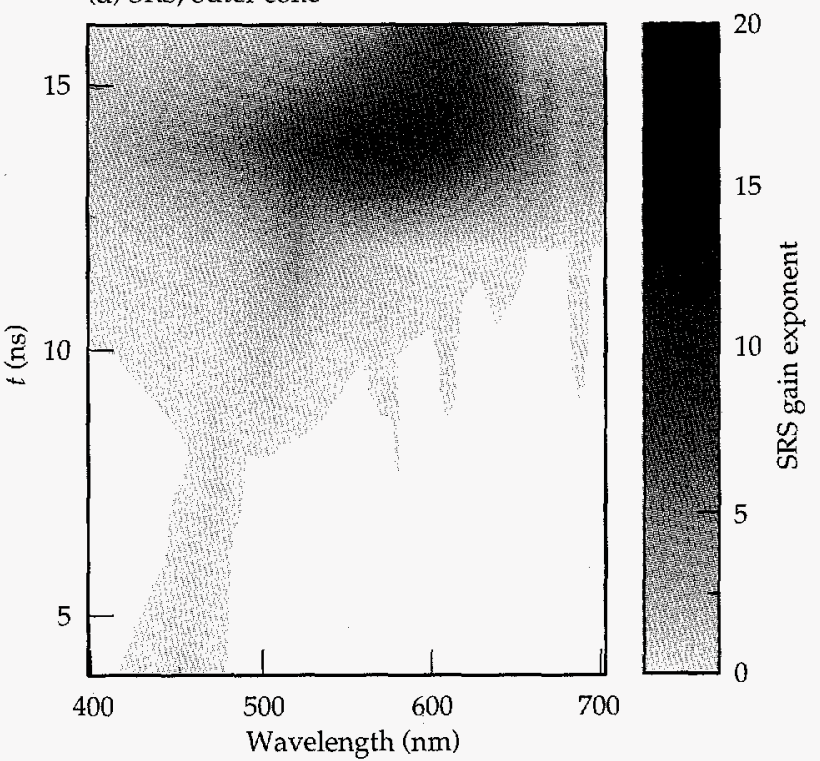

FiguRE 2. Post-processed (LIP) gain exponents along laser beam paths using plasma parameters from 2-D LASNEX hydrodynamics calculations of NIF. (a) SBS for inner cone;

(b) SBS for outer cone;

(c) SRS for inner cone;

(d) SRS for outer cone.

(20-07-1095-2435pb01) 
where $\mathbf{k}_{0}$ and $\mathbf{k}_{s}$ are the incident and scattered light wave vectors in the plasma. The wave number of the ion-acoustic wave $\mathbf{k}_{i a}$ has a magnitude equal to $2 k_{0}$ for direct backscattering (since $\mathbf{k}_{s} \approx-\mathbf{k}_{0}$ ), where $k_{0}$ is related to the vacuum wave number $k_{v a c}$ of the light by

$k_{0}=k_{v a c}\left(1-n_{\mathrm{e}} / n_{\mathrm{c}}\right)^{1 / 2} ;$

this is a minor correction for densities near $0.1 n_{\mathrm{c}}$.

Although most of the incident photon energy goes into the backscattered photon, the ion-acoustic wave takes away some energy according to

$\omega_{0}=\omega_{\mathrm{s}}+\omega_{\mathrm{ia}} ;$

the frequency shift $\Delta \omega$ in the scattered light wave is therefore approximately

$\Delta \omega=\omega_{\mathrm{ia}} \approx 2 k_{0} c_{\mathrm{S}}$,

resulting in a wavelength shift

$\Delta \lambda \approx 2 \lambda_{\text {vac }}\left(1-n_{\mathrm{e}} / n_{\mathrm{c}}\right)^{1 / 2} c_{\mathrm{s}} / c$,

where $c_{\mathrm{S}}$ is the sound speed. For a plasma flowing towards the observer with Mach number $M=v / c_{\mathrm{s}^{\prime}}$, we have

$\Delta \lambda \approx 2 \lambda_{\text {vac }}\left(1-n_{\mathrm{e}} / n_{\mathrm{c}}\right)^{1 / 2}(1-M) \mathcal{c}_{\mathrm{s}} / c$.

The strongest dependence here is on the sound speed and Mach number of the plasma in which the scattering is occurring. The sound speeds in $\mathrm{Au}$ and $\mathrm{He} / \mathrm{H}$ are very different, because the sound speed scales as $\left[\left(Z_{\text {eff }} T_{\mathrm{e}}+3 T_{\mathrm{i}}\right) / A\right]^{1 / 2}$ and $\mathrm{Au}$ is a heavy atom $(Z<A / 2)$ that is not completely ionized (i.e., $Z_{\text {eff }}<Z$ ) while the low- $Z$ plasma is fully ionized and has $Z \geq A / 2$. Typical sound speeds are 3 to $4 \times 10^{7} \mathrm{~cm} \mathrm{~s}^{-1}$ for $\mathrm{Au}$ and 6 to $7 \times 10^{7} \mathrm{~cm} \mathrm{~s}^{-1}$ for $\mathrm{He} / \mathrm{H}$ for the temperatures expected in the NIF plasma. The shifts in wavelength for SBS backscattering from $\mathrm{Au}$ and $\mathrm{He} / \mathrm{H}$ are then 7 and $14 \AA$, respectively, for stationary plasmas. Note that flows in the plasma will also shift the scattered light wavelength according to the $[1-M]$ term.

The feature at $7 \AA$ appearing at $15 \mathrm{~ns}$ for the outerbeam SBS calculation [Fig. 2(b)] corresponds to SBS gain in the $\mathrm{Au}$, with a peak gain exponent of 30 . The broad gain peak at $14 \AA$ in the inner-beam case [Fig. 2(a)] represents gain in the low- $Z$ plasma with a peak gain exponent of 20. Although there is a small amount of gain at $6 \AA$ in the inner-beam case, the observation can be made that the main SBS threat is from the wall material in the outer-beam case and from the low- $Z$ fill gas in the inner-beam case. Hence we can break down the SBS problem into a high- $Z$ problem, which is best addressed in experiments with Au walls (e.g., hohIraums), and a large, low-Z plasma SBS problem, which can be addressed in gas-filled hohlraums or with open-geometry gas targets.

The SRS gain exponents calculated by LIP are shown in Fig. 2(c) and (d) for the inner and outer beams, respectively. Figure $1(\mathrm{c})$ indicates that when the beams reach the Au they are absorbed in a short distance $(\sim 300$ to $500 \mu \mathrm{m})$ in a region with steep density gradients. Since SRS gain is higher in large, homogeneous regions with shallow density gradients, most of the calculated SRS gain is in the low-density, low- $Z$ plasma. Beams in the inner cone have higher expected SRS gain [with a maximum of 26, shown in Fig. 2(c)], because the beams have longer paths in the low- $Z$ plasma, and the total exponentiation is a product of the spatial gain rate with the length over which that exponentiation can occur. The outer beams traverse less low- $Z$ fill plasma and so have a lower calculated gain exponent for SRS [with a maximum of 11 , shown in Fig. 2(d)].

The SRS plots show the calculated gain exponent for SRS as a function of the wavelength of the scattered photon. The wavelength of the scattered optical light carries information about the density and temperature of the plasma from which it scattered, because the electron plasma wave from which it scatters must satisfy

$\omega_{\mathrm{p}}^{2}=\omega_{\mathrm{pe}}^{2}+v_{T}^{2} k_{\mathrm{p}}^{2}$,

where $\omega_{p}$ and $k_{p}$ are the frequency and wave number of the electron plasma wave, $\omega_{\mathrm{pe}}$ is the plasma frequency (which introduces density dependence), and $v_{\mathrm{T}}$ is the electron thermal velocity (which introduces temperature dependence). The incident and scattered wave numbers are related by energy and momentum conservation:

$\omega_{0}=\omega_{\mathrm{s}}+\omega_{\mathrm{p}}$

$\mathbf{k}_{0}=\mathbf{k}_{\mathrm{s}}+\mathbf{k}_{\mathrm{p}}$,

where $\mathbf{k}_{0}\left(\omega_{0}\right)$ and $\mathbf{k}_{\mathrm{s}}\left(\omega_{\mathrm{s}}\right)$ are the incident and scattered light wave vectors (frequencies). The wavelength of the scattered light that satisfies these conditions is strongly dependent on density and less strongly dependent on electron temperature. The large gain exponent at $590 \mathrm{~nm}$ for the inner beam corresponds to SRS growing at $n_{e} \approx 0.1 n_{c}$ and a $T_{e}=3$ to $4 \mathrm{keV}$. The long-scalelength targets described below were designed to explore this gain region. The lower-gain parts of the plot correspond to different densities within the NIF plasma; longer wavelengths correspond to higher densities. The small region of gain near $690 \mathrm{~nm}$ for the inner beam represents light scattering from plasma at $n_{\mathrm{e}} \approx 0: 2 n_{\mathrm{c}}$ and $T_{\mathrm{e}}=4 \mathrm{keV}$. This region of plasma is low $-Z$ material that has ablated from the capsule and produces 
a slightly higher-density region of low- $Z$ plasma where it collides with the Au wall. This region is difficult to reproduce experimentally and is less significant to the NIF problem because the laser energy in the part of the beam that reaches this plasma is very low because the laser light is being rapidly absorbed by this higherdensity plasma.

As can be seen in Fig. 2, calculated gain exponents occasionally reach or exceed 20 . Amplification of thermal fluctuations by $20 e$-foldings is sufficient to produce both significant backscattering and plasma waves of sufficient amplitude that nonlinear saturation mechanisms begin to be important. The LIP calculations include no nonlinear effects, so the LIP results should be interpreted as telling us that there might be a significant scattering due to SBS and SRS in the NIF plasma for the period during which gain exponents exceed 20 , but that we cannot predict the saturation level of that scattering. Hence we were motivated to try to reproduce plasma conditions with such gain exponents on Nova (where LIP can be used to compare the gains to the NIF case) in order to include saturation and other plasma effects that are omitted by the simple linear gain analysis.

The NIF beams will interact with the plasma at intensities up to $2 \times 10^{15} \mathrm{~W} \mathrm{~cm}^{-2}$, with a variation of intensity along the beam path as shown in Fig. 1(c). In the transverse direction, the laser intensity is assumed to be averaged over the beam profile, and the LIP calculations use this spatially averaged intensity. However, each beam is composed of small ( $\sim 6 \mu \mathrm{m}$ diam) speckles with a distribution of intensities above and below the average. The speckles can self-focus as they interact with the plasma, increasing the intensity within individual "hot spots," although beam smoothing such as smoothing by spectral dispersion (SSD), which moves the speckle pattern around rapidly, can reduce filamentation. This nonlinearity in the laser-plasma interaction is also omitted from the LIP calculations, so a further requirement of understanding laser-plasma interactions in the NIF by means of Nova-scale experiments was to use a laser beam with the intensity, smoothing, and $f /$ number appropriate to a NIF beam. These experiments would address the legitimate concern that some fraction of each NIF beam will drive saturated levels of SBS and SRS and be reflected from the NIF hohlraum with the loss of energy and other consequences described above. The goals of these experiments were expressed in the HLP5/ 6 part of the 1993 NAS Nova Technical Contract as follows:

- HLP5: Demonstrate acceptable levels of scattering in large-scale plasmas that match the plasma conditions, beam geometry, and beam smoothing of ignition hohlraums as closely as possible. The plasmas should have density and velocity scalelengths $\approx 2 \mathrm{~mm}$, electron temperature $>1.5 \mathrm{keV}$, and $n_{\mathrm{e}} / n_{\mathrm{c}}<0.15$.

Acceptable levels of scattering were defined as follows:

- Stimulated Brillouin scattering fraction $\mathrm{f}_{\mathrm{SBS}}$ (back, side) $<5$ to $10 \%$.

- Stimulated Raman scattering fraction $f_{\text {SRS }}$ (back, side) $<5$ to $10 \%$ and $f_{\text {SRS }}$ (forward) $<5 \%$.

- HLP6: Evaluate the impact of laser beam filamentation on SBS and SRS and develop control techniques to the extent necessary to ensure acceptable levels of scattering.

\section{Target/Plasma Development}

To experimentally evaluate the severity of SRS and SBS scattering and filamentation on the propagation of an $f / 8$ beam traversing a long-scalelength plasma, we reproduced the laser beam conditioning ( $f /$ number and smoothing) to be used on the NIF. At the same time, it was necessary to reproduce the plasma conditions expected within the NIF using plasmas produced by the Nova laser. Late 1993 and early 1994 saw a significant effort to produce plasmas that met the criteria expressed in the first part of the HLP5 statement of work. It was necessary to both reproduce the density $\left(\sim 0.1 n_{c}\right)$ and temperature (at least $\left.3 \mathrm{keV}\right)$ of the NIF target but also to do so over a volume large enough that the total gain exponent of the NIF target for SBS and SRS could be approached.

Various targets were explored, including low-density foams and thin exploding foils. The most promising targets were large gas-filled hohlraums (of which two types were developed) and "gasbags." The plasmas were created by irradiating a thin-walled gas balloon or a sealed hohlraum ${ }^{7}$ containing of order $1 \mathrm{~atm}$ of a low- $\mathrm{Z}$ gas (e.g., $\mathrm{C}_{5} \mathrm{H}_{12}, \mathrm{C}_{5} \mathrm{D}_{12}$, or $\mathrm{CO}_{2}$ ). All three of these targets relied on heating a large volume of a heavy gas that, when ionized, produced the appropriate plasma conditions and was large enough to last for a reasonable time and to provide the right amount of SRS and SBS amplification.

Figure 3 shows the three targets developed to investigate laser-plasma interactions in NIF-scale plasmas. Figure 3(a) shows the GASBIG hohlraum, which is larger than the typical Nova hohlraum. Normal (scale1) Nova hohlraums are the same length $(-2.5 \mathrm{~mm})$ but have a smaller diameter $(1.6 \mathrm{~mm})$, with the laser beams pointed so that they hit the wall before they reach the midplane of the hohlraum. The GASBIG hohlraums maximized the length over which the beams interacted with the plasma by pointing them at the opposite end of the hohlraum so that they crossed in the hohlraum mid plane. The hohlraum was filled with $\mathrm{C}_{5} \mathrm{H}_{12}$ gas retained with a thin $(6500 \AA)$ polyimide window. 
Figure 3(b) shows the toroidal hohlraum developed by LANL. This target produced a large length of plasma with which to interact by moving the wall of the standard Nova hohlraum radially outward to produce a more doughnut-shaped hohlraum. Figure 3(c) shows the open-geometry gasbag target, which was essentially two thin windows on either side of a thin washer. The target is inflated to make an almost spherical volume of gas. Figures $3(d)$ and (e) show density and temperature plots for the GASBIG and gasbag plasmas after they have been heated by the Nova laser beams, at the time at which the interaction with the NIF-like probe beam was studied ( $1 \mathrm{~ns}$ for the GASBIG, $750 \mathrm{ps}$ for the gasbag). Both Figs. 3(d) and (e) show a relatively flat region of plasma near $0.1 n_{c}$ at a temperature of $3 \mathrm{keV}$; this is the portion of the plasma that is approximating the region of high gain for SBS and SRS in the NIF inner beam.

The concept of producing a large, hot plasma by irradiating a gas-filled target has been discussed by Denavit and Phillion. ${ }^{8}$ A cold gas volume of a few millimeters dimension can be heated by pulsed laser beams (typical duration $1 \mathrm{~ns}$, intensity of order $10^{14} \mathrm{~W} \mathrm{~cm}^{-2}$ ). When the laser beams strike the cold gas, a plasma is formed by multiphoton ionization. The initial low temperature of the plasma leads to energy deposition through inverse bremsstrahlung at the edge of the plasma. As that plasma is heated and becomes more transmissive, the laser beams propagate further into the gas. Our targets contained a high-molecular-weight gas (typically $\mathrm{C}_{5} \mathrm{H}_{12}$ ) that on ionization produced a plasma (electron) density $n_{e}=10^{21} \mathrm{~cm}^{-3}$. The velocity of propagation of the laser light into such a gas was measured for various laser conditions and is typically a few millimeters per nanosecond for $3 \omega$ light. For a 2.75-mm-diam gasbag plasma, the laser beams propagate to the center of the plasma by about 400 ps. Thereafter the laser beams steadily heat the plasma that they traverse; thermal conduction heats parts of the gas not directly irradiated by the laser beams. For the gasbag targets, the edge of the plasma is free to expand into the surrounding vacuum, so an isothermal rarefaction wave propagates towards the middle of the plasma at the ion sound speed $(\sim 0.5 \mathrm{~mm} / \mathrm{ns})$. The density plateau in the center of the plasma is therefore eroded by the converging rarefaction wave at a rate of about $1 \mathrm{~mm} / \mathrm{ns}$. After the plasma is formed and heated, we have a few hundred picoseconds to perform laser-plasma interaction experiments on our 2-mm size plasma before the rarefaction wave shrinks the
FIGURE 3. Targets developed to study laser-plasma interactions in long-scalelength plasmas similar to those of NIF (a) GASBIG hohlraum; (b) toroidal hohlraum; (c) opengeometry gasbag target; (d) density and temperature for GASBIG hohlraum targets at a time (1 ns) at which interaction with NIFlike probe beam was studied. Lighter part of each curve indicates $\mathrm{Au}$ plasma. (e) Density and temperature for gasbag target at a time ( $750 \mathrm{ps})$ at which interaction with NIF-like probe beam was studied. (20-07-1095-2436pb01) (a) GASBIG hohlraum

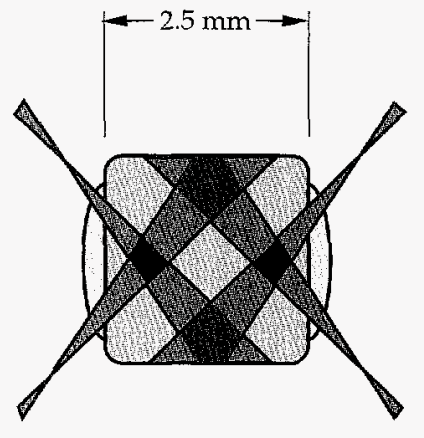

(d)

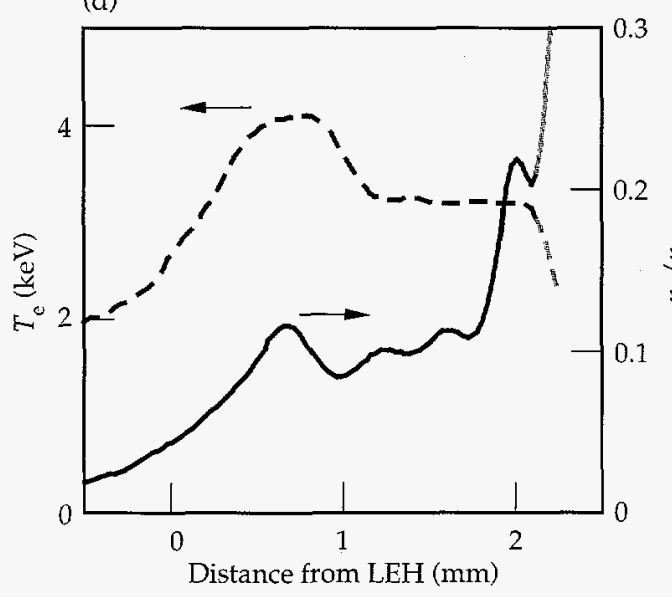

(b) Toroidal hohlraum

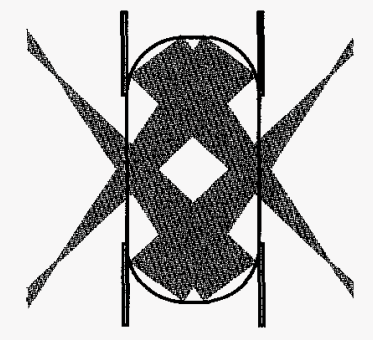

(c) Open-geometry gasbag

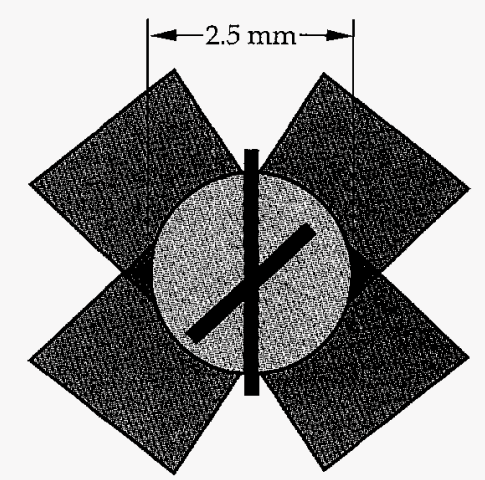

(e)

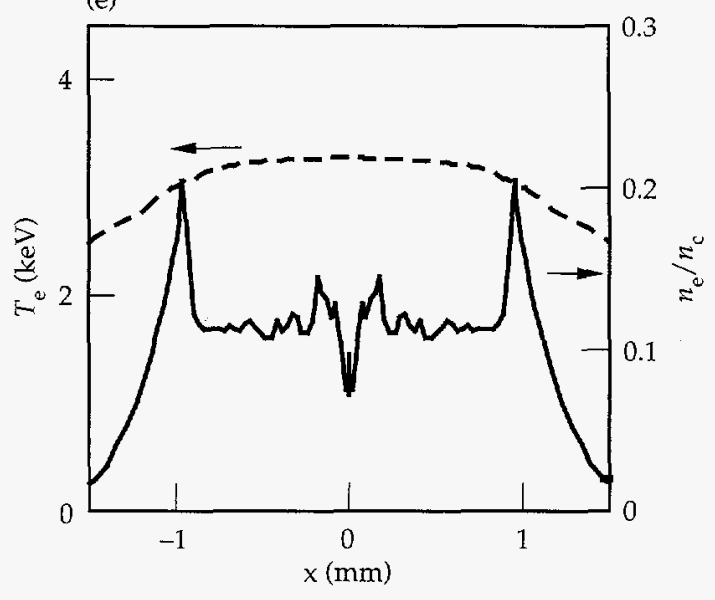


density plateau and so reduces the length available for amplification of SBS and SRS.

For each of the three targets, there was a period during which the plasma was ionized and heated using nine of the Nova beams. For the gasbags these "heater" beams were on for $1 \mathrm{~ns}$; the interaction beam was turned on at $t=500 \mathrm{ps}$ and also lasted for $1 \mathrm{~ns}$. There is therefore a 500-ps period during which the heaters and interaction beams overlap in time. During this period the plasma temperature is still rising. When the heater beams turn off there is a 500-ps period during which the plasma is cooling but the interaction beam remains on.

The balloon targets were constructed by placing a thin membrane of polyimide $\left(\mathrm{C}_{14} \mathrm{H}_{6} \mathrm{O}_{4} \mathrm{~N}_{2}\right)$ on each side of a thin $(400 \mu \mathrm{m})$ washer. The membranes were then inflated to an almost hemispherical shape through hypodermic fill tubes that penetrated the washer. Figure 3(c) shows the target and the laser-beam focusing geometry used to heat the plasma. Nine heater beams with a total energy of 18 to $22 \mathrm{~kJ}$ in a 1-ns pulse were used to create the plasma.

Unlike the more traditional targets for high-density laser-plasma interactions (solid or exploding-foil targets) the gas balloon targets convert most of the incident heater laser energy into thermal energy of the electrons and less into kinetic energy of bulk plasma motion. The energy budget for a typical gas balloon simulation is $22 \mathrm{~kJ}$ incident, $12.5 \mathrm{~kJ}$ absorbed, $8.8 \mathrm{~kJ}$ in electron thermal energy $\left(T_{e}=3.2 \mathrm{keV}\right)$ and only $2.6 \mathrm{~kJ}(21 \%$ of absorbed energy) in kinetic energy. These numbers should be compared with those for typical explodingfoil plasmas, for which similar calculations (to produce a $0.1 n_{\mathrm{c}}$ plasma) show $22 \mathrm{~kJ}$ incident, $8 \mathrm{~kJ}$ absorbed, $3.1 \mathrm{~kJ}$ in electron thermal energy $\left(T_{\mathrm{e}}=2.4 \mathrm{keV}\right)$ and $4.5 \mathrm{~kJ}$ in kinetic energy ( $56 \%$ of absorbed energy). The heating of as stationary low-density target is therefore a much more efficient way of producing a high-temperature plasma, and it has the added benefit that density and velocity gradients are much less severe and therefore more suitable for interaction experiments that mimic laser-plasma interactions within indirect-drive ICF hohlraums. Exploding-foil targets are excellent for producing the kind of flowing plasmas found in the corona around direct-drive ICF pellets.

Figure 4(a) shows a gated $x$-ray pinhole image of the plasma. The image is one of a series of gated images,

(b) L.ASNEX calculation at $750 \mathrm{ps}$
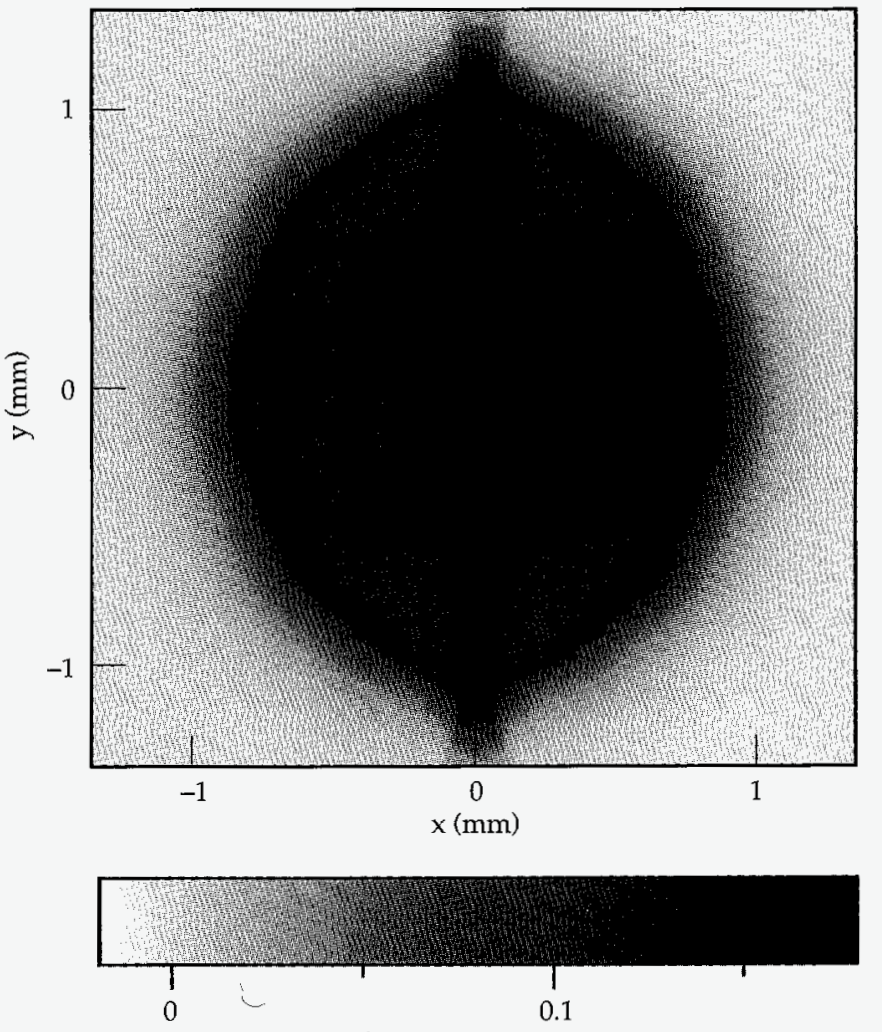

Relative x-ray intensity

FIGURE 4. (a) Gated x-ray pinhole image of gasbag at $750 \mathrm{ps}$; large stripe across image is the shadow of a shield that is well away from the plane of the plasma. Support washer (shown in Fig 3c) is only $400 \mu \mathrm{m}$ wide. (b) Simulated x-ray image from a LASNEX calculation at 750 ps. In both images, the gray scale indicates $x$-ray intensity integrated along the pinhole camera line of sight. Note the bright edge in this simulated image that is not seen in the data (a). The bright vertical stripe in the middle of the simulated image corresponds to a density peak that is propagating radially inwards in the plane of the washer. $\quad(20-07-1095-2437 \mathrm{pb} 02)$ 
with 100 ps time resolution, and was recorded 750 ps after the start of the heater pulses. The large vertical stripe across the image is not the support washer (shown in Fig. 3c) which is only $400 \mu \mathrm{m}$ wide, but is the shadow of a shield that is well away from the plane of the plasma. The x-ray emission looks uniform within the limits of the microchannel plate noise; the camera was filtered to view $\mathrm{x}$ rays at photon energies above $1.5 \mathrm{keV}$, where the target was optically thin. The image represents the integration of $x$-ray emissivity along a chord through the plasma and could average changes in the emissivity caused by density fluctuations. Abel inversion of images such as this can show us if the plasma has a cold (less emissive) center, as is the case before the heater beams propagate to the center of the target. Some kind of 3-D tomographic imaging would be preferable, and we might eventually develop such a capability. Another alternative for better quantifying the uniformity of our plasmas is to do space-resolved thermal Thomson scattering using a $4 \omega$ $(264 \mathrm{~nm})$ probe beam. Again, this capability may be developed for future experiments.

The membrane that represents the initial bag wall perturbs the situation somewhat. The membrane is $\sim 2000$ to $3000 \AA$ thick after the bag has been inflated and the material has stretched. The lasers heat the membrane, causing it to expand and rarefy, much like an exploding foil. ${ }^{9}$ That process launches a weak shock into the bag, which propagates into the center of the target at about the sound speed. The peak density in this shock is calculated to be $2 \times 10^{21} \mathrm{~cm}^{-3}$ for a $\mathrm{C}_{5} \mathrm{H}_{12}$ gas fill [Fig. 3(e)]; the density scales with the fill density of the gas. Figure $4(\mathrm{~b})$ shows a simulated image taken from a post-processed LASNEX calculation. A brightening due to this density peak is visible at the edge of the plasma. Although such a feature is seen at early times in our experiments, we do not see such a feature after the plasma has achieved its full size (at $\sim 500 \mathrm{ps}$ ). The area in front of the shock wave is the region that has a flat density profile with no significant velocity gradients. The structure visible near the middle of the profile in Fig. 3(e) is produced by the symmetry of the calculation and has not been observed in experiments (with framing cameras viewing down the washer axis). The bright vertical stripe in the middle of the simulated image corresponds to a density peak that is propagating radially inwards in the plane of the washer. This peak has been seen in axial images, but it is never in the path of the interaction beam, which enters the plasma shown in Fig. 4(b) from $50^{\circ}$ below the horizontal, crossing the image almost diagonally.

Electron temperatures were measured ${ }^{10,11}$ by $x$-ray spectroscopy using $\mathrm{K}$-shell line spectra from mid- $Z$ elements (such as $2000 \AA$ of cosputtered Ti and $\mathrm{Cr}$ or $2500 \AA$ of $\mathrm{KCl}$ ) placed in different locations inside the gas-filled targets. Analysis of the line intensity ratios uses a time-dependent collisional-radiative model of the plasma. ${ }^{12}$ Isoelectronic ratios of the He-like emission were used because they are less affected by timedependent ionization effects. Density variations are not expected to introduce large effects in the analysis and are included in the error estimates of the results.

The electron temperature in the $\mathrm{C}_{5} \mathrm{H}_{12}$-filled gasbag was measured with $\mathrm{TiCr}$ dopants and with $\mathrm{Ar} / \mathrm{Cl}$ gas dopants. These spectroscopic measurements, using three different line ratios, consistently indicate that peak temperatures of 2.8 to $3.2 \mathrm{keV}$ are achieved in the gasbags. Recent measurements of $\mathrm{Ar}$ and $\mathrm{Cl}$ satellite and resonance lines over several shots have enabled us to obtain a history of the temperature rise; these measurements show a peak temperature of $2.8 \pm 0.5 \mathrm{keV}$, slightly below the LASNEX predictions. The measured temperature lags behind the temperature rise indicated by the simulation. Gasbags attain their peak electron temperature at $1 \mathrm{~ns}$, when the heater beams turn off, but they are homogeneous after 400 ps when they are heated with $3 \omega$ heater beams.

The quoted electron temperature was measured in the gasbag plasmas with $3 \omega$ heater beams. Early experiments using $2 \omega(527 \mathrm{~nm})$ heater beams (which would give higher temperatures because of increased collisional absorption) showed poor coupling of the laser energy into the initially cold plasma, which then stayed cold. Since $n_{\mathrm{e}}=10^{21} \mathrm{~cm}^{-3}$ is $0.25 n_{c}$ for $2 \omega$ light, it was suspected that $2 \omega_{\mathrm{pe}}$ decay and /or SRS at the quarter-critical density was converting much of the incident energy to fast electrons, ${ }^{13,14}$ which then deposited their energy in the target superstructure without heating the plasma effectively. Hence most of the experiments described here used $3 \omega$ heater beams and a $3 \omega$ interaction beam.

The electron temperature of the large gas-filled hohlraums ("GASBIGS") was determined using dopants deposited on 150- $\mu$ m-wide, $800-\AA$-thick CH substrates. The gas volume of the hohlraums is not uniformly heated by the laser beams as in the gasbags, so the measurements were performed in and out of the beam path. Peak electron temperatures in the beam path of $3.6 \mathrm{keV}$ were measured by the isoelectronic line intensity ratios of the He-like emission. Figure 5 shows the temperature history calculated by LASNEX and the corresponding measured temperatures. Measurements earlier than $1 \mathrm{~ns}$ are not included because accounting for the transient effects of the ablation and equilibration of the foil with the surrounding plasma introduces large uncertainties in the measurements. The temperature in the GASBIG is also observed to lag at early times. This discrepancy may be related to the heat capacity of the gas, because volumes not heated by the laser light must be heated by lateral heat flow; alternatively, it may imply that beam propagation is not as fast as the LASNEX calculation predicts. The out-of-beam measurements (not shown) show a temporal lag, but still achieve peak temperatures of $3 \mathrm{keV}$. Similar measurements using $\mathrm{Ti} / \mathrm{Cr}$ spectroscopy made to characterize the toroidal hohlraum 
plasmas ${ }^{15}$ yielded temperatures of $3 \pm 0.5 \mathrm{keV}$ for the time at which the interaction beam would probe the plasma.

For all three of the targets shown in Fig. 3, we found temperatures of about $3 \mathrm{keV}$ for plasmas of order $2 \mathrm{~mm}$ in size at $0.1 n_{c}$. Since this approximates the region of the NIF plasma producing the highest calculated gain for SBS and SRS on the inner beam, we then turned to using these targets to perform interaction experiments. Note that the problem of producing a target with a gain similar to that seen on the outer beam in the Au [Fig. 2(b)] is not solved with these targets. None of them has sufficient radiation temperature to drive off a significant

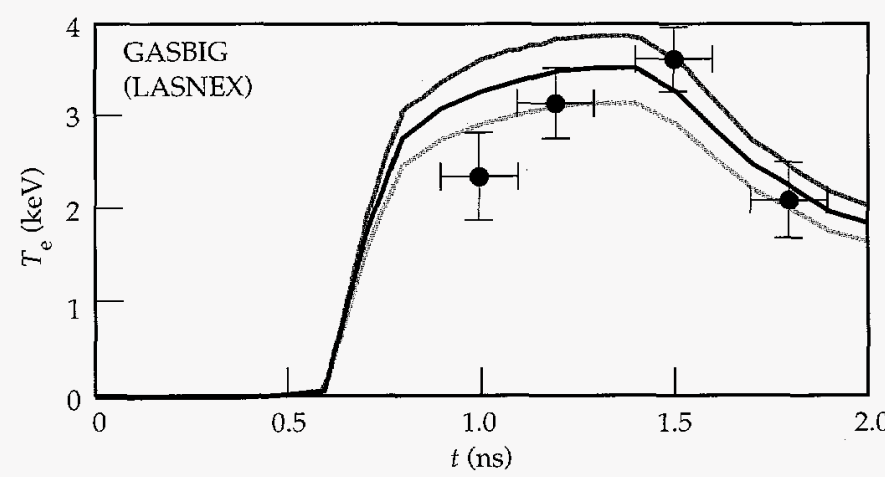

FIGURE 5. GASBIG hohlraum electron temperature history calculated by LASNEX, and corresponding measured temperatures. Top and bottom curves represent $\pm 10 \%$ temperature variation. (20-07-1095-2438pb01)

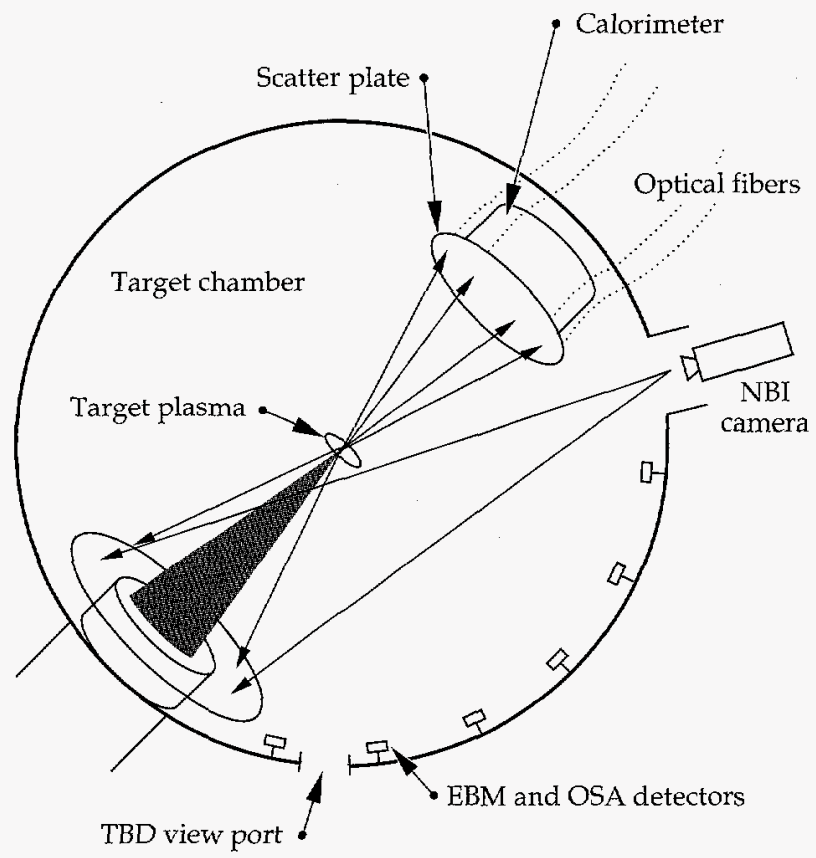

FIGURE 6. Nova target chamber, showing placement of scatteredlight diagnostics. Beam shown is the interaction beam (beamline 7), on which the NIF beam smoothing and $f /$ number were replicated. The other nine beams (used to heat the plasma) are not shown. (20-07-1095-2439pb01) plateau of low-density Au plasma to mimic that region for the NIF outer beam. In fact, the scale-1 Nova hohlraums, when gas-filled, actually produce SBS gain exponents in the Au similar to that in the NIF outer beam. This occurs because inverse bremsstrahlung absorption in the $\mathrm{Au}$, and not the hohlraum size, determines the scalelength in the Au. For gas-filled Nova scale-1 and NIF hohlraums, this absorption length is a few hundred micrometers. This issue is discussed towards the end of this article.

\section{Diagnostic Development}

The development of diagnostics for the long-scalelength plasma experiments was motivated by the desire to better account for the scattered light and at the same time to quantify the light transmitted by the plasma. Figure 6 shows the placement of the various diagnostics in the Nova target chamber. The beam shown is the interaction beam (beamline 7), on which the NIF beam smoothing and $f /$ number were replicated. The other nine beams (used to heat the plasma) are not shown.

Before 1993, measurements of SBS were made by taking a subaperture sample using a Fresnel reflector (uncoated beam splitter) within one of the ten Nova beams, and it was assumed that the backscatter was uniform within the beam aperture. The gain for both SBS and SRS peaks in the direct backscatter direction, so the scattered light should be collinear with the incident beam. In setting up for the long-scalelength plasma experiments, it was decided to move this diagnostic capability to the beam that we would use to mimic the NIF smoothing and focusing geometry, while making the system integrate backscatter over the full beam aperture. Figure 7 shows the new system, called the FABS (full-aperture backscatter diagnostic). The FABS views the scattered light that propagates back down the beamline and is transmitted by the $1 \omega(1054 \mathrm{~nm})$ turning mirror closest to the chamber [which of course has high reflectivity $(\sim 95 \%)$ for the incident $1 \omega$ light but transmits $\sim 30 \%$ of backscattered $3 \omega$ light]. A large hole was cut in the back of the mirror mounting box, allowing access to the scattered light transmitted by the mirror. Since light scattered from the target passes back through the Nova focusing lens, it is recollimated and is $70 \mathrm{~cm}$ in diameter when it emerges from the back of the mirror box. A second $f / 4.3$ Nova lens was then used to focus the beam into the various diagnostic packages to allow analysis of the scattered light. A time-resolved SBS spectrometer, normalized with a time-integrating calorimeter, allowed the power history for SBS to be recorded; the time-resolved SBS spectrometer provided additional information on the spectral features of the SBS. A CCD camera also recorded a time-integrated image of the SBS backscatter angularly resolved within the diagnostic acceptance angle (i.e., SBS imaged in the plane of the Nova lens). 
Viewing light scattered from the target through the Nova focus lens and refocusing it with a similar Nova lens introduces a large amount of chromatic aberration. The Nova lenses were intentionally designed with large chromatic aberration to separate the foci of $1 \omega, 2 \omega$, and $3 \omega$ light (wavelengths 1054, 527, and $351 \mathrm{~nm}$, respectively) in the target plane. This property causes problems when trying to focus the scattered light spectrum, which extends from 350 to $700 \mathrm{~nm}$, into the diagnostic packages. A later version of the FABS will use a collecting mirror in place of the second lens and will correct the chromatic aberration through a series of small-aperture optics. This system ${ }^{16}$ was not ready for the experiments described here; when it is implemented, it will have the capabilities of the current FABS but also will have the ability to image the target plane using the scattered light. The primary mission of the FABS in its original inception was to measure the SBS scattered light from the target. Because the SBS spectrum is quite narrow ( 1 to $2 \mathrm{~nm}$ ), the issue of chromatic aberration was not as severe as it is for broader-band SRS measurements.

SRS diagnosis was initially made through monitoring diodes placed $27^{\circ}$ from the incident laser beam together with a streaked optical spectrometer. This setup was complemented by measurements of the fast-electron yield using the FFLEX filter-fluorescer, viewing $x$ rays produced by electrons slowing down in the target material. As discussed below, the suspicion that SRS yields from targets were higher than implied by FFLEX led to the development of improved monitoring of the light scattered by SRS. The FABS diagnostic was modified (as indicated in Fig. 7) by having a diffuser placed soon after the focusing lens that scattered the light, which was then recorded by a filtered diode. This reduced the problem with chromatic aberration discussed above by making the measurement as close to the second focusing lens as possible, before the disparities in focal length could significantly change the beam size as a function of wavelength.

A further complication was introduced by severe modulations in the transmission of the turning mirror (inset in Fig. 7). The modulation is due to the multilayer coating on the mirror, which is optimized to reflect narrow-band $1 \omega$ laser light efficiently, not to transmit a broad-band signal. This modulation makes quantitative measurements of SRS difficult. The difficulty was addressed by using a streaked optical spectrometer to spectrally resolve the SRS signal seen by the diode. The spectrum could then be corrected using the calibrated mirror transmission function, and the diode signal (which represents an integral of the spectrum) could be corrected accordingly. This spectrometer also allowed us to record the spectrally resolved SRS reflectivity for comparison with our expectations [e.g., Figs. 2(c) and (d)] and also to study changes in the spectrum as a function of beam smoothing and laser $f /$ number. All of the SRS spectra shown below were corrected to remove the modulation of the mirror transmission.

The FABS diagnostic was calibrated by using an uncoated spherical mirror placed on the far side of the chamber from the instrumented beamline (beamline 7). Beam 7 was then focused through a large hole $(\sim 6 \mathrm{~mm}$ diam) in a plate positioned at chamber center. The plate absorbed the unwanted harmonics of the laser beam (e.g., $1 \omega$ and $2 \omega$ in the case of a $3 \omega$ calibration shot), because the chromatic aberration in the Nova lens meant that only one color was focused at the hole. The light then diverged before it was reflected by the uncoated mirror and sent back down the beamline, where is was recorded as a known amount $(\sim 6 \%)$ of backscattered light.

The SBS detectors were calibrated using the $3 \omega$ calibration shots that are run routinely to maintain confidence in the precision Nova incident-beam diagnostics. To calibrate the SRS detectors, $2 \omega$ light was used, since its wavelength is in the middle of the spectral range of SRS light from most of our experiments. The sensitivity of the other components in the diagnostic relative to their sensitivity at $2 \omega$ were calibrated off-line. This process resulted in a typical uncertainty of $10 \%$ in the SBS and $20 \%$ in the FABS SRS measurements.

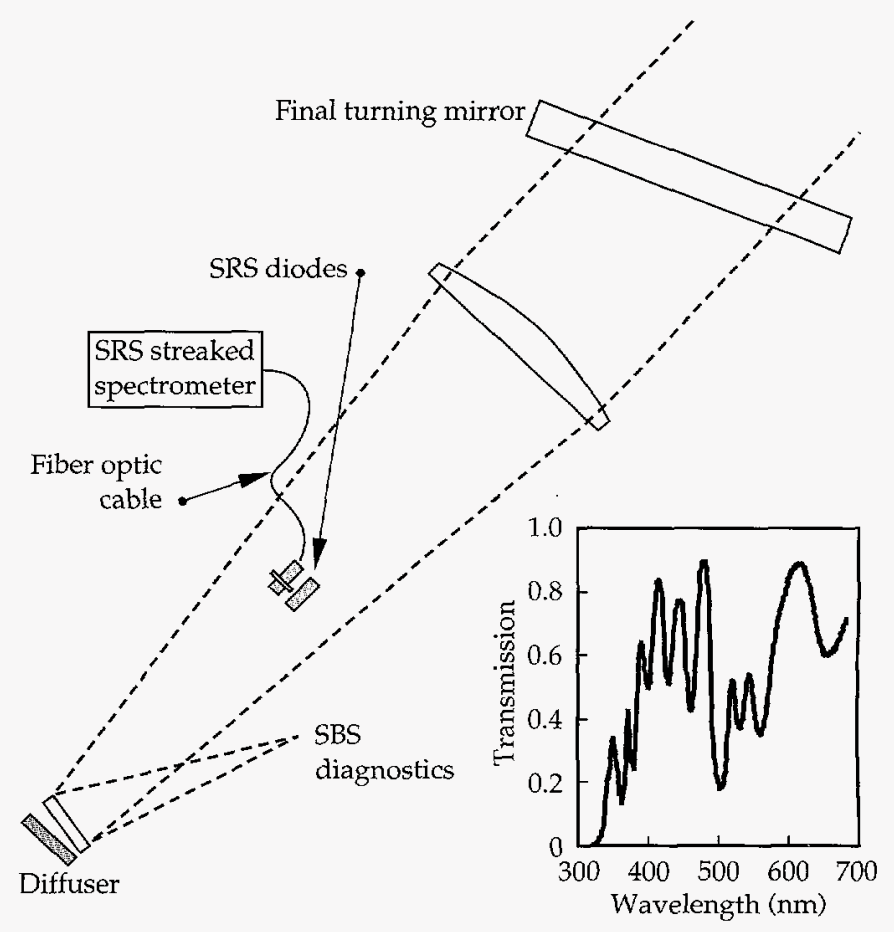

FIGURE 7. Full-aperture backscattering station (FABS) diagnostic, and (inset) transmission of final $1 \omega$ turning mirror through which FABS views scattered light. SBS light is reflected from a beam-splitter before it is injected into the SBS diagnostic package. A diffuser behind the beam-splitter reflects broader-band light into the SRS diagnostics. (20-07-1095-2440pb01) 
To complement the FABS, the diagnostic set included time-integrated diodes (Fig. 6 ) spaced at $\sim 10^{\circ}$ intervals around the chamber to look at sidescattered light. As discussed below, there were indications in the first experiments with these diagnostics that the scattered SBS was coming back outside the collection angle of the FABS (defined by the incident beam $f$ /number). Initial estimates were therefore made of the energy outside the lens using similar time-integrated diodes. An estimate of the spreading of the backscattered SBS was also obtained by restricting the aperture of the incident beam and using the FABS to image the light that scattered outside the angle of the incident subaperture beam. These estimates led us to conclude that up to three times as much scattered SBS energy may have been outside the lens as inside it for gasbag targets. At the time, the precision of this measurement was sufficient, because the amount of SBS in the lens was negligible. However, in the second phase of the experiments we planned to push the amount of SBS upward, so we expected that the energy outside the lens would play a large part in determining the energy budget of the interaction beam. Therefore a new diagnostic for near backscattered light was developed for the second series of experiments.

The near-backscattering imager (NBI) extended the continuous coverage of SBS and SRS backscattering outside the lens to $20^{\circ}$ from the lens axis. This was achieved by placing a large annular plate of bead-blasted aluminum around the outside of the lens assembly within the target chamber (Fig. 6). The near-backscattered light from the target scatters from the plate as if from a diffuse screen and is then imaged by two TV cameras on the far side of the target chamber. Filters on the TV cameras allowed us to separately measure SBS (near $351 \mathrm{~nm}$ ) and SRS (400 to $700 \mathrm{~nm}$ ). Off-line calibration of the plate reflectivity and the known filter and TV camera throughput allowed us to spatially integrate an image frame at shot time to determine the total amount of scattered light. The images could also be combined with the images recorded by the FABS SBS and SRS cameras to provide an image of the angular distribution of scattering inside and outside the interaction beam solid angle. The diagnostic was supplemented by time-integrated diodes and fiber optics viewing the target through holes in the plate. The fiber optics allowed samples of the scattered light to be transported to an optical streak camera, where differences in time history as a function of scattering angle could be quantified.

The calibration of the plate reflectivity plus camera sensitivity could be checked in situ by comparing the scattered intensity with that recorded on the diodes. We could also compare the sensitivity of the FABS and the NBI by using a Au foil target at the chamber center to reflect energy from one of the other beam lines towards beam 7 . This technique uses the "glint" reflected from the $\mathrm{Au}$ foil to fill both diagnostics with light. Comparison of the glint imaged in both diagnostics then revealed any inconsistencies as sharp jumps in image intensity where the coverage of the two diagnostics abutted one another. Contamination of the plate by target debris is a potential source of inconsistency. Initially a large effort was made to compare NBI and FABS to keep confidence in the calibration of NBI. As the use of the NBI diagnostic has become more routine, we have instituted a schedule of installation, removal, and recalibration of sections of the plate, and we use the diodes inset in the plate to maintain confidence in the evolution of the calibration. Problems with the time-integrating diodes (described below) have been eliminated by using more linear diodes with better dynamic range. The uncertainty in the NBI SRS and SBS numbers is about $30 \%$ for the data discussed below.

Sidescattered SBS light in these experiments was studied with a diode array. The diodes were time-integrating, so they could not distinguish the contributions due to sidescattering from the heater beams. The solution to this problem was to put fiber optics inside the chamber beside each diode to relay the SBS light from there to an optical streak camera, which then provided a time-resolved history of the sidescattering that could be normalized with the time-integrated diode signal. The contributions due to sidescattering from the heater beams could then be seen occurring early in time; those due to the interaction beam would come later. This system of combined diodes and fiber optics was called the oblique scattering array (OSA) ${ }^{16}$ The system was not operational during the first $f / 8$ campaign (April 1994) and was only partially operational during the second f/8 campaign (February 1995). In all applications involving diode measurements, it was necessary to absolutely calibrate the detectors, filters, and blast shields off-line and then monitor how their calibration changed in the target chamber due to debris buildup by frequent recalibration and replacement of debris shields and filters. During the first $f / 8$ campaign, when the diodes were being used to quantify SBS near backscatter outside the lens cone, the diodes became nonlinear in their sensitivity to $3 \omega$ light at distressingly low fluences. This effect required characterization of the diode sensitivity over a wide range of incident fluences and also reduced the effective dynamic range of the diodes. ${ }^{17}$ Individual diode readings fluctuated from shot to shot, and diodes next to one another often gave conflicting measurements. This may have been due to diode nonlinearity or damage or to small-scale structure in the scattered light intensity, which is difficult to resolve with single-point diode measurements. This latter consideration was one motivation for making the imaging NBI system for near backscatter. We may build a similar imaging system for sidescatter that will avoid the poor statistics of the single-point diode 
measurements. New diodes were identified that had better dynamic range and that were linear up to higher fluences. These diodes were installed in the NBI plate as described above and were retrofitted into the OSA array.

The last diagnostic shown in Fig. 6 is the Transmitted Beam Diagnostic (TBD), whose purpose is to measure the amount of light transmitted by our open-geometry plasmas and to study the effect of processes such as diffraction from filaments on the divergence of the transmitted beam. This diagnostic was first set up during the $1994 \mathrm{f} / 8$ campaign. The device was a frosted glass plate mounted on the opposite side of the chamber from Beam 7 (see Fig. 6). In its original form, the plate intercepted, transmitted, and forward-scattered light over an angle equivalent to f/7.2. However, it was quickly realized that the beam expanded beyond the $\mathrm{f} / 8$ original beam divergence as a consequence of refraction, diffraction in filaments, or forward SBS. For the second $f / 8$ campaign, the TBD was enlarged to detect light over a $f / 3.6$ cone. The plate was viewed by an optical framing camera that recorded the beam angular spread at four distinct times, together with a streak camera that resolved the divergence in one dimension continuously in time. Absolute measurement of the transmitted light was made using a fast diode that gave the history of the beam integrated in both spatial dimensions. The system was calibrated by firing a shot with known $3 \omega$ laser energy through an aperture placed at chamber center to remove the $1 \omega$ and $2 \omega$ light. $A$ secondary measurement made with the TBD system was quantification of forward Raman scattering by injecting some of the transmitted light through a fiber optic into a low-resolution streaked optical spectrometer. Fiber-optic coupling was also used to inject the forward-scattered light into a high-resolution spectrometer to investigate the effects of filamentation on laser wavelength.

The main purpose of the TBD was to reveal changes in transmission due to the onset of scattering processes. Quantifying all the loss mechanisms due to parametric processes is very difficult. However, since the main objective of our experiments is to show that we can propagate a NIF-like beam through a NIF-like plasma without adverse effects, it was decided that by looking at the beam after it has traversed such a plasma we can directly discern if some as-yet unquantified process is serving as a loss mechanism. By looking at the characteristics of the beam after it has passed through one of our open-geometry gasbag plasmas, as a function of beam smoothing and laser intensity, we can ensure that the laser will propagate according to design in the NIF hohlraum.

\section{NIF Experiments}

In April 1994 the NIF beam geometry was reproduced on Nova by modifying one beam to have the $f /$ number and smoothing characteristics of a cluster of four NIF beamlets. Nine of the Nova beams were used to produce the long-scalelength plasma; the tenth was configured as an interaction beam that was sent through the preformed plasma after a 500-ps delay. The SRS and SBS scattered from the plasma, together with the effects of the plasma on the transmitted beam, were studied as a function of the interaction beam intensity, beam smooth ing, and plasma constituents. The interaction beam was smoothed by using RPPs, ${ }^{18}$ and four different colors within the $f / 8$ beam to mimic the NIF laser architecture ${ }^{19}$ The four-color setup divided the $f / 8$ beam into four separate quadrants, each of which had its wavelength shifted relative to the others. The wavelength separation of the colors was approximately $1.4 \AA$ at $3 \omega$. Since each beam quadrant could have its frequency conversion crystals individually tuned for its wavelength, the four-color scheme approximated "bandwidth" on the interaction beam without losing $3 \omega$ conversion efficiency. We also studied the use of additional laser bandwidth of approximately $1.6 \AA$ at $1 \omega$ on each color, coupled with SSD, ${ }^{20}$ to further reduce reflected SBS. These studies were performed with both $f / 4.3$ and $f / 8$ interaction beam focusing.

The main results of the $1994 \mathrm{f} / 8$ campaign were the observation of low amounts of SBS, with indications of more SBS scattered around the lens (a factor 2 or 3 in gasbags), but not enough to be a significant loss mechanism. Figure 8 shows the total SBS backscatter as a function of laser beam intensity and beam smoothing for the gasbag targets. The data are separated into the three smoothing conditions that were studied: fourcolor as described above, "one color," in which the four quadrants were set to be the same color, and "onecolor SSD," which had an additional bandwidth of $1.6 \AA$ at $1 \omega$ put on the one-color setup. The data plotted is the peak SBS reflectivity in time (reflected power/incident power), not corrected to account for SBS light reflected outside the lens acceptance angle. Our estimates based on the use of diodes to measure the energy outside the lens indicate that these numbers should be increased by a factor of 2 to 3 , making the maximum SBS seen in these experiments only $\sim 6 \%$.

Low levels of SBS were observed for irradiances up to $6 \times 10^{15} \mathrm{~W} \mathrm{~cm}^{-2}$ (well above that planned for the NIF). There was little difference in the levels observed with one-color or four-color irradiation; however, the addition of SSD beam smoothing to the one-color case reduced the SBS to below $0.5 \%$. The observation of these low 
scattering levels did raise the interesting possibility that the highest intensity planned for the NIF was too conservative, and that a design with higher laser beam intensity and hence higher hohlraum radiation temperature might be viable. The benefit in higher capsule gain and relaxed surface roughness specifications for instability growth made experiments at higher intensity very attractive.

The low level of SBS backscatter and the scaling of the SBS with intensity are perplexing. The apparent observation that four-color beam smoothing produces more SBS backscatter than one-color smoothing at high intensity was contrary to our expectation that fourcolor smoothing should suppress filamentation at least as well as one-color smoothing, if not better. The observation that SBS is reduced with the addition of a small amount of bandwidth and SSD beam smoothing is more sensible.

During this 1994 campaign we made the first transmitted-beam measurements, but these were limited to collecting light over an $f / 7.2$ aperture; the transmitted $f / 8$ probe beam broadened to overfill the detector, making quantification of the transmitted fraction uncertain.

Quantification of SRS backscattering from the longscalelength plasmas was limited to large-angle diode measurements and measurements of the hard $x$-ray

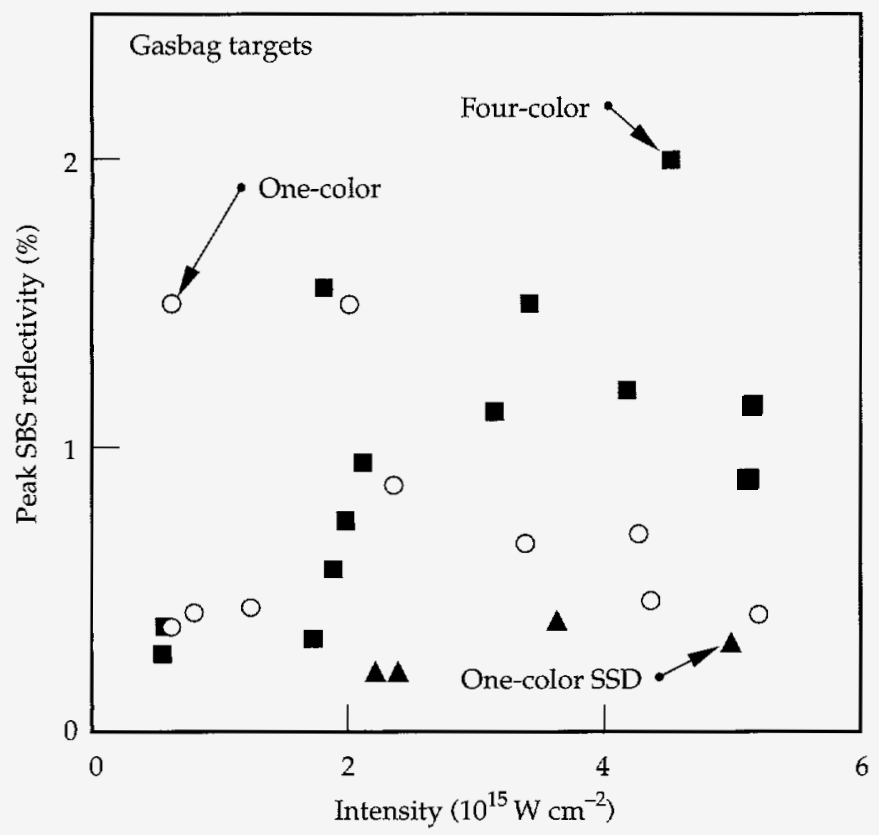

FIGURE 8. Measured SBS reflectivity from gasbag targets from April $1994 \mathrm{f} / 8$ campaign. Quantity plotted is peak reflectivity (scattered power/incident power) into an $\mathrm{f} / 8$ collection angle; contributions due to scattering outside this solid angle are estimated to increase these numbers by a factor of 2 to 3 . (20-07-1095-2441pb01) spectrum produced by fast electrons striking the $\mathrm{Au}$ hohlraum wall. These measurements showed that at most $1 \%$ of the incident energy was converted to fast electrons, with the limitation that the time-integrated technique did not distinguish between electrons produced by the heater beams and those produced by the probe beam. Experiments with the probe beam energy set to zero showed fast-electron yields similar to those with high power on the probe. In all of the interaction experiments carried out at $f / 4.3$ and $f / 8$ during this period, the inferred levels of SRS were low $(<1 \%)$. This was perplexing, because a few experiments with gasbag targets in the Two Beam chamber area of Nova produced larger levels of SRS (of the order of $4 \%$ ). However, those experiments were done with plasmas that were cooler $(1.5 \mathrm{keV})$ than the plasmas heated by nine Nova beams ( $3.2 \mathrm{keV})$. This observation is consistent with the higher temperature, in the plasmas heated with nine beams, resulting in strong electron Landau damping of the electron plasma waves at $0.1 n_{c}$ for $3 \omega$ and so reducing SRS. In either case, however, the plasma should have had a peak gain exponent of at least 20 for SRS [e.g., Fig. 10(d), below]. The Two Beam experiments did not have a filter-fluorescer measurement for fast electrons produced by SRS. The SRS was measured by observing the scattered optical light that went back into the interaction beam lens. This difference motivated the installation of a similar optical SRS detection system in the Ten Beam chamber for the second $f / 8$ campaign to better quantify SRS.

Several issues were left unresolved by the first f/8 campaign:

1. The effectiveness of SSD beam smoothing in suppressing filamentation and how it compared with four-color smoothing.

2. The effectiveness of four-color versus one-color beam smoothing in suppressing filamentation.

3. The low level of SBS.

4. The low level of SRS inferred from FFLEX.

5. No real quantification of the characteristics of the transmitted beam.

6. Sidescattering was not quantified.

Furthermore, the possibility of beams with different colors (e.g., four-color) transferring energy when crossing within the plasma had been raised by experiments that showed such an effect in long-wavelength $(10.6 \mu \mathrm{m})$, low-density experiments. ${ }^{21}$ This possibility merited investigation in more NIF-relevant plasmas using $3 \omega$ light.

For the initial $f / 8$ experiments, the peak SBS observed from the $\mathrm{C}_{5} \mathrm{H}_{12}$ plasmas was less than $6 \%$ for all irradiation conditions tested. It was therefore decided to perform a second series of $f / 8$ experiments 
with improved diagnostic capability to explore higher intensities than those expected in the NIF baseline design. These experiments would also attempt to resolve the issue of what beam smoothing is most effective for the NIF; however, these initial results were encouraging.

\section{Ion-Landau Damping Experiments}

To address the issue of low levels of SBS in our NIFlike plasmas, we looked at data obtained from plasmas that had a reduced level of ion-Landau damping. Some experiments carried out at $f / 4.3$ showed that changing the material in the target to one with a lower expected ion Landau damping of sound waves led to a strong increase in SBS. Figure 9 shows the results of an experiment performed at $f / 4.3$ that investigated the effect of removing the light ions (protons) from the plasma. Since the hydrogen ions in $\mathrm{C}_{5} \mathrm{H}_{12}$ are lighter than the carbon ions, their average velocity is significantly higher than that of carbon ions at the same ion temperature. The presence of these faster protons near the phase velocity of the ion-acoustic wave, which is pumped by the SBS process, allows them to extract energy from the wave and so damp it.

The data in Fig. 9(a) are from gasbag targets with a variety of gas fills. Backscattering levels as high as $35 \%$ were observed for $\mathrm{CO}_{2}$ plasmas, while subtly changing (a) SBS reflectivity measured

FIGURE 9. (a) Timeintegrated SBS reflectivity from gasbag targets containing mixtures of $\mathrm{CO}_{2}$ and $\mathrm{C}_{3} \mathrm{H}_{8}$ (crosses) or $\mathrm{C}_{5} \mathrm{D}_{12}$ and $\mathrm{C}_{5} \mathrm{H}_{12}$ (solid squares). The amount of the second material is varied and is expressed as percentage of ions that are protons vs proton concentration. (b) PIC calculation of SBS reflectivity from a $\mathrm{CH}$ plasma (normalized to unity for pure carbon) vs proton concentration. (c) Time integrated SBS backscatter from toroidal hohlraums, f/8 experiments; (d) SBS back-scatter from toroidal hohlraums, $f / 4$ experiments. (20-07-1095-2442pb01)

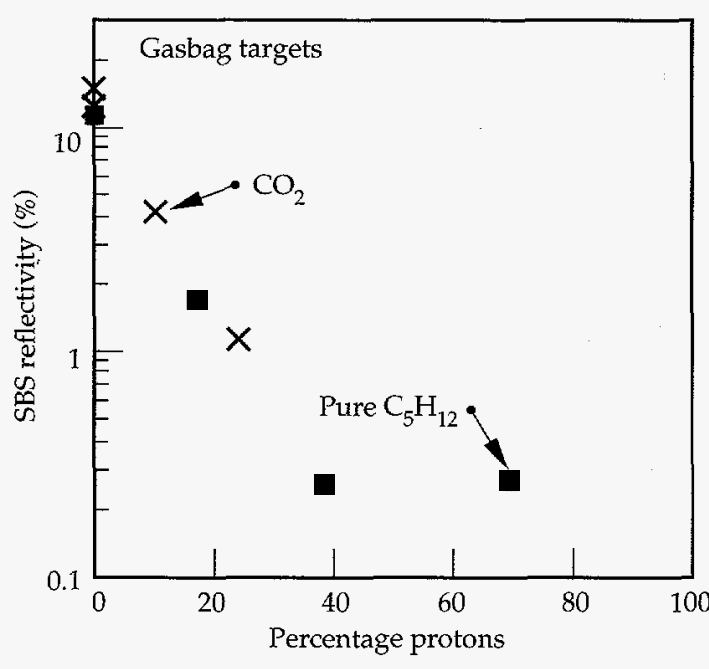

(c) Large gas-filled hohlraums, f/8, RPP

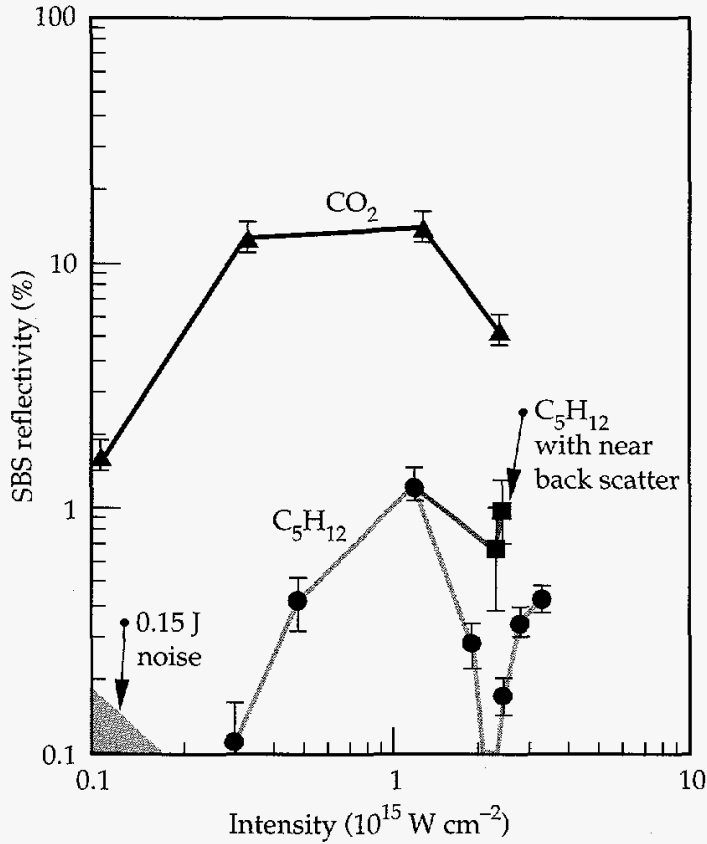

(b) PIC-calculated reflectivity

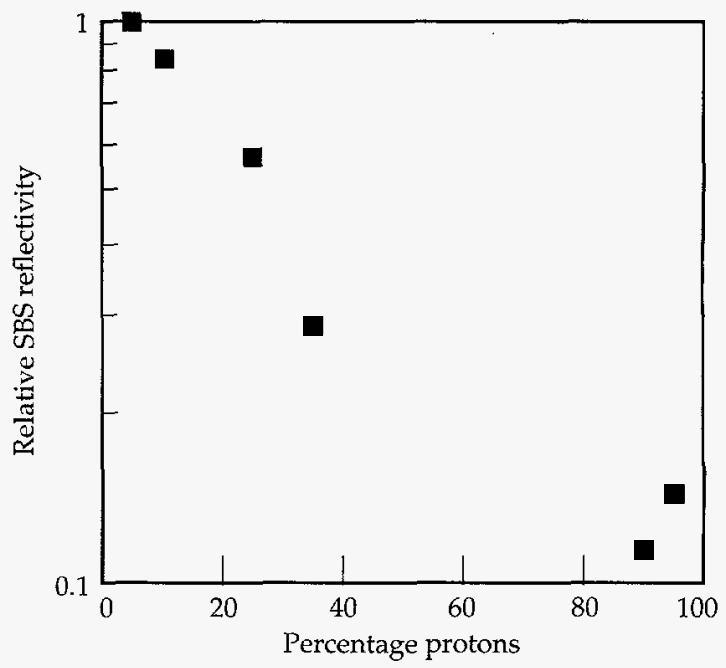

(d) Large gas-filled hohlraums, f/4, RPP

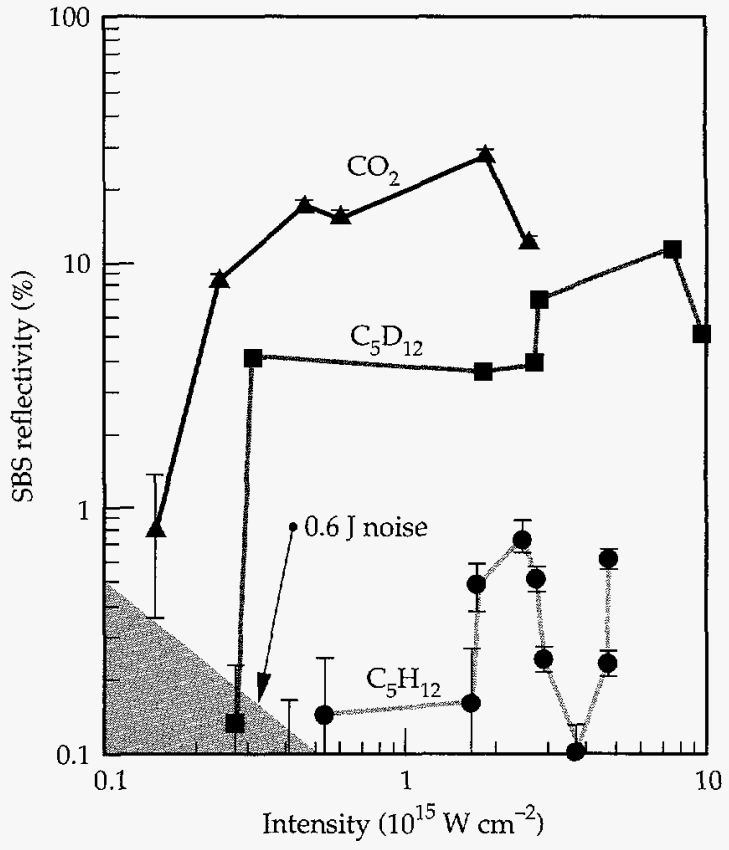


the plasma from $\mathrm{C}_{5} \mathrm{H}_{12}$ to $\mathrm{C}_{5} \mathrm{D}_{12}$ (i.e., changing protons to deuterons) increased the peak SBS from $\sim 3 \%$ to $\sim 25 \%$. Both $\mathrm{CO}_{2}$ and $\mathrm{C}_{5} \mathrm{D}_{12}$ are expected to have lower ion Landau damping than $\mathrm{C}_{5} \mathrm{H}_{12}$ at the range of ion temperatures expected in the experiments. ${ }^{22}$ The higher damping of the $\mathrm{C}_{5} \mathrm{H}_{12}$ plasma arises because of the mixing of a light ion $(\mathrm{H})$ that does the damping with a heavier ion $(\mathrm{C})$ that determines the sound speed. The high thermal speed of the hydrogen relative to the ion-acoustic wave speed allows the many protons moving at the wave speed to efficiently extract energy from the wave, damping the wave and reducing the SBS gain. This phenomenon was first reported by Clayton ${ }^{23}$ in low- $T_{e}$, low-density discharge plasmas irradiated with 10.6- $1 \mathrm{~m} \mathrm{CO}_{2}$ laser light. The experiment reported here was the first observation of this effect in a high- $T_{e^{\prime}}$ high-density plasma relevant to ICF. To demonstrate that the protons within the plasma provided the damping, Clayton altered the concentration of hydrogen in the plasma and observed the effect on the SBS threshold. We performed similar measurements with our $\mathrm{C}_{5} \mathrm{D}_{12}$ and $\mathrm{CO}_{2}$ plasmas by adding small concentrations of $\mathrm{C}_{5} \mathrm{H}_{12}$ or $\mathrm{C}_{3} \mathrm{H}_{8}$ to provide more protons. The gasbag experiments did not attempt to measure an intensity threshold for SBS, but instead kept the average intensity in the target fixed and varied the proton concentration.

Figure 9(a) shows the results, which illustrate the reduction in total SBS with increasing proton density. The SBS that we see has essentially saturated. This result has been modeled and essentially reproduced with PIC simulations [see Fig. 9(b)], ${ }^{24}$ which is interesting because the ion-Landau damping argument is an argument for a reduction in the linear gain for SBS. The PIC calculations obviously include this linear mechanism plus important nonlinear kinetics effects such as particle trapping and nonlinear frequency shifts. These latter effects reduce the gain beyond what would be calculated with the linear damping only. Figure 9(b) shows the calculated SBS reflectivity for a $\mathrm{CH}$ plasma, as a function of proton concentration, normalized to unity for pure carbon. The calculation gave a reflectivity of about $70 \%$ for this case. Note that a 1-D PIC simulation always overestimates the SBS reflectivity, because 2-D and 3-D effects that can also limit ion wave growth are obviously omitted.

Figures 9(c) and (d) show SBS reflectivity data (reflected energy/incident energy of the interaction beam) for the toroidal hohlraum experiments. These data are from the $1994 \mathrm{f} / 8$ series and from subsequent experiments carried out at $f / 4$. The figures also show the effect of the ion species on SBS reflectivity. The reflectivity appears to correlate inversely with the Landau damping rates of the SBS ion wave. For these hohlraum conditions $\left(T_{i} / T_{e} \approx 0.2\right)$, the estimated Landau damping rates normalized to the ion-acoustic frequency $\left(v_{\mathrm{i}} / \omega_{\mathrm{i}}\right)$ for $\mathrm{He} / \mathrm{H}, \mathrm{C}_{5} \mathrm{H}_{12}, \mathrm{C}_{5} \mathrm{D}_{12}, \mathrm{He}$, and $\mathrm{CO}_{2}$ are $0.35,0.25,0.15,0.01$, and 0.01 , respectively.

The plasmas we have produced seem homogeneous enough that the SBS is sensitive to the damping from different materials. This effect was not observed in exploding-foil experiments, ${ }^{25}$ in which the SBS reflectivities of $\mathrm{CH}$ and Ti plasmas were similar, presumably because of the large flow gradients $\left(L_{v} \approx 300 \mu \mathrm{m}\right)$, which reduce the effective interaction length because of dephasing proportional to the acoustic wave damping rate. The total gain is equal to the product of the gain rate $\left(\sim 1 / \nu_{\mathrm{a}}\right)$ and the interaction length $\left(\sim v_{\mathrm{a}} L_{v} / \omega_{\mathrm{a}}\right)$ and is then independent of the damping rate. Since our results show an SBS reflectivity that is sensitive to damping, they may indicate that the interaction length is being determined by something other than the velocity gradient scalelength $L_{v}$. We are continuing to study the relation between velocity gradients in our long-scalelength targets and those expected in NIF plasmas. Because our results show an SBS reflectivity that is sensitive to damping, they suggest that the interaction length is not dominated by inhomogeneities such as the global velocity-gradient scalelength $L_{v}$. Fluctuations in the velocity cannot be ruled out, but they should be similar in NIF plasmas to those in our long-scalelength targets, because the initial gases are uniform and nonuniformities are produced by similar laser heating processes.

The phenomenon of enhanced damping by adding protons to the plasma will be used in NIF hohlraums by filling them with mixtures of $\mathrm{H}_{2}$ and He. Calculations indicate that this mixture will provide even higher ionLandau damping than in our $\mathrm{C}_{5} \mathrm{H}_{12}$ experiments. ${ }^{22}$ The LIP calculations for the NIF plasma use the damping appropriate for this $\mathrm{He} / \mathrm{H}$ mixture. The gain exponent of 20 for the inner beam SBS taking place in the low- $Z$ plasma already takes into account the large ion-Landau damping in such a plasma.

\section{NIF Experiments}

As discussed above, the first round of $f / 8$ laser-plasma interaction experiments left many issues unresolved. After the diagnostic development described above, we embarked on a second $f / 8$ campaign with the intent of finding the optimum smoothing required for the NIF and of determining the beam. intensity above which laser-plasma instabilities prevented efficient beam propagation.

In this section we describe these most recent experiments and present some of the details of the experimental observations that were omitted in previous sections. Gasbag, GASBIG hohlraums, and toroidal hohlraum targets were all used in these experiments. The main results were that the levels of SRS backscattering were higher than inferred previously with the FFLEX 
technique. The SRS was collimated so that it came back straight into the lens. The levels of SBS were even lower than those observed previously; in fact, there is the distinct possibility that those measurements were contaminated by SRS getting into the SBS calorimeter. The levels of backscattering were sensitive to beam smoothing in the form of SSD and insensitive to differences between four-color or one-color beam smoothing. Scattering at $2 \times 10^{15} \mathrm{~W} \mathrm{~cm}^{-2}$ was at a level acceptable in NIF designs, but higher intensities produced far more SRS. The NBI diagnostic showed that the SBS scattered outside the lens multiplied the FABS-only number by a factor of 2 to 3 for gasbags, but GASBIG hohlraums produced much less symmetric SBS backscattering that had as much as a factor of 10 more SBS outside $f / 8$ than inside it.
Figures 10(a) and (b) show gasbag SBS and SRS spectra recorded in the direct backscatter direction with the FABS diagnostic. The data are for a $\mathrm{C}_{5} \mathrm{H}_{12}$-filled gasbag irradiated with $2.2 \times 10^{15} \mathrm{~W} \mathrm{~cm}^{-2}$ with a fourcolor $f / 8$ beam with $1.6 \AA$ of SSD beam smoothing. Figure 10(a) shows SBS spectra recorded from the gasbag plasma. Early in time ( 0 to 500 ps) the spectrum shows sidescattering from the nine heater beams scattering from the cold gasbag. The sidescattering contribution is small $(\sim 20 \mathrm{~J} / \mathrm{sr})$ and fades at later time $(\sim 500 \mathrm{ps})$ as the plasma heats up and the density peaks at the edge subside. Data taken with only heater beams (i.e., no interaction beam) show the same signals up to 500 ps. At 500 ps, the interaction beam turns on and produces a narrow SBS spectrum with a red shift of about $10 \AA$. This shift is consistent with that expected
FIGURE 10. Gasbag SBS and SRS spectra. (a) Time-resolved SBS spectrum; features appearing up to $500 \mathrm{ps}$ are sidescattering contributions from the nine heater beams interacting with a bag that is initially cold with high density at the edge. (b) Timeresolved SRS spectrum; (c) LIP-calculated gain exponent for SBS; (d) LIP-calculated gain exponent for SRS. (20-07-1095-2443pb01) (a) Gasbag SBS spectrum

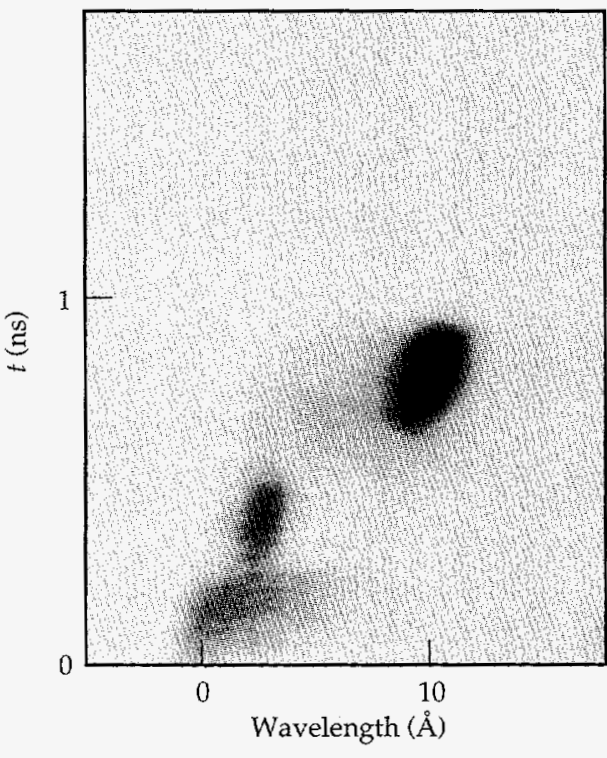

(c) SBS gain exponent (LIP)

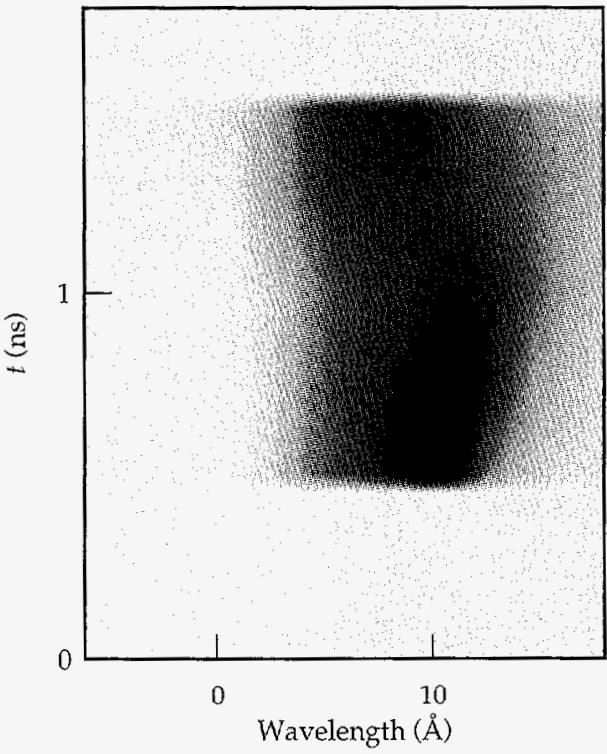

(b) Gasbag SRS spectrum

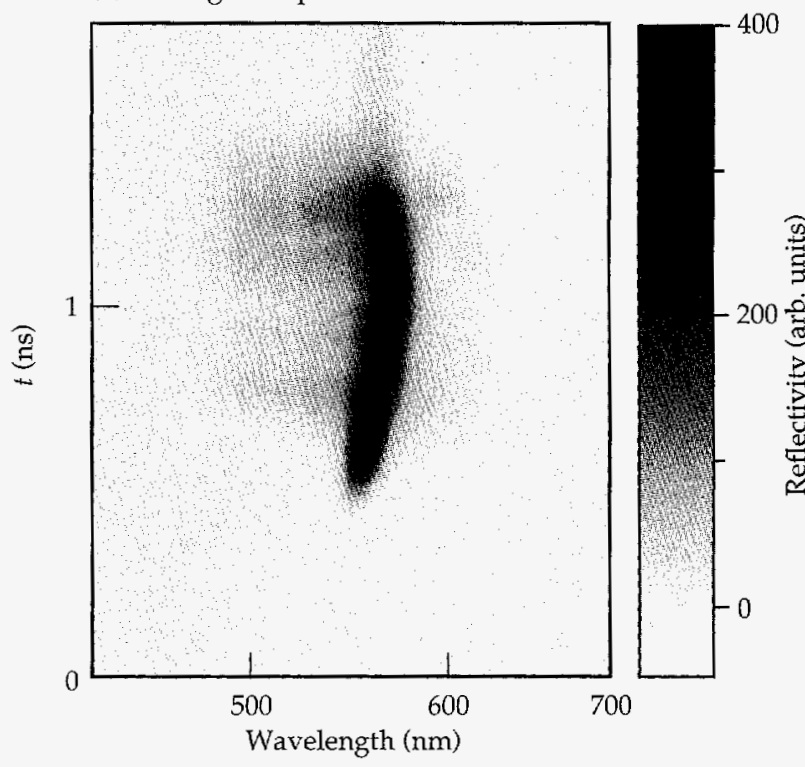

(d) SRS gain exponent (LIP)

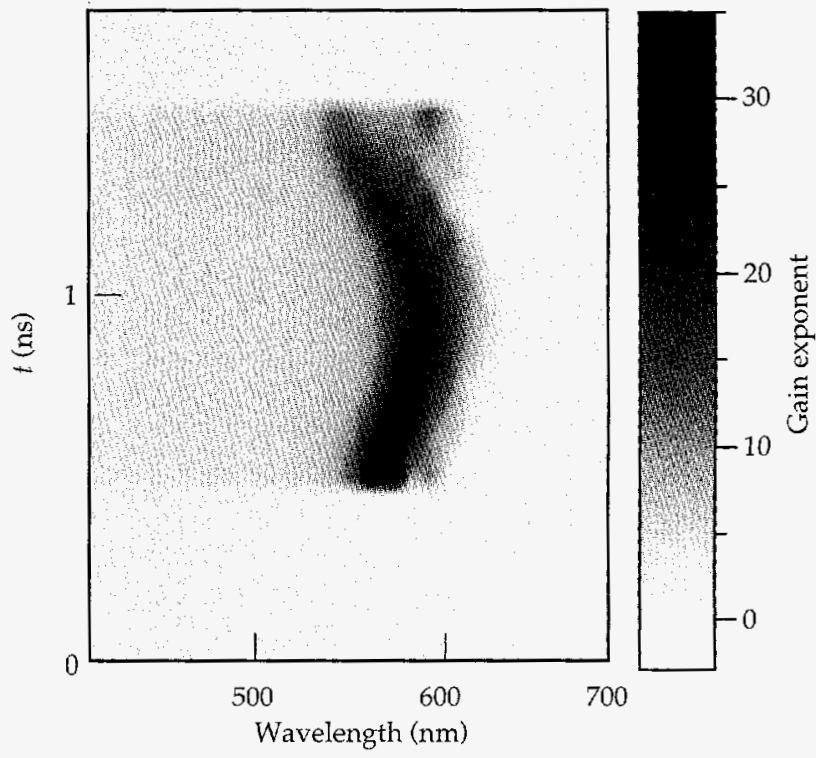


for SBS from a stationary $\mathrm{C}_{5} \mathrm{H}_{12}$ plasma at $3 \mathrm{keV}$. This result can be compared with spectra from exploding-foil plasmas, for which the SBS spectrum is usually broad and blue-shifted, indicating that it may be coming from a plasma that is flowing, giving various amounts of Doppler shift to the backscattered light wave. The fact that there is no shift other than that expected from conservation of energy is a good indication that the plasma is stagnant and that velocity gradients are low. After $1 \mathrm{~ns}$, the data show a strong reduction in the SBS signal.

The spectrum in Fig. 10(a) should be compared with the LIP gain exponent calculation using plasma parameters from a gasbag simulation, shown in Fig. 10(c). The calculation shows no gain before $500 \mathrm{ps}$, because the interaction beam is off LIP does not calculate sidescattering from the heater beams. After $500 \mathrm{ps}$, the calculation shows a narrow peak in the gain, which is 30 to 50 until 1 ns and then decreases. At late time, the gain exponent is reduced because the plasma is shrinking, making less interaction length available for amplification. The gain exponent is also decreasing because the ratio of ion to electron temperature is changing. The damping of SBS by ion-Landau damping is a strong function of this ratio (since the ion temperature determines the magnitude and slope of the ion distribution function at the ion wave speed, while the electron temperature determines the speed of the ion wave). At $1 \mathrm{~ns}$, when the heater beams turn off, the electron temperature starts to fall and, since the ion temperature responds much more slowly, $T_{\mathrm{i}} / T_{\mathrm{e}}$ rises rapidly, increasing the damping of SBS.

The correspondence between the gasbag SBS data and the LIP calculation is encouraging. The narrowness, red shift, and time history of the SBS spectrum are consistent, implying that we can predict the fundamental plasma parameters in the experiments. Since the gain exponent calculated at early time is 30 to 50 , well in excess of that calculated in the NIF inner beam (20), the measured SBS reflectivity of $\sim 0.2 \%$ for this experiment is promising for the NIF.

The LIP calculations [Fig. 10(d)] reproduce a number of features in the gasbag SRS spectrum shown in Fig. 10(b). The time history is interesting: there is a bright peak at $570 \mathrm{~nm}$, which is consistent with SRS scattering from electron plasma waves in plasma at $9 \times 10^{20} \mathrm{~cm}^{-3}$ (the average density of the plasma) and a temperature of $3.2 \mathrm{keV}$. The narrowness of the peak is consistent with a small range of densities that have sufficiently shallow density gradients to permit the growth of SRS. This narrow peak is reproduced in the LIP calculation shown in Fig. 10(d), but that calculation shows the peak at slightly longer wavelength $(585 \mathrm{~nm})$. Because the peak position depends on both temperature and density, it is possible that the disagreement arises because the plasma density or temperature is lower in the experiment than in the calculation. The peak moves towards long wavelengths from 500 ps to $1 \mathrm{~ns}$, consistent with an increasing temperature, as the heater beams continue to deposit energy in the plasma. After $1 \mathrm{~ns}$, when the heater beams turn off, the peak moves back to shorter wavelengths as the plasma cools. The peak movement associated with the rise and fall of the temperature is visible in the data, but the movement is not as great as in the simulation. The narrow SRS spectrum shown in Fig. 10(b) is reminiscent of those seen from exploding-foil plasmas, in which only the flat top of the density profile results in SRS because of the shallow density gradient there. ${ }^{26}$

Another remarkable feature in Fig. $10(\mathrm{~b})$ is the scattered light that appears between 470 and $550 \mathrm{~nm}$; this represents scattering from lower densities ( 0 to $0.1 n_{c}$ ), which may be present as a result of filamentation. The short-wavelength SRS appears and disappears as a function of beam smoothing and intensity (as shown in Fig. 16, below). Both these parameters affect filamentation of hot spots in the beam. The short wavelengths also appear in Fig. 10(b) to increase as the plasma cools; again, this is consistent with a reduction of filamentation thresholds as the temperature is reduced. These observations are therefore consistent with the short-wavelength light coming from filaments where the high intensities produced by filamentation are needed to overcome the heavy electron-Landau damping of the electron plasma wave at such low densities.

For comparison, Figs. 11(a) and (b) show SBS and SRS spectra from $\mathrm{C}_{5} \mathrm{H}_{12}$-filled GASBIG hohlraum targets irradiated with $2.3 \times 10^{15} \mathrm{~W} \mathrm{~cm}^{-2}$ with a four-color $\mathrm{f} / 8$ beam with $1.6 \AA$ of SSD beam smoothing. The small amount of scattering seen at early time in Fig. 11(a) is light from the heater beams scattering from the thin membrane that constitutes the window of the hohlraum. The interaction beam is $1 \mathrm{~ns}$ long and turns on $1 \mathrm{~ns}$ after the heater beams; the heater beams have a long, shaped pulse that is on for the entire duration of the experiment. Once the SBS turns on, it is red-shifted as was the gasbag SBS, but it is not red-shifted as much and is broader than for the gasbag. Both these observations are consistent with a more ramped profile [such as that shown in Fig. 3(d)], which has plasma flowing towards the observer (back towards the laser entrance hole), giving a range of Doppler shifts to the SBS light. The SRS spectrum shown in Fig. 11(b) is also consistent with scattering from such a ramped profile. The lower wavelength SRS ( 500 to $550 \mathrm{~nm}$ ) corresponds to densities below $0.1 n_{c}\left(0.04\right.$ to $\left.0.1 n_{c}\right)$. These lower densities may be on the profile or they may be present in filaments, as discussed above for the gasbag data. The time-integrated SBS and SRS reflectivities for this shot were 1.3 and $4.8 \%$, respectively.

Figure 12 illustrates some of the combined FABS and NBI data showing the collimation of the SRS backscattering and the large blob of SBS from the hohlraum that 
lies outside the FABS acceptance. The images are made by superposing the near-field image of the backscatter as seen by the FABS ( 0 to $\left.3.6^{\circ}\right)$ on the image recorded on the NBI camera $\left(5.3\right.$ to $\left.20^{\circ}\right)$ to produce an image that covers 0 to $20^{\circ}$ from the beam axis (a small annulus is missing between 3.6 and $5.3^{\circ}$ ). The SRS measurement is complicated by parallax in the view of the SRS camera inside the FABS, which results in distortion in the central part of the image. The examples shown in Fig. 12 are (a) gasbag SBS, (b) gasbag SRS, (c) GASBIG hohlraum SBS, and (d) GASBIG hohlraum SRS. The SRS images show that the SRS is well collimated. While the gasbag SBS image shows that the SBS is centered on the lens, that from the GASBIG [Fig. 12(c)] is mostly in a blob that is to one side of the lens. This energy was missed in our earlier (1994) experiments. Figures 12(e) and (f) are more informative, in that they show lineouts through the images and express the result in joules per steradian per incident joule. The lineouts are taken diagonally, such that they pass through the blob in Fig. 12(c). This direction is the radial direction where the center of symmetry is the long axis of the hohlraum. The lineouts show the collimated nature of the SBS and SRS for the gasbag target [Fig. 12(e)]. The GASBIG lineouts in Fig. 12(f) show that although SRS is collimated, the SBS appears about $8^{\circ}$ from the beam axis. The appearance of the SBS at such an angle may be due to deflection of the incoming beam as it filaments in the low-density plasma near the laser entrance hole. Filamentation in a plasma that is flowing at near the sound speed can introduce deflection of the beam. 27,28 If after deflection the beam continues to higher density, where direct SBS backscatter is produced, that SBS will not necessarily deflect again on the way out of the hohlraum and so will not retrace its path back out of the hohlraum. Hence there can be a net deflection of SBS that occurs after beam deflection. The direction of the deflection is consistent with the direction of deflection of the forward-propagating beam in $\mathrm{x}$-ray spot motion experiments performed with gas-filled hohlraums. ${ }^{29}$

Figure 13 summarizes the SBS backscattering data for gasbags and GASBIG hohlraums as a function of laser intensity and beam smoothing. Figure 13(a) shows the new SBS data for gasbags; it is noticeable that the backscattering levels are still low and in fact are lower than those observed in 1994 (Fig. 8). One explanation for this is that the time-integrated SBS signal in the earlier experiments was contaminated by stray light or by contributions from short-wavelength SRS. In these newer experiments, we used a diode that (1) was filtered to reject everything but SBS light (near 3 $\omega$ ), (2) had enough time resolution to discriminate against stray light reflected from objects such as the KDP crystal array, within the beam line, and (3) that could be calibrated in a similar manner to the SRS diode. The original motivation for using the diode in place of the calorimeter was that there was a finite reflection from the KDP array that had increased in severity since the first experiments and seemed to vary strongly with crystal tuning. In the 1994 experiments this stray reflection (quantified by observing the calorimeter signal when the beamline was fired with no target present) was small enough to ignore, but with the new experiment it produced random offsets in the signals recorded by the calorimeter. The new diode eliminated this problem and had the side benefit that it was insensitive to any SRS light that would be seen by the SBS calorimeter. (a) GASBIG SBS spectrum

FIGURE 11. GASBIG hohIraum SBS and SRS spectra. (a) Timeresolved SBS spectrum (b) Time-resolved SRS spectrum; data from FABS direct backscattering diagnostic. (20-07-1095-2444pb01)

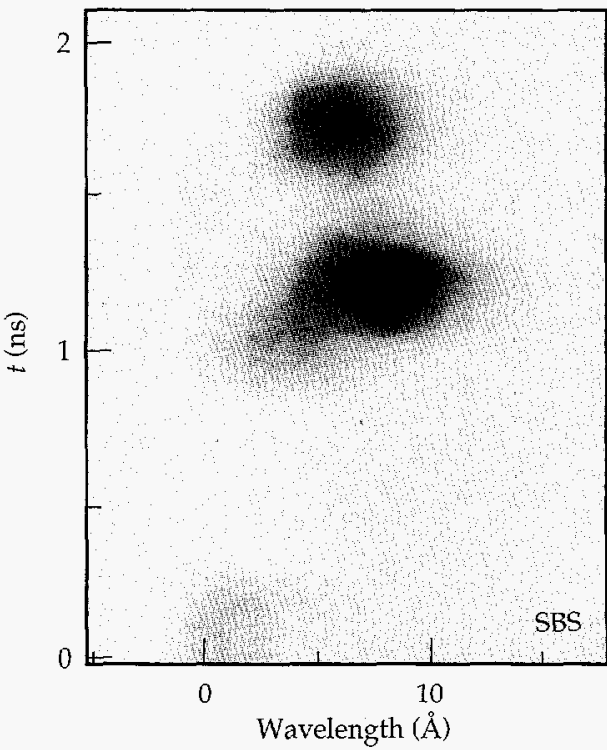

SBS (b) GASBIG SRS spectrum

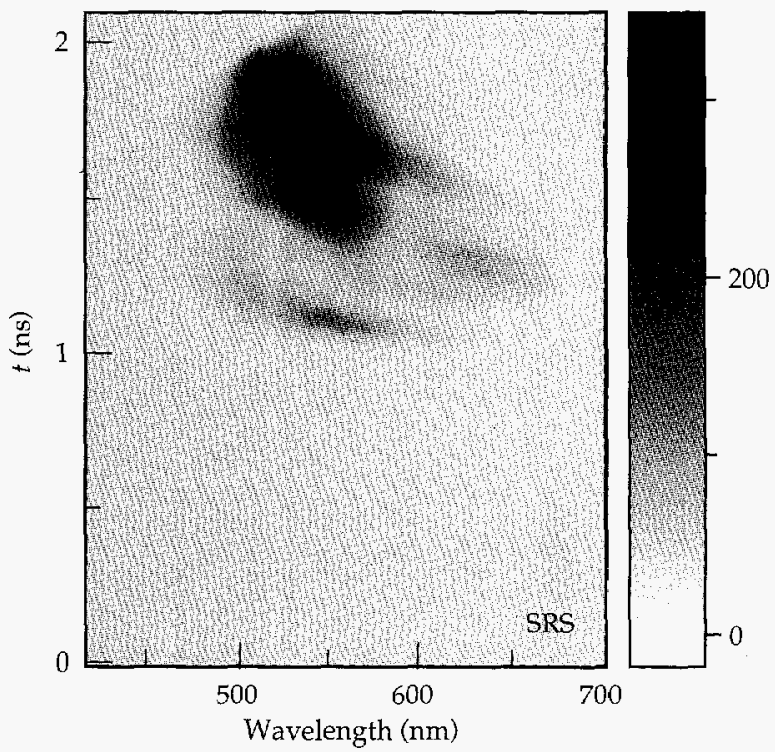


(a) Gasbag SBS

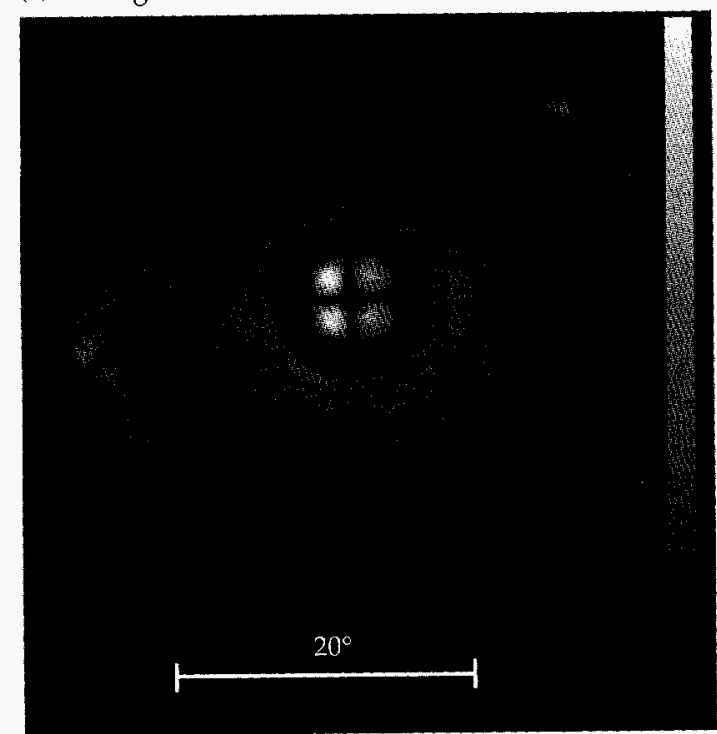

(c) GASBIG SBS

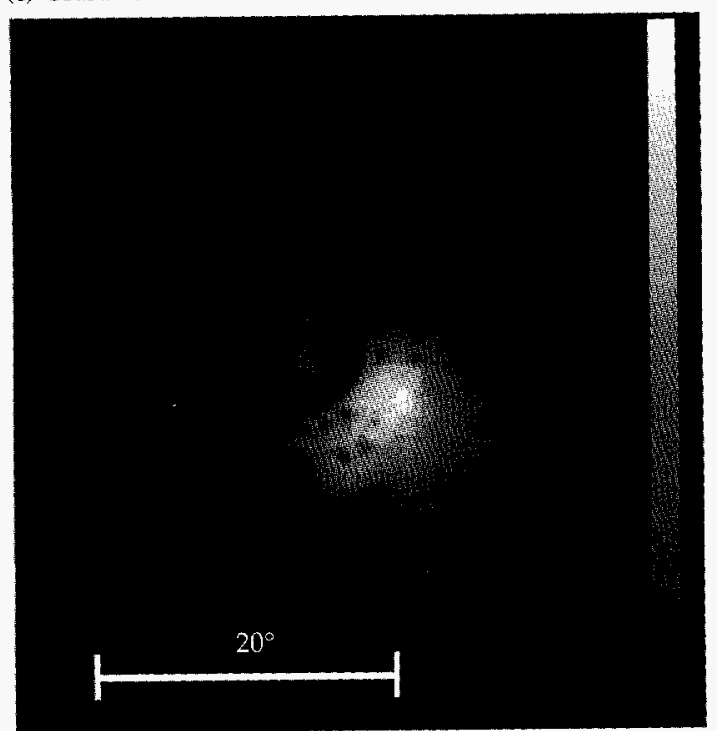

(e)

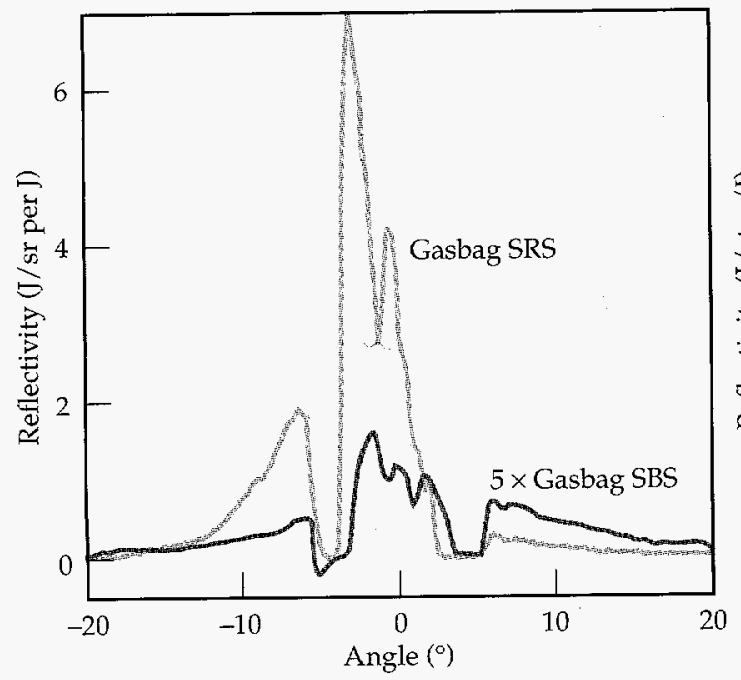

(b) Gasbag SRS

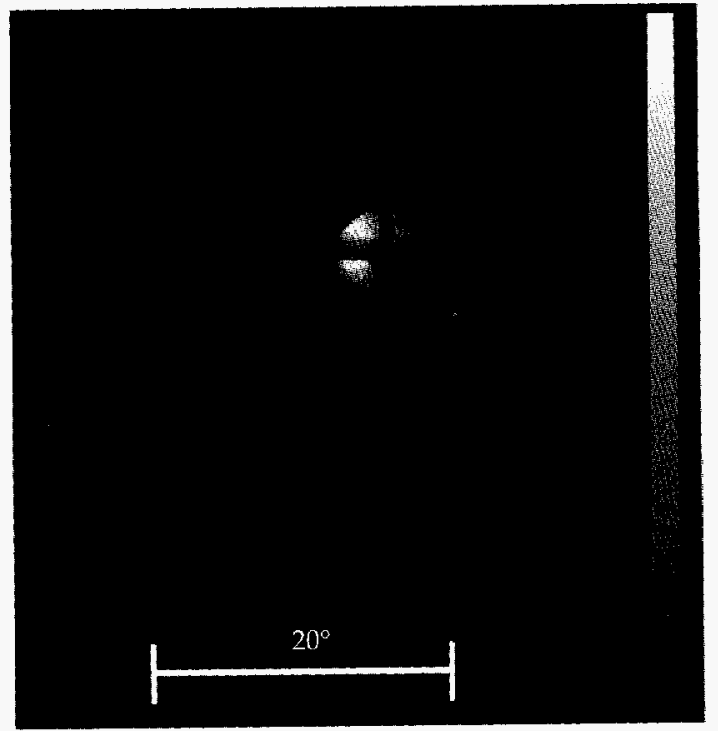

(d) GASBIG SRS

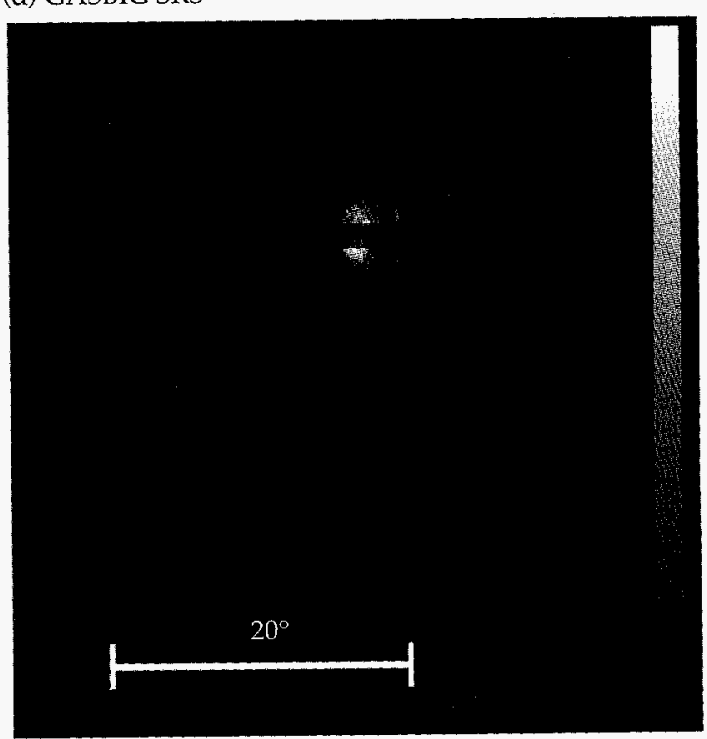

(f)

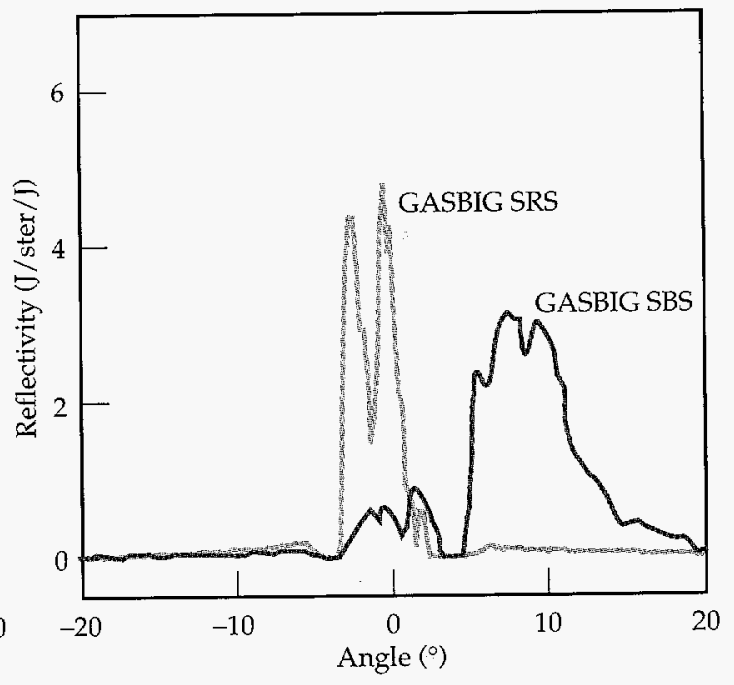

FIGURE 12. Angular distribution of scattered light gasbags and hohlraums. (a) Gasbag SBS; (b) gasbag SRS; (c) GASBIG hohlraum SBS; (d) GASBIG

hohlraum SRS; (e), (f) lineouts showing angular dependence of SRS and SBS: (e) gasbag target; (f) GASBIG target. (20-07-1095-2445pb01) 
Figure 13(a) shows the peak SBS reflectivity into $f / 8$, for the gasbag targets. There has been no attempt to include the contribution due to near-backscattered light. Again, that contribution increases these numbers by a factor of 2 to 3 , but the scattered light levels are so low that stray light transmitted by the target striking the NBI plate reduces the precision of the NBI measurement, which then is only good on high-SBS shots. Therefore it is not possible to show the intensity scaling of the data. Figure 13(b) shows similar SBS data for GASBIGS hohIraums; again the NBI contribution has been omitted (the detector malfunctioned on some of the shots), but here the correction factor for the NBI is 6 to 10 . In both sets of data it is evident that the addition of SSD reduces the SBS backscattering but that there is no obvious benefit to using four-color smoothing.

Figure 14 summarizes the SRS backscatter data for gasbags and GASBIG hohlraums as a function of laser intensity and beam smoothing. Figure 14(a) shows the data for gasbags. The quantity plotted is the timeresolved SRS reflectivity at the point of maximum plasma temperature (i.e., when the heater beams turn off at $1 \mathrm{~ns}$ ). The reflectivities for four-color and one-color beam smoothing approach $10 \%$ for the NIF intensity of $2 \times 10^{15} \mathrm{~W} \mathrm{~cm}^{-2}$; the values for experiments that used additional SSD smoothing are closer to $5 \%$. Figure 14(b) shows similar data for the GASBIG hohlraums; the SRS levels at $2 \times 10^{15} \mathrm{~W} \mathrm{~cm}^{-2}$ are similar to those for the gasbag plasmas. The data in both Figs. 14(a) and (b) have had the contributions due to the NBI added and have been scaled to account for energy that would fall into the annulus between 3.6 and $5.3^{\circ}$. These SRS numbers therefore represent the total reflectivity into a cone up to $20^{\circ}$ around the beam axis; however (for SRS), most of that energy is within the original $f / 8$ solid angle.

The SRS signals are stronger than previously inferred using the FFLEX $x$-ray technique. An explanation of this discrepancy is that the low phase velocity of electron
FIGURE 13. SBS vs intensity for various kinds of smoothing. (a) Gasbags. (b) GASBIGS; numbers do not include scattering outside the $f / 8$ lens and are the peak reflectivity in time (reflected power/incident power). (20-07-1095-2446pb01) (a) Gasbag SBS

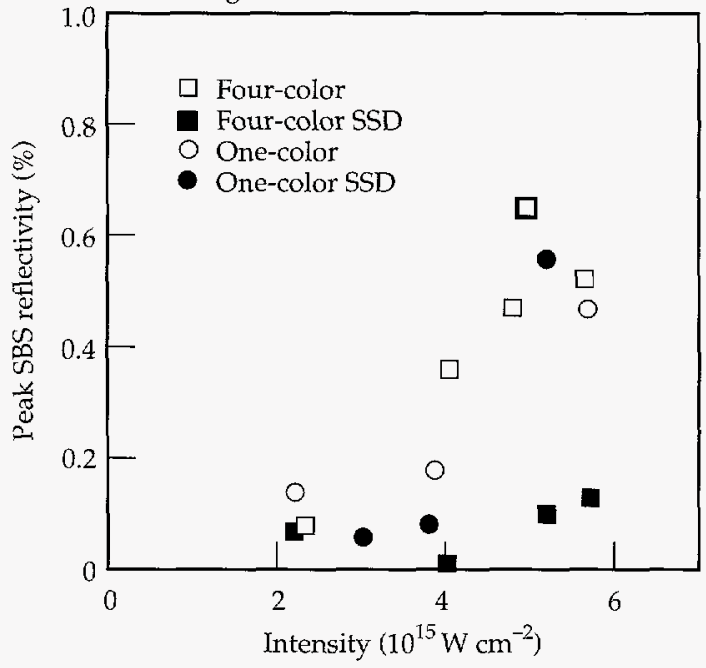

(b) GASBIG SBS

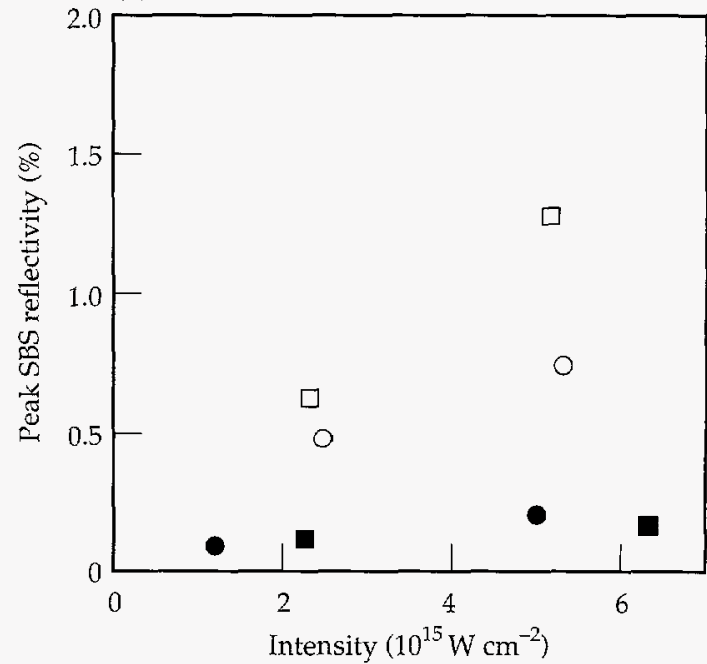

(a) Gasbag SRS

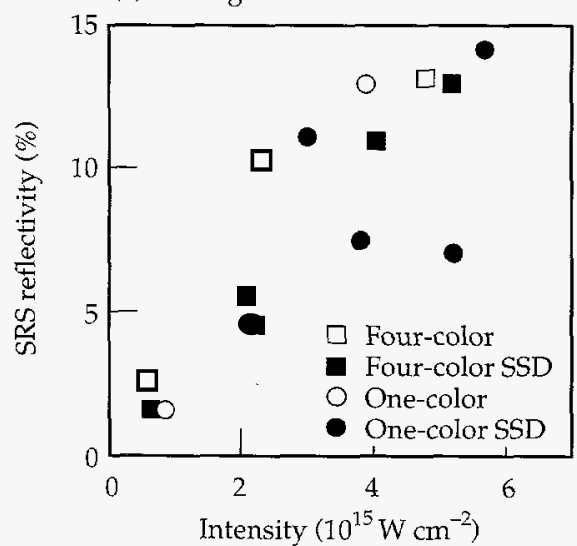

(b) GASBIGS

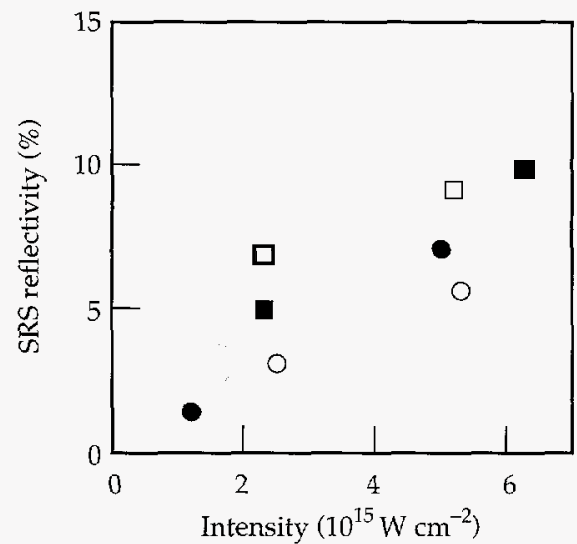

(c) $0.1 n_{c}$ gasbag (FFLEX)

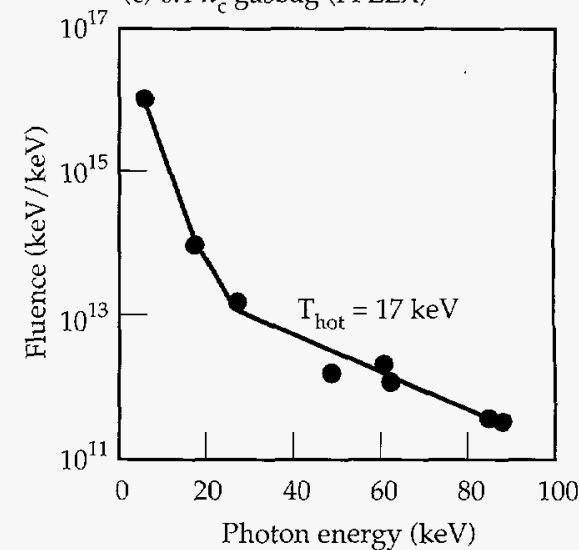

FIGURE 14. SRS vs intensity for various kinds of smoothing. (a) Gasbags; (b) GASBIGS (c) FFLEX data from $0.1 n_{\mathrm{c}}$ gasbag. (20-07-1095-2447pb01) 
plasma waves in $0.1 n_{c}$ plasma produces low-velocity electrons as the electrons damp the wave. Hence we expect electron energies of 10 to $20 \mathrm{keV}$ to be produced by the SRS that we are detecting. The FFLEX technique is flawed in a gas-filled hohlraum because a significant fraction of any $10-\mathrm{keV}$ electrons will be stopped in the gas (range $400 \mu \mathrm{m}$ ) before they reach the wall. Thus if they produce $x$ rays as they are slowed, it will be through bremsstrahlung resulting from interactions with the lower- $Z$ gas ions rather than with $A u$. The radiation production scales as $Z$, so carbon is much less efficient than $\mathrm{Au}$. (The FFLEX analysis requires a knowledge of the $Z$ of the plasma ion.) Even worse, the $x$ rays that are produced cannot then get out of the hohlraum, because the opacity of the Au wall is high for $x$ rays below $40 \mathrm{keV}$. Hence the FFLEX diagnostic was useful when the fast electrons that were being detected were $100 \mathrm{keV}$, such as are produced at $0.25 n_{\mathrm{C}^{\prime}}$ FFLEX is not useful at the low $n_{\mathrm{e}} / n_{\mathrm{c}}$ encountered in $3 \omega$ experiments unless the target has a well-defined $Z$ and has a low opacity to the $x$ rays that are produced. The up side of all this is that 10 - to $20-\mathrm{keV}$ electrons are not a preheat threat. The FFLEX is sensitive to electrons that are a preheat threat, because significant quantities of $100-\mathrm{keV}$ electrons could preheat a capsule while $10-$ to $20-\mathrm{keV}$ electrons would not penetrate the capsule ablator.

Figure 14(c) shows $x$-ray filter-fluorescer (FFLEX) data from a gasbag shot fired with 10 heater beams (i.e., Beam 7 was also configured as a heater beam, with no RPP or bandwidth and with the same $f / 4.3$ focus as

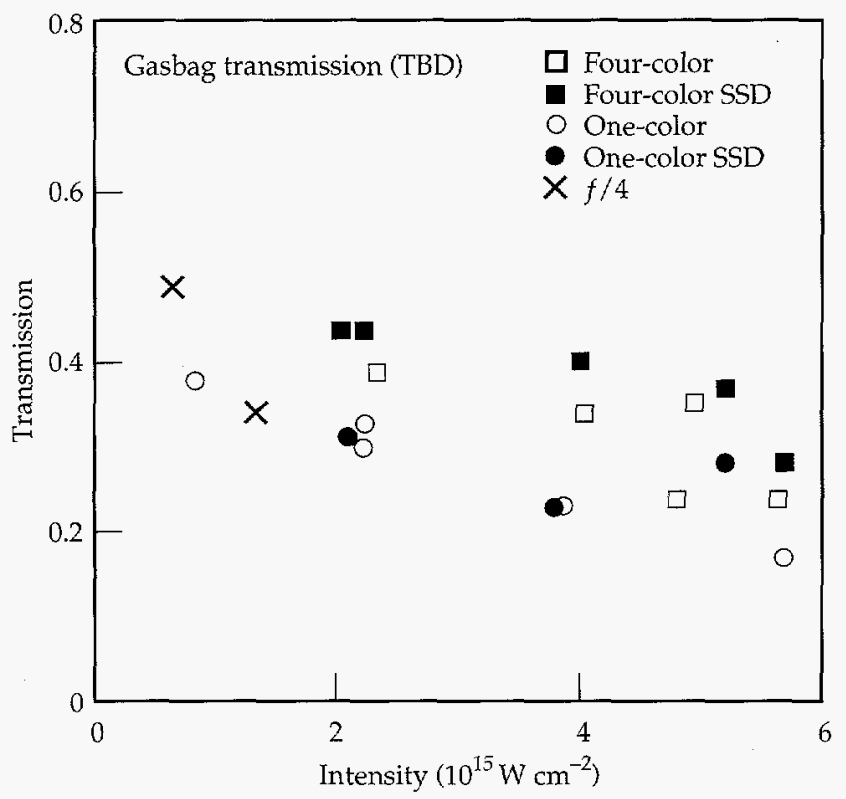

FIGURE 15. Time-integrated transmission of gasbag targets from TBD diagnostic for various kinds of beam smoothing. Expected gasbag transmission is $41 \%$ for $T_{\mathrm{e}}=3.2 \mathrm{keV}$ and $37 \%$ for $T_{\mathrm{e}}=2.8 \mathrm{keV}$. (20-07-1095-2448pb01) the other heater beams) with a total energy of $25 \mathrm{~kJ}$. This setup allowed us to measure the optically scattered SRS on Beam $7(150 \mathrm{~J}, 6 \%)$ and then (by assuming that all beams were the same) to estimate a total scattered SRS level of $1500 \mathrm{~J}$ for the target. The FFLEX data in Fig. 14(c) were fitted to a suprathermal-electron temperature of $17 \mathrm{keV}$, which is consistent with electrons accelerated by damping of electron plasma waves due to SRS at $0.1 n_{\mathrm{C}^{\prime}}$ which is the plasma density expected. The SRS optical spectrum (not shown) peaked at $560 \mathrm{~nm}$, also consistent with $0.1 n_{c}$.

The amount of fast electrons inferred from the spectrum, in Fig. 14(c), is $680 \mathrm{~J}$ assuming that the $\mathrm{x}$ rays are produced by electrons colliding with the $\mathrm{C}$ and $\mathrm{H}$ in the $\mathrm{C}_{5} \mathrm{H}_{12}$. Hence the ratio of electron energy to SRS optical light is 1:2.2. The expected division of energy between the electron plasma wave and the scattered light wave at $0.1 n_{c}$ is $1: 1.7$, implying that there should be $880 \mathrm{~J}$ of fast electrons. Although we detected $680 \mathrm{~J}$, our estimate of the energy transferred to the electrons does not take into account ways, other than electron Landau damping, by which energy can be removed from the electron plasma waves. We conclude that the FFLEX provides a useful time-integrated diagnostic of SRS losses if the converting material is known and the target is optically thin to the radiation produced by the electrons. Even in hohlraums, however, the FFLEX is a useful monitor of the electrons ( 50 to $100 \mathrm{keV}$ ) that are capable of preheating the capsule.

As mentioned above, the final test for beam propagation through a NIF plasma is to show that there are no adverse effects to the light that has propagated through such a plasma. Figure 15 shows a summary of the transmitted light from the $f / 8$ experiments with gasbag plasmas. The quantity plotted is the time-integrated transmission of the interaction beam through the gasbag. Since the laser light is being absorbed by inverse bremsstrahlung, the transmission at any instant is a function of the temperature and density of the plasma. Using LASNEX-generated density profiles, the expected transmission of the gasbag is $41 \%$ for a peak temperature of $T_{e}=3.2 \mathrm{keV}$ and $37 \%$ for $T_{e}=2.8 \mathrm{keV}$. The transmission values in Fig. 15 are consistent with these numbers for NIF-relevant intensities (up to $2 \times 10^{15} \mathrm{~W} \mathrm{~cm}^{-2}$ ) but drop below this value as the intensity is increased above $2 \times 10^{15} \mathrm{~W} \mathrm{~cm}^{-2}$. Possible explanations for the decrease in transmission at higher intensity are increased losses due to SRS backscattering and spreading of the transmitted beam outside the detector solid angle. The time-integrated SRS for many of the shots above $4 \times 10^{15} \mathrm{~W} \mathrm{~cm}^{-2}$ approached $15 \%$. Adding in losses due to fast electron generation ( $1 / 1.7$ times the scattered optical energy) results in a total time-integrated "loss" to SRS of order $25 \%$ for the higher-intensity shots. This $25 \%$ "loss" will reduce the expected transmission to $31 \%$ and $28 \%$ for peak temperatures of 3.2 and $2.8 \mathrm{keV}$. 
(The energy put into fast electrons is not really a loss, because the energy will stay in the plasma as the electrons are rapidly thermalized). The time-resolved aspects of these data are still being studied to correlate the SRS and transmitted beam as a function of time.

One interesting aspect of the data shown in Fig. 15 is that the highest transmission is seen in experiments with four-color beam smoothing. This is consistent with four-color smoothing providing better suppression of filamentation and therefore less losses due to parametric processes or beam breakup due to filamentation.

However, none of the backscattering data shows any reduction in reflectivity with four-color smoothing.

This inconsistency is under study.

A secondary goal of the TBD was to quantify forward Raman scattering. Spectral measurements of forward SRS ( $400 \mathrm{~nm}<\lambda<900 \mathrm{~nm}$ ) within 0.2 sr of the probe beam center show spectra that do not appear to depend on the presence of the probe beam. We observe no detectable change in the spectra for similar experiments with the probe beam on or off. The measured spectrum appears to come almost entirely from the sidescattered SRS produced by the heater beams. The level of this sidescattering is about $10 \mathrm{~J} / \mathrm{sr}$ (wavelength-integrated). We estimate an upper bound on the forward SRS level only from the probe beam to be less than about $5 \%$ of the heater beam sidescattering, or about $<0.5 \mathrm{~J} / \mathrm{sr}$ (wavelength-integrated).

Figure 16(a) shows time-integrated. backscattered SRS spectra from targets shot with different intensity or beam smoothing. The short-wavelength SRS appears and disappears with changes in the beam smoothing and laser intensity. The lowest intensity case shows very little short-wavelength SRS, while the case with four-color smoothing and $2 \times 10^{15} \mathrm{~W} \mathrm{~cm}^{-2}$ shows significant reflected light at short wavelength. The addition of $1.6 \AA$ of bandwidth reduces the peak reflectivity but also greatly reduces the short-wavelength SRS. Since the short-wavelength SRS is probably associated with low densities in filaments, the above observations are consistent with suppression of filamentation in the lower two curves by low intensity or by SSD. Figure 16(b) shows the results of an F3D calculation of the filamentation of an $f / 8$ beam in a $0.1 n_{c}$ plasma at $3 \mathrm{keV}$. The plot shows the lowest density present within filaments as a function of average laser intensity for four-color smoothing and four-color with additional SSD smoothing. On the axis at right the density is converted to an SRS wavelength assuming $3 \mathrm{keV}$. The onset of filamentation in F3D occurs at $2 \times 10^{15} \mathrm{~W} \mathrm{~cm}^{-2}$ and correlates with the appearance of the short-wavelength SRS in the data. Hence we may be able to use the short-wavelength SRS as a measure of beam filamentation.

A similar effect is seen in the late-time behavior of the SRS spectra, where the short-wavelength SRS appears as the plasma cools and filamentation thresholds go down. This increase in filamentation sometimes coincides with an increase in the SRS reflectivity, because intensities are higher in filaments, but also the damping of SRS by electron Landau damping is a strong function of temperature near $3 \mathrm{keV}$ (where $k \lambda_{D} \approx 0.3$ ). Hence the higher intensities from increased filamentation can conspire with the reduced damping to increase SRS reflectivity as the plasma cools, making time resolved data analysis important.

(a) Backscattered SRS spectra

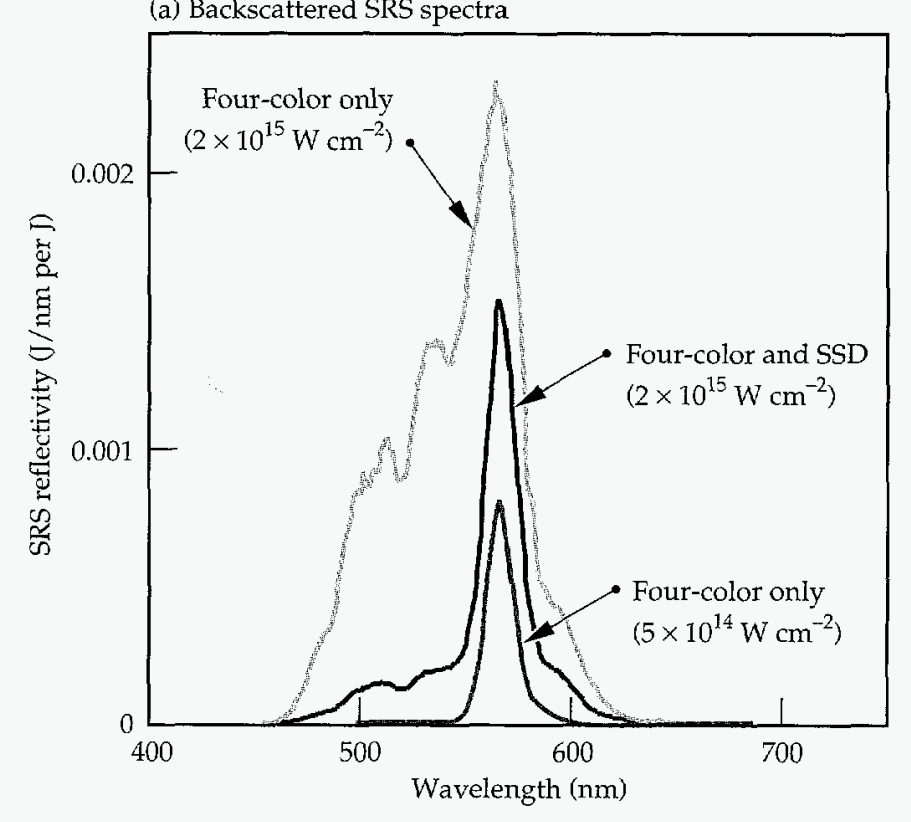

(b) F3D filamentation calculation

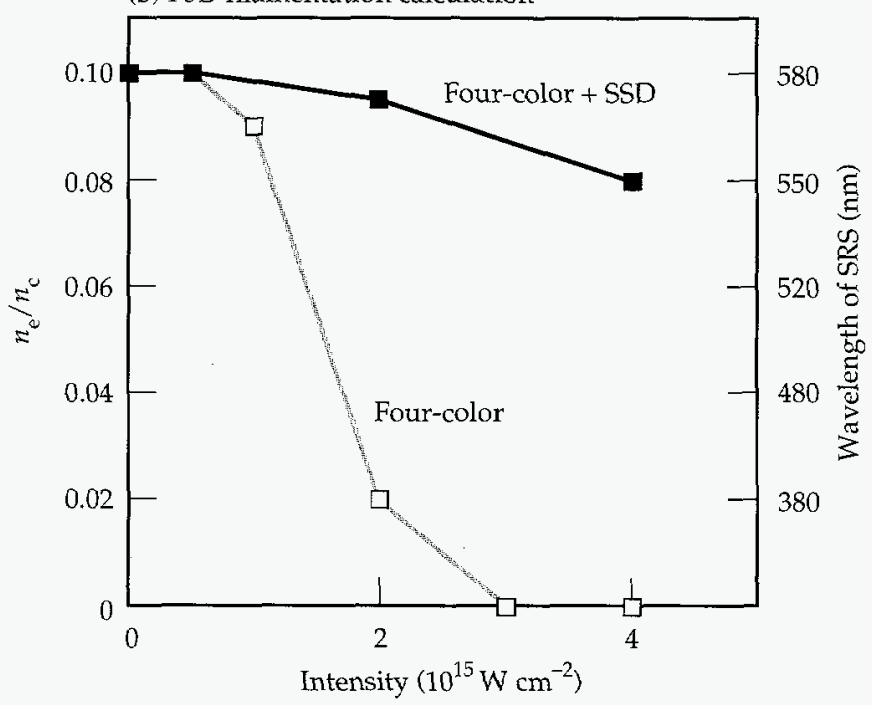

FIGURE 16. (a) Time-integrated backscattered SRS from targets with various intensity or beam smoothing. (b) F3D calculation of filamentation of an $f / 8$ beam in a $0.1 n_{c}$ plasma at $3 \mathrm{keV}$. Plot shows lowest density present within filaments vs average laser intensity for fourcolor smoothing with and without additional SSD smoothing. (20-07-1095-2449pb01) 


\section{Crossed-Beam Experiments}

The presence of multiple laser beams with substantial bandwidth in the next generation of ICF experiments will allow a new class of laser-plasma instabilities to take place in which energy from a high-frequency beam is scattered into a low-frequency beam. The mechanisms for these interactions are the same as for the stimulated backscattering instabilities that have been studied for many years in single-color, single-beam experiments. These instabilities grow from plasma wave noise that

(a) Crossed-beam expts: transmission

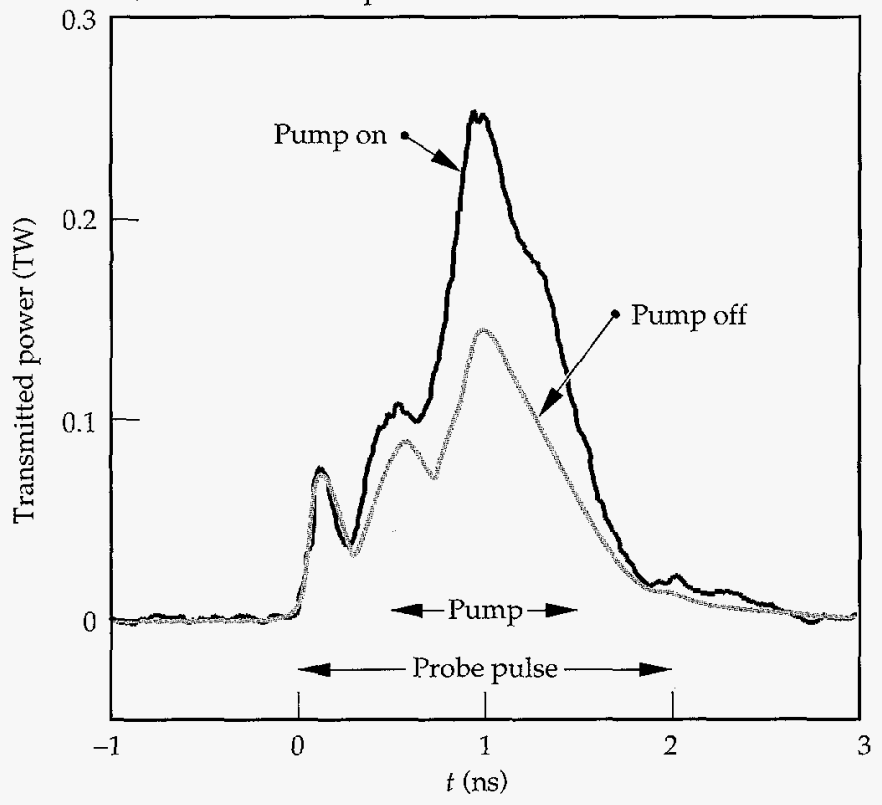

(b) Amplification

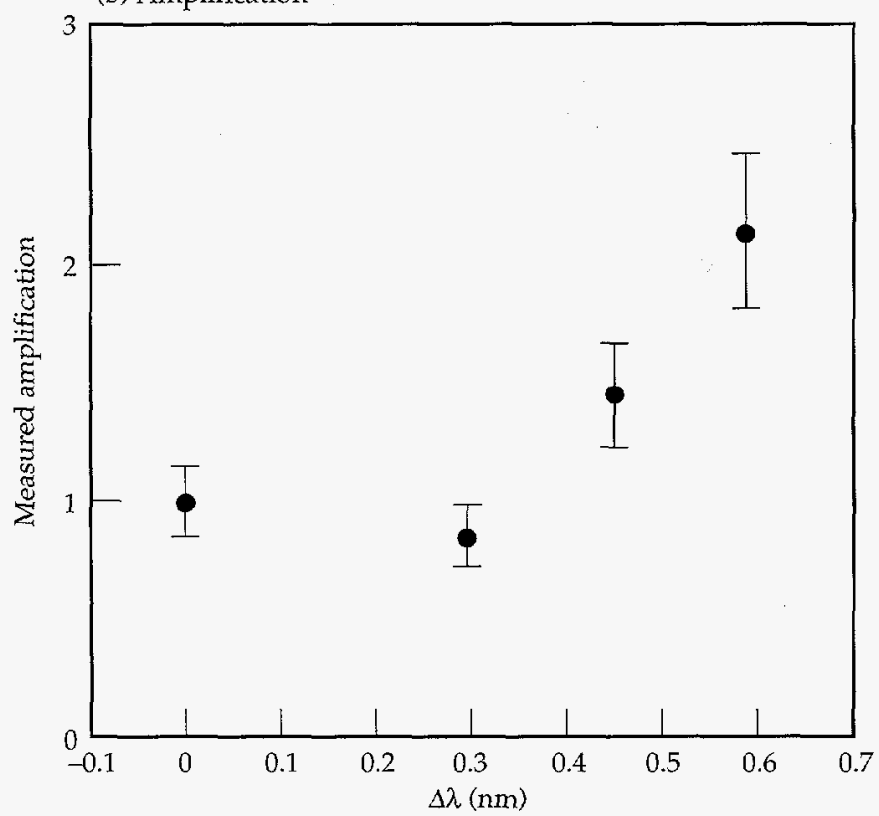

FIGURE 17. Crossed-beam experiments. (a) Typical transmitted light signal; (b) amplification vs tuning of pump with respect to probe beam. (20-07-1095-2450pb01) scatters the incident beam. The interference of the incident and scattered light creates a ponderomotive force on the plasma that reinforces the amplitude of the scattering plasma waves and causes both scattered and plasma waves to grow. The new instability arises when two laser beams cross with different frequency spectra. When the frequencies and crossing angles match, the lower-frequency beam can act as a seed for the sidescattering from the higher-frequency beam. The lower-frequency beam is then amplified by the transfer of energy from the higher-frequency beam. This type of interaction is important both because it can potentially occur near the laser entrance hole of an indirect-drive target and affect the drive symmetry and because it provides an opportunity to study fundamental backscattering processes in experiments in which both the incident and scattered frequencies can be controlled.

Experiments have been carried out at Nova in the past year to study the energy of two beams crossing in a plasma with parameters similar to those expected in NIF hohlraums. The beams cross at a $53^{\circ}$ angle and have slightly mismatched frequencies, so that the frequency difference is close to the frequency of the resonant ionacoustic wave, and Brillouin sidescattering is seeded. The power transmitted through the plasma by the lowfrequency probe beam is measured as a function of time by the TBD. The amplification is determined by comparing an experiment in which the high-frequency pump beam is present with one in which it is absent. As shown in Fig. 17(a), the transmitted power rises rapidly after the pump beam is turned on to a value well above what is measured when the pump is off. It has been demonstrated that as much as several hundred joules of energy can be transferred during a 1-ns interaction time, resulting in an amplification of the probe beam by a factor of as much as 2.5. Further, it has been shown that energy transfer occurs only when the difference in the beam frequencies is as large as the frequency of the resonant ion wave, corresponding to $\Delta \lambda=0.5 \mathrm{~nm}$ in Fig. 17(b). Further experiments are planned to measure the gain vs frequency curve and to directly study the spatial gain rate of Brillouin scattering in laser plasmas for the first time. ${ }^{30}$

\section{Nova Scale-1 Hohlraum Experiments}

Most of the diagnostics described above were specifically developed for the long-scalelength plasma experiments, but their larger field of detection made them very useful in other experiments. There is a significant, ongoing effort to understand radiation conversion in gas-filled hohlraums. These experiments use standard "scale- 1 " Nova hohlraums and a shaped drive pulse that has rapid rise to a "foot" power of $\sim 1$ TW/beam, followed by a gradual ramp up to a peak of 
about 2 TW/beam at $\sim 1.5 \mathrm{~ns}$. This is the standard PS22 pulse used in the symmetry experiments described in the article "Nova Symmetry: Experiments, Modeling, and Interpretation (HLP3 and HLP4)" on p. 293 of this Quarterly. The total pulse duration is $~ 2.2$ ns; Fig. 20 shows examples. Measurement of the backscattering from such hohlraums showed that energy loss due to reflection by SBS and SRS played a significant role in determining the hohlraum energetics.

Table 1 summarizes the (time-integrated) backscattering levels observed from the gas-filled hohlraums for various gases and the standard, empty Nova hohlraum. The scale-1 results are all for unsmoothed Nova beams. The quoted reflectivities include contributions from the NBI. For the scale-1 gas-filled hohlraums, the NBI detects a large fraction of the SBS, which appears in a blob similar to that seen with the GASBIG hohlraums [Fig. 12(c)]. The total backscattering losses for gas-filled hohlraums often exceed $15 \%$. Time-resolved measurements show that the peak loss to SBS and SRS combined can be as high as $40 \%$ in some targets. LIP

TABLE 1. Summary of scattered light levels from NIF-scale and Nova scale-1 hohlraums.

\begin{tabular}{|c|c|c|c|c|c|c|c|}
\hline \multicolumn{4}{|c|}{ NIF scale ${ }^{a}$} & \multirow{2}{*}{\multicolumn{4}{|c|}{$\begin{array}{c}\text { Nova scale }^{b} \\
\text { Scale-1 hohlraum }\end{array}$}} \\
\hline \multicolumn{2}{|c|}{ Gasbag } & \multicolumn{2}{|c|}{ Large hohlraum } & & & & \\
\hline $0.5-1.010^{15}$ & $2 \times 10^{15}$ & $1 \times 10^{15}$ & $2 \times 10^{15}$ & $\mathrm{Au}$ & $\mathrm{CH}_{4}$ & $\mathrm{C}_{3} \mathrm{H}_{8}$ & $\mathrm{C}_{5} \mathrm{H}_{12}$ \\
\hline SBS $<1 \%$ & $<1 \%$ & $\sim 0.1 \%$ & $1.3 \%$ & $6.5 \%$ & $9 \%$ & $11 \%$ & $4 \%$ \\
\hline SRS $1-2 \%$ & $4-8 \%$ & $1.5 \%$ & $5 \%$ & $2.5 \%$ & $5 \%$ & $12 \%$ & $14 \%$ \\
\hline
\end{tabular}

aAll with smoothed "NIF" beam.

bAll with unsmoothed beams. calculations of the expected SBS and SRS gain, using plasma conditions from 2-D LASNEX hydrodynamic calculations and average beam intensities, show that although peak gains for SBS are quite high ( 30 for both propane- and methane-filled hohlraums), the SRS gain exponents are lower $(\sim 20)$.

The SBS gain exponents calculated for the scale- 1 gas-filled hohlraums are similar to or higher than that calculated for the NIF outer beams [Fig. 2(c)] within the Au part of the plasma. Hence in some ways the scale- 1 hohlraums let us study that particular problem. The gasbag plasmas mimic the low- $Z$ part of the NIF SBS problem, but of course have no plateau of Au to grow SBS at shorter wavelength. The LIP calculations of the SBS gain exponent are shown in Figs. 18(a) and (b) for methane and propane, respectively; both show a peak gain late in time that is red-shifted by 2 to $3 \AA$ from $3 \omega$ and corresponds to SBS occurring in the $\mathrm{Au}$ shelf that has been ablated from the Au wall by radiative and laser ablation. A time-resolved SBS spectrum from each hohlraum is shown in Figs. 19(a) and (b). The data do not bear much resemblance to the spectral features of the LIP calculations. In particular, the SBS peak sweeps across the spectrum from $8 \AA$ red shifted to $3 \AA$ blue shifted. This feature could be due to SBS growing in the slow-moving Au near the wall at early time. As the pulse intensity ramps up, the point at which SBS grows moves out towards the laser entrance hole, where the faster-moving plasma imposes a blue shift on the scattered light. Such a motion would be consistent with the beam filamenting further out (at lower density) as the intensity ramps up.

The LIP calculations assume a beam intensity spatially averaged in the transverse direction, while the (a) $\mathrm{CH}_{4}$ (LIP)

FIGURE 18. Scale-1 hohlraum experiments. (a) LIP gain calculation for a methane-filled scale-1 hohlraum; peak gain exponent is $\sim 30$ late in the pulse; (b) LIP calculation for propane; peak gain exponent is $\sim 20$ late in the pulse. (20-07-1095-2451pb01)
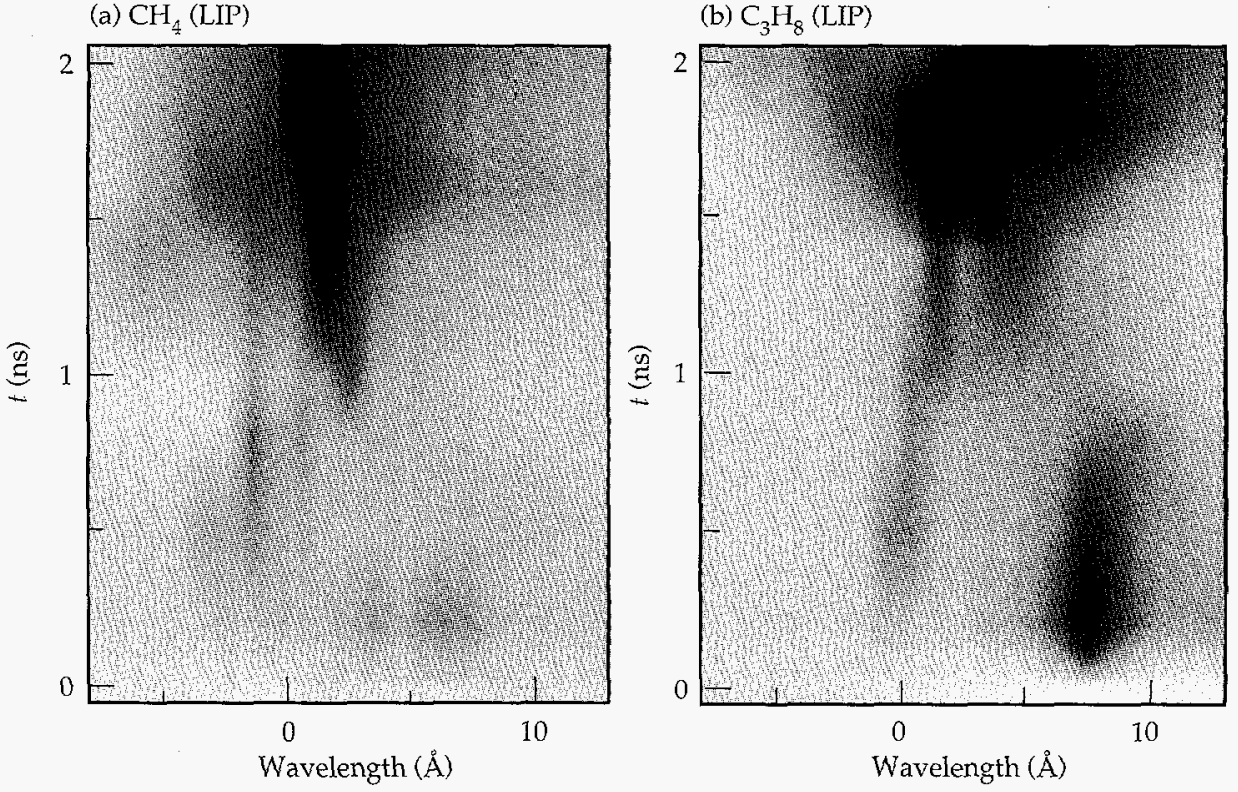

20

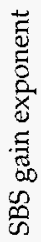


experiments not only have the short-wavelength speckle structure described earlier but also have longer-wavelength intensity modulations due to the aberration of the Nova beam when it is used without a random phase plate. If we clean up the Nova beam to make it look more like the LIP assumptions, i.e., put on a random phase plate to get rid of the long-wavelength structure and use SSD beam smoothing to reduce the short-wavelength structure, we get the results shown in Figs. 19(c) and 19(d). These figures show the time-resolved SBS spectra from gas-filled hohlraums irradiated with such a smoothed beam. As can be seen by comparing Figs. 18 and 19 for methane and propane, the smoothed-beam data approaches the LIP calculation both in terms of time history and spectral location. The unsmoothed data showed reflected SBS that swept from red shifted to blue shifted as a function of time. The SRS data from these shots (not shown) also showed a significant amount of the SRS coming from low density $\left(\sim 0.02 n_{c}\right)$, where the LIP calculations showed very low gain exponents. The application of beam smoothing moved the density at which SRS peaked closer to $0.1 n_{c^{\prime}}$ more consistent with the LIP calculations.

The SBS and SRS reflectivity from the scale-1 hohlraums also dropped when beam smoothing was applied. Figure 20 summarizes the time history of the scattered light from the data shown in Fig. 19. For methane, with standard Nova beams [Fig. 20(a)], the SRS and SBS are $6 \%$ and $11 \%$ respectively; with beam smoothing [Fig. 20(c)], the time-integrated SRS and SBS (a) SBS backscattering $\mathrm{CH}_{4}$

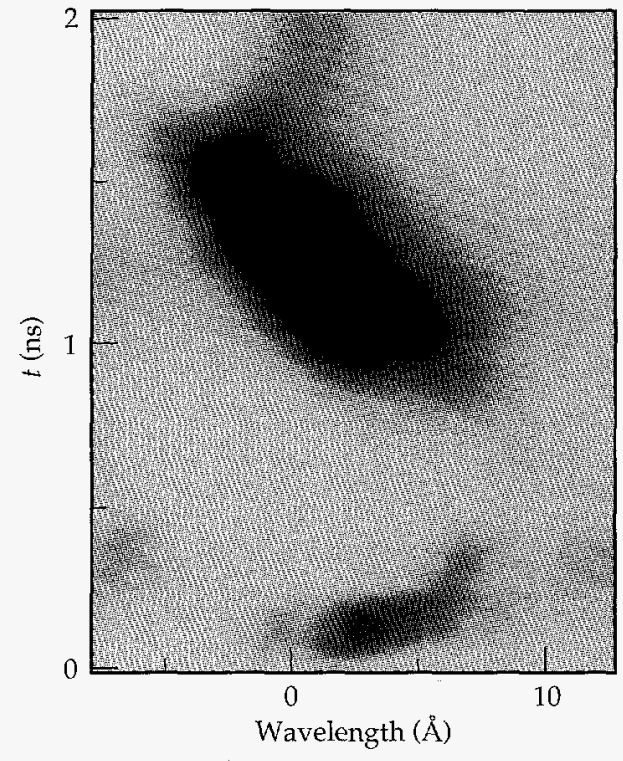

(c) $\mathrm{CH}_{4}$

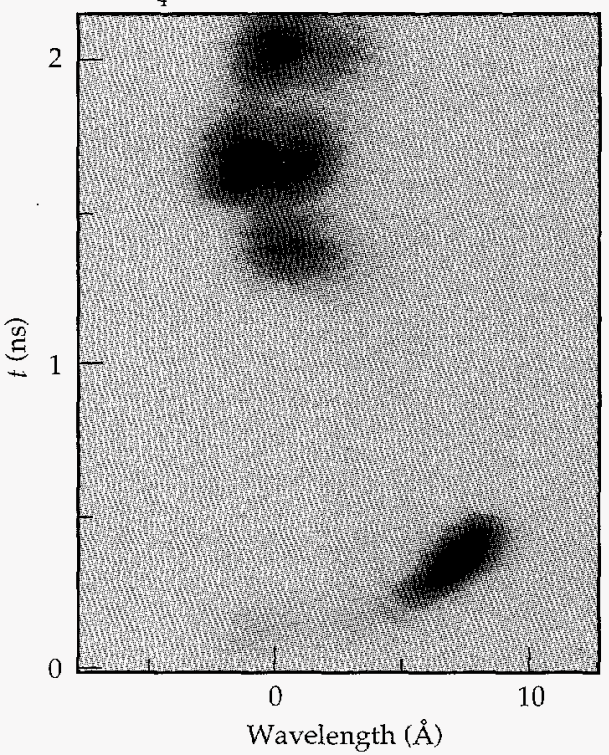

(b) $\mathrm{C}_{3} \mathrm{H}_{8}$

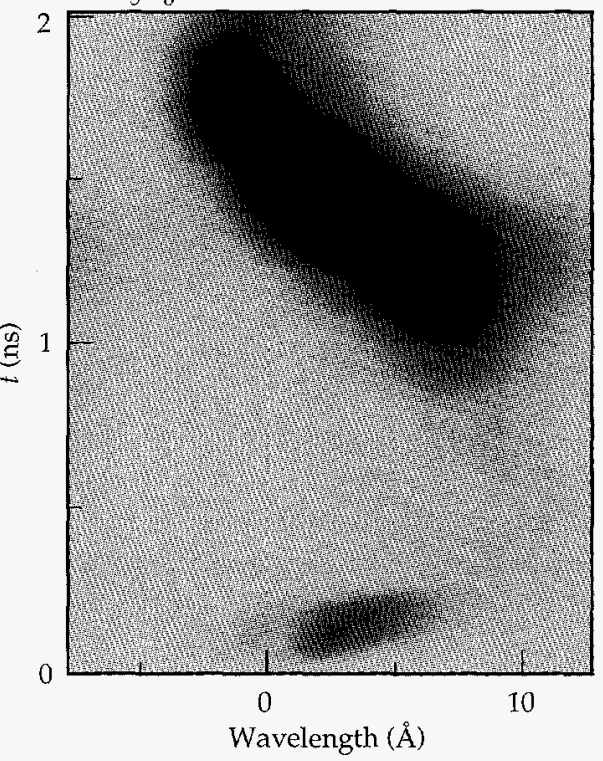

(d) $\mathrm{C}_{3} \mathrm{H}_{8}$

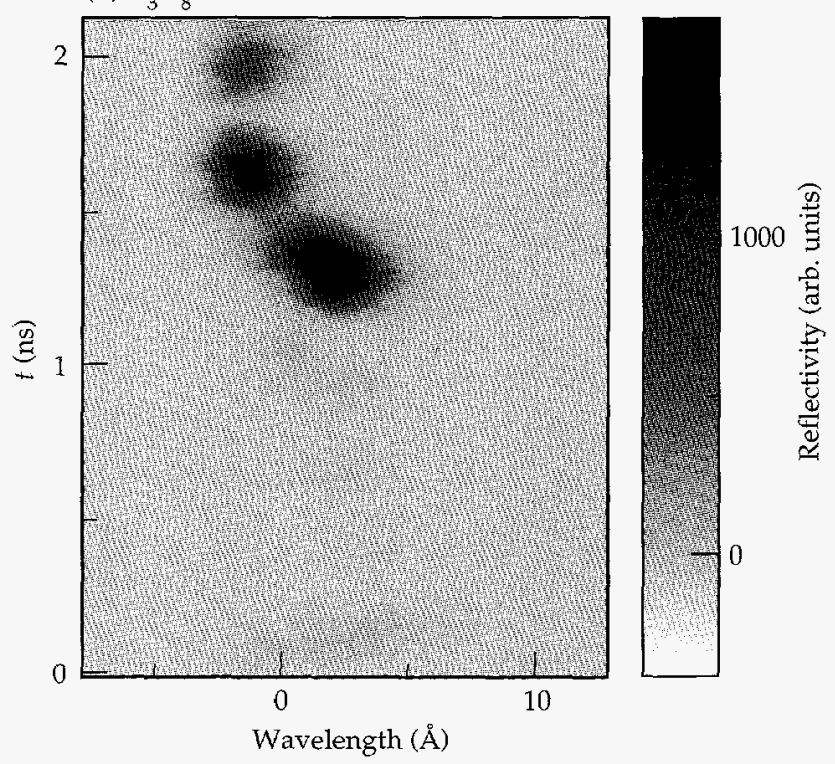

FIGURE 19. Observed SBS backscattering from scale-1 hohlraums. (a) methane-filled; (b) propane-filled; (c) methane-filled, with SSD beam smoothing; (d) propane-filled, with SSD beam smoothing. (20-07-1095-2452pb01) 
are $0.1 \%$ and $1.4 \%$. For propane the numbers are $11 \%$ and $13 \%$ before smoothing [Fig. 20(b)] and $1.7 \%$ and $1.5 \%$ after smoothing Fig. 20(d)] for SRS and SBS, respectively.

These results are encouraging for two reasons:

1. They indicate that if beam smoothing were applied to all ten arms of Nova, we would be able to couple significantly more energy into the gas-filled hohlraums. The implication (which we will explore in future experiments) is that backscattering losses from higher-temperature hohlraums that have higher densities and higher laser intensities may be reduced by smoothing.

2. The fact that the gain in the Au plasma predicted by LIP calculations for scale-1 hohlraums is comparable with that for the NIF outer-beam SBS implies that the smoothed-beam results also apply to the NIF SBS. Note that on the NIF, the beam intensity at the Au wall will be $10^{15} \mathrm{~W} \mathrm{~cm}^{-2}$ [Fig. 1(c)], while in our smoothed-beam hohlraum experiments we have an intensity of $2.5 \times 10^{15} \mathrm{~W} \mathrm{~cm}^{-2}$. Hence with a smaller target we are mimicking the expected gain exponent on the NIF outer beam in the Au and find that with beam smoothing we can produce low SBS reflectivity.

\section{Conclusion}

We have demonstrated that, with appropriate plasma composition and levels of beam smoothing, stimulated scattering in NIF-scale hohlraums can be limited to a few percent for the NIF range of intensities, $I \approx 5 \times 10^{14}$ to $2 \times 10^{15} \mathrm{~W} \mathrm{~cm}^{-2}$. At this level, stimulated scattering is not expected to have a significant effect on either the energetics or the symmetry of NIF hohlraums. Also, in the NIF intensity range, the light transmitted through the NIF-scale plasmas is consistent with calculations.

Using specially constructed gas-filled plastic balloons and large-scale hohlraums, we have been able to produce plasmas, using 8-9 of Nova's beams, whose density, electron temperature, velocity gradient, and integral interaction path length reproduce the conditions in the low-Z interior of NIF hohlraums. A single Nova beamline was modified to simulate an $f / 8$ cluster of four NIF beams in which the wavelength of each beam could differ from that of the others by a few angstroms. Diagnostics were implemented to measure the transmitted laser light and light scattered by SBS and SRS. We measured scattering levels for a variety of smoothing techniques that could be implemented on
FIGURE 20. Scale-1 hohlraum backscattering pulse shapes. (a) Standard beam conditioning, methanefilled; (b) standard conditioning, propanefilled; (c) SSD, methane-filled (d) SSD, propane-filled. (20-07-1095-2453pb01) (a) Scale-1 backscattering: methane

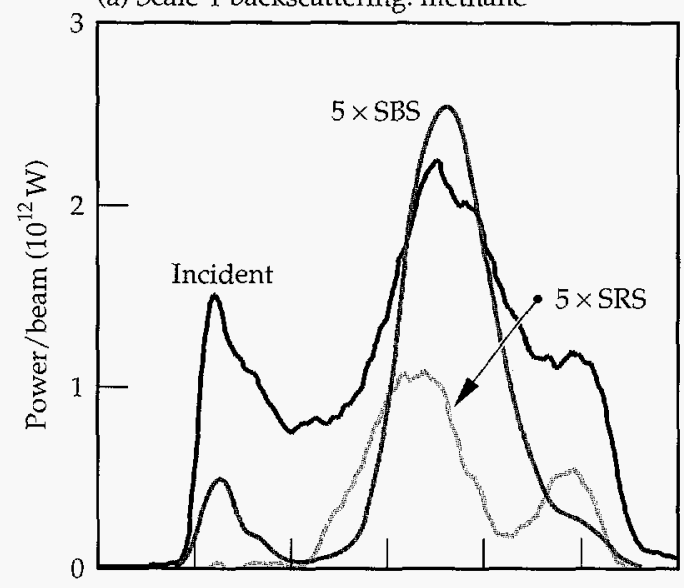

(c) Propane

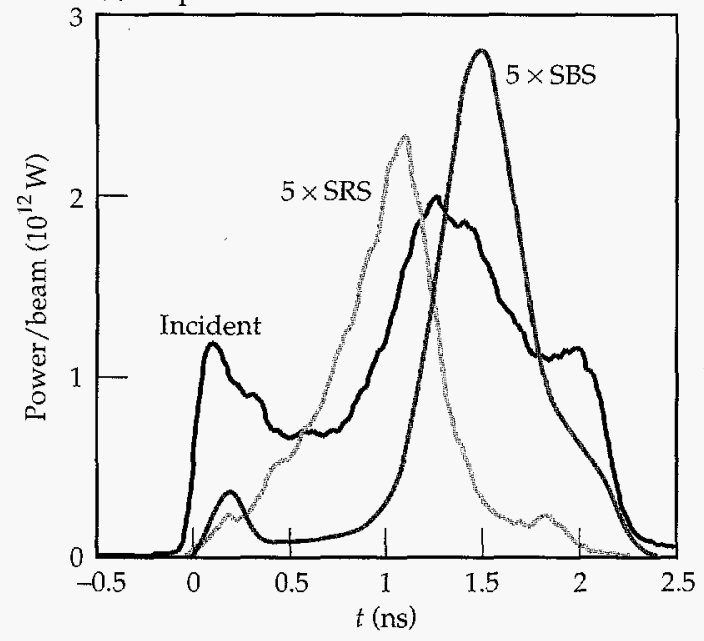

(b) Methane RPP and SSD

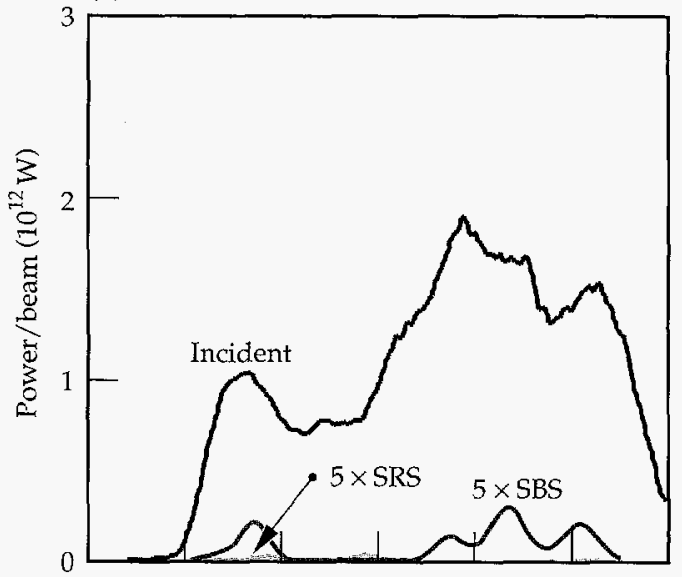

(d) Propane RPP and SSD

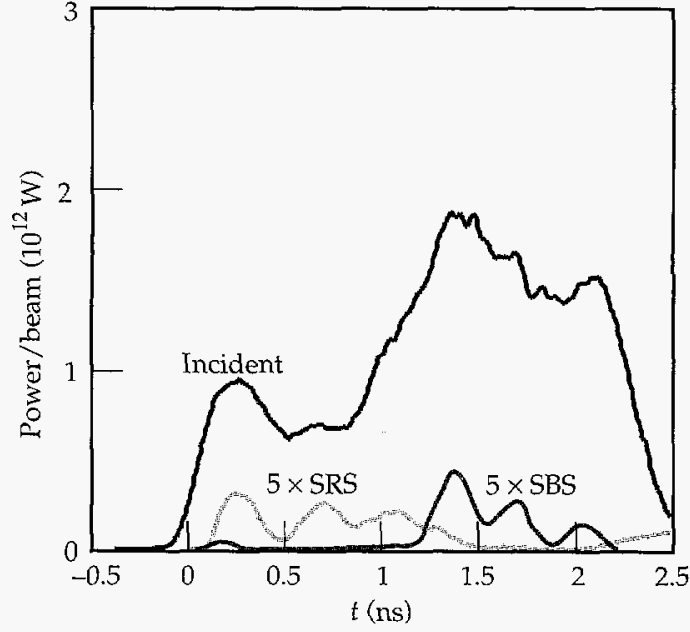


the NIF, including one- and four-color beams with RPPs only or with $1.6 \AA$ of SSD for temporal smoothing.

For all beam-smoothing schemes and for all intensities of interest to NIF, we find that SBS levels can be limited to $\sim 1 \%$ if the ion-acoustic wave is sufficiently damped. This can be achieved by adding hydrogen as a minority species in the low- $Z$ gas fill.

At an intensity of $5 \times 10^{14} \mathrm{~W} \mathrm{~cm}^{-2}$ (the low end of the NIF range), SRS is also limited to a few percent for all smoothing schemes. However, at an intensity of $2 \times 10^{15} \mathrm{~W} \mathrm{~cm}^{-2}$, near the upper end of the NIF range, SRS is about $10 \%$ with RPPs only. With $1.6 \AA$ of SSD, the SRS is limited to about 4 to $5 \%$. With SSD, the frequency spread of SRS is reduced, consistent with theoretical expectations of reduced plasma filamentation with SSD. There appears to be no benefit to having separate frequencies for each of the four beams, with or without SSD. In fact, experiments to explore coupling between beams crossing in the laser entrance hole show that it is possible to transfer energy from one beam to another, potentially affecting hohlraum symmetry, when the frequency difference between beams matches the ionacoustic frequency.

Although the gasbags and large hohlraums provide a plasma that is a good match to the low- $Z$ interior of a NIF hohlraum, neither reproduces the conditions of a NIF hohlraum near the high- $Z$ wall. The gas balloon has no high- $Z$ wall, and (because of their size) little laser energy reaches the wall in the large hohlraums. However, we find that standard Nova scale-1 hohlraums have conditions in the high- $Z$ wall that are very similar to those of NIF hohlraums. This is because the high- $Z$ conditions are set by inverse bremsstrahlung and $x$-ray emission and not by the hohlraum scale. Since standard Nova gas-filled hohlraums have a laser intensity comparable to that of the NIF, the inverse bremsstrahlung absorption lengths (of a few hundred micrometers) are also comparable. Although the high- $Z$ shelf is short, it is relatively high density $\left(\sim 0.2 n_{c}\right)$, has only weak velocity gradients, and has weak Landau damping, so that the gain for SBS can be quite high. Without smoothed beams, SBS levels in Nova gas-filled hohlraums are about $10 \%$, mostly from the Au shelf. As in the case of the low- $Z$ interior, a low-mass minority plasma can provide increased Landau damping to suppress SBS in the high$Z$ plasma near the wall. We are presently studying this idea, which has application to the NIF. We also find that SBS in the scale-1 hohlraums can be suppressed with sufficient beam smoothing, even without adding a low- $Z$ minority species.

More work remains to be done to fully characterize the range of operating conditions for NIF ignition hohlraums. There would be a substantial benefit if we could extend the range of hohlraum temperatures above that specified for the nominal ignition targets. This would require that hohlraums be operated above the nominal intensity and plasma density range. We have not yet determined the optimum combination of beam smoothing and plasma composition for controlling stimulated scattering, but the techniques examined so far result in low levels of stimulated scattering, and can all be implemented on the NIF.

\section{Notes and References}

1. L. J. Suter, A. A. Hauer, L. V. Powers, D. B. Ress, et al., Phys. Rev. Lett. 73 (17), 2328-2331; A. Hauer, L. Suter, N. Delameter, D. Ress, et al., Phys. Plasmas 2 (6), 2488-2494 (1995).

2. W. L. Kruer, Phys. Fluids B 3, 2356 (1991); also "Interaction Physics for Megajoule Laser Targets," in Laser Interaction and Related Plasma Phenomena, Vol. 10 (Plenum, New York, 1993), p. 503.

3. See, e.g., M. N. Rosenbluth and R. Z. Sagdeev. Handbook of Plasma Physics, Volume 3: Physics of Laser Plasma. A. M. Rubenchik and S. Witkowski, Eds. (Elsevier, Amsterdam, 1991), ch. 9, and references therein.

4. R. L. Berger, B. F. Lasinski, T. B. Kaiser, E. A. Williams, A. B. Langdon, and B. I. Cohen, Phys. Fluids B 5, 2243 (1993); R. L. Berger, T. B. Kaiser, B. F. Lasinski, C. W. Still, et al., "Modeling the Effects of Laser-Beam Smoothing on Filamentation and Stimulated Brillouin Scattering," ICF Quarterly Report 5 (3), 130, Lawrence Livermore National Laboratory, Livermore, CA, UCRL-LR-105821-95-3 (1995).

5. J. D. Lindl, Phys. Plasmas 2 (11), 3933-4024 (Nov. 1995); S. W. Haan, S. M. Pollaine, J. D. Lindl, L. J. Suter, et al., Phys. Plasmas 2 (6), 2480-2487 (June 1995).

6. R. L. Berger et al., Phys. Fluids 1, 414 (1989).

7. L. V. Powers, R. L. Berger, R. L. Kauffman, B. J. MacGowan, et al., Phys. Plasmas 2 (6), 2473 (1985); L. V. Powers, R. L. Berger, D. H. Munro, B. F. Lasinski, et al., Phys. Rev. Lett. 74, 2957 (1995).

8. J. Denavit and D. W. Phillion, Phys. Plasmas 1, 1971 (1994).

9. M. D. Rosen and J. H. Nuckolls, Phys. Fluids 22, 1393 (1979).

10. D. H. Kalantar, B. J. MacGowan, T. P. Bernat, D. E. Klem, et al., Rev. Sci. Instrum. 66, 782 (1995); D. H. Kalantar et al., Phys. Plasmas 2, 1361 (1995).

11. D. E. Klem, Lawrence Livermore National Laboratory, Livermore, CA, personal communication (1995).

12. R. W. Lee, B. L. Whitten, and R. E. Strout, II, J. Quant. Spectros. Radiat. Transfer 32, 91 (1984).

13. J. D. Lindl, "Cairn Hohlraum Scaling Study," in Laser Program Annual Report-1979, Lawrence Livermore National Laboratory, Livermore, CA, UCRL-50055-79 (1979), p. 5-80.

14. J. M. Auerbach, K. R. Manes, and D. W. Phillion, "Cairn Scaling Study-The Experiments," in Laser Program Annual Report-1979, Lawrence Livermore National Laboratory, Livermore, CA, UCRL-50055-79 (1979), p. 5-62.

15. B. H. Wilde, J. C. Fernandez, W. W. Hsing, J. A. Cobble, et al., "The Design and Characterization of Toroidal-Shaped Nova Hohlraums that Simulate National Ignition Facility Plasma Conditions for Plasma Instability Experiments," presented at the 12th International Conference on Laser Interaction and Related Plasma Phenomena, Los Alamos National Laboratory, Los Alamos, NM, LA-UR-95-1439 (April 1995), to be published.

16. J. C. Fernandez et al., Rev. Sci. Instrum. 66, 626 (1995).

17. R. Kirkwood, J. Moody, and B. MacGowan, "Analysis of Schottky Barrier Diodes for Detection of Pulsed UV Light," Lawrence Livermore National Laboratory, Livermore, CA, internal memorandum (June 1994). 
18. Y. Kato, K. Mima, N. Miyanaga, S. Aringa, et al., Phys. Rev. Lett. 53, 1057 (1984); H. T. Powell, S. N. Dixit, and M. A. Henesian, Laser Program Annual Report-1991, Lawrence Livermore National Laboratory, Livermore, CA, UCRL-LR-105820-91 (1991), pp. 28-38.

19. See National Technical Information Service Document Nos. DE95017671 through DE95017673 and DE95017676 through DE95017700 (J. A. Paisner, E. M. Campbell, and W. J. Hogan, The National Ignition Facility Project, UCRL-JC-117397 and UCRL-PROP-117093, May 1994). Copies may be obtained from the National Technical Information Service, Springfield, VA 22161; D. M. Pennington, ICF Quarterly Report 5 (2), 130, Lawrence Livermore National Laboratory, Livermore, CA, UCRL-LR-105821-95-2 (1995).

20. S. Skupsky, R. W. Short, T. Kessler, R. S. Craxton, et al., J. Appl. Phys. 66, 3456 (1989).

21. K. Marsh, C. Joshi, and C. McKinstrie, Bull. Am. Phys. Soc. 39 (1994).

22. E. A. Williams et al., Physics of Plasmas. 2, 1 (1995); H. X. Vu, J. M. Wallace, and B. Bezzerides, Phys. Plasmas 1 (11), 3542-3556 (1994).

23. C. E. Clayton, C. Joshi, A. Yasuda, and F. F. Chen, Phys. Fluids 24, 2312 (1981).
24. S. C. Wilks, W. L. Kruer, J. Denavit, K. Estabrook, et al., Phys. Rev. Lett. 74, 5048 (1995).

25. J. D. Moody, H. A. Baldis, D. S. Montgomery, R. L. Berger, et al., Phys. Plasmas 2, 4285 (1995).

26. D. S. Montgomery, J. D. Moody, H. A. Baldis, B. B. Afeyan, et al., "Effects of Laser Beam Smoothing on Stimulated Raman Scattering in Exploding Foil Plasmas," Lawrence Livermore National Laboratory, Livermore, CA, UCRL-JC-123014; submitted to Phys. Plasmas (1995).

27. R. W. Short, R. Bingham, and E. A. Williams, Phys. Fluids 25 (12), 2302-2303 (1982); D. E. Hinkel, E. A. Williams, R. L. Berger, and L. V. Powers, Bull. Am. Phys. Soc. 40 (11) (Oct. 1995), p. 1778 (Annual Meeting of the Division of Plasma Physics).

28. D. F. Dubois and H. A. Rose, Bull. Am. Phys. Soc. 40 (11), 1824 (1995); H. A. Rose, Bull. Am. Phys. Soc. 40 (11), 1778 (1995).

29. S. G. Glendinning, L. V. Powers, R. L. Kauffman, O. L. Landen, et al., "Measurements of Wall X-Ray Emission Patterns in GasFilled Hohiraums," Lawrence Livermore National Laboratory, Livermore, CA, UCRL-JC-123021; to be submitted to Phys. Rev. Lett. (1996).

30. R. K. Kirkwood, B. B. Afeyan, W. L. Kruer, B. J. MacGowan, et al., "Observation of Energy Transfer Between Frequency Mismatched Laser Beams in a Large-Scale Plasma," Lawrence Livermore National Laboratory, Livermore, CA, UCRL-JC-122102; submitted to Phys. Rev. Lett. (1995). 


\section{Appendix: Nova Technical Contract as presented to the 1990 NAS Review of ICF}

\section{Physics Program}

The primary focus of the Lawrence Livermore National Laboratory (LLNL) Program has been, and will continue to be, $x$-ray driven "indirect-drive" inertial confinement fusion (ICF). The physics of this approach is naturally divided into capsule physics and driver/ hohlraum development. As a result, the LLNL target physics program has two principal elements:

1. The Hydrodynamically Equivalent Physics (HEP) program to address the capsule physics associated with ignition and gain. Elements of this program include:

- hydrodynamic stability,

- the effects of drive nonuniformity on capsule performance, and

- the physics associated with ignition (energy gain/ loss to the fuel) in the absence of alpha deposition.

2. Hohlraum Laser-Plasma Physics (HLP) to address driver-plasma coupling, generation and transport of $x$-rays, and the development of energy-efficient (i.e., coupling of the driver energy to the capsule) hohlraums that provide the appropriate $x$-ray drive (spectral, temporal, and spatial) to a high-performance capsule.

While meeting specific milestones is important, the emphasis of the target physics program is to demonstrate and refine our ability to model/predict target performance, particularly those aspects that scale to, and influence, ignition and gain. The LLNL Program is thus directed toward instilling confidence in the successful outcome of ICF and of specifying the performance and characteristics of both the driver and target required to achieve these objectives.

We expect to undertake and complete the HEP and HLP elements described here in 2 to 3 years, depending upon the funding level available.

\section{Capsule Physics (HEP)}

An extensive program addressing the implosion physics of ignition and gain is presently underway on Nova. The goal of this effort is to further develop and demonstrate a quantitative and predictive understanding of the performance of capsules (properly scaled from ignition/gain designs) including the effects of hydrodynamic instability and $x$-ray drive nonuniformities (with known initial fabrication tolerances of the target and a detailed knowledge of the hohlraum environment). The extensive diagnostics used in the HEP program also allows confirmation of our ability to model ignition physics in imploding capsules, i.e., the balance between PdV work and electron conduction and radiative losses in the fuel in the absence of alpha heating.

The HEP experiments use both planar targets and capsules with/without prescribed perturbations (in capsule fabrication or x-ray flux uniformity). The targets do not include cryogenic fuel configurations. The experiments are extensively diagnosed with $x$-ray and neutron diagnostics including $x$-ray backlighting. The experiments also emphasize pulse-shaped drive to enable minimum entropy implosion trajectories to be examined. The pulse shapes are properly scaled from ignition/gain target requirements.

Although not principal objectives of the HEP program, the pulse shaping and the reduced levels of preheat from high-energy photons and superthermal electrons lead to noncryogenic implosions with final fuel and pusher $\left(\mathrm{CH}_{2}\right.$ or $\left.\mathrm{S}_{\mathrm{i}} \mathrm{O}_{2}\right)$ densities in the range of $40 \mathrm{~g} / \mathrm{cm}^{3}$ and $100-200 \mathrm{~g} / \mathrm{cm}^{3}$, respectively.

The HEP program is divided into five specific goals (HEP1-HEP5) described below. 


\section{HEP1 Summary of Tasks}

To demonstrate increased fuel/pusher compressions with high contrast pulse shaping using noncryogenic targets. These experiments have been successfully conducted, but due to the high compressions achieved, limited diagnostics were employed. For example, the pusher and fuel density increase were inferred from a measurement of the pusher areal density $\rho \Delta R$.

\section{HEP1 Goals}

To diagnose fuel densities in the range of $20-40 \mathrm{~g} / \mathrm{cm}^{3}$ inferred from measurements of fuel areal density $\rho R$ using advanced neutron-based diagnostics. This quantity and other features of target performance (neutron yield, ion temperature, fusion burnwidth) will be modeled considering both hydrodynamic stability and x-ray flux nonuniformities.

\section{HEP2 Summary of Tasks}

To measure reduced growth and early nonlinear behavior of the Rayleigh-Taylor (RT) instability at the ablation surface for $x$-ray driven targets. The goals of HEP2 are to experimentally demonstrate and model reductions in the growth rate of the RT instability due to finite density gradients and mass advection at the ablation surface. Planar targets with single fourier component areal density variations will be examined over a range of peak $T_{\mathrm{R}}$ and with pulse shaping. Initial perturbations will be small and the acceleration history designed so that linear analysis is $\left(k a_{0}<<1\right)$ valid for large growth factors ( 3 to $5 e$-folds). Successful experiments to date, on which the HEP2 project is based, have observed $\sim 1 e$-fold of growth rather than the 2 to $3 e$-folds predicted if finite density gradients and mass advection are not included. Perturbation wavelength and material opacity will be varied to establish an extensive database for comparison with detailed numerical simulation. The tasks in HEP2 will also include planar targets to simulate the ablation physics of HEP4 and HEP5 capsules. These targets use $\mathrm{CH}$ ablators doped with intermediate atomic number elements to control hydrodynamic stability. Experiments will include shock wave propagation and other áppropriate measurements to confirm our overall understanding of the hydrodynamic behavior of such targets.

The experiments will also address early nonlinear behavior such as harmonics (due to bubble and spike growth) and examine mode coupling. In these latter experiments, the foils will nominally have two initial perturbation wavelengths.

\section{HEP2 Goals}

To observe single-mode growth factors at the ablation surface $>30$ from which reductions in the classical $R T$ growth rate of 2 to 3 are inferred. The experiments will use planar targets driven by $x$-ray ablation at several peak $T_{\mathrm{R}}$ ranging from $150 \mathrm{eV}<T_{\mathrm{R}}<220 \mathrm{eV}$. Experiments will be compared with detailed simulations to confirm our ability to model RT growth at the ablation surface. Targets of various composition (including doped $\mathrm{CH}$ ablators) will be used to confirm our ability to properly account for plasma opacity in modeling the $x$-ray driven hydrodynamics.

To observe early nonlinear behavior (harmonics) and mode coupling. Planar targets with single or multiple fourier components will be used. Detailed comparison with numerical simulations to demonstrate our ability to model this nonlinear behavior will be made.

\section{HEP3 Summary of Tasks}

To analyze implosion experiments using capsules with deliberately perturbed surface finishes to validate mix models with a multimode initial noise spectrum. These "bumpy ball" experiments are designed to validate our multimode hydrodynamic mix models. Detailed time-resolved $x$-ray spectroscopy of dopant materials in both the fuel and pusher are used to infer mix and to compare with our modeling of both mix and its effect on the imploded state of the fuel. While the growth factors for these experiments are approximately 10 to 30 for $10 \lesssim l \lesssim 30$ (compared with growth factors of $\sim 500$ for ignition/ gain targets), the initial surface perturbation amplitudes are sufficiently large $\left(0.1<\mathrm{a}_{0} \leq 0.2 \mu \mathrm{m}\right.$ for $\left.10 \leq l \leq 30\right)$ to result in diagnosable mixing between the pusher and fuel. The target convergence is limited to $\lesssim 10$ so that the effects of drive asymmetry on target performance are limited. Targets with no deliberately fabricated surface perturbations are used for control and a well defined "null."

\section{HEP3 Goals}

To observe pusher/fuel mix that is dependent on initial target surface quality using $x$-ray spectroscopy. Targets use an initial multimode spectrum of surface perturbations. The implosion is designed for overall hydrodynamic mix growth factors of 10 to 30 . Peak hohlraum temperatures of $>200 \mathrm{eV}$ will be used. Detailed comparison of experimental results with numerical simulations employing multimode mix modeling will be performed. Experiments will include targets with no deliberately perturbed surface finish as "null" comparisons.

In addition to the HEP1 through HEP3 objectives, to conduct a series of sophisticated experiments, which directly address the hydrodynamic stability of the Nova Upgrade ignition targets. For example, these experiments will diagnose the performance of capsules when the instability growth factors (including both linear and, where appropriate, nonlinear behavior) as a function of $l$-mode 
number are similar to that of ignition gain target designs. Other features of the experiments, such as achievable implosion velocity and convergence, will be either limited or influenced by the existing capabilities of Nova. For example, the limited number of Nova beams (10) will limit the drive uniformity and thus capsule convergence before degradation from onedimensional (1-D) performance will be expected. The Nova Upgrade addresses this limitation by the use of a large number $(N>200)$ of independent beamlets. Specific laser technology issues addressable on Nova that impact drive uniformity and that are required for the Nova Upgrade will be demonstrated as part of the Precision Nova program. Issues include beam-to-beam power balance and beam pointing.

These experiments, HLP4, and the associated modeling will specifically identify the performance and flexibility required of the Nova Upgrade and will further validate our predictive capability of capsule performance including both hydrodynamic stability and drive nonuniformity.

The HEP4 and HEP5 experimental programs address these issues. The two projects differ primarily in the associated capsule convergences, which impact the choice and applicability of the target diagnostics.

\section{HEP4 Summary of Tasks}

To analyze implosion experiments to convergences of $\sim 10$ with overall hydrodynamic mix growth factors of 100 to 500 for an l-mode spectrum similar to ignition target designs. These experiments, which will make use of techniques developed in HEP3, will use plastic $\mathrm{D}_{2}$-filled capsules whose hydrodynamic stability (number of growth factors) is varied by target design and by selective doping of the ablator and/or fuel. The targets will use pulse-shaped drive with peak $T_{R} \lesssim 230 \mathrm{eV}$. Capsule convergences will be limited to $\sim 10$ so that the effects of drive nonuniformity on capsule performance will be minimized. The growth factors will be systematically varied from $\sim 10$ to levels exceeding that of ignition targets (peak growth factor $\sim 500$ ). This systematic variation in hydrodynamic stability will also ensure that the effects of drive asymmetry are isolated.

\section{HEP4 Goals}

To compare detailed diagnosis and modeling of $\mathrm{D}_{2}$-filled plastic capsules with doped ablators to control hydrodynamic stability. Diagnostics will be primarily (but not exclusively) $x$-ray based. Hydrodynamic instability growth factors will range from 10 to 500 in an $l$-mode spectrum, similar to ignition designs (i.e., maximum growth at $l \approx 30$ ).

Targets will be imploded with pulse-shaped drive at a peak $T_{\mathrm{R}}<230-250 \mathrm{eV}$ and will be limited to convergences of $\leqslant 10$ to minimize the effects of $x$-ray flux nonuniformity.

\section{HEP5 Summary of Tasks}

To analyze implosion experiments to convergences of $\sim 20$ to 40 with overall hydrodynamic mix growth factors of 100 to 500 for an l-mode spectrum similar to ignition target designs. These experiments, which will make use of techniques developed in HEP3 and HEP4, will use plastic $D_{2}$ - and DT-filled capsules whose hydrodynamic stability (number of growth factors) is varied by target design and by selective doping of the ablator and fuel. The targets will use pulse-shaped drive with peak $T_{\mathrm{R}} \lesssim 230 \mathrm{eV}$.

HEP5 is similar to HEP4 with the exceptions of higher target convergence ( 20 to 40 ) and a reliance on both $\mathrm{x}$-ray and advanced neutron-based diagnostics to measure imploded core conditions. As stated above, the convergence will be limited by Nova's finite number of beams and will be as large as possible commensurate with diagnosability (the majority of measurements will rely on target emission). Convergences in the range of 20 to 40 are expected. As in HEP4, the growth factors will be systematically varied from $\sim 10$ to levels exceeding that of ignition targets (peak growth factor $~ 500$ ). This systematic variation in hydrodynamic stability will enable the effects of drive asymmetry to be isolated from that due to mix from higher $l$-mode target fabrication "noise" sources.

\section{HEP5 Goals}

To compare detailed diagnosis and modeling of $\mathrm{D}_{2}$-and DT-filled plastic capsules with doped ablators to control hydrodynamic stability. Diagnostics will be neutron and $x$-ray based. Hydrodynamic instability growth factors will range from 10 to 500 in an $l$-mode spectrum, similar to ignition designs (i.e., maximum growth at $l \approx 30$ ).

Targets will be imploded with pulse-shaped drive at a peak $T_{R}<230-250 \mathrm{eV}$. Convergences will be in the range of 20 to 40 , but at yields degraded from 1-D performance due to the uniformity limitations of Nova. The achievable convergence will be commensurate with experimental diagnosability.

\section{Hohlraum Laser-Plasma (HLP) Physics}

An extensive experimental and modeling hohlraum physics effort has been underway at LLNL for over a decade. In particular, substantial progress has been made on Nova. As a result, the Nova Upgrade hohlraums are in many aspects an extension of the ongoing experiments. For example, the ignition/moderate gain hohlraums are typically scale 2.9 to 4.5 that of the 
nominal Nova targets. Nova scale 1 targets are right circular cylinders $1600 \mu \mathrm{m}$ diam, $2550 \mu \mathrm{m}$ long, with typical laser entrance holes (LEHs) $800 \mu \mathrm{m}$ diam. The Nova Upgrade has LEHs $1.5-4 \mathrm{~mm}$ diam.

Present calculations give the following performance specifications for the Nova Upgrade hohlraums:

1. Peak $T_{R} \approx 300-350 \mathrm{eV}$ in a picket fence pulse or continuous pulse of overall duration 10 to $20 \mathrm{~ns}$.

2. Time average drive uniformity with $P_{2}, P_{4}$ components of $<2 \%$ (peak to peak) with instantaneous values several times larger. While meeting this uniformity requirement, the hohlraum must deliver 8 to $15 \%$ of the laser energy to the capsule.

3 . Hot electron energy fraction $f_{\text {hot }} \leqslant 5 \%$ at a temperature $T_{\text {hot }} \gtrsim 50 \mathrm{keV}$. The exact levels are dependent on capsule design details, the time and location of the hot electron generation, and their transport to the capsule.

4. Scattered light from stimulated Brillouin and Raman scattering $<5-10 \%$. The exact values depend primarily on the influence of this scattering on drive symmetry control. For symmetry/coupling efficiency, the hohlraums are lined with low to moderate $Z(Z \lesssim 28)$ material.

The objectives of the Nova experiments are to experimentally demonstrate and predictively model hohlraum performance in properly scaled targets consistent with Nova's performance limitations and to further develop our understanding (experimental and theoretical) of laser-plasma interaction physics in the millimeter-size plasmas associated with ignition hohlraums. Where appropriate, the hohlraums will also contain capsules to better simulate the Nova Upgrade targets.

The hohlraum/plasma physics program will also continue to explore, within the limits of Nova, the limits of achievable peak $T_{\mathrm{R}}$ in laser-driven hohlraums. While specific objectives will not presently be assigned to this task, these experiments will help define the "operating experimental parameters" of the Nova Upgrade. The availability of peak $T_{R}{ }^{\prime} s>300$ to $350 \mathrm{eV}$ is of general interest to the weapons community.

The HLP program is divided into seven specific goals (HLP1-HLP7) described below.

\section{HLP1 Summary of Tasks}

To demonstrate acceptable hohlraum-plasma coupling and gross hohlraum energetics with targets (properly scaled from Nova Upgrade designs) having temporally shaped pulses. Equivalent $T_{\mathrm{R}}$ during the pulse will range from $\sim 100$ to $210 \mathrm{eV}$ and both continuous and picket fence pulses of overall duration $\lesssim 4 \mathrm{~ns}$ will be used. The high- $Z$ hohlraums will be lined with low- $Z$ material, whose peak $n_{\mathrm{e}}$ within the hohlraum at the peak of the pulse is 0.03 to $0.25 n_{\mathrm{c}}$.

\section{HLP1 Goals}

To achieve acceptable hohlraum coupling for a pulseshaped drive with an equivalent $T_{R}$ ranging from $\sim 100$ to $210 \mathrm{eV}$, as follows:

- Absorption fraction $f_{\mathrm{abs}}>90 \%$

- Stimulated Brillouin scattering fraction $f_{\mathrm{SBS}}<5-10 \%$

- Hot electron fraction $f_{\text {hot }}<5 \%$ at $T_{\text {hot }} \geq 50 \% \mathrm{keV}$

- Stimulated Raman scattering fraction $f_{\mathrm{SRS}}<5 \%$

\section{HLP2 Summary of Tasks}

To demonstrate acceptable hohlraum-plasma coupling with peak equivalent $T_{R} \gtrsim 270 \mathrm{eV}$ using lined hohlraums. It is important to experimentally demonstrate $T_{\mathrm{R}} \approx 300 \mathrm{eV}$ to establish credibility for Nova Upgrade target designs. Nova's power capability of $\sim 40$ TW limits the target scale and pulse duration for these high temperatures. Laser pulse formats will be limited to square $1 \mathrm{~ns}$ and appropriately scaled pulses i.e., shorter overall pulses or more limited shaped pulses (both continuous and picket fence) compared with those used in HLP1. Experiments will include hohlraums with capsules. "First bounce" laser intensities will be equivalent to that planned for the Nova Upgrade $\left(1-3 \times 10^{15} \mathrm{~W} / \mathrm{cm}^{2}\right)$.

\section{HLP2 Goals}

To achieve acceptable hohlraum-plasma coupling, the following values must be attained in hohlraums that have a peak $T_{R}>270 \mathrm{eV}$.

- $f_{\mathrm{abs}}>90 \%$

- $f_{\mathrm{SBS}}<5-10 \%$

- $f_{\text {hot }}<5 \%$ at $T_{\text {hot }} \geq 50 \mathrm{keV}$

- $f_{\mathrm{SRS}}<5 \%$

\section{HLP3 Summary of Tasks}

To obtain hohlraum experiments with peak $T_{R}$ ranging from 100 to $\lesssim 230 \mathrm{eV}$ with a variety of pulse formats and targets (lined and unlined hohlraums). Examine hohlraums up to ignition scale albeit at lower temperature and shorter pulse duration. When appropriate, capsules will be contained in the hohlraum. Measurements will focus on the $x$-ray environment (including photons with $h v>2 \mathrm{keV}$ ) within the hohlraum. The deliverable will be confirmation of our ability to calculate energy balance in a hohlraum with emphasis on wall loss/ albedo and to diagnose and predict the position of the time-dependent laser produced $x$-ray source within the hohlraum. Both of these topics are important to understand drive symmetry in hohlraums. These experiments (and the tasks in HLP4) will demonstrate our knowledge of the hohlraum physics required to implement beam phasing on the Nova Upgrade to control timedependent drive symmetry. 
This task will also include characterization of x-ray and laser-driven ablation plasmas. The experiments will verify detailed models of plasma formation at the laser aperture, the tamping of the high- $Z$ hohlraum plasma by the low to moderate $Z$ liner plasma, and plasma stagnation within the hohlraum (including the effects of capsules). Measurements of $n_{\mathrm{e}}(\bar{x}, t) T_{\mathrm{e}}(\bar{x}, t) Z(\bar{x}, t)$ will be included.

\section{HLP3 Goals}

To confirm our ability to calculate energy balance in a hohlraum (with emphasis on wall loss/albedo) and to model the details of the plasma and $x$-ray emission within the hohlraum.

\section{HLP4 Summary of Tasks}

To demonstrate symmetry control with low and intermediate Z-lined hohlraums. Experiments will use a variety of $x$-ray imaging techniques to measure time-integrated $\mathrm{x}$-ray drive asymmetry in low-order $l$ modes, particularly $P_{2}$ and $P_{4}$. Supporting experiments, measuring/ inferring $\mathrm{x}$-ray source-spot motion, and time-dependent $\mathrm{x}$-ray albedo will also be conducted.

\section{HLP4 Goals}

To achieve low-order l-mode asymmetry (time integrated) $\lesssim 2-4 \%\left(P_{2}, P_{4}\right)$ in a lined hohlraum with pulse shaping properly scaled to ignition targets. In these experiments, the hohlraum case area will nominally be $\sim 16 \times$ that of the capsule, and LEH losses will be comparable to the capsule absorbed energy. Peak $T_{R}<230 \mathrm{eV}$, consistent with Nova's power and focusing capabilities, will be used. A limited number of experiments with peak $T_{\mathrm{R}} \approx 250 \mathrm{eV}$ (and corresponding shaped pulses consistent with Nova's capabilities) will also be conducted. The achievable flux uniformity is limited by the number of Nova's beams (10) and potentially by the individual beam quality. Experiments to measure time-dependent laser produced $x$-ray source motion and hohlraum wall albedo will further establish our understanding of time-dependent drive asymmetry. The experiments will reach the stated level of flux uniformity with Nova and demonstrate our quantitative understanding of the limitations (physics and technology) so that flux uniformities (both instantaneous and time integrated) required for ignition/moderate gain will be achieved on the Nova Upgrade.

To experimentally demonstrate time-integrated flux asymmetry control by varying $P_{2}$ nonuniformity from 10 to $20 \%$ (waist high) to 10 to $20 \%$ (pole high). These experiments, supported by quantitative modeling, will further demonstrate our ability to control and achieve the flux uniformity required for ignition/gain targets. The majority of the experiments will use lined hohlraums and shaped pulses with peak $T_{\mathrm{R}}<230 \mathrm{eV}$. A limited number of experiments with $T_{\mathrm{R}} \approx 230 \mathrm{eV}$ will also be included. The capsule convergences that we employ will be limited by our diagnostic resolution and sensitivity, but we expect it will range from 15 to 20 .

\section{HLP5 Summary of Tasks}

To demonstrate acceptable levels of stimulated Brillouin (back and side scatter) and Raman scattering (back, side, and forward) in plasmas where the overall gain and $I / I_{\text {th }}$ ratio are equal to that of Nova Upgrade targets. (Here, $I$ is the laser intensity and $I_{t h}$ is the threshold intensity for the particular process.) The present Nova hohlraum experiments, in which good laser-plasma coupling is observed, have many relevant features that are nearly equivalent to an ignition hohlraum but differ in scale lengths and plasma dimensions ( $1 \mathrm{~mm}$ compared with 3-4 mm). As such, the experimental goals described in HLP1, HLP2, and HLP4 will continue to be relevant.

Experiments will also involve exploiting opengeometry targets such as exploding foils/disks of varying atomic number and will focus on long scale length plasmas where $n_{\mathrm{e}} \lesssim 0.15 n_{\mathrm{c}^{\prime}}$ at which collisional damping of the various instabilities will not be effective. Experimental techniques will exploit the use of 8 to 9 Nova beams (operating at 0.53 or $0.35 \mu \mathrm{m}$ ) to independently create large $(L>2 \mathrm{~mm})$, hot $\left(T_{e}>1.5 \mathrm{keV}\right)$ plasmas $n_{\mathrm{e}} / n_{\mathrm{c}}<0.15$, which will then be irradiated by an independently controlled interaction beam (operating at $0.53,0.35$, or $1.05 \mu \mathrm{m}$ ). Plasma conditions will be characterized and experimentally controlled (e.g., allowing sufficient delay between the plasma forming beams and interaction beam to allow hydrodynamic smoothing of density ripples and bumps and to reduce fluctuation and other noise sources to thermal levels). Freely expanding and interpenetrating plasma sources will be examined. A variety of pulse shapes including continuous and picket fence pulses will be studied.

\section{HLP5 Goals}

To quantify:

- $f S B S$ (back, side) $<5-10 \%$

- $f S R S$ (back, side) $<5-10 \%$

- $f S R S$ (forward) $<5 \%$,

In long scale length plasmas with the following characteristics of $L$ (density and velocity scale length $2 \mathrm{~mm}$ ), $T_{\mathrm{e}} \approx 1.5 \mathrm{keV}$, $n_{\mathrm{e}} / n_{\mathrm{c}}<0.15$, and $<3<Z><28$.

These experiments will initially be conducted with the narrow frequency $\left(\Delta v / v<10^{-5}\right)$, spatially modulated 
Nova beams and thus represent a "worst case" scenario. Experiments using spatially smooth beams (with smoothing times $\leqslant 20 \mathrm{ps}$ ) are planned as part of the Nova program. Initial experiments will be conducted on the 2-beam facility in parallel with HLP6 and will eventually be performed on the 10 -beam chamber where larger interaction plasmas are possible. Only the interaction beam, however, would have a smooth intensity profile. These latter experiments would be completed after the approval for the Nova Upgrade (provided the successful completion of HLP1, HLP2, HLP4, and HLP6) and would be complete in time to impact the Nova Upgrade configuration. Since laser wavelength effects are generally well understood and the purpose of this program is to examine laser coherence effects, the experiments will use 0.53- $\mu \mathrm{m}$ light where, at minimal facility cost, we can study relevant phenomena at laser energy/power $>10$ to 100 times that presently available on other facilities. Experiments will be conducted at both the 2-beam (spatial and temporal coherence variations) and 10-beam (temporal coherence) target areas. Continuous and comb spectra will be used with total fractional bandwidth of 0.2 to $0.3 \%$ ( $\tau_{\text {coh }} \approx 0.6-0.8 \mathrm{ps}$ ). The experiments will use both open geometry (disks, foils) and hohlraums. Extensive plasma characterization (e.g., an optical interferometer to measure plasma $n_{\mathrm{e}}$ profiles is nearing completion on the 2-beam facility) will be part of this program so that quantitative plasma physics experiments will be performed (i.e., well-defined laser intensities interacting with known plasmas).

\section{HLP6 Summary of Tasks}

\author{
To study the influence/coupling of filamentation with \\ large scale length plasmas.
}

\section{HLP6 Goals}

To experimentally characterize the nonlinear state and the influence/coupling of filamentation with other instabilities $(S B S, S R S)$ in the low-density $\left(n_{e} / n_{c}<0.15\right)$ plasma within the hohlraum channel. Experiments will also examine the predicted stabilizing effects of multiple beams, angular divergence, and beam smoothing. This effort will proceed in parallel but extend beyond the completion dates of HLP1, HLP2, HLP4, and HLP5. If objectives in these other tasks are not met, then the priority of the HLP6 activity would be increased with a resulting completion date before the Nova Upgrade construction starts. The results would then be of importance in establishing the baseline beam smoothing requirements for the new facility.

\section{HLP7 Summary of Tasks}

To achieve the hohlraum temperatures required for ignition/moderate gain within the output performance of the Nova Upgrade, $x$-ray conversion efficiencies $>35$ to $50 \%$ are required for $I \geq 10^{15} \mathrm{~W} / \mathrm{cm}^{2}$. Disk experiments on Nova have already demonstrated these efficiencies with measured values exceeding $70 \%$ (and instantaneous values exceeding $80 \%$ ) at relevant intensities and pulse lengths equal to that of the main pulse of the ignition target. Temperatures achieved in recent hohlraum experiments have implied conversion efficiencies $\geq 50 \%$ at laser $I>3$ to $5 \times 10^{15} \mathrm{~W} / \mathrm{cm}^{2}$.

In addition, experiments with disks have demonstrated or inferred that $x$-ray conversion efficiency (at a given wavelength, plasma composition, and intensity) increases with pulse length, spot size, and potentially beam quality. Furthermore, Nova experiments have also implied that the effective conversion efficiency in a hohlraum environment is also larger than that obtained in open-geometry targets. While our present modeling can match much of the data, some of the trends seen in the data (e.g., spot-size dependence) are not quantitatively understood.

$X$-ray conversion experiments planned over the next several years will further build upon our extensive database and attempt to develop an improved modeling capability. Experiments will focus on areas of applicability to the Nova Upgrade, i.e., long pulse, $I>5 \times 10^{14} \mathrm{~W} / \mathrm{cm}^{2}$, hohlraum environment, and beam uniformity. The power and energy capabilities of Nova will limit the overall parameter range that can be experimentally addressed. Irradiations will include both continuous and picket fence pulse shapes.

\section{HLP7 Goals}

Since the required conversion efficiency $\eta$ (more importantly $\eta I$ ) has already been achieved and the favorable trends in conversion efficiency as a function of the parameters associated with the Nova Upgrade (compared with Nova) have also been observed, we feel no additional milestones are required. The experiments will be used primarily to improve our understanding of the appropriate physics to enable better predictions in the range of hohlraum performance available on the Nova Upgrade. 


\title{
FACILITY REPORT JULY-SEPTEMBER 1995
}

\author{
G. Hermes
}

Nova Operations Group

During this quarter, Nova Operations fired a total of 279 system shots resulting in 301 experiments. These experiments were distributed among ICF experiments, Defense Sciences experiments, X-Ray Laser experiments, Laser Sciences, and facility maintenance shots.

This is the final report for FY 1995. During the past year, Nova fired a total of 1101 system shots resulting in a total of 1228 experiments. There were 856 target experiments done in the 10-beam chamber and 128 experiments done in the 2-beam chamber. As a result of the declassification of ICF, there were only six target shots that were classified. There were 92 experiments conducted in support of laser science work, including precision pointing, failsafe chirp activation, and miscellaneous beam propagation experiments. We fired 150 calibration shots in support of routine and precision operations.

The first $8 \times$ CCD camera was installed and activated on the 10-beam chamber. This camera, plus one more, will eventually replace the film-based pinhole cameras currently being used to acquire $x$-ray images from precision pointing shots. Using the CCD cameras will greatly reduce the time required to analyze data from the pointing shots.

A set of cylinder lenses was removed from the 2-beam chamber and installed on beamline 3 of the 10 -beam chamber in support of a series of $x$-ray laser experiments. This lens set was removed after several days of experiments and reinstalled on the 2-beam chamber. These lenses will be reinstalled next quarter with an additional spacer to allow for a longer line focus on the $x$-ray laser targets.

The 100-TW system was activated this quarter. We fired a shot at $\sim 129$ TW with a pulse width of $395 \mathrm{fs}$ and an energy of $51 \mathrm{~J}$. Work will continue to complete the target system activation to support target shots with the 100-TW system next quarter.

Work continues in support of the Petawatt system. The support frames for the compressor vacuum chamber and turning mirrors have been installed in the 10-beam target bay. The fabrication of the three major sections of the compressor tank is well under way. We are planning to install the compressor chamber in mid-November. 


\section{Publications}

A

Afeyan, B. B., and Williams, E. A., Unified Theory of Stimulated Raman Scattering and Two Plasmon Decay in Inhomogeneous Plasmas: the High Frequency Hybrid Instability, Lawrence Livermore National Laboratory, Livermore, CA, UCRL-JC-121846 (1995). Submitted to Phys. Rev. Lett.

Afeyan, B., Berger, R. L., Kaiser, T. B., and Kruer, W. L., Theory and Simulations of the Effects of Long Wavelength Fluctuations on Parametric Instabilities, Lawrence Livermore National Laboratory, Livermore, CA, UCRL-JC-121299 ABS (1995). Prepared for the 37th Annual Mtg of the American Physical Society Div of Plasma Physics, Louisville, KY, Nov 6-10, 1995.

Aikens, D., Roussel, A., and Bray, M., Derivation of Preliminary Specifications for Transmitted Wavefront and Surface Roughness for Large Optics Used in Inertial Confinement Fusion, Lawrence Livermore National Laboratory, Livermore, CA, UCRL-ID-121339 (1995).

Aikens, D., The Origin and Evolution of the Optics Specifications for the National Ignition Facility, Lawrence Livermore National Laboratory, Livermore, CA, UCRL-ID-121333 (1995).

Amendt, P., and Murphy, T. J., Symmetry Tuning and Increased X-Ray Drive in Modified Nova Hohlraums, Lawrence Livermore National Laboratory, Livermore, CA, UCRL-JC-121632 (1995). Submitted to Phys. Rev. Lett.

Amendt, P., Glendinning, S. G., Hammel, B. A., Landen, O., Suter, L. J., Laffite, S., and Jadaud, J. P., Nova Hohlraum Time-Dependent Drive Asymmetry Diagnostic with Surrogate Foam Targets, Lawrence Livermore National Laboratory, Livermore, CA, UCRL-JC-121602 ABS (1995). Prepared for the 37th Annual Mtg of American Physical Society Div of Plasma Physics, Louisville, KY, Nov 6-10, 1995.
Auerbach, J. M., Eimerl, D., Hunt, J. T., Milam, D., Trenholme, J. B., and Milonni, P. W., Perturbation Theory for Frequency Doubling and Tripling of Electric Field Amplitude and Phase Ripples, Lawrence Livermore National Laboratory, Livermore, CA, UCRL-JC-121452 (1995). Prepared for the 1st Annual Int'l Conf on SolidState Lasers for Application to Inertial Confinement Fusion, Monterey, CA, May 30-Jun 2, 1995.

Auerbach, J. M., Lawson, J. K., Rotter, M. D., Sacks, R. A., Van Wonterghem, B. M., and Williams, W. H., Performance Modeling of Beamlet, Lawrence Livermore National Laboratory, Livermore, CA, UCRL-JC-121453 (1995). Prepared for the 1st Annual Int'l Conf on Solid-State Lasers for Application to Inertial Confinement Fusion, Monterey, CA, May 30-Jun 2, 1995.

\section{B}

Back, C. A., Berger, R. L., Estabrook, K., Failor, B. H., Hsing, W. W., Hsieh, E. J., Hockaday, R., Kalantar, D. H., Kauffman, R. L., Keane, C. J., Klem, D. E., MacGowan, B. J., Montgomery, D. S., Moody, J. D., Powers, L. V., Shepard, T. D., Stone, G. F., Suter, L. J., and Turner, R. E., Measurements of Large Scale-Length Plasmas Produced from Gas-Filled Targets, Lawrence Livermore National Laboratory, Livermore, CA, UCRL-JC-118834 (1995). Prepared for the 6th Int'l Workshop on Radiative Properties of Hot Dense Matter, Sarasota, FL, Oct 31-Nov 4, 1994.

Back, C. A., Glenzer, S. H., Estabrook, K., Kauffman, R. L., Landen, O. L., MacGowan, B. J., Powers, L. V., Shepard, T. D., and Stone, G. F., Localized Measurements of Electron Temperature in Hohlraum Targets by X-Ray Spectroscopy, Lawrence Livermore National Laboratory, Livermore, CA, UCRL-JC-121290 ABS (1995). Prepared for the 37th Annual Mtg of the American Physical Society Div of Plasma Physics, Louisville, KY, Nov 6-10, 1995. 
Barnard, J. J., Cable, M. D., Callahan, D. A., Fessenden, T. J., Friedman, A., Grote, D. P., Judd, D. L., Lund, S. M., Newton, M. A., Sharp, W. M., and Yu, S. S., Physics Design and Scaling of Recirculating Induction Accelerators: From Benchtop Prototypes to Drivers, Lawrence Livermore National Laboratory, Livermore, CA, UCRL-JC-121457 ABS (1995). Prepared for the Int'l Symp on Heavy Ion Fusion, Princeton, NJ, Sept 6-9, 1995.

Beach, R. J., A Classical Geometric Theory of Matter and Electromagnetism, Lawrence Livermore National Laboratory, Livermore, CA, UCRL-JC-121633 (1995). Submitted to Phys. Rev. B.

Beach, R. J., Sutton, S. B., Skidmore, J. A., and Emanuel, M. A., High Power 2 um Diode-Pumped Tm:YAG Laser, Lawrence Livermore National Laboratory, Livermore, CA, UCRL-JC-121647 ABS (1995). Prepared for the Society of Photo-Optical Instrumentation Engineers 1996 Symp on Lasers and Integrated Optoelectronics, San Jose, CA, Jan 27-Feb 2, 1996.

Berger, R. L., Afeyan, B. B., Cohen, B. I., Hinkel, D., Kaiser, T. B., Kirkwood, R., Langdon, A. B., Lasinski, B. F, MacGowan, B. J., Montgomery, D. S., Moody, J. D., Powers, L. V., Still, C. W., Williams, E. A., and Bonnaud, G., Influence of Filamentation on the Growth and Angular Width of SBS and SRS, Lawrence Livermore National Laboratory, Livermore, CA, UCRL-JC-121477 ABS (1995). Prepared for the 37th Annual Mtg of the American Physical Society Dio of Plasma Physics, Louisville, KY, Nov 6-10, 1995.

Berger, R., The Influence of Spatial and Temporal Laser Beam Smoothing on Stimulated Brillouin Scattering in Filamentary Laser Light, Lawrence Livermore National Laboratory, Livermore, CA, UCRL-JC-119259 (1995); Phys. Rev. Lett. 75(8), 1078-1081 (1995).

Bliss, E. S., Feldman, M., Murray, J. E., and Vann, C. S., Laser Chain Alignment with Low Power Local Light Sources, Lawrence Livermore National Laboratory, Livermore, CA, UCRL-JC-120520 (1995). Prepared for the 1st Annual Int'l Conf on Solid-State Lasers for Application to Inertial Confinement Fusion, Monterey, CA, May 30-Jun 2, 1995.

Boley, C. D., Rhodes, M. A., and Bauer, B. S., Modeling of Plasma-Electrode Pockels Cells, Lawrence Livermore National Laboratory, Livermore, CA, UCRL-JC-116684 ABS Rev 1 (1995). Prepared for the 37th Annual Mtg of the American Physical Society Div of Plasma Physics, Louisville, KY, Nov 6-10, 1995.
Boyd, R. D., Britten, J. A., Decker, D. E., Shore, B. W., Stuart, B. C., Perry, M. D., and Li, L., High-Efficiency Metallic Diffraction Gratings for Laser Applications, Lawrence Livermore National Laboratory, Livermore, CA, UCRL-JC-115589 (1994); Appl. Opt. 34(10), 1697-1706 (1995).

Britten, J. A., Boyd, R. D., Perry, M. D., Shore, B. W., and Thomas, I. M., Low Efficiency Gratings for 3rd Harmonic Diagnostics Applications, Lawrence Livermore National Laboratory, Livermore, CA, UCRL-JC-120707 (1995). Prepared for the 1st Annual Int'l Conf on SolidState Lasers for Application to Inertial Confinement Fusion, Monterey, CA, May 30-Jun 2, 1995.

Brusasco, R. M., Dittrich, T., and Cook, R. C., Feasibility of Organo-Beryllium Target Mandrels Using Organo-

Germanium PECVD as a Surrogate, Lawrence Livermore National Laboratory, Livermore, CA, UCRL-JC-119533 Rev 1 (1995). Submitted to Fusion Technology.

Burkhart, S. C., Beach, R. J., Crane, J. H., Davin, J. M., Perry, M. D., and Wilcox, R. B., The National Ignition Facility Front-End Laser System, Lawrence Livermore National Laboratory, Livermore, CA, UCRL-JC-119019 (1995). Prepared for the 1st Annual Int'l Conf on SolidState Lasers for Application to Inertial Confinement Fusion, Monterey, CA, May 30-Jun 2, 1995.

\section{C}

Cable, M. D., Barnard, J. J., Callahan, D. A., Deadrick, F. J., Eylon, S., Fessenden, T. J., Friedman, A., Grote, D. P., Hopkins, H. A., Judd, D. L., Hawkins, S. A., Karpenko, V. P., Kirbie, H. C., Logan, B. G., Longinotti, D. B., Lund, S. M., Nattrass, L. A., Nelson, M. B., Newton, M. A., and Ollis, C. W., Recirculating Heary Ion Accelerator Experiment, Lawrence Livermore National Laboratory, Livermore, CA, UCRL-JC-121479 ABS (1995). Prepared for the 37th Annual Mtg of the American Physical Society Div of Plasma Physics, Louisville, KY, Nov 6-10, 1995.

Callahan, D. A., and Langdon, A. B., Chamber Transport Physics for Heavy Ion Fusion, Lawrence Livermore National Laboratory, Livermore, CA, UCRL-JC-121480 ABS (1995). Prepared for the 37th Annual Mtg of the American Physical Society Div of Plasma Physics, Louisville, KY, Nov 6-10, 1995.

Callahan, D. A., Chamber Propagation Physics for Heavy Ion Fusion, Lawrence Livermore National Laboratory, Livermore, CA, UCRL-JC-121279 (1995). Prepared for the Int'l Symp on Heary Ion Fusion, Princeton, NJ, Sept 6-9, 1995. 
Callahan, D. A., Interactions between Neighboring Beams in a Heavy Ion Fusion Reactor Chamber, Lawrence Livermore National Laboratory, Livermore, CA, UCRL-JC-121132 (1995). Submitted to Appl. Phys. Lett.

Campbell, J. H., Edwards, G. J., and Marion, J. E., Damage and Fracture in Large Aperture, Fused Silica, Vacuum Spatial Filter Lenses, Lawrence Livermore National Laboratory, Livermore, CA, UCRL-JC-120780 (1995). Prepared for the 1st Annual Int'l Conf on SolidState Lasers for Application to Inertial Confinement Fusion, Monterey, CA, May 30-Jun 2, 1995.

Celliers, P., Da Silva, L. B., Dane, C. B., Mrowka, S., Norton, M., Harder, J., Hackel, L., Matthews, D., Fiedorowicz, H., Barnick, A., and Maldonado, J., Optimization of X-Ray Sources for Proximity Lithography Produced by a High Average Power Nd:Glass Laser, Lawrence Livermore National Laboratory, Livermore, CA, UCRL-CR-121294 (1995).

Celliers, P., Fringe Formation and Coherence of a Soft X-Ray Laser Beam Illuminating a Mach-Zehnder Interferometer, Lawrence Livermore National Laboratory, Livermore, CA, UCRL-JC-120921 (1995). Submitted to Opt. Lett.

Chang, J. J., Warner, B. E., Boley, C. D., and Dragon, E. P., High-Power Copper Vapour Lasers and Applications, Lawrence Livermore National Laboratory, Livermore, CA, UCRL-JC-121626 (1995). Prepared for the NATO Advanced Research Workshop on Pulsed Metal Vapour Lasers, St Andrews, Scotland, Aug 7-10, 1995.

Chang, T., and Havstad, M., Design of a Substrate Heater for Calcium Hydroxyapatite Coating by Pulsed Laser Ablation, Lawrence Livermore National Laboratory, Livermore, CA, UCRL-ID-121808 (1995).

Chenais-Popovics, C., Rancu, O., Renaudin, P., Gauthier, J. C., Gilleron, F., Lindenmeyer, O., Kawagoshi, H., Dirksmoller, M., Uschmann, I., Missalla, T., Sondhauss, P., Forster, E., Renner, O., Krousky, E., Pepin, H., Larroche, O., Peyrusse, O., and Shepard, T. D., X-Ray Spectroscopy and Imaging of a Plasma Collision, Lawrence Livermore National Laboratory, Livermore, CA, UCRL-JC-121582 (1995). Prepared for the Society of Photo-Optical Instrumentation Engineers Conf, San Diego, CA, Jul 12-14, 1995.

Cohen, B. I., Lasinski, B. F., Langdon, A. B., and Williams, E. A., 2D Hybrid Simulations of Ponderomotively Driven Nonlinear Ion Waves, Lawrence Livermore National Laboratory, Livermore, CA, UCRL-JC-121297 ABS (1995). Prepared for the 37th Annual Mtg of the American Physical Society Div of Plasma Physics, Louisville, KY, Nov 6-10, 1995.
Correll, D. L., Vergino, E. S., and Bibeau, C., Laser Fusion Education at LLNL, Lawrence Livermore National Laboratory, Livermore, CA, UCRL-JC-121496 ABS (1995). Prepared for the 37th Annual Mtg of the American Physical Society Div of Plasma Physics, Louisville, KY, Nov 6-10, 1995.

Coverdale, C. A., Darrow, C. B., Decker, C. D., Mori, W. B., Tzeng, K.-C., Marsh, K. A., Clayton, C. E., and Joshi, C., Propagation of Intense Subpicosecond Laser Pulses Through Underdense Plasmas, Lawrence Livermore National Laboratory, Livermore, CA, UCRL-JC-119542 (1994); Phys. Rev. Lett. 74(23), 4659-4662 (1995).

Coverdale, C. A., Darrow, C. B., Decker, C. D., Naumova, N., Bulanov, S., Mori, W. B., and Tzeng, K. C., Temporal Bursting of Stimulated Raman Backscatter in Underdense Plasmas, Lawrence Livermore National Laboratory, Livermore, CA, UCRL-JC-121490 ABS (1995). Prepared for the 37th Annual Mtg of the American Physical Society Div of Plasma Physics, Louisville, KY, Nov 6-10, 1995.

\section{D}

Da Silva, L. B., Barbee Jr., T. W., Cauble, R., Celliers, P., Ciarlo, D., Libby, S., London, R. A., Matthews, D., Mrowka, S., Moreno, J. C., Ress, D., Trebes, J. E., Wan, A. S., and Weber, F., Electron Density Measurements of High Density Plasmas Using Soft X-Ray Laser Interferometry, Lawrence Livermore National Laboratory, Livermore, CA, UCRL-JC-120243 (1995); Phys. Rev. Lett. 74(20), 3991-3994 (1995).

Da Silva, L. B., Barbee, T. W., Cauble, R., Celliers, P., Ciarlo, D., Libby, S., London, R. A., Matthews, D. L., Mrowka, S., Moreno, J. C., Trebes, J. E., Wan, A. S., and Weber, F., Development of XUV-Interferometry (155 A) Using a Soft X-Ray Laser, Lawrence Livermore National Laboratory, Livermore, CA, UCRL-JC-121629 (1995). Prepared for the Society of Photo-Optical Instrumentation Engineers 40th Annual Mtg, San Diego, CA, Jul 9-14, 1995.

Da Silva, L. B., Barbee, T. W., Cauble, R., Celliers, P., Kalantar, D. H., Key, M. H., Moreno, J. C., Trebes, J. E., Wan, A. S., and Weber, F., Application of X-Ray Lasers to Probe High Density Plasmas, Lawrence Livermore National Laboratory, Livermore, CA, UCRL-JC-121623 ABS (1995). Prepared for the 37th Annual Mtg of the American Physical Society Div of Plasma Physics, Louisville, KY, Nov 6-10, 1995. 
Dahlburg, J., Fyfe, D. E., Gardner, J. H., Haan, S. W., Bodner, S. E., and Doolen, G. D., Three Dimensional Multimode Simulations of the Ablative Rayleigh-Taylor Instability, Lawrence Livermore National Laboratory, Livermore, CA, UCRL-JC-118845 (1994); Phys. Plasmas 2(6), 2453-2459 (1995).

Darrow, C., Hay, G., Zakharenkow, Y., Nguyen, H., Hammel, B., and Perry, M. D., Experimental Investigation of the Interaction of $1 \mu \mathrm{m}, 0.7 \mathrm{ps}, 10^{19} \mathrm{~W} / \mathrm{cm}^{2}$ Laser Pulses with Solid Targets, Lawrence Livermore National Laboratory, Livermore, CA, UCRL-JC-120245 ABS Rev 1 (1995). Prepared for the 37th Annual Mtg of the American Physical Society Div of Plasma Physics, Louisville, KY, Nov 6-10, 1995.

De Yoreo, J. J., Malkin, A. J., Land, T. A., Kuznetsov, Y. G., Lee, J. D., and McPherson, A., An AFM Study of the Growth Mechanisms and Morphologies of Solution-Based Crystals, Lawrence Livermore National Laboratory, Livermore, CA, UCRL-JC-121610 ABS (1995). Prepared for the Metallurgical Society Annual Mtg, Anaheim, CA, Feb 4-8, 1996.

DeGroot, J. S., Estabrook, K. G., Williams, E. A., Wilks, S. C., Wallace, R. J., and Stone, G. F., High Z Hydrides to Simultaneously Suppress Raman and Brillouin in Laser-Plasmas, Lawrence Livermore National Laboratory, Livermore, CA, UCRL-JC-121459 ABS (1995). Prepared for the $37 t h$ Annual Mtg of the American Physical Society Div of Plasma Physics, Louisville, KY, Nov 6-10, 1995.

Ditmire, T., Crane, J. K., Nguyen, H., Da Silva, L. B., and Perry, M. D., Energy-Yield and Conversion-Efficiency Measurements of High Order Harmonic Radiation, Lawrence Livermore National Laboratory, Livermore, CA, UCRL-JC-118146 (1994); Phys. Rev. A 51(2), R902R905 (1995)

Ditmire, T., Hutchinson, M. H. R., Key, M. H., Lewis, C. L. S., Mac Phee, A., Mercer, I., Neely, D., Perry, M. D., Smith, R. A., Wark, J. S., and Zepf, M., Amplification of XUV Harmonic Radiation in a Gallium Amplifier, Lawrence Livermore National Laboratory, Livermore, CA, UCRL-JC-119550 (1994); Phys. Rev. A 51(6), R4337-R4340 (1995).

Ditmire, T., Kulander, K., Crane, J. K., Nguyen, H., and Perry, M. D., Calculation and Measurement of High Order Harmonic Energy Yields in Helium, Lawrence Livermore National Laboratory, Livermore, CA, UCRL-JC-121468 (1995). Submitted to J. Opt. Soc. Am. B.
Ditmire, T., Nguyen, H., and Perry, M. D., Amplification of Femtosecond Pulses to $1 \mathrm{~J}$ in Cr:LiSrAlF ${ }_{6}$, Lawrence Livermore National Laboratory, Livermore, CA, UCRL-JC-118531 (1995); Opt. Lett. 20(10), 1142-1144 (1995).

Dittrich, T. R., and Haan, S. W., Some Criteria for Designing Robust NIF Capsules, Lawrence Livermore National Laboratory, Livermore, CA, UCRL-JC-121300 ABS (1995). Prepared for the 37th Annual Mtg of the American Physical Society Div of Plasma Physics, Louisville, KY, Nov 6-10, 1995.

Dixit, S. M., Rushford, M. C., Thomas, I. M., and Perry, M. D., Continuous Contour Phase Plates for Tailoring the Focal Plane Irradiance Profile, Lawrence Livermore National Laboratory, Livermore, CA, UCRL-JC-121127 (1995). Prepared for the 1st Annual Int'l Conf on Solid-State Lasers for Application to Inertial Confinement Fusion, Monterey, CA, May 30-Jun 2, 1995.

\section{$\mathrm{E}$}

Eddleman, J. L., DeGroot, J. S., Speilman, R. B., Hartman, C. W., Gee, M., Hammer, J. H.,

Estabrook, K. G., Harte, J. A., Bateson, W. B., and Hewett, D. W., Effect of Lower Hybrid Instabilities on Radiating Z-Pinches, Lawrence Livermore National Laboratory, Livermore, CA, UCRL-JC-121461 ABS (1995). Prepared for the 37th Annual Mtg of the American Physical Society Div of Plasma Physics, Louisville, KY, Nov 6-10, 1995.

Eimerl, D., Configuring the National Ignition Facility for Direct-Drive Experiments, Lawrence Livermore National Laboratory, Livermore, CA, UCRL-ID-120758 (1995).

Eimerl, D., Rothenberg, J., Key, M., Weber, S., Verdon, C., Skupsky, S., Soures, J., and Craxton, S., Configuring NIF for Direct Drive Experiments, Lawrence Livermore National Laboratory, Livermore, CA, UCRL-JC-121271 (1995). Prepared for the 1st Annual Int'l Conf on SolidState Lasers for Application to Inertial Confinement Fusion, Monterey, CA, May 30-Jun 2, 1995.

English, R. E., Aikens, D. M., Whistler, W. T., Walmer, C. D., and Maney, R. T., Implementation of ISO 10110 Optics Drawing Standards for the National Ignition Facility, Lawrence Livermore National Laboratory, Livermore, CA, UCRL-JC-121608 (1995). Prepared for the Society of Photo-Optical Instrumentation Engineers 40th Annual Mtg, San Diego, CA, Jul 9-14, 1995. 
Estabrook, K. G., Glenzer, S., Back, C., Montgomery, D., Kornblum, H., Kirkwood, B., MacGowan, B., and Moody, J., Quarter Critical Resonance in Gasbags, Lawrence Livermore National Laboratory, Livermore, CA, UCRL-JC-121460 ABS (1995). Prepared for the 37th Annual Mtg of the American Physical Society Div of Plasma Physics, Louisville, KY, Nov 6-10, 1995.

\section{$\mathbf{F}$}

Foster, J. M., Rosen, P. A., Dunne, A. M., Stevenson, R. M., Shepard, T. D., and Orzechowski, T. J., Interface Motion in Gas-Tamped Laser-Heated Hohlraums, Lawrence Livermore National Laboratory, Livermore, CA, UCRL-JC-121601 ABS (1995). Prepared for the 37th Annual Mtg of the American Physical Society Div of Plasma Physics, Louisville, KY, Nov 6-10, 1995.

\section{G}

Glendinning, S. G., Amendt, P., Budil, K. S., Hammel, B. A., Kalantar, D. H., Key, M. H., Landen, O. L., Remington, B. A., and Desenne, D. E., Laser-Plasma Diagnostics of Dense Plasmas, Lawrence Livermore National Laboratory, Livermore, CA, UCRL-JC-121494 (1995). Prepared for the Society of Photo-Optical Instrumentation Engineers Conf on Applications of Laser Plasma Radiation II, San Diego, CA, Jul 9-14, 1995.

Glendinning, S. G., Powers, L. V., Landen, O. L., Hammel, B. A., Kauffman, R. A., Suter, L. J., Richard, A. L., and Stone, G., Drive Symmetry Measurements in Gas-Filled Hohlraum, Lawrence Livermore National Laboratory, Livermore, CA, UCRL-JC-121609 ABS (1995). Prepared for the 37th Annual Mtg of the American Physical Society Div of Plasma Physics, Louisville, KY, Nov 6-10, 1995.

Glenzer, S. H., Back, C. A., Estabrook, K., MacGowan, B. J., Montgomery, D. S., Moody, J. D., and Stone, G. F., Electron Temperature and Density Measurements in Gasbag Plasmas by X-Ray Spectroscopy, Lawrence Livermore National Laboratory, Livermore, CA, UCRL-JC-121498 ABS (1995). Prepared for the 37th Annual Mtg of the American Physical Society Div of Plasma Physics, Louisville, KY, Nov 6-10, 1995.

Glinsky, M., Regimes of Suprathermal Electron Transport, Lawrence Livermore National Laboratory, Livermore, CA, UCRL-JC-118514 (1994); Phys. Plasmas 2(7), 27962806 (1995).
Grote, D. P., Friedman, A., Haber, I., and Yu, S., ThreeDimensional Simulations of High-Current Beams in Induction Accelerators with WARP3D, Lawrence Livermore National Laboratory, Livermore, CA, UCRL-JC-121280 (1995). Prepared for the Int'l Symp on Heavy Ion Fusion, Princeton, NJ, Sept 6-9, 1995.

\section{$\mathbf{H}$}

Haan, S. W., Modeling of NIF Ignition Targets with Irradiation Asymmetry and Short-Wavelength

Hydrodynamic Instabilities, Lawrence Livermore National Laboratory, Livermore, CA, UCRL-JC-121810 ABS (1995). Prepared for the Nuclear Explosives Design Physics Conf (NEDPC), Los Alamos, NM, Oct 30-Nov 3, 1995.

Haan, S. W., Pollaine, S. M., Chandler, E. A., and Strobel, G. L., Implosion Modeling of Indirect Drive Ignition Targets for the National Ignition Facility, Lawrence Livermore National Laboratory, Livermore, CA, UCRL-JC-121464 ABS (1995). Prepared for the 37th Annual Mtg of the American Physical Society Div of Plasma Physics, Louisville, KY, Nov 6-10, 1995.

Haan, S. W., Pollaine, S. M., Lindl, J. D., Suter, L. J., Berger, R. L., Powers, L. V., Alley, W. E., Amendt, P. A., Futterman, J. J., Levedahl, W. K., Rosen, M. D., Rowley, D. P., Sacks, R. A., Shestakov, A. I.,

Strobel, G. L., Tabak, M., Weber, S. V., Zimmerman, G. B., Krauser, W. J., Wilson, D. C., Coggeshell, S. V., Harris, D. B., Hoffman, N. M., and Wilde, B. H., Design and Modeling of Ignition Targets for the National Ignition Facility, Lawrence Livermore National Laboratory, Livermore, CA, UCRL-JC-117034 (1994); Phys. Plasmas 2(6), 2480-2487 (1995).

Haddad, W. S., Trebes, J. E., Goodman, D. M., Lee, H.-R., McNulty, I., Anderson, E. H., and Zalensky, A. O., Ultra High Resolution Soft X-Ray Tomography, Lawrence Livermore National Laboratory, Livermore, CA, UCRL-JC-121622 (1995). Prepared for the Society of Photo-Optical Instrumentation Engineers 1995 Int'l Society for Optical Engineering and Instrumentation, San Diego, CA, Jul 9-14, 1995.

Hammer, J. H., and Mattor, N., Magnetic Fast Ignition, Lawrence Livermore National Laboratory, Livermore, CA, UCRL-JC-121809 ABS (1995). Prepared for the Advanced Approaches to Economical Fusion Power Conf, Monterey, CA, Sept 11-15, 1995. 
Hammer, J., Eddleman, J., Estabrook, K., Osterheld, A., Springer, P., Tabak, M., Toor, A., Wilson, B., Zimmerman, G., DeGroot, J., Allshouse, G., Humphries, R., Matsen, K., Sanford, T., and Speilman, R., 2D MHD Simulations of Radiating Z-Pinch Dynamics with LASNEX, Lawrence Livermore National Laboratory, Livermore, CA, UCRL-JC-121482 ABS (1995). Prepared for the 37th Annual Mtg of the American Physical Society Div of Plasma Physics, Louisville, KY, Nov 6-10, 1995.

Hermes, G. L., Nova Experimenters Users Guide, Lawrence Livermore National Laboratory, Livermore, CA, UCRL-MA-121291 Rev 1 (1995).

Hinkel, D. E., Williams, E. A., Berger, R. L., and Powers, L. V., Laser Beam Deflection Induced by Transverse Plasma Flow, Lawrence Livermore National Laboratory, Livermore, CA, UCRL-JC-121493 ABS (1995). Prepared for the 37th Annual Mtg of the American Physical Society Div of Plasma Physics, Louisville, KY, Nov 6-10, 1995.

Ho, D., Autoneutralization of Space-Charge-Dominated Beams for Heary-Ion Fusion, Lawrence Livermore National Laboratory, Livermore, CA, UCRL-JC-120830 (1995). Submitted to Nuclear Fusion.

\section{K}

Kaiser, T. B., Afeyan, B. B., Berger, R. L., and Kruer, W. L., Near-Forward Stimulated Brillouin Scattering and Filamentation Simulations with F3D, Lawrence Livermore National Laboratory, Livermore, CA, UCRL-JC-121298 ABS (1995). Prepared for the 37th Annual Mtg of the American Physical Society Div of Plasma Physics, Louisville, KY, Nov 6-10, 1995.

Kalantar, D. H., Barbee, T. W., Da Silva, L. B., Glendinning, S. G., Key, M. H., Knauer, J. P., Weber, F, and Weber, S. V., XUV Laser Radiography of Perturbations due to Imprint of Laser Speckle in $0.35 \mu \mathrm{m}$ Laser Irradiation of a Thin Si Foil, Lawrence Livermore National Laboratory, Livermore, CA, UCRL-JC-121625 (1995). Submitted to Rev. Sci. Instrum.

Kalantar, D. H., Budil, K. S., Hammel, B. A., Landen, O. L., and Keane, C. J., X-Ray Backlit Imaging of an Implosion Core to Measure the In-Flight Pusher Density of an Indirect Drive Capsule, Lawrence Livermore National Laboratory, Livermore, CA, UCRL-JC-121491 ABS (1995). Prepared for the 37th Anmual Mtg of the American Physical Society Div of Plasma Physics, Louisville, KY, Nov 6-10, 1995.
Kalantar, D. H., Da Silva, L. B., Glendinning, S. G., Key, M. H., Weber, F., Weber, S. V., and Knauer, J. P., XUV Probing of Laser Imprint Using an Yttrium X-Ray Laser, Lawrence Livermore National Laboratory, Livermore, CA, UCRL-JC-121492 ABS (1995). Prepared for the 37th Annual Mtg of the American Physical Society Div of Plasma Physics, Louisville, KY, Nov 6-10, 1995.

Kalantar, D., Production and Characterization of Large Plasmas from Gas Bag Targets on Nova, Lawrence Livermore National Laboratory, Livermore, CA, UCRL-JC-119910 (1995); Phys. Plasmas 2(8), 3161-3168 (1995).

Kane, J., Arnett, D., Remington, B. A., Dearborn, D., Rubenchik, A., Castor, J., Woosley, S., Wood-Vasey, M., Liang, E. P., and London, R., Simulations of a SupernovaRelevant Hydrodynamic Instability Experiment on the Nova Laser, Lawrence Livermore National Laboratory, Livermore, CA, UCRL-JC-121487 ABS (1995). Prepared for the 37th Annual Mtg of the American Physical Society Div of Plasma Physics, Louisville, KY, Nov 6-10, 1995.

Keane, C. J., Pollak, G. W., Cook, R. C., Dittrich, T. R., $X-R a y$ Spectroscopic Diagnostics of Mix in High Growth Factor Spherical Implosions, Lawrence Livermore National Laboratory, Livermore, CA, UCRL-JC-119917 (1995); J. Quant. Spectros. \& Radiat. Transfer 54(7/8), 207-220 (1995).

Kershaw, D. S., Prasad, M. K., Shaw, M. J., 3D Unstructured Mesh ALE Hydrodynamics with the Upwind Discontinuous Finite Element Method, Lawrence Livermore National Laboratory, Livermore, CA, UCRL-JC-122104 (1995). Submitted to J. Comput. Phys.

Key, M. H., Barbee, T. W., Da Silva, L. B., and Glendinning, S. G., New Plasma Diagnostic Possibilities from Radiography with XUV Lasers, Lawrence Livermore National Laboratory, Livermore, CA, UCRL-JC-118848 (1995); J. Quant. Spectros. E Radiat. Transfer 54(1/2), 221-226 (1995).

Key, M. H., Kalantar, D. H., Barbee, T. W., Da Silva, L. B., Glendinning, S. G., Knauer, J. P., Remington, B. A., Rose, S. J., Weber, F. A., and Weber, S. V., Measurement by XUV Laser Radiography of Hydrodynamic Perturbations in Laser Accelerated Thin Foil Targets, Lawrence Livermore National Laboratory, Livermore, CA, UCRL-JC-121586 (1995). Prepared for the Society of Photo-Optical Instrumentation Engineers, Soft X-Ray Lasers and Applications, San Diego, CA, Jul 9-14, 1995. 
Kirkwood, R. K., Afeyan, B. B., Kruer, W. L., MacGowan, B. J., Montgomery, D. S., Moody, J. D., Pennington, D. M., and Wilks, S. C., Observation of Energy Transfer between Frequency Mismatched Laser Beams in a Large Scale Plasma, Lawrence Livermore National Laboratory, Livermore, CA, UCRL-JC-120249 ABS Rev 1 (1995). Prepared for the 37th Annual Mtg of the American Physical Society Div of Plasma Physics, Louisville, KY, Nov 6-10, 1995.

Kirkwood, R. K., Afeyan, B. B., Kruer, W. L., MacGowan, B. J., Moody, J. D., Montgomery, D. S., Pennington, D. M., Weiland, T. L., and Wilks, S. C., Observation of Energy Transfer between Frequency Mismatch Laser Beams in a Large Scale Plasma, Lawrence Livermore National Laboratory, Livermore, CA, UCRL-JC-122102 (1995). Submitted to Phys. Rev. Lett.

Kruer, W. L., Control of Laser-Plasma Instabilities in Hohlraums, Lawrence Livermore National Laboratory, Livermore, CA, UCRL-JC-121584 ABS (1995). Prepared for La Jolla Summer School '95, La Jolla Int'l School of Physics, La Jolla, CA, Aug 8-18, 1995.

Kruer, W. L., Laser Beam Deflection in Self-Consistent Flow Profiles, Lawrence Livermore National Laboratory, Livermore, CA, UCRL-JC-121481 ABS (1995). Prepared for the 37th Annual Mtg of the American Physical Society Div of Plasma Physics, Louisville, KY, Nov 6-10, 1995.

Kruer, W. L., Wilks, S. C., Afeyan, B. B., and Kirkwood, R. K., Energy Transfer between Crossing Laser Beams, Lawrence Livermore National Laboratory, Livermore, CA, UCRL-JC-120776 Rev 1 (1995). Submitted to Phys. of Plasmas.

$\mathbf{L}$

Lambert, S., Overturf, G., Cook, B., and Schroen-Carey, D., EGDM Foam Shell Status Report (TAT 95-049), Lawrence Livermore National Laboratory, Livermore, CA, UCRL-ID-121332 (1995).

Landen, O. L., Bell, P. M., Kalantar, D. H., and Bradley, D. K., X-Ray Framing Cameras for $>5 \mathrm{keV}$ Imaging, Lawrence Livermore National Laboratory, Livermore, CA, UCRL-JC-119326 (1995). Prepared for the Society of Photo-Optical Instrumentation Engineers' 1995 Int'l Symp on Optical Science, Engineering, and Instrumentation, San Diego, CA, Jul 9-14, 1995.

Landen, O. L., Large Rayleigh-Taylor Growth, IndirectlyDriven Implosions at Nova, Lawrence Livermore

National Laboratory, Livermore, CA, UCRL-JC-121497 ABS (1995). Prepared for the 37th Annual Mtg of the American Physical Society Div of Plasma Physics,

Louisville, KY, Nov 6-10, 1995.
Langer, S. H., Keane, C. J., and Scott, H. A., Emission Line Ratios from ICF Target Implosions with Rayleigh-Taylor Perturbations, Lawrence Livermore National Laboratory, Livermore, CA, UCRL-JC-121462 ABS (1995). Prepared for the 37th Annual Mtg of the American Physical Society Div of Plasma Physics, Louisville, KY, Nov 6-10, 1995.

Larson, D., Anderson, R., and Boyes, J., Fault Tolerance of the NIF Power Conditioning System, Lawrence Livermore National Laboratory, Livermore, CA, UCRL-JC-121817 (1995). Prepared for the 1st Annual Int'l Conf on Solid-State Lasers for Application to Inertial Confinement Fusion, Monterey, CA, May 30-Jun 2, 1995.

Lasinski, B. F., Langdon, A. B., Cohen, B. I., and Williams, E. A., Ion Wave Modeling in PIC-Fluid Simulations, Lawrence Livermore National Laboratory, Livermore, CA, UCRL-JC-120775 ABS Rev 1 (1995). Prepared for the $37 t h$ Annual Mtg of the American Physical Society Div of Plasma Physics, Louisville, KY, Nov 6-10, 1995.

Latkowski, J. F., Sanz, J., Vujic, J. L., and Tobin, M. T., Charged Particle and Neutron Activation of Flibe in HYLIFE-II Inertial Fusion Energy Power Plant, Lawrence Livermore National Laboratory, Livermore, CA, UCRL-JC-121292 SUM (1995). Prepared for the American Nuclear Society 1995 Winter Mtg, San Francisco, CA, Oct 29-Nov 2, 1995.

Lerche, R. A., Cable, M. D., and Dendooven, P. G., Fusion Gamma Rays Observed in ICF Experiments, Lawrence Livermore National Laboratory, Livermore, CA, UCRL-JC-121485 ABS (1995). Prepared for the 37th Annual Mtg of the American Physical Society Div of Plasma Physics, Louisville, KY, Nov 6-10, 1995.

Logan, B. G., Role of the U.S. National Ignition Facility within an Overall Plan for Inertial Fusion Energy,

Lawrence Livermore National Laboratory, Livermore, CA, UCRL-JC-121579 ABS (1995). Prepared for the 16th Institute for Electrical and Electronics Engineers/Nuclear and Plasma Sciences Society Symp on Fusion Engineering, Champaign, IL, Sept 30-Oct 5, 1995.

London, R. A., Decker, C., Powers, L. V., Harte, J. A., Trebes, J. E., Cauble, R., Wan, A. S., and Da Silva, L. B., Simulations of X-Ray Laser Probing of Hohlraums, Lawrence Livermore National Laboratory, Livermore, CA, UCRL-JC-121484 ABS (1995). Prepared for the 37th Annual Mtg of the American Physical Society Div of Plasma Physics, Louisville, KY, Nov 6-10, 1995. 


\section{M}

MacGowan, B. J., Laser-Plasma Interactions in IgnitionScale Hohlraum Plasmas, Lawrence Livermore National Laboratory, Livermore, CA, UCRL-JC-121499 ABS (1995). Prepared for the 37th Annual Mtg of the American Physical Society Div of Plasma Physics, Louisville, KY, Nov 6-10, 1995.

Marinak, M. M., Three-Dimensional Simulations of Nova Capsule Implosions and Planar Hydrodynamic Experiments, Lawrence Livermore National Laboratory, Livermore, CA, UCRL-JC-121475 ABS (1995). Prepared for the 37th Annual Mtg of the American Physical Society Div of Plasma Physics, Louisville, KY, Nov 6-10, 1995.

Marshall, C. D., Payne, S. A., Emanuel, M. A., Smith, L. K., Powell, H. T., and Krupke, W. F., DiodePumped Solid-State Laser Driver Experiments for Inertial Fusion Energy, Lawrence Livermore National Laboratory, Livermore, CA, UCRL-JC-121614 (1995). Prepared for the 1st Annual Int'l Conf on Solid-State Lasers for Application to Inertial Confinement Fusion, Monterey, CA, May 30-Jun 2, 1995.

Marshall, C. D., Payne, S. A., Smith, L. K., Beach, R. J., Emanuel, M. A., Powell, H. T., and Krupke, W. F., Diode-Pumped $\mathrm{Yb}: \mathrm{Sr}_{5}\left(\mathrm{PO}_{4}\right)_{3} \mathrm{~F}$ Laser Performance, Lawrence Livermore National Laboratory, Livermore, CA, UCRL-JC-118194 (1995). Prepared for the Advanced Solid State Lasers 10th Topical Mtg, Memphis, TN, Jan 30-Feb 2, 1995.

Marshall, C. D., Smith, L. K., Beach, R. J., Emanuel, M. A., Schaffers, K. I., Payne, S. A., and Chai, B. H. T., DiodePumped Ytterbium-Doped $\mathrm{Sr}_{5}\left(\mathrm{PO}_{4}\right)_{3}$ F Laser Performance, Lawrence Livermore National Laboratory, Livermore, CA, UCRL-JC-122010 (1995). Submitted to the J. Appl. Phys.

Marshall, C. D., Speth, J. A., DeLoach, L. D., and Payne, S. A., Neutron and Gamma Irradiated Optical Property Changes for the Final Optics of the National Ignition Facility, Lawrence Livermore National Laboratory, Livermore, CA, UCRL-JC-120213 (1995). Prepared for the 1st Annual Int'l Conf on Solid-State Lasers for Application to Inertial Confinement Fusion, Monterey, CA, May 30-Jun 2, 1995.

Maxon, S., and Suter, L., Prediction of Energy Radiated and Spectra of Laser-Irradiated Xe Gas Bags, Lawrence Livermore National Laboratory, Livermore, CA, UCRL-JC-121466 ABS (1995). Prepared for the 37th Annual Mig of the American Physical Society Div of Plasma Physics, Louisville, KY, Nov 6-10, 1995.
Moir, R. W., Feasibility and Advantages of Liquid Walls for MFE Power Plants, Lawrence Livermore National Laboratory, Livermore, CA, UCRL-JC-122007 ABS (1995). Prepared for Advanced Approaches to Economical Fusion Power, Monterey, CA, Sept 11-15, 1995.

Moir, R. W., Liquid First Wall and Blanket Development on the NIF and Other Facilities, Lawrence Livermore National Laboratory, Livermore, CA, UCRL-JC-121580 ABS (1995). Prepared for the 16th Institute for Electrical and Electronic Engineers/Nuclear and Plasma Sciences Society Symp on Fusion Engineering, Champaign, IL, Sept 30-Oct 5, 1995.

Moir, R. W., Liquid Wall IFE Power Plants, Lawrence Livermore National Laboratory, Livermore, CA, UCRL-JC-121588 ABS (1995). Submitted to the J. Fusion Engineering Design.

Moir, R. W., The Inertial Fusion Energy Approach to Economical Fusion Power: HYLIFE-II, Lawrence Livermore National Laboratory, Livermore, CA, UCRL-JC-122008 ABS (1995). Prepared for Advanced Approaches to Econornical Fusion Power, Monterey, CA, Sept 11-15, 1995.

Montesanti, R. C., and Thompson, S. L., A Procedure for Diamond Turning KDP Crystals, Lawrence Livermore National Laboratory, Livermore, CA, UCRL-ID-121651 (1995).

Montgomery, D. S., Kirkwood, R. K., MacGowan, B. J., Moody, J. D., Afeyan, B. B., Berger, R. L., and Munro, D. H., Effects of Laser Smoothing on Strongly Damped SRS from Gasbag Plasmas, Lawrence Livermore National Laboratory, Livermore, CA, UCRL-JC-121289 ABS (1995). Prepared for the 37th Annual Mtg of the American Physical Society Div of Plasma Physics, Louisville, KY, Nov 6-10, 1995.

Moody, J. D., MacGowan, B. J., Kirkwood, R. K., Montgomery, D. S., Berger, R. L., Hinkel, D. E., Shepard, T. D., and Williams, E. A., Experimental Studies of Beam Deflection through an Exploding Foil Plasma, Lawrence Livermore National Laboratory, Livermore, CA, UCRL-JC-121486 ABS (1995). Prepared for the 37th Annual Mtg of the American Physical Society Div of Plasma Physics, Louisville, KY, Nov 6-10, 1995.

Moon, S. J., and Eder, D. C., Generation of X-Ray Pulses with Rapid Rise Times to Pump Inner-Shell Photo-Ionized $X$-Ray Lasing in Carbon at $45 \AA$, Lawrence Livermore National Laboratory, Livermore, CA, UCRL-JC-118103 (1995). Prepared for the Society of Photo-Optical Instrumentation Engineers '95 Int'l Society for Optical Engineering, San Diego, CA, Jul 10-11, 1995. 
Munro, D. H., Using the Yorick Interpreted Language, Lawrence Livermore National Laboratory, Livermore, CA, UCRL-JC-121811 (1995). Submitted to Computers and Physics.

Murphy, T. J., Landen, O. L., Keane, C. J., Hammel, B. A., Brusasco, R. M., Cable, M. D., Cook, R. C., Dittrich, T., Haan, S., Hatchett, S. P., Marinak, M. M., McEachern, R. L., Nelson, M. B., Suter, L., Wallace, R. J., Chrien, R., Colvin, J., Hoffman, N., and Hsing, W., High Growth Factor Implosion Experiments on Nova, Lawrence Livermore National Laboratory, Livermore, CA, UCRL-JC-116982 ABS Rev 1 (1995). Prepared for the 37th Annual Mtg of the American Physical Society Div of Plasma Physics, Louisville, KY, Nov 6-10, 1995.

Murray, J. E., Van Wonterghem, B., Seppala, L., Speck, D. R., and Murray, J. R., Parasitic Pencil Beams Caused by Lens Reflections in Laser Amplifier Chains, Lawrence Livermore National Laboratory, Livermore, CA, UCRL-JC-121125 (1995). Prepared for the 1st Annual Int'l Conf on Solid-State Lasers for Application to Inertial Confinement Fusion, Monterey, CA, May 30-Jun 2, 1995.

\section{$\mathbf{N}$}

Newton, M. A., Deadrick, F. J., Hanks, R. L., Hawkins, S. A., Holm, K. A., Kirbie, H. C., Karpenko, V. P., Longinotti, D. B., and Nattrass, L. A., Engineering Development for a Small-Scale Recirculator Experiment, Lawrence Livermore National Laboratory, Livermore, CA, UCRL-JC-121456 (1995). Prepared for the Int'l Symp on Heavy Ion Fusion, Princeton, NJ, Sept 6-9, 1995.

\section{$\mathrm{O}$}

Orzechowski, T. J., Kauffman, R. L., Kirkwood, R. K., Kornblum, H. N., MacGowan, B. J., Montgomery, D. S., Powers, L. V., Stone, G. F., Suter, L. J., Wallace, R. J., Desenne, D., Dulieu, A. G., Juraszek, D., and Richard, A. L., Energetics of Gas-Filled Hohlraums, Lawrence Livermore National Laboratory, Livermore, CA, UCRL-JC-120760 ABS Rev 1 (1995). Prepared for the 37th Annual Mtg of the American Physical Society Div of Plasma Physics, Louisville, KY, Nov 6-10, 1995.

\section{$\mathbf{P}$}

Page, R. H., DeLoach, L. D., Wilke, G. D., Payne, S. A., Beach, R. J., and Krupke, W. F., $\mathrm{Cr}^{2+}$-Doped II-VI Crystals: New Widely-Tunable, Room-Temperature Mid-IR Lasers, Lawrence Livermore National Laboratory, Livermore, CA, UCRL-JC-120815 SUM (1995). Prepared for the Lasers and Electro Optics Society '95, San Francisco, CA, Oct 30-Nov 2, 1995.
Payne, S. A., Krupke, W. F., Beach, R. J., DeLoach, L. D., Emanuel, M. A., Marshall, C. D., Page, R. H., Schaffers, K. I., and Skidmore, J. A., New Solid State Lasers from the Ultraviolet to the Mid-infrared, Lawrence Livermore National Laboratory, Livermore, CA, UCRL-JC-121646 (1995). Prepared for the American Ceramic Society Annual Mtg, Cincinnati, OH, Apr 30-May 4, 1995.

Payne, S. A., Powell, H. T., and Krupke, W. F., Can Solid-State Laser Technology Serve Usefully beyond Fusion Ignition Facilities?, Lawrence Livermore National Laboratory, Livermore, CA, UCRL-JC-120887 (1995).

Prepared for the 1st Annual Int'l Conf on Solid-State Lasers for Application to Inertial Confinement Fusion, Monterey, CA, May 30-Jun 2, 1995.

Payne, S., Smith, L. K., and Krupke, W. F., Cross Sections and Quantum Yields of the $3 \mu \mathrm{m}$ Emission for $\mathrm{Er}^{3+}$ and $\mathrm{Ho}^{3+}$ Dopants in Crystals, Lawrence Livermore National Laboratory, Livermore, CA, UCRL-JC-117882 (1994); J. Appl. Phys. 77(5), 4274-4279 (1995).

Pennington, D. M., Henesian, M. A., Milam, D., and Eimerl, D., Efficient Broadband Third Harmonic Frequency Conversion via Angular Dispersion, Lawrence Livermore National Laboratory, Livermore, CA, UCRL-JC-121197 (1995). Prepared for the 1st Annual Int'l Conf on SolidState Lasers for Application to Inertial Confinement Fusion, Monterey, CA, May 30-Jun 2, 1995.

Petzoldt, R. W., and Moir, R. W., Target Injection, Tracking, and Beam Pointing Requirements and Feasibility Considerations for Inertial Fusion Energy, Lawrence Livermore National Laboratory, Livermore, CA, UCRL-JC-120880 (1995). Submitted to Fusion Engineering and Design.

Pollaine, S. M., Haan, S. W., Amendt, P. A., Moreno, J. C., and Wan, A. S., National Ignition Facility Point Design, Lawrence Livermore National Laboratory, Livermore, CA, UCRL-JC-121473 ABS (1995). Prepared for the 37th Annual Mtg of the American Physical Society Div of Plasma Physics, Louisville, KY, Nov 6-10, 1995.

Powers, L. V., Berger, R. L., Kauffman, R. L., MacGowan, B. J., Amendt, P. A., Back, C. A., Bernat, T. P., Dixit, S. N., Eimerl, D., Estabrook, K. G., Harte, J. A., Kalantar, D. H., Klem, D. E., Lasinski, B. F., Montgomery, D. S., Moody, J. D., Munro, D. H., Shepard, T. D., Suter, L. J., Turner, R. E., Williams, E. A., Fernandez, J. C., Hsing, W. W., Wilde, B. H., and Failor B. H., Gas-Filled Targets for Large Scale-Length Plasma Interaction Experiments on Nova, Lawrence Livermore National Laboratory, Livermore, CA, UCRL-JC-117735; Phys. Plasmas 2(6), 2473-2479 (1995). 
Powers, L. V., Turner, R. E., Kauffman, R. L., Berger, R. L., Amendt, P., Back, C. A., Bernat, T. P., Dixit, S. N., Eimerl, D., Harte, J. A., Henesian, M. A., Kalantar, D. H., Lasinski, B. F., MacGowan, B. J., Montgomery, D. S., Munro, D. H., Pennington, D. M., Shepard, T. P., Stone, G. F., Suter, L. J., and Williams, E. A., Low Stimulated Brillouin Backscatter Observed from Large, Hot Plasmas in Gas-Filled Hohlraums, Lawrence Livermore National Laboratory, Livermore, CA, UCRL-JC-118394 (1994); Phys. Rev. Lett. 74(4), 2957-2960 (1995).

\section{$\mathbf{R}$}

Rambo, P., A Comparison of Kinetic and Multifluid Simulations of Laser-Produced Colliding Plasmas, Lawrence Livermore National Laboratory, Livermore, CA, UCRL-JC-117498 Rev 1 (1995); Phys. Plasmas 2(8), 31303145 (1995).

Ratowsky, R., Propagation of Mutual Coherence in Refractive X-Ray Lasers Using a WKB Method, Lawrence Livermore National Laboratory, Livermore, CA, UCRL-JC-116772 (1994); Phys. Rev. A 51(3), 2361-2370 (1995).

Remington, B. A., Marinak, M. M., Weber, S. V., Budil, K. S., Landen, O. L., Shepard, T., Peyser, T., Kalantar, D., Wallace, R. J., and Rothman, S., Planar Hydrodynamics Experiments in Indirect-Drive, Lawrence Livermore National Laboratory, Livermore, CA, UCRL-JC-121489 ABS (1995). Prepared for the 37th Annual Mtg of the American Physical Society Div of Plasma Physics, Louisville, KY, Nov 6-10, 1995.

Remington, B., Single-Mode and Multimode Rayleigh-Taylor Experiments, Lawrence Livermore National Laboratory, Livermore, CA, UCRL-JC-116897 (1994); Phys. Plasmas 2(1), 241-255 (1995).

Rhodes, M. A., Boley, C. D., Tarditi, A. G., and Bauer, B. S., Plasma-Electrode Pockels Cell for ICF Lasers, Lawrence Livermore National Laboratory, Livermore, CA, UCRL-JC-121180 (1995). Prepared for the 1st Annual Int'l Conf on Solid-State Lasers for Application to Inertial Confinement Fusion, Monterey, CA, May 30-Jun 2, 1995.

Rosen, M. D., Recent Results in Inertial Confinement Fusion with Lasers, Lawrence Livermore National Laboratory, Livermore, CA, UCRL-JC-121472 (1995). Submitted to 1997 McGraw-Hill Yearbook of Science $\mathcal{E}$ Technology.

Rosen, M. D., The Physics of Radiation Driven ICF Hohlraums, Lawrence Livermore National Laboratory, Livermore, CA, UCRL-JC-121585 (1995). Prepared for the Plasma Physics and Technology, La Jolla, CA, Aug 8-18, 1995.
Rosen, M. D., The Science Applications of the High Energy Density Plasmas Created on the Nova Laser, Lawrence Livermore National Laboratory, Livermore, CA, UCRL-JC-121476 ABS (1995). Prepared for the 37th Annual Mtg of the American Physical Society Div of Plasma Physics, Louisville, KY, Nov 6-10, 1995.

Rothenberg, J. E., Eimerl, D., Key, M. H., and Weber, S. V., Illumination Uniformity Requirements for Direct Drive Inertial Confinement Fusion, Lawrence Livermore National Laboratory, Livermore, CA, UCRL-JC-121195 (1995). Prepared for the 1st Annual Int'l Conf on SolidState Lasers for Application to Inertial Confinement Fusion, Monterey, CA, May 30-Jun 2, 1995.

Rothenberg, J. E., Two Dimensional Beam Smoothing by Spectral Dispersion for Direct Drive Inertial Confinement Fusion, Lawrence Livermore National Laboratory, Livermore, CA, UCRL-JC-121198 (1995). Prepared for the 1st Annual Int'l Conf on Solid-State Lasers for Application to Inertial Confinement Fusion, Monterey, CA, May 30-Jun 2, 1995.

\section{S}

Schmitt, A. J., and Afeyan, B. B., Filamentation Dynamics with Full Nonlinear Transverse and Axial Hydrodynamics, Lawrence Livermore National Laboratory, Livermore, CA, UCRL-JC-121621 ABS (1995). Prepared for the 37th, Annual Mtg of the American Physical Society Div of Plasma Physics, Louisville, KY, Nov 6-10, 1995.

Schroen-Carey, D., Overturf, G. E., Letts, S. A., Cook, R. C., and Lambert, S. M., Polymer Overcoated ResorcinolFormaldehyde Foam Microshells for Inertial Confinement Fusion Targets, Lawrence Livermore National Laboratory, Livermore, CA, UCRL-JC-122015 ABS (1995). Prepared for the 10th Int'l Symp on Microencapsulation, Austin, TX, Sept 26-28, 1995.

Sheehan, L., and Kozlowski, M., Detection of Inherent and Laser-Induced Scatter in Optical Materials, Lawrence Livermore National Laboratory, Livermore, CA, UCRL-JC-119613 (1995). Prepared for the Society of Photo-Optical Instrumentation 1995 Int'l Symp on Optical, Science, Engineering and Instrumentation, Tucson, AZ, Jul 9-14, 1995.

Sheehan, L., Kozlowski, M., Rainer, F., Runkel, M., Stolz, C., and Schwartz, S., Large Area Damage Testing of Optics, Lawrence Livermore National Laboratory, Livermore, CA, UCRL-JC-122006 ABS (1995). Prepared for Optical Systems Design and Projection II, Glasgow, Scotland, May 12-16, 1996. 
Shepard, T. D., Back, C. A., Kalantar, D. H., Kauffman, R. L., Keane, C. J., Klem, D. E., Lasinski, B. F, MacGowan, B. J., Powers, L. V., Suter, L. J., Turner, R. E., Failor, B. H., and Hsing, W. W., Isoelectronic X-Ray Spectroscopy to Determine Electron Temperatures in LongScale-Length ICF Plasmas, Lawrence Livermore National Laboratory, Livermore, CA, UCRL-JC-121575 (1995). Submitted to Phys. Rev. E.

Shepard, T. D., Orzechowski, T. J., Murphy, T. J., Suter, L. J., Delamater, N. D., Richard, A. L., Foster, J. M., and Rosen, P. A., Gas-Hohlraum Interface-Motion Modeling, Lawrence Livermore National Laboratory, Livermore, CA, UCRL-JC-121465 ABS (1995). Prepared for the 37th Annual Mtg of the American Physical Society Div of Plasma Physics, Louisville, KY, Nov 6-10, 1995.

Shore, B. W., Stuart, B. C., Feit, M. D., Rubenchik, A. M., and Perry, M. D., Laser Induced Damage in Multilayer Dielectric Gratings due to Ultrashort Laser Pulses, Lawrence Livermore National Laboratory, Livermore, CA, UCRL-JC-120187 Rev 1 (1995). Prepared for the 1st Annual Int'l Conf on Solid-State Lasers for Application to Inertial Confinement Fusion, Monterey, CA, May 30-Jun 2, 1995.

Skidmore, J., High-Power CW Operation of Al Gain P Laser-Diode Arrays, Lawrence Livermore National Laboratory, Livermore, CA, UCRL-JC-115945 (1995); IEEE J. Quantum Electron. 66(2), 133-135 (1995).

Skidmore, J., High-Power CW 690 nm Al Gain P LaserDiode Arrays, Lawrence Livermore National Laboratory, Livermore, CA, UCRL-JC-117679 (1995); Appl. Phys. Lett. 66(3), 1163-1165 (1995).

Smolski, I., De Yoreo, J. J., Zaitseva, N. P., Lee, J. D., and Land, T. A., Oriented Liquid Inclusions in KDP Crystals, Lawrence Livermore National Laboratory, Livermore, CA, UCRL-JC-122011 (1995). Submitted to Crystal Growth.

Still, C. H., Berger, R. L., Langdon, A. B., Miller, D. S., and Williams, E. A., Nonlinear Eulerian Hydrodynamics in 3D, Lawrence Livermore National Laboratory, Livermore, CA, UCRL-JC-121122 ABS (1995). Prepared for the 37th Annual Mtg of the American Physical Society Div of Plasma Physics, Louisville, KY, Nov 6-10, 1995.

Stolz, C., Genin, F., Kozlowski, M., Lalazari, R., and Wu, Z., Influence of Microstructure on Laser Damage Threshold of IBS Coatings, Lawrence Livermore National Laboratory, Livermore, CA, UCRL-JC-121627 ABS (1995). Prepared for the Annual Symp on Optical Materials for High Power Lasers, Boulder, CO, Oct 30-Nov 1, 1995.
Stolz, C., Tench, R., Kozlowski, M., and Fornier, A., A Comparison of Nodular Defect Seed Geometries from Different Deposition Techniques, Lawrence Livermore National Laboratory, Livermore, CA, UCRL-JC-121628 ABS (1995). Prepared for the Annual Symp on Optical Materials for High Power Lasers, Boulder, CO, Oct 30-Nov 1, 1995.

Stone, G., Fabrication and Testing of Gas Filled Targets for Large Scale Plasma Experiments on Nova, Lawrence Livermore National Laboratory, Livermore, CA, UCRL-JC-119390 (1995). Submitted to Fus. Tech.

Stuart, B., Herman, S., and Perry, M. D., Chirped-Pulse Amplification in Ti-Sapphire Beyond $1 \mu \mathrm{m}$, Lawrence Livermore National Laboratory, Livermore, CA, UCRL-JC-115176 (1995); IEEE J. Quantum Electron. 31(3), 528-538 (1995).

Suter, L. J., Kauffman, R. L., Darrow, C. B., Hauer, A. A., Kornblum, H., Landen, O. L., Orzechowski, T. J., Phillion D. W., Porter, J. L., Powers, L. V., Rosen, M. D., and Thiessen, A. R., Radiation Drive in Laser Heated Hohlraums, Lawrence Livermore National Laboratory, Livermore, CA, UCRL-JC-121483 ABS (1995). Prepared for the 37th Annual Mtg of the American Physical Society Div of Plasma Physics, Louisville, KY, Nov 6-10, 1995.

Sutton, S. B., and Albrecht, G. F., Thermal Management in Inertial Fusion Energy Slab Amplifiers, Lawrence Livermore National Laboratory, Livermore, CA, UCRL-JC-120220 (1995). Prepared for the 1st Annual Int'l Conf on Solid-State Lasers for Application to Inertial Confinement Fusion, Monterey, CA, May 30-Jun 2, 1995.

\section{$\mathbf{T}$}

Tabak, M., Old and New Trick to Symmetrize Hohlraum Radiation, Lawrence Livermore National Laboratory, Livermore, CA, UCRL-JC-121474 ABS (1995). Prepared for the 37th Annual Mtg of the American Physical Society Div of Plasma Physics, Louisville, KY, Nov 6-10, 1995.

Tobin, M., Anderson, A., Diaz de la Rubia, T., Peterson, P., Peterson, B., and Perlado, M., Phenomenology of Chamber Responses to Target Emissions: NIF Experiments, Lawrence Livermore National Laboratory, Livermore, CA, UCRL-JC-121293 ABS (1995). Prepared for the 16th Institute for Electrical and Electronic Engineers/Nuclear and Plasma Sciences Society Symp on Fusion Engineering, Champaign, IL, Sept 30-Oct 5, 1995. 
Trebes, J. E., Barbee, T. W., Cauble, R., Celliers, P., Da Silva, L., Decker, C., London, R., Moreno, J. C., Ress, D., Wan, A. S., and Weber, F., Soft X-Ray Interferometry, Lawrence Livermore National Laboratory, Livermore, CA, UCRL-JC-121631 ABS (1995). Prepared for the Synchrotron Research and Instrumentation '95 Mtg of American Physical Society X-Ray Centennial Symp, Argonne, IL, Oct 18-20, 1995.

\section{V}

Van Wonterghem, B. M., Barker, C. E., Campbell, J. H., Caird, J. A., Murray, J. R., and Speck, D. R., Recent Results of the National Ignition Facility Beamlet Demonstration Project, Lawrence Livermore National Laboratory, Livermore, CA, UCRL-JC-120917 (1995). Prepared for the 1st Annual Int'l Conf on Solid-State Lasers for Application to Inertial Confinement Fusion, Monterey, CA, May 30-Jun 2, 1995.

Vu, B.-T. V., Landen, O. L., and Szoke, A., Time-Resolved Probing of Femtosecond-Laser-Produced Plasmas in Transparent Solids by Electron Thermal Transport, Lawrence Livermore National Laboratory, Livermore, CA, UCRL-JC-117456 (1995); Phys. Plasmas 2(2), 476485 (1995).

Vu, B.-T. V., Lee, R. W., Landen, O. L., and Price, D. F., Temporally and Radially-Resolved Femtosecond Optical Measurement of Solid Density Plasma Reflectivities and Transmissivities, Lawrence Livermore National Laboratory, Livermore, CA, UCRL-JC-118332 (1995); J. Quant. Spectros. \& Radiat. Transfer 54(1/2), 413-418 (1995).

\section{W}

Walton, C. C., Genin, F. Y., Chow, R., Kozlowski, M. R., Piece, E., and Loomis, G., Laser Damage Threshold Dependence on Overlayer Film Thickness for Non-normal Incidence Hafria-Silica High Reflectors, Lawrence Livermore National Laboratory, Livermore, CA, UCRL-JC-121638 ABS (1995). Prepared for the Annual Symp on Optical Materials for High Power Lasers, Boulder, CO, Oct 30-Nov 1, 1995.

Wang, P., Orzechowski, T. J., MacFarlane, J. J., and Moses, G. A., An Investigation of the Opacity of High-Z Mixtures and Implications for ICF Hohlraums Design, Lawrence Livermore National Laboratory, Livermore, CA, UCRL-JC-121500 ABS (1995). Prepared for the 37th Annual Mtg of the American Physical Society Div of Plasma Physics, Louisville, KY, Nov 6-10, 1995.
Warner, B. E., Boley, C. D., Chang, J. J., Dragon, E. P., Havstad, M. A., Martinez, M., and McLean, W., Ablative Material Removal Utilizing the Copper Vapor Laser, Lawrence Livermore National Laboratory, Livermore, CA, UCRL-JC-121130 (1995). Prepared for LEOS '95 8th Annual Mtg Institute of Electrical and Electronic Engineers, San Francisco, CA, Oct 30-Nov 2, 1995.

Weber, S. V., Dalhed, S., Eimerl, D., Key, M. H., and Verdon, C. P., Hydrodynamic Stability of NIF Direct Drive Capsules, Lawrence Livermore National Laboratory, Livermore, CA, UCRL-JC-121451 ABS (1995). Prepared for the 37th Annual Mtg of the American Physical Society Div of Plasma Physics, Louisville, KY, Nov 6-10, 1995.

Wilemski, G., and Wyslouzil, B. E., Binary Nuclear Kinetics 1. Self-Consistent Size Distribution, Lawrence Livermore National Laboratory, Livermore, CA, UCRL-JC-JC-118536 Pt 1 (1995); J. Chem. Phys. 103(3), 1127-1136 (1995).

Wilks, S. C., Introduction to Ultra-Intense, Short Pulse Laser-Plasma Interactions, Lawrence Livermore National Laboratory, Livermore, CA, UCRL-JC-121611 ABS (1995). Prepared for Plasma Physics and Technology, La Jolla, CA, Aug 8-18, 1995.

Wilks, S. C., Kruer, W. L., Denavit, J., Estabrook, K., Hinkel, D. E., Kalantar, D., Langdon, A. B.,

MacGowan, B. J., Montgomery, D. S., and Williams, E. A., Nonlinear Theory and Simulations of Stimulated Brillouin Backscatter in Multispecies Plasmas, Lawrence Livermore National Laboratory, Livermore, CA, UCRL-JC-117313; Phys. Rev. Lett. 74(25), 5048-5051 (1995).

Wilks, S. C., Kruer, W. L., Williams, E. A., and Amendt, P., Stimulated Raman Backscatter in Ultraintense, Short Pulse Laser Plasma Interactions, Lawrence Livermore National Laboratory, Livermore, CA, UCRL-JC-118111 (1994); Phys. Plasmas 2(1), 274-279 (1995).

Wilks, S. C., Kruer, W. L., Young, P. E., Tabak, M., Hammer, J., and Feit, M. D., Laser-Plasma Interactions Relevant to the Fast Ignitor, Lawrence Livermore National Laboratory, Livermore, CA, UCRL-JC-121478 ABS (1995). Prepared for the 37th Annual Mtg of the American Physical Society Div of Plasma Physics, Louisville, KY, Nov 6-10, 1995.

Wilks, S. C., Kruer, W., Hammer, J., Tabak, M., and Young, P., Fast Ignitor Plasma Physics Issues, Lawrence Livermore National Laboratory, Livermore, CA, UCRL-JC-120884 ABS Rev 1 (1995). Prepared for Plasma Physics and Technology, La Jolla, CA, Aug 8-18, 1995. 
Williams, E. A., and Hinkel, D. E., Laser Filamentation and Deflection in a Transverse Plasma Flow, Lawrence Livermore National Laboratory, Livermore, CA, UCRL-JC-121495 ABS (1995). Prepared for the 37th Annual Mtg of the American Physical Society Div of Plasma Physics, Louisville, KY, Nov 6-10, 1995.

Williams, E. A., Parametric Instabilities in Inertial Confinement Fusion: Basic Theory, Lawrence Livermore National Laboratory, Livermore, CA, UCRL-JC-121612 ABS (1995). Prepared for Plasma Physics and Technology, La Jolla, CA, Aug 8-18, 1995.

Wolfe, C. R., Lawson, J. K., Kellam, M., Maney, R. T., and Demiris, A., Measurement of Wavefront Structure from Large Aperture Optical Components by Phase Shifting Interferometry, Lawrence Livermore National Laboratory, Livermore, CA, UCRL-JC-121471 (1995). Prepared for the Society of Photo-Optical Instrumentation Engineers' 1995 Int'l Symp on Optical Science, Engineering, and Instrumentation, San Diego, CA, Jul 9-14, 1995.

Wood-Vasey, M., Remington, B. A., Kane, J., Arnett, D., Dearborn, D., Rubenchik, A., Glendinning, S. G., Budil, K. S., Castor, J., Woosley, S., Liang, E. P., and London, R., A Supernova-Relevant Hydrodynamic Instability Experiment on the Nova Laser, Lawrence Livermore National Laboratory, Livermore, CA, UCRL-JC-121488 ABS (1995). Prepared for the 37th Annual Mtg of the American Physical Society Div of Plasma Physics, Louisville, KY, Nov 6-10, 1995.

Woods, B., Runkel, M., Yan, M., De Yoreo, J., and Kozlowski, M., Optical Scatter as a Diagnostic Tool for Studying Bulk Defects Which Cause Laser Damage in Conventional and Rapid Growth KDP and DKDP, Lawrence Livermore National Laboratory, Livermore, CA, UCRL-JC-121652 (1995). Prepared for the Society of Photo-Optical Instrumentation Engineers San Diego 1995, San Diego, CA, Jul 10-14, 1995.

Wyslouzil, B. E., and Wilemski, G., Binary Nucleation Kinetic 2. Numerical Solution of the Birth-Death Equations, Lawrence Livermore National Laboratory, Livermore, CA, UCRL-JC-118536 (1995); J. Chem. Phys. 103(3), 1137-1151 (1995).

\section{$\mathbf{Y}$}

Yan, M., De Yoreo, J., Zaitseva, N., and Torres, R., Impurity Contamination in Fast Grown KDP, Lawrence Livermore National Laboratory, Livermore, CA, UCRL-JC-122004 ABS (1995). Prepared for the 1995 Boulder Damage Symp, Boulder, CO, Oct 30-Nov 1, 1995.
Yan, M., Rothberg, L., Galvin, M. E., Miller, T. M., and Padimitrakopoulos, F, Defects and Morphological Concerns in Electroluminescent Polymers, Lawrence Livermore National Laboratory, Livermore, CA, UCRL-JC-121620 (1995). Prepared for the 27th Int'l Society for the Advancement of Materials and Process Engineering, Albuquerque, NM, Oct 9-12, 1995.

Yang, T.-Y. B., Kruer, W. L., More, R. M., and Langdon, A. B., Absorption of Laser Light in Overdense Plasmas by Sheath Inverse Bremsstrahlung, Lawrence Livermore National Laboratory, Livermore, CA, UCRL-JC-119068 (1995); Phys. Plasmas 2(8), 3146-3154 (1995).

Yang, T.-Y. B., Kruer, W. L., and Langdon, A. B., Collisionless Absorption of Light Waves Incident on Overdense Plasmas with Steep Density Gradients, Lawrence Livermore National Laboratory, Livermore, CA, UCRL-JC-121624 (1995). Prepared for Plasma Physics and Technology, La Jolla, CA, Aug 8-18, 1995.

Yang, T.-Y. B., Kruer, W. L., and Langdon, A. B., Collisionless Absorption of Light Waves Obliquely Incident on Overdense Plasmas with Steep Density Gradients, Lawrence Livermore National Laboratory, Livermore, CA, UCRL-JC-121296 ABS (1995). Prepared for the 37 th Annual Mtg of the American Physical Society Div of Plasma Physics, Louisville, KY, Nov 6-10, 1995.

Young, P., Experimental Observation of Filamentation Growth in Laser-Produced Plasmas, Lawrence Livermore National Laboratory, Livermore, CA, UCRL-JC-119827 (1995); Phys. Plasmas 2(7), 2815-2824 (1995).

Young, P., Foord, M. E., Hammer, J. H., Kruer, W. L., Tabak, M., and Wilks, S. C., Time-Dependent Channel Formation in a Laser-Produced Plasma, Lawrence Livermore National Laboratory, Livermore, CA, UCRL-JC-120577 (1995); Phys. Rev. Lett. 75(8), 1082-1085 (1995).

Young, P., Hammer, J. H., Wilks, S. C., and Kruer, W. L., Laser Beam Propagation and Channel Formation in Underdense Plasmas, Lawrence Livermore National Laboratory, Livermore, CA, UCRL-JC-119828 (1995); Phys. Plasmas 2(7), 2825-2834 (1995). 